Final Report

FHWA/IN/JTRP-2001/5

\title{
A REAL-TIME OFFSET TRANSITIONING ALGORITHM FOR COORDINATING TRAFFIC SIGNALS
}

\author{
By \\ Darcy Bullock \\ Professor of Civil Engineering \\ Principal Investigator \\ and \\ Montasir M. Abbas \\ Research Assistant \\ School of Civil Engineering \\ Purdue University \\ Joint Transportation Research Program \\ Project No. C-36-37II \\ File No. 8-4-52 \\ SPR-2391 \\ Prepared in Cooperation with the \\ Indiana Department of Transportation and the \\ U.S. Department of Transportation \\ Federal Highway Administration
}

The contents of this report reflect the views of the authors who are responsible for the facts and the accuracy of the data presented herein. The contents do not necessarily reflect the official views of or policies of the funding agencies. This report does not constitute a standard, specification, or regulation.

Purdue University

West Lafayette, Indiana 47907

November 2001 
TECHNICAL REPORT STANDARD TITLE PAGE

\begin{tabular}{|c|c|c|}
\hline $\begin{array}{l}\text { 1. Report No. } \\
\text { FHWA/IN/JTRP-2001/5 }\end{array}$ & 2. Government Accession No. & 3. Recipient's Catalog No. \\
\hline \multirow{2}{*}{\multicolumn{2}{|c|}{$\begin{array}{l}\text { 4. Title and Subtitle } \\
\text { A Real-Time Offset Transitioning Algorithm for Coordinating Traffic Signals }\end{array}$}} & $\begin{array}{l}\text { 5. Report Date } \\
\text { November } 2001\end{array}$ \\
\hline & & 6. Performing Organization Code \\
\hline \multicolumn{2}{|l|}{$\begin{array}{l}\text { 7. Author(s) } \\
\text { Darcy Bullock and Montasir Abbas }\end{array}$} & $\begin{array}{l}\text { 8. Performing Organization Report No. } \\
\text { FHWA/IN/JTRP-2001/5 }\end{array}$ \\
\hline \multirow{2}{*}{\multicolumn{2}{|c|}{$\begin{array}{l}\text { 9. Performing Organization Name and Address } \\
\text { Joint Transportation Research Program } \\
1284 \text { Civil Engineering Building } \\
\text { Purdue University } \\
\text { West Lafayette, Indiana 47907-1284 }\end{array}$}} & 10. Work Unit No. \\
\hline & & $\begin{array}{l}\text { 11. Contract or Grant No. } \\
\text { SPR-2391 }\end{array}$ \\
\hline \multirow{2}{*}{\multicolumn{2}{|c|}{$\begin{array}{l}\text { 12. Sponsoring Agency Name and Address } \\
\text { Indiana Department of Transportation } \\
\text { State Office Building } \\
100 \text { North Senate Avenue } \\
\text { Indianapolis. IN } 46204\end{array}$}} & $\begin{array}{l}\text { 13. Type of Report and Period Covered } \\
\text { Final Report }\end{array}$ \\
\hline & & 14. Sponsoring Agency Code \\
\hline \multicolumn{3}{|c|}{$\begin{array}{l}\text { 15. Supplementary Notes } \\
\text { Prepared in cooperation with the Indiana Department of Transportation and Federal Highway Administration. }\end{array}$} \\
\hline \multicolumn{3}{|c|}{$\begin{array}{l}\text { This report introduces an adaptive real-time offset transitioning algorithm that can be viewed as an integrated optimization approach } \\
\text { designed to work with traditional coordinated-actuated systems. The Purdue Real-Time Offset Transitioning Algorithm for Coordinating } \\
\text { Traffic Signals (PRO-TRACTS) adds to the controllers the ability to adaptively change their offsets in response to changes in traffic } \\
\text { pattern, providing an intermediate solution between traditional coordinated-actuated control systems and adaptive control systems. To } \\
\text { facilitate implementation, a new National Transportation Communication for ITS Protocol (NTCIP) object for capturing detector } \\
\text { actuation at the controller's level is defined in this report. The unique cycle-based tabulation of volume and occupancy profiles at } \\
\text { upstream detectors is used by a newly defined metric to examine the existence of shockwaves generated due to a poor offset downstream. } \\
\text { The procedure is modeled after the analysis of variance testing. This procedure is performed on cycle-by-cycle basis to evaluate the offset } \\
\text { performance and adjust it accordingly. Simulations of two case studies revealed 0-16\% savings in total travel time and up to } 44 \% \text { saving } \\
\text { in total number of stops for the coordinated movement when applied to systems with poor offsets. The algorithm is best suited for arterials } \\
\text { with primarily through traffic. Heavy movements from the side streets onto the arterial make it difficult for the algorithm to determine } \\
\text { which movement should be favored. PRO-TRACTS mitigates problems such as early-return-to-green, waiting queues, and improperly } \\
\text { designed offsets using current setups of traffic signals/detectors in the US. The algorithm capitalizes on the existing knowledge and } \\
\text { familiarity of traffic engineers and personnel with the current actuated control system to provide a cost-effective solution to improving } \\
\text { signal coordination. Future research is needed to improve the stability of the algorithm with highly dispersed platoons and oscillatory } \\
\text { traffic patterns caused by situations such as controllers skipping phases due to light traffic volume. It is also recommended that the } \\
\text { algorithm should be extended to improve two-way signal progression instead of one-way progression. }\end{array}$} \\
\hline
\end{tabular}

Offset Tuning, Traffic Signal Coordination, CoordinatedActuated Operation, NTCIP, ITS, Shockwave, PRO-TRACTS, Purdue, Real-Time, Platoon, CORSIM, Case Study.

No restrictions. This document is available to the public through the National Technical Information Service, Springfield, VA 22161

\begin{tabular}{|c|c|c|c|}
\hline $\begin{array}{c}\text { 19. Security Classif. (of this report) } \\
\text { Unclassified }\end{array}$ & $\begin{array}{c}\text { 20. Security Classif. (of this page) } \\
\text { Unclassified }\end{array}$ & 21. No. of Pages \\
\end{tabular}




\section{TECHNICAL Summary}

INDOT Research

Technology Transfer and Project Implementation Information

TRB Subject Code: 54-5 Traffic Control Devices

Publication No.: FHWA/IN/JTRP-2001/5, SPR-2391

November 2001

Final Report

\section{A REAL-TIME OFFSET TRANSITIONING ALGORITHM FOR COORDINATING TRAFFIC SIGNALS}

\section{Introduction}

Over the last few decades, extensive efforts have been made in the development of adaptive and responsive traffic control systems. The United States Department of Transportation (US DOT) has sponsored a great amount of research and field deployment of new traffic adaptive algorithms, such as OPAC and RHODES. Internationally, systems like SCOOT and SCATS, developed in the United Kingdom and Australia respectively, have seen increasing deployments. These adaptive control systems are based on applying specific proprietary software to a signal control system. It is estimated that travel time savings from these adaptive systems could range between $8 \%$ and $25 \%$.

Adaptive control systems typically require an extensive input of system parameters and weighting factors for favoring individual movements plus a large number of vehicle detectors to collect movement-specific traffic data. A major drawback of these systems is the extensive effort required for training personnel on the new proprietary architecture. Operation and maintenance of these systems can also be very costly and time consuming.

In a parallel track, the private sector has developed closed loop systems that are operated by coordinated-actuated controllers. These systems provide actuated control capabilities through their ability to respond to cycle-by-cycle variation in traffic demand while still being able to provide progression for the arterial movement. Unfortunately though, the quality of vehicle progression depends on both the initial setting of offsets and the stochastic nature of traffic on individual intersections, causing waiting queues and what is known as the early-return-to-green phenomenon.

Expanding the control logic for modern coordinated- actuated systems to account for problems such as early-return-to-green, waiting queues, and improperly designed offsets would address many of the day to day problems associated with closed loop systems. Additional training would be minimized, in comparison to fully adaptive systems, since traffic engineers and technicians managing traffic signal operating systems are already familiar with coordinatedactuated logic. Fundamental concepts and communication systems for coordinated-actuated systems would also remain the same. Additionally, extra cost would be kept to a minimum since no extra detectors or infrastructure would be required.

The objective of this research is to introduce an adaptive real-time offset transitioning expansion to actuated controllers to improve coordination of traffic signals. This algorithm, the Purdue Real-time Offset Transitioning Algorithm for Coordinating Traffic Signals (PRO-TRACTS) is designed to mitigate the effect of early-returnto-green problems experienced with coordinatedactuated controllers and accounts for downstream vehicle queues that may impede vehicle progression. PRO-TRACTS fine-tunes the offsets at signalized intersections continuously to improve progression regardless of the offsets' initial values. The algorithm presented in this report works with both the internal model of the CORSIM simulation package and actual NTCIP controllers. 


\section{Findings}

The report introduced a new metric by which offset performance can be evaluated. The new metric, namely the F' model, makes use of the fact that bad offsets at downstream intersections generate backward shockwaves that can extend to the upstream detector location. The metric developed in this research tests for the significance of the presence of shockwaves by calculating a proxy to the variance of the reciprocal of the speed at the upstream detector. The philosophy behind this procedure is that a large variance in speed suggests that a shockwave has a significant effect on the traffic flow. This procedure is modeled after the analysis of variance $\mathrm{F}$ distribution testing. Discriminant analysis is used to develop thresholds to which the F' value is compared to determine the amount of offset adjustment needed.

A new NTCIP object for capturing a cyclebased detector actuation profile at the controller level was defined in this report. The defined object will enable the implementation of PROTRACTS in the field by allowing real-time exchange of the data required to fine-tune the offsets.

It was found that in some situations, PROTRACTS caused an increase in total network delay compared to system-optimum timings when activated with optimum timing plans. These results were not surprising since favoring a directional movement is an additional constraint in the system optimization function. When enforcing a constraint such as favoring a certain traffic movement, the best that can be achieved in the overall system objective function is to keep it constant. However, if the current timing plans were not the system optimum plans, PROTRACTS can result in a reduction in total system delay as well.

Adjusting offsets in real-time caused some stability problems when PRO-TRACTS was run with CORSIM's internal control model. A hardware-in-the-loop simulations of the US 31 traffic network in Kokomo, Indiana revealed that field traffic controllers are more stable than CORSIM's internal model in responding to realtime offset adjustment.

The platooning phenomenon has an effect on all coordination schemes since platoon dispersion can make it impossible to fit the whole platoon within a downstream green window. The platooning phenomenon was found to have significant impacts on the performance of PROTRACTS. When the traffic turning from the side streets is a low percentage compared to the arterial's through-movement, a good coordination scheme would align the arterial through-movement with the green window, leaving the turning traffic from the side streets to face the red window. This situation causes only a weak shockwave resulting from the turning traffic compared to the strong shockwave that results from a bad offset that align the platoon with the red window. However, if the side street traffic volume approaches that of the arterial's through, even the turning traffic from the side streets will generate a strong shockwave that could cause PRO-TRACTS activation.

The ideal location for a PRO-TRACTS detector is between the termination of the shockwave generated by the side street turning traffic and the shockwave generated by the arterial's traffic. The algorithm is best suited for arterials with primarily through-traffic since a wider separation is provided between the two shockwaves.

Another factor that affected the stability of PRO-TRACTS was the frequency of phase skips and oscillatory traffic patterns caused by certain situations, such as spillbacks or lane blockages. Since PRO-TRACTS is a reactive algorithm, it assumes that the next cycle's arrivals are comparable to the current cycle's arrivals. With phase skips, the discrepancy in arrival patterns of succeeding cycles causes PRO-TRACTS offsets to fluctuate, reducing its efficiency. Future research should address this issue.

\section{Implementation}

Two simulation case studies were conducted on two traffic networks: SR 26 in Lafayette, Indiana and US31 in Kokomo, Indiana. The studies showed that for a network with poor or sub-optimal offsets, PRO-TRACTS consistently resulted in an improvement in both the travel time and the number of stops in the arterial direction to which it was applied, with a greater impact in reducing the number of stops. In the SR26 case study, PRO-TRACTS achieved a performance level close to that of Synchro's timing plans, while it achieved a better 
performance than Synchro's plans in the US 31 case study. The magnitude of such an improvement depends on the existing performance of the network offsets. Obviously, if there is only minor room for improvement, one can only expect that much. On the other hand, when starting with a system with poor offsets, computer simulations revealed up to $16 \%$ savings in the total travel time and up to $43 \%$ savings in the total number of stops for the coordinated movement.
The case studies investigated in this research found that PRO-TRACTS has always caused an improvement in the direction to which it was applied. However, the impact on the other direction was typically an increase in the travel time. Although coordination usually takes place during the peak period when traffic is typically at its highest in one direction, there might be situations when the two direction of traffic are almost equal, in which a case, it is difficult to decide which direction to coordinate. Future research should also consider this situation by expanding the algorithm to work in two directions.

\section{Contacts}

\section{For more information:}

\author{
Prof. Andrzej Tarko \\ Principal Investigator \\ Purdue University \\ 1284 Civil Engineering Building \\ West Lafayette, IN 47907-1284 \\ Phone: 765-494-5027 \\ Fax: $765-496-1105$
}

\author{
Prof. Darcy Bullock \\ Principal Investigator \\ Purdue University \\ 1284 Civil Engineering Building \\ West Lafayette, IN 47907-1284 \\ Phone: 765-494-2226 \\ Fax: $\quad 765-496-1105$
}

\section{Indiana Department of Transportation}

Division of Research

1205 Montgomery Street

P.O. Box 2279

West Lafayette, IN 47906

Phone: 765-463-1521

Fax: 765-497-1665

\section{Purdue University}

Joint Transportation Research Program School of Civil Engineering

West Lafayette, IN 47907-1284

Phone: 765-494-9310

Fax: 765-496-1105 


\section{Implementation Report}

Over the last few decades, extensive efforts have been made in the development of adaptive and responsive traffic control systems. The Federal Highway Administration has estimated that travel timesavings from these adaptive systems could range between $8 \%$ and $25 \%$. However, adaptive control systems typically require an extensive input of system parameters and weighting factors for favoring individual movements plus a large number of vehicle detectors to collect movementspecific traffic data. A major drawback of these systems is the extensive effort required for training personnel on the new proprietary architecture. Operation and maintenance of these systems can also be very costly and time consuming. Consequently, deployment of these fully adaptive systems is not very likely in Indiana.

In a parallel track, the private sector has developed closed loop systems that are operated by coordinated-actuated controllers. These systems are widely used in Indiana. These systems provide actuated control capabilities through their ability to respond to cycle-by-cycle variation in traffic demand while still being able to provide progression for the arterial movement. Unfortunately though, the quality of vehicle progression depends on both the initial setting of offsets and the stochastic nature of traffic on individual intersections, causing waiting queues and what is known as the early-return-to-green phenomenon. It takes a significant amount of man power to continually retime these systems.

Expanding the control logic for modern coordinated- actuated systems to account for problems such as early-return-to-green, waiting queues, and improperly designed offsets would address many of the day to day problems associated with closed loop systems. Additional training would be minimized, in comparison to fully adaptive systems, since traffic engineers and technicians managing traffic signal operating systems are already familiar with coordinated-actuated logic. Fundamental concepts and communication systems for coordinated-actuated systems would also remain the same. Additionally, extra cost would be kept to a minimum since no extra detectors or infrastructure would be required.

The objective of this research was to evaluate an adaptive real-time offset transitioning expansion to actuated controllers to improve coordination of traffic signals. This algorithm, the Purdue Real-time Offset Transitioning Algorithm for Coordinating Traffic Signals (PROTRACTS) was designed to mitigate the effect of early-return-to-green problems experienced with coordinated-actuated controllers and accounts for downstream vehicle queues that may impede vehicle progression (Figures 5-3 and 5-4). PRO-TRACTS fine-tunes the offsets at signalized intersections continuously to improve progression regardless of the offsets' initial values. The US 31 corridor in Kokomo, IN was selected for evaluating the feasibility of this algorithm since this corridor had INDOT funding programmed for improving the traffic signal system. The generality of the algorithm was also evaluated by applying it to the SR 26 corridor in Lafayette, IN.

In order to ensure that this algorithm could be directly implemented on any controller, the algorithm was implemented using the National Transportation Communications For ITS Protocol (NTCIP) and new data objects were defined with the framework of the NEMA TS 3.5 MIB. The 
algorithm presented in this report was evaluated using both CORSIM controller models, and Econolite Controllers running the NTCIP Protocol.

For the SR 26 evaluation, the algorithm performance was as almost as good as offsets generated with Synchro. For the US 31 corridor, the algorithm performed similar to Synchro for the morning peak, but reduced delay between $1 \%$ and $11 \%$ (Table 11-1).

Although this algorithm shows considerable promise because offsets fine tune themselves automatically, it is not clear that these relatively modest benefits warrant the cost of a full scale implementation in Indiana at this point. 


\section{TABLE OF CONTENT}

Page

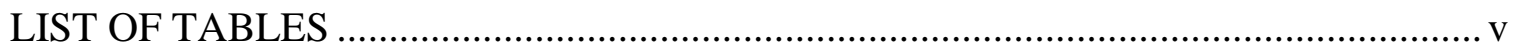

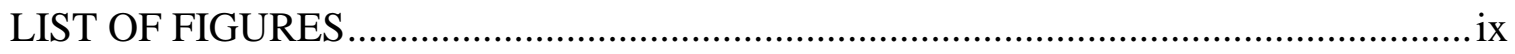

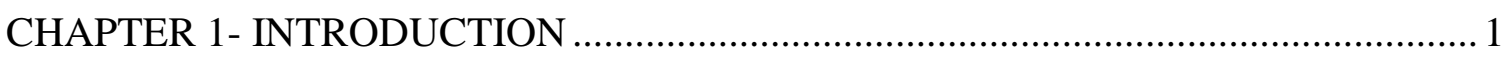

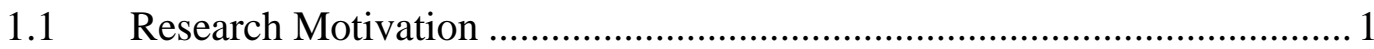

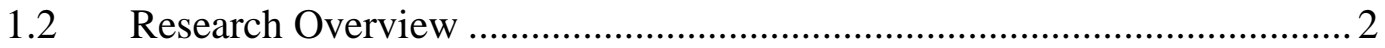

CHAPTER 2- LITERATURE REVIEW: CURRENT STATE OF TRAFFIC CONTROL

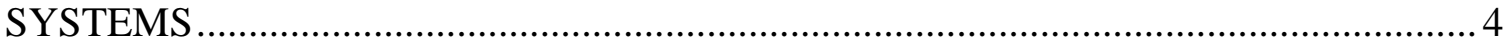

2.1 A Historical Perspective of Traffic Control Systems.................................. 4

2.1.1 Microprocessor-Based Controllers................................................. 5

2.1.2 Model 170 Controllers ................................................................ 5

2.1.3 Advanced Transportation Controller (ATC) …………………........ 6

2.1.4 Urban Traffic Control Software (UTCS) ...................................... 6

2.2 Adaptive Control Systems and Algorithms............................................... 7

2.2.1 History of Adaptive Control Systems ……………......................... 7

2.2.2 Adaptive Control Algorithms ........................................................ 8

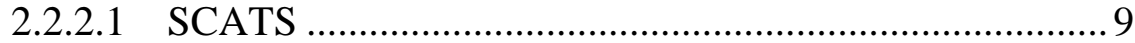

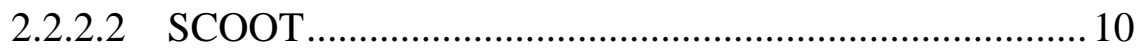

2.2.2.3 Optimized Policies for Adaptive Control (OPAC) ......... 11

2.2.2.4 Real-Time, Hierarchical, Optimized, Distributed, and

Effective System (RHODES) ..................................................... 12

2.3 Actuated Traffic Control Systems ......................................................... 15

2.3.1 Actuated Control Parameters ....................................................... 15

2.3.2 Coordinated-Actuated Control .................................................. 18

2.4 Current Signal Timing Procedures .........................................................2 23

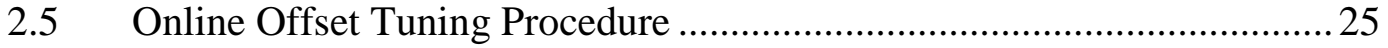

2.6 Definition of Adaptive Offset Algorithm Opportunity ……...................... 26

CHAPTER 3- COMPARATIVE STUDY OF THEORETICAL, SIMULATION, AND

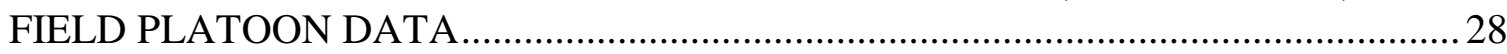

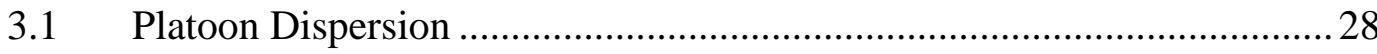

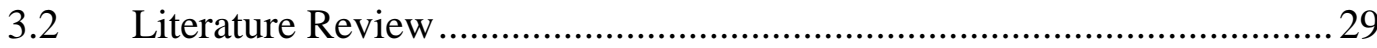

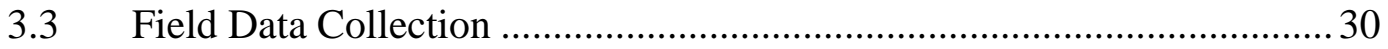




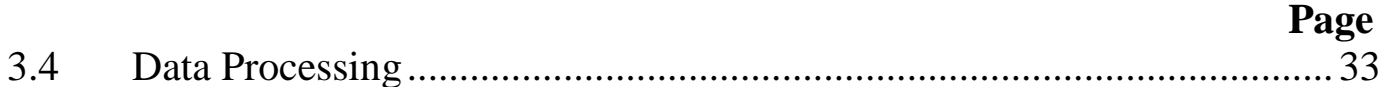

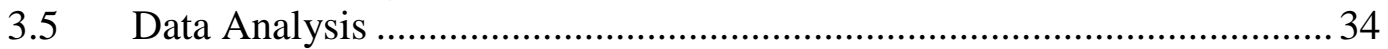

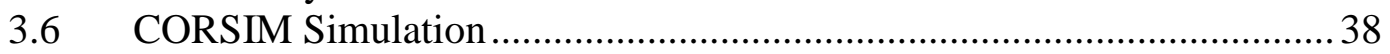

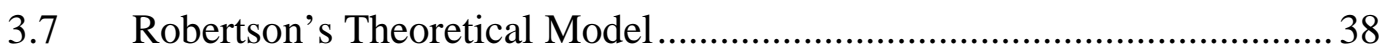

3.8 Determination of Green Windows for Particular Percentage of Platoons 41

3.9 Plots of Green Windows Required by Different Percentages of the Platoon 42

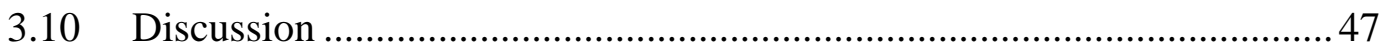

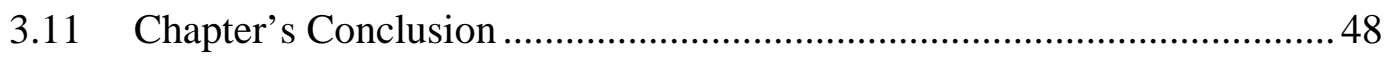

CHAPTER 4- CALIBRATION OF CORSIM PLATOON DISPERSION MODEL....... 50

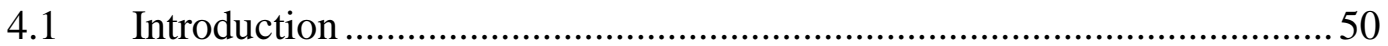

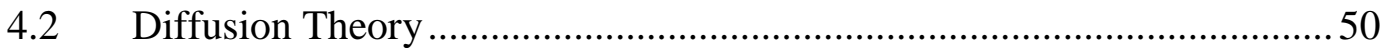

4.3 Calculation of Dispersion Factor from Speed Study...............................51

4.4 Calculation of Dispersion Factor Using Pacey's Model ..........................52

4.5 Conversion of the Normal Distribution Into A Decile Distribution...........53

4.6 Effect of Platoon Dispersion on Signal Coordination Benefits.................56

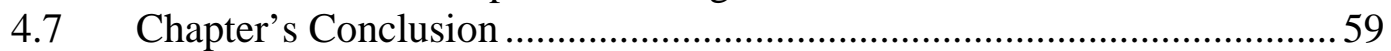

CHAPTER 5- RELATIONSHIP BETWEEN OFFSETS AND SHOCKWAVES ..........61

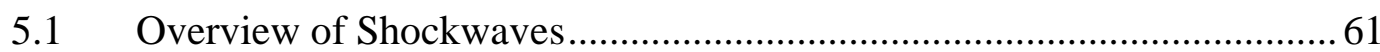

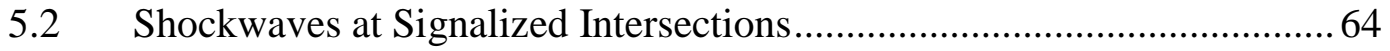

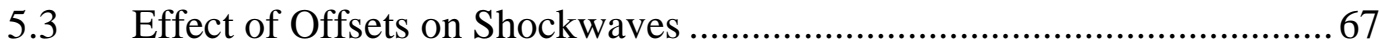

5.4 Effect of High Turning Percentage on Shockwave Formation .................. 73

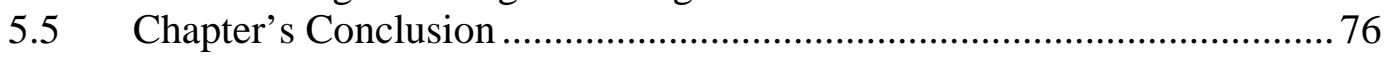

CHAPTER 6- PRO-TRACTS CONCEPT AND STRUCTURE.................................... 77

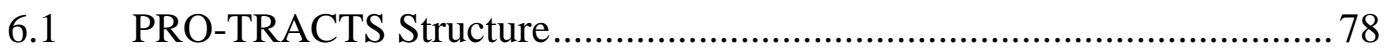

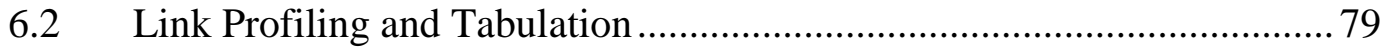

6.3 PRO-TRACTS Concept: The Skewness Model....................................... 86

6.3.1 The need for an Activation Mechanism ......................................8 88

6.3.2 Modified Skewness Functions .................................................... 94

6.3.3 Calculation of Skewness Around the Count Profile's Mean....... 102

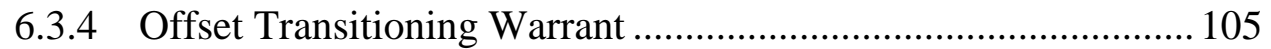

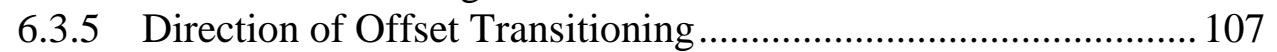

6.3.6 Network Level Coordination................................................... 111

6.3.7 Effect of Detector Location..................................................... 111

6.4 Evaluation Network and Procedure..................................................... 111

6.4.1 Adapting from Incorrect Offsets ........................................... 115

6.4.2 Adjusting Offsets to Adapt for Early-Return-to-Green............... 121

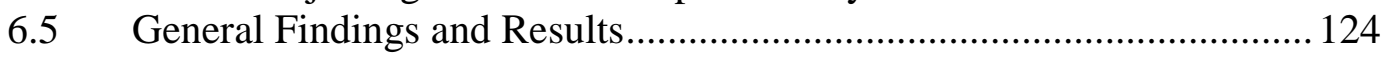

CHAPTER 7- THE F' MODEL AND DISCRIMINANT ANALYSIS ....................... 125

7.1 Limitation of the Skewness Model.................................................. 125

7.1.1 False Alarms with the Skewness Model ................................. 126 
7.1.2 Singularity Cases

7.2 Factors Affecting Offset Performance

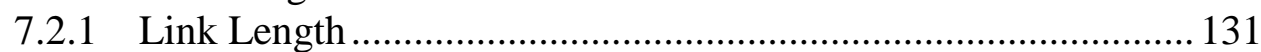

7.2.2 Cycle Length/Green Split..........................................................133

7.2.3 Traffic Volumes ..................................................................... 134

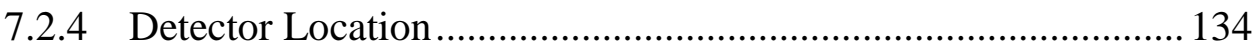

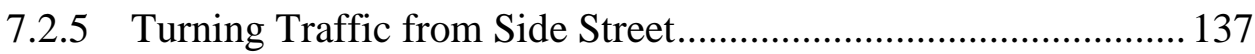

7.3 Design of Experiment and Statistical Testing .......................................... 137

7.3.1 Design of Experiment................................................................. 137

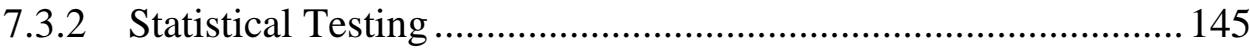

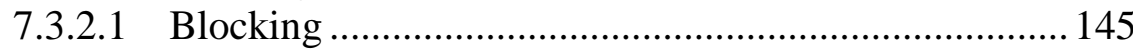

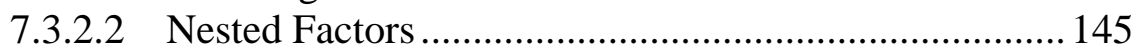

7.3.2.3 Split-Plot Factorial Design ............................................ 146

7.3.3 Expected Mean Squares and Testing for Significance................. 147

$7.4 \quad$ The F' Model...................................................................................... 157

7.4.1 ANOVA Results of the F' Model .................................................. 160

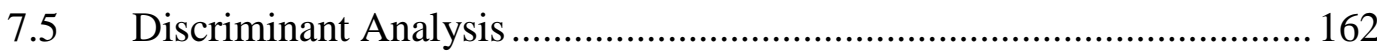

7.5.1 Overview of Discriminant Analysis ............................................ 163

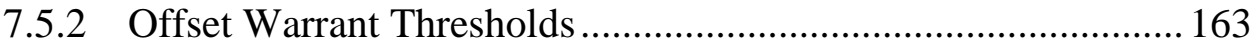

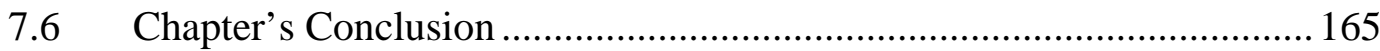

CHAPTER 8- CASE STUDY: SR 26, LAFAYETTE, INDIANA.................................... 167

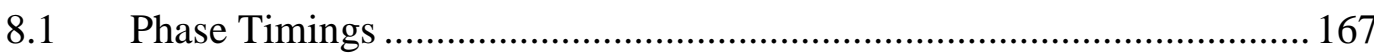

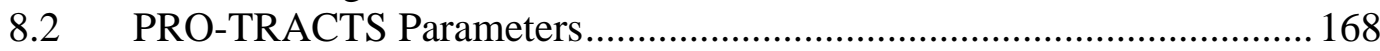

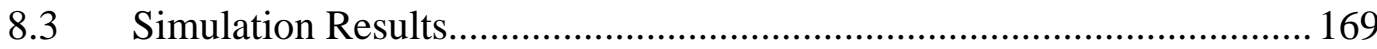

8.3.1 A.M. Travel Time, Stops and Delay ………………………….... 170

8.3.2 A.M. Offset and Cycle Transitioning.......................................... 177

8.3.3 P.M. Travel Time, Stops and Delay ............................................. 184

8.3.4 P.M. Offset and Cycle Transitioning .......................................... 190

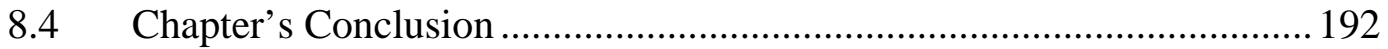

CHAPTER 9- HARDWARE-IN-THE-LOOP SIMULATION WITH NTCIP

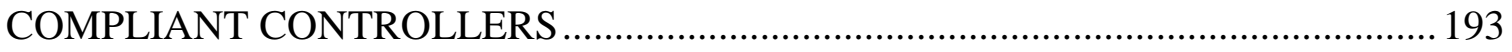

9.1 Overview of Hardware-in-the-Loop Simulation ........................................ 194

9.1.1 Motivation of Hardware-in-the-Loop Simulation ......................... 194

9.1.2 Basic Concepts of Hardware-in-the-Loop Simulation .................. 195

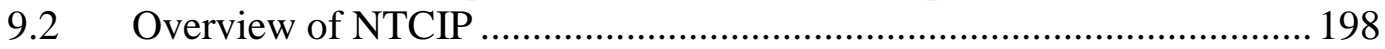

9.2.1 NTCIP Protocols for Center to Field Communications ……....... 202

9.2.2 Application Message Bits and Bytes...........................................2203

9.3 Hardware-in-the-Loop Simulation with NTCIP Offset Download......... 206

$9.4 \quad$ Proposed NTCIP Object.......................................................................2 211

9.4.1 Proposed Field Implementation …………………………….....211 
9.4.2 NTCIP Object Definitions for PRO-TRACTS Application Support 213

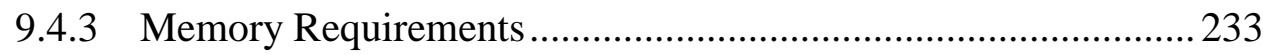

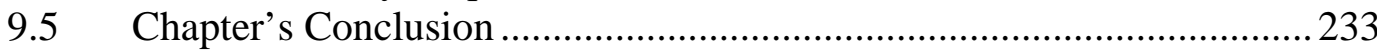

CHAPTER 10- CASE STUDY: US31, KOKOMO, INDIANA ……………………......235

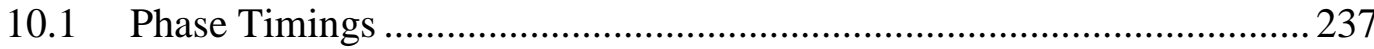

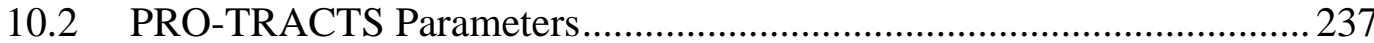

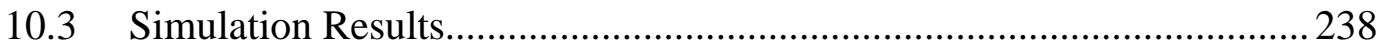

10.3.1 A.M. Travel Time, Stops, and Delay …………….................... 242

10.3.1.1 CORSIM's Internal Model Simulation ........................2242

10.3.1.2 Hardware-in-the-Loop Simulation ...............................246

10.3.2 A.M. Offset and Cycle Transitioning .......................................2.250

10.3.3 P.M. Travel Time, Stops, and Delay ………………………......2. 253

10.3.4 P.M. Offset and Cycle Transitioning ........................................2.260

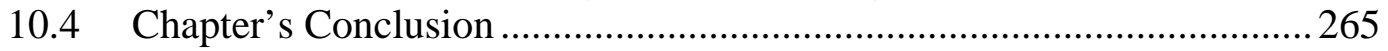

CHAPTER 11- CONCLUSION AND FUTURE RESEARCH ………………….......... 266

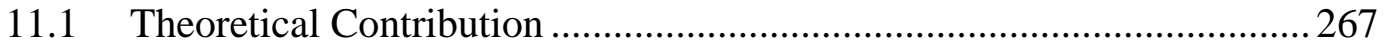

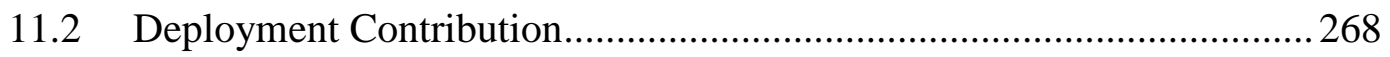

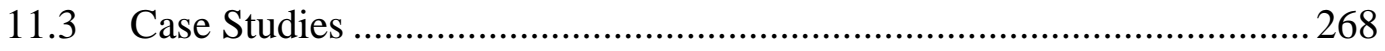

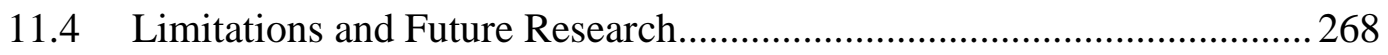

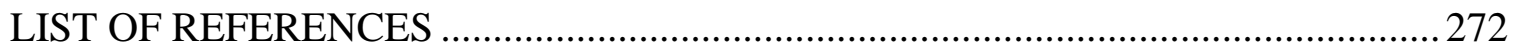

Appendix A- SR 26 Study Network and Controller Parameters..........................2278

APPENDIX B- US 31 NETWORK and CONTROLLER PARAMETERS .......291

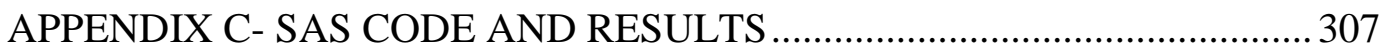

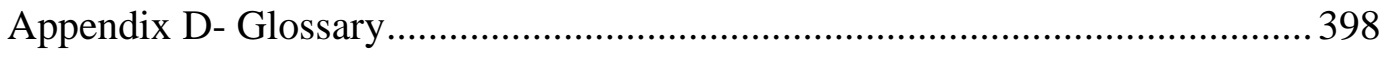




\section{LIST OF TABLES}

\section{Table}

Table 2-1 Common Actuated Traffic Controller Parameters [Source: Econolite, 1996].. 18

Table 3-1Number of Data Points Collected at Each Distance for Varying Speeds and Initial Discharge 32

Table 3-2 Regression Analysis Coefficients for Field Data, CORSIM Simulation, and Robertson's Model

Table 4-1 $\beta$ Parameter Values for Computing the Decile Distribution-UFPCT(I) ............55

Table 6-1 Sample Occupancy Actuation.........................................................................96

Table 6-2 Descriptive Statistics for Occupancy in Table 6-1 Obtained by Direct

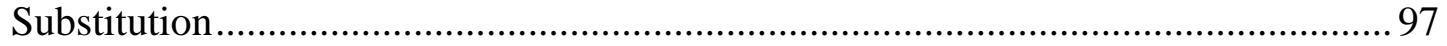

Table 6-3 Repetition of Bin Values According to Occupancy Value in........................... 99

Table 6-4 A histogram analysis for bin values in Table 6-3 ……………………........... 99

Table 6-5 Descriptive Statistics for Bin Values in Table 6-3 …...................................... 100

Table 6-6 Test Bed General Phase Parameters (All Intersections) ................................... 114

Table 6-7 Test Bed Coordination Parameters ................................................................... 114

Table 6-8 Test Bed Entry Volumes per Period (VPH)................................................... 115

Table 7-1 Offset Values for 3000 Feet Link- 90 Seconds Cycle ....................................139

Table 7-2 Offset Values for 3000 Feet Link- 120 Seconds Cycle .................................. 140

Table 7-3 Offset Values for 3000 Feet Link- 150 Seconds Cycle .................................... 141

Table 7-4 Offset Values for 1000 feet Link-90 Seconds Cycle ....................................... 142

Table 7-5 Offset Values for 1000 Feet Link-120 Seconds Cycle ..................................... 143

Table 7-6 Offset Values for 1000 Feet Link-150 Seconds Cycle .................................... 144

Table 7-7 Expected Mean Square Derivation for Nested Split-Plot Design (Table 1 of 6) 
Table

Table 7-8 Expected Mean Square Derivation for Nested Split-Plot Design (Table 2 of 6)

Table 7-9 Expected Mean Square Derivation for Nested Split-Plot Design (Table 3 of 6) 150

Table 7-10 Expected Mean Square Derivation for Nested Split-Plot Design (Table 4 of 6)

Table 7-11 Expected Mean Square Derivation for Nested Split-Plot Design (Table 5 of 6)

Table 7-12 Expected Mean Square Derivation for Nested Split-Plot Design (Table 6 of 6)

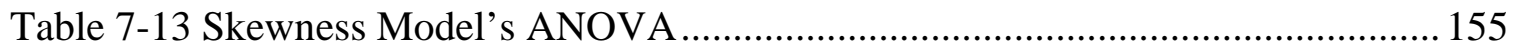

Table 7-14 Offset Volume Interactions for the Skewness Model................................ 156

Table 7-15 Offset Turn Interaction for the Skewness Model...................................... 157

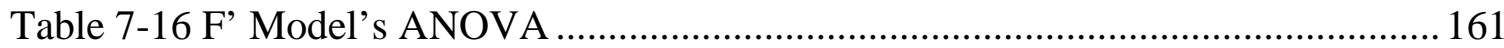

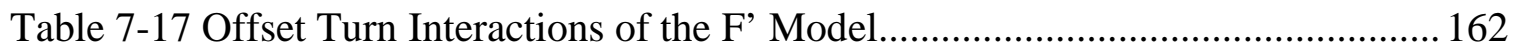

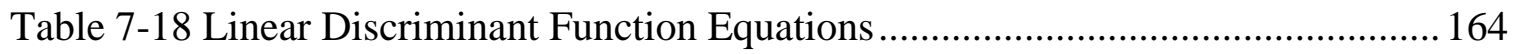

Table 8-1 SR 26 (South) Coordination Plans and Splits ............................................ 168

Table 8-2 SR 26 (South) Entry Volumes per Period (VPH) ....................................... 170

Table 8-3 SR 26 (South) Turning Movements (Percentages - Table 1 of 2)................. 180

Table 8-4 SR 26 (South) Turning Movements (Percentages -Table 2 of 2)................. 181

Table 9-1 TEA21 Requirements for Conformity with National Architecture [Source:

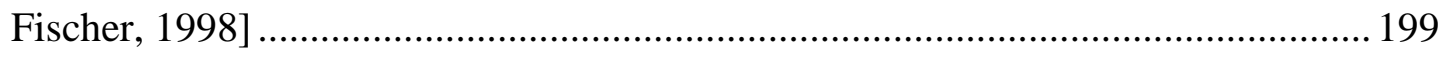

Table 9-2 TEA21 Requirements for NTCIP [Source: NEMA, 1999A] ......................... 200

Table 9-3 SNMP and STMP Comparison [Source: NEMA, 1999A] …....................... 203

Table 9-4 Example NTCIP message for Download an Offset Change of 10 Seconds to

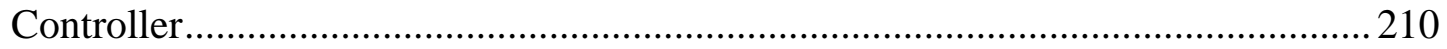

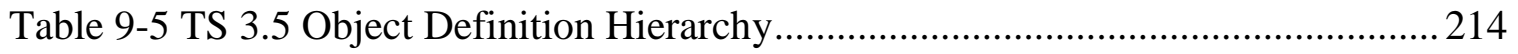

Table 9-6 Table of Tables for New Object's ASN Definitions and Example Data........214

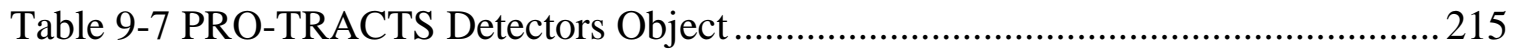

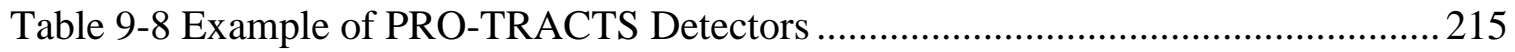


Table 9-9 PRO-TRACTS Physical Channel Table

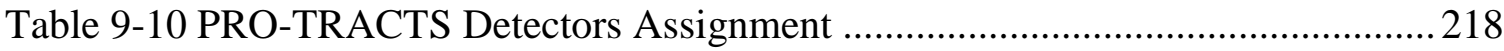

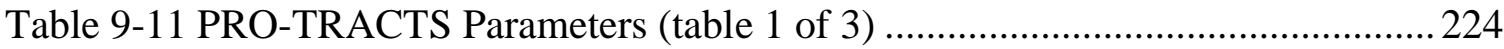

Table 9-12 PRO-TRACTS Parameters (table 2 of 3 ) ................................................... 225

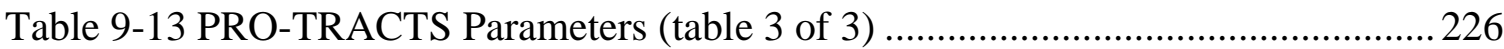

Table 9-14 Example of PRO-TRACTS Parameters...................................................227

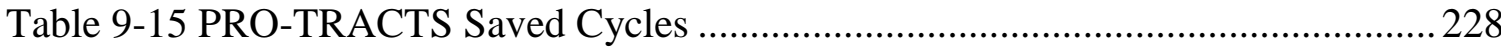

Table 9-16 Example of PRO-TRACTS Saved Cycles..................................................2228

Table 9-17 PRO-TRACTS Occupancy Table .......................................................... 230

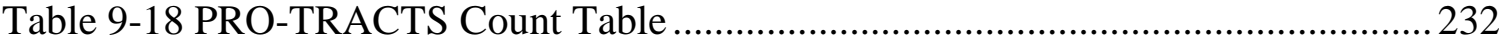

Table 9-19 Example Memory Requirements ...............................................................233

Table 10-1 US 31 Coordination Plans and Splits....................................................... 237

Table 10-2 US 31 Detailed Traffic Volume (vph Table 1 of 3) ....................................239

Table 10-3 US 31 Detailed Traffic Volume (vph Table 2 of 3) ...................................240

Table 10-4 US 31 Detailed Traffic Volume (vph Table 3 of 3)..................................241

Table 11-1 Summary of PRO-TRACTS Improvements Using Internal and Hardware-in-

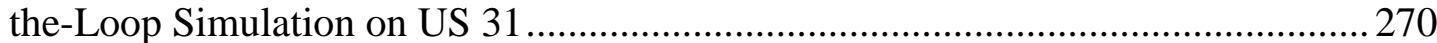

\section{Appendix Tables}

Table A- 1 Intersection Pocket Lengths and Detector Locations................................. 284

Table A- 2 Intersection Detector Configuration (CID Logic Only)...........................2285

Table A- 3 General Phase Parameters (SR 26 - South) ............................................. 286

Table A- 4 SR 26 (South) Coordination Plans and Splits .......................................... 287

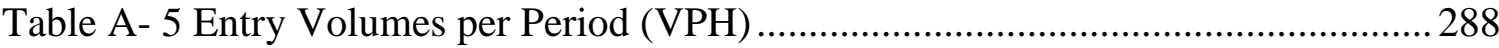

Table A- 6 Turning Movements (Percentages - Table 1 of 2)...................................289

Table A- 7 Turning Movements (Percentages -Table 2 of 2)..................................... 290

Table B- 1 Intersection Pocket Lengths and Detector Locations (Table 1 of 2)............ 299

Table B- 2 Intersection Pocket Lengths and Detector Locations (Table 2 of 2)............ 300

Table B- 3 Intersection Detector Configuration (CID Logic Only) ............................. 301

Table B- 4 General Phase Parameters (US 31) ............................................................. 302 
Table

Page

Table B- 5 US 31 Coordination Plans and Splits ....................................................... 303

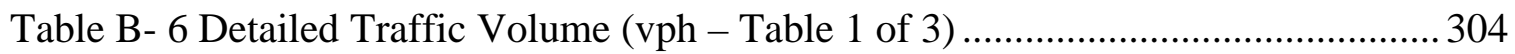

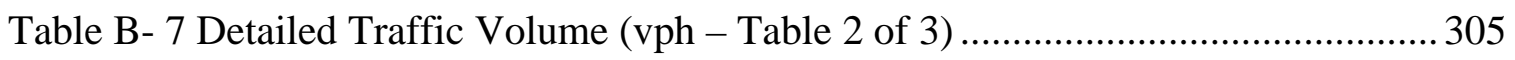

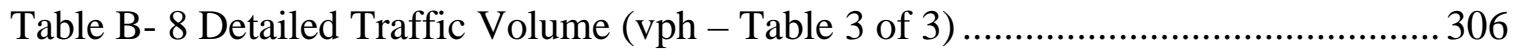




\section{LIST OF FIGURES}

\section{Figure}

Figure 2-1 Hierarchy Framework of RHODES [Source: Head et al, 1992]. ................... 14

Figure 2-2 Typical Dual Ring Structure for Coordinated Actuated Operation................. 18

Figure 2-3 Coordinated-actuated ring structure [Source: Shoup, 1998] .........................21

Figure 2-4 Coordinated-actuated time-space diagrams [Source: Shoup, 1998]................ 22

Figure 2-5 NAZTEC Algorithm [Source: Black, 1998] ........................................... 26

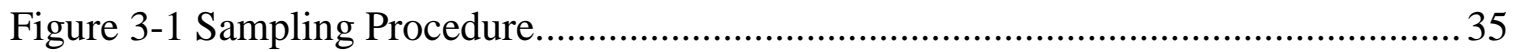

Figure 3-2 Platoon Extraction Algorithm ............................................................ 37

Figure 3-3 Flow Profiles of Field Data and as Predicted by Robertson's Model ............ 40

Figure 3-4 Field Data Platoon Distribution (20 Second Saturated Platoon) .................... 44

Figure 3-5 CORSIM Simulation Platoon Distribution (20 Second Saturated Platoon) .... 45

Figure 3-6 Robertson's Model Platoon Distribution (20 Second Saturated Platoon) ....... 46

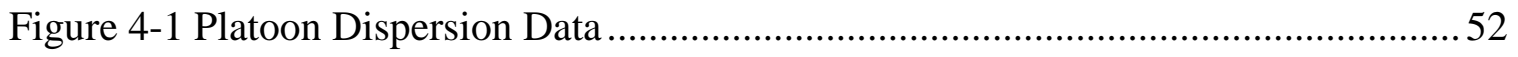

Figure 4-2 Pacey's Expected Flow versus Actual Flow .............................................. 53

Figure 4-3 CORSIM Speed Distribution Parameters ..................................................56

Figure 4-4 Flow Profile at Different Downstream Locations ......................................57

Figure 4-5 SR26 Travel Time With Calibrated Versus Default Distribution ................... 58

Figure 4-6 US31 Travel Time with Calibrated Versus Default Distribution ....................59

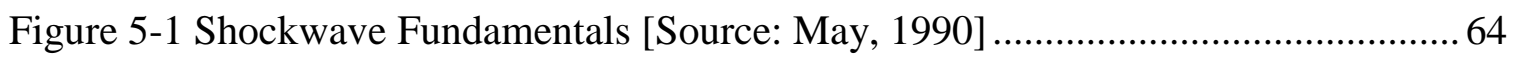

Figure 5-2 Shockwaves at Signalized Intersections [Source: May, 1990]....................... 66

Figure 5-3 A Shockwave Originated by a Bad Offset ............................................. 70

Figure 5-4 A Shockwave Originated by a Good Offset ............................................. 72

Figure 5-5 A Shockwave Originated by a Bad Offset and High Turning Traffic............. 74

Figure 5-6 A Shockwave Originated by a Good Offset and High Turning Traffic .......... 75 
Figure

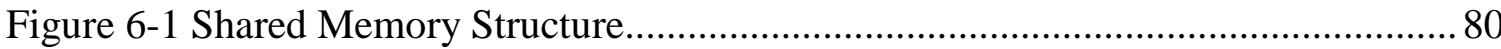

Figure 6-2 LinkPro Screen Capture for a tuned Offset ............................................ 82

Figure 6-3 Detectors Occupancy Data at Selected Downstream Locations..................... 83

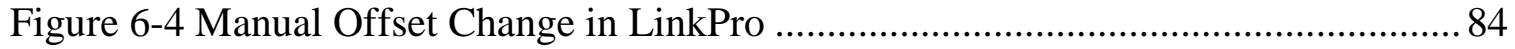

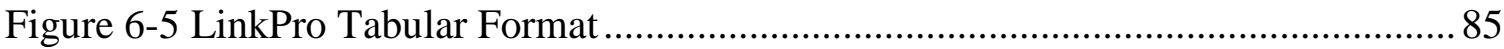

Figure 6-6 LinkPro Screen Capture for an un-tuned offset......................................... 87

Figure 6-7 Condensed Platoon's Occupancy and Count Profiles Over 25 Cycles, Green

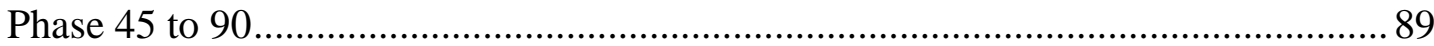

Figure 6-8 An End-Dispersed Platoon's Occupancy and Count Profiles Over 25 Cycles,

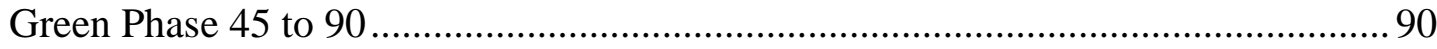

Figure 6-9 An End-Dispersed Platoon's Occupancy and Count Profiles Over 25 Cycles

With a Wide Green Window, Green Phase 25 to 90 ................................................ 91

Figure 6-10 A Dispersed Platoon's Occupancy and Count Profiles Over 25 Cycles, Green

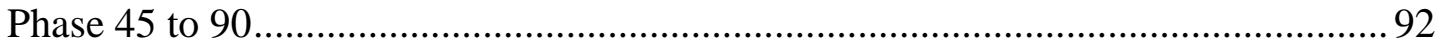

Figure 6-11 A Dispersed Platoon's Occupancy and Count Profiles Over 25 Cycles With a

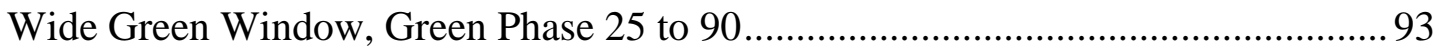

Figure 6-12 Distribution of an Artificially Generated Sample Occupancy Actuation in

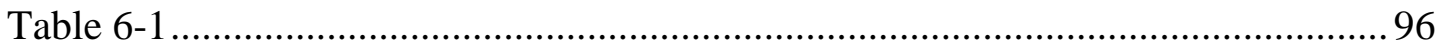

Figure 6-13 Distribution of Occupancy when directly substituted ...............................98

Figure 6-14 A Histogram Graph for Values in Table 6-4 ........................................... 100

Figure 6-15 Good Offset With a Turning Traffic Profiles .......................................... 103

Figure 6-16 Bad Offset With a Turning Traffic Profiles ............................................ 104

Figure 6-17 Differential Skewness between Occupancy and Counts Histograms.......... 106

Figure 6-18 Determination of Offset Transitioning Direction in PRO-TRACTS .......... 109

Figure 6-19 Validation of Offset Transitioning Direction in PRO-TRACTS ................ 110

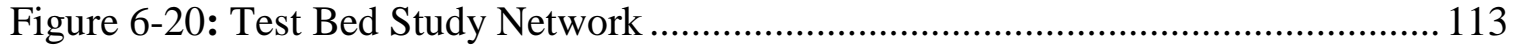

Figure 6-21 Test Bed Phase Sequence Ring Diagram for Nodes 2, 3, 4, and 5............ 114

Figure 6-22 Test Bed North Bound Cumulative Travel Time-90 sec Cycle ................. 116

Figure 6-23 Test Bed South Bound Cumulative Travel Time-90 sec Cycle .................. 117 
Figure

Figure 6-24 Test Bed Offset and Cycle Transitions-Bad offset Study 119

Figure 6-25 Test Bed Total Delay and Number of Stops-90 sec Cycle 120

Figure 6-26 Test Bed North Bound Cumulative Travel Time-Case 1-5, 120 sec Cycle 122 Figure 6-27 Test Bed Second Hour Cumulative Travel Time-Case 5, 120 sec Cycle.... 123 Figure 7-1 False Alarm With Low Traffic Volume 127

Figure 7-2 Singularity of Differential Skewness- Case 1 129

Figure 7-3 Singularity of Differential Skewness- Case 2 130

Figure 7-4 Effect of Platoon Dispersion on Progression 132

Figure 7-5 Tuning Offsets for Purpose of Reducing Platoon Dispersion 133

Figure 7-6 Effect of Detector Location on PRO-TRACTS Activation Mechanism ....... 136

Figure 7-7 Experiment Network 138

Figure 7-8 Effect of offset on Speed variance 160

Figure 7-9 Linear Discriminant Functions for Different Offset Classes. 165

Figure 8-1 SR 26 (South) Network - Earl Avenue to Creasy Lane 167

Figure 8-2 SR 26 (South) East Bound Cumulative Travel Time-A.M. Period............... 172

Figure 8-3 SR 26 (South) West Bound Cumulative Travel Time-A.M. Period ............. 173

Figure 8-4 SR 26 (South) East Bound Cumulative Number of Stops-A.M. Period ....... 174

Figure 8-5 SR 26 (South) West Bound Cumulative Number of Stops-A.M. Period ...... 175

Figure 8-6 SR 26 (South) Total Delay and Number of Stops-A.M. Period.................... 176

Figure 8-7 SR 26 (South) Offset and Cycle Transitions-A.M. Period............................ 179

Figure 8-8 Effect of Phase Skips on Progression ..................................................... 182

Figure 8-9 Offset Correction for Phase Skips ......................................................... 183

Figure 8-10 SR 26 (South) East Bound Cumulative Travel Time-P.M. Period.............. 185

Figure 8-11 SR 26 (South) West Bound Cumulative Travel Time-P.M. Period ............ 186

Figure 8-12 SR 26 (South) East Bound Cumulative Number of Stops-P.M. Period ...... 187

Figure 8-13 SR 26 (South) West Bound Cumulative Number of Stops-P.M. Period..... 188

Figure 8-14 SR 26 (South) Total Delay and Number of Stops-P.M. Period................... 189

Figure 8-15 SR 26 (South) Offset and Cycle Transitions-P.M. Period.......................... 191 
Figure

Figure 9-1 Schematic of the Hardware-in-the-Loop Simulation Environment [Source:

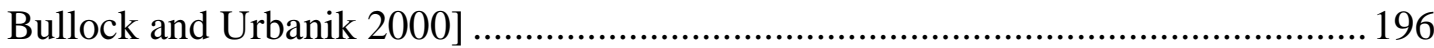

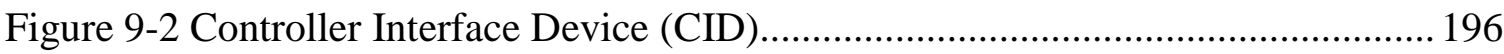

Figure 9-3 Hardware-in-the-Loop Concept.................................................................. 197

Figure 9-4 SNMP Bits and Bytes [Source: NEMA, 1999A] ...................................... 205

Figure 9-5 STMP Bits and Bytes [Source: NEMA, 1999A] ....................................... 206

Figure 9-6 Hardware-in-the-Loop with NTCIP Offset Download................................208

Figure 9-7 Sample of NTCIP Lab Equipment ............................................................ 209

Figure 9-8 Schematic Proposal of PRO-TRACTS Implementation ............................. 212

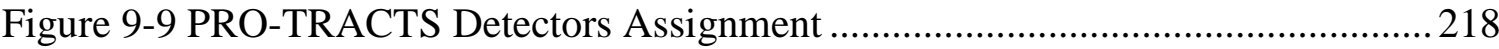

Figure 9-10 Effect of the updateFrequency Parameter on Offset Transitioning............ 222

Figure 9-11 PRO-TRACTS Parameters ................................................................ 223

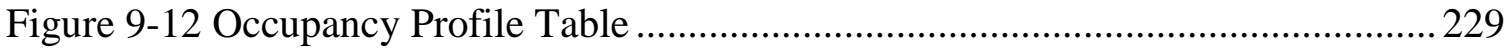

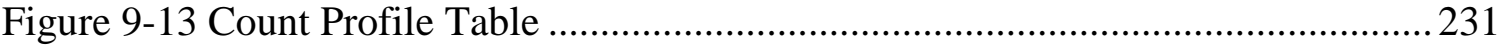

Figure 10-1 US 31 - Lincoln Street and Sycamore Drive.......................................... 236

Figure 10-2 US 31 North Bound Cumulative Travel Time-A.M. Period .......................243

Figure 10-3 US 31 South Bound Cumulative Travel Time-A.M. Period ....................... 244

Figure 10-4 US 31 Total Delay and Number of Stops-A.M. Period............................. 245

Figure 10-5 US 31 North Bound Cumulative Travel time-A.M. Period with Hardware-inthe-loop 247

Figure 10-6 US 31 South Bound Cumulative Travel time-A.M. Period with Hardware-inthe-loop 248

Figure 10-7 US 31 Total Delay and Number of Stops- A.M. Period with Hardware-inthe-Loop 249

Figure 10-8 US 31 Offset and Cycle Transitions-A.M. Period, Internal CORSIM Model .251

Figure 10-9 US 31 Offset and Cycle Transitions-A.M. Period with Hardware-in-the-Loop 
Figure

Figure 10-10 US 31 North Bound Cumulative Travel Time-P.M. Period, Internal Model

Figure 10-11 US 31 South Bound Cumulative Travel Time-P.M. Period, Internal Model 255

Figure 10-12 US 31 Total Delay and Number of Stops-P.M. Period, Internal Model ... 256

Figure 10-13 US 31 North Bound Cumulative Travel time-P.M. Period with Hardwarein-the-loop 257

Figure 10-14 US 31 South Bound Cumulative Travel time-P.M. Period with Hardwarein-the-loop 258

Figure 10-15 US 31 Total Delay and Number of Stops-P.M. Period with Hardware-inthe-Loop 259

Figure 10-16 US 31 Offset and Cycle Transitions-P.M. Period 261

Figure 10-17 US 31 Offset and Cycle Transitions-P.M. Period with Hardware-in-the-

Loop. 262

Figure 10-18 Main Street Green at Nodes 8 and 9 263

Figure 10-19 Lane Blockage at Node 9 264

\section{Appendix Figures}

Figure A-1 SR 26 (South) Network - Earl Avenue to Creasy Lane 279

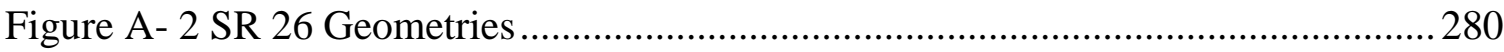

Figure A- 3 Intersection Ring Structures for SR 26.............................................. 281

Figure A- 4 SR 26 (South) Approach Labels ................................................................. 282

Figure A- 5 External Control File (CID) for Phasing .............................................. 283

Figure B- 1 US 31 Network - US 26 to Road 450.....................................................292

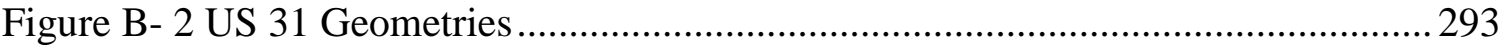

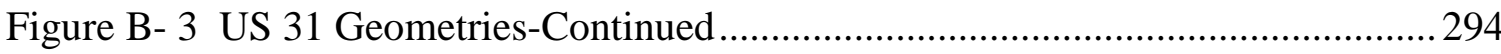

Figure B- 4 Intersection Ring Structures for US 31 ….........................................295

Figure B- 5 Intersection Ring Structures for US 31-Continued...................................296

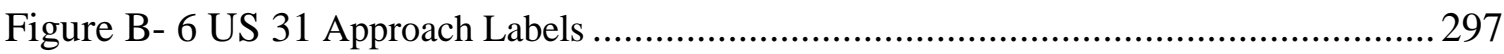

Figure B- 7 External Control File (CID) for Phasing .............................................. 298 


\section{CHAPTER 1- INTRODUCTION}

\subsection{Research Motivation}

Over the last few decades, extensive efforts have been made in the development of adaptive and responsive traffic control systems. The United States Department of Transportation (US DOT) has sponsored a great amount of research and field deployment of new traffic adaptive algorithms, such as OPAC [Gartner, 1983] and RHODES [Head et al., 1992]. Internationally, systems like SCOOT [Hurt et al., 1991] and SCATS [Lowrie, 1992], developed in the United Kingdom and Australia respectively, have seen increasing deployments. These adaptive control systems are based on applying specific proprietary software to a signal control system [Pooran, 1997]. It is estimated that travel time savings from these adaptive systems could range between $8 \%$ and $25 \%$ [FHWA, 1996].

Adaptive control systems typically require an extensive input of system parameters and weighting factors for favoring individual movements plus a large number of vehicle detectors to collect movement-specific traffic data. A major drawback of these systems is the extensive effort required for training personnel on the new proprietary architecture. Operation and maintenance of these systems can also be very costly and time consuming. 
In a parallel track, the private sector has developed closed loop systems that are operated by coordinated-actuated controllers. These systems provide actuated control capabilities through their ability to respond to cycle-by-cycle variation in traffic demand while still being able to provide progression for the arterial movement. Unfortunately though, the quality of vehicle progression depends on both the initial setting of offsets and the stochastic nature of traffic on individual intersections, causing waiting queues and what is known as the early-return-to-green phenomenon.

Expanding the control logic for modern coordinated- actuated systems to account for problems such as early-return-to-green, waiting queues, and improperly designed offsets would address many of the day to day problems associated with closed loop systems. Additional training would be minimized, in comparison to fully adaptive systems, since traffic engineers and technicians managing traffic signal operating systems are already familiar with coordinated-actuated logic. Fundamental concepts and communication systems for coordinated-actuated systems would also remain the same. Additionally, extra cost would be kept to a minimum since no extra detectors or infrastructure would be required.

\subsection{Research Overview}

The objective of this research is to introduce an adaptive real-time offset transitioning expansion to actuated controllers to improve coordination of traffic signals. This algorithm, the Purdue Real-time Offset Transitioning Algorithm for Coordinating Traffic Signals (PRO-TRACTS) is designed to mitigate the effect of early-return-to-green problems experienced with coordinated-actuated controllers and accounts for downstream 
vehicle queues that may impede vehicle progression. PRO-TRACTS fine-tunes the offsets at signalized intersections continuously to improve progression regardless of the offsets' initial values. The algorithm presented in this report works with both the internal model of the CORSIM simulation package [Kaman, 1997A] and actual NTCIP controllers.

Chapter 2 includes a review of traffic control systems, including adaptive traffic control, from a historical perspective. Chapter 2 also discusses the current state of actuated controllers and addresses the issues associated with their operation. Due to the close relationship between the quality of progression and vehicle platooning effects, Chapter 3 presents a comparative study between theoretical, simulation, and field platoon data and Chapter 4 addresses the calibration of the CORSIM platoon dispersion model. The theory of shockwaves and how it relates to the introduced offset tuning algorithm are discussed in Chapter 5, while Chapter 6 discusses the basic structure and concept of the introduced algorithm. Chapter 7 introduces a more general model underlining PROTRACTS activation and Chapter 8 evaluates the performance of PRO-TRACTS with a real traffic network of SR26 in Lafayette, IN. Chapter 9 discusses the integration of hardware-in-the-loop simulation with NTCIP controllers and defines a new NTCIP object for capturing detector actuation at the controller's level. The database needed for deployment of the algorithm in the field can be implemented at the controller's level, using the new NTCIP object defined in Chapter 9 of this report. A before-and-after study with both CORSIM's internal model and hardware-in-the-loop simulation of US 31 in Kokomo, Indiana is presented in Chapter 10. Finally, Chapter 11 summarizes the conclusions drawn from the research and provides suggestions for future work. 


\section{CHAPTER 2- LITERATURE REVIEW: CURRENT STATE OF TRAFFIC CONTROL SYSTEMS}

This chapter provides an overview of the current state of traffic control systems and their historical development through the past few decades. The state of the art in adaptive traffic control systems is discussed and their key features are highlighted. The chapter also describes the operation of actuated controllers and their parameters. Issues associated with coordinated-actuated control systems such, as the early-return-to-green phenomenon, are discussed as well as the efforts made to address them. Finally, current signal timing procedures are discussed to show the need for an algorithm that adapts offsets in real-time in response to change in traffic pattern and early-return-to-green.

\subsection{A Historical Perspective of Traffic Control Systems}

Traffic control concepts were first introduced when manually turned semaphores were developed in London, England in 1868 [Wolkomir, 1986]. Forty years later, similar devices were introduced to the U.S. in New York. The first electrically operated traffic signal was installed in Cleveland, Ohio in 1914. Five years later, New York began converting from hand-cranked semaphores to electromechanical controllers. By 1932, "the last hand-cranked semaphore on Parkside Avenue in Brooklyn was replaced by an electromechanical controller" [Bullock and Urbanik, 2000]. Electromechanical 
controllers dominated the traffic signal market from the 1920s until the 1970s [Kell and Fullerton, 1991].

\subsubsection{Microprocessor-Based Controllers}

The first computerized traffic signal control system was installed in Toronto, Canada in 1963 [Bullock and Urbanik, 2000]. In 1970, advancements in microprocessor technology and standardization effort in hardware and software led to the introduction of many new controller features. In order to standardize those new features, a group of vendors drafted a standard specification commonly referred to as TS1 [NEMA, 1989], which defined the operation and electrical pins on the $\mathrm{A}, \mathrm{B}$, and $\mathrm{C}$ connectors for actuated controllers.

TS1 provided a basic set of features and standard connectors. However, to provide more advanced operations such as coordinated-actuated operation, it was necessary for manufacturers to provide a set of complementary features. These proprietary features created incompatibility between controllers supplied from different vendors. In response, the NEMA TS1 specification was updated in the late 1980s and early 1990s to provide coordinated-actuated operation, preemption, and an optional serial bus to simplify cabinet wiring [NEMA TS2].

\subsubsection{Model 170 Controllers}

The Model 170 controllers were developed in a parallel track to the NEMA developments. The California Department of Transportation (Caltrans) and the New York 
Department of Transportation adopted a standard for providing precise specifications for a generic traffic control microcomputer, and specifications for the Model 170 controllers provide definitions for microprocessors, memory, input and output addresses, serial ports, mechanical form factor, and electrical connectors. This standard allowed agencies to purchase the controller software and competitively procure additional Model 170 controllers based on their need. This strategy, however, led to only a limited number of software vendors staying in the market for developing and updating Model 170 software.

\subsubsection{Advanced Transportation Controller (ATC)}

In 1989, Caltrans prepared a report documenting some of the Model 170 deficiencies and recommended a new platform, which was to embrace commercial standards rather than static technology [Quinlan, 1989]. The new model was called the 2070 model and was anticipated to benefit from new technology at the same rate as desktop computers. The broadened interest in this new development effort led to the emergence of the new ATC, which is less dependent on the microprocessor and operating system [Bullock and Urbanik, 2000].

\subsubsection{Urban Traffic Control Software (UTCS)}

In the 1970's, as centralized control of traffic signals became more popular around the globe, the Federal Highway Administration (FHWA) began to develop a structured approach to centralized traffic signal control, called urban traffic control software (UTCS). Various levels of traffic control, ranging from time of day plan selection to real-time adaptive signal timing were defined in the UTCS; and although the 
UTCS did not achieve many of its objectives, the UTCS effort resulted in the development of many concepts and system displays that are currently used in traffic operation centers.

\subsection{Adaptive Control Systems and Algorithms}

There are two primary motivations for the development of adaptive control algorithms. The first motivation is the need for the controllers to react to unexpected deviations from historical traffic patterns, either as a result of incidents or day-to-day random variations of the magnitude and temporal distribution of the demand peaks. The second motivation is that even for predicted traffic conditions there are a finite number of time-of-day plans that can be handled by current controllers. Also, pre-selected plans do not typically perform well during periods of temporal transitions in traffic patterns [Stewart et al., 1998]. Adaptive strategies usually respond to changes in traffic patterns in real-time, either reactively or proactively. Reactive adaptive strategies "follow" the change in traffic patterns and therefore are always lagging. Whereas, proactive strategies try to "predict" the change in traffic pattern, aiming at a better performance.

\subsubsection{History of Adaptive Control Systems}

The UTCS project was directed toward the development and testing of a variety of advanced network control concepts and strategies developed over three generations:

The first generation control (1-GC) used a library of pre-stored signal timing plans calculated off-line, based on historical traffic data, in the same way as the pre-timed 
control strategies. The original 1-GC selected a particular timing plan by either time-ofday or pattern matching every 15 minutes. A later modification of the 1-GC control consisted of the system automatically selecting a timing plan when conditions warrant its implementation. This strategy became known as 1.5-generation control (1.5-GC) [Gartner et al., 1995; McShane and Roess, 1990].

The second generation control (2-GC) used surveillance data and predicted values to compute and implement timing plans in real-time. Timing plans were updated no more than once per 10-minute period to avoid transition disturbances from one implemented plan to the next, [Gartner et al.,1995; McShane and Roess, 1990].

The third generation control (3-GC) used on-line optimization to update the cycle lengths, splits, and offsets in real-time, with a sampling period duration of 60-120 seconds [McShane and Roess, 1990]. The 3-GC differs from the 2-GC in its shorter periods after which the timing plans are revised. The cycle length in 3-GC was also allowed to vary among the signals, as well as the same signal, during the control period [Gartner et al., 1995].

\subsubsection{Adaptive Control Algorithms}

The experience with the 3-GC control in the UTCS experiments of the 1970's revealed that new strategies for adaptive control needed to be developed [Gartner et al., 1995]. Adaptive control attempts to achieve real-time optimization of signal operations by using current short-term vehicle information obtained from advanced detectors. However, the performance of the adaptive control system response is entirely dependent 
on the quality of the prediction model [Gartner, 1995]. The implementation of adaptive control logic is not always superior to pre-timed and actuated control, especially when traffic is highly peaked [Stewart et al., 1998].

Significant advances in adaptive traffic control were achieved with the introduction of four control strategies. Namely SCOOT, SCATS, OPAC, and RHODES. SCOOT (Split, Cycle, Offset Optimization Technique) was developed in the United Kingdom [Hunt et al., 1981] and is considered a UTCS-3-GC, although some authors put it into the 2-GC category [Gartner et al., 1995]. SCATS (Sydney Coordinated Adaptive Traffic System) was developed in Australia [Lowrie, 1992] and is considered to be a variant of the UTCS 1.5-GC variant. OPAC (Optimized Policies for Adaptive Control) was introduced by Gartner in the U.S. and involved the determination of when to switch between successive phases based on actual arrival data at the intersection [Sen and Head, 1997]. RHODES (Real-Time, Hierarchial, Optimized, Distributed and Effective System) consists of a distributed hierarchical framework that operates in real-time to respond to the natural stochastic variation in traffic flow [Head et el., 1992].

\subsubsection{1 $\underline{\text { SCATS }}$}

Outside of Australia, SCATS has seen limited deployment. Each SCATS controller has a micro-controller that uses stop line detector information. The philosophy of SCATS is that it has no comprehensive plans, rather, it selects from a library of offsets and phase splits to optimize timing plans in real-time. SCATS divides the network into sub-regions with homogeneous flow characteristics. In each region, the intersection with the highest saturation determines the cycle length of the region. As traffic patterns 
change, a computer at a higher level "marries" and "divorces" intersections by reassigning them to regions with similar flow characteristics. Each subsystem makes independent decisions regarding timing parameters involving cycle, offset, and phase lengths. The timing plans are incrementally adjusted to varying traffic conditions [Head et al., 1992]. SCATS has limited deployment in the U.S., with the most recent in Minneapolis. However, Minneapolis operators have not been particularly comfortable with the system because of the significant differences between SCATS conventions and NEMA standards.

\subsubsection{2 $\underline{\text { SCOOT }}$}

SCOOT is widely deployed all over the world but has had limited deployment in the U.S.. The main advantage of SCOOT is that it is based on the TRANSYT optimization model that runs in the background called the SCOOT Kernel. SCOOT uses a central computer and immediate downstream detectors to optimize timing plans in realtime. SCOOT uses cyclic flow profiles (CFP) the same way TRANSYT [Robertson, 1969] does. Since SCOOT has evolved from an on-line model of TRANSYT, it can be used to optimize performance indices such as the number of stops, delay, or a mix of both. SCOOT timing parameters are communicated to the controller immediately. The controller makes incremental adjustments to the cycle lengths, phase lengths, and offsets for the current and next cycles [Head et al., 1992]. 


\subsubsection{Optimized Policies for Adaptive Control (OPAC)}

OPAC is different from other models that try to optimize timing parameters using average traffic volumes. Instead, OPAC uses actual traffic data at intersections to decide when to switch to different phases. [Gartner, 1983].

OPAC uses detectors placed far upstream the intersections to predict vehicle arrivals at the intersection and proactively determines the phase timings [Gartner, 1981]. The algorithm has gone through several development efforts ranging from OPAC I through OPAC-RT [Gartner, 1995]

OPAC breaks the signal optimization problem into sub-problems with the use of dynamic programming (DP), an approach that leads to more efficient computation. OPAC-1 assumed an infinite horizon and used DP to optimize the performance index (minimizes delay). The main limitation of OPAC I was its need for elaborate, and most likely very costly, surveillance detectors since it needs the arrival data for the entire planning horizon. It was also impossible to implement the DP on-line in real-time because of the extensive time required to compute the optimal settings [Gartner, 1983].

OPAC II used an optimal sequential constraint search (OSCO) to calculate the total delay for all possible phase switching options. The optimal solution was determined as the phase switching that produces the lowest total delay values, and OPAC-2 was found to derive solutions with performance indices within $10 \%$ of those generated with OPAC-I [Gartner, 1983]. 
Although OPAC-II was faster than OPAC-I, it still suffered from the need for vehicle arrival information for the entire planning stage, which was 50-100 seconds in length. Gartner developed the rolling horizon approach, which he called ROPAC, to overcome this problem [Gartner, 1983]. ROPAC was later converted to an algorithm that can be implemented in real-time called, OPAC-RT version 1.0. OPAC-RT was developed for a simple two-phase fully actuated isolated intersection. The algorithm was found to be superior to actuated control with high traffic volumes [Gartner et al., 1991].

OPAC-RT version 2.0 was an extension of version 1.0 that allowed the control of an eight-phase intersection. OPAC-RT version 2.0 controlled the through-phases only, and the minor phases (left-turn phases) were controlled by the gap/out or max/out functionality of the actuated controller. OPAC-RT 2.0 showed an improvement in terms of total delay and percent stops [Gartner et al., 1991].

OPAC-RT version 3.0 had several enhancements and added features, which include the ability to optimize all eight phases, skip phases, and an algorithm to coordinate adjacent signals. The OPAC-RT version 3.0 performed best under oversaturated conditions and demand conditions change for the arterial [Andrews et al., 1997].

\subsubsection{Real-Time, Hierarchical, Optimized, Distributed, and Effective System $\underline{(\text { RHODES })}$}

Head et al. introduced an adaptive control strategy entitled RHODES in 1992. RHODES is reported to be better than SCOOT and SCATS in the way it responds to the 
natural stochastic variation in traffic flow proactively [Head et al., 1992]. RHODES is entirely based on dynamic programming, and it formulates a strategy that makes phase switching decisions based on vehicle arrival data [Sen and Head, 1997]. Like SCOOT, RHODES has the ability to use a variety of performance measures, including delays, queues, and stops. In addition, it allows for phase sequencing to be optimized in addition to the various timing parameters.

The design of RHODES is based on dividing the traffic control problem into subproblems by use of network hierarchy. The sub-problems include network-loading problem, network flow control problem, and intersection control problem.

At the top of the hierarchy (Figure 2-1) is the network-loading problem. At this level, link loads and the prediction of the trends in the change of loads from real-time data are estimated. RHODES uses this information proactively to predict future platoon sizes near the boundaries of the system [Head et al., 1992]. 


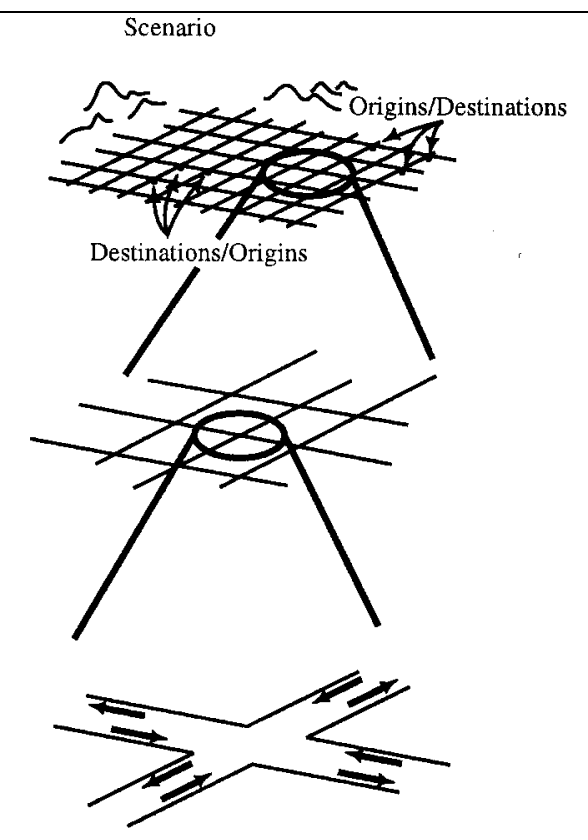

Figure 2-1 Hierarchy Framework of RHODES [Source: Head et al, 1992].

The middle level consists of the network flow problem and involves the selection of signal timing to optimize the overall flow of vehicles in the network [Head et al., 1992]. The decisions are made in this level every 200-300 seconds. A platoon prediction logic model called REALBAND, which was created by Dell Olmo and Mirchandani [1995], is used at this level, using both the information in Level One and the actual detector data. Network optimization is also established at this level and its results are used as constraints for the decision made in the next level [Head et al., 1998].

The lowest level of the control strategy is the one at the intersection and is responsible for making the final second-by-second decisions regarding traffic signal operation. This level uses two sublevels of logic. The first is the Link Flow Prediction Logic that uses data from upstream detectors' target timings of the upstream signal to estimate vehicle arrivals at the intersection being optimized. The other level is the 
Controlled Optimization of Phases (COP) [Sen and Head, 1997], which uses the information from the network flow problem, in addition to the results from the link prediction logic, to determine whether the current phase should be extended or terminated.

Although there are several adaptive control strategies that attempts to adapt to traffic patterns either reactively or proactively, each of these strategies perform differently under different type of conditions. [Head et al., 1992].

\subsection{Actuated Traffic Control Systems}

There have been tremendous improvements in traffic signal control hardware since its evolution from one-ring logic and pre-determined green splits into the multi-ring control logic controllers. This evolution led to the development of actuated traffic controllers in their current form.

An actuated traffic controller uses both detector information and a set of control parameters to operate the intersection in a more efficient way. With the use of actuated controllers, intersection approaches are allocated green times based on their current demand. If there is low demand on an intersection approach, less green time is allocated to the approach, providing the opportunity for other approach demands to be served.

\subsubsection{Actuated Control Parameters}

Although today's control strategies have numerous features and configuration parameters, three parameters have the most impact on system performance. These 
parameters are cycle length, splits, and offset. The cycle length is the time it takes the controller to cycle through all phases. Phase splits are the maximum percent of cycle allocated to each of the various phases. Offset is the time at which the main street green ends referenced to system cycle.

The cycle, split, and offset parameters are used in two different control architectures; pre-timed control and actuated control. In both cases, plans are usually implemented by either a time of day (TOD) or by traffic responsive approaches. The TOD approach typically divides the day into morning peak, afternoon peak, and off peak periods and timing plans are selected according to a global clock. The Traffic Responsive Program (TRP) selects plans based on the traffic demand defined by detector counts and occupancy and are based on user pre-set thresholds. The TRP collects system detector information, scales it, and smoothes it by factoring the current values with the previous values. Each detector is pre-assigned to a detector group. Information coming from each detector group is then combined into a group value. The information from all groups' is then combined into a final value and the master controller compares the final value to user pre-selected thresholds to determine the appropriate plan for the current demand. Besides the difficulty in choosing the optimum set of thresholds, "TRP can be very unpredictable, particularly when traffic conditions are rapidly changing, or do not closely resemble any of the existing case scenarios" [Nelson, 2000].

Downtown grid systems are still predominantly using fixed-time control. However, actuated control is used much more than fixed time control on arterials, 
especially when arrival patterns are less predictable or when intersections are spaced far apart.

Table 2-1 shows the basic parameters for operating an actuated controller in its simplest mode or what is called a "free" operation. The "Minimum Green" time as defined in the table is allocated to a phase once a detector associated with that phase registers a call. If vehicles continue to pass over the detector while the phase is green, an additional green time equal to the "Vehicle Extension" time is added to the phase. The green can be extended until it reaches the "Maximum Green" at which time the phase terminates in a condition called a "Max Out." However, if no detector call is registered within a "Vehicle Extension" period, the phase is terminated in a condition called a "Gap Out." The "Maximum Green" parameter is only active when there is a detector call for a conflicting phase. If no call is present, the phase green continues until the phase gaps out. Non-conflicting phases, e.g. southbound and northbound through movements, can show green simultaneously. The sequence by which the green is allocated to phases is determined by their sequence in the ring structure as shown in Figure 2-2. 
Table 2-1 Common Actuated Traffic Controller Parameters [Source: Econolite, 1996]

\begin{tabular}{|l|l|}
\hline CONTROLLER ACTUATED PARAMETER & \multicolumn{1}{c|}{ DESCRIPTION } \\
\hline Minimum Green & $\begin{array}{l}\text { Shortest possible green time prior to vehicle } \\
\text { extensions. }\end{array}$ \\
\hline Maximum Green & Maximum green time allowed for a phase. \\
\hline Vehicle Extension & $\begin{array}{l}\text { Length of added time periods to minimum green } \\
\text { based on frequency of actuations. }\end{array}$ \\
\hline
\end{tabular}

\subsubsection{Coordinated-Actuated Control}

Figure 2-2 shows a typical dual ring structure that corresponds to the standards of the National Electrical Manufacturing Agency (NEMA) phase numbers [NEMA, 1992]. The figure shows an additional parameter, the force off, which is a very important parameter for the coordinated actuated operation.

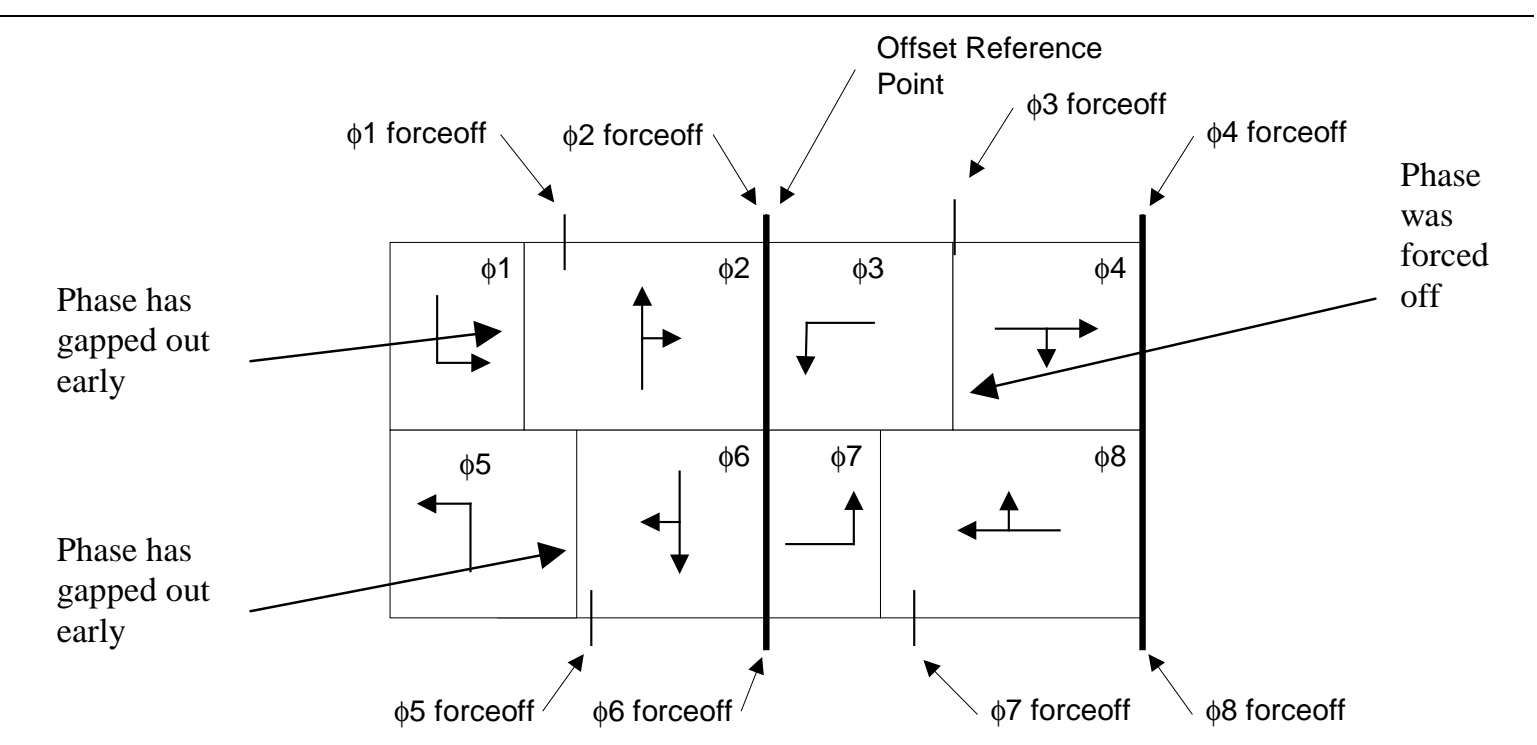

Figure 2-2 Typical Dual Ring Structure for Coordinated Actuated Operation 
A force off point is a point in time beyond which the phase cannot be extended and is computed from the split. However, a phase is allowed to terminate prior to its force off point if there is not enough demand to warrant the phase extension. This force off parameter overrides the "Maximum Green" parameter. The engineer usually "inhibits" the "Maximum Green" parameter when operating the controller in a coordinated actuated logic. Alternatively, the "Maximum Green" parameter can be set to a very high value so that it does not interfere with the operation of the force off parameter. It should be noted in some traffic controllers, splits are entered instead of force offs. However, the force offs are calculated and used internally.

Unlike the "free" operation, the coordinated actuated operation must operate under a constant cycle length to ensure coordination between adjacent signalized intersections. Another important setting for the coordinated actuated operation is the declaration of a coordinated phase in each ring as a coordinated phase. This coordinated phase will receive all the extra green not used by previous phases.

In Figure 2-2, phases 2 and 6 are the coordinated phases and typically, those phases would be assigned to the main street through movements. Note that the offset is referenced to the end of the coordinated phases, which is necessary because the beginning of the coordinated phases are not deterministic since they receive the unused green from previous phases in their ring. This means that the force off points immediately preceding the coordinated phase corresponds to a latest start time. However, the coordinated phases can start earlier in what is called "the-early-return-to-green" phenomenon. 
Early-return-to-green can have very negative impacts on arterial coordination. If an upstream signal experiences an early-return-to-green, a vehicle platoon will be released earlier, only to be stopped by a red interval downstream. This problem violates the driver's expectations and causes unnecessary delays. Figure 2-3 and Figure 2-4 illustrate the negative impacts of early-return-to-green. Figure 2-3-a shows a case where traffic demand is high enough for all phases to reach their force off points. Note that if traffic volume is constant at that level, the start of green on the main street through movement will be deterministic. Figure 2-3-b shows a case where the non-coordinated phases gap out causing all the excess green time to be allocated to the coordinated phases. In such a case, the coordinated phases experience an early-return-to-green.

Figure 2-4 illustrates the effect of early-return-to-green on the arterial progression and Figure 2-4-a shows a case where traffic volume on the actuated phases is high enough for the phases to extend to their force offs. In such a case, green on the coordinated phases occur at its expected time and the offset perform the way it was originally designed. However, in Figure 2-4-b, early-return-to-green at different intersections causes disruption of signal progression, mainly due to the fact that different intersections will experience different amount of early return to green because of the difference in their traffic conditions. 


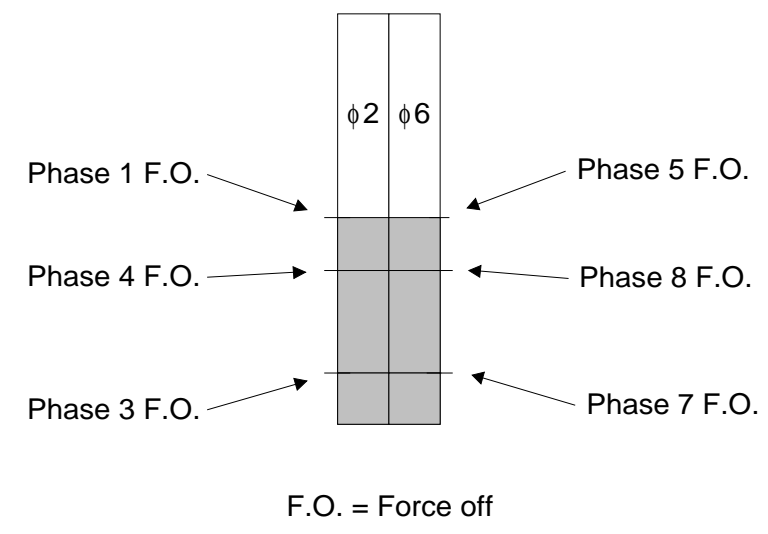

(a) All phases max out and are forced off

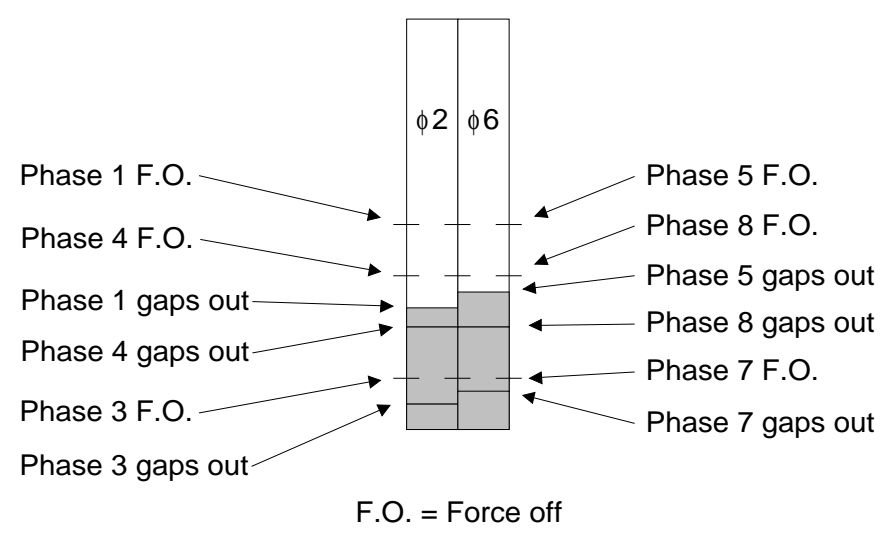

(b) Phases gap out before reaching force offs and extra green time is allocated to $\phi 2$ and $\phi 6$

Figure 2-3 Coordinated-actuated ring structure [Source: Shoup, 1998] 


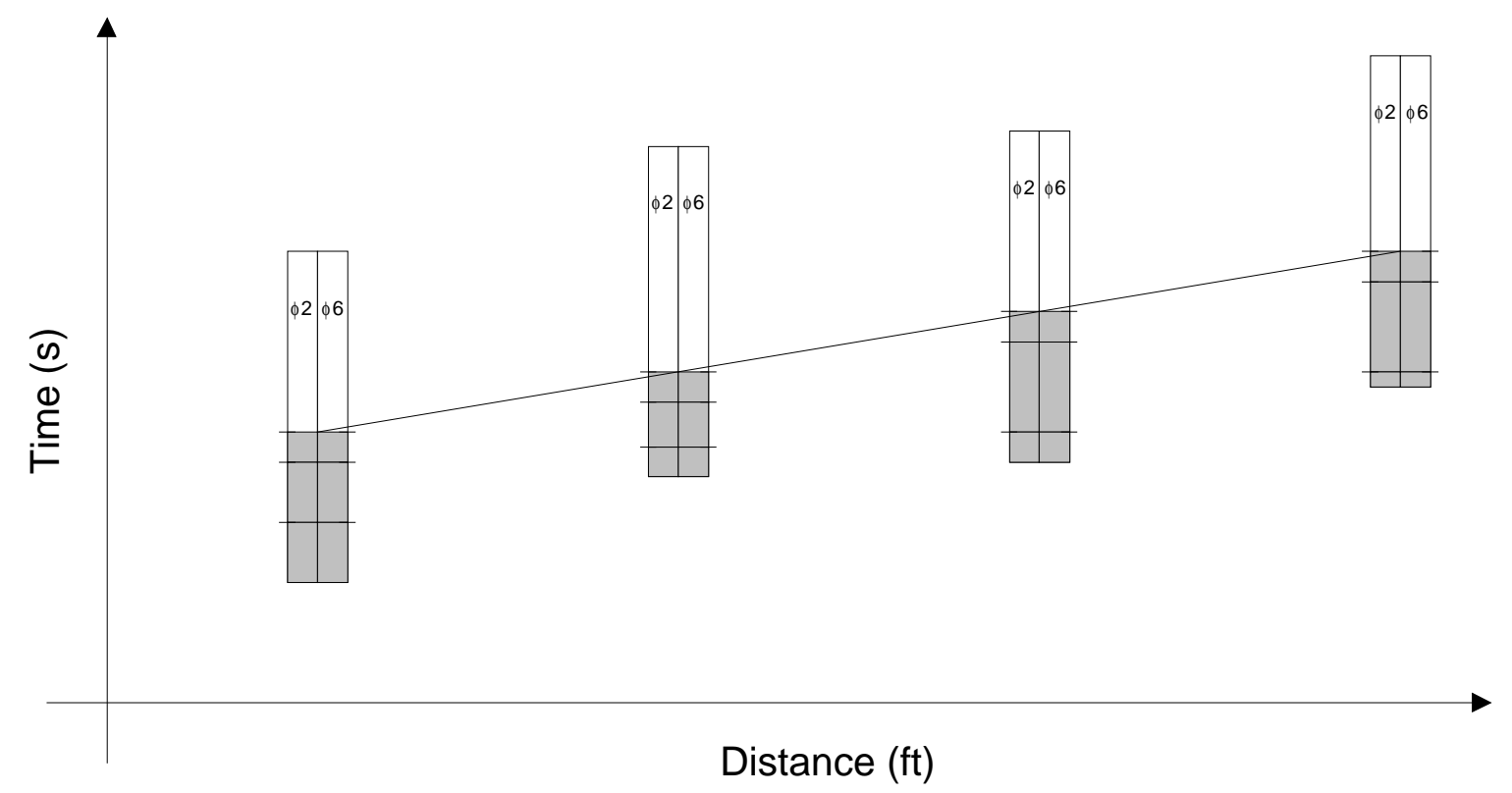

(a) Start of green band when all phases max out

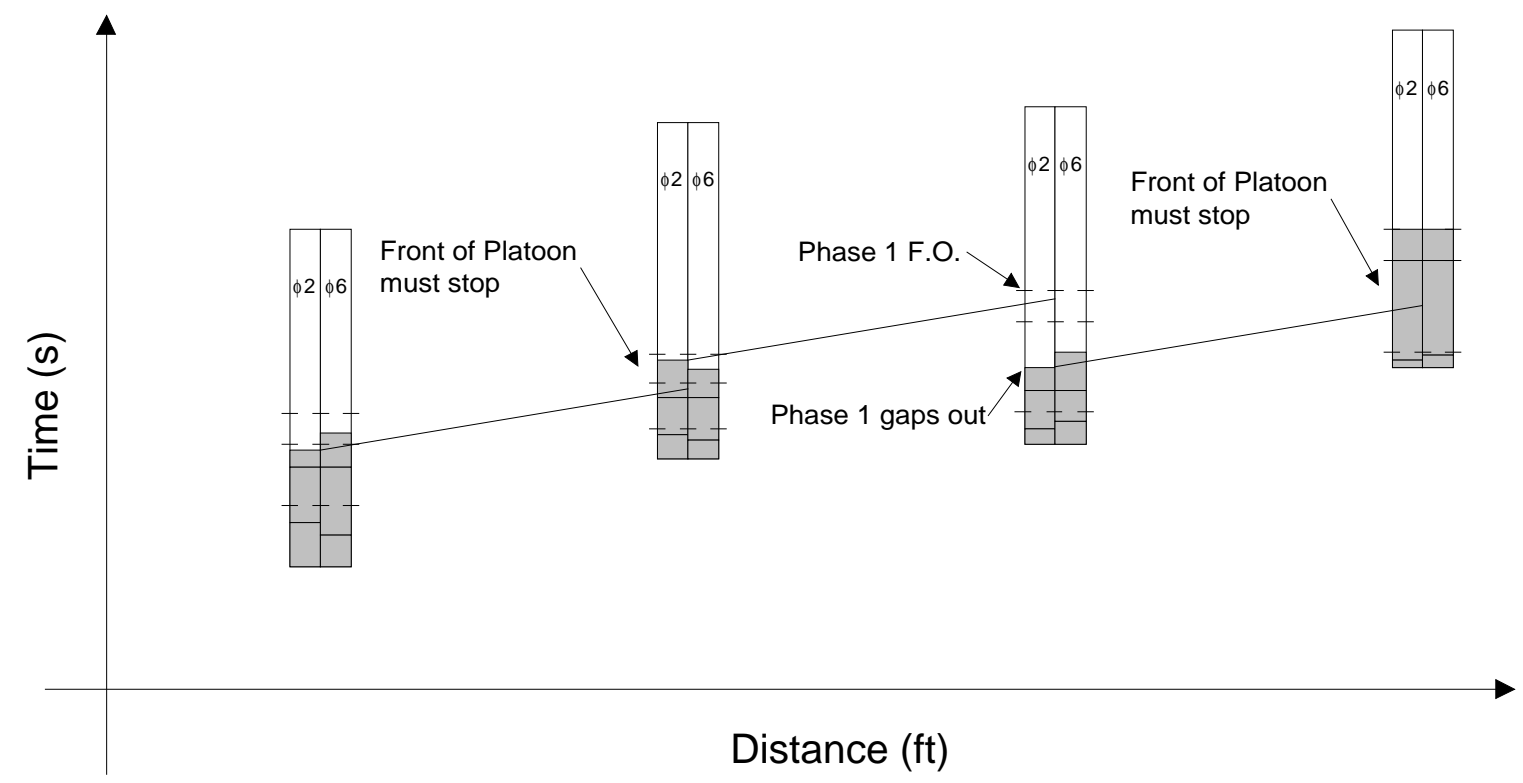

(b) Start of green band when some phases gap out early

Figure 2-4 Coordinated-actuated time-space diagrams [Source: Shoup, 1998] 


\subsection{Current Signal Timing Procedures}

The two oldest, popular signal timing programs, PASSER and TRANSYT-7F, were designed to model fixed-time controllers. The stochastic behavior of actuated signals, in response to the stochastic nature of traffic patterns, is not modeled in these [Tighe, 1987], although some features of modern actuated controllers have been incorporated in recent versions of TRANSYT-7F [Skabardonis, 1996; Husch, 1998].

An iterative actuated control design procedure for phase splits based on empirical formulas is included in Chapter 9, Appendix 2, of the 1997 Highway Capacity Manual. These formulas are developed to account for the inherent characteristics of dual-ring logic structure [Pei-Sung, 1996]. However, they do not address cycle offset parameters.

More recently, the signal timing software, SYNCHRO, has introduced procedures that design traffic signal offsets based on the probability of the time when an "earlyreturn-to-green" will occur in a controller given the input traffic demands [Husch, 1998].

It is very important to note that although some of the traffic signal design software programs have started to model the early-return-to-green and stochastic nature of traffic, they can only provide an offset value that will perform best "on average". If the traffic demand varies significantly over the course of the time period, an algorithm that adapts offsets in real-time in response to a change in traffic pattern and early-return-to-green will be of great value.

This phenomenon has been identified and investigated by several researchers [Chang, 1996; Ficklin, 1973; Jovanis et al., 1986; Kuzbari, 1996; Skabardonis, 1996]. 
Skabardonis used the average green times allocated to the non-coordinated phases in computing offsets with TRANSYT-7F [Skabardonis, 1988]. This study assumed that actuated phases are always experiencing a high degree of saturation for the critical movement. The method provided encouraging results when evaluated with NETSIM models based upon actual arterial conditions. These promising results led to the inclusion of the option to estimate actuated green times based on a high degree of saturation rates in TRANSYT-7F.

Skabardonis suggested two strategies. The first strategy was to operate the signalized intersections at the boundaries as fixed-time to provide a fixed leading edge for the arterial bandwidth [1996]. Although this strategy would ensure that through vehicles entering the arterial at the arterial boundary edges would not be released prematurely, it does not account for vehicles entering the arterial from mid-link arterial sources or arterial boundaries. Another problem with this procedure is that the operation of the boundary signal in a fixed-time mode reduces its efficiency.

Skabardonis' second strategy was to set the coordinated through phase as actuated and the non-coordinated phases as fixed-time. Although this method provides a fixed leading edge for the coordinated phase and removes the "early-return-to-green" effect, it allocates the extra green to the side street rather than the more demanding arterial, which defies the basic concept of actuated traffic signal operation.

Chang suggested the design of offsets with a fixed-time signal optimization package such as PASSER-II and observing the average green times for the non-actuated phases at each intersection in the field. Once the average green times are entered, a 
second optimization run for the offsets could be performed with the expected green times entered as constraints for the maximum green times [1996]. Chang did not provide quantitative data about the results of this strategy.

Kuzbari [1996] suggested recording the actual times for when the coordinated phase begins and ends in the background cycle at each of the signalized intersections of an arterial. A time-space diagram based on the average times for the coordinated phase was used to improve an arterial bandwidth for one direction. Actual field data in California were collected and significant improvements in the arterial bandwidth were reported for one direction.

It should be noted that all of these procedures discussed to this point are off-line procedures that solely depend on collected data, none of them adapt to the current traffic conditions in real-time.

\subsection{Online Offset Tuning Procedure}

NAZTEC implemented an adaptive signal control algorithm [Black, 1998] to modify the time-of-day splits according to the actuated phases demand (Figure 2-5). The algorithm allows the controller to reduce each actuated split by one second each cycle if the phase gaps out prior to its force off point. The "slack" time is added to the end of the coordinated phases to reduce the early-return-to-green problem. Since the offsets in actuated controllers are measured from the end of green, offset values may hence be increased by a maximum of this slack time. If the actuated phases are later forced off, then one second is added back to their splits. Although this procedure adapts responsively 
to the side street demand, it counts on perfectly designed offsets. Moreover, since the leading edge positions of the coordinated phases always remain constant, the algorithm will not be able to adapt to waiting queues or different traffic conditions in the arterial, e.g. slower travel speed on the arterial due to high arterial volume or vice versa [Shoup, 1998].

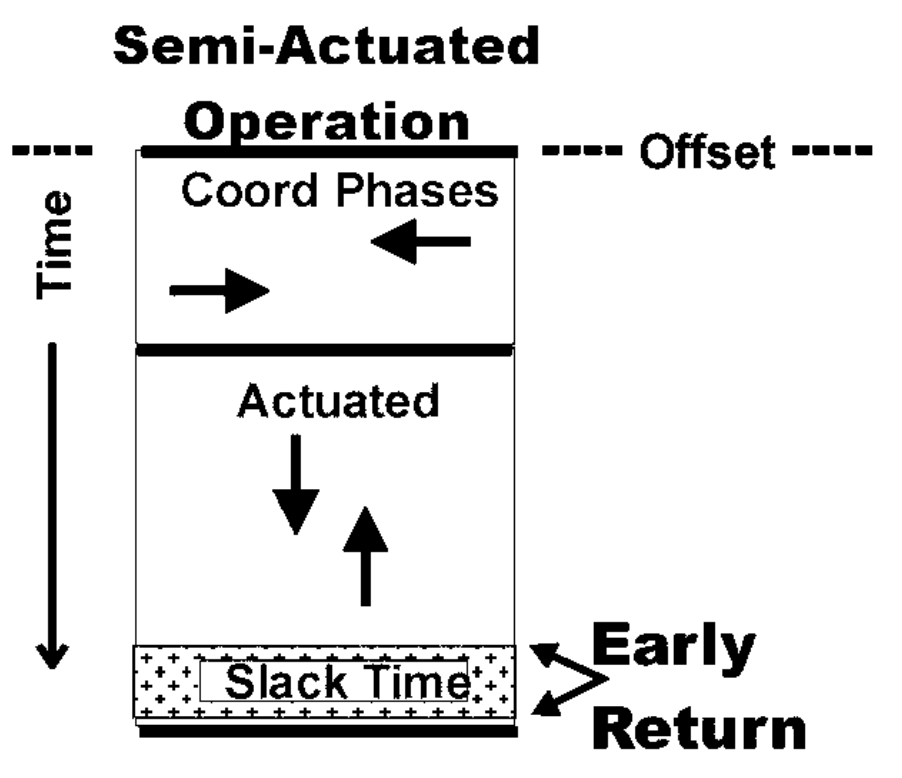

Adaptive Split

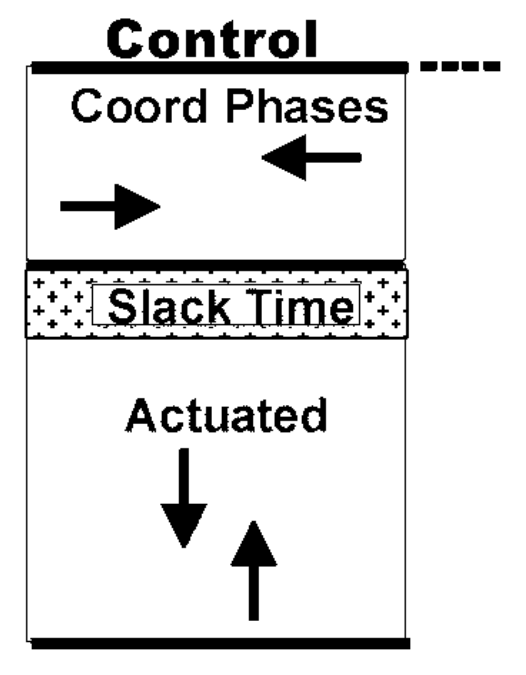

Figure 2-5 NAZTEC Algorithm [Source: Black, 1998]

\subsection{Definition of Adaptive Offset Algorithm Opportunity}

To date, there has not been any real-time offset tuning algorithm that accounts for early-return-to-green phenomenon as well as changes in the coordinated phases travel time. Such an adaptive offset algorithm would be of significant benefit to drivers because of its ability to reduce delay times, travel times, number of stops, and vehicular emissions 
in response to the change in traffic patterns. The objective of this research is to develop an integrated adaptive control algorithm that can be readily added to current actuated traffic controllers. This would provide a cost effective procedure to improve the performance of current traffic control systems without adding major cost to the system. Additionally, such an approach would capitalize on the existing knowledge and familiarity of traffic engineers and personnel with the current actuated control system. Such an algorithm would not only mitigate the "early-return-to-green" problem and account for downstream queues, but it would also respond to both short and long-term changes in traffic patterns. 


\section{CHAPTER 3- COMPARATIVE STUDY OF THEORETICAL, SIMULATION, AND FIELD PLATOON DATA}

\subsection{Platoon Dispersion}

Vehicle platoons are initially limited in size by the green window at the upstream location. However, as the platoon travels downstream, it disperses because of vehicles traveling at different speeds. Platoon dispersion along arterial links is an important factor in determining whether adjacent signals should be coordinated. The amount of dispersion also determines, to a great extent, the number of vehicular stops and total delay in the coordinated movements. The objective of this chapter is to compare and contrast the percentage of platoon arriving within particular green windows as portrayed by theoretical models, simulation, and field data: 1) to evaluate the impact of distance on the dispersion of platoons discharged from intersections using data collected in the field and 2) to determine if similar conditions could be modeled with reasonable accuracy using the theoretical or simulation models. This is important because the performance of the model is heavily influenced by platoon dispersion.

Both field data and CORSIM simulation were used to estimate the amount of platoon dispersion downstream from traffic signals. Field data was also used to calibrate the Robertson's theoretical model and the results were tabulated and plotted such that comparison could be made [Wasson et al., 1999]. 


\subsection{Literature Review}

There have been several published papers that describe various platoon dispersion models. The earliest reported work is on diffusion theory, developed by Pacey [1956]. That model assumes that speeds in a platoon are normally distributed and, hence, the dispersion of vehicles in the platoon can be described by the dispersion in speeds. Subsequent research led to the development of the recurrence model, an empirical platoon dispersion model using a discrete iterative technique. The recurrence model, also known as the Robertson's model [Robertson, 1969; Rouphail et al., 1992], is utilized in the TRANSYT software package. Robertson's model operates under the assumption of the binomial distribution of vehicle travel time and is considered an improvement on the Pacey model [Denney, 1989] because of the ease of computation.

Much of the previous research focused on calibrating the platoon dispersion parameters of Robertson's model [Manar and Baass, 1996; Baass and Lefebvre, 1988; Castle and Bonniville, 1985; McCoy et al., 1983; Seddon P.A., 1972; Collins and Gower, 1974]. Denney [1989] tested the diffusion and recurrence models that are used to replicate platoon dispersion. Manar and Baass [1996] and Baass and Lefebvre [1988] researched the effects of external and internal friction on the calibration factors respectively. Castle and Bonniville [1985] researched the calibration of TRANSYT dispersion factors with respect to long road links.

Robertson's model can be represented by the following equation:

$$
q_{2}(j)=\frac{1}{1+\alpha \beta \tau} q_{1}(j)+\left(1-\frac{1}{1+\alpha \beta \tau}\right) q_{2}(j-1)
$$


Where:

$$
\begin{aligned}
& \alpha=\text { platoon dispersion factor, } \\
& \beta=\text { travel time factor, } \\
& \tau=\text { average travel time, } \\
& q 2(j)=\text { discharge volume at downstream location } 2 \text { for bin } j, \\
& q 1(j)=\text { discharge volume at upstream location } 1 \text { for bin } j, \\
& q 2(j-1)=\text { discharge volume at downstream location } 2 \text { for bin }(j-1)
\end{aligned}
$$

TRANSYT software uses Robertson's model to transform the flow histogram at the upstream traffic stop bar into an arrival pattern at the downstream signal. The model can be varied by changing the platoon dispersion factor, $\alpha$, and the travel time factor, $\beta$. The TRANSYT manual suggests values for these two parameters for a variety of conditions and many researchers have performed additional research to calibrate and analyze these parameters [McCoy et al., 1983].

Although previous research is invaluable for users of the TRANSYT software, its results are not tabulated in a manner that allows comparison with microscopic simulation models. This chapter presents the platoon dispersion data in a graphical format, by which the percentage of traffic arriving at a downstream traffic signal during an allocated green time can be estimated.

\subsection{Field Data Collection}

Several field sites were selected for platoon data collection in this study. To insure that a downstream-signalized intersection would not impact the platoon, sites were chosen with a distance of at least 5,000 feet between signalized intersections. It was also 
desirable to have a minimal impact on the platoon from merging and diverging vehicles, and therefore, sites with a minimum number of side streets and driveways were selected. Data collection occurred during peak and off-peak hours in order to gain platoon dispersion data for varying sizes of platoons. The detailed data collection procedure is described in the report by Wasson et al. [1999].

Table 3-1a shows the number of platoons collected in the field for each combination of speed, platoon discharge, and downstream distance. Table 3-1b shows the number of vehicles collected for the same combinations of speed, platoon discharge, and downstream distance. 
Table 3-1Number of Data Points Collected at Each Distance for Varying Speeds and Initial Discharge

\begin{tabular}{|c|c|c|c|c|c|c|c|c|c|c|}
\hline \multirow{2}{*}{$\begin{array}{l}\text { Speed } \\
\text { (mph) }\end{array}$} & \multirow{2}{*}{$\begin{array}{l}\text { Saturated } \\
\text { Discharge } \\
\text { Interval } \\
(\mathrm{sec})\end{array}$} & \multicolumn{9}{|c|}{ D/S Distance (feet) } \\
\hline & & 500 & 1000 & 1500 & 2000 & 2500 & 3000 & 3500 & 4000 & 4500 \\
\hline \multirow[t]{4}{*}{30} & 10 & 22 & 22 & 20 & 11 & 17 & 14 & 2 & - & - \\
\hline & 20 & 8 & 8 & 19 & 26 & 19 & 15 & 8 & - & - \\
\hline & 30 & 4 & 1 & 3 & 15 & 10 & 10 & 5 & - & - \\
\hline & 40 & 11 & 17 & 4 & 7 & 1 & 1 & 2 & - & - \\
\hline \multirow[t]{2}{*}{40} & 10 & 20 & 7 & 23 & 23 & 20 & 19 & 21 & 21 & - \\
\hline & 20 & 7 & 2 & 11 & 11 & 11 & 11 & 18 & 17 & - \\
\hline \multirow[t]{2}{*}{50} & 10 & 8 & 9 & 9 & 10 & 9 & 9 & 9 & 8 & 8 \\
\hline & 20 & 6 & 5 & 5 & 9 & 10 & 8 & 5 & 5 & 5 \\
\hline \multirow[t]{2}{*}{55} & 10 & 10 & - & 11 & 9 & 5 & 4 & 7 & 8 & - \\
\hline & 20 & 3 & - & 1 & 2 & 1 & 1 & 1 & - & - \\
\hline \multirow[t]{2}{*}{60} & 10 & 5 & 8 & 12 & 12 & 8 & 8 & 5 & 5 & 5 \\
\hline & 20 & 10 & 10 & 9 & 11 & 12 & 11 & 5 & 5 & 5 \\
\hline
\end{tabular}

a) Number of Platoons in Each Cell

\begin{tabular}{|c|c|c|c|c|c|c|c|c|c|c|}
\hline \multirow{2}{*}{$\begin{array}{l}\text { Speed } \\
\text { (mph) }\end{array}$} & \multirow{2}{*}{$\begin{array}{l}\text { Saturated } \\
\text { Discharge } \\
\text { Interval } \\
\text { (sec) }\end{array}$} & \multicolumn{9}{|c|}{ D/S Distance (feet) } \\
\hline & & 500 & 1000 & 1500 & 2000 & 2500 & 3000 & 3500 & 4000 & 4500 \\
\hline \multirow[t]{4}{*}{30} & 10 & 101 & 108 & 92 & 67 & 77 & 73 & 8 & - & - \\
\hline & 20 & 66 & 70 & 184 & 323 & 188 & 159 & 64 & - & - \\
\hline & 30 & 50 & 15 & 39 & 250 & 142 & 159 & 63 & - & - \\
\hline & 40 & 215 & 338 & 65 & 144 & 15 & 35 & 37 & - & - \\
\hline \multirow[t]{2}{*}{40} & 10 & 78 & 31 & 87 & 108 & 79 & 79 & 88 & 88 & - \\
\hline & 20 & 92 & 27 & 117 & 116 & 92 & 99 & 150 & 143 & - \\
\hline \multirow[t]{2}{*}{50} & 10 & 35 & 37 & 40 & 41 & 37 & 42 & 41 & 60 & 60 \\
\hline & 20 & 53 & 64 & 64 & 113 & 132 & 109 & 67 & 67 & 67 \\
\hline \multirow[t]{2}{*}{55} & 10 & 36 & - & 45 & 17 & 13 & 14 & 17 & 31 & - \\
\hline & 20 & 25 & - & 7 & 15 & 10 & 11 & 4 & - & - \\
\hline \multirow[t]{2}{*}{60} & 10 & 33 & 32 & 63 & 63 & 46 & 46 & 29 & 29 & 34 \\
\hline & 20 & 131 & 130 & 84 & 126 & 144 & 132 & 62 & 62 & 53 \\
\hline
\end{tabular}

b) Number of Vehicles in Each Cell

The Hewlett Packard 48GX scientific calculators were used to record the observations; one was used to record the signal transition times and the second was used to record the downstream arrival times of every vehicle. The two calculators were 
synchronized to a common reference time before they were used for data collection. The signal observer used three pre-programmed keys to collect data at the intersection: start of green, end of green, and vehicle departure time. The signal observer used the vehicle departure time key to record each time a vehicle entered the downstream side of the arterial. Vehicle arrivals included vehicles performing through movements through the intersection, as well as vehicles making left and right turns onto the downstream side of the arterial.

The calculator used by the downstream observer was programmed to record vehicle arrival times at the downstream observation location, which occurred at 500-foot intervals from the upstream traffic signal. Vehicle arrivals at those locations were correlated with the departure times recorded by the signal state observer to generate downstream histograms for the platoon.

\subsection{Data Processing}

The data collected by the calculators were downloaded to a desktop computer and extracted into a database. The database for this study consisted of six tables: the intersection table, the observation table, the field green table, the field data table, the CORSIM green table, and the CORSIM data table. The intersection table included a sketch of the intersection and the names of intersection legs. The observation table included information about the location and start time of the downstream observation. The location was recorded as the distance downstream from the upstream signal location. The table also contained information regarding the physical characteristics of the site, including the number of lanes and the width of each lane (typically two and 12 feet 
respectively). The field green table contained the green start times and green end times for the upstream traffic signal. The field data table contained the times each vehicle arrived at the downstream observer. The CORSIM green and CORSIM data tables were used to save the simulation data generated by the CORSIM traffic simulation software.

Organizing the data into a database allowed easy retrieval for analysis. Using the query tool within the database, the data needed for analysis was extracted and analyzed in a spreadsheet.

\subsection{Data Analysis}

Vehicle platoons disperse as they travel downstream on the arterial link. However, a platoon is expected to reach a downstream location within an expected range of time depending on the downstream distance, the average travel speed, and the initial platoon discharge length. The time $t_{a}$ in Figure 3-1 represents the projected time window during which the dispersed platoon is expected to arrive, which is obtained by projecting the upstream green window using a speed higher than the speed limit to locate the start of $t_{a}$ and a speed lower than the speed limit when locating the end of $t_{a}$. Field data reduction was therefore performed in two steps. First, the vehicle arrival times at the observation location lying within the time period $t_{a}$ were extracted from the database for each discharged platoon using a data base query, and the second step was to extract the platoon from that window. 


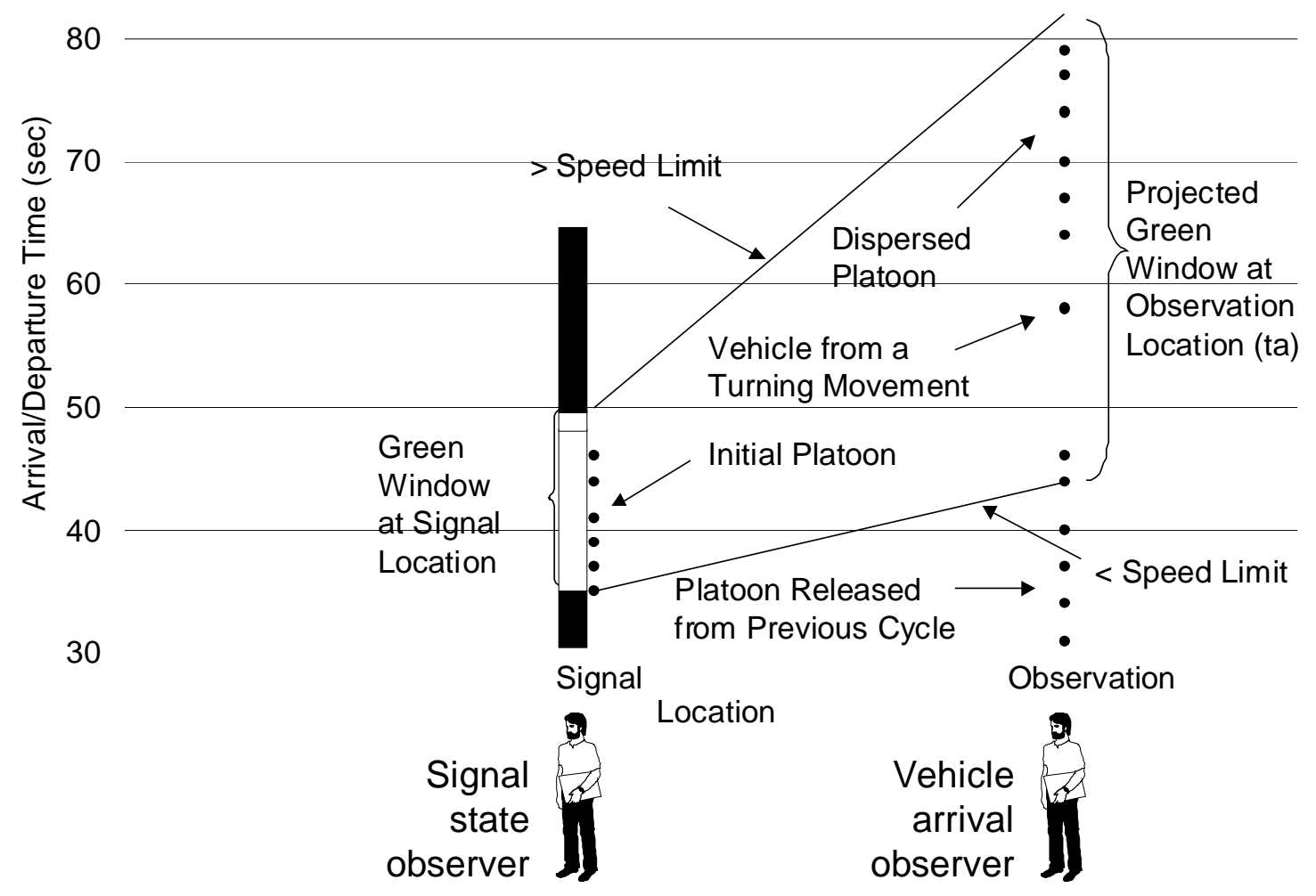

Figure 3-1 Sampling Procedure.

Different traffic conditions (gaps between successive platoons) were observed to affect the speed of the platoon, making it a difficult task to predict the exact time of platoon arrival at the downstream location within the extracted window. Extracting a wider range of projected upstream green window reduced the problem to the recognition of the platoon from the turning movement traffic preceding and following it. If the window was too large, the projected window would also contain the trailer of the platoon released from the previous cycle and the start of the platoon released from the following cycle as illustrated in Figure 3-1. 
The platoon within the projected green window was recognized using the algorithm illustrated in Figure 3-2. The narrow gap and wide gap thresholds used were three and eight seconds respectively. 

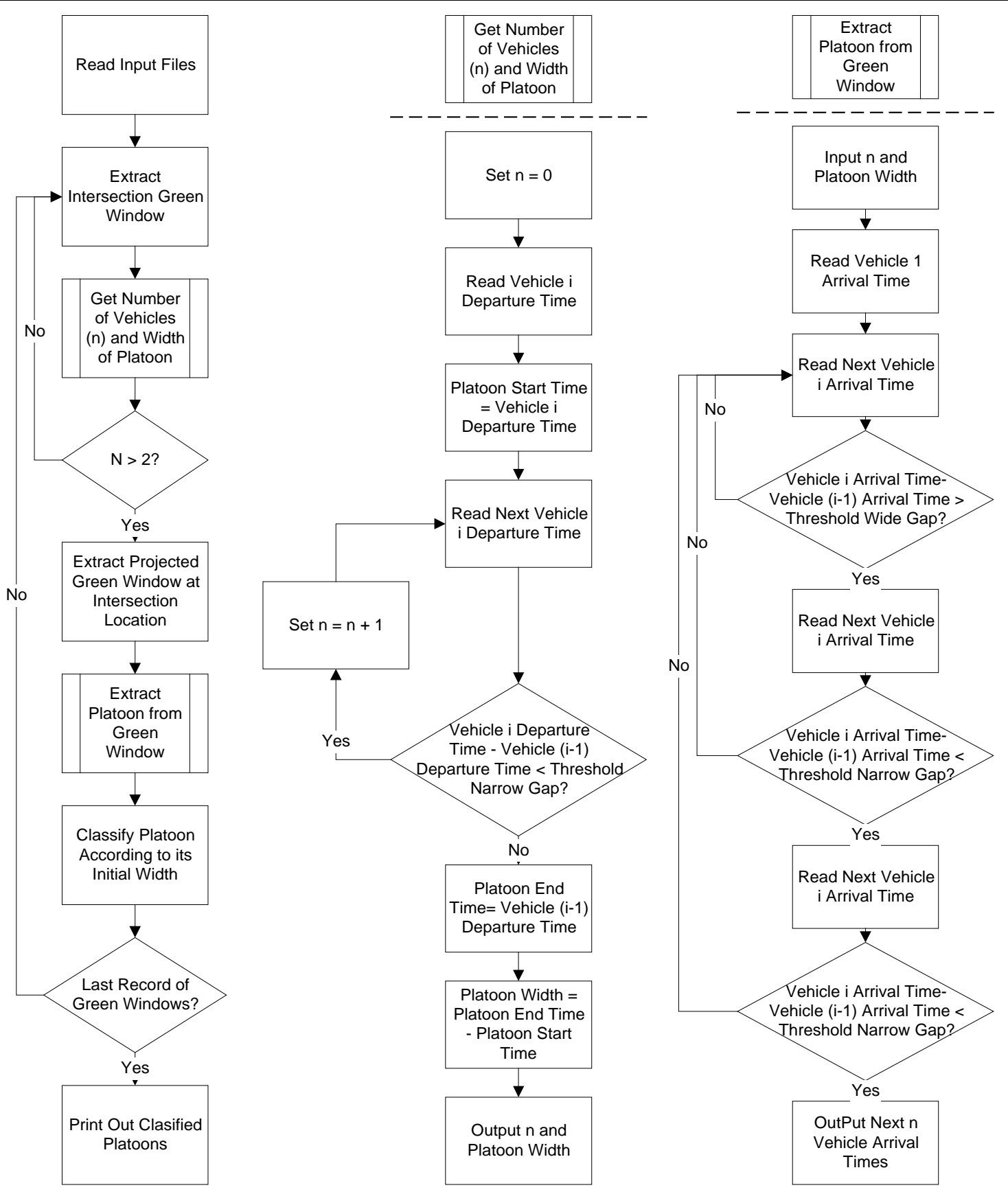

Figure 3-2 Platoon Extraction Algorithm 


\subsection{CORSIM Simulation}

CORSIM simulations were run for different combinations of travel speed and initial platoon discharge lengths that replicated observed field data conditions. Initial platoon discharge predetermined lengths were obtained by putting the main street traffic in an oversaturated condition and setting the main street green time to the required length. This setup insured that the green time would be fully utilized by the waiting vehicles. The arrival time of vehicles at particular downstream distances, at 500 feet intervals were obtained by extracting vehicle positions from the CORSIM animation file. The start of aplatoon was easily identified in the simulation part of the analysis since no right turns on red were allowed in simulation. The headway gap between different platoons was made distinguishable by setting long side-street greens with no turning movement traffic.

\subsection{Robertson's Theoretical Model}

Robertson's model was calibrated for the filed data histograms at each observation point. Histograms were predicted for each pair of $\alpha$ and $\beta$ values. $\alpha$ and $\beta$ were varied over the ranges of 0.0-1.0 and 0.5-1.0 respectively with an increment of 0.01 . The values of $\alpha$ and $\beta$ that produced the minimum sum of square at all downstream locations were picked as the calibrated values. It was observed that very close values of the minimum sum of square could be obtained with different pairs of $\alpha$ and $\beta$. To provide a basis of comparison with earlier research about platoon dispersion, the $\beta$ parameter was fixed at 0.8 and the $\alpha$ parameter was varied to obtain the minimum sum of squares values. The sum of squares for models calibrated for a fixed $\beta$ parameter of 0.8 were not 
observed to vary significantly from those calibrated over the whole range of $\beta$. Calibrated $\alpha$ parameters for $\beta=0.8$ for speeds of 30 and $40 \mathrm{mph}$ were found to be 0.18 and 0.12 respectively. Figure 3-3 shows example comparisons between field data flow profiles and profiles predicted by Robertson's model for different speeds and at different locations. Figure 3-3-a shows the flow profiles at 2,000 feet downstream for $30 \mathrm{mph}$ speed, while Figure 3-3-b shows the flow profiles at 1,500 feet downstream for $40 \mathrm{mph}$ speed. 
$\square$ Robertson Model $\mathbf{\square}$ Field Data

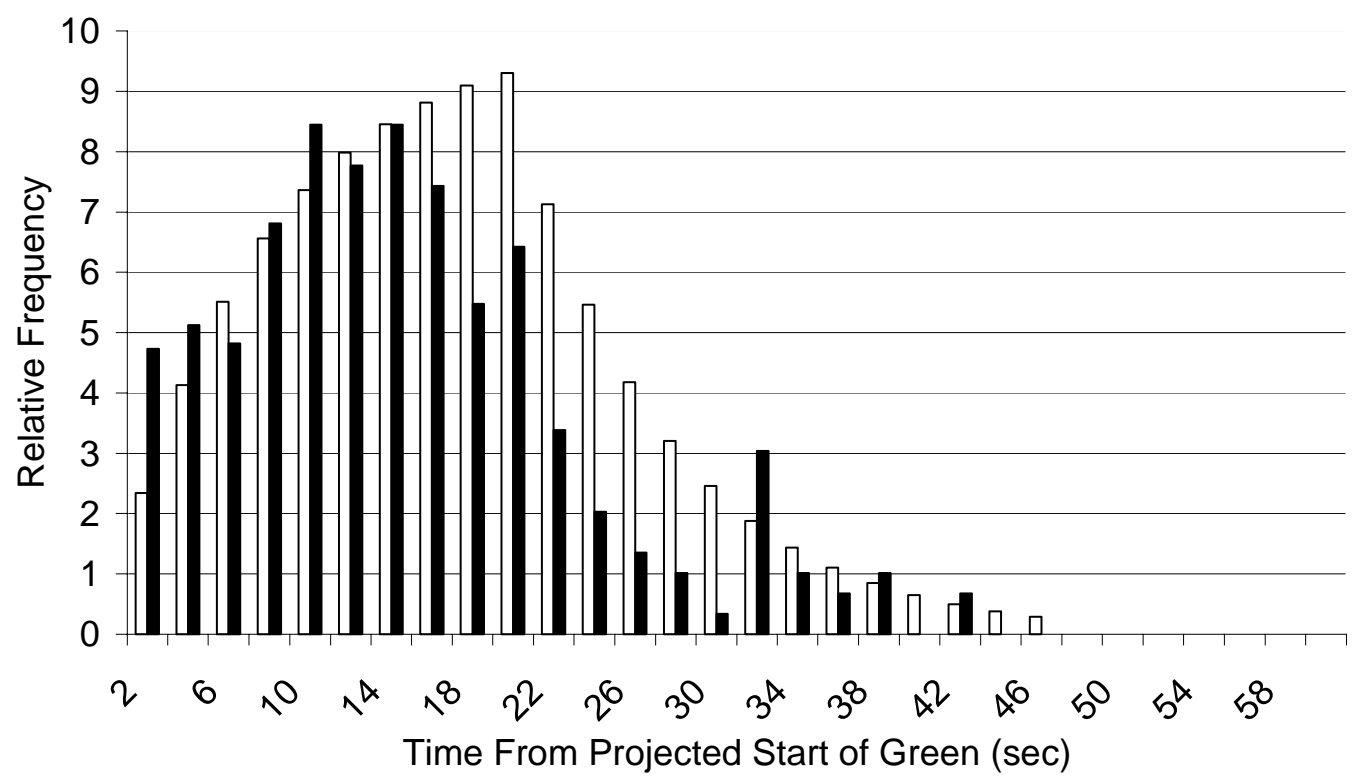

a) Flow Profile at 2000 feet Down Stream, $30 \mathrm{mph}$ speed

$\square$ Robertson Model $\mathbf{\square}$ Field Data

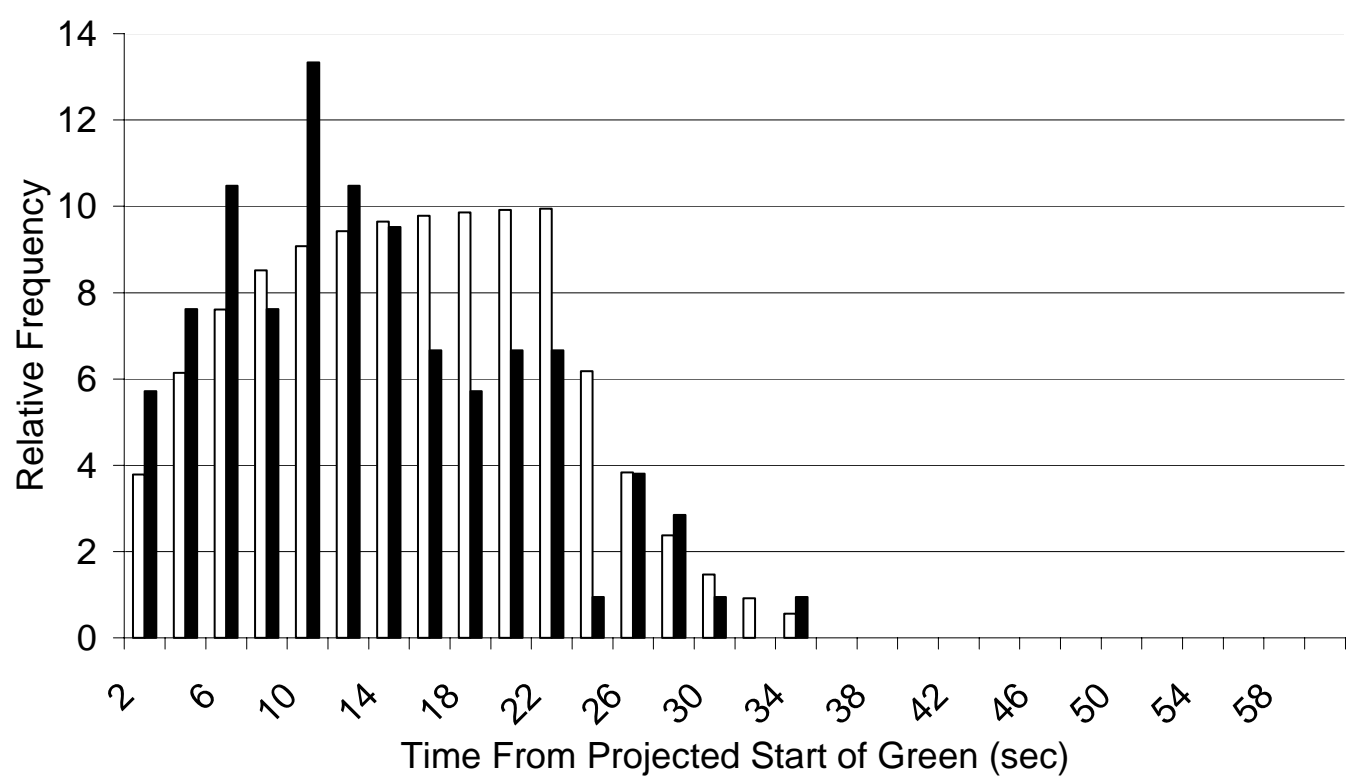

a) Flow Profile at 1500 feet Down Stream, $40 \mathrm{mph}$ speed

Figure 3-3 Flow Profiles of Field Data and as Predicted by Robertson's Model 


\subsection{Determination of Green Windows for Particular Percentage of Platoons}

The green windows needed for particular percentages of a platoon to pass through were determined by analyzing the vehicle arrival times. After the different platoons were identified and classified, the arrival times of vehicles in each platoon were referenced to the arrival time of the start of their platoon. A histogram analysis was then conducted for all the platoons at different downstream locations to determine the percentage of the platoon length arriving at different points in time, measured from the start of the platoon.

The histogram analysis was useful in finding the green window required for different percentages of the platoon at varying downstream distances. The green window needed represents the time required for a certain percentage of the platoon to pass when the first vehicle in the platoon arrives at the start of green at a certain downstream location. For example, $t_{50}$ would represent the time difference between the passage of the $50^{\text {th }}$ percentile vehicle and the first vehicle of the platoon at a certain downstream location.

In order to obtain the percentage of the platoon passing at each cumulative bin time, the histograms were standardized by dividing the number of vehicles in each bin range by the total number of vehicles in the analysis period. The time at which specific percentages of the platoon passed, e.g. $100 \%, 95 \%, 75 \%, 50 \%$, and $25 \%$, passed were then determined by interpolation. 


\subsection{Plots of Green Windows Required by Different Percentages of the Platoon}

Times at which predetermined percentages $(100 \%, 95 \%, 75 \%, 50 \%$, and $25 \%)$ of the platoon passed when the first vehicle in the platoon arrived at the start of green were tabulated and plotted versus several downstream locations. The plots were produced for 30 and $40 \mathrm{mph}$ posted speed limits and an initial platoon discharge of 20 seconds. A regression analysis was then conducted for arrival times for the specified percentages of the platoon versus the downstream distance, and resulting regression models were used to plot the relationships. For example, the time at which $75 \%$ of the platoon passed could be determined by the regression model:

$$
t_{75}=a_{75}+b_{75} X
$$

Where:

$$
\begin{aligned}
& \mathrm{a}_{75}=\text { intercept for } 75 \% \text { of platoon model, } \\
& \mathrm{b}_{75}=\text { linear coefficient for } 75 \% \text { of platoon model, } \\
& \mathrm{t}_{75}=\text { time at which } 75 \% \text { of the platoon pass, } \\
& \mathrm{X}=\text { downstream distance in thousand feet. }
\end{aligned}
$$

Table 3-2 summarizes the regression analysis coefficients for the reduced data, the CORSIM simulation, and the histograms produced by Robertson's model. Figure 3-4, Figure 3-5, and Figure 3-6 show plots of average values of raw data with regression lines for the observed field data, the CORSIM data, and the histograms produced by Robertson's model respectively. 
Table 3-2 Regression Analysis Coefficients for Field Data, CORSIM Simulation, and Robertson's Model

\begin{tabular}{|c|c|c|c|}
\hline \multirow{2}{*}{$\begin{array}{l}\text { Green Window required to pass } \\
\mathrm{X} \% \text { of the platoon }\left(t_{x}\right)\end{array}$} & \multicolumn{2}{|c|}{ Equation $t=a+b X$ Coefficients } & \multirow[t]{2}{*}{ R Squared Value } \\
\hline & $\mathrm{A}$ & $\mathrm{b}$ & \\
\hline \multicolumn{4}{|c|}{ a) Field Data (20 second saturated platoon, Speed: $30 \mathrm{MPH})$} \\
\hline 100 & 20.00 & 10.24 & 0.92 \\
\hline 95 & 19.00 & 6.29 & 0.85 \\
\hline 75 & 15.00 & 1.42 & 0.85 \\
\hline 50 & 10.00 & 0.5 & 0.64 \\
\hline 25 & 5.00 & 1.0 & 0.64 \\
\hline \multicolumn{4}{|c|}{ b) Field Data (20 second saturated platoon, Speed: $40 \mathrm{MPH}$ ) } \\
\hline 100 & 20.00 & 9.41 & 0.93 \\
\hline 95 & 19.00 & 4.90 & 0.92 \\
\hline 75 & 15.00 & 1.55 & 0.76 \\
\hline 50 & 10.00 & 0.31 & 0.73 \\
\hline 25 & 5.00 & 0.22 & 0.78 \\
\hline \multicolumn{4}{|c|}{ c) CORSIM Simulation (20 second saturated platoon, Speed: $30 \mathrm{MPH}$ ) } \\
\hline 100 & 20.00 & 12.34 & 0.98 \\
\hline 95 & 19.00 & 10.12 & 0.99 \\
\hline 75 & 15.00 & 9.09 & 0.99 \\
\hline 50 & 10.00 & 8.24 & 0.98 \\
\hline 25 & 5.00 & 6.02 & 0.94 \\
\hline \multicolumn{4}{|c|}{ d) CORSIM Simulation (20 second saturated platoon, Speed: $40 \mathrm{MPH}$ ) } \\
\hline 100 & 20.00 & 10.74 & 0.99 \\
\hline 95 & 19.00 & 8.25 & 0.99 \\
\hline 75 & 15.00 & 7.35 & 0.99 \\
\hline 50 & 10.00 & 6.69 & 0.99 \\
\hline 25 & 5.00 & 5.13 & 0.99 \\
\hline \multicolumn{4}{|c|}{ a) Robertson's Model (20 second saturated platoon, Speed: $30 \mathrm{MPH}$ ) } \\
\hline 100 & 20.00 & 13.54 & 0.99 \\
\hline 95 & 19.00 & 6.95 & 0.98 \\
\hline 75 & 15.00 & 3.28 & 0.99 \\
\hline 50 & 10.00 & 2.73 & 0.99 \\
\hline 25 & 5.00 & 2.25 & 0.98 \\
\hline \multicolumn{4}{|c|}{ b) Robertson's Model (20 second saturated platoon, Speed: $40 \mathrm{MPH})$} \\
\hline 100 & 20.00 & 6.57 & 0.99 \\
\hline 95 & 19.00 & 3.11 & 0.97 \\
\hline 75 & 15.00 & 1.56 & 1.00 \\
\hline 50 & 10.00 & 1.46 & 0.99 \\
\hline 25 & 5.00 & 1.24 & 0.99 \\
\hline
\end{tabular}




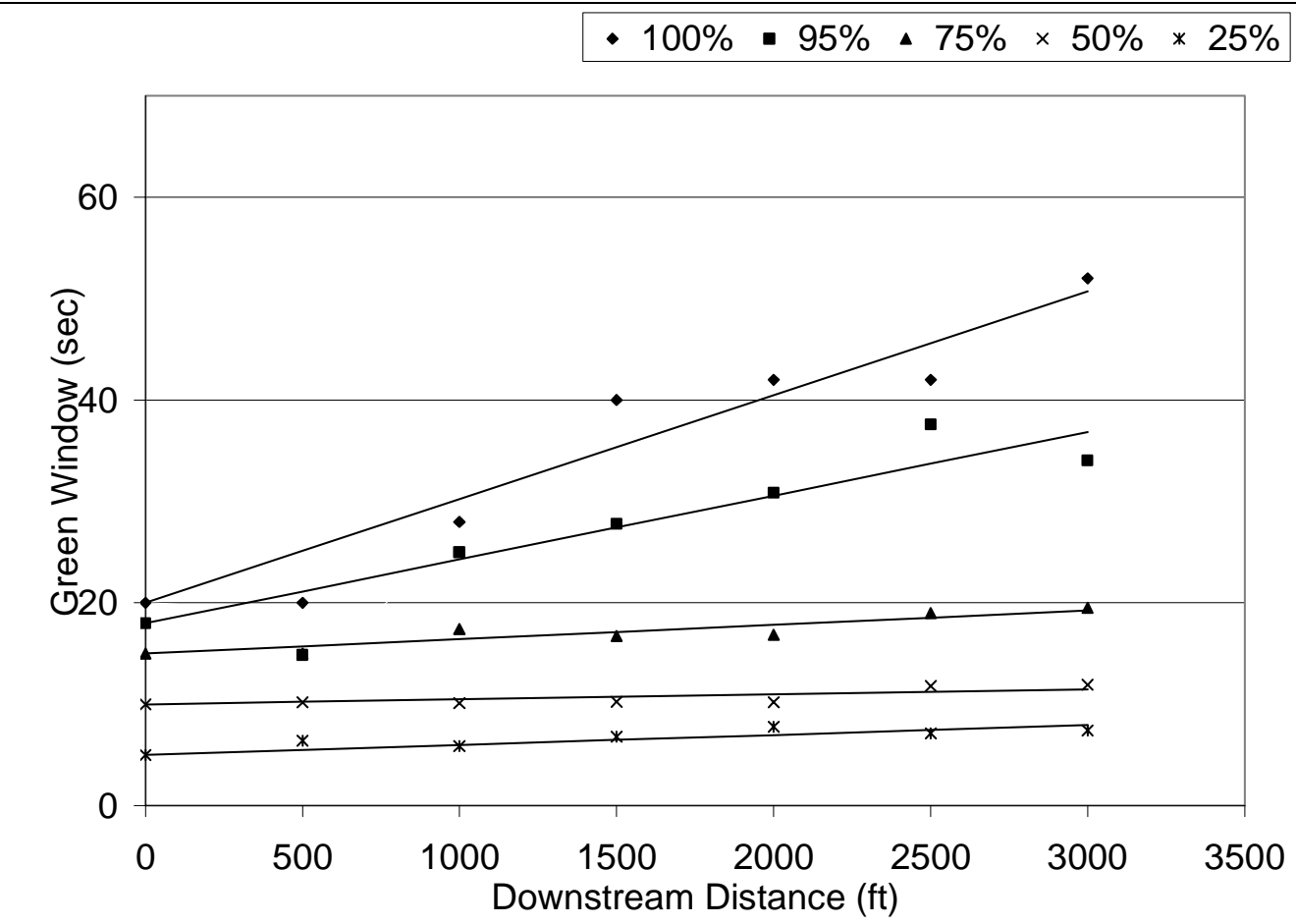

a) $30 \mathrm{mph}$ speed

- $100 \% \cdot 95 \%$ - $75 \% \times 50 \% * 25 \%$ - Series 9

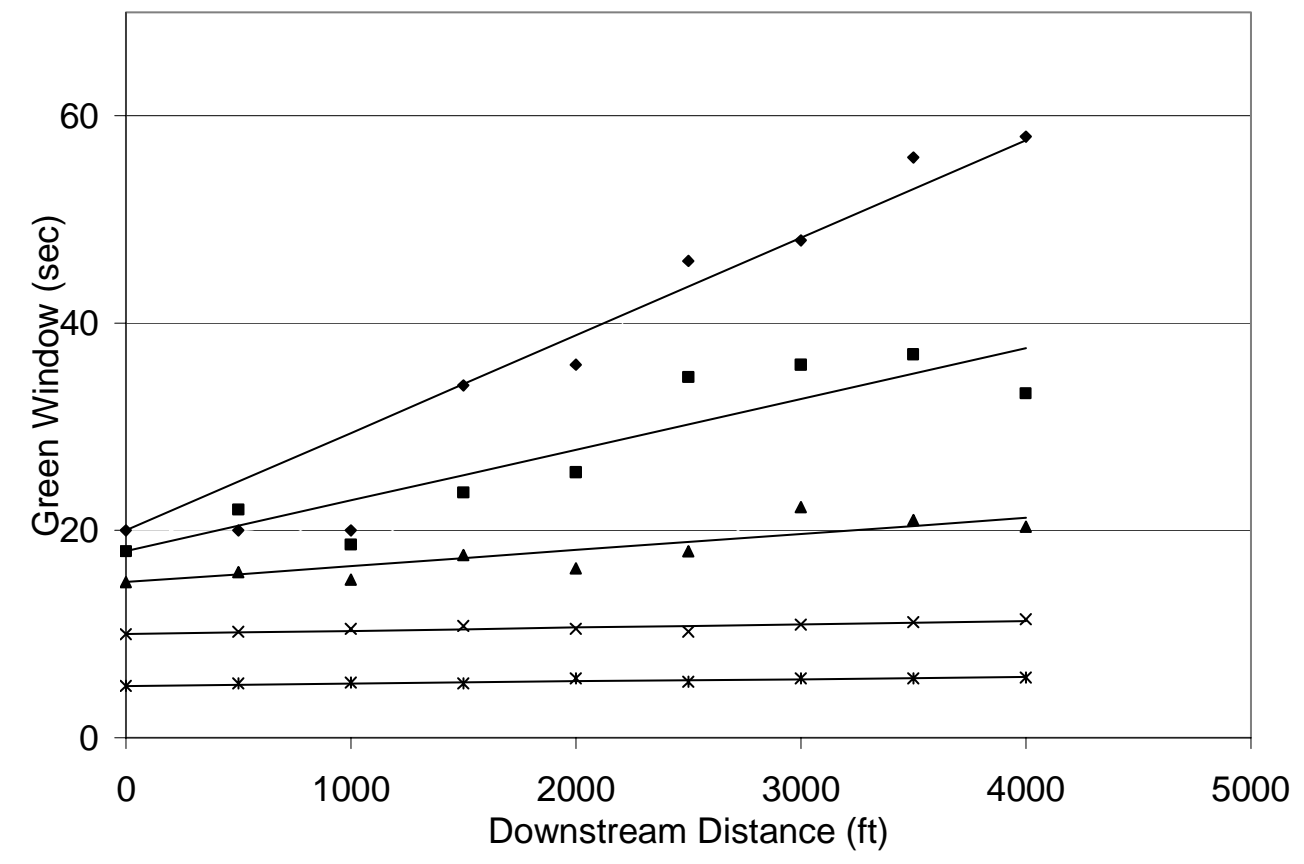

b) $40 \mathrm{mph}$ speed

Figure 3-4 Field Data Platoon Distribution (20 Second Saturated Platoon) 


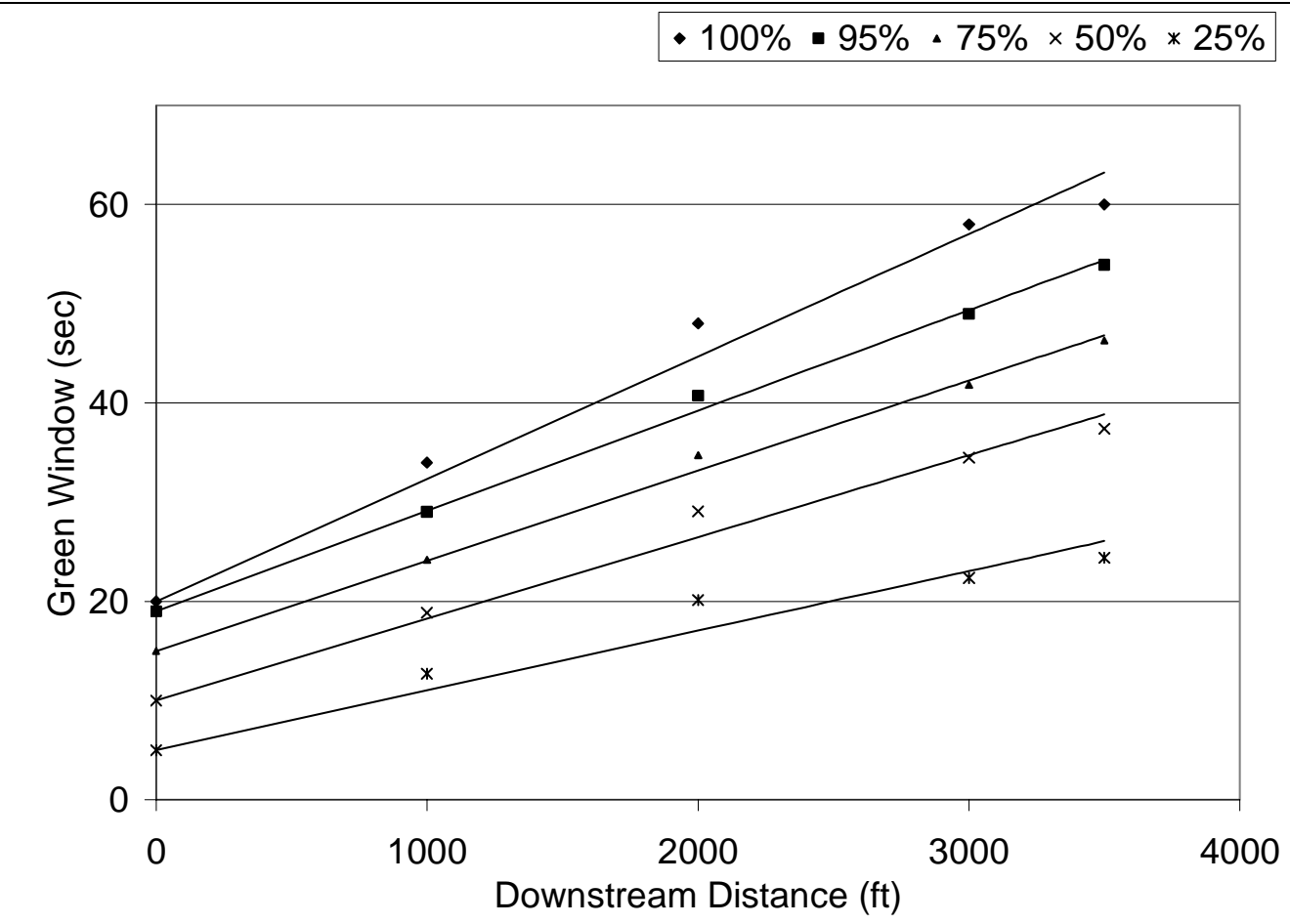

a) $30 \mathrm{mph}$ speed

$\cdot 100 \% \cdot 95 \% \cdot 75 \% \times 50 \% * 25 \%$

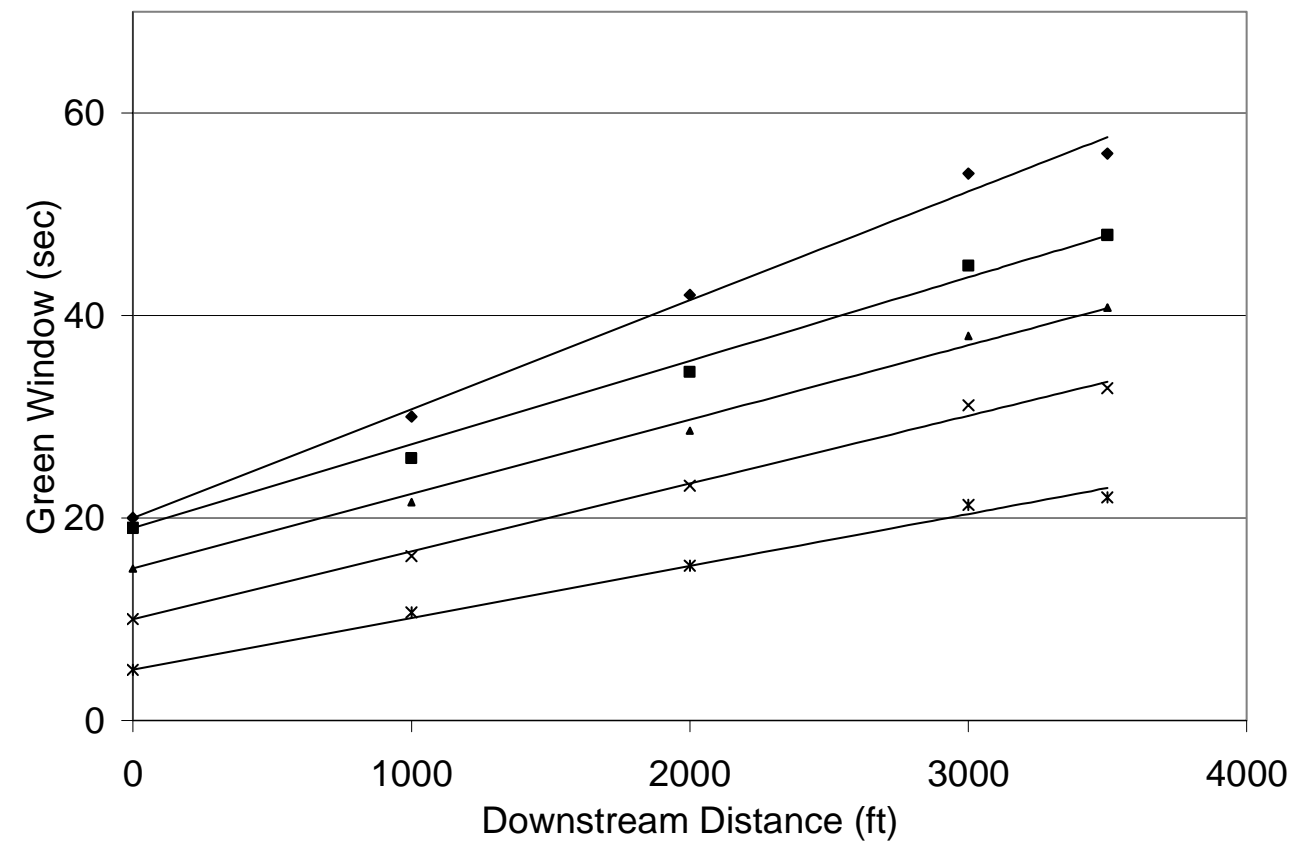

b) $40 \mathrm{mph}$ speed

Figure 3-5 CORSIM Simulation Platoon Distribution (20 Second Saturated Platoon) 


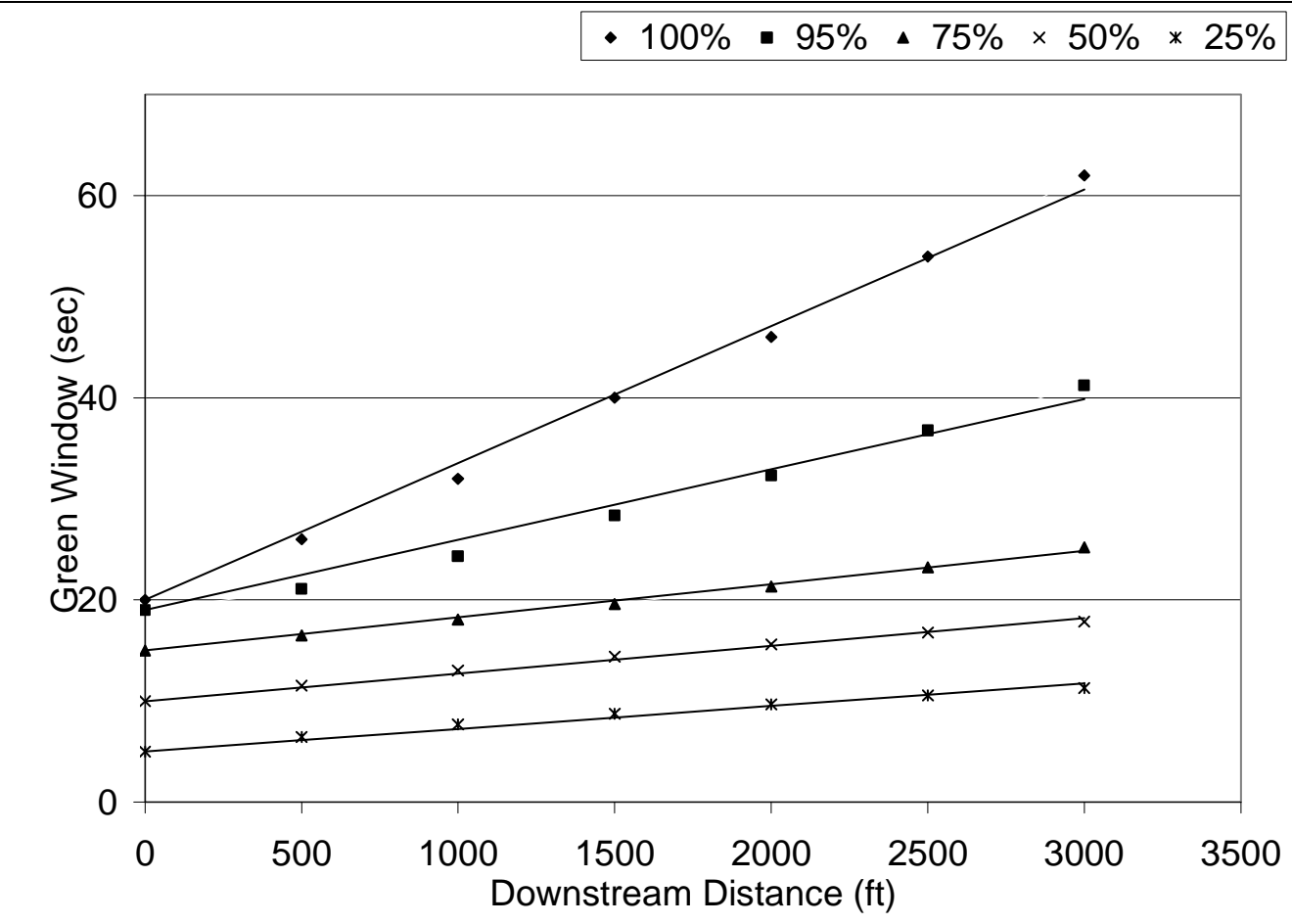

a) $30 \mathrm{mph}$ speed

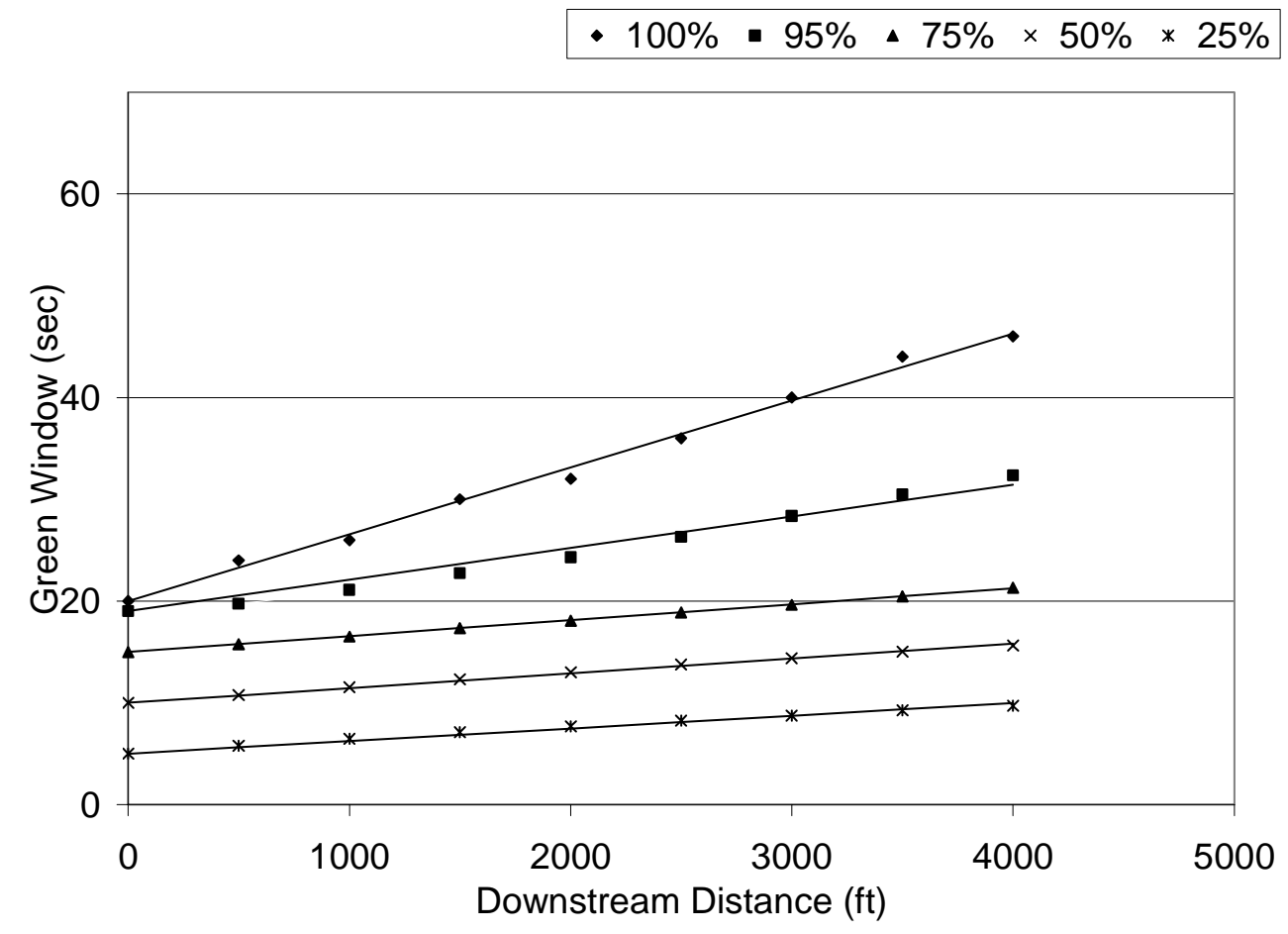

b) $40 \mathrm{mph}$ speed

Figure 3-6 Robertson's Model Platoon Distribution (20 Second Saturated Platoon) 


\subsection{Discussion}

Platoons with a saturated discharge of 20 seconds were analyzed because the best sample size distribution was obtained for the field data collected for that discharge, which is illustrated in Table 3-1.

CORSIM simulation demonstrated an overall platoon dispersion similar to the field data, as can be seen by examining Figure 3-4 and Figure 3-5. However, CORSIM simulation tended to have more platoon dispersion in the beginning of the platoon when compared to the field data. For example, Figure 3-5-a and Figure 3-5-b show that the lines for $25 \%$ of the platoon are much higher than their equivalents in both Figure 3-4-a and Figure 3-4-b. This trend is probably due to the car following logic in the CORSIM simulation that tends to aggressively disperse the front vehicles in the platoon. This logic makes CORSIM simulation somewhat conservative in quantifying the benefits of coordinating downstream signals for smaller green windows downstream as it suggests that a smaller percentage of the platoon benefits from that coordination.

Since Robertson's model was calibrated for the same set of data, graphs of that model were very similar to those of the field data. However, it should be noted that when the $\alpha$ parameter increases, Robertson's model predicts that most of the dispersion occurs at the end of the platoon. This can be observed when comparing Figure 3-6-a to Figure 3-6-b in that the lines for 95\% and $100 \%$ of the platoon in Figure 3-6-a are much higher than their equivalents in Figure 3-6-b. When studying Figure 3-3-a and Figure 3-3-b, which have $\alpha$ values of 0.18 and 0.12 respectively, one can observe that higher values of the $\alpha$ parameter cause platoon tails to extend much longer than platoon beginnings. This 
phenomenon makes Robertson's model conservative in quantifying the benefits of coordinating downstream signals for large green windows downstream with higher $\alpha$ values as it predicts that a smaller percentage of the platoon benefits from that coordination.

\subsection{Chapter's Conclusion}

This chapter compared field platoon profiles to those produced by simulation and Robertson's model. Graphs showing arrival times of different percentages of platoons relative to the first vehicle in the platoon were produced for field, simulation, and Robertson's model. Also, these graphs were presented to quantify the percentage of a platoon that can pass through a particular green window downstream if the first vehicle of the platoon arrived at the start of the green according to each model's parameters. Such graphs can provide valuable information when evaluating the benefits from coordinating two traffic signals, by quantifying the percentage of a platoon that would benefit of a particular green window provided at the downstream intersection. Alternatively, they may suggest the green time required at the downstream intersection if a certain percentage of a platoon's passage was desired.

When compared to the field platoon data, the CORSIM was observed to be more conservative in quantifying the benefit of traffic signal coordination for small green windows. This observation suggests that the CORSIM platoon dispersion model need to be calibrated before analysis of different coordination plans is conducted. The following 
chapter discusses the calibration of CORSIM platoon dispersion model in order to evaluate different coordination plans. 


\section{CHAPTER 4- CALIBRATION OF CORSIM PLATOON DISPERSION MODEL}

\subsection{Introduction}

The CORSIM model is one of the most widely-used simulation programs in the U.S. and is used quite often for analyzing ITS projects involving traffic signal systems. Although the calculations of total delay and vehicular stops in the arterial systems depend on the platoon dispersion parameters, very little is explained about calibrating the CORSIM platoon dispersion model in the literature. The objective of this chapter is to 1) quantitatively show the impact of dispersion on the results generated by CORSIM and 2) contribute to the platoon dispersion calibration literature by showing a systematic approach for calibrating the CORSIM platoon dispersion model. The dispersion factor calculations are demonstrated using both speed study analysis and Pacey's model.

\subsection{Diffusion Theory}

As a platoon of vehicles moves downstream of traffic signals, it diffuses and increases in length. This phenomenon significantly affects the benefits that can be achieved by coordinating traffic signals. The basic assumption of the diffusion theory [Pacey, 1956] is that the speeds of the cars in the platoon are distributed normally so that the spread of the platoon can be described by the dispersion of car speeds. Pacey's model had some simplifications, such as 1) it allows free passing of cars; 2) it assumes that the 
speed of the car is constant and 3) it assumes that speed of the car is independent of its location in the platoon. Nevertheless, Pacey was able to show that his model gave a good fit to moderate traffic volume data. This can be explained by considering that the platoon behaves as if it diffused from an extrapolated initial condition in accordance with the kinematical model [Muriel and Renfrey, 1985]. Seddon [1972] summarized the diffusion theory with the formula:

$$
q_{2}(j)=\sum_{i} q_{1}(i) g(j-i)
$$

Where $\mathrm{i}$ and $\mathrm{j}$ are counts of discrete intervals of time at the first and second observation points respectively, i.e, the flow at the jth interval at the second point is equal to the sum over all the values of $i$, of the flow at the ith interval at the first observation location multiplied by the probability of a travel time of $(j-i)$ intervals.

\subsection{Calculation of Dispersion Factor from Speed Study}

The dispersion factor $(\alpha)$ can be defined as the standard deviation of the free flow speed divided by the mean free flow speed [Seddon, 1972]. The dispersion factor can be directly calculated if a speed study is conducted between two observation locations and each car was identified. Figure 4-1 below shows some platoon data analysis that was conducted on SR26 in Lafayette, Indiana. Careful investigation of the platoon data allowed correlation of the arrival time of each individual car. In such a case, mean free flow speed and standard deviation of free flow speed could be easily calculated. The $\alpha$ 
parameter could then be calculated as the standard deviation divided by the mean of the free flow speed. In the SR26 case, the $\alpha$ parameter was calculated as 0.14 .

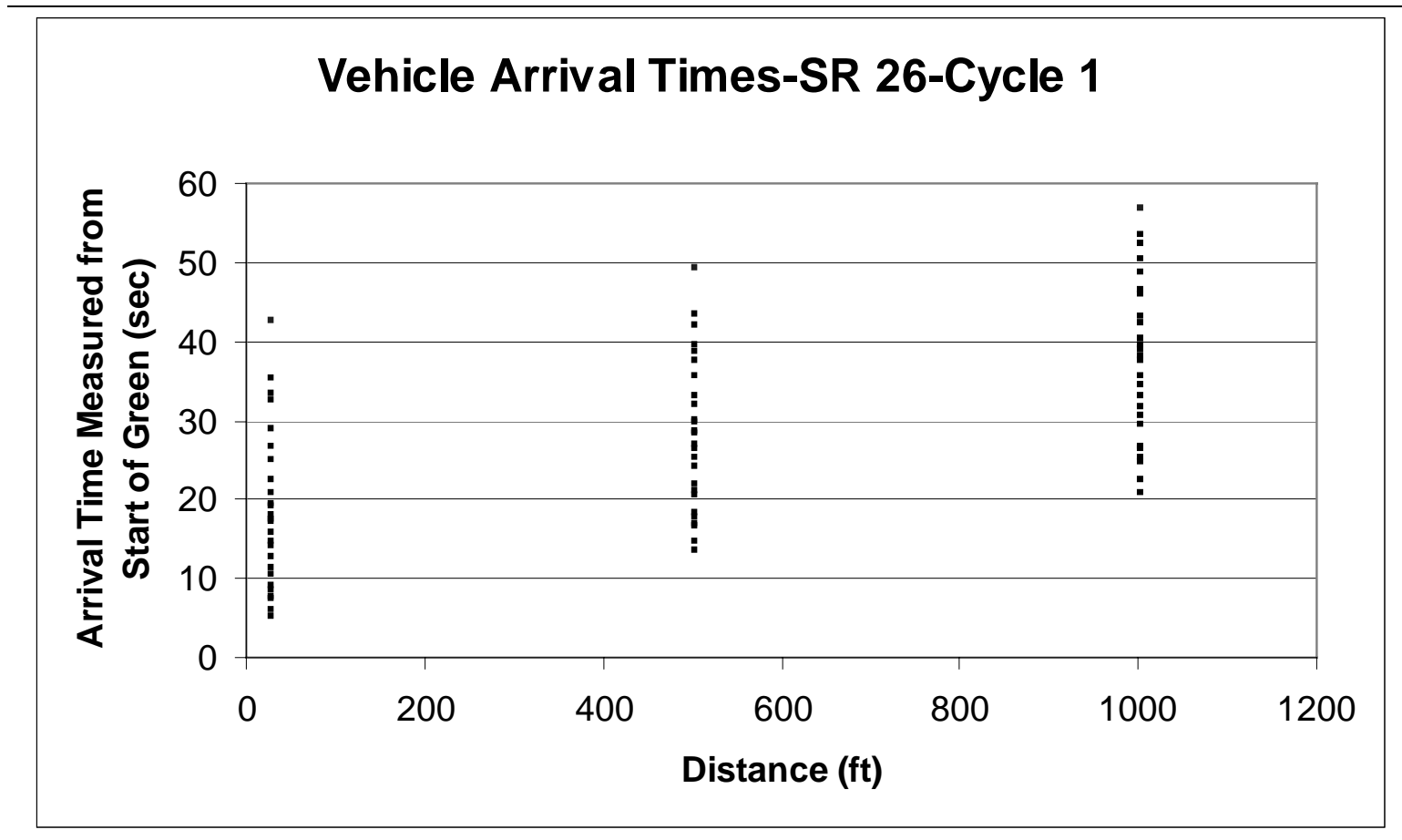

Figure 4-1 Platoon Dispersion Data

\subsection{Calculation of Dispersion Factor Using Pacey's Model}

Alternatively, if the arrival time of each car could not be identified, the diffusion equation could be used to estimate the mean speed and the standard deviation. The mean speed and standard deviation of the arrival profiles are identified as the mean and standard deviation that correspond to the least mean square error between the expected and observed profile at the second observation location, as can be seen in Figure 4-2. The $\alpha$ parameter is then calculated as the standard deviation divided by the mean of the free 
flow speed as previously defined. Pacey's model was used to find the dispersion factor for a site in US3 in Kokomo, Indiana, which was found to be 0.03 . US31 will be studied in Chapter 10.

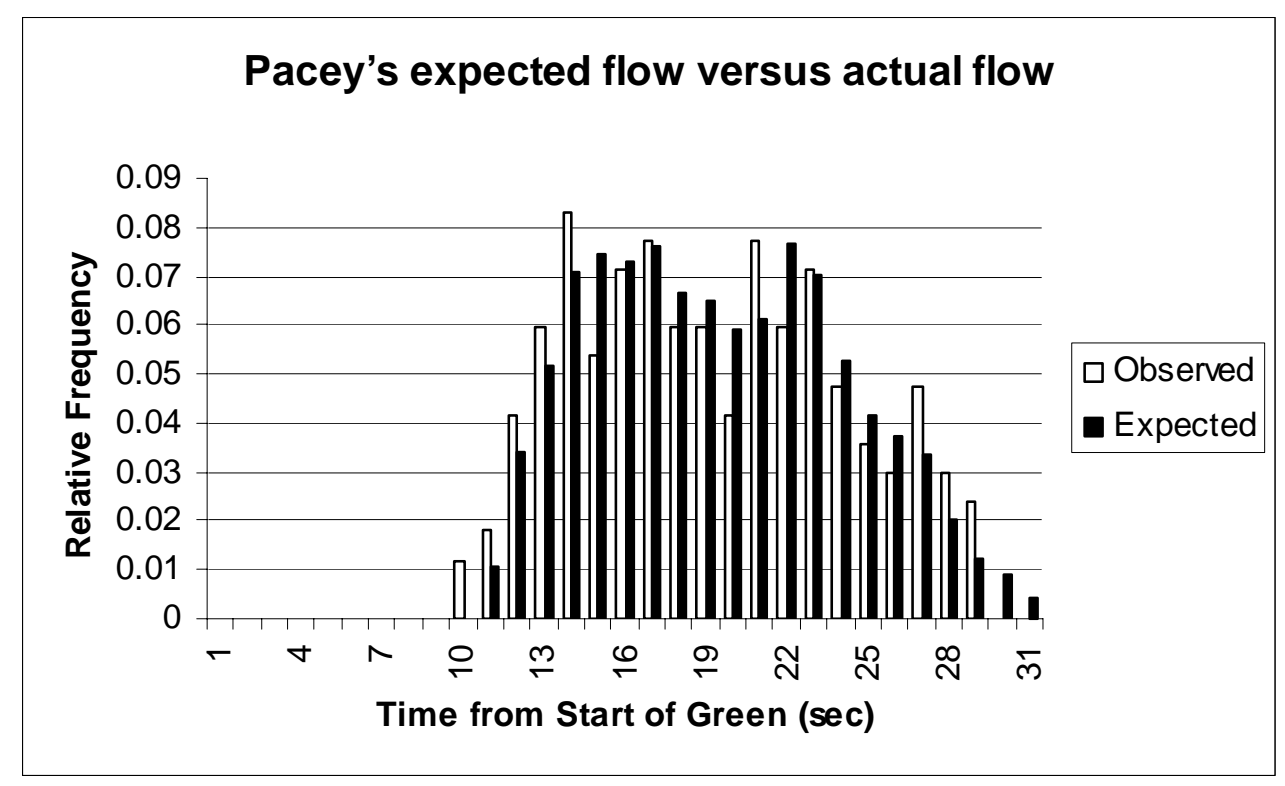

Figure 4-2 Pacey's Expected Flow versus Actual Flow

\subsection{Conversion of the Normal Distribution Into A Decile Distribution}

CORSIM uses a decile distribution to assign free flow speeds to vehicles entering the network. The decile distribution used is based on the types of drivers of the vehicles, where the first decile corresponds to the least aggressive driver and the last decile is the most aggressive. The free flow speed of each vehicle is determined by multiplying the decile value assigned to the vehicle's driver type times the mean free flow speed for the link. The decile value assigned to each driver type is specified in record type 147 in the 
simulation file. The record has default values ranging between $75 \%$ and $127 \%$ of the mean free flow speed.

In order to transform the normal distribution of the free flow speed into a decile distribution, each 10 percentile of the normal distribution curve is replaced with the center of gravity of the percentile area, which was calculated using numerical integration. Each percentile area was divided into 1,000 segment, and the area was calculated by multiplying each segment by the density function, where the center of gravity for each segment was calculated by multiplying the segment by its $\mathrm{x}$-axis value. The center of gravity of the area was then calculated by dividing the sum of gravity of the 1,000 segments by the total area. Since the center of gravity of the percentile depends on the dispersion factor, the center of gravity for each percentile area was calculated for four different platoon dispersion factors $(\alpha)(5,10,15$, and 20$)$ and a regression analysis was conducted to obtain an equation for calculation of the center of gravity for each percentile. The ten percentiles' center of gravity can then be entered in record 147 in the CORSIM simulation file as the decile distribution for the free flow speed.

Although the normal distribution shape implies that the center of gravity equation is a curvilinear function, the increase of the center of gravity in relation to the dispersion factor $(\alpha)$ was found to be linear with an $R^{2}$ of 1 . Equation (4-2) shows the general equation for the decile distribution where the $\beta$ parameter values for each of the 10 decile values are shown in Table 4-1.

$$
\operatorname{UFPCT}(I)=100+\beta_{I} * \alpha
$$


Where

UFPCT (I): Free-Flow speed percentage for driver type I,

$\beta_{\mathrm{I}}$ : Linear parameter value for driver type I,

and $\alpha$ is the dispersion factor in percent

Table 4-1 $\beta$ Parameter Values for Computing the Decile Distribution-UFPCT(I)

\begin{tabular}{|l|l|l|l|l|l|l|l|l|l|l|}
\hline $\mathrm{I}$ & 1 & 2 & 3 & 4 & 5 & 6 & 7 & 8 & 9 & 10 \\
\hline$\beta$ & -1.745 & -1.044 & -0.677 & -0.386 & -0.126 & 0.126 & 0.387 & 0.677 & 1.045 & 1.760 \\
\hline
\end{tabular}

Figure 4-3 shows the speed decile distribution for two sites, SR26 and US31, in Indiana and compares them to the CORSIM default decile distribution. 


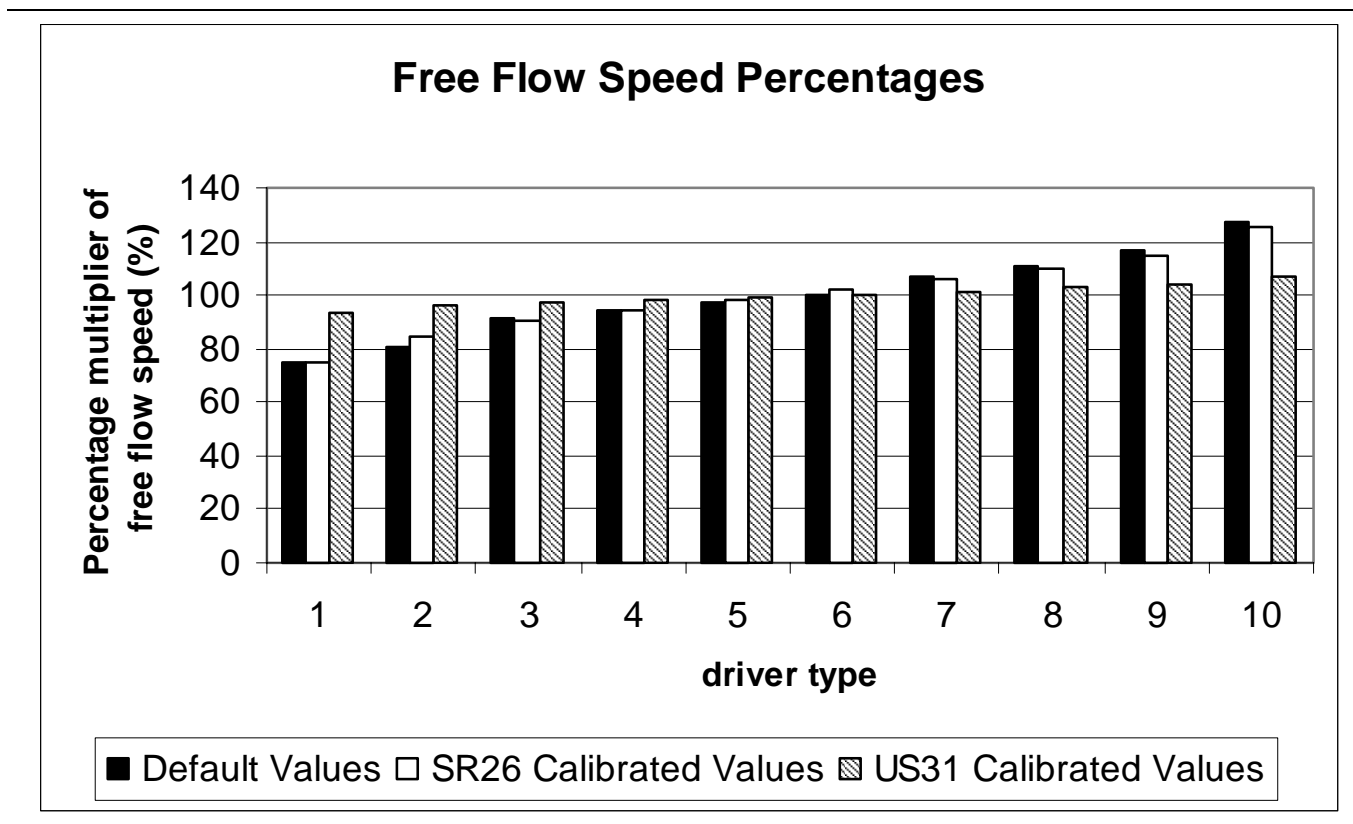

Figure 4-3 CORSIM Speed Distribution Parameters

\subsection{Effect of Platoon Dispersion on Signal Coordination Benefits}

Platoon dispersion significantly affects the benefits attained by coordinating traffic signals. Figure 4-4 shows the flow profile at several locations downstream for a traffic signal with both the default and calibrated speed distributions. Figure 4-4-a through Figure 4-4-d show the flow profile at different downstream locations when CORSIM default parameters are used. Figure 4-4-e and Figure 4-4-f show the flow profile calibrated for the US31 site at the stop bar and 3,000 feet downstream. By comparing Figure 4-4-d and Figure 4-4-f, it can be observed that the flow profiles at 3,000 feet are significantly different when using the calibrated parameters versus the default ones. 


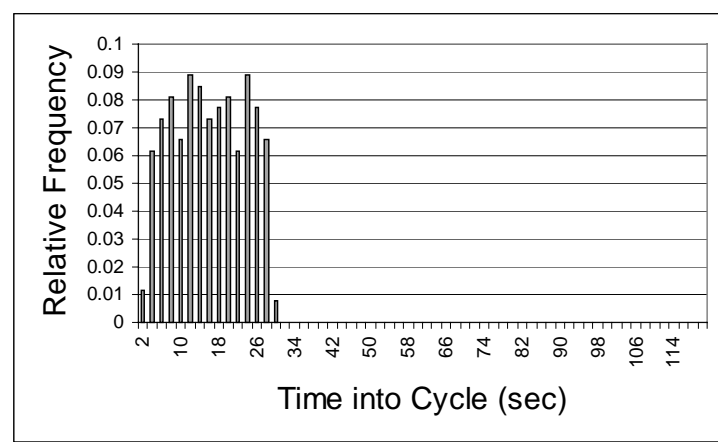

a) Stop bar-Default Speed Distribution

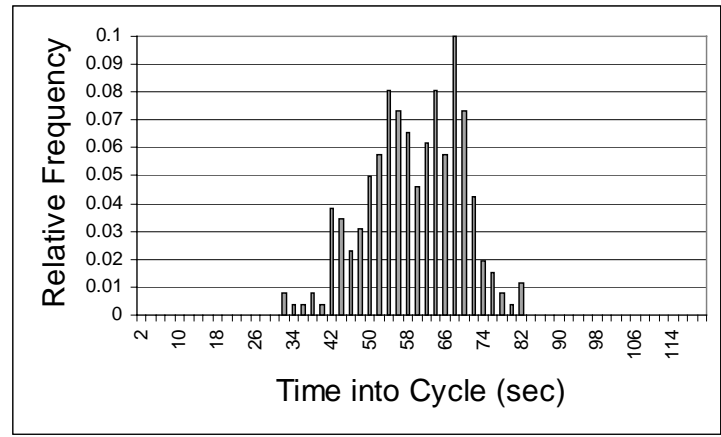

c) 2000 feet Downstream-Default Speed Distribution

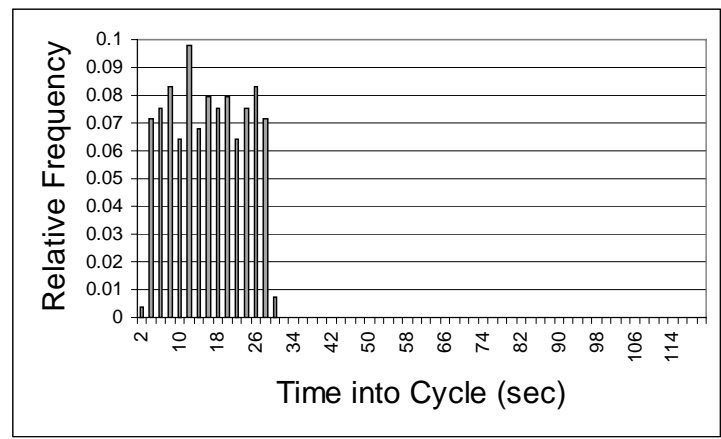

e) Stop bar-Calibrated Speed Distribution

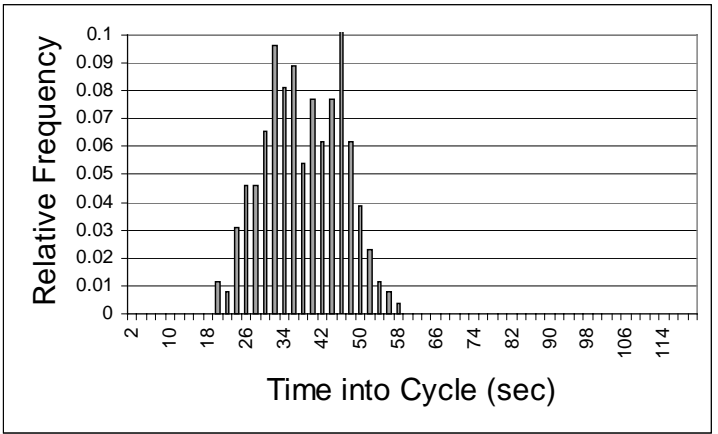

b) 1000 feet Downstream-Default Speed Distribution

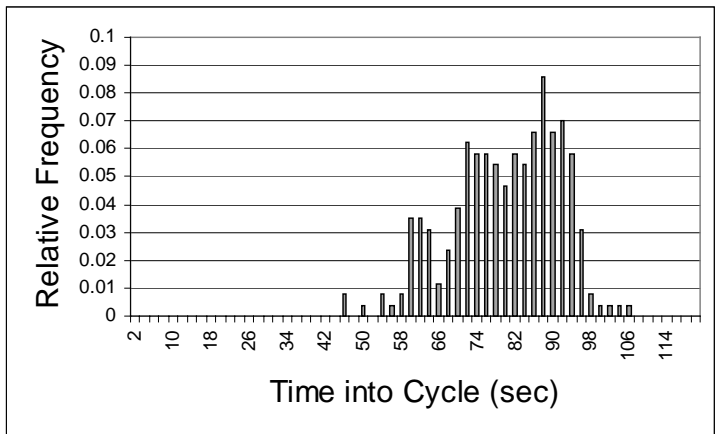

d) 3000 feet Downstream- Default Speed Distribution

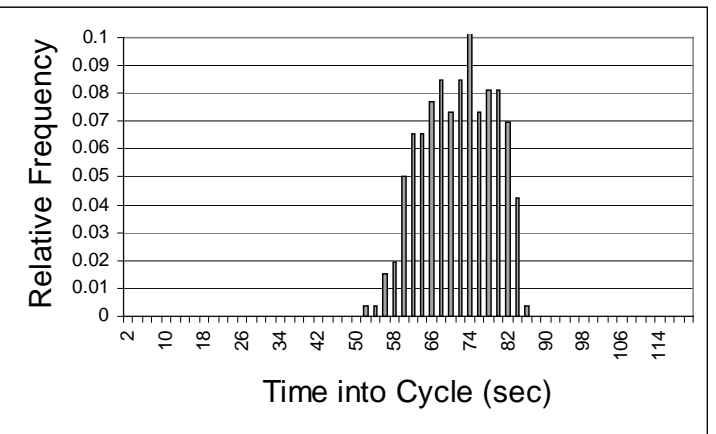

f) 3000 feet Downstream-Calibrated Speed Distribution

Figure 4-4 Flow Profile at Different Downstream Locations

To study the effect of platoon dispersion on signal coordination benefits, the travel time along the corridor was used as a measure of performance for the coordinated traffic signal system (10). Figure 4-5 shows the tabulated CORSIM output for SR26 with 
the default speed parameters and with the calibrated value, while Figure 4-6 shows the results for US31. It should be noted that the results differed significantly for US31 case, but no significance difference was found for SR 26. This is due to the fact that the calibrated parameters for SR 26 were very close to the default values (Figure 4-3). These results illustrate that it is very important to calibrate the parameters before a conclusion regarding signal coordination can be drawn.

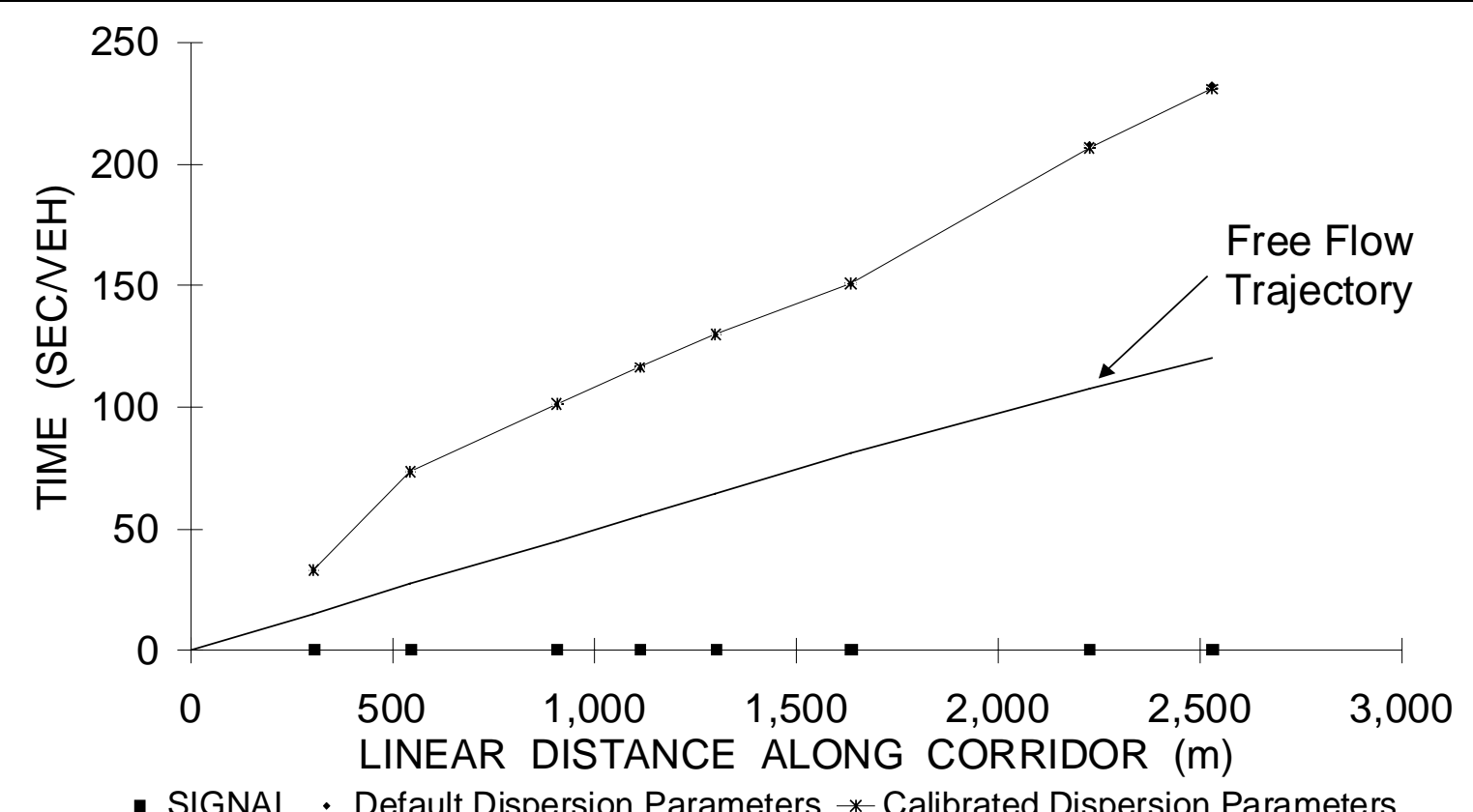

Figure 4-5 SR26 Travel Time With Calibrated Versus Default Distribution 


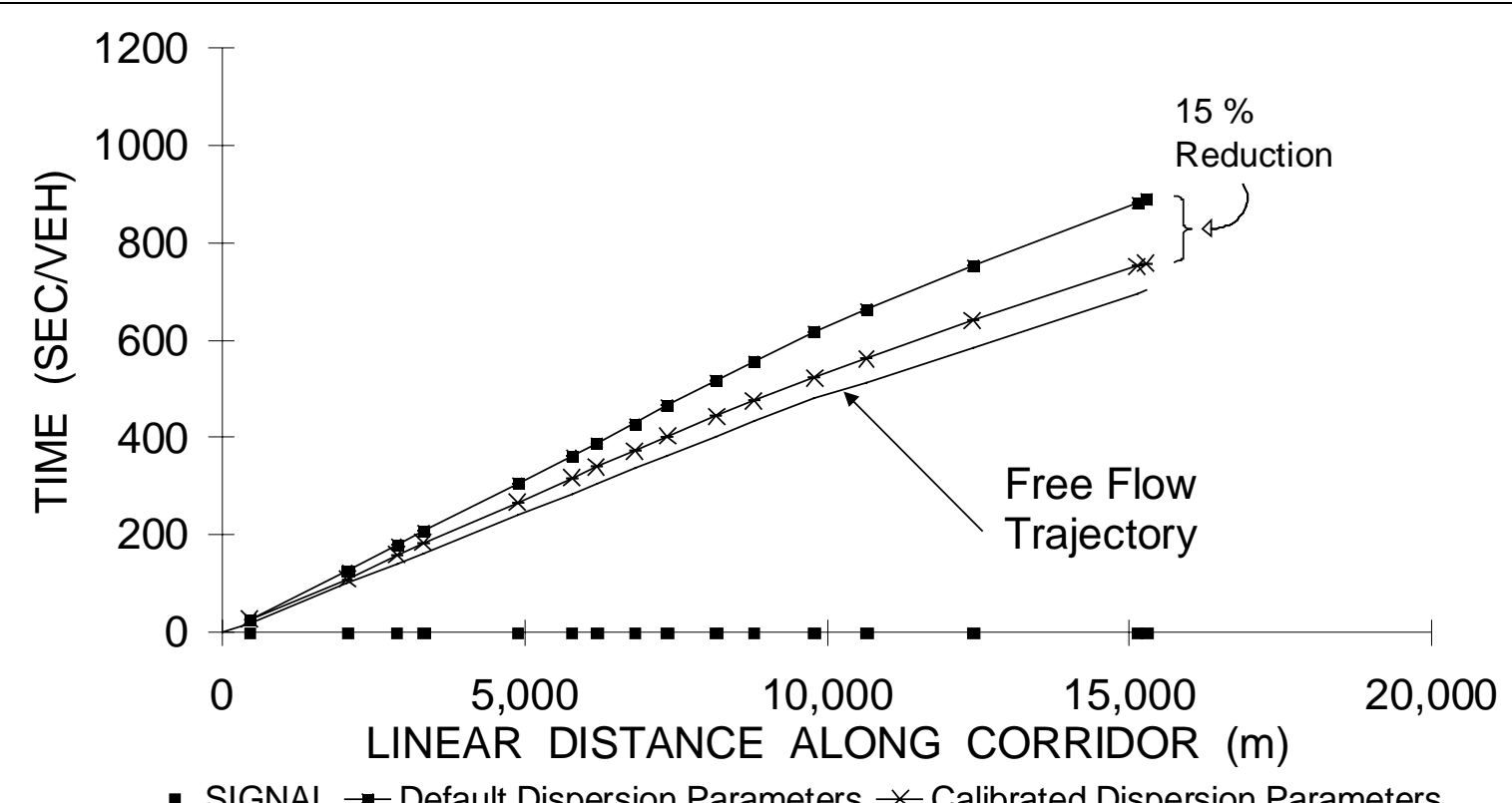

- SIGNAL $\rightarrow$ Default Dispersion Parameters $*$ Calibrated Dispersion Parameters

Figure 4-6 US31 Travel Time with Calibrated Versus Default Distribution

\subsection{Chapter's Conclusion}

This chapter discussed the importance of calibrating the CORSIM platoon dispersion model and demonstrated two methods by which the platoon dispersion factor $(\alpha)$ can be calculated, from speed study data or alternatively by using Pacey's formula. Once the dispersion factor is calculated, the decile distribution used by CORSIM to replicate platoon dispersion can be calculated using Equation (4-2) and Table 1. The chapter also showed the effect of calibrating the CORSIM model by comparing the results of a calibrated model versus a model using default dispersion values for two sites in Indiana (SR26 and US31). The US31 study showed that the effect of platoon 
dispersion calibration in CORSIM resulted in a $15 \%$ reduction in travel time in comparison to the default calibration values. 


\section{CHAPTER 5- RELATIONSHIP BETWEEN OFFSETS AND SHOCKWAVES}

This chapter lays the theoretical and conceptual foundations for the algorithm developed in this report providing an overview of the shockwave theory and how it relates to the platooning phenomenon and the offset at the downstream signalized intersection. The conceptual foundation of this chapter is carried through Chapters 6 and 7, where new metrics are defined and used to capture the effects of shockwaves using upstream detector actuation.

\subsection{Overview of Shockwaves}

Several researchers and engineers have studied the traffic flow-density-speed relationship and have provided insights into the phenomenon [May, 1990; Lighthill and Whitham, 1955]. When traffic conditions change in time or space, boundaries demarking different flow states in the time-space domains are formed. Those boundaries are called shockwaves, and there are several situations where shockwaves form including the increase in traffic demand, lane drops, incidents, etc.

Figure 5-1-a shows the fundamentals of shockwave analysis as illustrated by May [1990]. State A in the figure represents a steady-state flow with flow $\left(q_{a}\right)$, density $\left(k_{a}\right)$, and speed $\left(\mathrm{u}_{\mathrm{a}}\right)$. If the traffic volume decreases after some period of time, its state will be represented by state B with flow $\left(\mathrm{q}_{\mathrm{b}}\right)$, density $\left(\mathrm{k}_{\mathrm{b}}\right)$, and speed $\left(\mathrm{u}_{\mathrm{b}}\right)$. Note how the new state 
will have a higher speed because it has a lower density and vehicles will travel more freely. The traffic of state B will eventually catch up with the traffic of state A.

Figure 5-1-b shows a space-distance diagram of the two conditions. Note that, for illustration purposes, the scales of time and distance diagram were selected such that lines representing speeds in the space-distance diagram are parallel to those in the flow distance diagram. The heavy line $\left(\mathrm{W}_{\mathrm{AB}}\right)$ in Figure 5-1-b represents the shockwave between states A and B. Figure 5-1-c shows a slice of the highway at time $t$ as well as the upstream traffic speed $u_{b}$, the downstream traffic speed $u_{a}$ and the speed of the shockwave $\mathrm{W}_{\mathrm{ab}}$. This case shows a forward moving shockwave.

Note that a line originating from the origin of the flow density curve and ending at any point in the same curve represents the speed at that point or state. Where the chord between any two points represents the shockwave forming if the system states transitioned between the two points.

The continuity condition enforces that, as the shockwave progresses, the number of vehicles leaving state $A\left(N_{a}\right)$ must be equal to the number of vehicles entering state $B$ $\left(\mathrm{N}_{\mathrm{b}}\right)$. This is certainly true since no cars are created or destroyed in the process. The number of vehicles leaving state $B$ in time $t$ is determined by the speed of the shockwave relative to the speed of the vehicles in state $\mathrm{B} . \mathrm{N}_{\mathrm{b}}$ can therefore be calculated by the equation:

$$
N_{B}=q_{B} t=\left(u_{B}-w_{A B}\right) k_{B} t
$$

Similarly, the number of vehicles entering state A can be calculated by the equation: 


$$
N_{A}=q_{A} t=\left(u_{A}-w_{A B}\right) k_{A} t
$$

From Equations (5-1) and (5-2) and solving for $w_{A B}$ :

$$
w_{A B}=\frac{q_{A}-q_{B}}{k_{A}-k_{B}}
$$

therefore, the speed of the shockwave traveling between different flow-density states is equal to the change in flow divided by the change in density of those two states. 


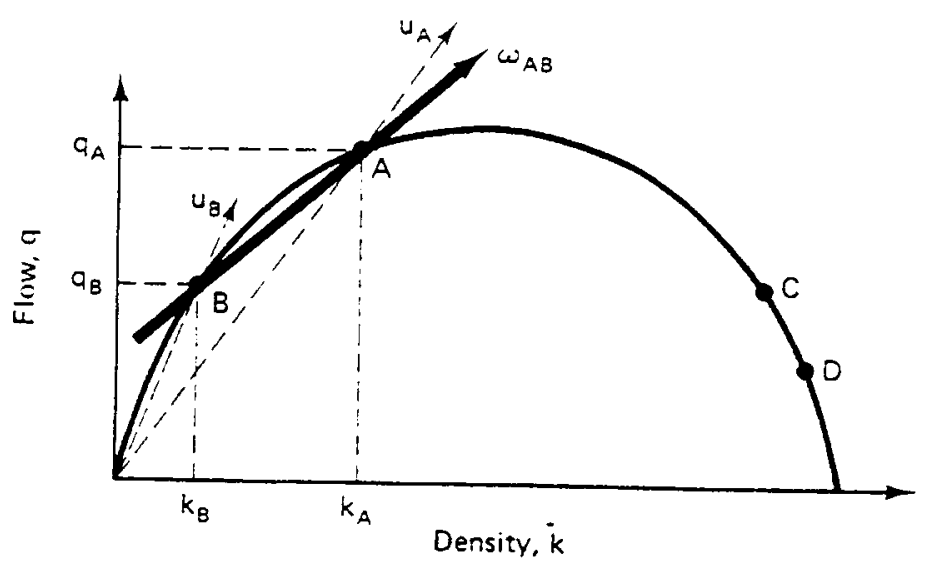

(a)

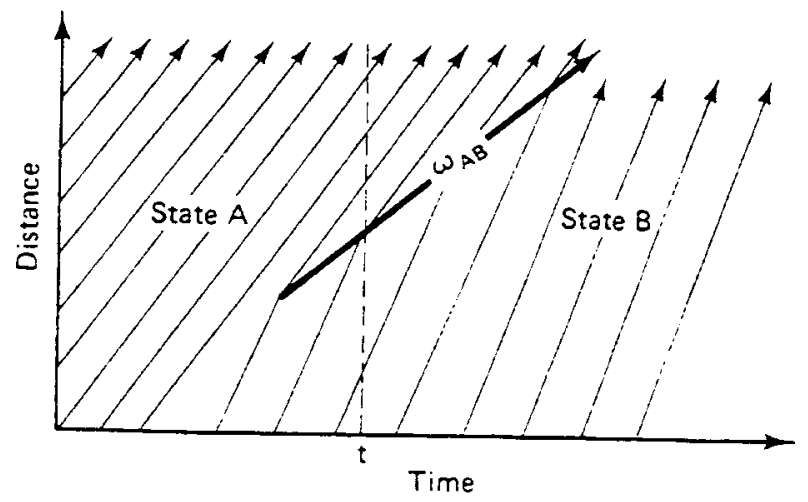

(b)

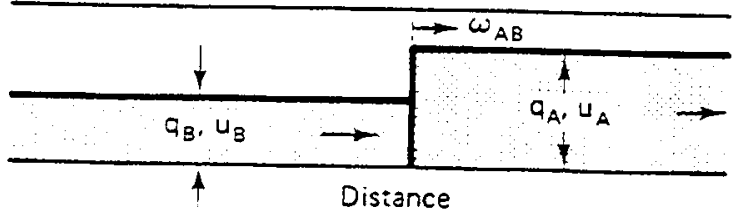

(c)

Figure 5-1 Shockwave Fundamentals [Source: May, 1990]

\subsection{Shockwaves at Signalized Intersections}

Of particular interest to this research is the formation of shockwaves at signalized intersections. Consider a case where the traffic signal has enough capacity to 
accommodate the demand. However, traffic can only pass through the signal during the green interval. Figure 5-2 illustrates an example of traffic flow approaching a traffic signal. When the signal interval is green (between times $t_{0}$ and $t_{1}$ ), the same traffic condition exists both upstream and downstream of the signal (traffic condition A). At time $t_{1}$, the signal turns red causing three different traffic conditions to form: a) traffic condition B upstream of the signal where queues start to form; b) traffic condition D downstream of the signal with zero density; and c) and traffic condition A of the traffic that has not been affected by the queues yet. At the start of the red interval $\left(t_{1}\right)$, the forward moving shockwave $w_{A D}$, the frontal stationary shockwave $w_{D B}$, and the backward moving shockwave $w_{A B}$ form. The direction of shockwave movements can also be determined by looking at Figure 5-2-b. The speed of those shockwaves can be calculated by applying Equation (5-3). 


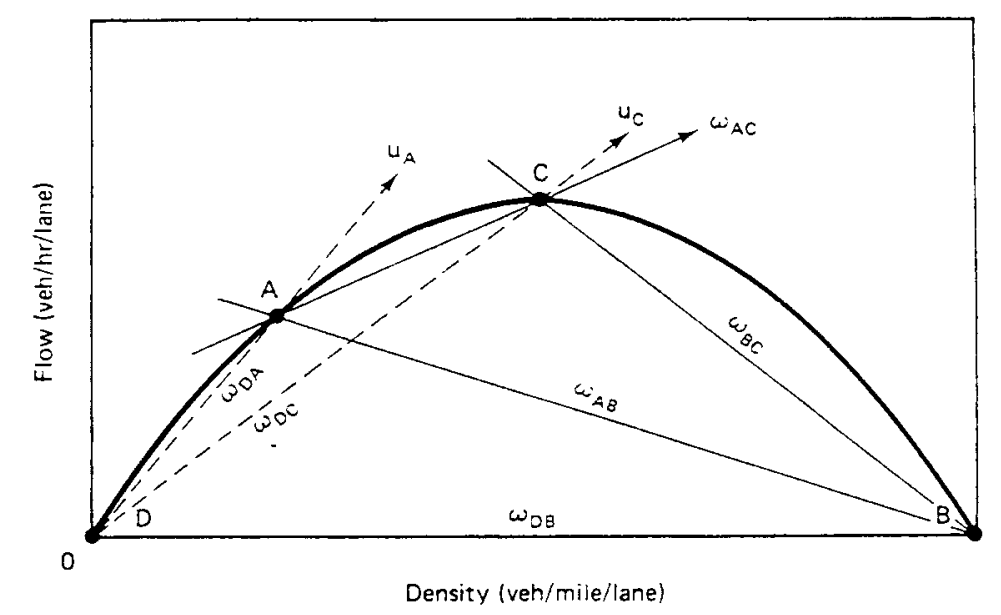

(a)

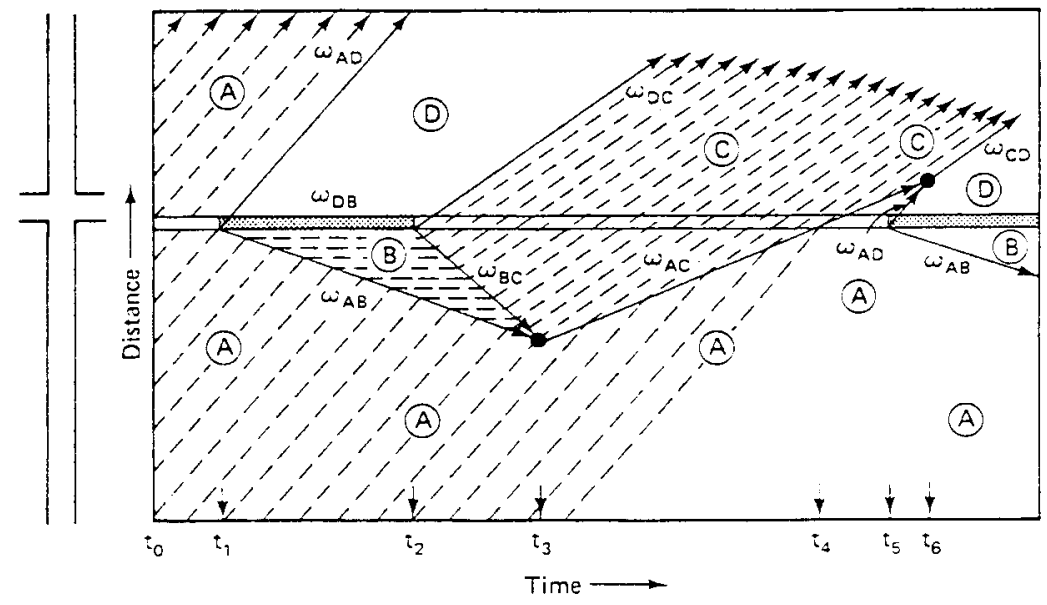

(b)

Figure 5-2 Shockwaves at Signalized Intersections [Source: May, 1990]

At time $t_{2}$, the signal indication changes to green and traffic starts to depart from the intersection with the saturation flow rate. At that point, shockwave $w_{D B}$ terminates and $w_{B C}$ and $w_{D C}$ start forming, separating the different regions of traffic flow conditions $\mathrm{B}, \mathrm{C}$, and $\mathrm{D}$. At time $\mathrm{t}_{3}$, the two backward moving shockwaves $w_{A B}$ and $w_{B C}$ meet and terminate. A new forward moving shockwave $w_{A C}$ is formed. This is the time 
at which the queues start to dissipate. At time $\mathrm{t}_{4}$, the shockwave $w_{A C}$ crosses the stop bar and the flow decreases from saturation flow to arrival flow.

At time $\mathrm{t}_{5}$, the shockwave $w_{A D}$ is created in a similar pattern to the previous cycle. At time $\mathrm{t}_{6}$, the two shockwaves $w_{A C}$ and $w_{A D}$ meet together and form the shockwave $w_{C D}$. From that point on and as long as the traffic pattern remains constant, the shockwave pattern will repeat itself.

This introduction illustrates that when traffic faces a red interval at a downstream signalized intersection, a backward moving shock wave is formed and extended upstream of the traffic signal. If a detector is placed within the range of that shockwave, it can capture its existence. This information can be very useful in determining whether the traffic signal offset is properly adjusted.

\subsection{Effect of Offsets on Shockwaves}

The greater the proportion of the traffic that faces the red interval, the farther back the shockwave extends. Therefore, if the offset was not performing well, e.g. due to early-return-to-green or poor initial design, a long queue will form due to the shockwave.

Figure 5-3 illustrates a poor offset at the downstream signal where the platoon arrives during the red interval. The figure shows a relatively low traffic volume passing through the upstream detector between times $t_{1}$ and $t_{2}$ from side street turning traffic. The red interval at the signal causes three different traffic conditions to form: a) traffic condition $\mathrm{C}$ upstream of the signal where queues start to form; b) traffic condition D 
downstream of the signal with zero density; and c) traffic condition A of the traffic that has not been affected by the queues yet. This situation causes the formation of three shockwaves: the forward moving shockwave $w_{A D}$, the frontal stationary shockwave $w_{D C}$, and the backward moving shockwave $w_{C A}$. The arrival of the platoon (traffic condition B) causes the formation of the shockwave $w_{A B}$ that meets the shockwave $w_{C A}$ and they both terminate forming a new shockwave $w_{C B}$. The new shockwave $w_{C B}$ extends faster than the shockwave $w_{C A}$ because of the higher traffic volume of the platoon. The low traffic volume from the side street follows the platoon, causing the shockwave $w_{A B}$ to form again. When the shockwave $w_{A B}$ meets the shockwave $w_{C B}$, they cause the formation of shockwave $w_{C A}$.

When the signal turns green, traffic starts to depart from the intersection with the saturation flow rate. At that point, shockwave $w_{D C}$ terminates, and $w_{D E}$ and $w_{C E}$ start forming separating the different regions of traffic flow conditions D, E, and C. The two backward moving shockwaves $w_{C E}$ and $w_{C A}$ meet and terminate, and a new forward moving shockwave $w_{A E}$ is formed. When the shockwave $w_{A E}$ crosses the stop bar and the flow decreases from saturation flow to arrival flow, the shockwave $w_{A D}$ is created in a similar pattern to the previous cycle. The two shockwaves $w_{A E}$ and $w_{A D}$ meet and form the shockwave $w_{E D}$, and from that point on and as long as the traffic pattern remains constant, the shockwave pattern will repeat itself.

Note the effect of the shockwave at the detector's occupancy shown at the bottom of the figure. Between times $t_{1}$ and $t_{2}$, low traffic volume turning from the side street 
passes through the upstream detector at a higher speed, theoretically speaking. Although they could, in practicality, be traveling at a lower speed than the arterial through movement, which causes low occupancy at the detector. As the shockwave $w_{A B}$ crosses the upstream detector at time $t_{2}$, the platoon starts to arrive with higher volume and lower speed, causing an increase in the occupancy between times $t_{2}$ and $t_{3}$. The traffic queues over the detector between times $t_{3}$ and $t_{4}$, raising the occupancy to $1.0(100 \%)$. The queue starts to dissipate between times $t_{4}$ and $t_{5}$ with the saturation flow rate causing the occupancy to drop from 1.0. Finally, as the low traffic volume from the side street passes through the detector between times $t_{5}$ and $t_{6}$, the occupancy drops to its lowest value. This variation in occupancy at the upstream detector denotes the existence of a strong shockwave due to a bad offset. 

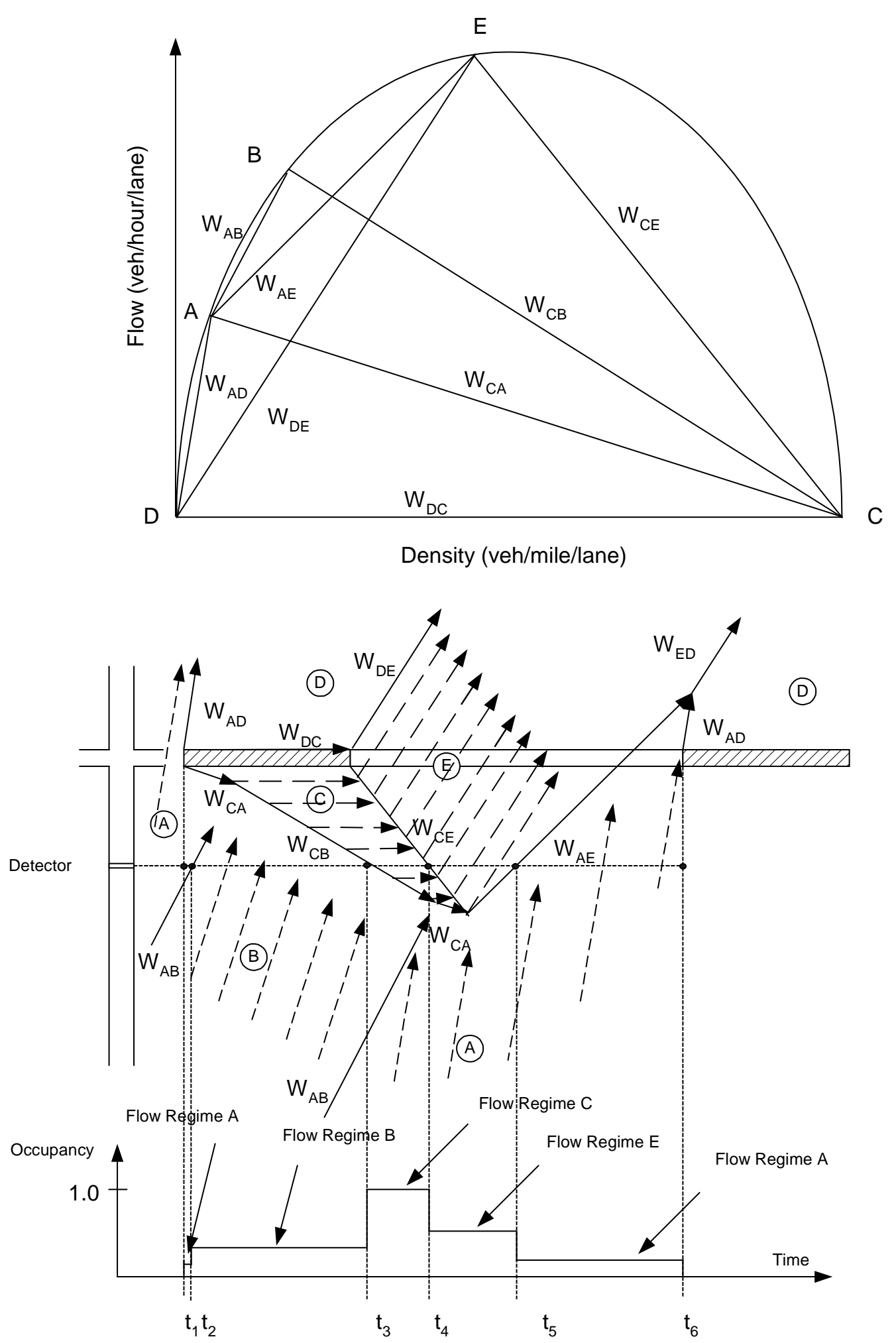

Figure 5-3 A Shockwave Originated by a Bad Offset 
A well-designed offset however, will align the green window with the platoon, minimizing the proportion of traffic arriving at the signal during the red interval. Figure 5-4 shows a comparatively good offset at the downstream signalized intersection. Note that the effect of the weak shockwave caused by the low traffic volume from the side street does not reach the upstream detector and the variation in occupancy at the detector therefore remains minimal. 

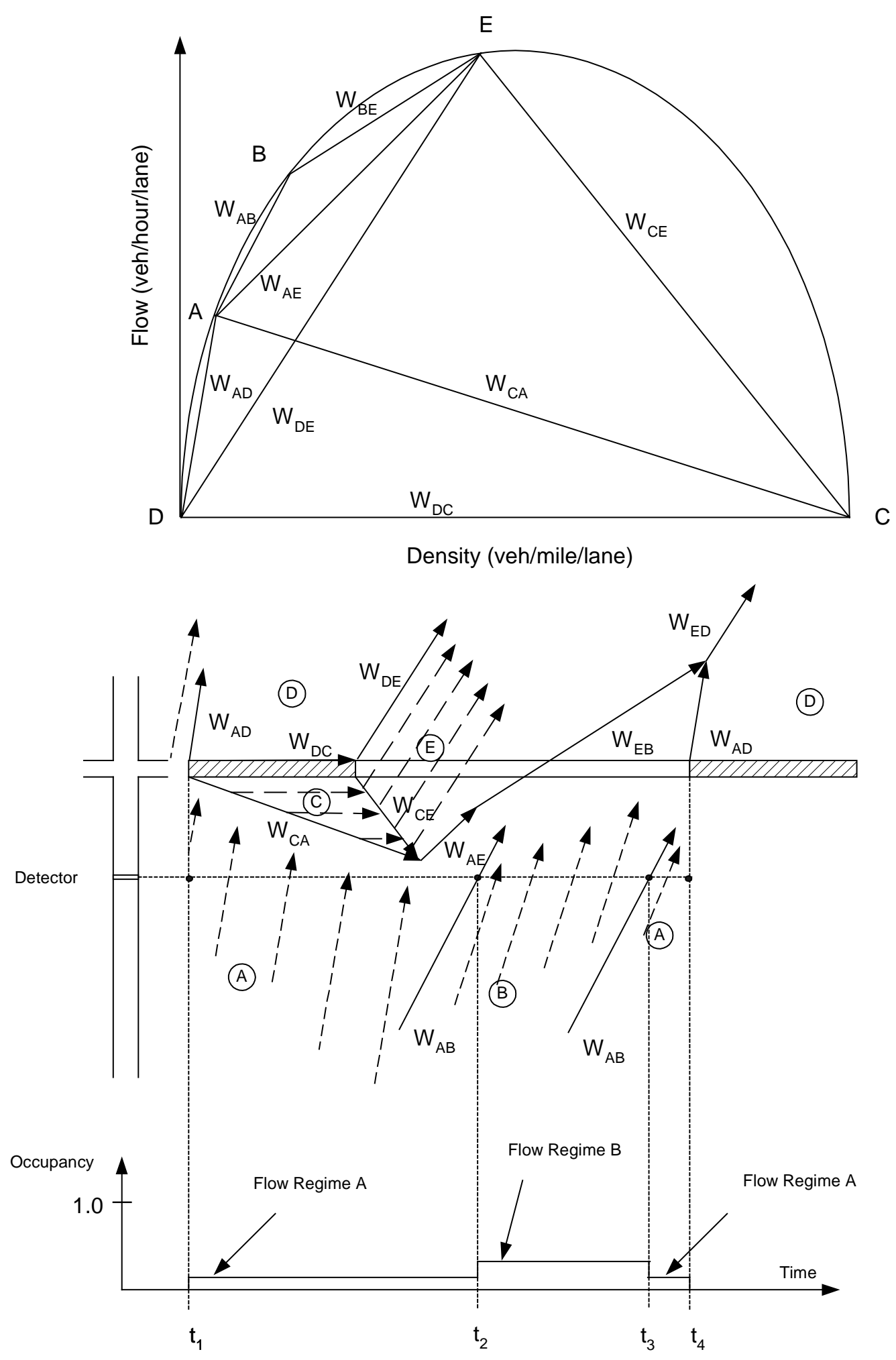

Figure 5-4 A Shockwave Originated by a Good Offset 


\subsection{Effect of High Turning Percentage on Shockwave Formation}

Traffic coordination benefits accrue due to the platooning phenomenon. The concentration of arterial traffic into platoons makes it feasible to align the green interval such that the platoon can pass through the intersection without stopping. If the vehicle platoons are highly dispersed or if vehicles arrive at the traffic signalized intersection in a random fashion, little or no benefits can be achieved from traffic signal coordination. In such a case, no matter how the green window is moved, almost an equal number of vehicles would arrive during the red interval.

Figure 5-5 and Figure 5-6 show a bad offset and a good offset respectively, where traffic volume turning from the side street approaches the arterial's traffic volume. Figure 5-5 shows a case of a bad offset where the platoon (traffic condition B) is aligned with the red interval, note that the shockwaves generated are very similar to the shockwaves in Figure 5-3, except that the occupancy during flow regime A is slightly higher due to the higher traffic volume and slower speed of traffic condition A.

On the other hand, Figure 5-6 shows a case of a good offset that attempts to align the platoon (traffic condition B) with the green window. However, one can observe that the figure shows a shockwave pattern is observed in the figure similar to the one generated due to the bad offset situation in Figure 5-5. This is due to the fact that the secondary traffic volume is very close in value to the primary traffic volume. 

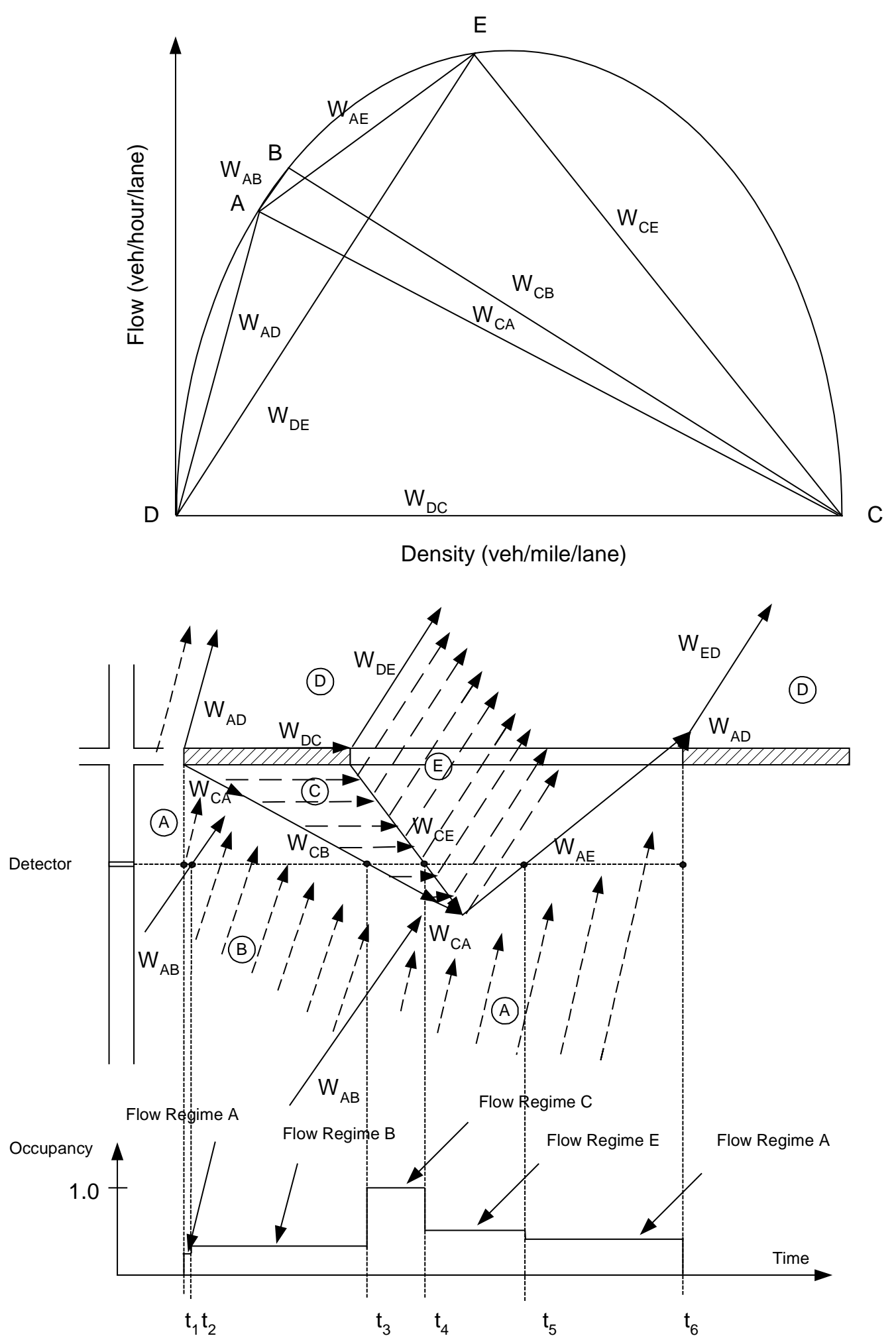

Figure 5-5 A Shockwave Originated by a Bad Offset and High Turning Traffic 

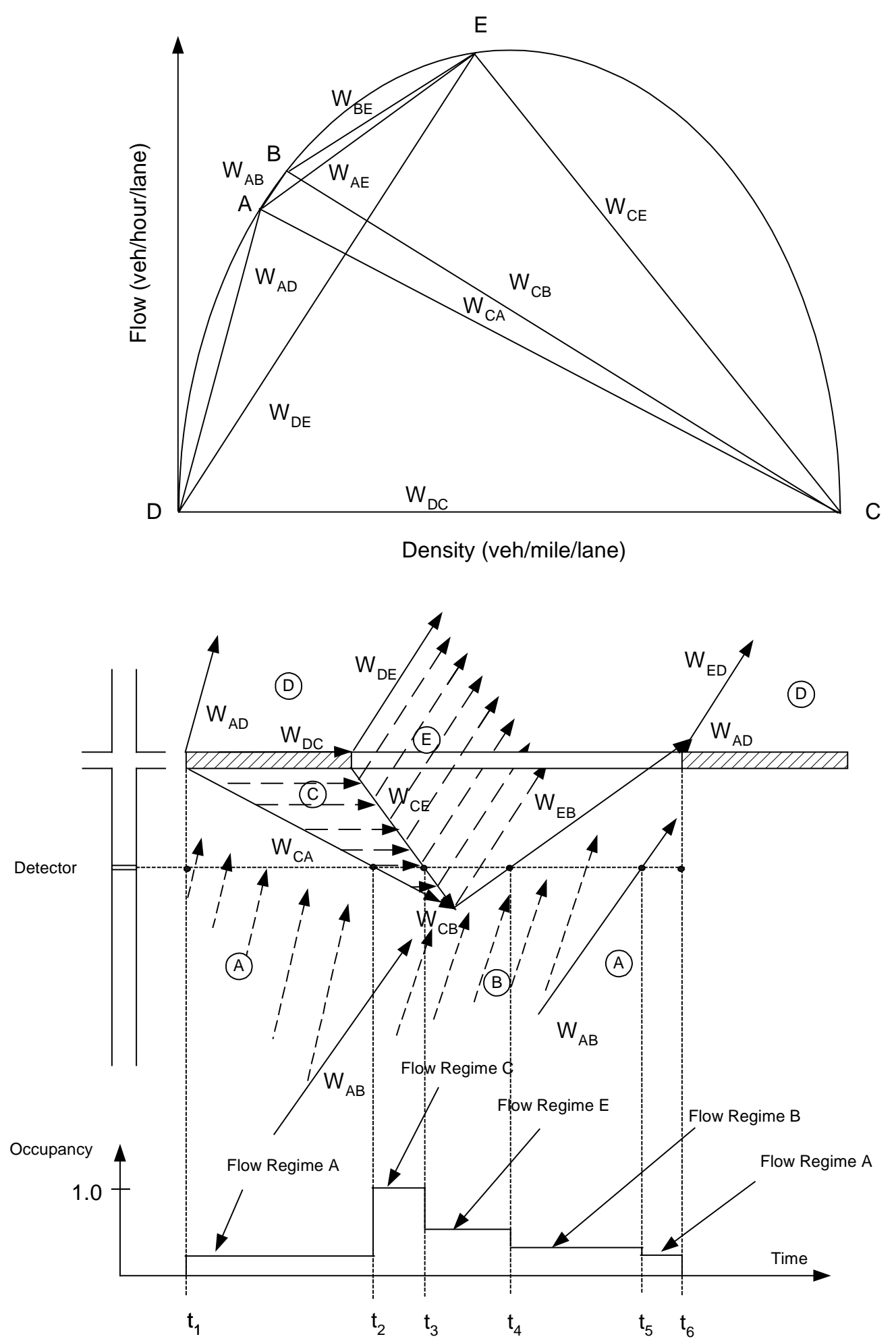

Figure 5-6 A Shockwave Originated by a Good Offset and High Turning Traffic 


\subsection{Chapter's Conclusion}

This chapter laid the theoretical and conceptual foundations for the algorithm developed in this research. The effect of offsets on shockwaves under different traffic conditions is discussed and how that reflects on the occupancy profile at the upstream detector. Chapters 6 and 7 will introduce two models by which the existence of shockwaves generated due to a bad offset can be captured and accounted for, namely, the skewness model and the F' model. The skewness model is discussed in Chapter 6 when the algorithm developed in this research is introduced. The limitations of the skewness model are discussed in Chapter 7 and the more general F' model is introduced. Chapter 7 also conducts an analysis of variance for the two models and compares their potential usefulness under different conditions that may affect the offset performance, including the case where the volume of turning traffic from the side street approaches that of the arterial's through traffic. 


\section{CHAPTER 6- PRO-TRACTS CONCEPT AND STRUCTURE}

This chapter introduces an adaptive real-time offset transitioning expansion to actuated controllers to improve coordination of traffic signals. This algorithm, Purdue Real-time Offset Transitioning Algorithm for Coordinating Traffic Signals (PROTRACTS), mitigates the effect of the early-return-to-green problem experienced with coordinated-actuated controllers and accounts for downstream vehicle queues that may impede vehicle progression. The chapter describes the basic concept and methods by which PRO-TRACTS captures the shockwave information through the use of advance detectors actuation. The skewness model is introduced and evaluated with a test bed case study and general findings and conclusions are presented at the end of the chapter.

PRO-TRACTS can be viewed as an integrated optimization approach that is designed to work with traditional coordinated-actuated systems. The objective of the proposed algorithm is to add to the actuated controllers the ability of adaptively changing their offsets in response to changes in an arterial's traffic demand. This model will provide an intermediate solution between traditional coordinated actuated control systems and adaptive control systems.

PRO-TRACTS assumes a fixed cycle length (selected by either the time of day or some traffic responsive technique) and splits decided by each local controller, subject to maximum and minimum constraints traditionally imposed by coordinated-actuated signal systems. The unique aspect of the proposed algorithm is that the end of green offsets at 
each intersection are continually adjusted in real-time with the objective of providing smooth progression of a platoon through an intersection using the volume and occupancy profile of advance detectors. This automatic tuning process is analogous to an engineer or technician standing beside the cabinet and "tuning" the offset so that the coordinated phase turns green at the appropriate time to facilitate smooth progression of the upstream platoon.

\subsection{PRO-TRACTS Structure}

PRO-TRACTS utilizes an internal structure that profiles the occupancy and count actuation on a second by second basis for approach detectors. The algorithm keeps records of the latest 30 cycles actuation, although for actual field implementation, the number of cycles used would likely be reduced to say 10 cycles. The occupancy and count actuation are stored in a bin-based storage $\left(\operatorname{occ}_{\mathrm{i}, \mathrm{j}}\right.$ and $\left.\mathrm{cnt} i, \mathrm{j}\right)$. For example, the occupancy at any time $(\mathrm{t})$ bin is stored as $\operatorname{occ}_{\mathrm{i}, \mathrm{j}}$ where $\mathrm{i}$ and $\mathrm{j}$ are the time into cycle and the current cycle number respectively, determined by the following equations:

$$
\begin{gathered}
i=\bmod \left(\left(t-t_{r e f}\right), \text { cycle }\right) \\
j=\operatorname{int}\left(\left(t-t_{r e f}\right) / c y c l e\right)
\end{gathered}
$$

where $t_{\text {ref }}$ is the reference time, which may be the start of simulation or perhaps midnight in a deployed system.

For development purposes, the algorithm is currently implemented as external control logic in CORSIM [Kaman, 1997B]. The algorithm has the ability to work with either CORSIM internal logic or alternatively with a set of NTCIP NEMA controllers 
through hardware-in-the-loop simulation [Bullock and Catarella 1998]. The algorithm exchanges information with the CORSIM simulation package through its run time extension DLL in real time.

\subsection{Link Profiling and Tabulation}

A utility module (LinkPro) has been developed to visually evaluate the effect of a particular offset in an arterial and/or to evaluate and visualize the impact of waiting queues in the arterial that might disrupt traffic progression. The implementation of the graphical utility requires a shared memory structure to allow for information exchange between the different programs involved in the simulation. The graphical utility (LinkPro) exchanges information with CORSIM through its run time extension DLL. Therefore there was a need for an intermediate shared memory structure was needed that contains the exchanged information. Figure 6-1 shows a sketch of the implementation of the shared memory structure that enables the graphical utility to interact with CORSIM and the real-time offset tuning program. 


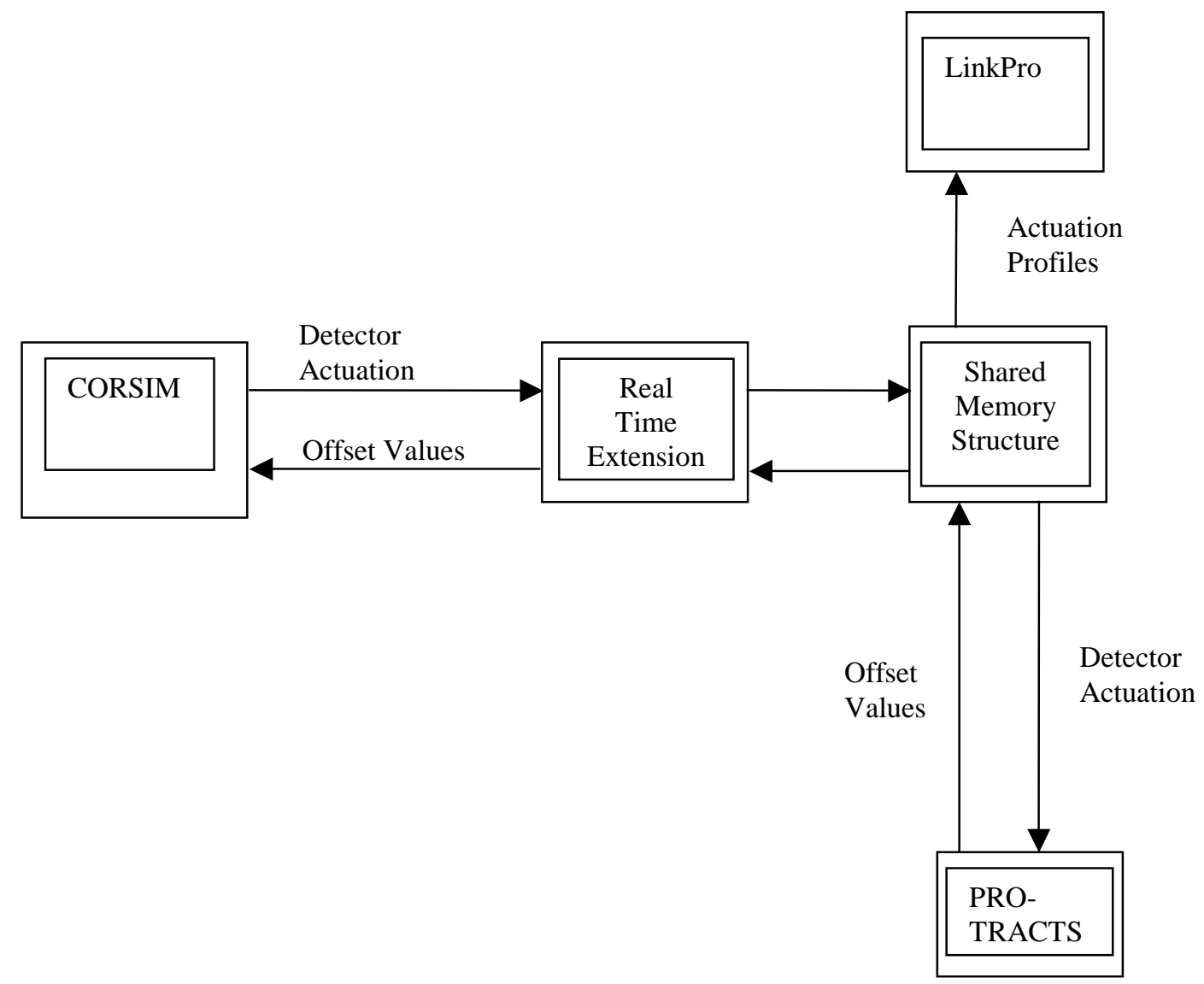

Figure 6-1 Shared Memory Structure

LinkPro allows the evaluation of the performance of the offsets currently set in the arterial system by selectively showing occupancy or traffic count measures over particular CORSIM system detectors in graphical and/or tabular format. The screen shows the traffic measures aggregated through several cycles (one to10 cycles) over the upstream detectors, along with the current green window at the traffic signal. The graphs have increments of cycle length on the $\mathrm{X}$-axis and the traffic measure (occupancy/count) 
on the Y-axis as shown in Figure 6-2. Actuation of other detectors in the system can also be shown by selecting the detector number from LinkPro menu, as shown in Figure 6-3. 


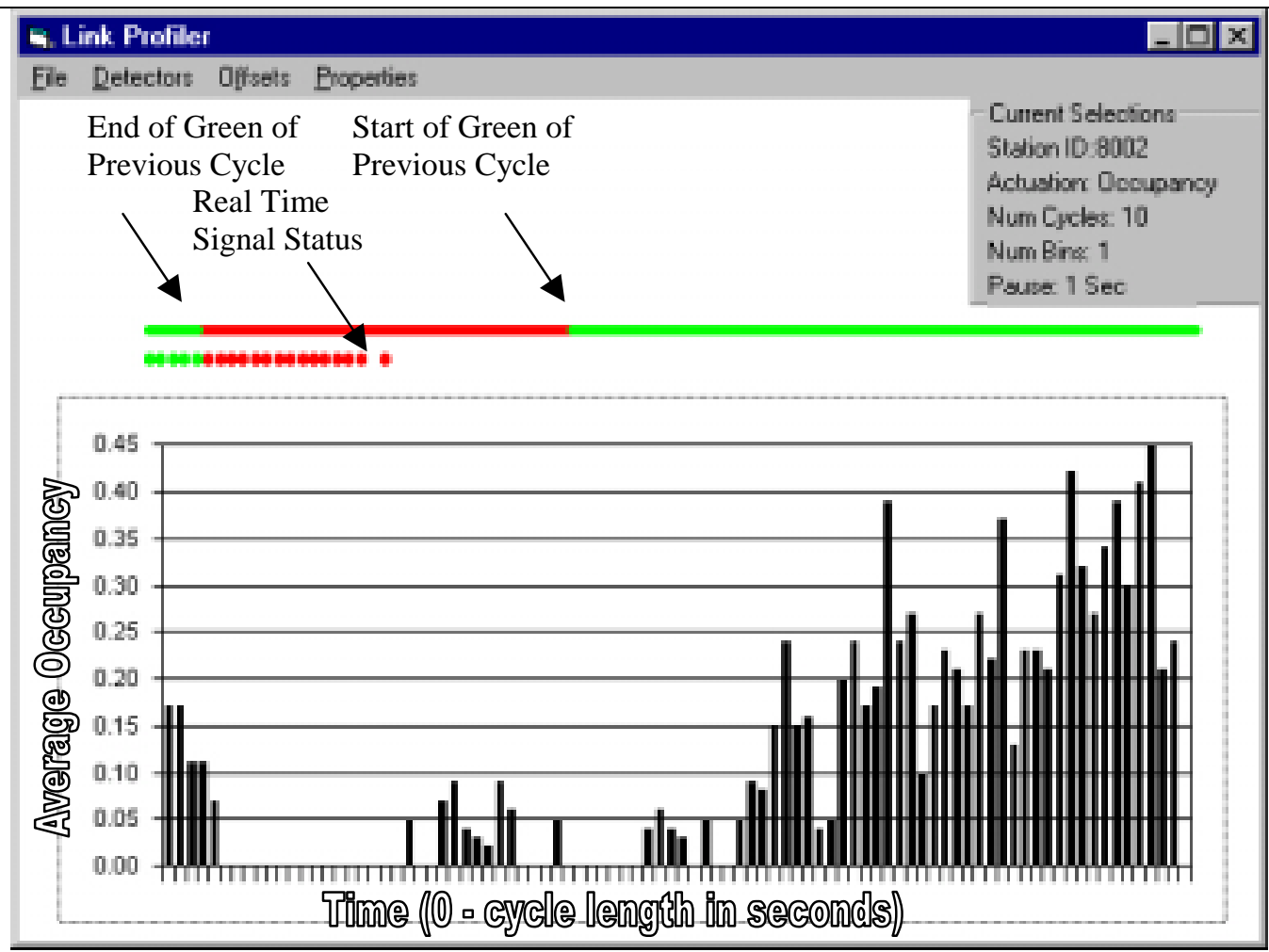

a) Average Occupancy in each bin

\section{G. Link Profiler}

Ele Detectors Offsets Buoperties

Cunrent Selections

Station 10.8002

Achustion Count

Num Cjelas: 10

Num Binc 1

Pouve. $1 \mathrm{Sec}$

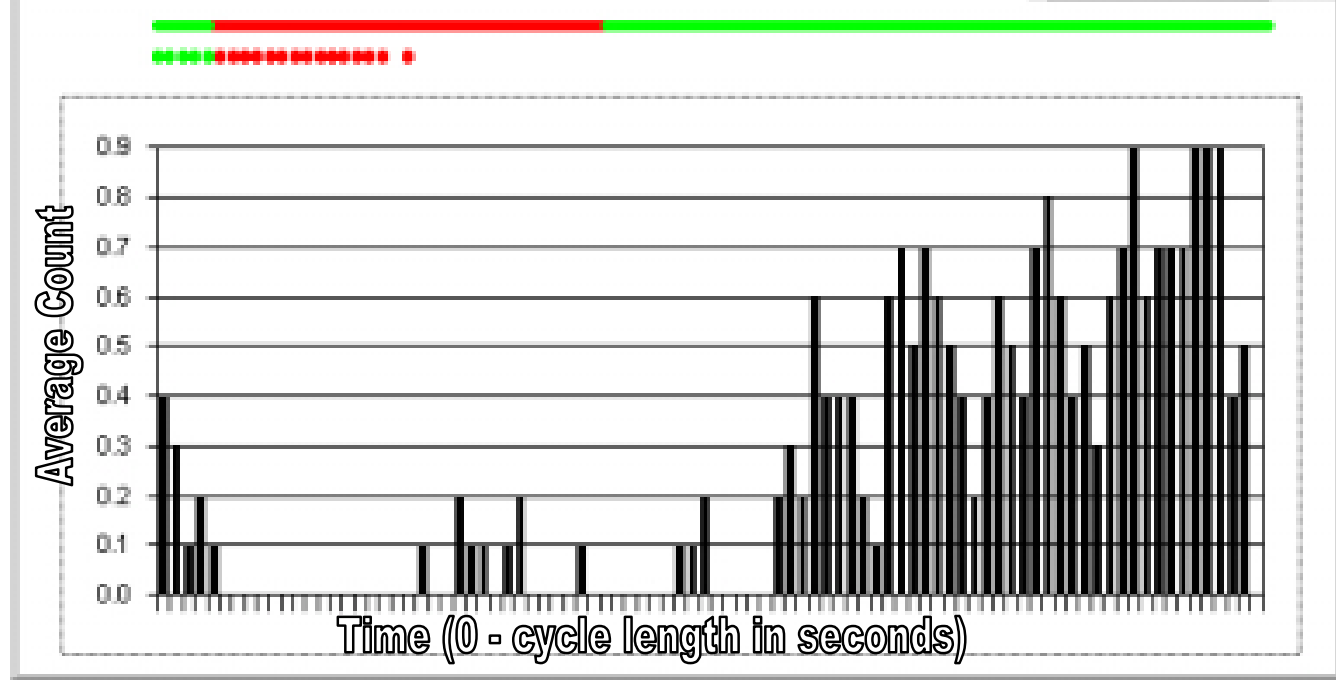

b) Average Count in each bin

Figure 6-2 LinkPro Screen Capture for a tuned Offset 


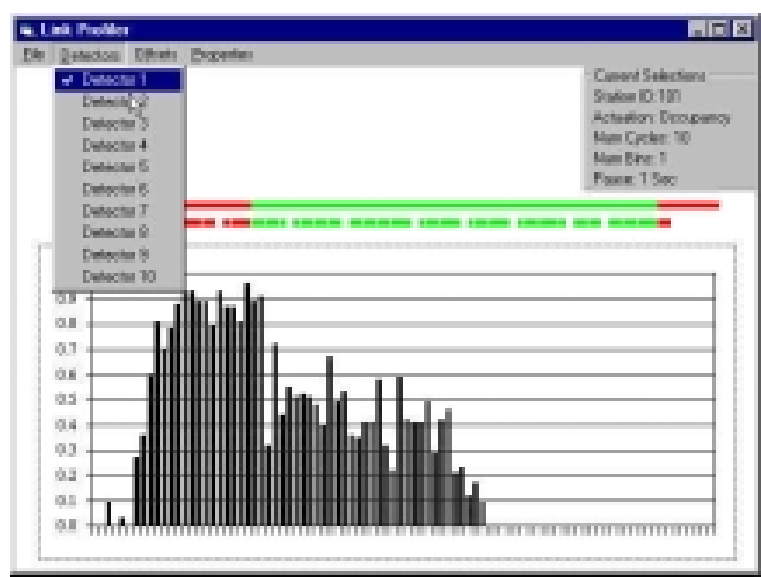

a) Stop Bar Detector

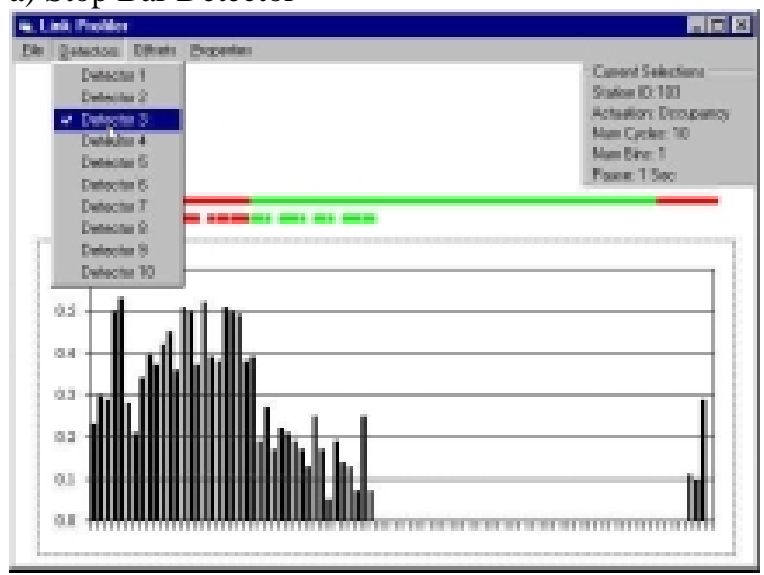

c) Advanced Detector 400 feet Upstream

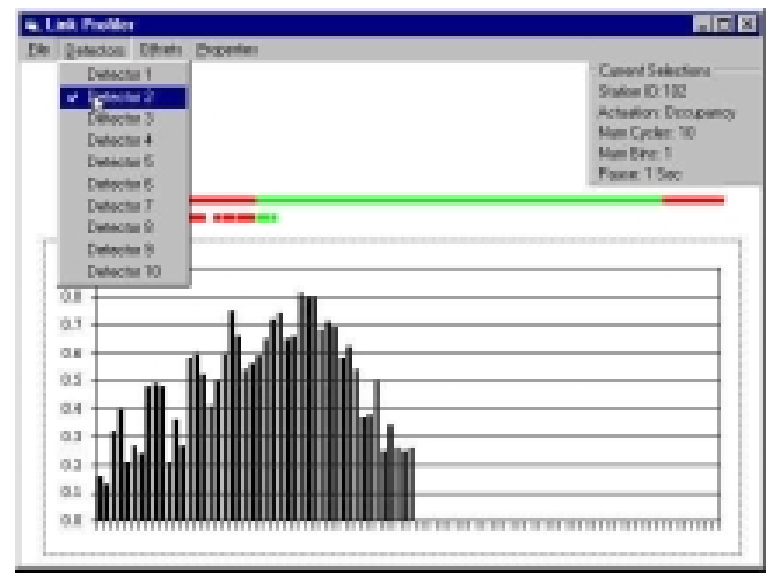

b) Advanced Detector 200 feet Upstream

Figure 6-3 Detectors Occupancy Data at Selected Downstream Locations

LinkPro also allows manual change of offset value at any intersection during the simulation for illustration purposes, as can be seen in Figure 6-4. 


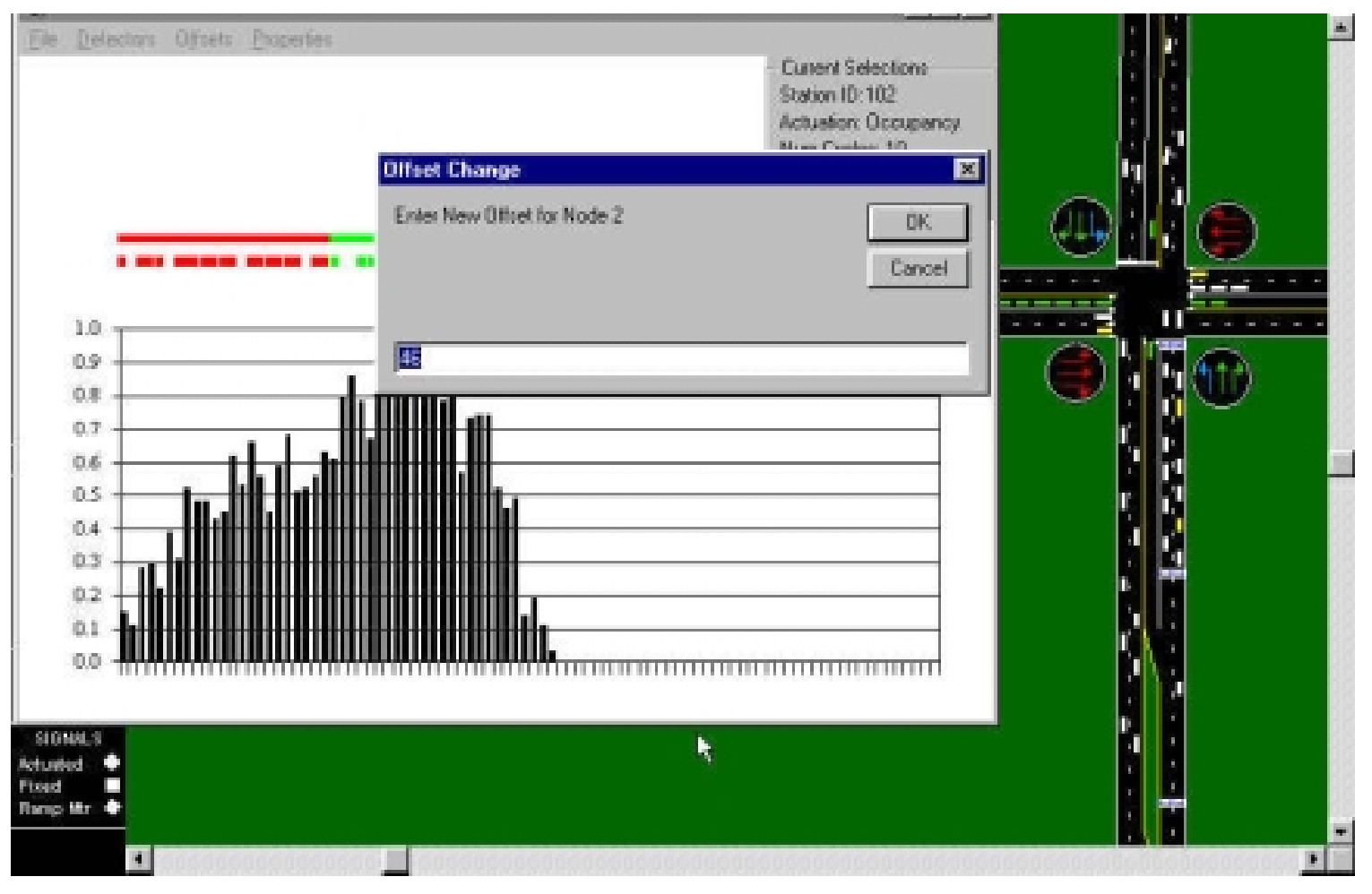

Figure 6-4 Manual Offset Change in LinkPro

By double-clicking the chart that shows the profile of any selected detector, a tabular format of the detector actuation is displayed. The actuation is displayed in a second by second basis. Vehicle counts/occupancy measures are output to the window in a one-second time increment referenced to the starting time of the cycle. The display window shows the current cycle and the previous cycles information (30 cycles). When the rows of the table are completely filled, all of the actuations of previous cycles are shifted down by one row and the current actuation is shown in the higher row. Figure 6-5 illustrates the display of the occupancy. Note that since the whole cycle does not fit in 
one row, a slider bar is used to shift the display in order to observe actuation at any point in the cycle.

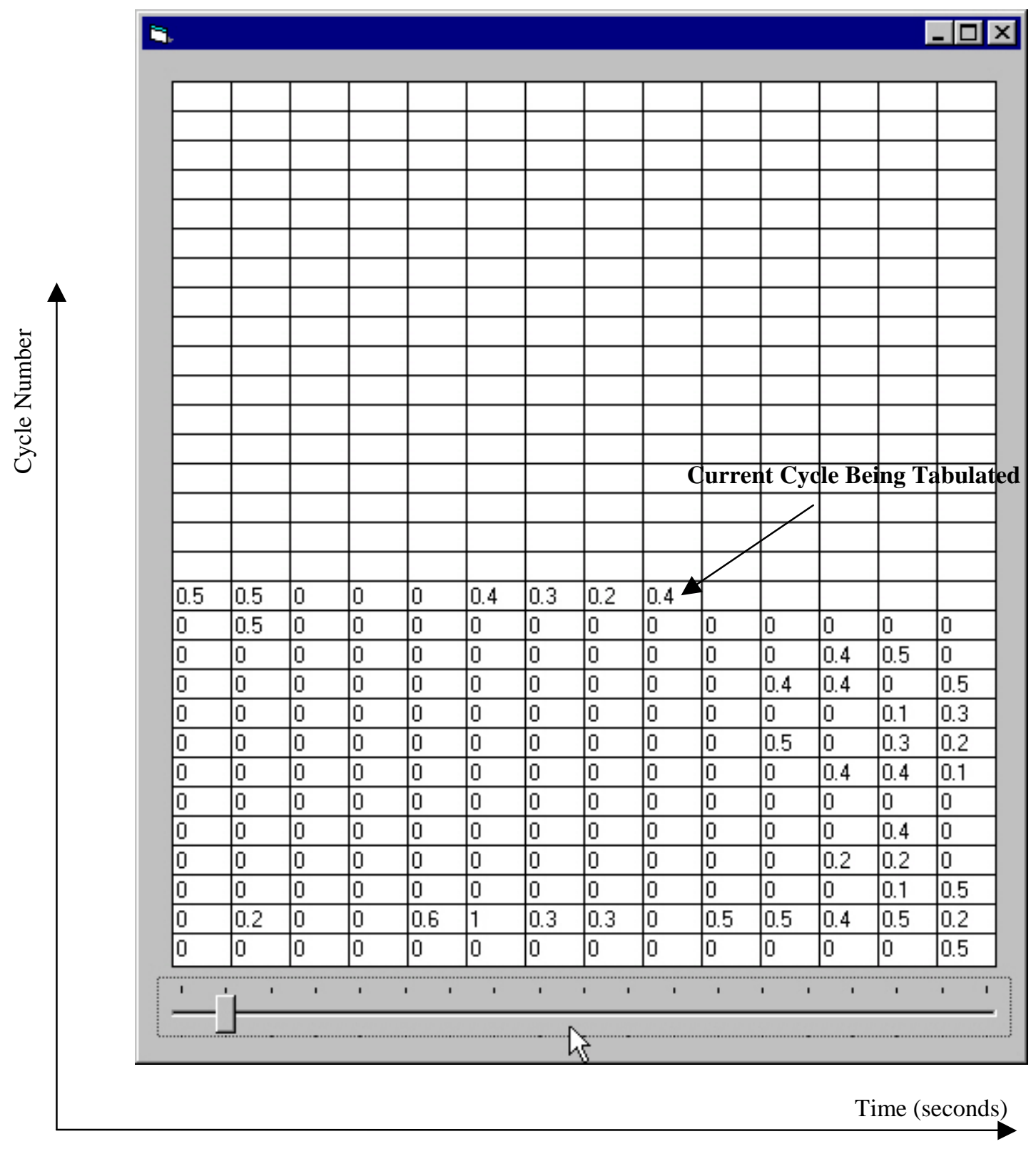

Figure 6-5 LinkPro Tabular Format 


\subsection{PRO-TRACTS Concept: The Skewness Model}

As discussed in the previous chapter, occupancy distribution over advanced detectors is significantly affected by the state of the downstream signal offset. A good offset causes a smaller variation in the occupancy distribution than a bad offset does since it does not generate a shockwave strong enough to reach the advanced detector. The small variation of occupancy distribution with a good offset will cause the occupancy profile to look very similar to the count profile when tabulated over the cycle. In the bad offset case, however, the platoon of vehicles starts to slow down as its the strong shockwave and eventually queues over the detector. This phenomenon results in the surveillance detector observing a larger number of slow moving, or stopped vehicles,

through the cycle. Consequently, the occupancy profile will have a significantly different shape from that of the count profile. To illustrate the point further, Figure 6-2 shows LinkPro screen captures that illustrate detector actuation for a surveillance detector 60 meters upstream of an intersection with a good offset. Figure 6-2-a shows the occupancy actuation and Figure 6-2-b shows the count actuation. Occupancy and count detector actuation are shown to have similar shapes in the figure when the downstream signal offset is tuned. Figure 6-6 shows LinkPro screen captures showing detector actuation for a surveillance detector upstream of an intersection with an un-tuned offset. Figure 6-6-a shows the occupancy actuation and Figure 6-6-b shows the count actuation. Occupancy and count detector actuation are shown to have different shapes when the downstream signal offset is un-tuned. This difference in actuation shapes is utilized by PRO-TRACTS as the basic concept for offset transitioning. 


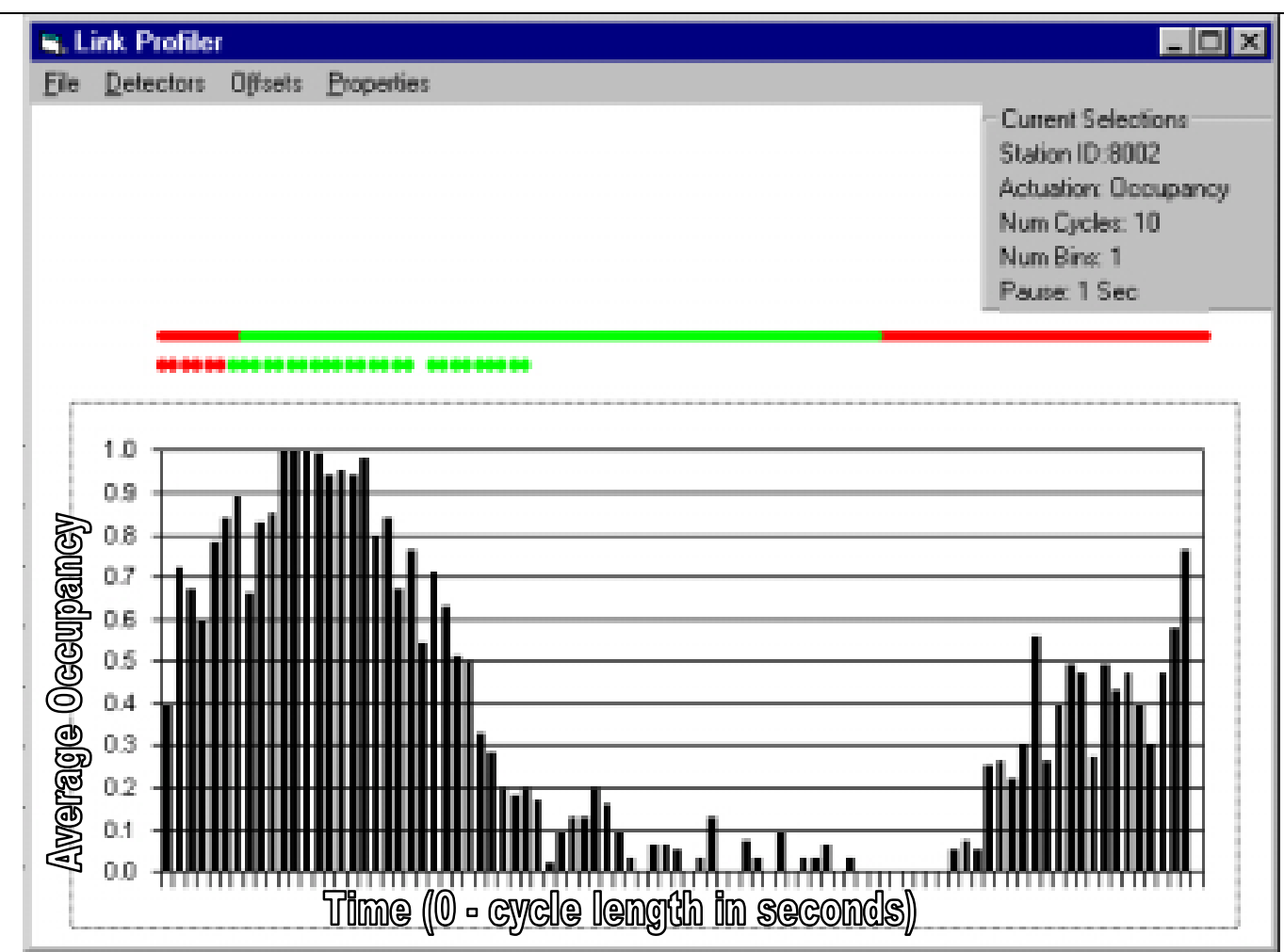

a) Average Occupancy in each bin

\section{G. Link Profiler}

Ele Detectors Offsets Broperties

Psure. $1 \mathrm{Sec}$

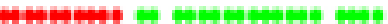

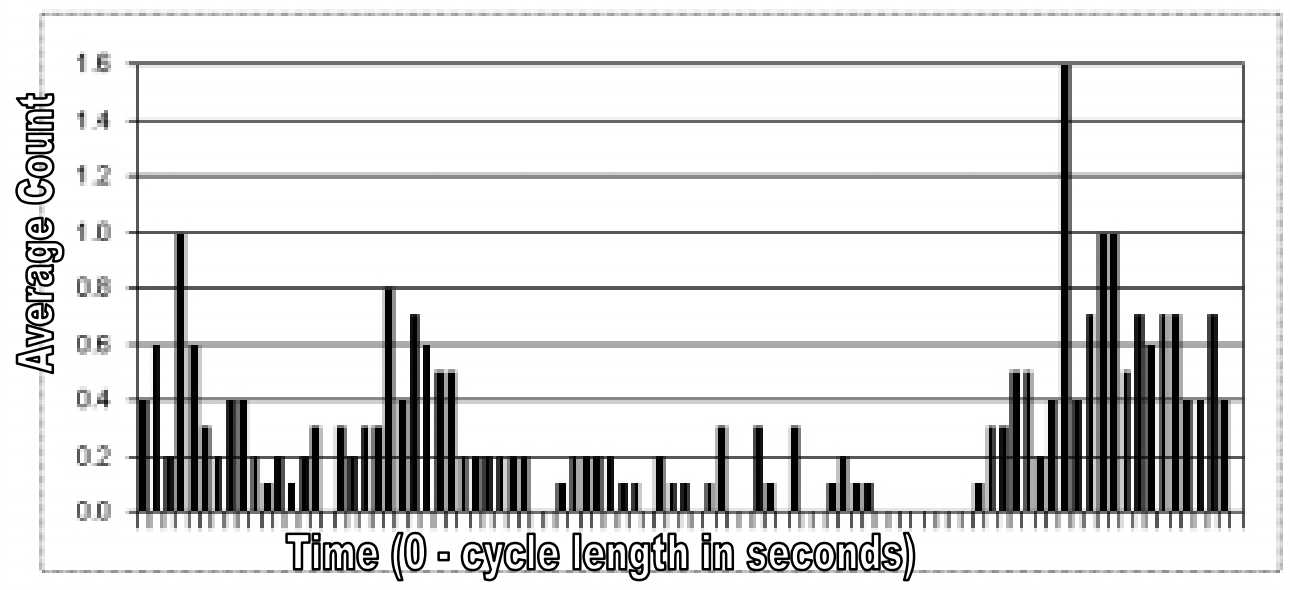

b) Average Count in each bin

Figure 6-6 LinkPro Screen Capture for an un-tuned offset 


\subsubsection{The need for an Activation Mechanism}

Poor offsets affect the shapes of the occupancy and count distributions in different ways, depending on 1) the direction and degree by which the offset is shifted from the ideal offset, and 2) the characteristics of the platoon approaching the signal. Figure 6-7 through Figure 6-11 show how the shapes of the occupancy and count profiles for different platoon conditions are affected by the offset deviation from the ideal value. Figure 6-7 shows a condensed platoon with a negatively shifted offset, an ideal offset, and a positively shifted offset. One can easily recognize in the figure the direction of the offset shift that has been applied to the signal by looking at the shapes of the profiles. Figure 6-8 shows an end-dispersed platoon, where vehicles are released from the upstream signal with the saturation flow rate followed by a lower flow rate. Note that in such a case, the direction of the offset shift is not as clear as it was with condensed platoons. Note that even with the ideal offset, not all vehicles are aligned within the green window. Figure 6-9 shows the same end-dispersed platoon but with a wide green window at the downstream signal that can easily accommodate the platoon, and one can easily recognize the offset shift type needed is easily seen. Figure 6-10 shows a dispersed platoon where the direction of movement is again not clear. Figure 6-11 shows the same dispersed platoon but with a wide green window where the shift type can be identified. All of these figures show that it is not always easy to decide whether the offset needs to be changed, nor its direction. The decision becomes even more complex with the effect of turning traffic from side street. Some activation mechanism therefore is needed that decides whether the offset at the downstream signal needs to be modified. 

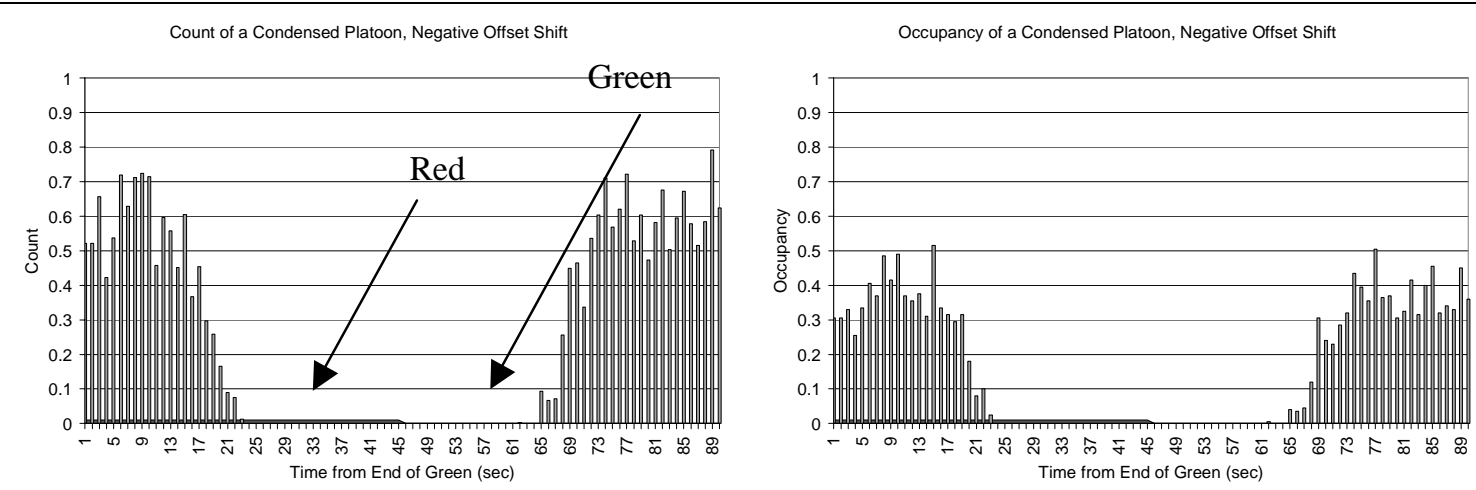

a) Negative Offset Shift

Occupancy of a Condensed Platoon, Good Offset

Count of a Condensed Platoon, Good Offset
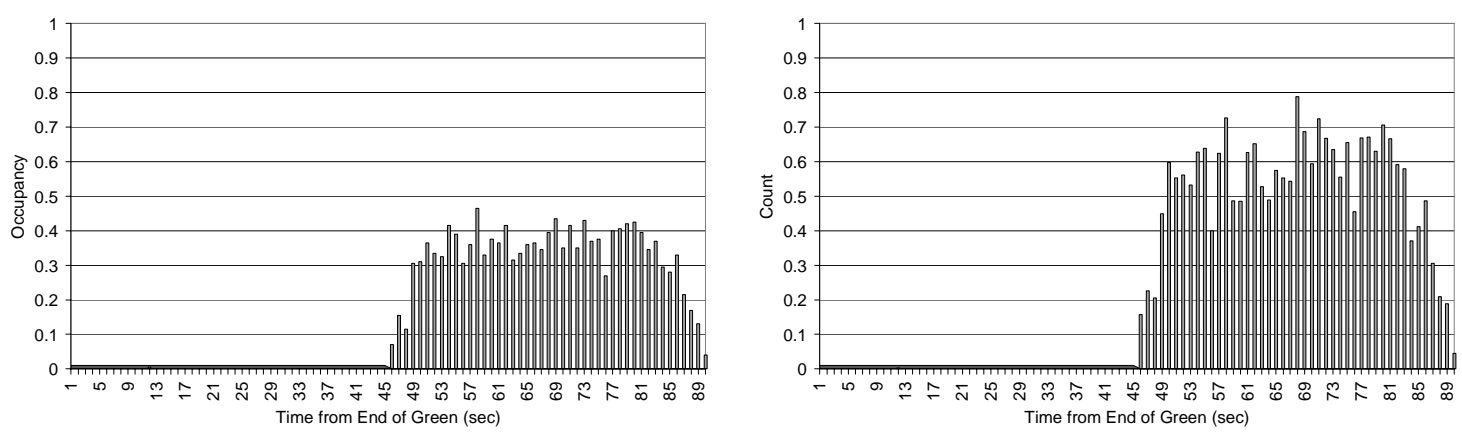

b) Good Offset

Occupancy of a Condensed Platoon, Positive Offset Shift

Count of a Condensed Platoon, Positive Offset Shift
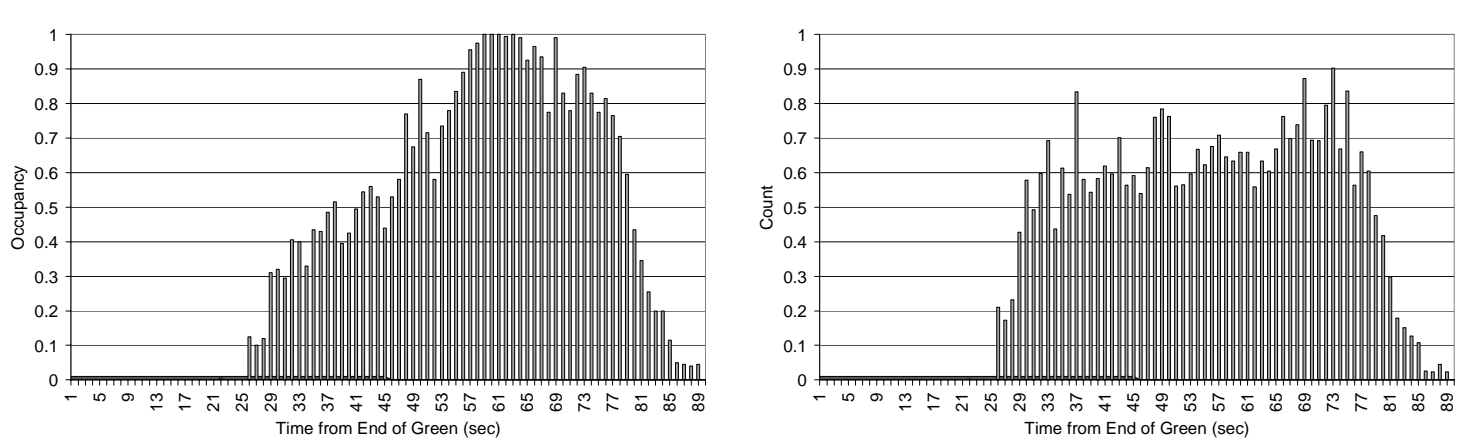

c) Positive Offset Shift

Figure 6-7 Condensed Platoon's Occupancy and Count Profiles Over 25 Cycles, Green Phase 45 to 90 

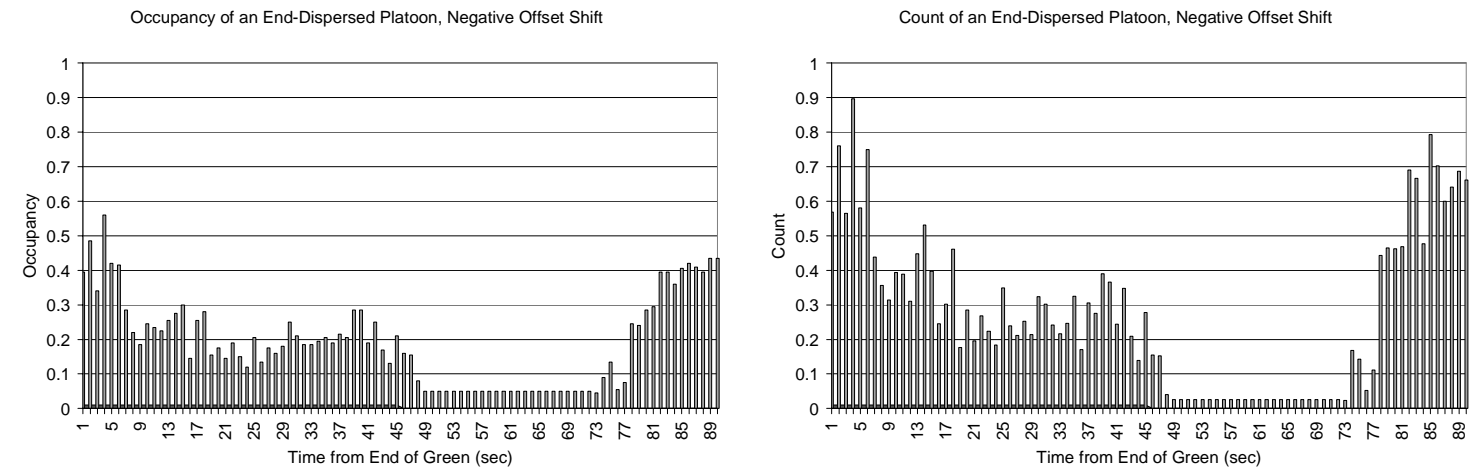

a) Negative Offset Shift

Occupancy of an End-Dispersed Platoon, Good Offset

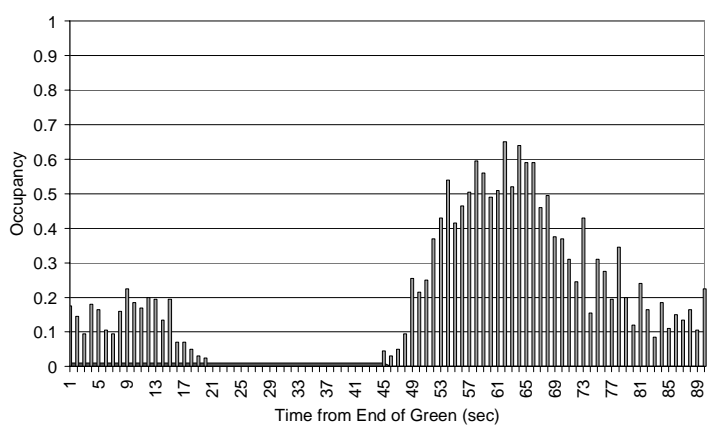

Count of an End-Dispersed Platoon, Good Offset

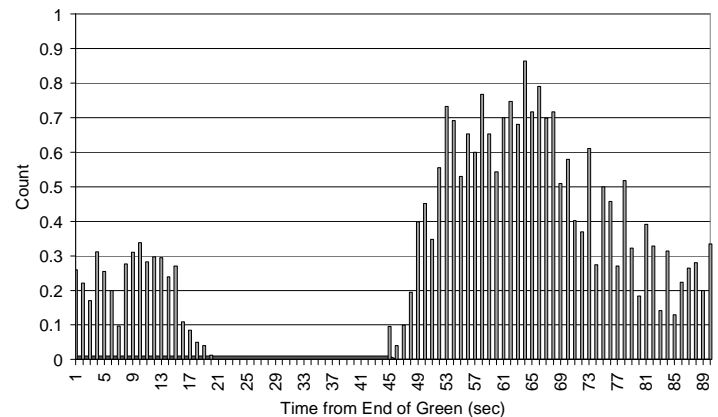

b) Good Offset

Occupancy of an End Dispersed Platoon, Positive Offset Shift

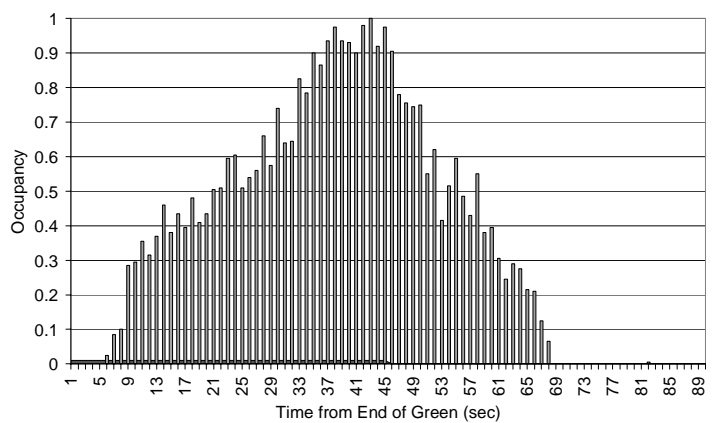

Count of an End-Dispersed Platoon, Positive Offset Shift

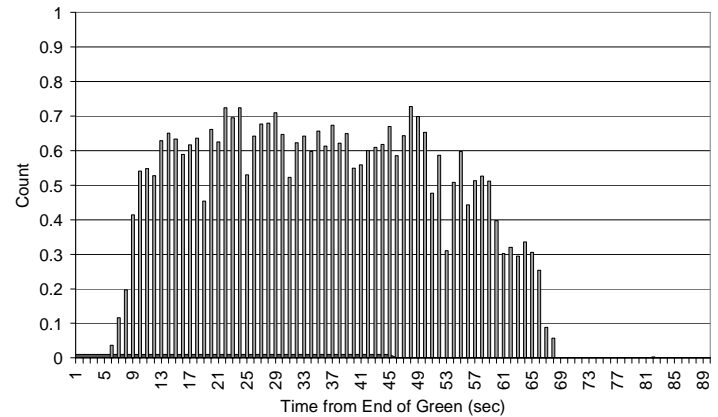

c) Positive Offset Shift

Figure 6-8 An End-Dispersed Platoon's Occupancy and Count Profiles Over 25 Cycles, Green Phase 45 to 90 

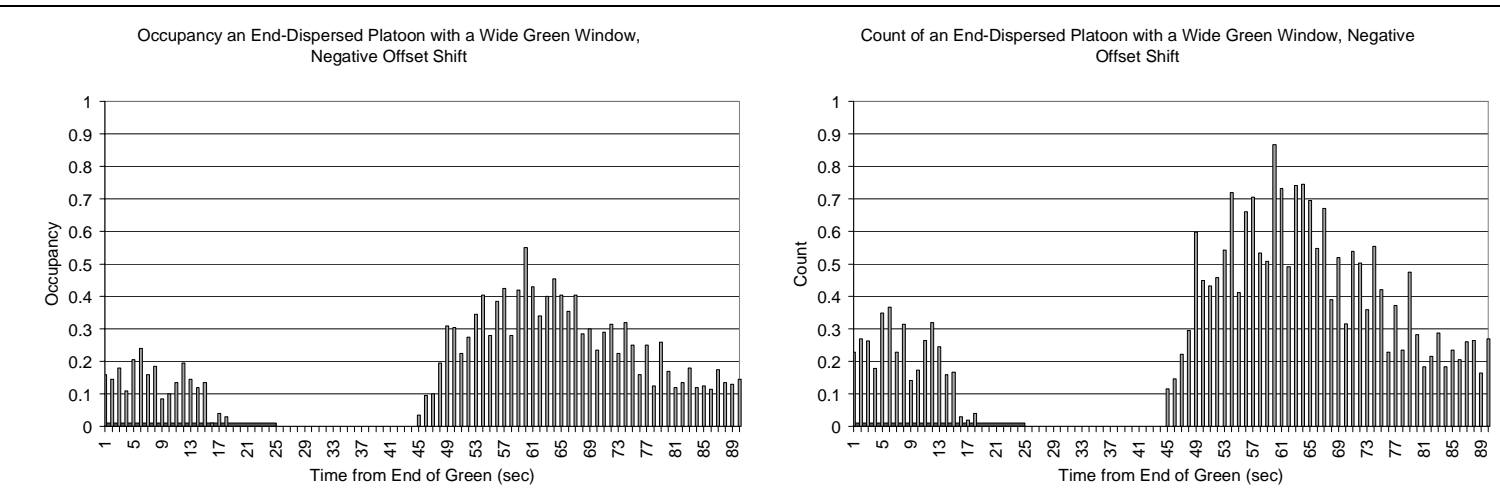

a) Negative Offset Shift
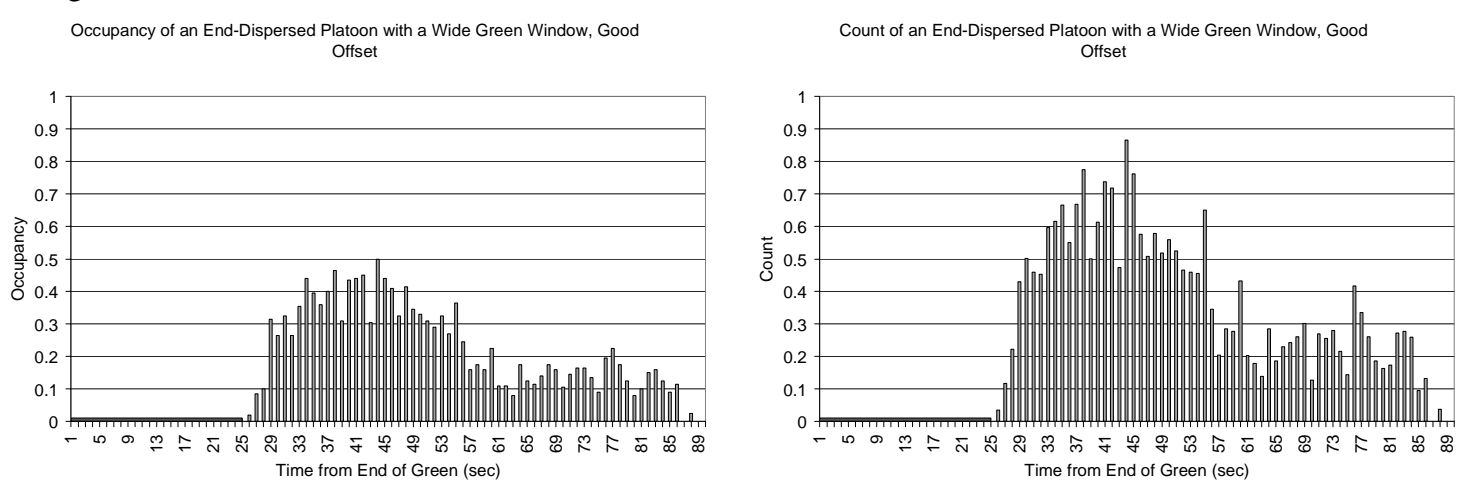

b) Good Offset

Occupancy of an End-Dispersed Platoon with a Wide Green Window, Positive Offset Shift

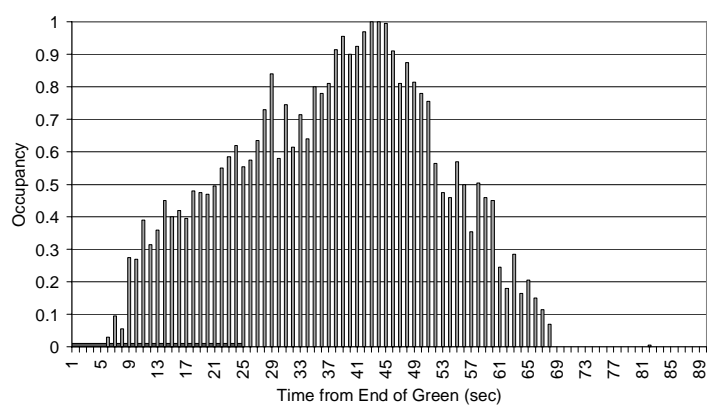

Count of an End-Dispersed Platoon with a Wide Green Window, Positive Offset Shift

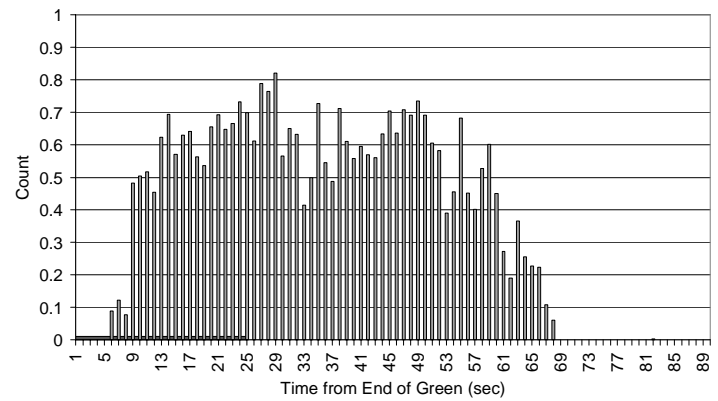

c) Positive Offset Shift

Figure 6-9 An End-Dispersed Platoon's Occupancy and Count Profiles Over 25 Cycles With a Wide Green Window, Green Phase 25 to 90 

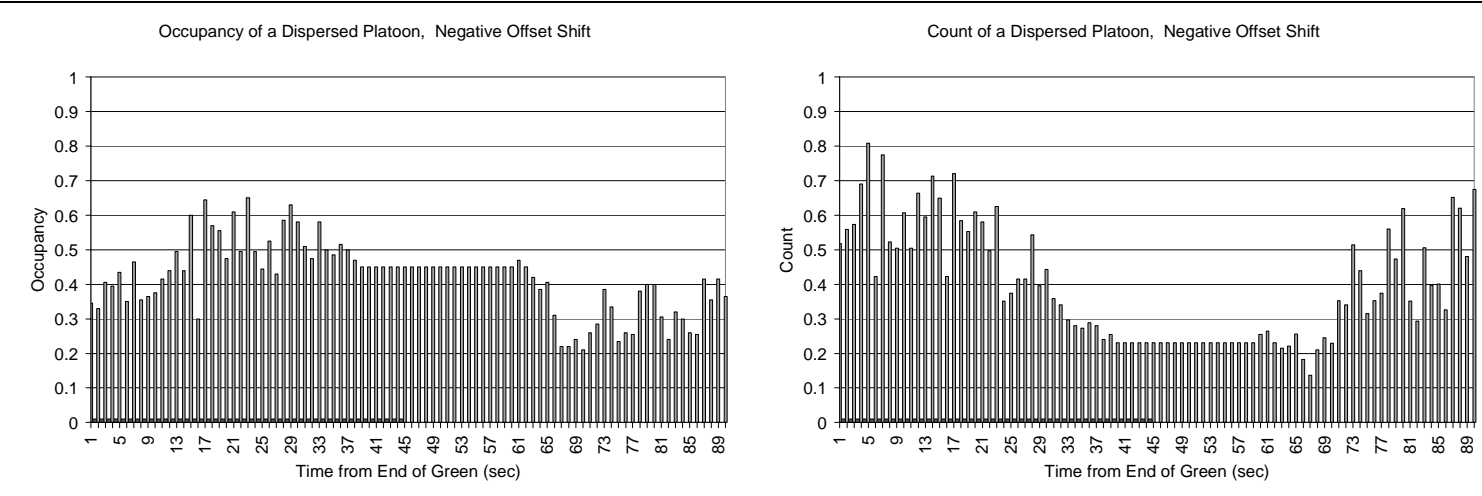

a) Negative Offset Shift

Occupancy of a Dispersed Platoon, Good Offset

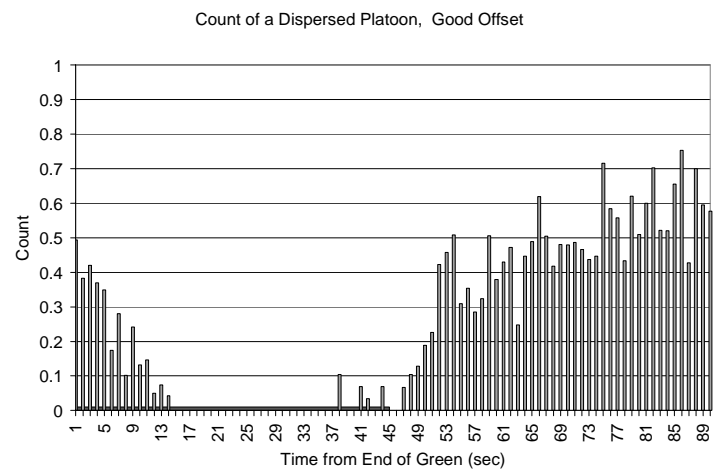

b) Good Offset

Occupancy of a Dispersed Platoon, Positive Offset Shift

Count of a Dispersed Platoon, Positive Offset Shift
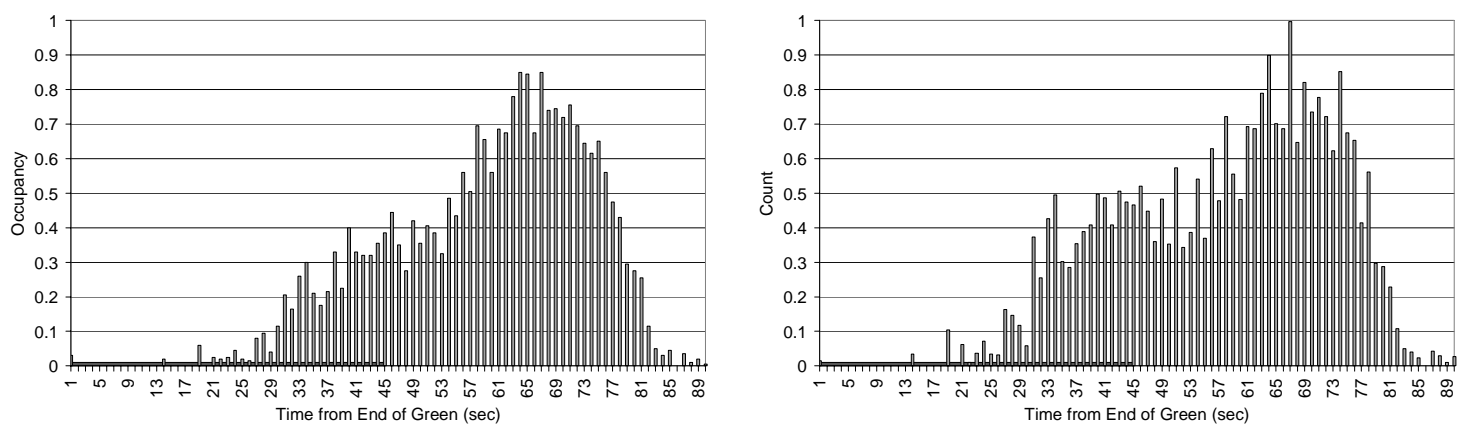

c) Positive Offset Shift

Figure 6-10 A Dispersed Platoon's Occupancy and Count Profiles Over 25 Cycles, Green Phase 45 to 90 

Occupancy of a Dispersed Platoon with a Wide Green Window, Negative

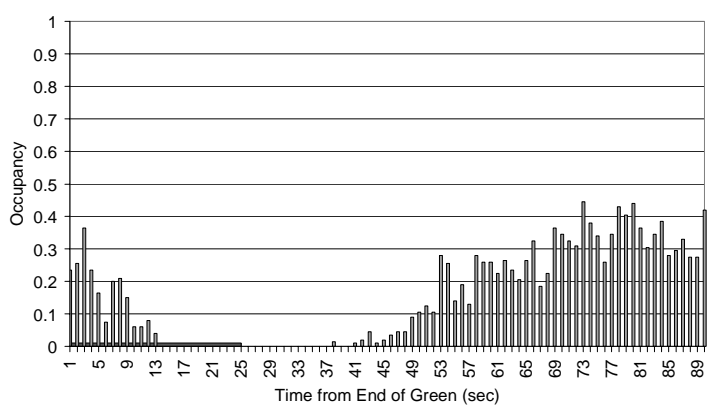

a) Negative Offset Shift

Occupancy of a Dispersed Platoon with a Wide Green Window, Good Offset

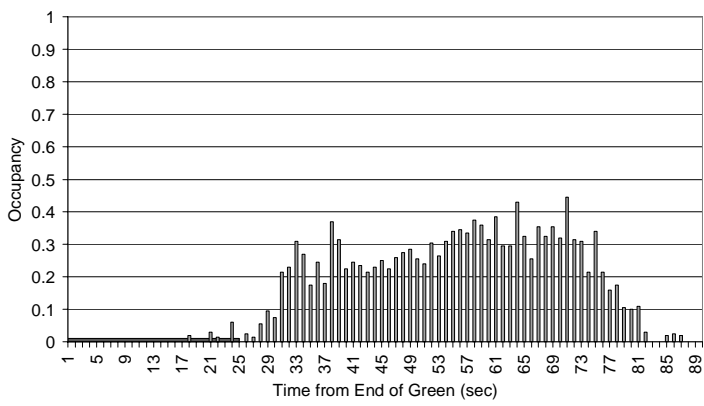

b) Good Offset

Occupancy of a Dispersed Platoon with a Wide Green Window, Positive Offset Shift

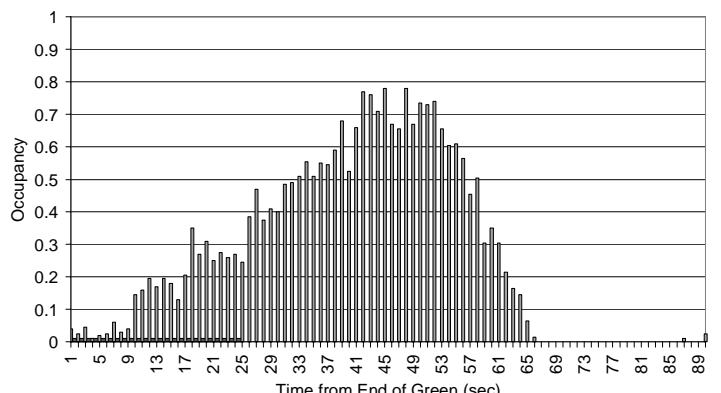

c) Positive Offset Shift
Count of a Dispersed Platoon with a Wide Green Window, Negative Offset

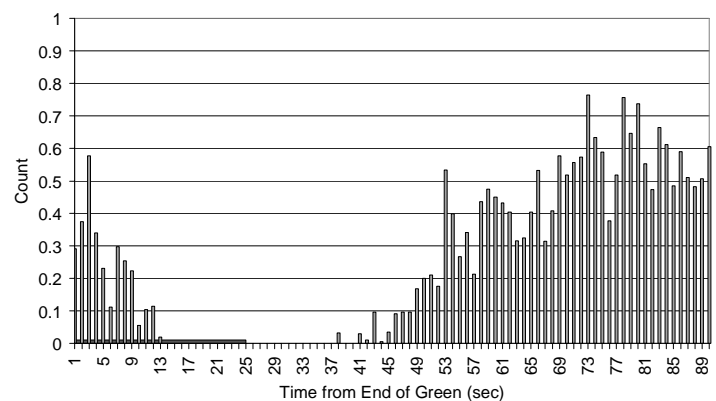

Count of an End-Dispersed Platoon with a Wide Green Window, Good Offset

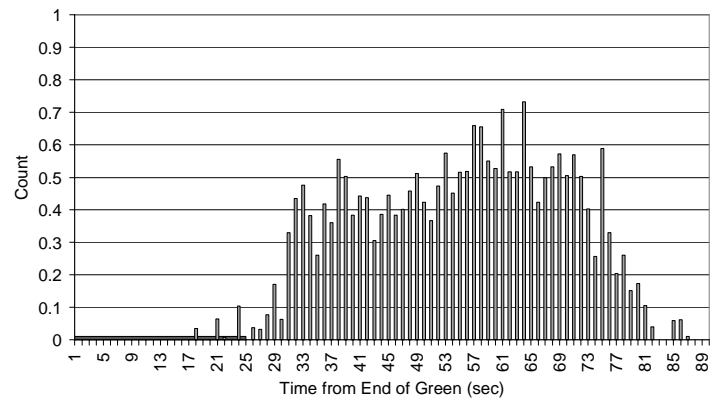

Count of a Dispersed Platoon with a Wide Green Window, Positive Offset
Shift Shift

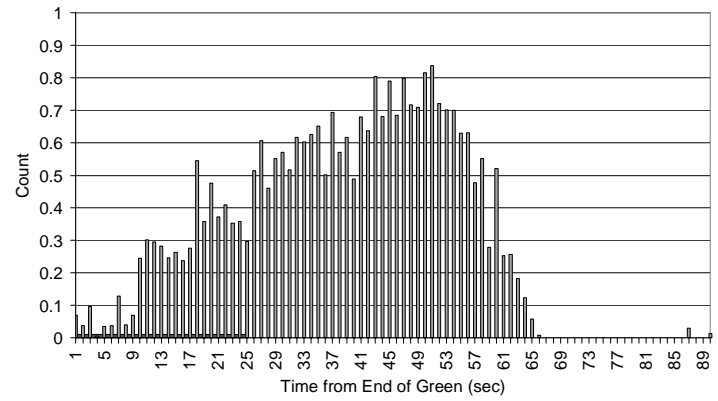

Figure 6-11 A Dispersed Platoon's Occupancy and Count Profiles Over 25 Cycles With a Wide Green Window, Green Phase 25 to 90 


\subsubsection{Modified Skewness Functions}

The difference in shapes between the occupancy and count profiles due to a bad offset can be quantified by means of the difference in the profiles' skewness. PROTRACTS calculates the absolute difference in occupancy and count actuation skewness to determine if offset transitioning is warranted. The calculation is performed at a predefined frequency that can be input at the user level.

Skewness characterizes the degree of asymmetry of a distribution around its mean where positive skewness indicates a distribution with an asymmetric tail extending toward more positive values and negative skewness indicates a distribution with an asymmetric tail extending toward more negative values.

The Skewness of a distribution can be calculated by Equation (6-1).

$$
\text { skewness }=\frac{n}{(n-1)(n-2)} \sum_{i=1}^{n}\left(\frac{x_{i}-\bar{x}}{s t d e v}\right)^{3}
$$

where $x_{i}$ is the ith observation of variable $\mathrm{x}, \bar{x}$ is the mean value, stdev is the standard deviation of variable $\mathrm{x}$, and $\mathrm{n}$ is the number of observations.

Equation (6-2) can be used for calculating the skewness for the detector actuation by substituting the detector actuation per bin (act) instead of $\mathrm{x}$ in Equation (6-1). The standard deviation can be calculated through Equation (6-3), where $n$ is the number of bins in the cycle. 


$$
\begin{aligned}
& \text { skewness }=\frac{n}{(n-1)(n-2)} \sum_{i=1}^{n}\left(\frac{a c t_{i}-a c t}{s t d e v}\right)^{3} \\
& \text { Stdev }=\left(\frac{n \sum a c t^{2}-\left(\sum a c t\right)^{2}}{n(n-1)}\right)^{1 / 2}
\end{aligned}
$$

However, looking more deeply into the skewness equation, one can observe that direct substitution of detector actuation into Equation (6-1) does not preserve the intended meaning of skewness. Using Equation 6-2 would statistically have a different meaning. To illustrate this, an artificial occupancy actuation is shown in Table 6-1 and is plotted in Figure 6-12. The distribution was obtained by the normal distribution function NORMDIST in Microsoft Excel for a mean value of 10 and a standard deviation value of 1.5 . 
Table 6-1 Sample Occupancy Actuation

\begin{tabular}{|r|r|}
\hline Bin & Occupancy (\%) \\
\hline 5 & 0.001 \\
\hline 6 & 0.008 \\
\hline 7 & 0.036 \\
\hline 8 & 0.109 \\
\hline 9 & 0.213 \\
\hline 10 & 0.266 \\
\hline 11 & 0.213 \\
\hline 12 & 0.109 \\
\hline 13 & 0.036 \\
\hline 14 & 0.008 \\
\hline 15 & 0.001 \\
\hline
\end{tabular}

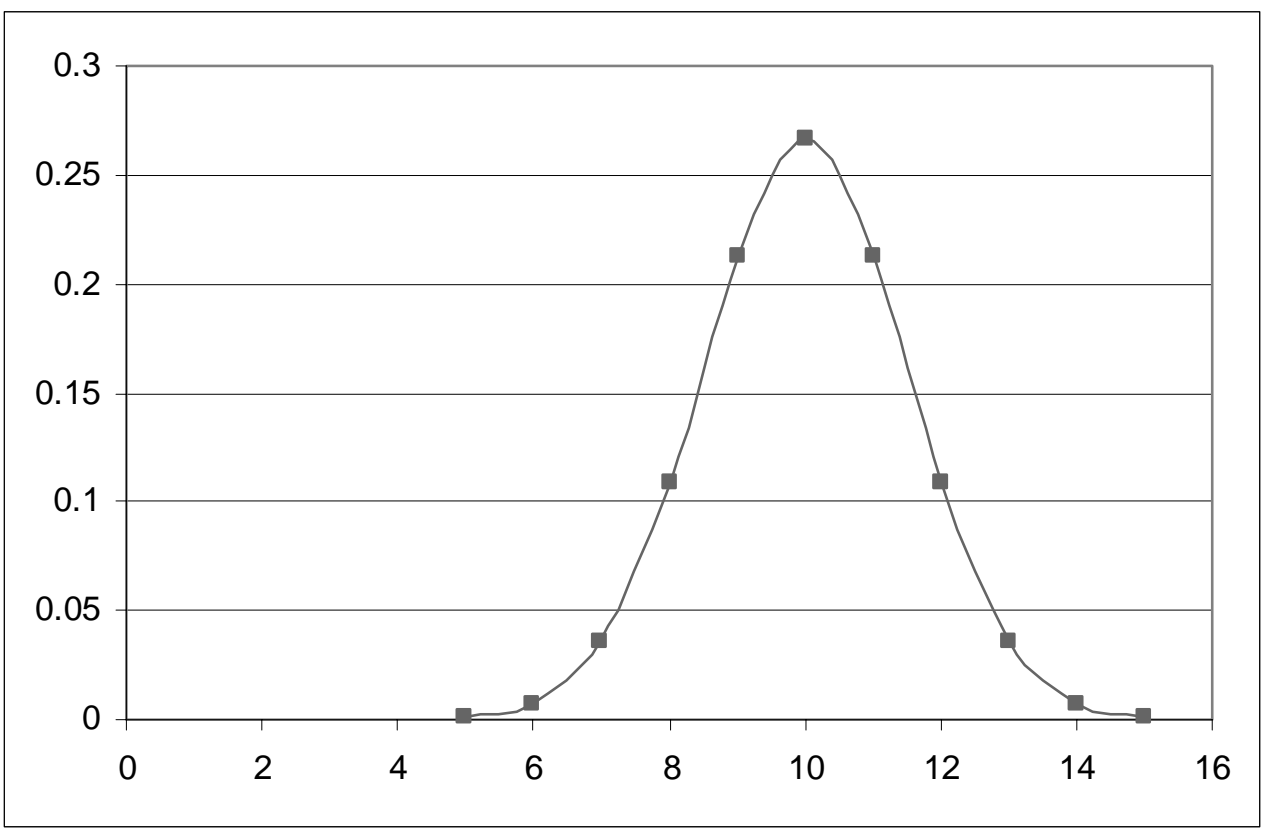

Figure 6-12 Distribution of an Artificially Generated Sample Occupancy Actuation in Table 6-1

Note that the normal distribution bell shape shown in Figure 6-12 is portrayed by drawing the actuation as an ordinate for each bin, which means that each actuation value stands for the number of repetitions of each bin value. For example, bin "9" was repeated 
0.213 times, whereas bin " 10 " was repeated " 0.266 " times (213 and 266 times in a 0.001 scale). This is not the same as substituting the actuation as the bin values.

Table 6-2 shows the descriptive statistics for the occupancy values in Table 6-1, where skewness could be obtained by direct substitution of occupancy in Equation (6-2) and (3) . Note that Table 6-2 shows a skewness value of 0.775 instead of 0 (for a perfectly normal distribution). Also, Table 6-2 shows values of 0.09 and 0.99 for the mean and standard deviation respectively, instead of the 10 and 1.5 used to generate the distribution. These values are correct for a different distribution where the occupancy values are treated as bin values and the ordinates are the number of repetitions of each bin value, as shown in Figure 6-13.

Table 6-2 Descriptive Statistics for Occupancy in Table 6-1 Obtained by Direct Substitution

\begin{tabular}{|l|r|}
\hline Mean & 0.091 \\
\hline Standard Error & 0.030 \\
\hline Median & 0.036 \\
\hline Mode & 0.001 \\
\hline Standard Deviation & 0.100 \\
\hline Sample Variance & 0.010 \\
\hline Kurtosis & -1.006 \\
\hline Skewness & 0.775 \\
\hline Range & 0.265 \\
\hline Minimum & 0.001 \\
\hline Maximum & 0.266 \\
\hline Sum & 1.000 \\
\hline Count & 11.000 \\
\hline
\end{tabular}




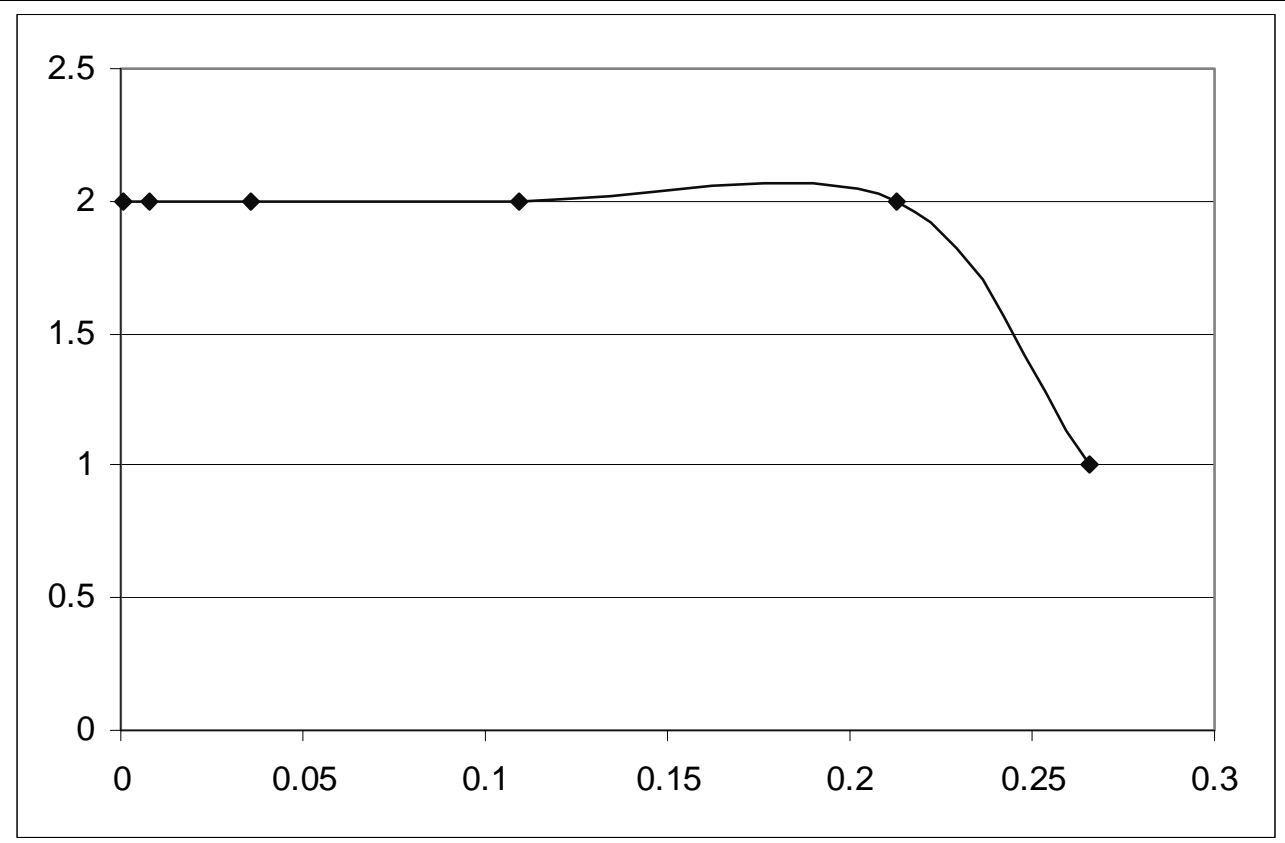

Figure 6-13 Distribution of Occupancy when directly substituted

In order to preserve the meaning of skewness as statistically defined, each bin value has to be repeated several times as determined by the occupancy, or count, actuation. This is illustrated in Table 6-3 where the bin values are repeated according to the occupancy value at each bin (multiplied by one thousand). Table 6-4 shows the histogram analysis for the values in Table 6-3; note that the frequency of each bin is the same as the occupancy values in Table 6-1 multiplied by one thousand. Figure 6-14 shows the histogram graph for the bin values; note that Figure 6-14 looks the same as Figure 6-12. 
Table 6-3 Repetition of Bin Values According to Occupancy Value in

Table 6-1 (occupancy multiplied by a thousand)

\begin{tabular}{|r|}
\hline 5 \\
\hline 6 \\
\hline 6 \\
\hline 6 \\
\hline 6 \\
\hline 6 \\
\hline 6 \\
\hline 6 \\
\hline 6 \\
\hline 7 \\
\hline 7 \\
\hline 7 \\
\hline 7 \\
\hline 7 \\
\hline Etc \\
\hline
\end{tabular}

Table 6-4 A histogram analysis for bin values in Table 6-3

\begin{tabular}{|c|c|}
\hline Bin & Frequency \\
\hline 5 & 1 \\
\hline 6 & 8 \\
\hline 7 & 36 \\
\hline 8 & 109 \\
\hline 9 & 213 \\
\hline 10 & 266 \\
\hline 11 & 213 \\
\hline 12 & 109 \\
\hline 13 & 36 \\
\hline 14 & 7 \\
\hline 15 & 1 \\
\hline More & 0 \\
\hline
\end{tabular}




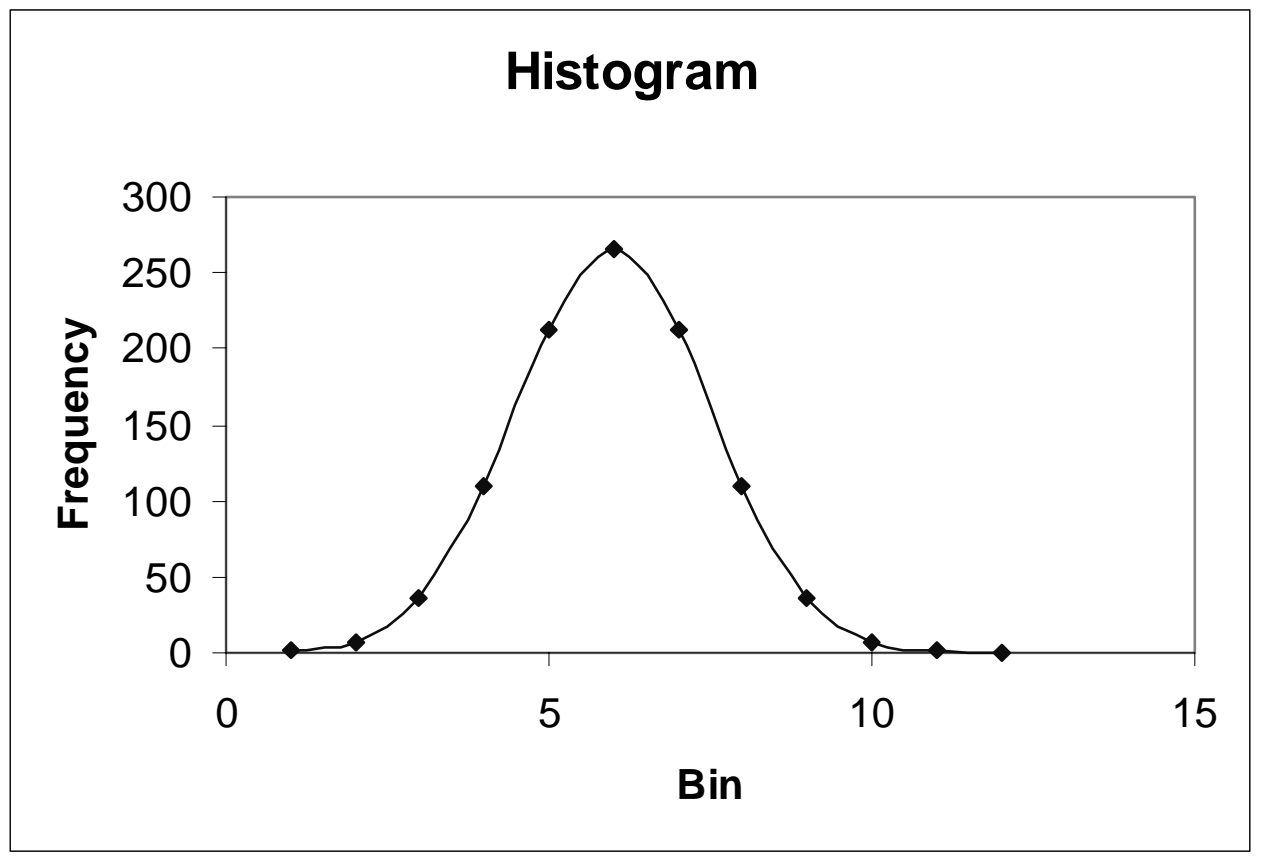

Figure 6-14 A Histogram Graph for Values in Table 6-4

Table 6-5 shows the descriptive statistics calculated for the bin values listed in Table 6-3. Note that the skewness, mean, and standard deviation values are now correct $(0,10$, and 1.5 with the rounding error $)$.

Table 6-5 Descriptive Statistics for Bin Values in Table 6-3

\begin{tabular}{|l|r|}
\hline Mean & 10 \\
\hline Standard Error & 0.047234969 \\
\hline Median & 10 \\
\hline Mode & 10 \\
\hline Standard Deviation & 1.492206433 \\
\hline Sample Variance & 2.22668004 \\
\hline Kurtosis & -0.041755857 \\
\hline Skewness & $4.28467 \mathrm{E}-17$ \\
\hline Range & 10 \\
\hline Minimum & 5 \\
\hline Maximum & 15 \\
\hline Sum & 9980 \\
\hline Count & 998 \\
\hline
\end{tabular}


In order to preserve the meaning of skewness, the skewness and standard deviation equations should be modified. The modified equations are as follows:

$$
\begin{aligned}
& \text { skewness' }=\frac{n^{\prime}}{\left(n^{\prime}-1\right)\left(n^{\prime}-2\right)} \sum_{i=1}^{n} a c t_{i}\left(\frac{\left(\text { bin }_{i}-m^{\prime}\right)}{s_{d e v^{\prime}}}\right)^{3} \\
& \text { Stdev' }=\left(\frac{n^{\prime} \sum a c t_{i}\left(\operatorname{bin}_{i}\right)^{2}-\left(\sum \operatorname{bin}_{i} * a c t_{i}\right)^{2}}{n^{\prime}\left(n^{\prime}-1\right)}\right)^{1 / 2}
\end{aligned}
$$

where:

$$
\begin{aligned}
& n^{\prime}=\sum_{i=1}^{n} a c t_{i} \\
& m^{\prime}=\frac{\sum_{i=1}^{n} \operatorname{bin}_{i} * a c t_{i}}{n^{\prime}}
\end{aligned}
$$

act $_{i}: \quad$ actuation at bin $i$ in the cycle

bin: interval over which actuation is aggregated, 5 seconds was used in this study.

and

$\mathrm{n}$ number of actuation bins (cycle length in seconds devided by bin size)

These new equations allow the calculation of the occupancy and count skewness from the bins/actuation values. It should be noted that multiple-second bins can be used instead of one-second bins. This can improve PRO-TRACTS performance in two ways: a) data normalization, where noise due to the stochastic arriaval of vehicles can be reduced, in contrast to real changes in travel pattern due to improper offset values; and b) histograms can be built from a smaller number of cycles, which leads to a more responsive performance (more frequent offset evaluation operation). 


\subsubsection{Calculation of Skewness Around the Count Profile's Mean}

A small percentage of traffic turning from the side street during the red period, which is the beginning of the cycle since the cycle is referenced to the end of green, usually shows similar occupancy and count profiles regardless of the offset value (Figure 6-16 and Figure 6-16). This is due to the fact that the gap between the stop bar and the algorithm detector can accommodate low traffic volumes before showing a significant change in the acuation profiles at the detector location. Althought the shapes of the profiles are similar, the effect of the turning traffic is not cancelled out if count and occupancy skewness are calculated around each distribution's mean, which has a different location for the two distributions. To cancel the effect of the turning movement and at the same time improve the ability of the algorithm to detect bad offsets, the skewness of both distributions is calculated around the count distribution's mean. 


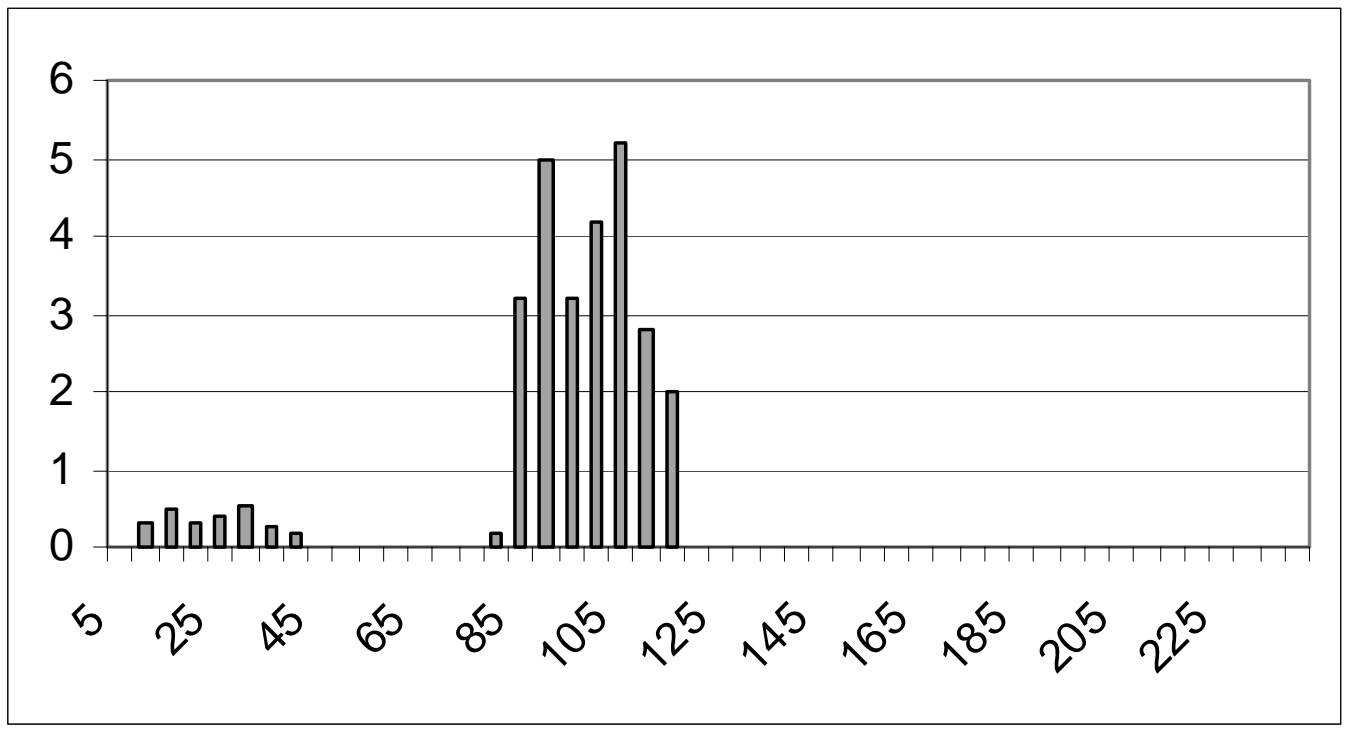

a) Count Profile

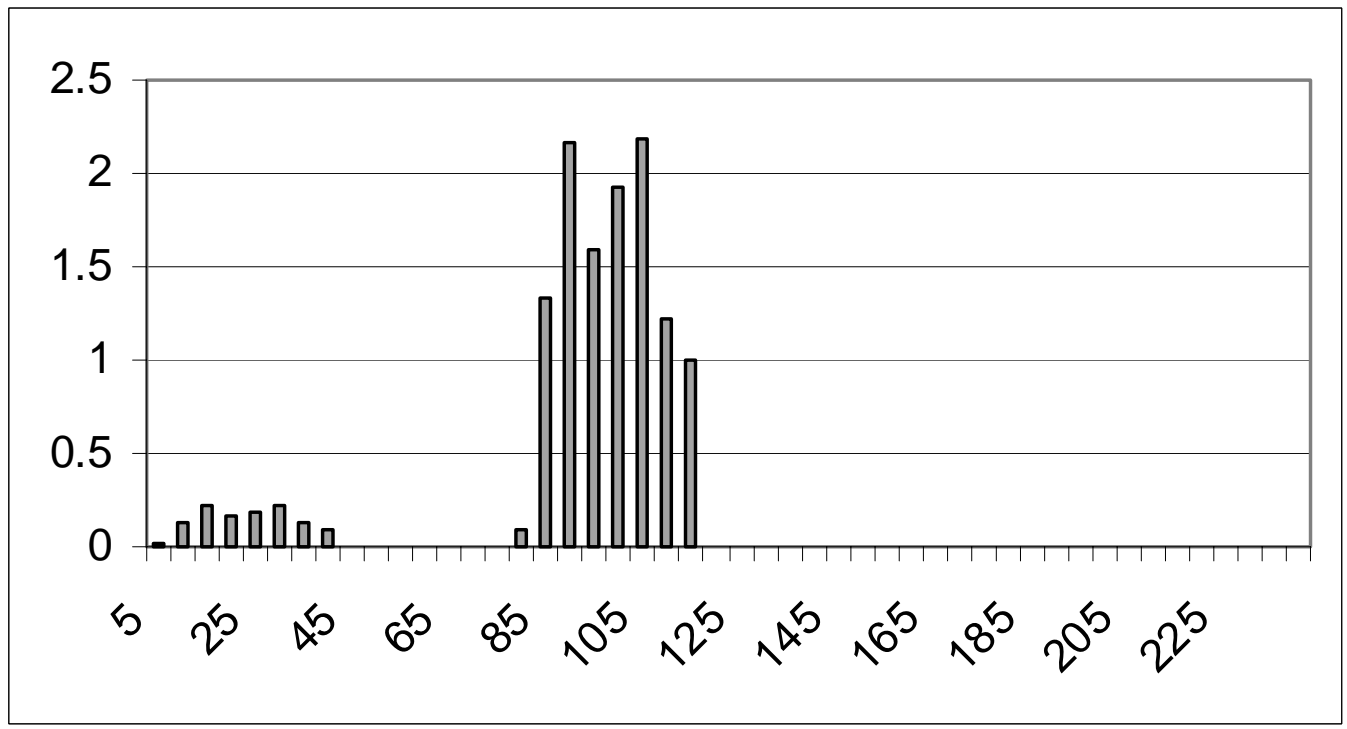

b) Occupancy Profile

Figure 6-15 Good Offset With a Turning Traffic Profiles 


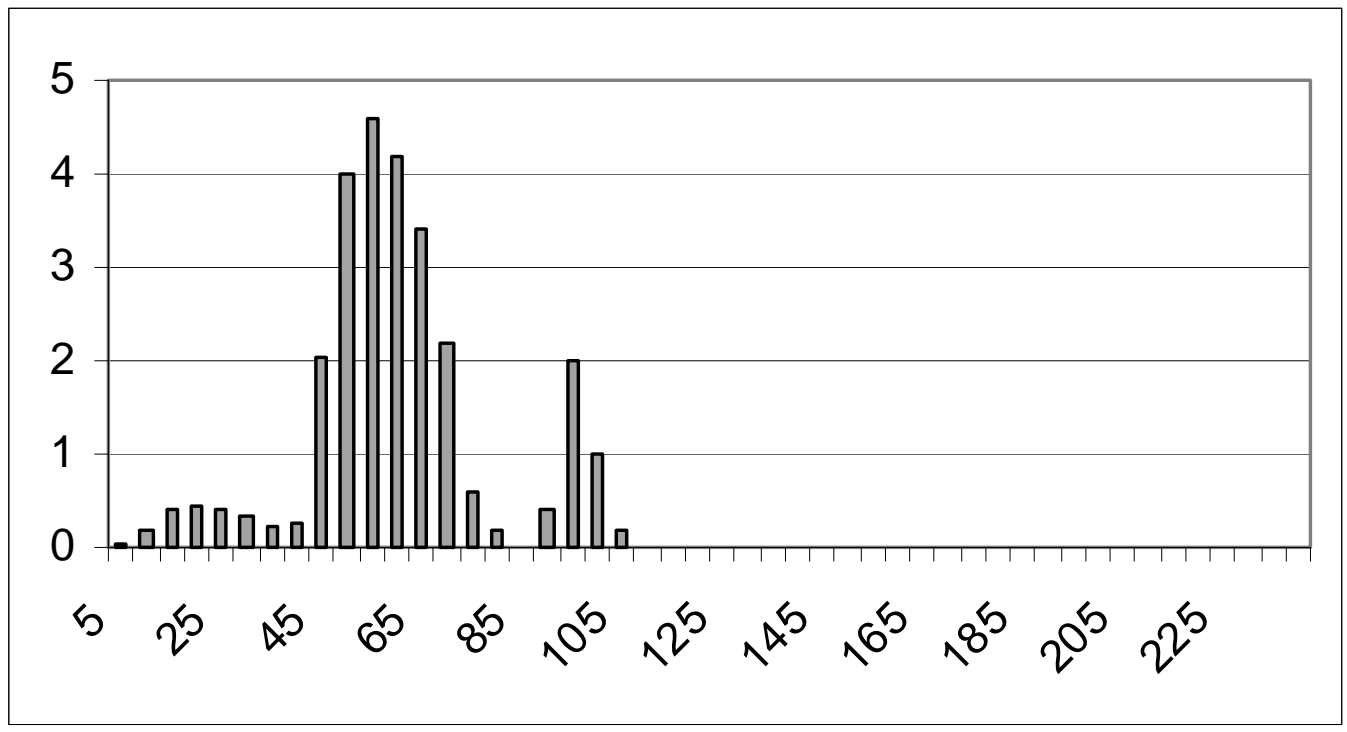

a) Count profile

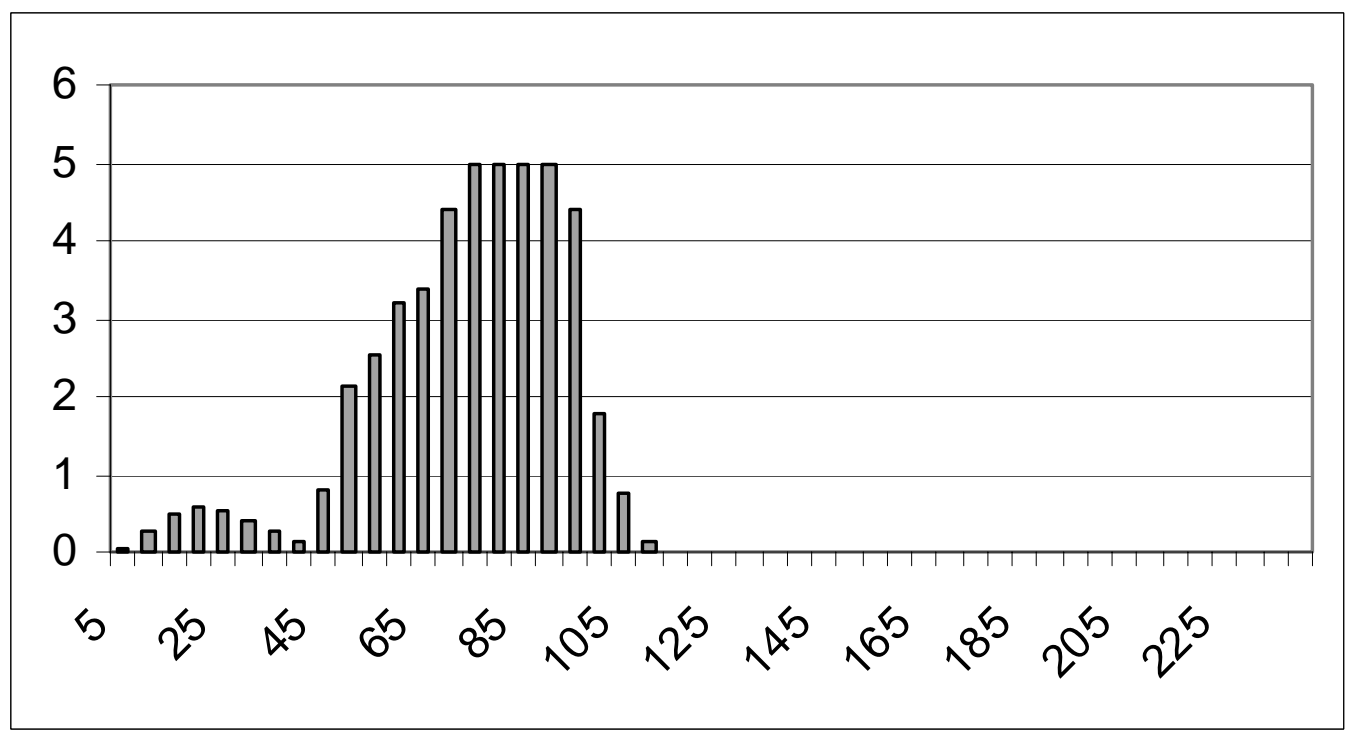

b) Occupancy profile

Figure 6-16 Bad Offset With a Turning Traffic Profiles 


\subsubsection{Offset Transitioning Warrant}

An experiment was conducted to quantify the concept of PRO-TRACTS with different sets of cycles, percent green ratios, and offsets. Figure 6-17-a shows the differential skewness values for the combinations of six different offset shift levels and three different percent greens for a surveillance detector 30 meter upstream and 60 seconds in cycle length. Figure 6-17-b shows the same histograms, but for a 120 second cycle length. Figure 6-17-c and Figure 6-17-d show the same histograms as in Figure 6-17-a and Figure 6-17-b but for a surveillance detector 60 meters upstream. The experiment illustrates that the more the offset shifts from the ideal range, the more pronounced the difference in actuation skewness. These figures empirically show that a differential skewness threshold of 0.25 can be used for triggering PRO-TRACTS. 


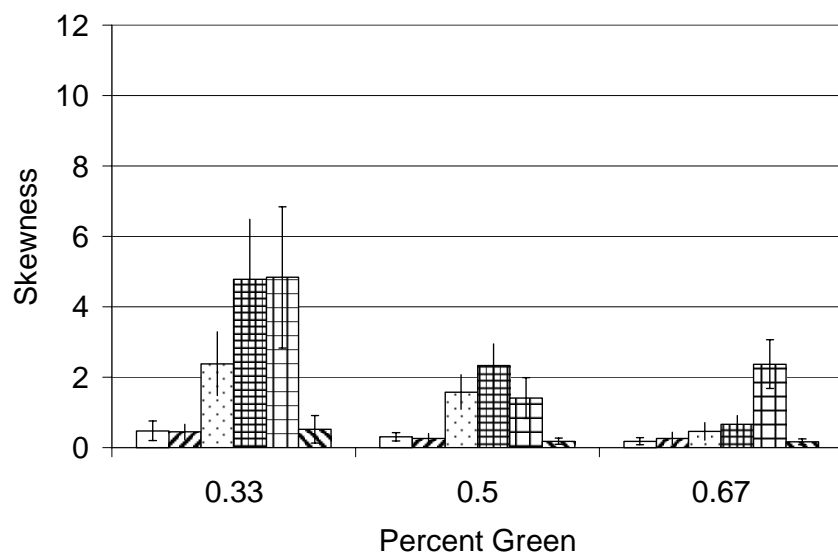

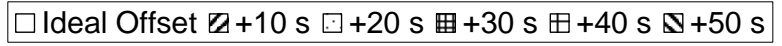

a) 30 meter Upstream Detector, $\mathrm{C}=60 \mathrm{sec}$

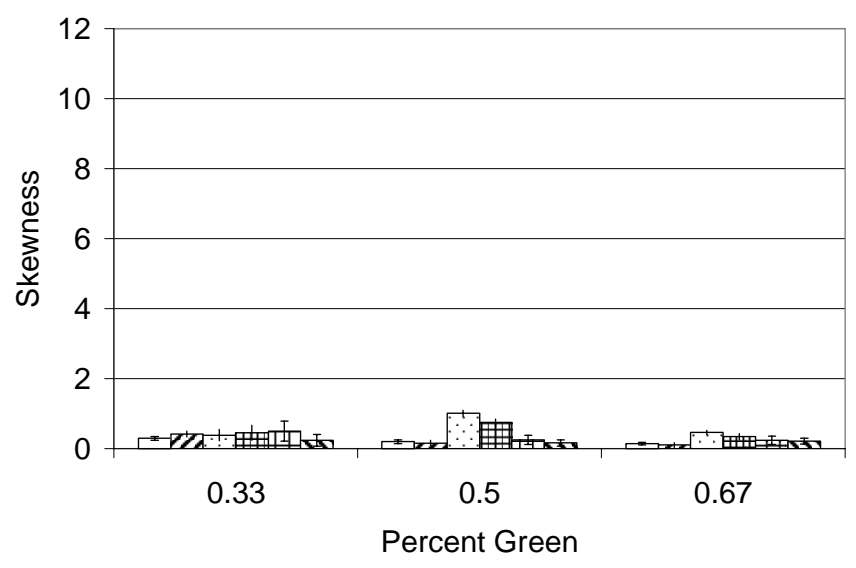

$\square$ Ideal Offset $\square+10 \mathrm{~s} \square+20 \mathrm{~s}$ 田+30 s $\mathbb{\nabla}+40 \mathrm{~s} \mathbf{\mathrm { V }}+50 \mathrm{~s}$

c) 60 meter Upstream Detector, $\mathrm{C}=60 \mathrm{sec}$

Figure 6-17 Differential Skewness between Occupancy and Counts Histograms

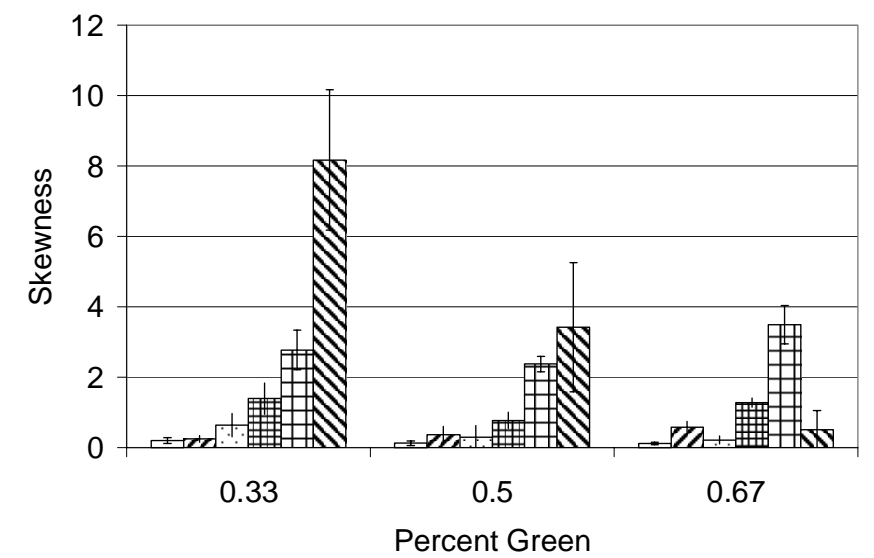

$\square$ Ideal Offset $\square+20 \mathrm{~s} \square+40 \mathrm{~s}$ 田+60s $\mathbb{\mathrm { A }}+80 \mathrm{~s} \mathbf{\mathrm { s } + 1 0 0 \mathrm { s }}$

b) 30 meter Upstream Detector, $\mathrm{C}=120 \mathrm{sec}$

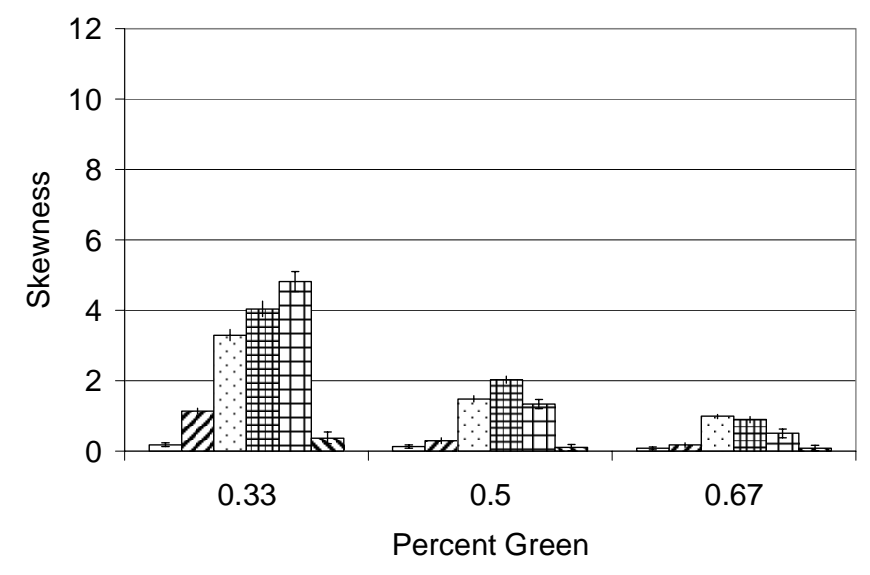

$\square$ Ideal Offset $\square+20 \mathrm{~s} \cdot+40 \mathrm{~s}$ 困+60 s $⿴+80 \mathrm{~s} \$+100 \mathrm{~s}$ d) 60 meter Upstream Detector, $\mathrm{C}=120 \mathrm{sec}$ 


\subsubsection{Direction of Offset Transitioning}

Once the application of PRO-TRACTS is warranted, the algorithm follows a greedy approach to determine the optimum direction of offset transitioning. PROTRACTS moves the current green window such that more of the current occupancy actuation is included within the new green window. The philosophy here is that for the same traffic volume, higher occupancy typically denotes slower moving, or even stopped, traffic. Therefore, lowering the occupancy actuation can be used as a proxy objective to obtain better progression. This objective can be achieved by moving the green window so that more of the current occupancy actuation histogram is included within the new window location. Figure 6-18-a illustrates how this decision is made. Note that if the green window was moved to the right, the estimated increase in occupancy histogram aligned within the green window will be area "d" - area "b." If the green window was moved to the left, the estimated increase in the occupancy histogram aligned within the green window will be area "a" - area "c." Following this approach, the green window should be moved to the left if $(a-c)>(d-b)$, which can also be algebraically expressed as $(a+b)>(c+d)$. Areas $(a+b)$ and $(c+d)$ in Figure 4-a are calculated as OccL and OccR respectively in Equations (6-8) and (6-9). PRO-TRACTS transitions the offset in the direction of the greater area calculated in Equations (6-8) and (6-9).

$$
O c c L=\sum_{l=1}^{n} \sum_{k=G s-\text { Shift }}^{G s+\text { Shift }} o c c_{k, j-l}
$$

and 


$$
O c c R=\sum_{l=1}^{n} \sum_{k=G e-S h i f t}^{G e+\text { Shift }} o c c_{k, j-l}
$$

where:

Occl: Estimated increase in occupancy area under the green window if offset was transitioned to the left

OccR: Estimated increase in occupancy area under the green window if offset was transitioned to the right

$\mathrm{n}$ : number of cycles included in PRO-TRACTS evaluation (1 cycle was used in this study)

Ge: green end time of the Last cycle

Gs: green start time. Calculated by subtracting the average green duration from green end $(\mathrm{Ge})$

Shift: PRO-TRACTS Window shift parameter (5 seconds is used in this study) and

$\mathrm{j}: \quad$ current cycle as defined in Equation (6-2)

Figure 6-18-b shows the occupancy actuation that resulted from moving the green window in Figure 6-18-a to the left. 


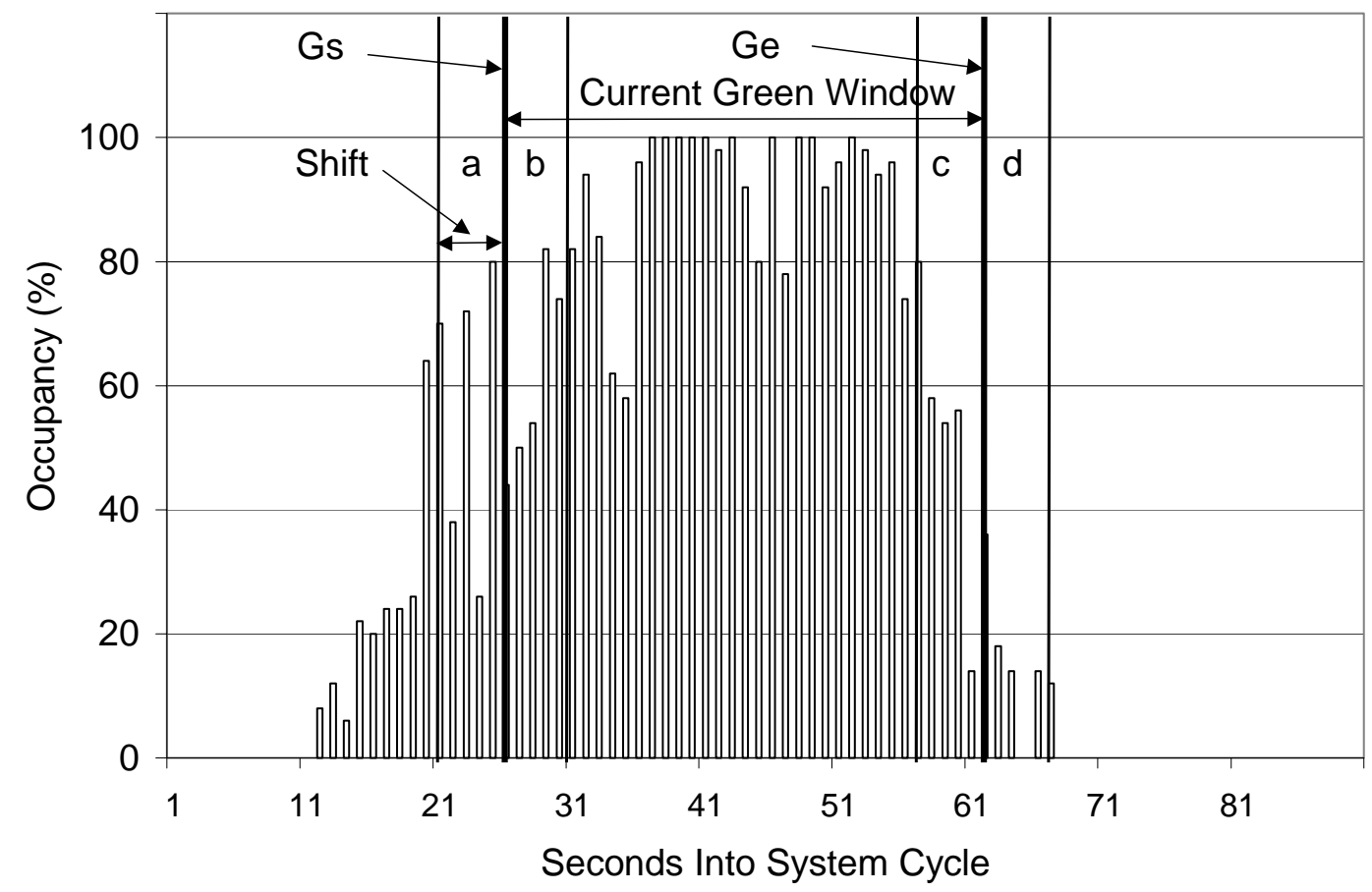

a) Occupancy Actuation Before Offset Transition

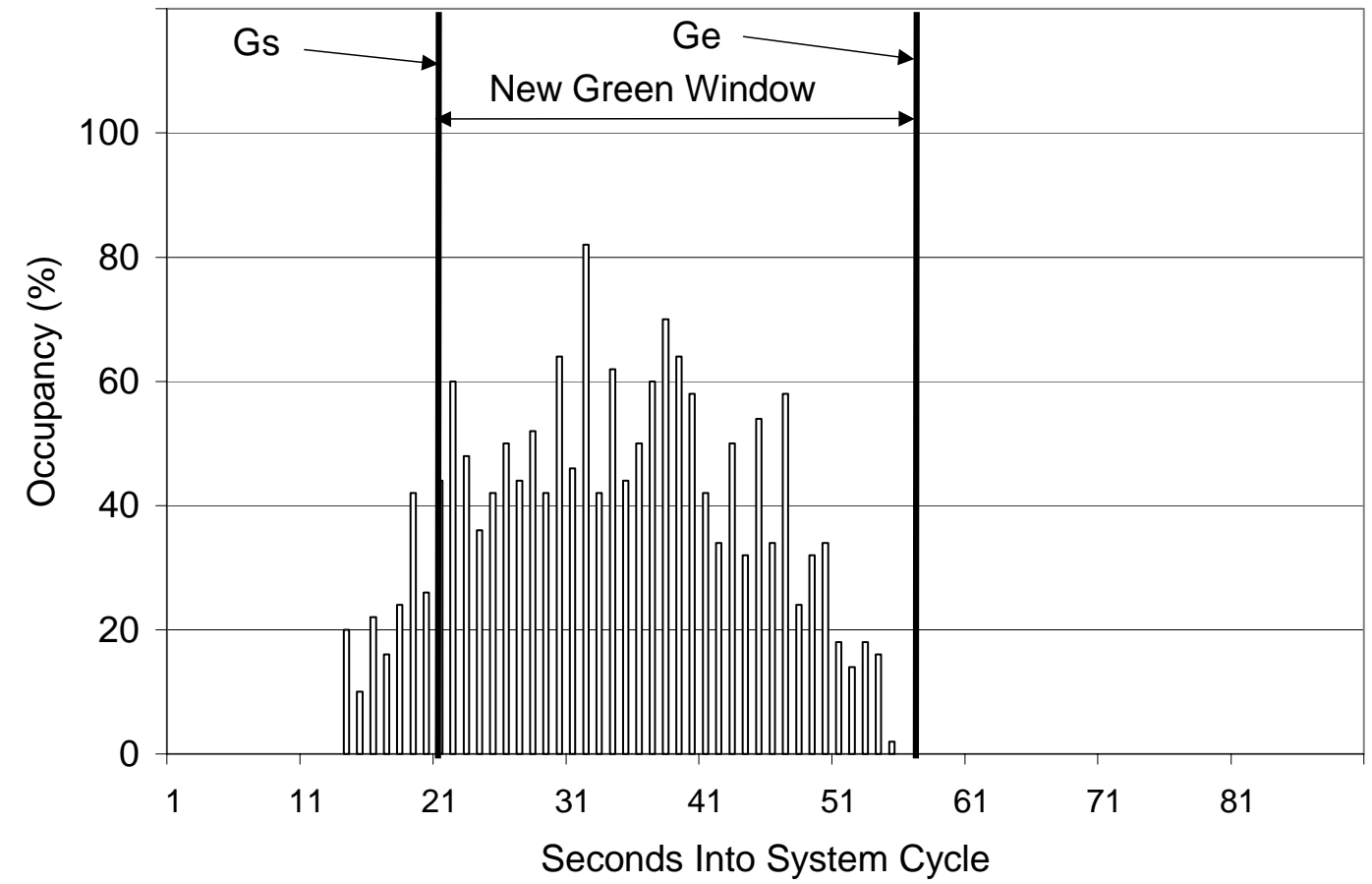

b) Occupancy Actuation After Offset Transition

Figure 6-18 Determination of Offset Transitioning Direction in PRO-TRACTS 
Figure 6-19 shows the results of an experiment to test and validate the offset transitioning direction algorithm in PRO-TRACTS. The figure shows the results of 18 simulation runs with offsets shifted by multiple of five seconds from the ideal between the runs. For each case, the area of the occupancy profile within the green window is calculated, along with the anticipated area if the green window was moved five seconds to the left and right (negative or positive shifts respectively). The figure shows that the shortest path to the optimum offset is in the direction of the maximum anticipated occupancy profile's area within the green window.

\section{Detector Occupancy Within the Green Window Vs Offset Shift Offset Step $=5 \mathrm{sec}, \mathrm{V}=1200 \mathrm{veh} / \mathrm{hr}$}

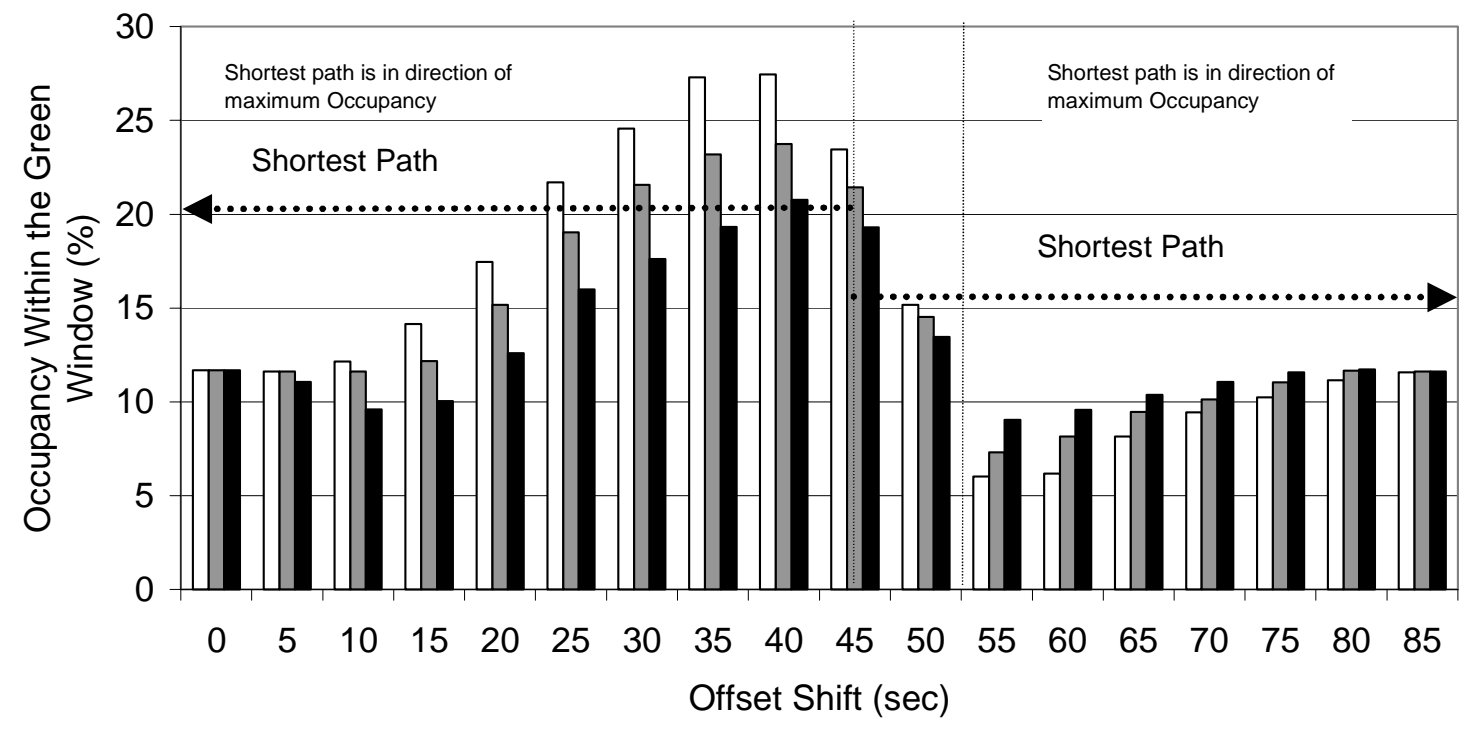

$\square$ Predicted Occupancy for a -ve Shift $\square$ Current Occupancy Value

Predicted Occupancy for a +ve Shift

Figure 6-19 Validation of Offset Transitioning Direction in PRO-TRACTS 


\subsubsection{Network Level Coordination}

Once an offset adjustment takes place at a particular intersection, PRO-TRACTS changes the offset at all the downstream intersections by the same amount such that the intersections do not loose coordination. This mechanism insures that the offset of any intersection referenced to its upstream intersection remains the same. If the downstream intersection needed an offset adjustment as well, the cumulative adjustment in offset is then applied.

\subsubsection{Effect of Detector Location}

The farther the surveillance detector location is set back, the less sensitive it becomes, especially for short cycle lengths because of the small queue size which is also the case with low traffic volume. This is clearly illustrated by comparing Figure 6-17-a and Figure 6-17-c, where a 60 meter upstream detector could not capture the effect of offset shifts where a 30 meter upstream detector did. It should be noted that it is also not desirable to have the detector too close to the downstream signal so as not to capture the turning-movement traffic noise from side streets. In this chapter, a surveillance detector 60 meter upstream was used.

\subsection{Evaluation Network and Procedure}

Two studies were conducted to test the basic concept of PRO-TRACTS. The first study evaluated PRO-TRACTS with a set of incorrect offsets at three intersections. The second study evaluated the adaptation of PRO-TRACTS to an offset previously tuned for an early-return-to-green. The evaluation network used to test the effect of PRO-TRACTS 
is shown in Figure 6-20. The network had 11-meter stop bar detectors at the side streets and the arterial left-turn pockets, and 1.8-meter advanced detectors $92 \mathrm{~s}$ meter3 upstream for the through-arterial phases. Figure 6-21 shows the common ring structure for all intersection in the studied network. Table 6-6 lists the general phase parameters for all intersections (minimum green, vehicle extension, etc). Table 6-7 and Table 6-8 list the coordination parameters for all intersections and the entry volumes per periods for all intersections for the two studies. Turning percentages were $80 \%$ through, $10 \%$ right, and $10 \%$, left for all approaches. 


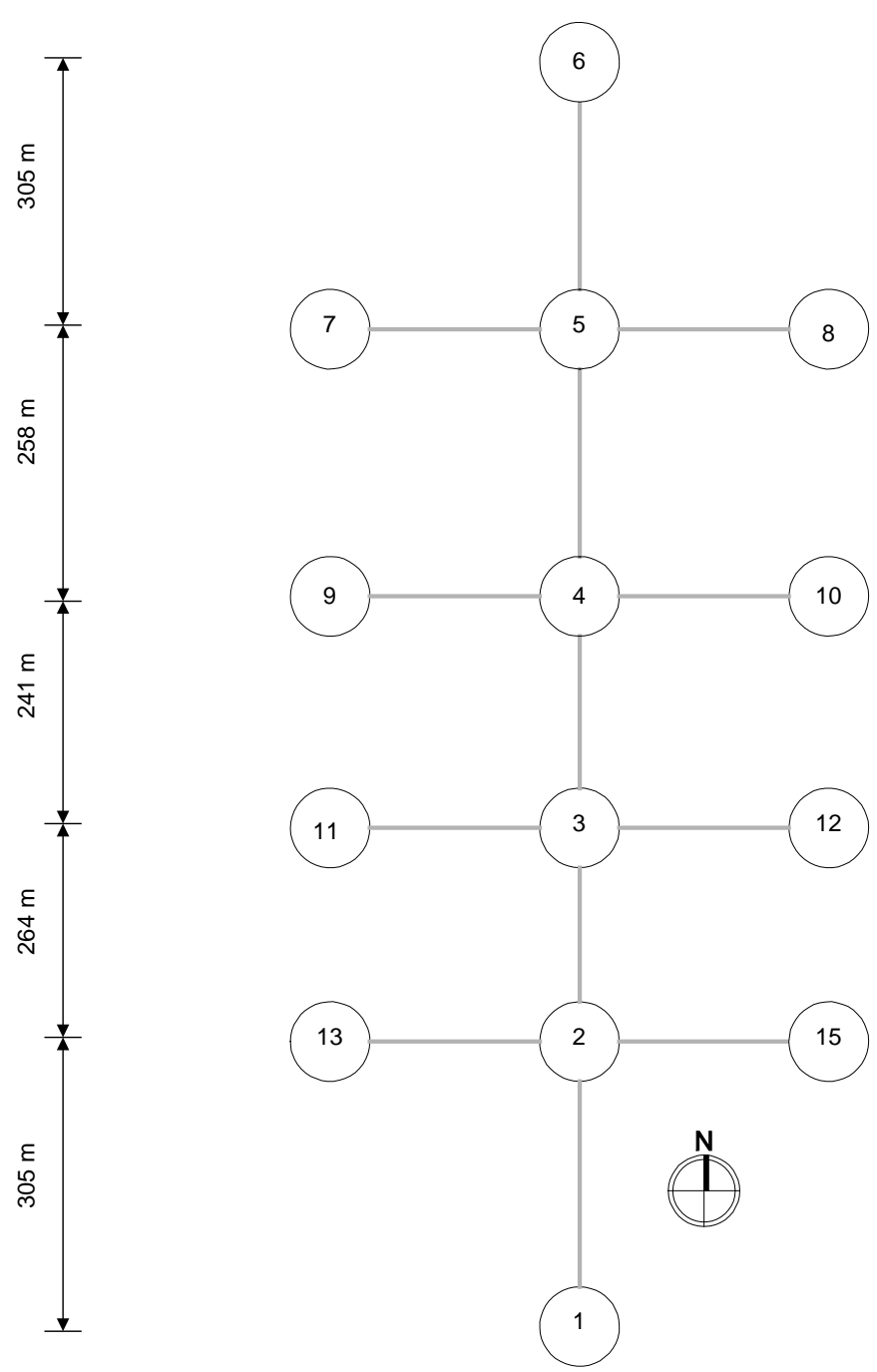

Figure 6-20: Test Bed Study Network 


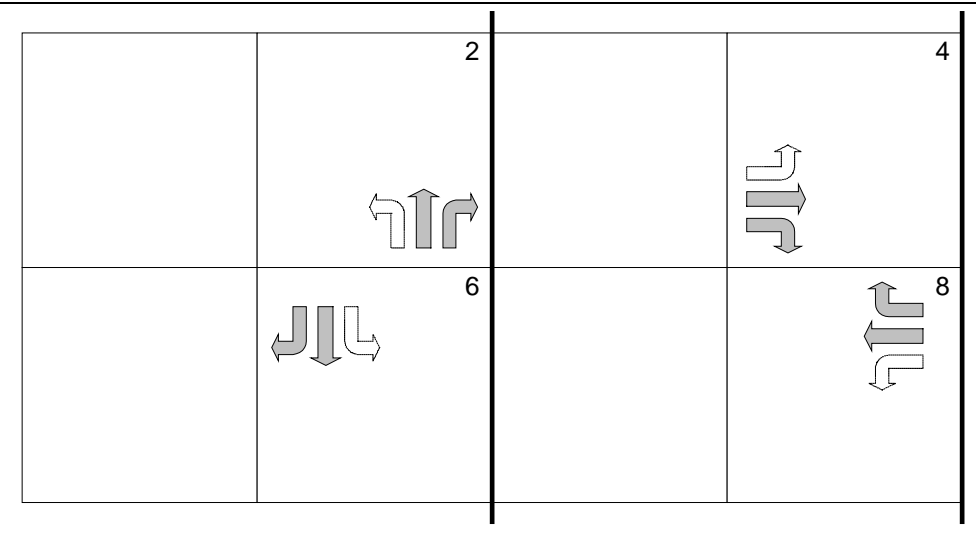

Figure 6-21 Test Bed Phase Sequence Ring Diagram for Nodes 2, 3, 4, and 5

Table 6-6 Test Bed General Phase Parameters (All Intersections)

\begin{tabular}{|l|c|c|c|c|c|c|c|c|}
\hline \multirow{2}{*}{ DESCRIPTION (SEC) } & \multicolumn{9}{c|}{ Phase Number } \\
\cline { 2 - 10 } & 1 & 2 & 3 & 4 & 5 & 6 & 7 & 8 \\
\hline MINIMUM GREEN & -- & 20 & -- & 10 & -- & 20 & -- & 10 \\
\hline VEHICLE EXTENSION & -- & 3.5 & -- & 3.5 & -- & 3.5 & -- & 2.0 \\
\hline MAXIMUM GREEN & -- & 99 & -- & 99 & -- & 99 & -- & 99 \\
\hline YELLOW CLEARANCE & -- & 3.0 & -- & 3.0 & -- & 3.0 & -- & 3.0 \\
\hline RED CLEARANCE & -- & 2.0 & -- & 2.0 & -- & 2.0 & -- & 2.0 \\
\hline MINIMUM GAP TIME & -- & 3.5 & -- & 3.5 & -- & 3.5 & -- & 3.5 \\
\hline
\end{tabular}

Table 6-7 Test Bed Coordination Parameters

\begin{tabular}{|c|c|c|c|c|c|c|c|c|c|c|c|}
\hline \multirow[t]{2}{*}{ Study } & \multirow[t]{2}{*}{ Cycle } & \multirow[t]{2}{*}{ INT } & \multirow[t]{2}{*}{ Offset } & \multicolumn{8}{|c|}{ Force Off for Each Phase } \\
\hline & & & & 1 & 2 & 3 & 4 & 5 & 6 & 7 & 8 \\
\hline \multirow[t]{4}{*}{ Cases 1-5 } & \multirow[t]{4}{*}{120} & 2 & 0 & -- & 120 & -- & 70 & -- & 120 & -- & 70 \\
\hline & & 3 & 23 & -- & 120 & -- & 65 & -- & 120 & -- & 65 \\
\hline & & 4 & 83 & -- & 120 & -- & 60 & -- & 120 & -- & 60 \\
\hline & & 5 & 70 & -- & 120 & -- & 55 & -- & 120 & -- & 55 \\
\hline \multirow[t]{4}{*}{ Bad Offsets } & \multirow[t]{4}{*}{90} & 2 & 0 & -- & 90 & -- & 45 & -- & 90 & -- & 45 \\
\hline & & 3 & 68 & -- & 90 & -- & 40 & -- & 90 & -- & 40 \\
\hline & & 4 & 30 & -- & 90 & -- & 35 & -- & 90 & -- & 35 \\
\hline & & 5 & 68 & -- & 90 & -- & 30 & -- & 90 & -- & 30 \\
\hline
\end{tabular}


Table 6-8 Test Bed Entry Volumes per Period (VPH)

\begin{tabular}{|c|c|c|c|c|c|c|c|}
\hline \multirow[t]{3}{*}{ INT } & Study & \multicolumn{5}{|c|}{ Early Return to Green } & Bad Offsets \\
\hline & Cycle & \multicolumn{5}{|c|}{120} & 90 \\
\hline & Case & 1 & 2 & 3 & 4 & 5 & -- \\
\hline \multirow[t]{4}{*}{2} & NB & 1150 & 1150 & 1150 & 1150 & 1150 & 1350 \\
\hline & EB & $\begin{array}{l}- \\
-\end{array}$ & - & -- & -- & -- & - \\
\hline & WB & 1800 & 1800 & 1800 & 1800 & 1800 & 1350 \\
\hline & EB & 850 & 850 & 850 & 850 & 850 & 850 \\
\hline \multirow[t]{2}{*}{3} & WB & 1600 & 1600 & 1600 & 1600 & 1600 & 1250 \\
\hline & EB & 100 & 100 & 100 & 100 & 100 & 100 \\
\hline \multirow[t]{2}{*}{4} & $\mathbf{W B}^{1}$ & 100 & 400 & 700 & 1000 & 1300 & 900 \\
\hline & EB & 400 & 400 & 400 & 400 & 400 & 400 \\
\hline \multirow[t]{2}{*}{5} & WB & 1300 & 1300 & 1300 & 1300 & 1300 & 600 \\
\hline & SB & 400 & 400 & 400 & 400 & 400 & 400 \\
\hline
\end{tabular}

1 volume changing at intersection approach

\subsubsection{Adapting from Incorrect Offsets}

The first study evaluated the effect of PRO-TRACTS applied to an arterial with poor offsets. Figure 6-22 shows the northbound cumulative travel time during four consecutive hours, with and without running PRO-TRACTS. This figure was obtained by setting the poor offsets in the three labeled intersections and running CORSIM simulation five times with different number seeds without PRO-TRACTS, and five times with PROTRACTS. The figures show a statistically significant savings in travel time with PROTRACTS as shown by the $95 \%$ error bars with PRO-TRACTS reducing total travel time by $27.9,32.7,33.7$, and 34.5 percents, during the first, second, third, and fourth hours respectively. Figure 6-23 shows the southbound cumulative travel time during four consecutive hours with and without running PRO-TRACTS for the same simulation. The figure shows a statistically non-significant decrease in travel time with PRO-TRACTS totaling $0,3.6,4.9$, and 5.1 percents during the first, second, third, and fourth hours respectively. 

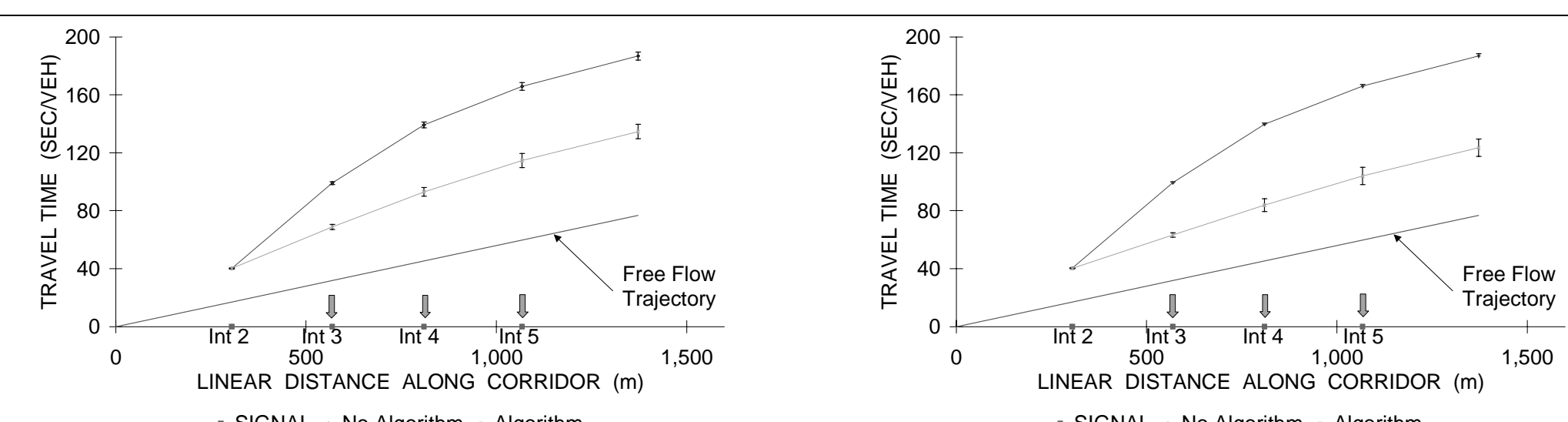

a) First Hour

- SIGNAL - No Algorithm - Algorithm

b) Second Hour
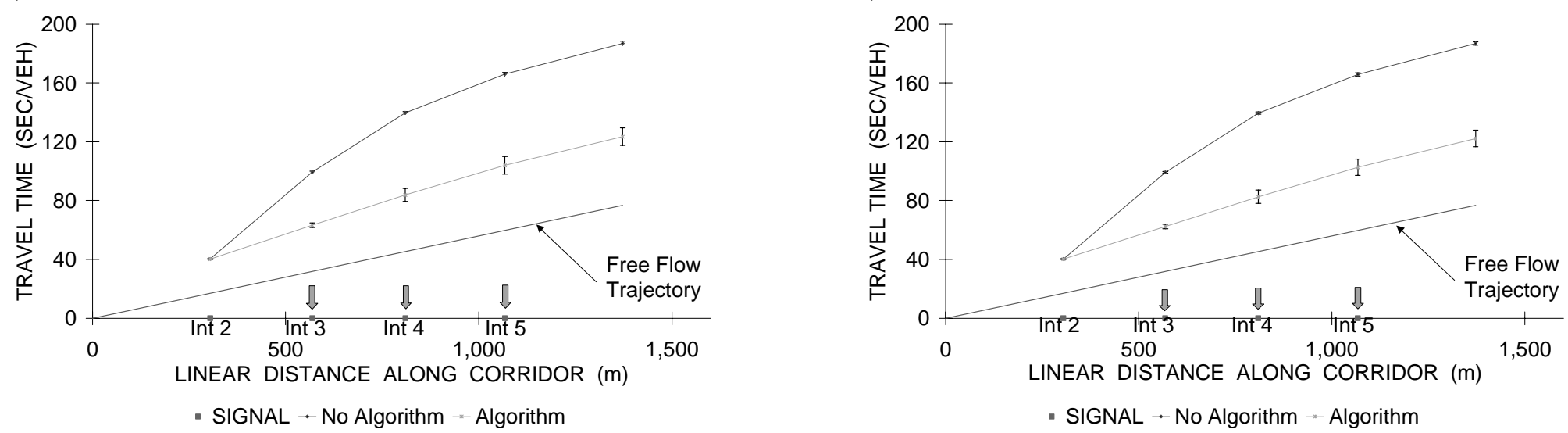

c) Third Hour

d) Fourth Hour

Figure 6-22 Test Bed North Bound Cumulative Travel Time-90 sec Cycle 

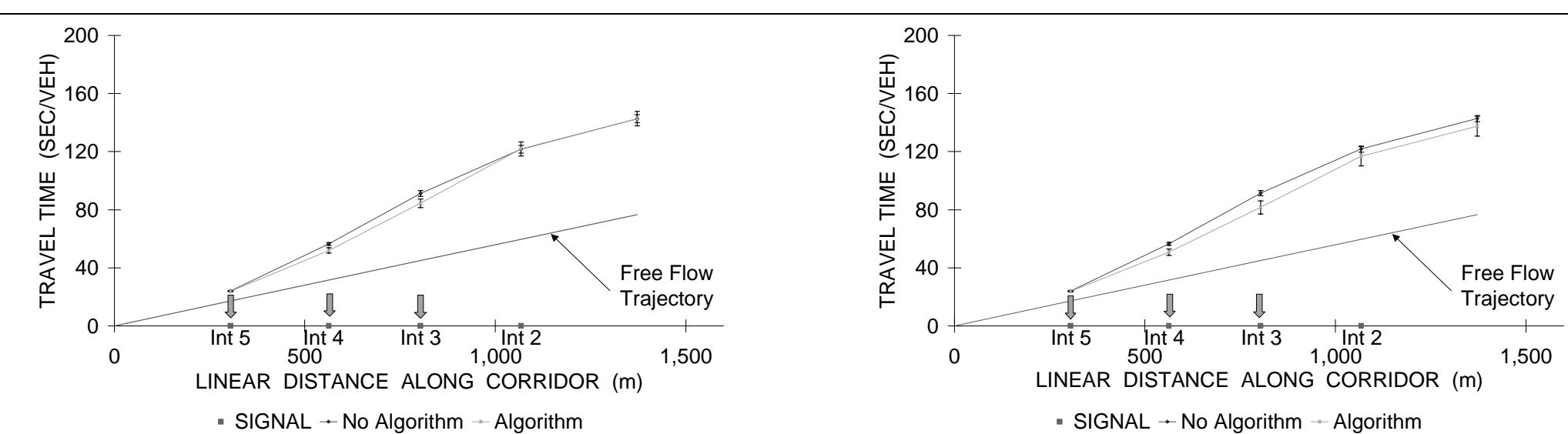

a) First Hour

- SIGNAL $\rightarrow$ No Algorithm - Algorithm

- SIGNAL - No Algorithm - Algorithm

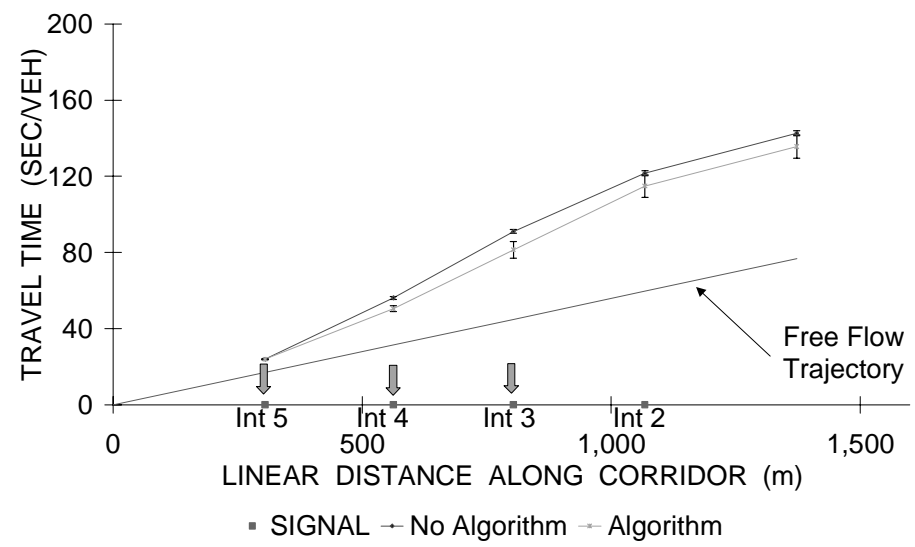

b) Second Hour

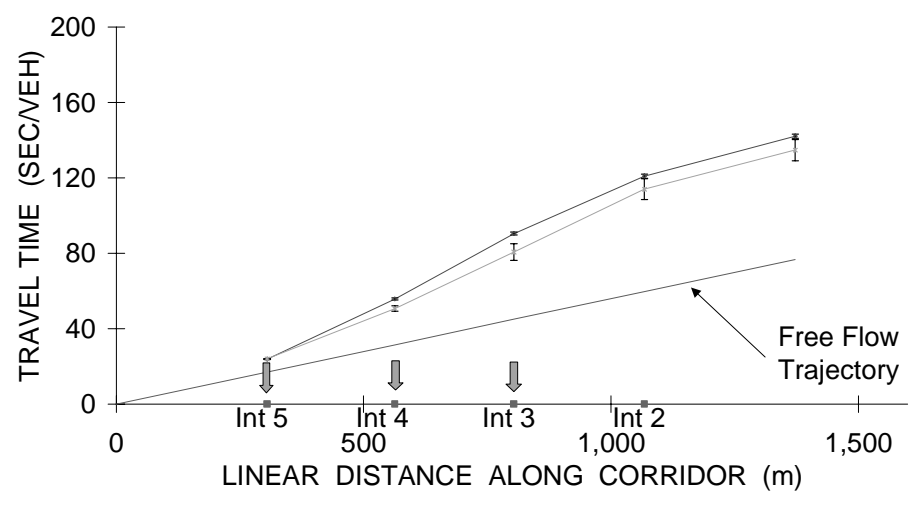

c) Third Hour

d) Fourth Hour

Figure 6-23 Test Bed South Bound Cumulative Travel Time-90 sec Cycle 
Figure 6-24-a shows how the offsets converged with PRO-TRACTS during one simulation run. Note that although the Node 5's offset transitioned from 68 to 73 seconds, the figure shows the initial offset value of 68 as 158 seconds (68 plus 90 seconds cycle length) indicating the path of the offset until it was stabilized. This long path was taken because the immediate upstream signal's offset was simultaneously decreasing. This long path was not taken, however, when the immediate upstream signal offset was constant as was the case with Node 3' offset. Figure 6-24-b shows the cycle length during the simulation. Note that because the offsets were changed by small increments, there were no major irregularities in the controllers' cycle lengths.

The total delay and number of stops, with and without PRO-TRACTS, are shown in Figure 6-25, where the delay and number of stops for all approaches are seen to have decreased with PRO-TRACTS, $29.0 \%$ and $27.6 \%$ percent respectively, by the end of the fourth hour. 


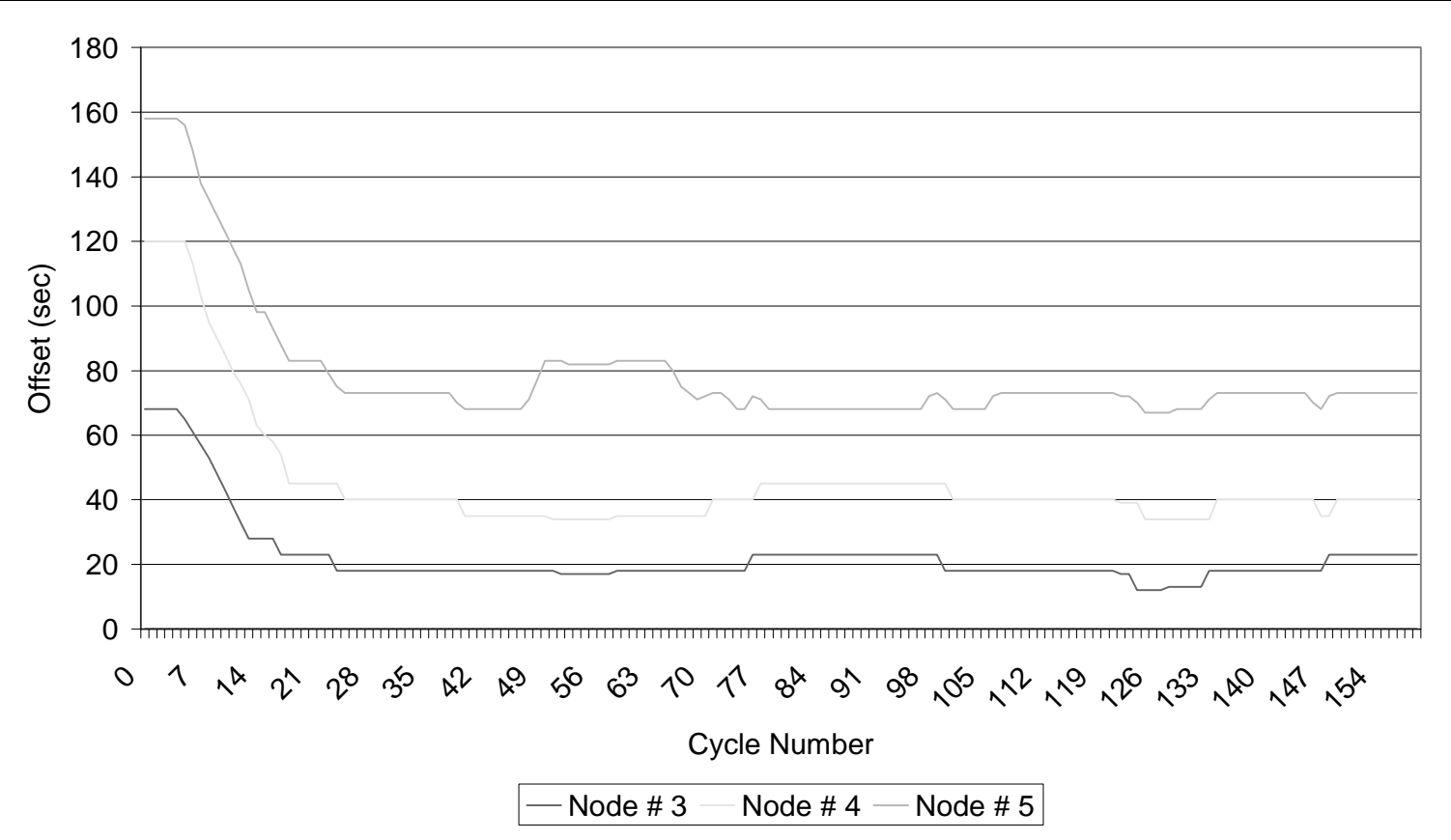

a) Offset Transitioning

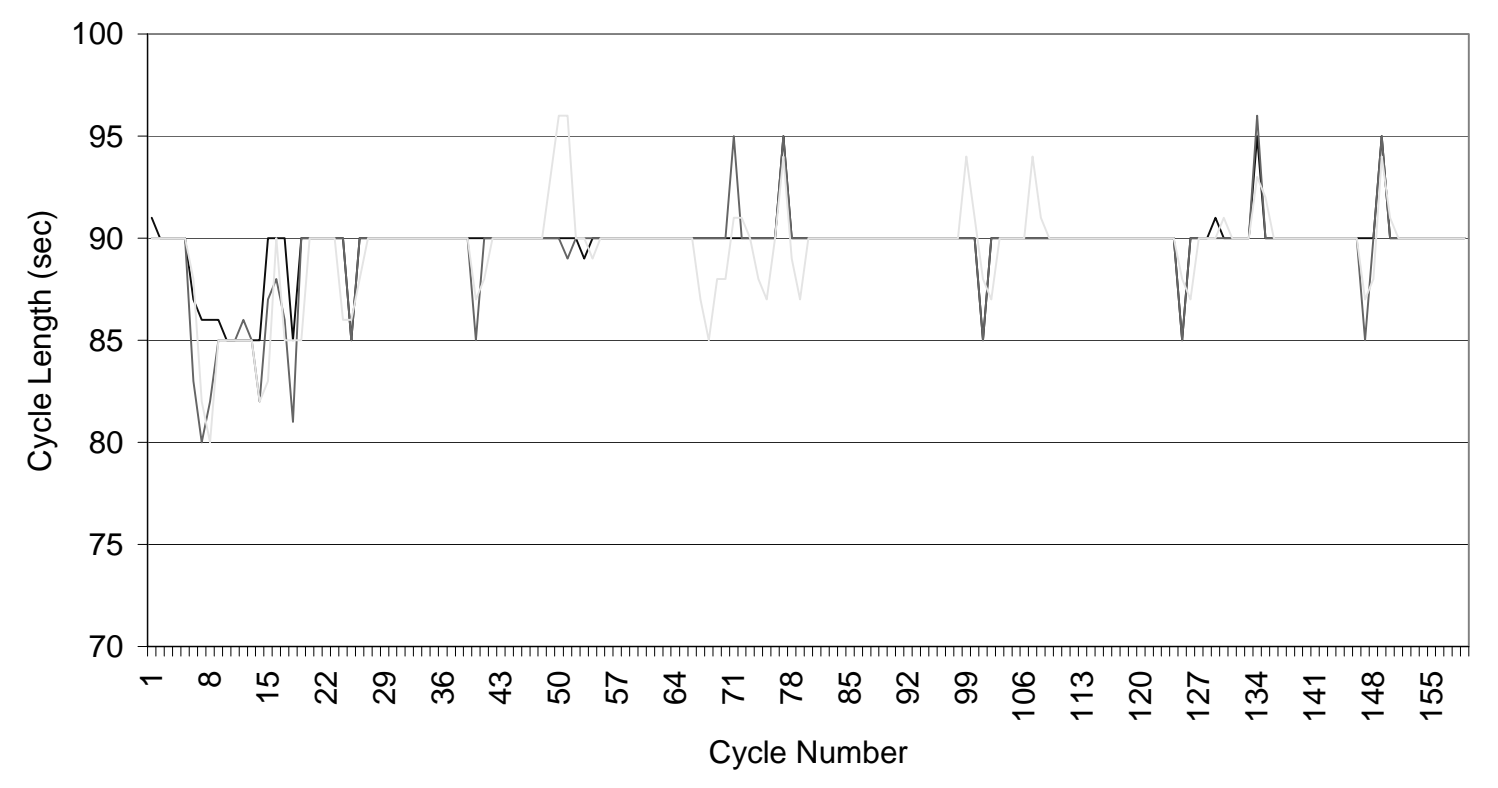

b) Cycle Transitioning

Figure 6-24 Test Bed Offset and Cycle Transitions-Bad offset Study 


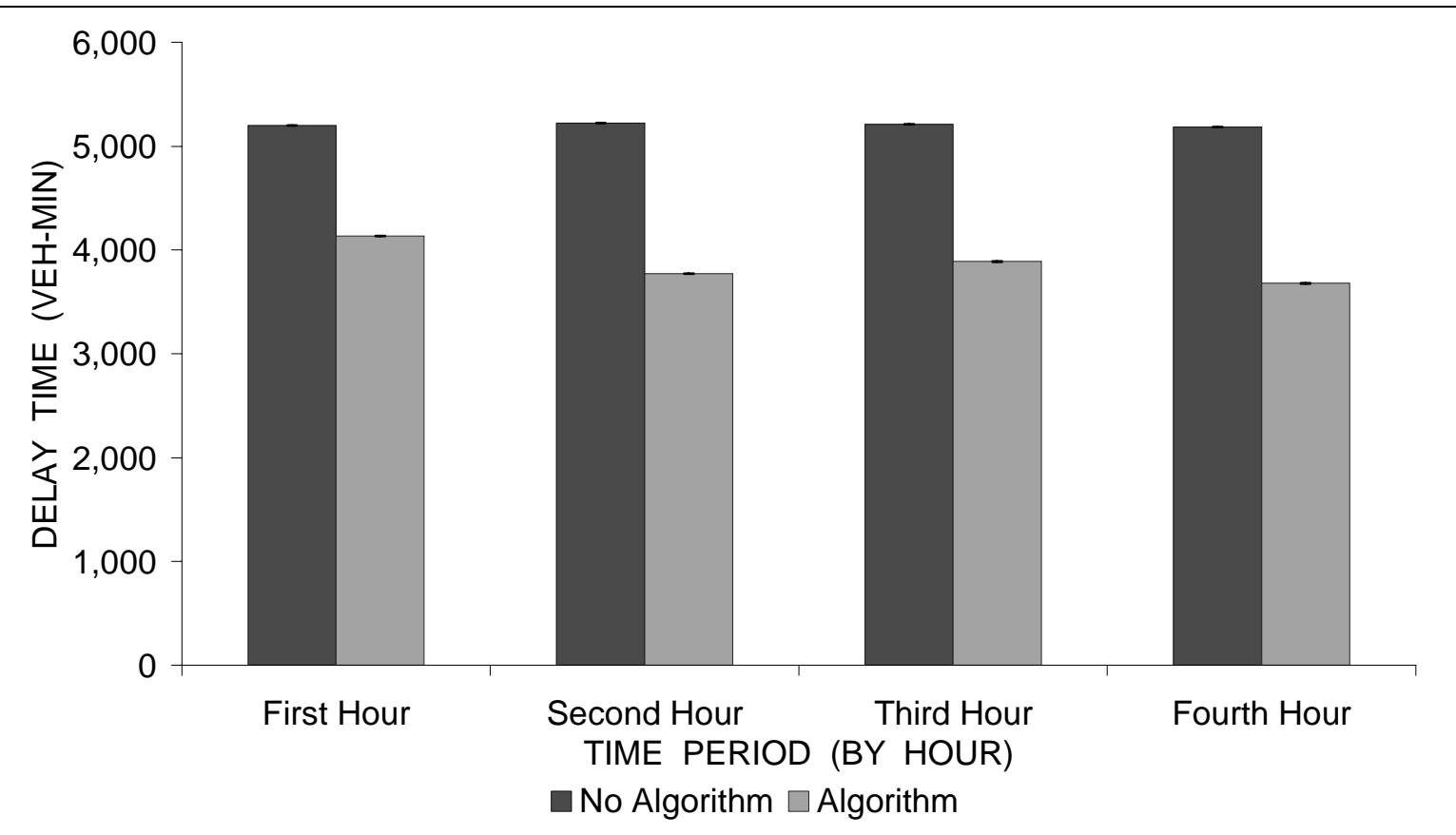

a) Total Delay Time

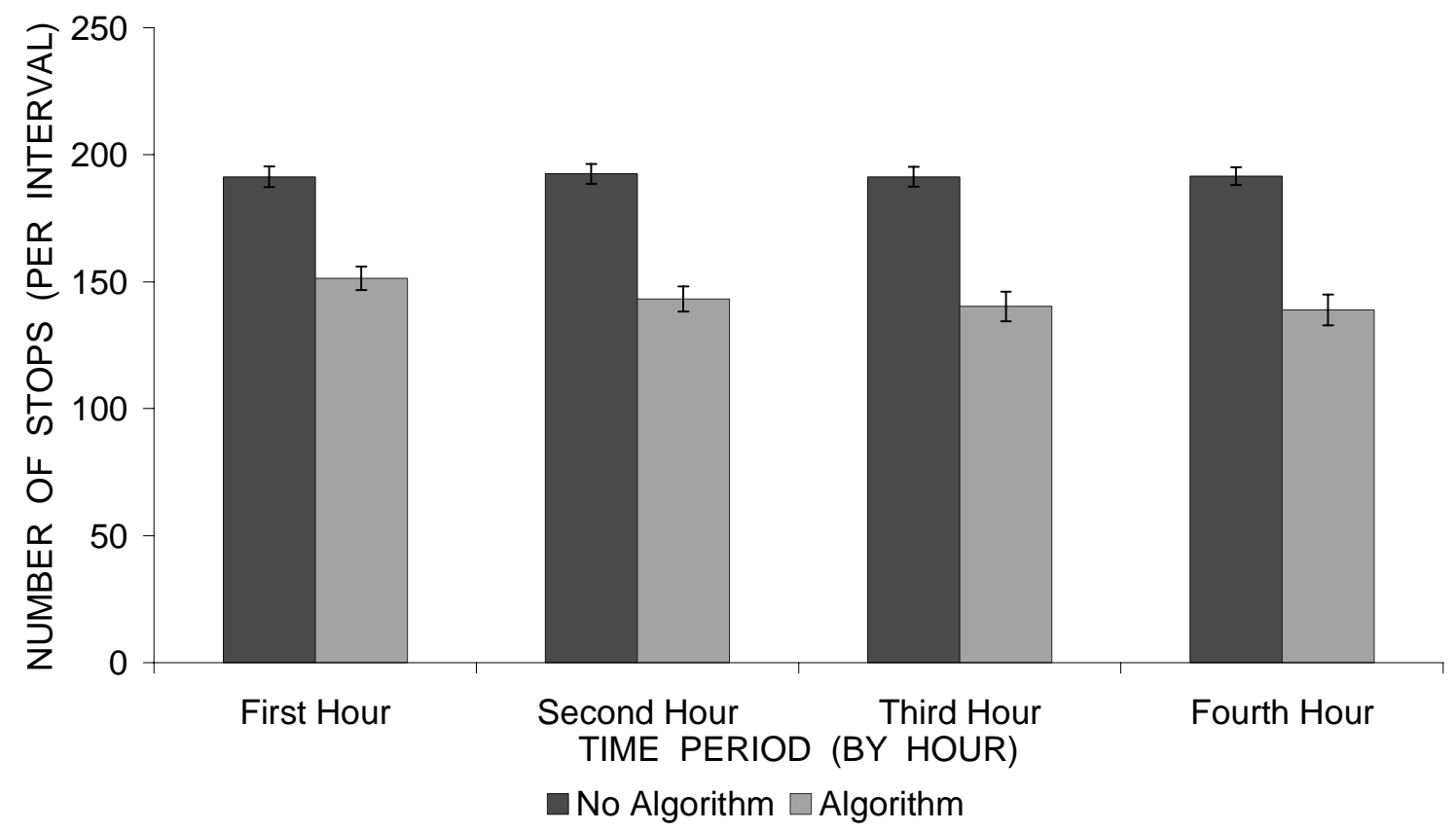

b) Total Number of Stops

Figure 6-25 Test Bed Total Delay and Number of Stops-90 sec Cycle 


\subsubsection{Adjusting Offsets to Adapt for Early-Return-to-Green}

Figure 6-26 shows the result when one intersection (Node 4) experienced different amounts of early-return-to-green. The Figure shows the northbound cumulative travel time for five different side street traffic volumes, with and without running PROTRACTS, at Node 4 . The offset at Node 4 was originally tuned for the early-return-togreen at the lowest traffic volume. Further, PRO-TRACTS increases the arterial travel speed to closely match the free trajectory speed for cases of high side street traffic volume.

Figure 6-27 further illustrates the case of the highest side street traffic volume of Figure 11, showing the north and southbound cumulative travel time at the second hour with and without PRO-TRACTS. Application of PRO-TRACTS is shown to significantly decrease the travel time in the north direction by $21.8 \%$ and in the south direction by 1.7 $\%$. It should also be noted that the total decrease in the north bound travel time was statistically significant while the total decrease in the south bound travel time was statistically insignificant. 


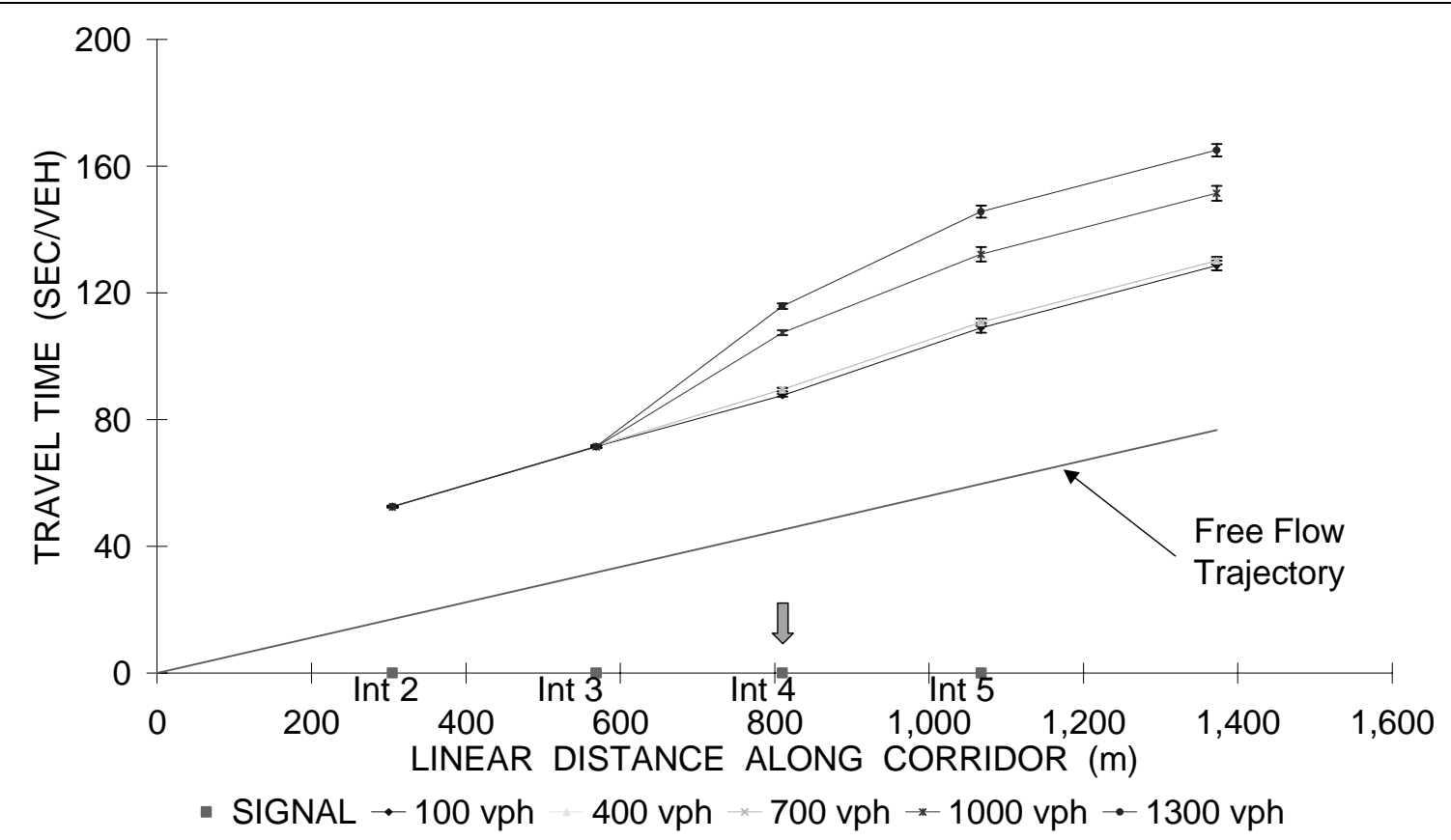

a) Without Algorithm

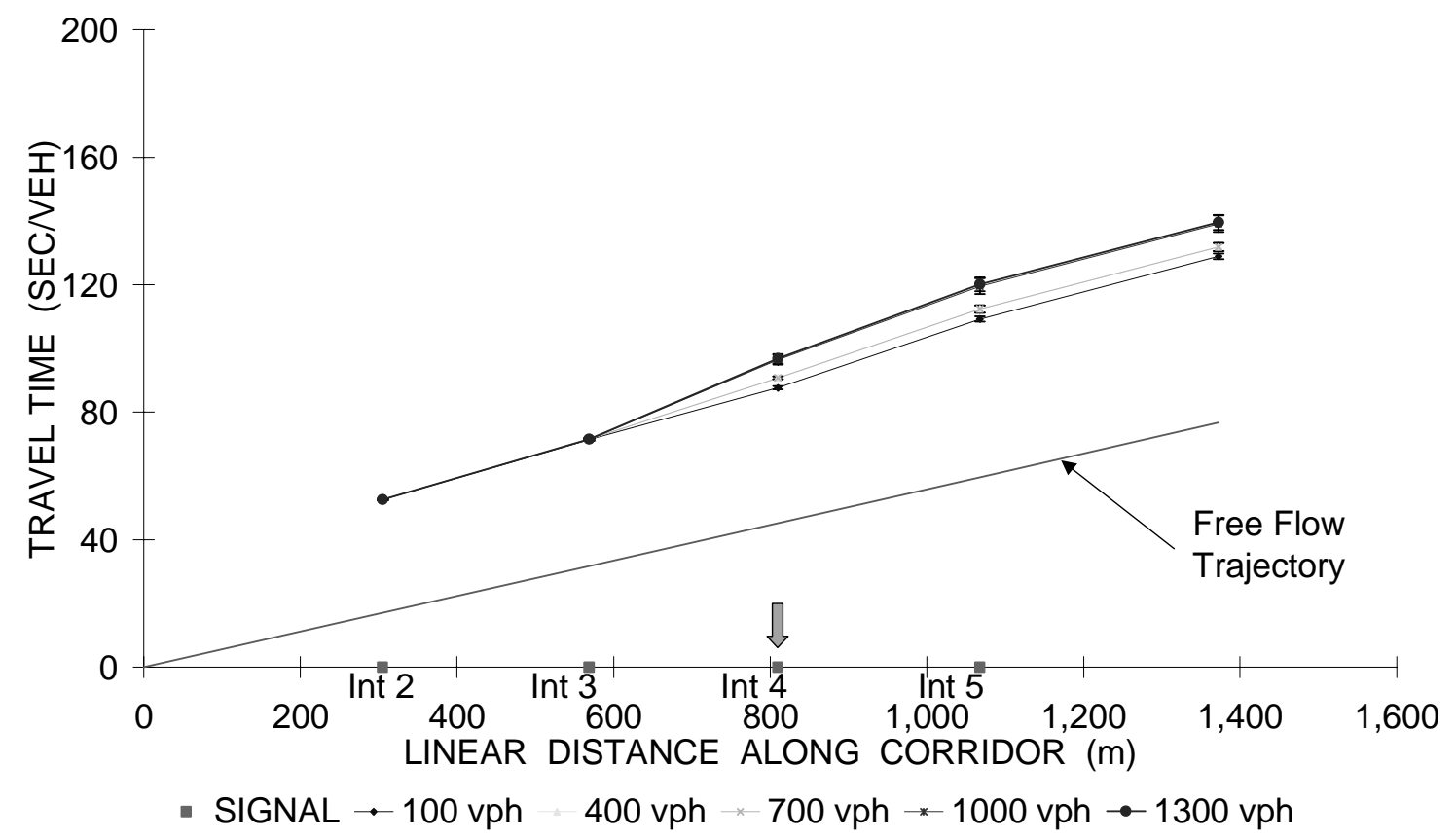

b) With Algorithm

Figure 6-26 Test Bed North Bound Cumulative Travel Time-Case 1-5, 120 sec Cycle 


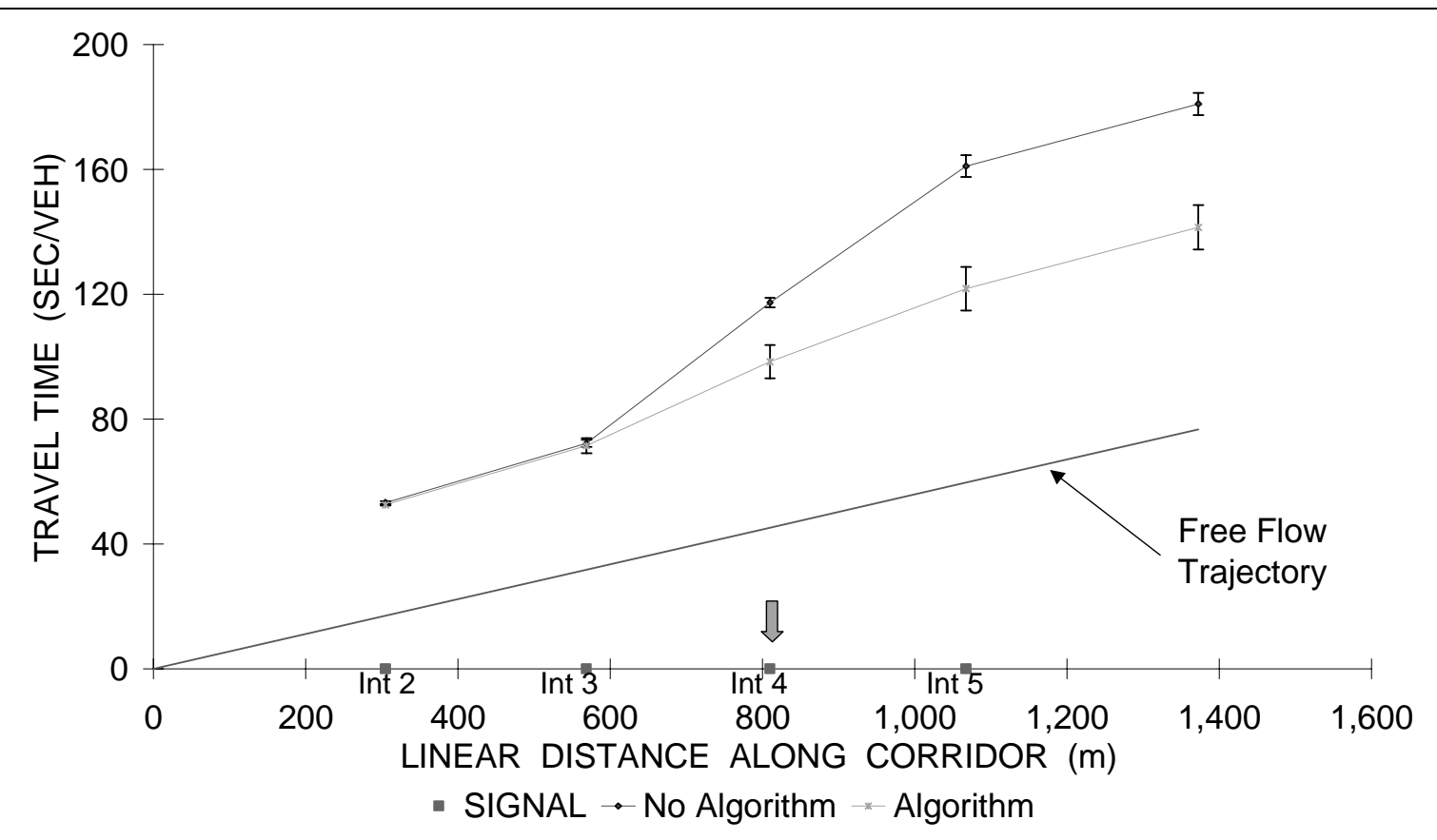

a) North Bound

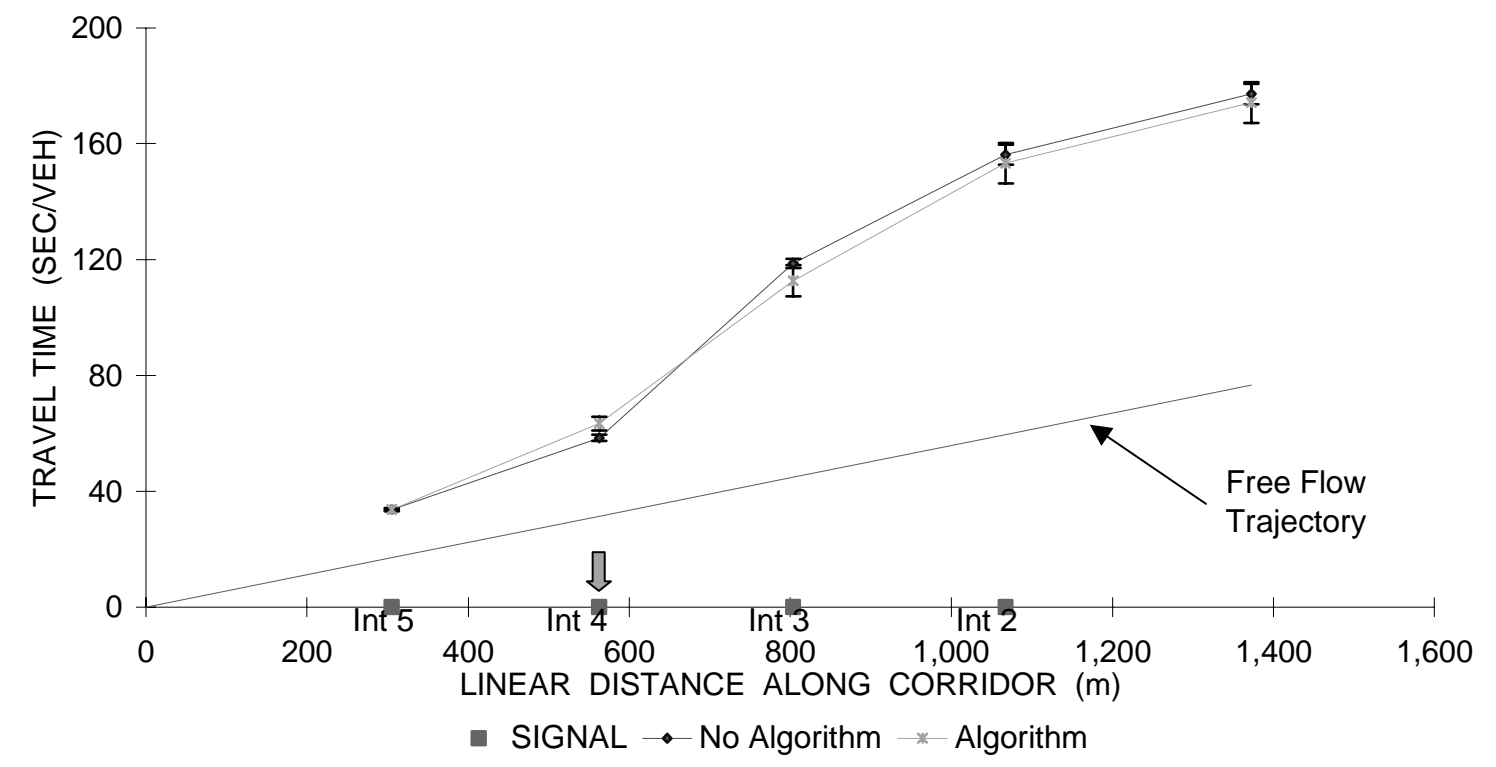

c) South Bound

Figure 6-27 Test Bed Second Hour Cumulative Travel Time-Case 5, 120 sec Cycle 


\subsection{General Findings and Results}

This chapter introduced the structure and basic concept of PRO-TRACTS. The unique aspect of PRO-TRACTS is its use of occupancy and count profiles to estimate the offset performance. Difference between the occupancy and count profiles skewness was calculated and compared to pre-determined thresholds to decide whether the algorithm is to be activated. Once activated, PRO-TRACTS uses a greedy approach to determine the direction of offset movement. This approach was evaluated with a set of incorrect offsets at several nodes and early return to green at one node. PRO-TRACTS was able to significantly reduce total travel time in the coordinated direction by up to $34.5 \%$ (Figure 6-22). The algorithm significantly decreased the total delay and the number of stops for all approaches by 29.0 and 27.6 percent respectively (Figure 6-25). 


\section{CHAPTER 7- THE F' MODEL AND DISCRIMINANT ANALYSIS}

As previously discussed in Chapters 5 and 6, a bad offset at the downstream intersection results in a strong backward shockwave that affects the relationship between the shapes of the occupancy and count profiles. Chapter 6 discussed the skewness model, which calculates the difference in occupancy and count profiles skewness to capture that information and use it in real-time to evaluate the offset performance. This chapter will discuss the limitations of the skewness model and will introduce the more general F' model. The two models are compared under different traffic network conditions using the analysis of variance, and a discriminant analysis is conducted to develop some thresholds to which F' values, calculated from the occupancy and count profiles, can be compared to estimate the offset performance.

\subsection{Limitation of the Skewness Model}

The skewness model proved very useful and effective when applied to the test bed network discussed in Chapter 6. However, only minor savings in travel time were observed when applied to networks with high turning traffic [Tompos, 2000]. In addition, several conditions were observed to have negative effects on the performance of the skewness model. 


\subsubsection{False Alarms with the Skewness Model}

One limitation of the skewness model observed was a tendency to be unstable with low traffic volumes. Figure 7-1 illustrates a case where a good offset is associated with a high differential skewness value, which occurred because of the sensitivity of the skewness to the shape of short platoons. 


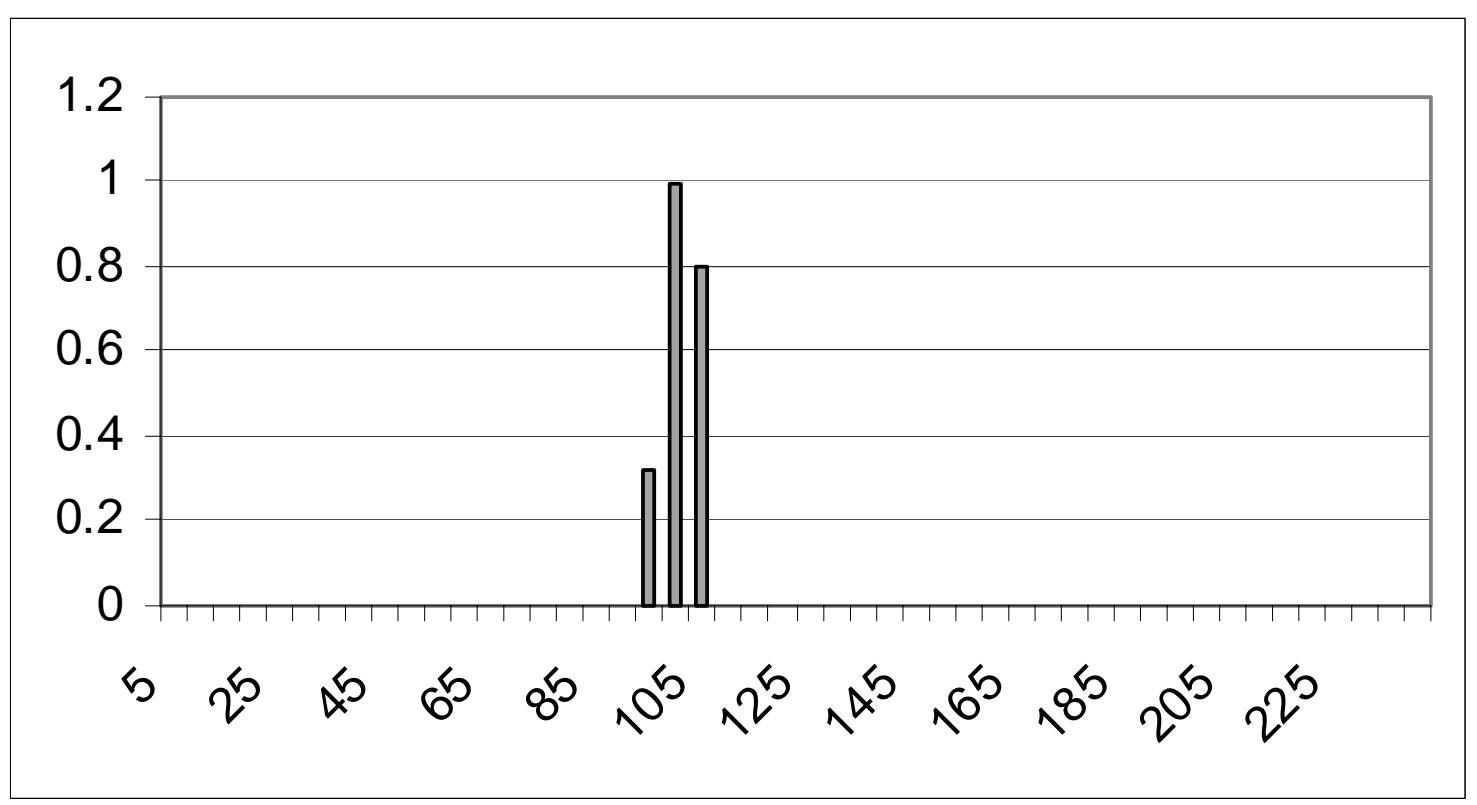

a) Count Profile, Skewness = -0.11

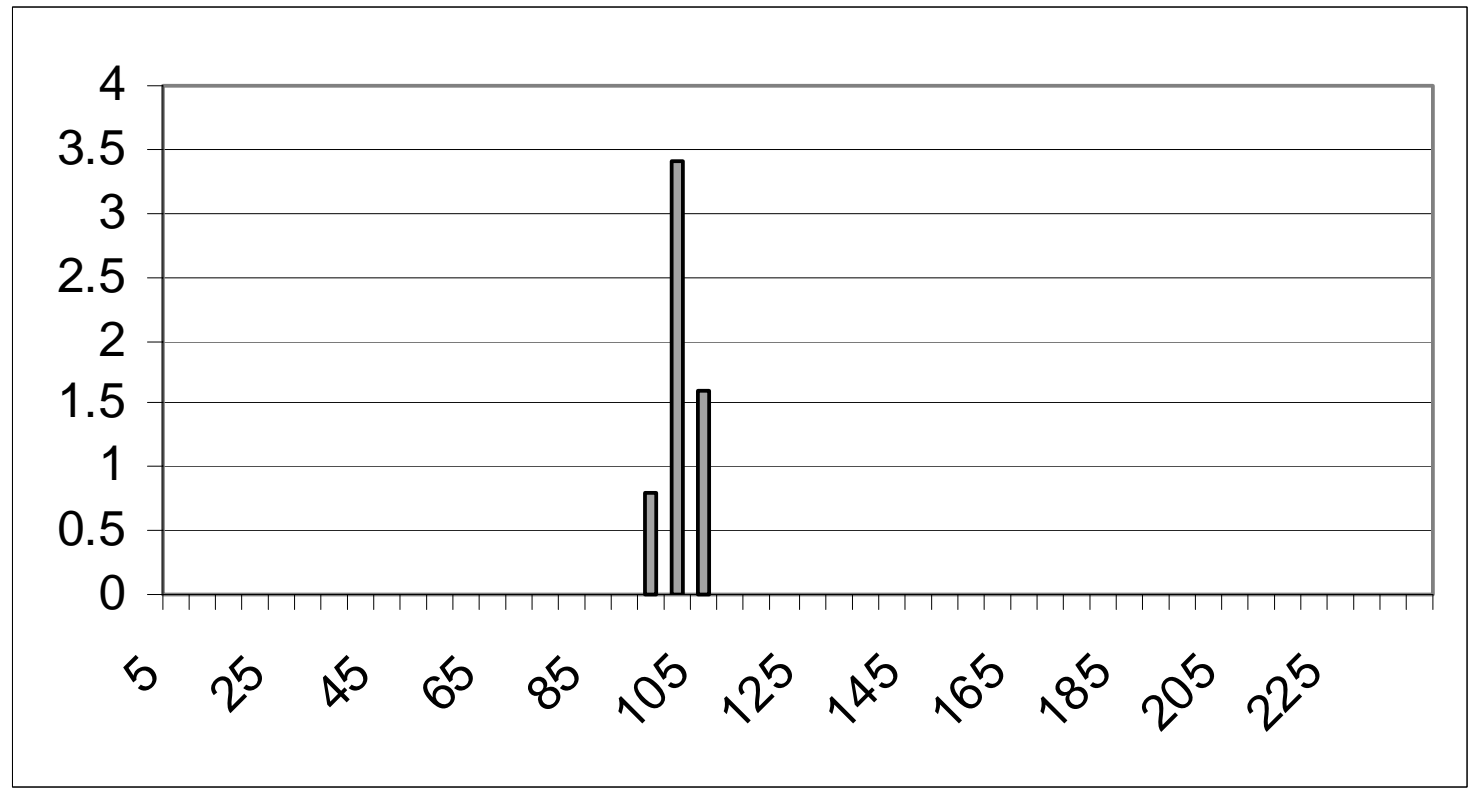

b) Occupancy Profile, Skewness $=-0.33$

Figure 7-1 False Alarm With Low Traffic Volume 


\subsubsection{Singularity Cases}

It was also observed that the differential skewness function was singular because of the singularity of the occupancy skewness function. Figure 7-2 and Figure 7-3 illustrate the singularity in the occupancy skewness function and show the occupancy and count profiles averaged over the last five cycles. A case where the last vehicle in the platoon queues over the advance detector during two of the five cycles is shown in Figure $7-2$, causing an average skewness value of ' 2 ' between times 65 and 95 in the cycle. Figure 7-3 shows another sample where the last vehicle in the platoon does not queue over the detector. Note that altough the two cases occurred with the same offset case, the first case has a much larger occupancy skewness value. The skewness function is threfore singular at the point where the shockwave extends back just to touch the advance detector's location. This critical point in the system occurs when average traffic volume is at the level where some cycle platoons queue over the advance detectors and some other cycle platoons do not. This situation causes instability in the algorithm activation mechanism. 


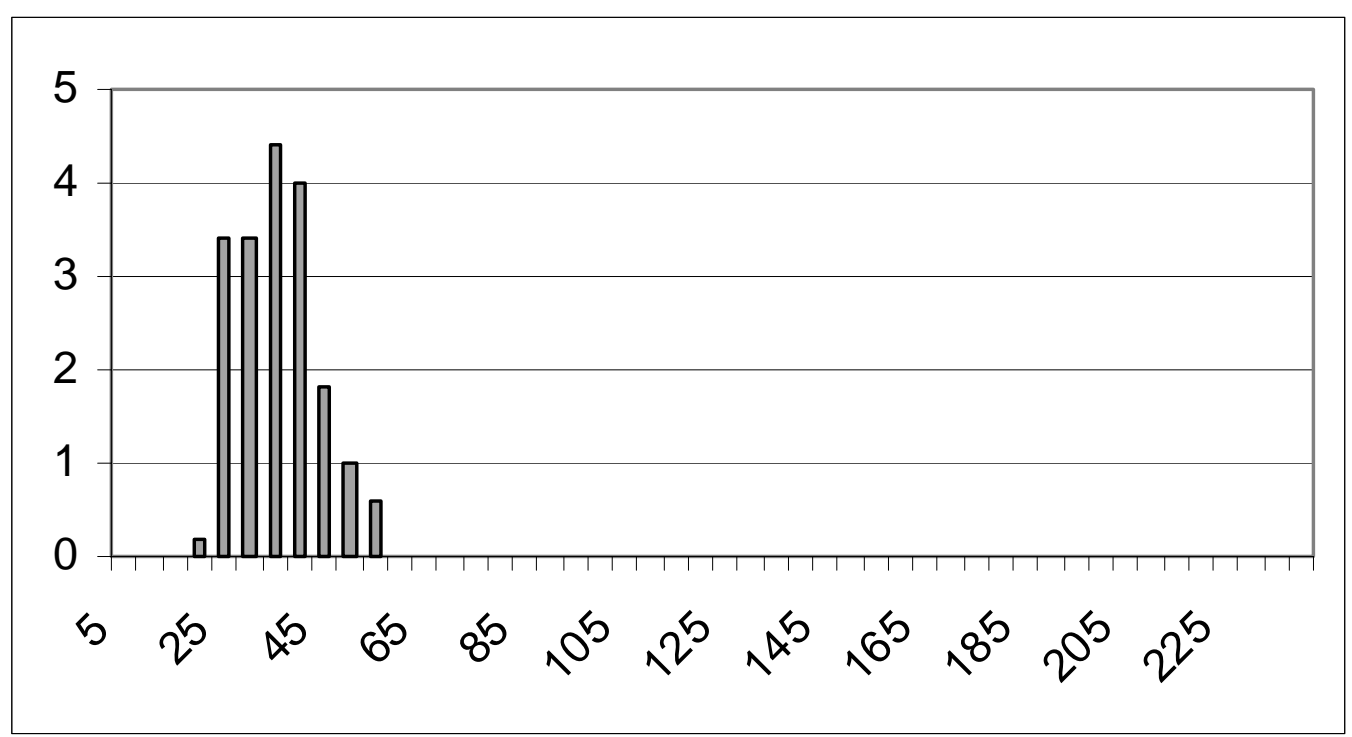

a) Count Profile, Skewness $=0.40$

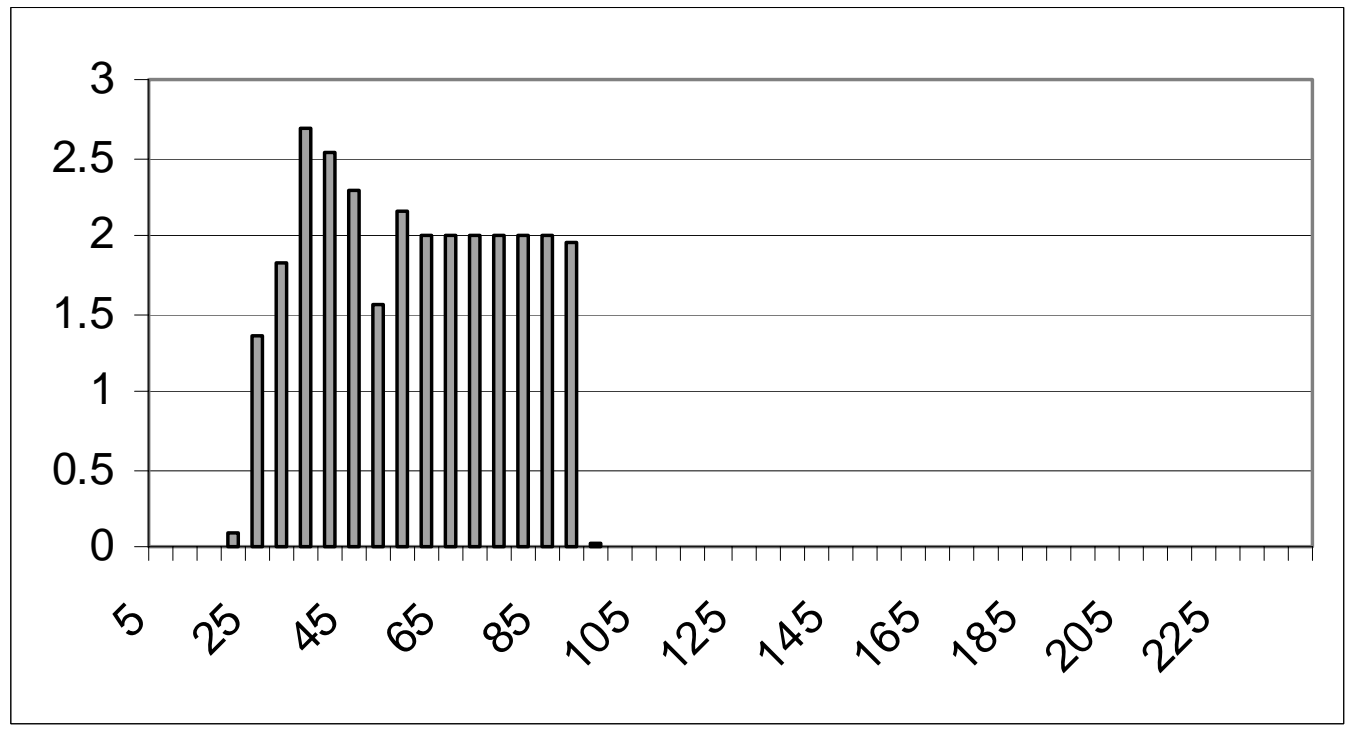

b) Occupancy Profile, Skewness = 4.69 (Differential Skewness=4.29)

Figure 7-2 Singularity of Differential Skewness- Case 1 


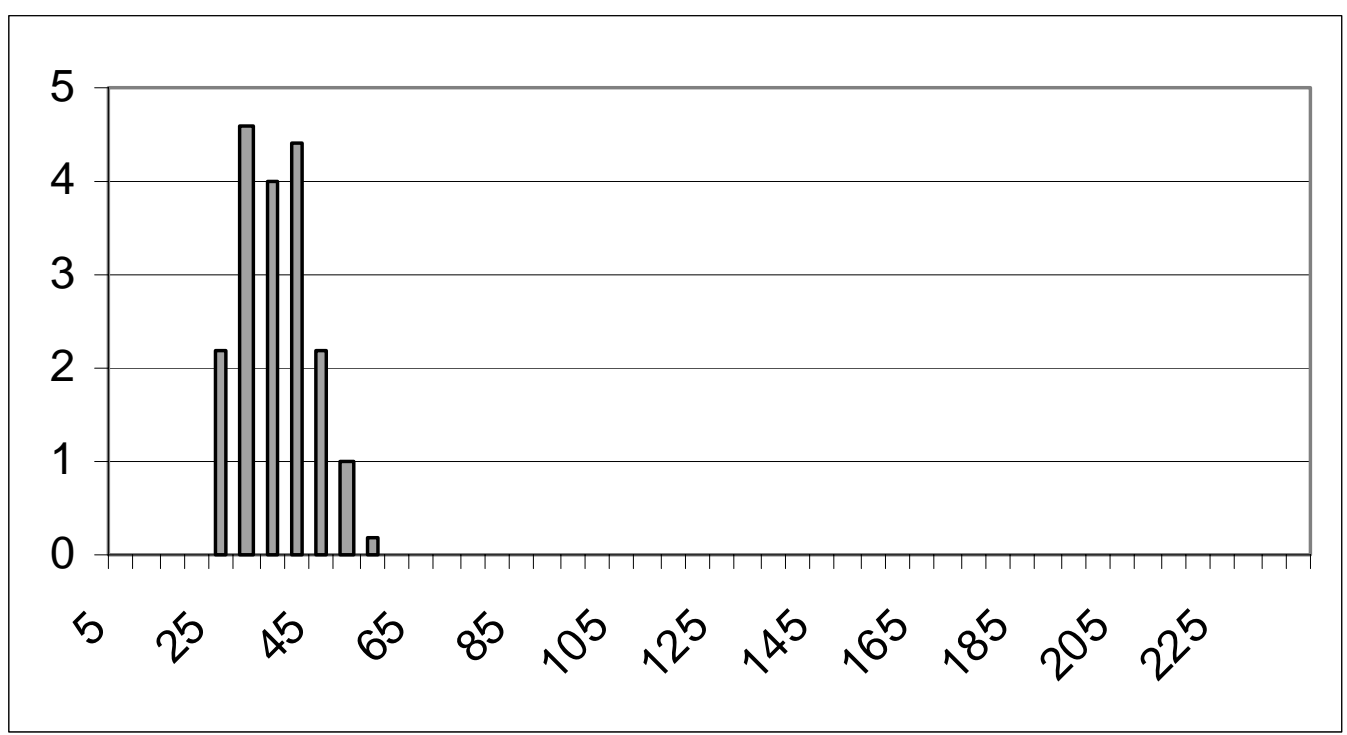

a) Count Profile, Skewness $=0.32$

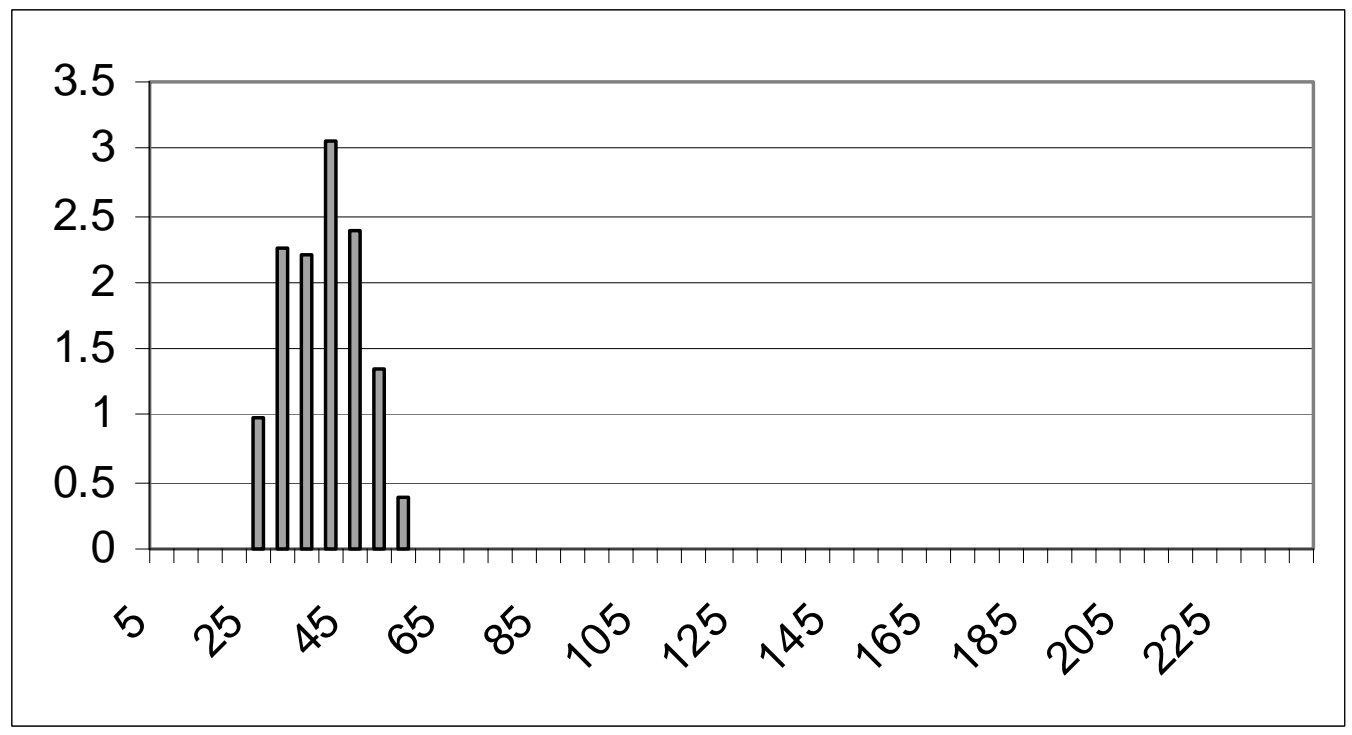

b) Occupancy Profile, Skewness= 1.11 (Differential skewness=0.79)

Figure 7-3 Singularity of Differential Skewness- Case 2

\subsubsection{Effects of Turning Traffic from Side Streets}

Although calculating the skewness of the occupancy profile around the count profile's mean, discussed in Section 6.3.3, tends to reduce the effect of turning traffic from side street, the method is not robust when the traffic volume from a side street is a 
large percentage of the total traffic volume. Turning traffic has a direct impact on the shape of the occupancy and count profiles, and the performance of PRO-TRACTS was observed to degrade when the percentage of turning traffic increases [Tompos, 2000].

\subsection{Factors Affecting Offset Performance}

\subsubsection{Link Length}

Link length has an effect on the amount of platoon dispersion, making it impossible to fit the whole platoon in the green window without introducing some delay at the signal. When platoons are dispersed to the extent that they do not fit within the downstream green window, it may be optimum to design the upstream signal timing such that the platoon is condensed before being allowed to pass through the signal.

For example, Figure 7-4-a shows a dispersed platoon released from intersection 1. The dispersed portion at the end of the platoon cannot pass through the downstream green window and it has to wait for the next cycle to pass through. This not only causes delay to that part of the platoon, but it also causes delay to the leading portion of the next cycle's platoon that has to wait for the waiting queue before it can progress. This case could be a result of an early-return-to-green situation at the signal upstream of intersection 1. The early-return causes the green window to be wider than expected, which in turn results in an end-dispersed platoon because of the random arrivals at the end of the green period at the upstream signal. Figure 7-4-b, on the other hand, shows a condensed platoon released from intersection 1 that can progress well through the arterial system. 


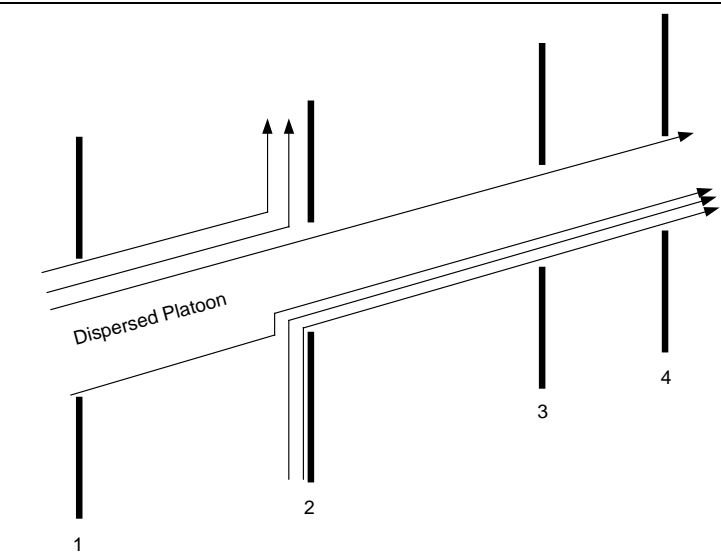

a) A Dispersed Platoon at intersection 1

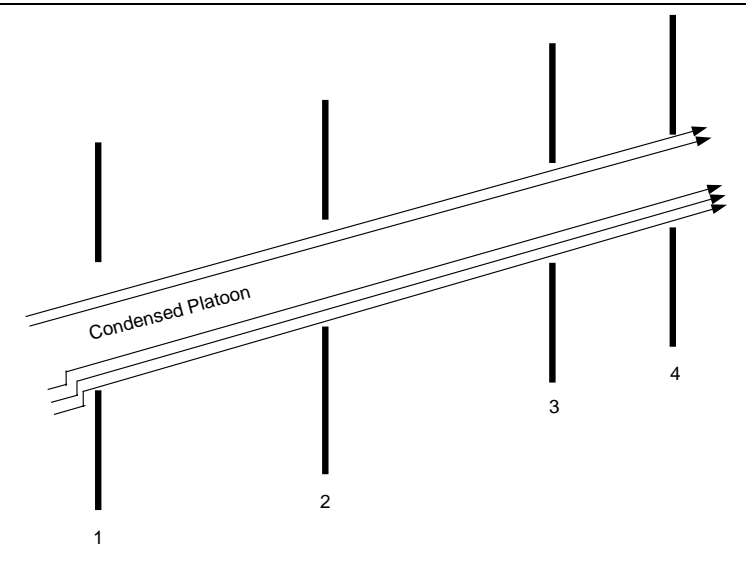

b) A Condensed Platoon at intersection 1

Figure 7-4 Effect of Platoon Dispersion on Progression

Condensing the platoon can be achieved by applying a positive delta shift to the ideal offset value at the upstream signal as shown in Figure 7-5. This will cause the vehicles in the leading edge of the platoon to slow down, causing a platoon condensation such that the platoon can pass through the arterial system. There is of course a trade-off between the amount of delay induced by this procedure on the leading platoon vehicles and the amount of delay saved from the lagging platoon vehicles. The net benefit of this procedure depends on both the degree of platoon dispersion and $\mathrm{g} / \mathrm{C}$ ratio at the upstream signal. 


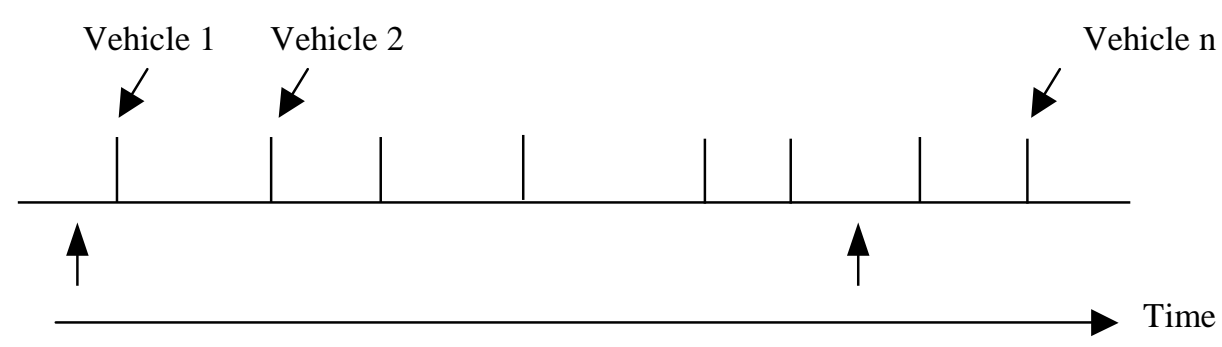

a) A Dispersed Platoon Can not fit Through a Narrow Green Window

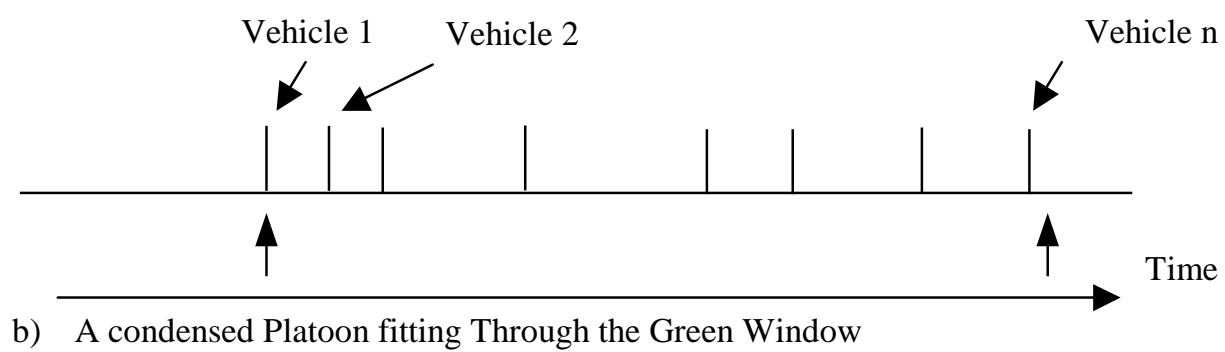

Figure 7-5 Tuning Offsets for Purpose of Reducing Platoon Dispersion

Testing the effect of the link length factor not only tests for the obvious effect of link length on dispersion, but it also tests platoon dispersion effect on PRO-TRACTS and examines whether there is a need to change its activation parameters to accommodate platoon dispersion.

\subsubsection{Cycle Length/Green Split}

The optimum cycle length in a traffic signal is the minimum cycle length that provides enough green to accommodate all the phases. Larger cycle lengths introduce extra delay to the red interval phases and smaller cycle lengths do not allow full dissipation of queues. Given that the Cycle Length/ Green split were designed efficiently, larger cycle lengths can cause larger queues and hence will interact with the upstream 
detector location's effect. The green split has to be large enough to offer enough capacity at the signal. It can either be just large enough to dissipate the queues or even larger to accommodate the whole dispersed platoon. The selection of the cycle length depends upon the network optimization criteria (minimizing the total delay versus minimizing the total number of stops). The red interval can affect the strength of the shockwave traveling upstream of the signal. Cycle Length/Green Split can have an effect similar to moving the detector closer to the signal.

\subsubsection{Traffic Volumes}

Larger traffic volumes require larger green splits and can cause stronger shockwaves when facing the red interval because of a bad offset. Larger traffic volumes can also construct rich profiles that are more robust for estimating offset performance.

\subsubsection{Detector Location}

Detector location significantly affects the activation mechanism in PROTRACTS. If the detector was very far upstream, PRO-TRACTS will not capture the existence of the shockwave caused by the downstream bad offset. On the other hand, if the detector was very close to the signal, PRO-TRACTS will be affected by the weaker shockwave caused by the turning traffic, which is illustrated in Figure 7-6. Note that detector 1 in Figure 7-6-a and Figure 7-6-b captures the existence of the strong shockwave generated by the bad offset, as well as the weaker shockwave generated by the turning traffic from side street, respectively. Detector 3, on the other hand, cannot capture any information since both shockwaves terminate before reaching its position. As 
the figure suggests, the ideal detector location, e.g. detector 2, lies between the point where the weak shockwave terminates and the point where the strong shockwave terminates. 


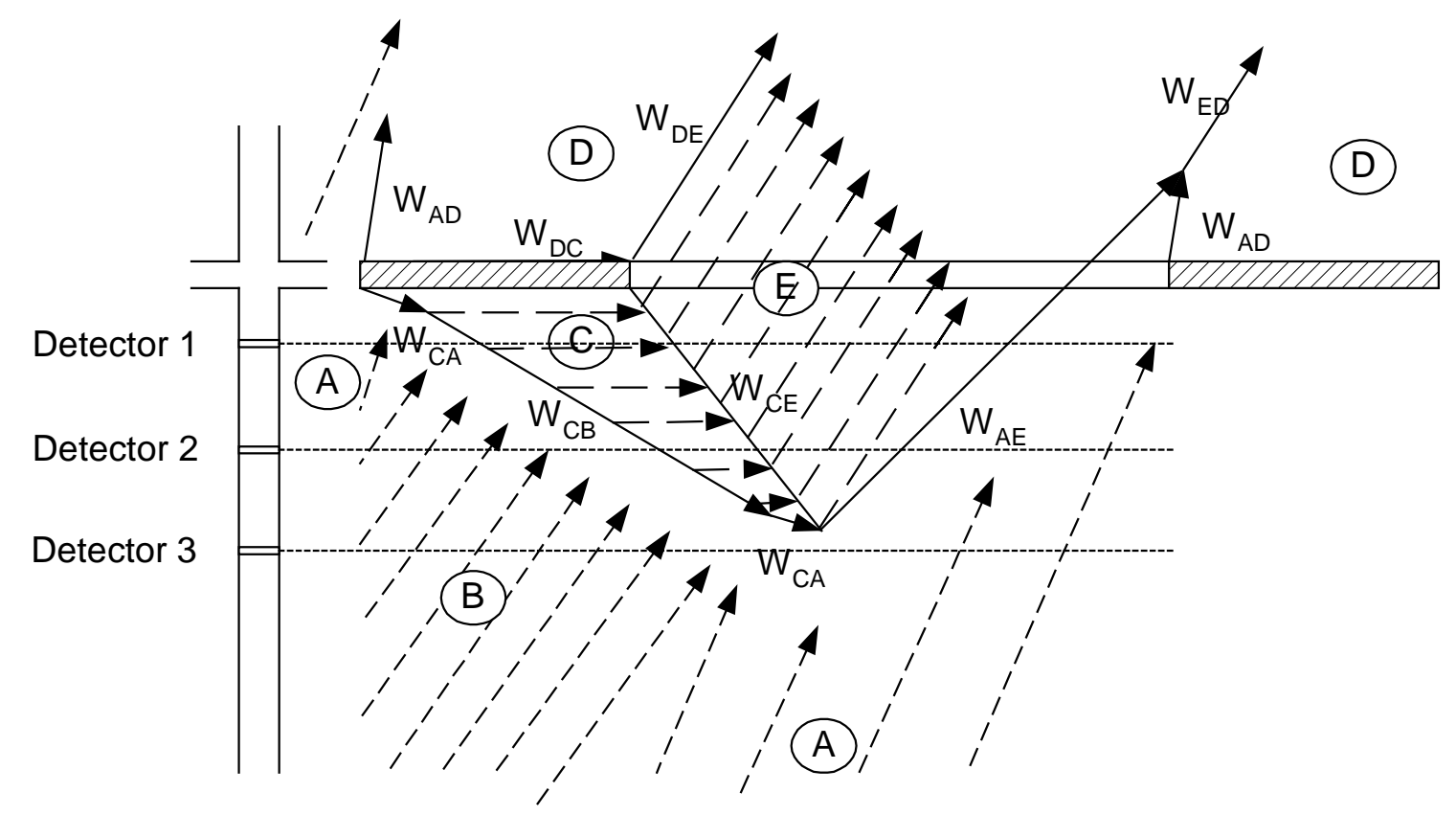

a) A Bad Offset Case

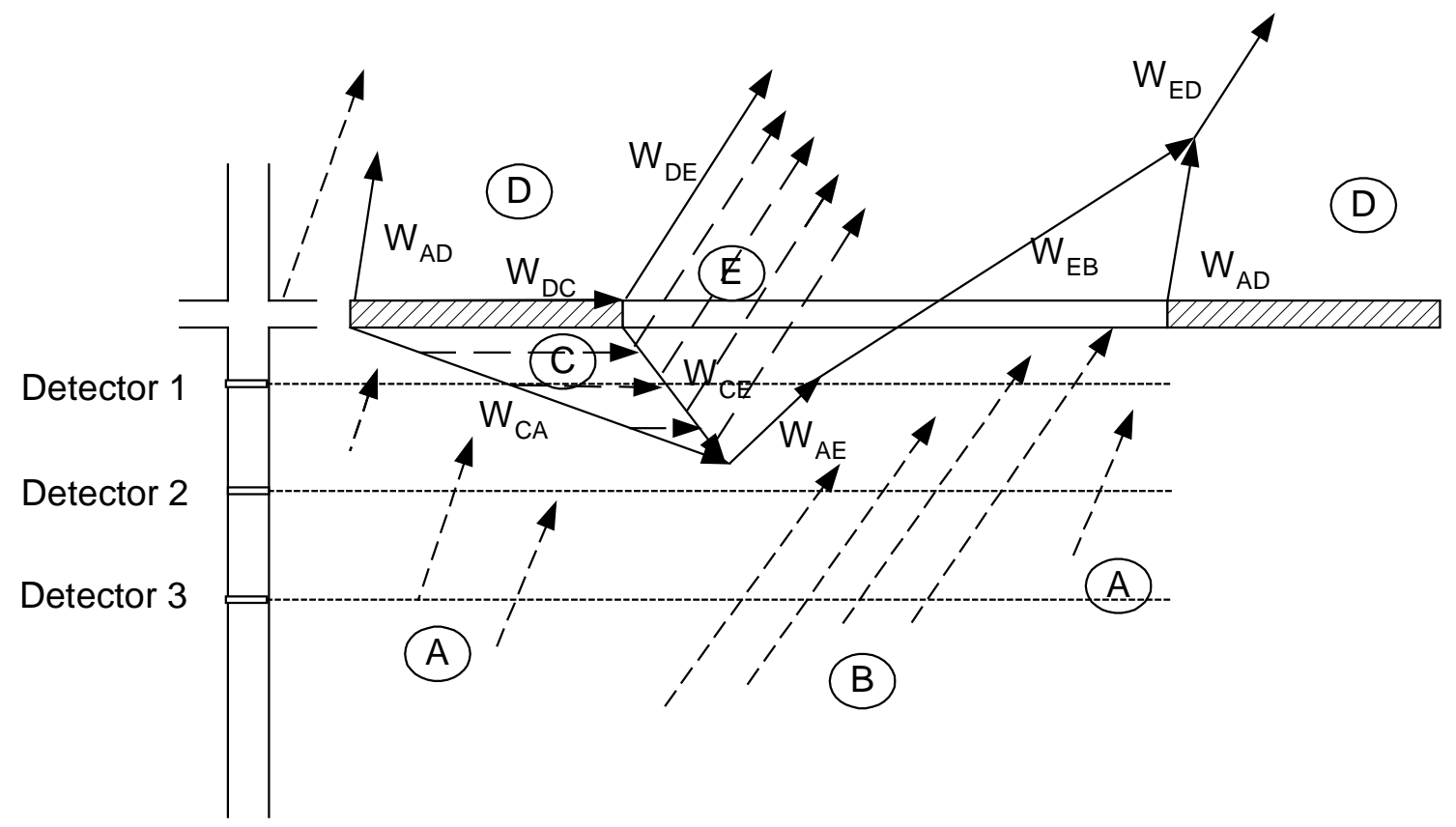

b) A Good Offset Case

Figure 7-6 Effect of Detector Location on PRO-TRACTS Activation Mechanism 


\subsubsection{Turning Traffic from Side Street}

Turning traffic from a side street can have a significant effect on both the optimum detector location and the algorithm performance. In fact, the turning traffic percentage can affect all coordination schemes. If the turning traffic volume approaches the volume of the primary traffic, little or no coordination benefits can be achieved from signal coordination. The effect of the turning traffic on the PRO-TRACTS detector location is based on the strength of the shockwave caused by the turning traffic, which will always face the red interval unless an abundant amount of green is available, relative to the shockwave caused by the arterial platoon if it faces a red interval. Generally speaking, PRO-TRACTS will perform best if the detector is placed such that it only captures the shockwave caused by the arterial's platoon.

\subsection{Design of Experiment and Statistical Testing}

\subsubsection{Design of Experiment}

In order to test the effect of all of the previous factors on the offset performance, 972 simulation files were prepared. To test the effect of cycle length, three levels of the cycle's length were evaluated: 90, 120, and 150-seconds cycle length (typical cycle ranges). For each cycle length, three green splits were tested: 1/3, 1/2, and 2/3. For each of these combinations, three levels of traffic volumes were tested: high $(1350 *(\mathrm{~g} / \mathrm{c})$ veh/hr/lane), medium $(900 *(\mathrm{~g} / \mathrm{c}) \mathrm{veh} / \mathrm{hr} / \mathrm{lane})$, and low $(450 *(\mathrm{~g} / \mathrm{c}) \mathrm{veh} / \mathrm{hr} / \mathrm{lane})$. These three traffic volume levels correspond to $82 \%, 55 \%$, and $27 \%$ of the arterial's capacity, respectively. Turning percent volume of $0,0.25$, and 0.5 for each combination of the 
above, were tested. The whole set was repeated at link length of 1,000 feet and 3,000 feet, and each simulation file had five detectors at 150, 200, 250, 300, and 350 feet upstream. The network used for conducting the experiment is shown in Figure 7-7.

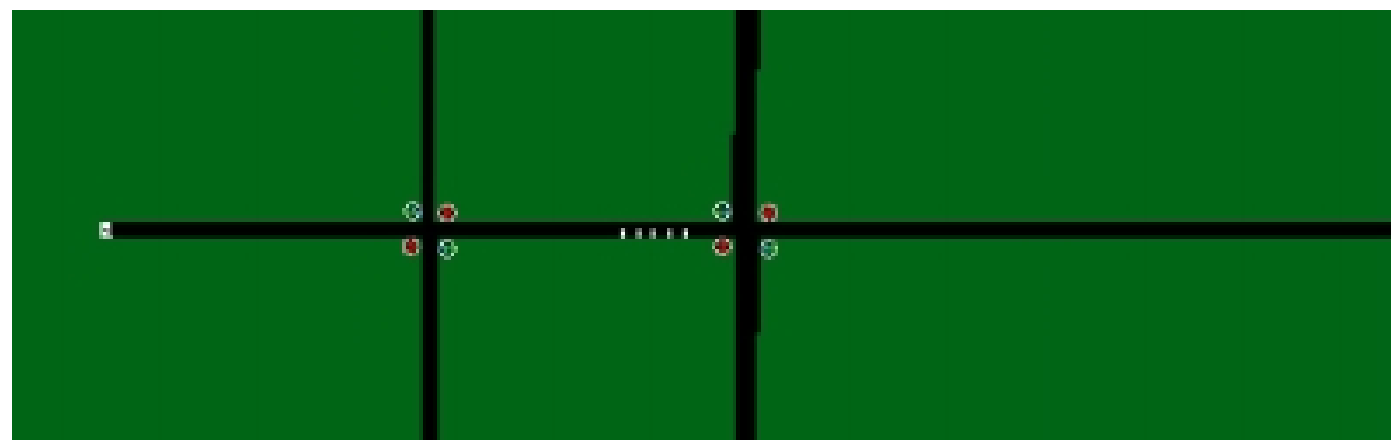

a) 1000 Feet Link Length

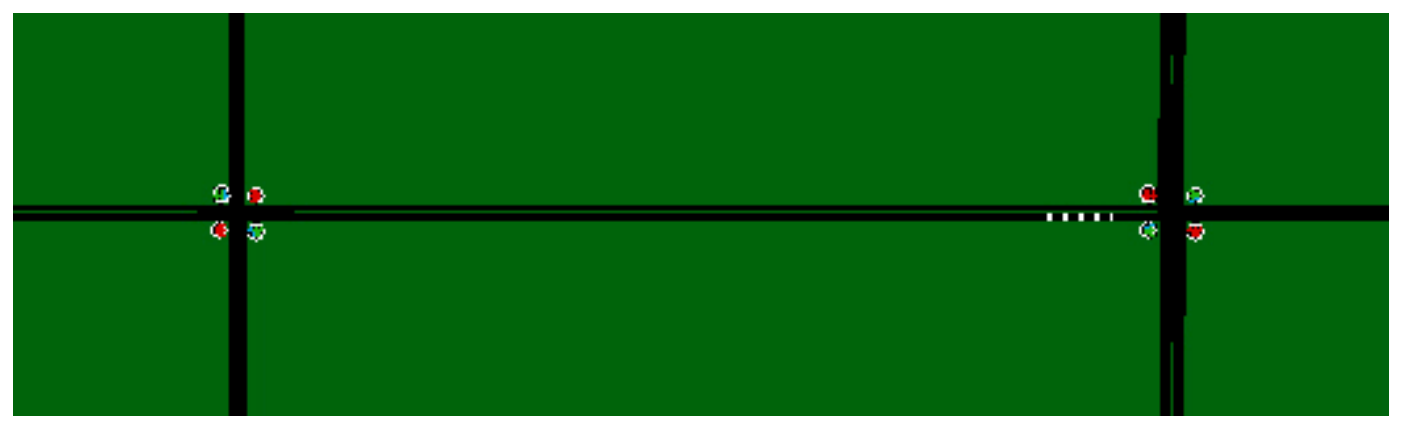

b) 3000 Feet Link Length

Figure 7-7 Experiment Network

Synchro was used to calculate the optimum offset for each combination of factors. Once the optimum offset was calculated, six different cases were prepared for each simulation file by incrementing the offset value by (Cycle Length/6). Table 7-1 through Table 7-6 list the offset values for each case for the 3,000 and 1,000 feet link lengths. 
Table 7-1 Offset Values for 3000 Feet Link- 90 Seconds Cycle

\begin{tabular}{|c|c|c|c|c|c|c|c|c|c|}
\hline \multicolumn{3}{|c|}{ Traffic Volume (veh/hr) } & \multirow[t]{2}{*}{$\mathrm{g} / \mathrm{c}$} & \multicolumn{6}{|c|}{ Offset Cases (Case 1 is Synchro Offset) } \\
\hline Thru & Turning & Total & & 1 & 2 & 3 & 4 & 5 & 6 \\
\hline 1800 & 0 & 1800 & 0.67 & 51 & 66 & 81 & 6 & 21 & 36 \\
\hline 1350 & 450 & 1800 & & 29 & 44 & 59 & 74 & 89 & 14 \\
\hline 900 & 900 & 1800 & & 12 & 27 & 42 & 57 & 72 & 87 \\
\hline 1350 & 0 & 1350 & 0.50 & 51 & 66 & 81 & 6 & 21 & 36 \\
\hline 1013 & 338 & 1351 & & 41 & 56 & 71 & 86 & 11 & 26 \\
\hline 675 & 675 & 1350 & & 27 & 42 & 57 & 72 & 87 & 12 \\
\hline 900 & 0 & 900 & 0.33 & 51 & 66 & 81 & 6 & 21 & 36 \\
\hline 675 & 225 & 900 & & 56 & 71 & 86 & 11 & 26 & 41 \\
\hline 450 & 450 & 900 & & 39 & 54 & 69 & 84 & 9 & 24 \\
\hline 1200 & 0 & 1200 & 0.67 & 51 & 66 & 81 & 6 & 21 & 36 \\
\hline 900 & 300 & 1200 & & 45 & 60 & 75 & 0 & 15 & 30 \\
\hline 600 & 600 & 1200 & & 6 & 21 & 36 & 51 & 66 & 81 \\
\hline 900 & 0 & 900 & 0.50 & 51 & 66 & 81 & 6 & 21 & 36 \\
\hline 675 & 225 & 900 & & 47 & 62 & 77 & 2 & 17 & 32 \\
\hline 450 & 450 & 900 & & 42 & 57 & 72 & 87 & 12 & 27 \\
\hline 600 & 0 & 600 & 0.33 & 51 & 66 & 81 & 6 & 21 & 36 \\
\hline 450 & 150 & 600 & & 48 & 63 & 78 & 3 & 18 & 33 \\
\hline 300 & 300 & 600 & & 44 & 59 & 74 & 89 & 14 & 29 \\
\hline 600 & 0 & 600 & 0.67 & 51 & 66 & 81 & 6 & 21 & 36 \\
\hline 450 & 150 & 600 & & 51 & 66 & 81 & 6 & 21 & 36 \\
\hline 300 & 300 & 600 & & 51 & 66 & 81 & 6 & 21 & 36 \\
\hline 450 & 0 & 450 & 0.50 & 51 & 66 & 81 & 6 & 21 & 36 \\
\hline 338 & 113 & 451 & & 50 & 65 & 80 & 5 & 20 & 35 \\
\hline 225 & 225 & 450 & & 51 & 66 & 81 & 6 & 21 & 36 \\
\hline 300 & 0 & 300 & 0.33 & 51 & 66 & 81 & 6 & 21 & 36 \\
\hline 225 & 75 & 300 & & 51 & 66 & 81 & 6 & 21 & 36 \\
\hline 150 & 150 & 300 & & 51 & 66 & 81 & 6 & 21 & 36 \\
\hline
\end{tabular}


Table 7-2 Offset Values for 3000 Feet Link- 120 Seconds Cycle

\begin{tabular}{|c|c|c|c|c|c|c|c|c|c|}
\hline \multicolumn{3}{|c|}{ Traffic Volume (veh/hr) } & \multirow[t]{2}{*}{$\mathrm{g} / \mathrm{c}$} & \multicolumn{6}{|c|}{ Offset Cases (Case 1 is Synchro Offset) } \\
\hline Thru & Turning & Total & & 1 & 2 & 3 & 4 & 5 & 6 \\
\hline 1800 & 0 & 1800 & 0.67 & 51 & 71 & 91 & 111 & 11 & 31 \\
\hline 1350 & 450 & 1800 & & 18 & 38 & 58 & 78 & 98 & 118 \\
\hline 900 & 900 & 1800 & & 117 & 17 & 37 & 57 & 77 & 97 \\
\hline 1350 & 0 & 1350 & 0.50 & 51 & 71 & 91 & 111 & 11 & 31 \\
\hline 1013 & 338 & 1351 & & 38 & 58 & 78 & 98 & 118 & 18 \\
\hline 675 & 675 & 1350 & & 17 & 37 & 57 & 77 & 97 & 117 \\
\hline 900 & 0 & 900 & 0.33 & 51 & 71 & 91 & 111 & 11 & 31 \\
\hline 675 & 225 & 900 & & 57 & 77 & 97 & 117 & 17 & 37 \\
\hline 450 & 450 & 900 & & 62 & 82 & 102 & 2 & 22 & 42 \\
\hline 1200 & 0 & 1200 & 0.67 & 51 & 71 & 91 & 111 & 11 & 31 \\
\hline 900 & 300 & 1200 & & 44 & 64 & 84 & 104 & 4 & 24 \\
\hline 600 & 600 & 1200 & & 26 & 46 & 66 & 86 & 106 & 6 \\
\hline 900 & 0 & 900 & 0.50 & 51 & 71 & 91 & 111 & 11 & 31 \\
\hline 675 & 225 & 900 & & 45 & 65 & 85 & 105 & 5 & 25 \\
\hline 450 & 450 & 900 & & 39 & 59 & 79 & 99 & 119 & 19 \\
\hline 600 & 0 & 600 & 0.33 & 51 & 71 & 91 & 111 & 11 & 31 \\
\hline 450 & 150 & 600 & & 47 & 67 & 87 & 107 & 7 & 27 \\
\hline 300 & 300 & 600 & & 51 & 71 & 91 & 111 & 11 & 31 \\
\hline 600 & 0 & 600 & 0.67 & 51 & 71 & 91 & 111 & 11 & 31 \\
\hline 450 & 150 & 600 & & 51 & 71 & 91 & 111 & 11 & 31 \\
\hline 300 & 300 & 600 & & 51 & 71 & 91 & 111 & 11 & 31 \\
\hline 450 & 0 & 450 & 0.50 & 51 & 71 & 91 & 111 & 11 & 31 \\
\hline 338 & 113 & 451 & & 51 & 71 & 91 & 111 & 11 & 31 \\
\hline 225 & 225 & 450 & & 51 & 71 & 91 & 111 & 11 & 31 \\
\hline 300 & 0 & 300 & 0.33 & 51 & 71 & 91 & 111 & 11 & 31 \\
\hline 225 & 75 & 300 & & 51 & 71 & 91 & 111 & 11 & 31 \\
\hline 150 & 150 & 300 & & 51 & 71 & 91 & 111 & 11 & 31 \\
\hline
\end{tabular}


Table 7-3 Offset Values for 3000 Feet Link- 150 Seconds Cycle

\begin{tabular}{|c|c|c|c|c|c|c|c|c|c|}
\hline \multicolumn{3}{|c|}{ Traffic Volume (veh/hr) } & \multirow[t]{2}{*}{$\mathrm{g} / \mathrm{c}$} & \multicolumn{6}{|c|}{ Offset Cases (Case 1 is Synchro Offset) } \\
\hline Thru & Turning & Total & & 1 & 2 & 3 & 4 & 5 & 6 \\
\hline 1800 & 0 & 1800 & 0.67 & 51 & 76 & 101 & 126 & 1 & 26 \\
\hline 1350 & 450 & 1800 & & 7 & 32 & 57 & 82 & 107 & 132 \\
\hline 900 & 900 & 1800 & & 132 & 7 & 32 & 57 & 82 & 107 \\
\hline 1350 & 0 & 1350 & 0.50 & 51 & 76 & 101 & 126 & 1 & 26 \\
\hline 1013 & 338 & 1350 & & 34 & 59 & 84 & 109 & 134 & 9 \\
\hline 675 & 675 & 1350 & & 7 & 32 & 57 & 82 & 107 & 132 \\
\hline 900 & 0 & 900 & 0.33 & 51 & 76 & 101 & 126 & 1 & 26 \\
\hline 675 & 225 & 900 & & 58 & 83 & 108 & 133 & 8 & 33 \\
\hline 450 & 450 & 900 & & 64 & 89 & 114 & 139 & 14 & 39 \\
\hline 1200 & 0 & 1200 & 0.67 & 51 & 76 & 101 & 126 & 1 & 26 \\
\hline 900 & 300 & 1200 & & 42 & 67 & 92 & 117 & 142 & 17 \\
\hline 600 & 600 & 1200 & & 32 & 57 & 82 & 107 & 132 & 7 \\
\hline 900 & 0 & 900 & 0.50 & 51 & 76 & 101 & 126 & 1 & 26 \\
\hline 675 & 225 & 900 & & 44 & 69 & 94 & 119 & 144 & 19 \\
\hline 450 & 450 & 900 & & 36 & 61 & 86 & 111 & 136 & 11 \\
\hline 600 & 0 & 600 & 0.33 & 51 & 76 & 101 & 126 & 1 & 26 \\
\hline 450 & 150 & 600 & & 46 & 71 & 96 & 121 & 146 & 21 \\
\hline 300 & 300 & 600 & & 51 & 76 & 101 & 126 & 1 & 26 \\
\hline 600 & 0 & 600 & 0.67 & 51 & 76 & 101 & 126 & 1 & 26 \\
\hline 450 & 150 & 600 & & 51 & 76 & 101 & 126 & 1 & 26 \\
\hline 300 & 300 & 600 & & 51 & 76 & 101 & 126 & 1 & 26 \\
\hline 450 & 0 & 450 & 0.50 & 51 & 76 & 101 & 126 & 1 & 26 \\
\hline 338 & 113 & 451 & & 51 & 76 & 101 & 126 & 1 & 26 \\
\hline 225 & 225 & 450 & & 51 & 76 & 101 & 126 & 1 & 26 \\
\hline 300 & 0 & 300 & 0.33 & 51 & 76 & 101 & 126 & 1 & 26 \\
\hline 225 & 75 & 300 & & 51 & 76 & 101 & 126 & 1 & 26 \\
\hline 150 & 150 & 300 & & 51 & 76 & 101 & 126 & 1 & 26 \\
\hline
\end{tabular}


Table 7-4 Offset Values for 1000 feet Link-90 Seconds Cycle

\begin{tabular}{|c|c|c|c|c|c|c|c|c|c|}
\hline \multicolumn{3}{|c|}{ Traffic Volume (veh/hr) } & \multirow[t]{2}{*}{$\mathrm{g} / \mathrm{c}$} & \multicolumn{6}{|c|}{ Offset Cases (Case 1 is Synchro Offset) } \\
\hline Thru & Turning & Total & & 1 & 2 & 3 & 4 & 5 & 6 \\
\hline 1800 & 0 & 1800 & 0.67 & 17 & 32 & 47 & 62 & 77 & 2 \\
\hline 1350 & 450 & 1800 & & 26 & 41 & 56 & 71 & 86 & 11 \\
\hline 900 & 900 & 1800 & & 36 & 51 & 66 & 81 & 6 & 21 \\
\hline 1350 & 0 & 1350 & 0.50 & 17 & 32 & 47 & 62 & 77 & 2 \\
\hline 1013 & 338 & 1351 & & 6 & 21 & 36 & 51 & 66 & 81 \\
\hline 675 & 675 & 1350 & & 83 & 8 & 23 & 38 & 53 & 68 \\
\hline 900 & 0 & 900 & 0.33 & 17 & 32 & 47 & 62 & 77 & 2 \\
\hline 675 & 225 & 900 & & 22 & 37 & 52 & 67 & 82 & 7 \\
\hline 450 & 450 & 900 & & 5 & 20 & 35 & 50 & 65 & 80 \\
\hline 1200 & 0 & 1200 & 0.67 & 17 & 32 & 47 & 62 & 77 & 2 \\
\hline 900 & 300 & 1200 & & 11 & 26 & 41 & 56 & 71 & 86 \\
\hline 600 & 600 & 1200 & & 62 & 77 & 2 & 17 & 32 & 47 \\
\hline 900 & 0 & 900 & 0.50 & 17 & 32 & 47 & 62 & 77 & 2 \\
\hline 675 & 225 & 900 & & 13 & 28 & 43 & 58 & $\overline{73}$ & 88 \\
\hline 450 & 450 & 900 & & 8 & 23 & 38 & 53 & 68 & 83 \\
\hline 600 & 0 & 600 & 0.33 & 17 & 32 & 47 & 62 & 77 & 2 \\
\hline 450 & 150 & 600 & & 14 & 29 & $\overline{44}$ & 59 & 74 & 89 \\
\hline 300 & 300 & 600 & & 10 & 25 & 40 & 55 & 70 & 85 \\
\hline 600 & 0 & 600 & 0.67 & 17 & 32 & 47 & 62 & 77 & 2 \\
\hline 450 & 150 & 600 & & 17 & 32 & 47 & 62 & 77 & 2 \\
\hline 300 & 300 & 600 & & 17 & 32 & 47 & 62 & 77 & 2 \\
\hline 450 & 0 & 450 & 0.50 & 17 & 32 & 47 & 62 & 77 & 2 \\
\hline $\begin{array}{l}338 \\
\end{array}$ & 113 & 451 & & 15 & 30 & 45 & 60 & 75 & 0 \\
\hline 225 & 225 & 450 & & 17 & 32 & 47 & 62 & 77 & 2 \\
\hline 300 & 0 & 300 & 0.33 & 17 & 32 & 47 & 62 & 77 & 2 \\
\hline 225 & 75 & 300 & & 17 & 32 & 47 & 62 & 77 & 2 \\
\hline 150 & 150 & 300 & & 17 & 32 & 47 & 62 & 77 & 2 \\
\hline
\end{tabular}


Table 7-5 Offset Values for 1000 Feet Link-120 Seconds Cycle

\begin{tabular}{|c|c|c|c|c|c|c|c|c|c|}
\hline \multicolumn{3}{|c|}{ Traffic Volume (veh/hr) } & \multirow[t]{2}{*}{$g / c$} & \multicolumn{6}{|c|}{ Offset Cases (Case 1 is Synchro Offset) } \\
\hline Thru & Turning & Total & & 1 & 2 & 3 & 4 & 5 & 6 \\
\hline 1800 & 0 & 1800 & 0.67 & 17 & 37 & 57 & 77 & 97 & 117 \\
\hline 1350 & 450 & 1800 & & 103 & 3 & 23 & 43 & 63 & 83 \\
\hline 900 & 900 & 1800 & & 83 & 103 & 3 & 23 & 43 & 63 \\
\hline 1350 & 0 & 1350 & 0.50 & 17 & 37 & 57 & 77 & 97 & 117 \\
\hline 1013 & 338 & 1351 & & 0 & 20 & 40 & 60 & 80 & 100 \\
\hline 675 & 675 & 1350 & & 103 & 3 & 23 & 43 & 63 & 83 \\
\hline 900 & 0 & 900 & 0.33 & 17 & 37 & 57 & 77 & 97 & 117 \\
\hline 675 & 225 & 900 & & 23 & 43 & 63 & 83 & 103 & 3 \\
\hline 450 & 450 & 900 & & 28 & 48 & 68 & 88 & 108 & 8 \\
\hline 1200 & 0 & 1200 & 0.67 & 17 & 37 & 57 & 77 & 97 & 117 \\
\hline 900 & 300 & 1200 & & 9 & 29 & 49 & 69 & 89 & 109 \\
\hline 600 & 600 & 1200 & & 112 & 12 & 32 & 52 & 72 & 92 \\
\hline 900 & 0 & 900 & 0.50 & 17 & 37 & 57 & 77 & 97 & 117 \\
\hline 675 & 225 & 900 & & 11 & 31 & 51 & 71 & 91 & 111 \\
\hline 450 & 450 & 900 & & 5 & 25 & 45 & 65 & 85 & 105 \\
\hline 600 & 0 & 600 & 0.33 & 17 & 37 & 57 & 77 & 97 & 117 \\
\hline 450 & 150 & 600 & & 13 & 33 & 53 & 73 & 93 & 113 \\
\hline 300 & 300 & 600 & & 17 & 37 & 57 & 77 & 97 & 117 \\
\hline 600 & 0 & 600 & 0.67 & 17 & 37 & 57 & 77 & 97 & 117 \\
\hline 450 & 150 & 600 & & 17 & 37 & 57 & 77 & 97 & 117 \\
\hline 300 & 300 & 600 & & 17 & 37 & 57 & 77 & 97 & 117 \\
\hline 450 & 0 & 450 & 0.50 & 17 & 37 & 57 & 77 & 97 & 117 \\
\hline 338 & 113 & 451 & & 17 & 37 & 57 & 77 & 97 & 117 \\
\hline 225 & 225 & 450 & & 17 & 37 & 57 & 77 & 97 & 117 \\
\hline 300 & 0 & 300 & 0.33 & 17 & 37 & 57 & 77 & 97 & 117 \\
\hline 225 & 75 & 300 & & 17 & 37 & 57 & 77 & 97 & 117 \\
\hline 150 & 150 & 300 & & 17 & 37 & 57 & 77 & 97 & 117 \\
\hline
\end{tabular}


Table 7-6 Offset Values for 1000 Feet Link-150 Seconds Cycle

\begin{tabular}{|c|c|c|c|c|c|c|c|c|c|}
\hline \multicolumn{3}{|c|}{ Traffic Volume (veh/hr) } & \multirow[t]{2}{*}{$\mathrm{g} / \mathrm{c}$} & \multicolumn{6}{|c|}{ Offset Cases (Case 1 is Synchro Offset) } \\
\hline Thru & Turning & Total & & 1 & 2 & 3 & 4 & 5 & 6 \\
\hline 1800 & 0 & 1800 & 0.67 & 17 & 42 & 67 & 92 & 117 & 142 \\
\hline 1350 & 450 & 1800 & & 123 & 148 & 23 & 48 & 73 & 98 \\
\hline 900 & 900 & 1800 & & 98 & 123 & 148 & 23 & 48 & 73 \\
\hline 1350 & 0 & 1350 & 0.50 & 17 & 42 & 67 & 92 & 117 & 142 \\
\hline 1013 & 338 & 1350 & & 145 & 20 & 45 & 70 & 95 & 120 \\
\hline 675 & 675 & 1350 & & 123 & 148 & 23 & 48 & 73 & 98 \\
\hline 900 & 0 & 900 & 0.33 & 17 & 42 & 67 & 92 & 117 & 142 \\
\hline 675 & 225 & 900 & & 24 & 49 & 74 & 99 & 124 & 149 \\
\hline 450 & 450 & 900 & & 30 & 55 & 80 & 105 & 130 & 5 \\
\hline 1200 & 0 & 1200 & 0.67 & 17 & 42 & 67 & 92 & 117 & 142 \\
\hline 900 & 300 & 1200 & & 8 & 33 & 58 & 83 & 108 & 133 \\
\hline 600 & 600 & 1200 & & 148 & 23 & 48 & 73 & 98 & 123 \\
\hline 900 & 0 & 900 & 0.50 & 17 & 42 & 67 & 92 & 117 & 142 \\
\hline 675 & 225 & 900 & & 10 & 35 & 60 & 85 & 110 & 135 \\
\hline 450 & 450 & 900 & & 2 & 27 & 52 & 77 & 102 & 127 \\
\hline 600 & 0 & 600 & 0.33 & 17 & 42 & 67 & 92 & 117 & 142 \\
\hline 450 & 150 & 600 & & 11 & 36 & 61 & 86 & 111 & 136 \\
\hline 300 & 300 & 600 & & 17 & 42 & 67 & 92 & 117 & 142 \\
\hline 600 & 0 & 600 & 0.67 & 17 & 42 & 67 & 92 & 117 & 142 \\
\hline 450 & 150 & 600 & & 17 & 42 & 67 & 92 & 117 & 142 \\
\hline 300 & 300 & 600 & & 17 & 42 & 67 & 92 & 117 & 142 \\
\hline 450 & 0 & 450 & 0.50 & 17 & 42 & 67 & 92 & 117 & 142 \\
\hline 338 & 113 & 451 & & 17 & 42 & 67 & 92 & 117 & 142 \\
\hline 225 & 225 & 450 & & 17 & 42 & 67 & 92 & 117 & 142 \\
\hline 300 & 0 & 300 & 0.33 & 17 & 42 & 67 & 92 & 117 & 142 \\
\hline 225 & 75 & 300 & & 17 & 42 & 67 & 92 & 117 & 142 \\
\hline 150 & 150 & 300 & & 17 & 42 & 67 & 92 & 117 & 142 \\
\hline
\end{tabular}




\subsubsection{Statistical Testing}

Analysis of variance was conducted to test for the significance of the factors discussed above on the skewness model. In order to properly test for significance, it was necessary to define to estimate the expected Mean Squares (EMS) tables. The experiment conditions are discussed below.

\subsubsection{Blocking}

Blocking design is used when the experiment is not completely randomized, i.e., when only a limited number of observations can be taken per day. However, it only applies when the blocked factor might introduce other confounding factors not accounted for in the experiment. In that case, the blocked factor is used as a block to eliminate the effect of the other factors. Although this experiment was conducted over several days, it was obvious that no blocking was needed since the computer simulation would produce the same results regardless of the sequence by which each individual file was run.

\subsubsection{Nested Factors}

Nested models in factorial design are generally used when for two levels A and B, different levels of factor B are not the same under different levels of factor A. In such a case, factor $\mathrm{B}$ is said to be nested within factor $\mathrm{A}$. The traffic volume factor in the experiment has three different levels: high, medium, and low. Each level has a different value under a different level of the green split. Recall that the volume is determined from the equation $v=x^{*} g / C$, where $x=1350$ for the high level, $x=900$ for the medium level, 
and $\mathrm{x}=450$ for the low level respectively. Therefore, the volume factor is nested within the green split factor. Similarly the split factor is also nested within the cycle length. This is due to the difference in the effect of a 0.5 split in a 90 -seconds cycle length from that of a 0.5 split in a 150 -second cycle length. The difference in the effect is due to the different duration of red interval in the two cases that can cause different shockwave strengths.

\subsubsection{Split-Plot Factorial Design}

The split plot design is another case of incomplete randomization in the experiment design. This type of design is typically recognized when different treatments are applied to the same subject without changing the other experiment factors when applying the different treatments. Typically, the within subject variation is much less than the between subject variation since the between subject variation might include some confounding factors that are not accounted for, where the within subject part is very controlled. Testing a factor in the plot part will generally give more accurate results.

Both the offset and detector location factors were tested in the within plot part of the split plot design. Although the computer simulator generates traffic randomly, the same traffic pattern is generated again when the computer simulation is rerun (for a given number seed). Randomness in the simulation run can be observed in the cycle length (due to uncalled actuated phases) or in the green split (due to early-return-to-green). Traffic pattern and behavior also change from cycle to cycle because every time a random number seed is used, another random number seed is generated. However, when the simulation was rerun, the same random number seeds sequence was reused and the same 
set of factor levels are repeated exactly, except for the offset factor. Since detector actuations at different detectors were collected for the same sample, the detector location factor was also tested in the within plot part of the experiment.

\subsubsection{Expected Mean Squares and Testing for Significance}

In order to test for the effect of the previous factors, a statistical model with the above factors and their interactions were used. The complete model is attached in Appendix C. The expected mean Squares were derived in Table 7-7. Note that an additional factor, rep, was added to the model to represent the replicate of each run. The addition of this factor allows for the split plot testing of factorial effects. By comparing the EMS in Table 7-7, it is apparent that the length, cycle, split, volume, and turn factors and their interactions should be tested by the replicate residuals. while the offset and detector location factors, and their interactions with the above five factors, should be tested by their interaction with the replicate factor. The interactions of the offset and detector location factors and their higher level interactions are to be tested by the threeway interaction of offset, detector location, and the replicate factor. 
Table 7-7 Expected Mean Square Derivation for Nested Split-Plot Design (Table 1 of 6)

\begin{tabular}{|c|c|c|c|c|c|c|c|c|c|c|c|c|c|}
\hline & & & 2 & 3 & 3 & 3 & 3 & 5 & 5 & 6 & 1 & & \\
\hline & & & $\mathrm{F}$ & $\mathrm{F}$ & $\mathrm{F}$ & $\mathrm{F}$ & $\mathrm{F}$ & $\mathrm{R}$ & $\mathrm{F}$ & $\mathrm{F}$ & $\mathrm{R}$ & & \\
\hline Num & Source & DF & I & $J$ & $\mathrm{~K}$ & $\mathrm{~L}$ & $\mathrm{~m}$ & $r$ & $\mathrm{~N}$ & 0 & e & EMS & $\begin{array}{l}\text { F Test by } \\
\text { Term \#: }\end{array}$ \\
\hline 1 & $\mathrm{~L}_{\mathrm{i}}$ & 1 & 0 & 3 & 3 & 3 & 3 & 5 & 5 & 6 & 1 & $3750 \phi L+30 \sigma^{2} R+\sigma^{2}$ & 20 \\
\hline 2 & $\mathrm{C}_{\mathrm{j}}$ & 2 & 2 & 0 & 3 & 3 & 3 & 5 & 5 & 6 & 1 & $8100 \phi C+30 \sigma^{2} R+\sigma^{2}$ & 20 \\
\hline 3 & $\mathrm{~S}_{(\mathrm{j}) \mathrm{k}}$ & 2 & 2 & 1 & 0 & 3 & 3 & 5 & 5 & 6 & 1 & $2700 \phi S+30 \sigma^{2} R+\sigma^{2}$ & 20 \\
\hline 4 & $\mathrm{~V}_{(\mathrm{k}) \mathrm{I}}$ & 6 & 2 & 3 & 1 & 0 & 3 & 5 & 5 & 6 & 1 & $2700 \phi \mathrm{V}+30 \sigma \mathrm{R}+\sigma^{2}$ & 20 \\
\hline 5 & $\mathrm{~T}_{\mathrm{m}}$ & 2 & 2 & 3 & 3 & 3 & 0 & 5 & 5 & 6 & 1 & $8100 \phi T+30 \sigma^{2} R+\sigma^{2}$ & 20 \\
\hline 6 & $\mathrm{LC}_{\mathrm{ij}}$ & 2 & 0 & 0 & 3 & 3 & 3 & 5 & 5 & 6 & 1 & $4050 \phi L C+30 \sigma^{2} R+\sigma^{2}$ & 20 \\
\hline 7 & $\mathrm{LS}_{\mathrm{i}(\mathrm{j}) \mathrm{k}}$ & 2 & 0 & 1 & 0 & 3 & 3 & 5 & 5 & 6 & 1 & $1350 \phi L S+30 \sigma^{2} R+\sigma^{2}$ & 20 \\
\hline 8 & $\mathrm{LV}_{\mathrm{i}(\mathrm{k}) !}$ & 6 & 0 & 3 & 1 & 0 & 3 & 5 & 5 & 6 & 1 & $1350 \phi L V+30 \sigma^{2} R+\sigma^{2}$ & 20 \\
\hline 9 & $\mathrm{LT}_{\mathrm{im}}$ & 2 & 0 & 3 & 3 & 3 & 0 & 5 & 5 & 6 & 1 & $4050 \phi \mathrm{LT}+30 \sigma^{2} \mathrm{R}+\sigma^{2}$ & 20 \\
\hline 10 & $\mathrm{CV}_{\mathrm{j}(\mathrm{k}) \mathrm{l}}$ & 12 & 2 & 0 & 1 & 0 & 3 & 5 & 5 & 6 & 1 & $900 \phi C V+30 \sigma^{2} R+\sigma^{2}$ & 20 \\
\hline 11 & $\mathrm{CT}_{\mathrm{jm}}$ & 4 & 2 & 0 & 3 & 3 & 0 & 5 & 5 & 6 & 1 & $2700 \phi C T+30 \sigma^{2} R+\sigma^{2}$ & 20 \\
\hline
\end{tabular}


Table 7-8 Expected Mean Square Derivation for Nested Split-Plot Design (Table 2 of 6)

\begin{tabular}{|c|c|c|c|c|c|c|c|c|c|c|c|c|c|}
\hline 12 & $\mathrm{ST}_{(\mathrm{j}) \mathrm{km}}$ & 4 & 2 & 1 & 0 & 3 & 0 & 5 & 5 & 6 & 1 & $900 \phi S T+30 \sigma^{2} R+\sigma^{2}$ & 20 \\
\hline 13 & $\mathrm{VT}_{(\mathrm{k}) \mathrm{Im}}$ & 12 & 2 & 3 & 1 & 0 & 0 & 5 & 5 & 6 & 1 & $900 \phi \mathrm{VT}+30 \sigma^{2} \mathrm{R}+\sigma^{2}$ & 20 \\
\hline 14 & $\operatorname{LCV}_{\mathrm{ij}(\mathrm{k}) !}$ & 12 & 0 & 0 & 1 & 0 & 3 & 5 & 5 & 6 & 1 & $450 \phi L C V+30 \sigma^{2} R+\sigma^{2}$ & 20 \\
\hline 15 & $\mathrm{LCT}_{\mathrm{ijm}}$ & 4 & 0 & 0 & 3 & 3 & 0 & 5 & 5 & 6 & 1 & $1350 \phi L C T+30 \sigma^{2} R+\sigma^{2}$ & 20 \\
\hline 16 & $\operatorname{LST}_{\mathrm{i}(\mathrm{j}) \mathrm{km}}$ & 4 & 0 & 1 & 0 & 3 & 0 & 5 & 5 & 6 & 1 & $450 \phi L S T+30 \sigma^{2} R+\sigma^{2}$ & 20 \\
\hline 17 & $\mathrm{LVT}_{\mathrm{i}(\mathrm{k}) \mathrm{Im}}$ & 12 & 0 & 3 & 1 & 0 & 0 & 5 & 5 & 6 & 1 & $1450 \phi L V T+30 \sigma^{2} R+\sigma^{2}$ & 20 \\
\hline 18 & $\mathrm{CVT}_{\mathrm{j}(\mathrm{k}) \mathrm{Im}}$ & 8 & 2 & 0 & 1 & 0 & 0 & 5 & 5 & 6 & 1 & $300 \phi C S T+30 \sigma^{2} R+\sigma^{2}$ & 20 \\
\hline 19 & $\mathrm{LCVT}_{\mathrm{ij}(\mathrm{k}) \mathrm{Im}}$ & 8 & 0 & 0 & 1 & 0 & 0 & 5 & 5 & 6 & 1 & $150 \phi L C V+30 \sigma^{2} R+\sigma^{2}$ & 20 \\
\hline 20 & $R_{(i j k l m) r}$ & 4 & 1 & 1 & 1 & 1 & 1 & 1 & 5 & 6 & 1 & $30 \sigma^{2} R+\sigma^{2}$ & -- \\
\hline 21 & $\mathrm{O}_{0}$ & 5 & 2 & 3 & 3 & 3 & 3 & 5 & 5 & 0 & 1 & $4050 \phi \mathrm{O}+5 \sigma^{2} \mathrm{OR}+\sigma^{2}$ & 41 \\
\hline 22 & $\mathrm{OL}_{\text {io }}$ & 5 & 0 & 3 & 3 & 3 & 3 & 5 & 5 & 0 & 1 & $2025 \phi \mathrm{OL}+5 \sigma^{2} \mathrm{OR}+\sigma^{2}$ & 41 \\
\hline 23 & $\mathrm{OC}_{\mathrm{jo}}$ & 10 & 2 & 0 & 3 & 3 & 3 & 5 & 5 & 0 & 1 & $1350 \phi \mathrm{OC}+5 \sigma^{2} \mathrm{OR}+\sigma^{2}$ & 41 \\
\hline 24 & $\mathrm{OS}_{(\mathrm{j}) \mathrm{ko}}$ & 10 & 2 & 1 & 0 & 3 & 3 & 5 & 5 & 0 & 1 & $450 \phi \mathrm{OS}+5 \sigma^{2} \mathrm{OR}+\sigma^{2}$ & 41 \\
\hline 25 & $\mathrm{OV}_{(\mathrm{k}) l \mathrm{o}}$ & 30 & 2 & 3 & 1 & 0 & 3 & 5 & 5 & 0 & 1 & $450 \phi O V+5 \sigma^{2} \mathrm{OR}+\sigma^{2}$ & 41 \\
\hline 26 & $\mathrm{OT}_{\mathrm{mo}}$ & 10 & 2 & 3 & 3 & 3 & 0 & 5 & 5 & 0 & 1 & $1350 \phi \mathrm{OT}+5 \sigma^{2} \mathrm{OR}+\sigma^{2}$ & 41 \\
\hline
\end{tabular}


Table 7-9 Expected Mean Square Derivation for Nested Split-Plot Design (Table 3 of 6)

\begin{tabular}{|c|c|c|c|c|c|c|c|c|c|c|c|c|c|}
\hline 27 & $\mathrm{OLC}_{\mathrm{ijo}}$ & 10 & 0 & 0 & 3 & 3 & 3 & 5 & 5 & 0 & 1 & $675 \phi O L C+5 \sigma^{2} \mathrm{OR}+\sigma^{2}$ & 41 \\
\hline 28 & OLS $_{i(j) k o}$ & 10 & 0 & 1 & 0 & 3 & 3 & 5 & 5 & 0 & 1 & $225 \phi \mathrm{OLS}+5 \sigma^{2} \mathrm{OR}+\sigma^{2}$ & 41 \\
\hline 29 & $\mathrm{OLV}_{\mathrm{i}(\mathrm{k}) / 0}$ & 30 & 0 & 3 & 1 & 0 & 3 & 5 & 5 & 0 & 1 & $225 \phi \mathrm{OLV}+5 \sigma^{2} \mathrm{OR}+\sigma^{2}$ & 41 \\
\hline 30 & $O L T_{\text {imo }}$ & 10 & 0 & 3 & 3 & 3 & 0 & 5 & 5 & 0 & 1 & $675 \phi \mathrm{OLT}+5 \sigma^{2} \mathrm{OR}+\sigma^{2}$ & 41 \\
\hline 31 & $O C V_{j(k) / 0}$ & 60 & 2 & 0 & 1 & 0 & 3 & 5 & 5 & 0 & 1 & $150 \phi O C V+5 \sigma^{2} \mathrm{OR}+\sigma^{2}$ & 41 \\
\hline 32 & $\mathrm{OCT}_{\mathrm{jmo}}$ & 20 & 2 & 0 & 3 & 3 & 0 & 5 & 5 & 0 & 1 & $450 \phi O C T+5 \sigma^{2} \mathrm{OR}+\sigma^{2}$ & 41 \\
\hline 33 & $\mathrm{OST}_{(\mathrm{j}) \mathrm{kmo}}$ & 20 & 2 & 1 & 0 & 3 & 0 & 5 & 5 & 0 & 1 & $150 \phi \mathrm{OST}+5 \sigma^{2} \mathrm{OR}+\sigma^{2}$ & 41 \\
\hline 34 & $\mathrm{OVT}_{(\mathrm{k}) / \mathrm{mo}}$ & 60 & 2 & 3 & 1 & 0 & 0 & 5 & 5 & 0 & 1 & $150 \phi \mathrm{OVT}+5 \sigma^{2} \mathrm{OR}+\sigma^{2}$ & 41 \\
\hline 35 & $\mathrm{OLCV}_{\mathrm{ij}(\mathrm{k}) / 0}$ & 60 & 0 & 0 & 1 & 0 & 3 & 5 & 5 & 0 & 1 & $75 \phi \mathrm{OLCV}+5 \sigma^{2} \mathrm{OR}+\sigma^{2}$ & 41 \\
\hline 36 & $\mathrm{OLCT}_{\mathrm{ijmo}}$ & 20 & 0 & 0 & 3 & 3 & 0 & 5 & 5 & 0 & 1 & $225 \phi \mathrm{OLCT}+5 \sigma^{2} \mathrm{OR}+\sigma^{2}$ & 41 \\
\hline 37 & $\mathrm{OLST}_{\mathrm{i}(\mathrm{j}) \mathrm{kmo}}$ & 20 & 0 & 1 & 0 & 3 & 0 & 5 & 5 & 0 & 1 & $75 \phi \mathrm{OLS}+5 \sigma^{2} \mathrm{OR}+\sigma^{2}$ & 41 \\
\hline 38 & $\mathrm{OLVT}_{\mathrm{i}(\mathrm{k}) \mathrm{mo}}$ & 60 & 0 & 3 & 1 & 0 & 0 & 5 & 5 & 0 & 1 & $75 \phi O L V T+5 \sigma^{2} \mathrm{OR}+\sigma^{2}$ & 41 \\
\hline 39 & $\mathrm{OCVT}_{\mathrm{j}(\mathrm{k}) \mid \mathrm{mo}}$ & 40 & 2 & 0 & 1 & 0 & 0 & 5 & 5 & 0 & 1 & $50 \phi O C V T+5 \sigma^{2} \mathrm{OR}+\sigma^{2}$ & 41 \\
\hline 40 & $\mathrm{OLCVT}_{\mathrm{ij}(\mathrm{k}) \mathrm{Imo}}$ & 40 & 0 & 0 & 1 & 0 & 0 & 5 & 5 & 0 & 1 & $25 \phi O L C V T+5 \sigma^{2} \mathrm{OR}+\sigma^{2}$ & 41 \\
\hline 41 & $\mathrm{OR}_{(\mathrm{j} \mathrm{kl} / \mathrm{m}) \mathrm{ro}}$ & 25 & 1 & 1 & 1 & 1 & 1 & 1 & 5 & 0 & 1 & $5 \sigma^{2} \mathrm{OR}+\sigma^{2}$ & -- \\
\hline
\end{tabular}


Table 7-10 Expected Mean Square Derivation for Nested Split-Plot Design (Table 4 of 6)

\begin{tabular}{|c|c|c|c|c|c|c|c|c|c|c|c|c|c|}
\hline 42 & $\mathrm{D}_{\mathrm{n}}$ & 4 & 2 & 3 & 3 & 3 & 3 & 5 & 0 & 6 & 1 & $4860 \phi D+6 \sigma^{2} \mathrm{DR}+\sigma^{2}$ & 62 \\
\hline 43 & $\mathrm{LD}_{\text {in }}$ & 4 & 0 & 3 & 3 & 3 & 3 & 5 & 0 & 6 & 1 & $2430 \phi L D+6 \sigma^{2} D R+\sigma^{2}$ & 62 \\
\hline 44 & $\mathrm{CD}_{\mathrm{jn}}$ & 8 & 2 & 0 & 3 & 3 & 3 & 5 & 0 & 6 & 1 & $1620 \phi C D+6 \sigma^{2} \mathrm{DR}+\sigma^{2}$ & 62 \\
\hline 45 & $\mathrm{SD}_{(\mathrm{j}) \mathrm{kn}}$ & 8 & 2 & 1 & 0 & 3 & 3 & 5 & 0 & 6 & 1 & $540 \phi S D+6 \sigma^{2} \mathrm{DR}+\sigma^{2}$ & 62 \\
\hline 46 & $\mathrm{VD}_{(\mathrm{k}) \ln }$ & 24 & 2 & 3 & 1 & 0 & 3 & 5 & 0 & 6 & 1 & $540 \phi V D+6 \sigma^{2} \mathrm{DR}+\sigma^{2}$ & 62 \\
\hline 47 & $\mathrm{TD}_{\mathrm{mn}}$ & 8 & 2 & 3 & 3 & 3 & 0 & 5 & 0 & 6 & 1 & $1620 \phi T D+6 \sigma^{2} \mathrm{DR}+\sigma^{2}$ & 62 \\
\hline 48 & $\mathrm{LCD}_{\mathrm{ijn}}$ & 8 & 0 & 0 & 3 & 3 & 3 & 5 & 0 & 6 & 1 & $810 \phi L C D+6 \sigma^{2} \mathrm{DR}+\sigma^{2}$ & 62 \\
\hline 49 & $\mathrm{LSD}_{\mathrm{i}(\mathrm{j}) \mathrm{kn}}$ & 8 & 0 & 1 & 0 & 3 & 3 & 5 & 0 & 6 & 1 & $270 \phi \mathrm{LSD}+6 \sigma^{2} \mathrm{DR}+\sigma^{2}$ & 62 \\
\hline 50 & $\mathrm{LVD}_{\mathrm{i}(\mathrm{k}) \ln }$ & 24 & 0 & 3 & 1 & 0 & 3 & 5 & 0 & 6 & 1 & $270 \phi L V D+6 \sigma^{2} D R+\sigma^{2}$ & 62 \\
\hline 51 & $\mathrm{LTD}_{\text {imn }}$ & 8 & 0 & 3 & 3 & 3 & 0 & 5 & 0 & 6 & 1 & $810 \phi L T D+6 \sigma^{2} \mathrm{DR}+\sigma^{2}$ & 62 \\
\hline 52 & $\mathrm{CVD}_{\mathrm{j}(\mathrm{k}) \mid \mathrm{n}}$ & 48 & 2 & 0 & 1 & 0 & 3 & 5 & 0 & 6 & 1 & $180 \phi C V D+6 \sigma^{2} D R+\sigma^{2}$ & 62 \\
\hline 53 & $\mathrm{CTD}_{\mathrm{jmn}}$ & 16 & 2 & 0 & 3 & 3 & 0 & 5 & 0 & 6 & 1 & $540 \phi$ CTD $+6 \sigma^{2} \mathrm{DR}+\sigma^{2}$ & 62 \\
\hline 54 & $\operatorname{STD}_{(j) k m n}$ & 16 & 2 & 1 & 0 & 3 & 0 & 5 & 0 & 6 & 1 & $180 \phi S T D+6 \sigma^{2} D R+\sigma^{2}$ & 62 \\
\hline 55 & $\operatorname{VTD}_{(\mathrm{k}) \mid \mathrm{mn}}$ & 48 & 2 & 3 & 1 & 0 & 0 & 5 & 0 & 6 & 1 & $180 \phi \mathrm{VTD}+6 \sigma^{2} \mathrm{DR}+\sigma^{2}$ & 62 \\
\hline 56 & $\operatorname{LCVD}_{\mathrm{ij}(\mathrm{k}) \ln }$ & 48 & 0 & 0 & 1 & 0 & 3 & 5 & 0 & 6 & 1 & $90 \phi L C V D+6 \sigma^{2} \mathrm{DR}+\sigma^{2}$ & 62 \\
\hline
\end{tabular}


Table 7-11 Expected Mean Square Derivation for Nested Split-Plot Design (Table 5 of 6)

\begin{tabular}{|c|c|c|c|c|c|c|c|c|c|c|c|c|c|}
\hline 57 & LCTD $_{\text {ijmn }}$ & 16 & 0 & 0 & 3 & 3 & 0 & 5 & 0 & 6 & 1 & $270 \phi \mathrm{LCTD}+6 \sigma^{2} \mathrm{DR}+\sigma^{2}$ & 62 \\
\hline 58 & LSTD $_{i(j) k m n}$ & 16 & 0 & 1 & 0 & 3 & 0 & 5 & 0 & 6 & 1 & $90 \phi \mathrm{LSTD}+6 \sigma^{2} \mathrm{DR}+\sigma^{2}$ & 62 \\
\hline 59 & LVTD $_{i(k) I m n}$ & 48 & 0 & 3 & 1 & 0 & 0 & 5 & 0 & 6 & 1 & $90 \phi \mathrm{LVTD}+6 \sigma^{2} \mathrm{DR}+\sigma^{2}$ & 62 \\
\hline 60 & $\mathrm{CVTD}_{\mathrm{j}(\mathrm{k}) \mid \mathrm{mn}}$ & 32 & 2 & 0 & 1 & 0 & 0 & 5 & 0 & 6 & 1 & $60 \phi C V T D+6 \sigma^{2} \mathrm{DR}+\sigma^{2}$ & 62 \\
\hline 61 & LCVTD $_{\mathrm{ij}(\mathrm{k}) \operatorname{lmn}}$ & 32 & 0 & 0 & 1 & 0 & 0 & 5 & 0 & 6 & 1 & $30 \phi L C V T D+6 \sigma^{2} \mathrm{DR}+\sigma^{2}$ & 62 \\
\hline 62 & $\mathrm{DR}_{(\mathrm{ijklm}) \mathrm{nr}}$ & 20 & 1 & 1 & 1 & 1 & 1 & 1 & 0 & 6 & 1 & $6 \sigma^{2} \mathrm{DR}+\sigma^{2}$ & -- \\
\hline 63 & $\mathrm{OD}_{\mathrm{no}}$ & 20 & 2 & 3 & 3 & 3 & 3 & 5 & 0 & 0 & 1 & $810 \phi O D+\sigma^{2} \mathrm{ODR}+\sigma^{2}$ & 83 \\
\hline 64 & $\mathrm{LOD}_{\text {ino }}$ & 20 & 0 & 3 & 3 & 3 & 3 & 5 & 0 & 0 & 1 & $405 \phi L O D+\sigma^{2} \mathrm{ODR}+\sigma^{2}$ & 83 \\
\hline 65 & $\mathrm{COD}_{\mathrm{jno}}$ & 40 & 2 & 0 & 3 & 3 & 3 & 5 & 0 & 0 & 1 & $270 \phi C O D+\sigma^{2} \mathrm{ODR}+\sigma^{2}$ & 83 \\
\hline 66 & $\mathrm{SOD}_{(j) k n o}$ & 40 & 2 & 1 & 0 & 3 & 3 & 5 & 0 & 0 & 1 & $90 \phi S O D+\sigma^{2} \mathrm{ODR}+\sigma^{2}$ & 83 \\
\hline 67 & $\operatorname{VOD}_{(\mathrm{k}) \operatorname{Ino}}$ & 120 & 2 & 3 & 1 & 0 & 3 & 5 & 0 & 0 & 1 & $90 \phi \mathrm{VOD}+\sigma^{2} \mathrm{ODR}+\sigma^{2}$ & 83 \\
\hline 68 & $\mathrm{TOD}_{\mathrm{mno}}$ & 40 & 2 & 3 & 3 & 3 & 0 & 5 & 0 & 0 & 1 & $270 \phi T O D+\sigma^{2} \mathrm{ODR}+\sigma^{2}$ & 83 \\
\hline 69 & $\operatorname{LCOD}_{\mathrm{ijno}}$ & 40 & 0 & 0 & 3 & 3 & 3 & 5 & 0 & 0 & 1 & $135 \phi \mathrm{LCOD}+\sigma^{2} \mathrm{ODR}+\sigma^{2}$ & 83 \\
\hline 70 & $\operatorname{LSOD}_{\mathrm{i}(\mathrm{j}) \mathrm{kno}}$ & 40 & 0 & 1 & 0 & 3 & 3 & 5 & 0 & 0 & 1 & $45 \phi \mathrm{LSOD}+\sigma^{2} \mathrm{ODR}+\sigma^{2}$ & 83 \\
\hline 71 & $\operatorname{LVOD}_{\mathrm{i}(\mathrm{k}) \operatorname{Ino}}$ & 120 & 0 & 3 & 1 & 0 & 3 & 5 & 0 & 0 & 1 & $45 \phi L V O D+\sigma^{2} O D R+\sigma^{2}$ & 83 \\
\hline
\end{tabular}


Table 7-12 Expected Mean Square Derivation for Nested Split-Plot Design (Table 6 of 6)

\begin{tabular}{|c|c|c|c|c|c|c|c|c|c|c|c|c|c|}
\hline 72 & LTOD $_{\text {imno }}$ & 40 & 0 & 3 & 3 & 3 & 0 & 5 & 0 & 0 & 1 & $135 \phi L T O D+\sigma^{2} \mathrm{ODR}+\sigma^{2}$ & 83 \\
\hline 73 & $\mathrm{CVOD}_{\mathrm{j}(\mathrm{k}) \mid \mathrm{no}}$ & 240 & 2 & 0 & 1 & 0 & 3 & 5 & 0 & 0 & 1 & $30 \phi C V O D+\sigma^{2}$ ODR $+\sigma^{2}$ & 83 \\
\hline 74 & $\mathrm{CTOD}_{\text {jmno }}$ & 80 & 2 & 0 & 3 & 3 & 0 & 5 & 0 & 0 & 1 & $90 \phi$ CTOD $+\sigma^{2}$ ODR $+\sigma^{2}$ & 83 \\
\hline 75 & $\operatorname{STOD}_{(j) k m n o}$ & 80 & 2 & 1 & 0 & 3 & 0 & 5 & 0 & 0 & 1 & $30 \phi S T O D+\sigma^{2}$ ODR $+\sigma^{2}$ & 83 \\
\hline 76 & $\operatorname{VTOD}_{(\mathrm{k}) \mid \mathrm{mno}}$ & 240 & 2 & 3 & 1 & 0 & 0 & 5 & 0 & 0 & 1 & $30 \phi \mathrm{VTOD}+\sigma^{2} \mathrm{ODR}+\sigma^{2}$ & 83 \\
\hline 77 & $\operatorname{LCVOD}_{\mathrm{ij}(\mathrm{k}) \mid \mathrm{no}}$ & 240 & 0 & 0 & 1 & 0 & 3 & 5 & 0 & 0 & 1 & $15 \phi L C V O D+\sigma^{2}$ ODR $+\sigma^{2}$ & 83 \\
\hline 78 & LCTOD $_{i j m n o}$ & 80 & 0 & 0 & 3 & 3 & 0 & 5 & 0 & 0 & 1 & $45 \phi L C T O D+\sigma^{2}$ ODR $+\sigma^{2}$ & 83 \\
\hline 79 & LSTOD $_{\mathrm{i}(j) \mathrm{kmno}}$ & 80 & 0 & 1 & 0 & 3 & 0 & 5 & 0 & 0 & 1 & $15 \phi$ LSTOD $+\sigma^{2} \mathrm{ODR}+\sigma^{2}$ & 83 \\
\hline 80 & LVTOD $_{\mathrm{i}(\mathrm{k}) \mid \mathrm{mno}}$ & 240 & 0 & 3 & 1 & 0 & 0 & 5 & 0 & 0 & 1 & $15 \phi L V T O D+\sigma^{2}$ ODR $+\sigma^{2}$ & 83 \\
\hline 81 & CVTOD $_{\mathrm{j}(\mathrm{k}) \mid \mathrm{mn} o}$ & 160 & 2 & 0 & 1 & 0 & 0 & 5 & 0 & 0 & 1 & $10 \phi C V T O D+\sigma^{2}$ ODR $+\sigma^{2}$ & 83 \\
\hline 82 & 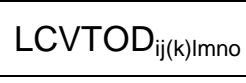 & 160 & 0 & 0 & 1 & 0 & 0 & 5 & 0 & 0 & 1 & $5 \phi \mathrm{LCVTOD}+\sigma^{2} \mathrm{ODR}+\sigma^{2}$ & 83 \\
\hline 83 & $\mathrm{ODR}_{(\mathrm{ijklm}) \mathrm{nor}}$ & 80 & 1 & 1 & 1 & 1 & 1 & 1 & 0 & 0 & 1 & $\sigma^{2} \mathrm{ODR}+\sigma^{2}$ & 84 \\
\hline 84 & $\varepsilon_{(\mathrm{ijklmnor}) \mathrm{e}}$ & 0 & 1 & 1 & 1 & 1 & 1 & 1 & 1 & 1 & 1 & $\sigma^{2}$ & -- \\
\hline
\end{tabular}


Table 7-13 shows the F statistic for the skewness model with an F value of 5.77. Most of the interaction terms were found significant at the 0.05 significance level and therefore the cell means were analyzed further. An interesting output to look at is the interaction between the offset and traffic volume and the offset and turn percentage shown in Table 7-14 and Table 7-15 respectively.

Table 7-14 shows that there were only four significant groups: offset class 4 with a high traffic volume; offset class 4 with medium volume and offset class 3 with a high volume; and offset class 3 with medium volume; and the remaining combinations formed a fourth group. The largest differential skewness was obtained with the case of the worst offset class and high volume (strongest shockwave) and was significantly different from all other group. The differential skewness obtained from the second worst offset class and high volume, or the worst offset class and medium volume, were not significantly different from each other (assigned the same letter under the SNK grouping column), but were significantly different from other groups. The second worst offset class and medium volume case was significantly different from all other cases. The results also showed that the rest of the offset-volume combinations were not found to be significantly different from one another, which means that the skewness model will not be able to distinguish between offset class 1 , offset class 2 , offset class 3 with low volume, or offset class 4 with low volume.

Table 7-15 shows a case where several groups were not found significantly different from the others. The trend in Table $7-15$ is that if the turning percentage 
increases, the offset classification is insignificantly different with the lower offset classification with less turning percentage.

Table 7-13 Skewness Model's ANOVA

The GLM Procedure

Dependent Variable: diff_skw

\begin{tabular}{lrrrrr} 
& \multicolumn{2}{c}{ Sum of } & & & \\
Source & DF & Squares & Mean Square & F Value & Pr $>$ F \\
Model & 809 & 5473.4 & 6.76568 & 5.77 & $<.0001$ \\
Error & 23490 & 27536.1 & 1.17225 & & \\
Corrected Total & 24299 & 33009.5 & & &
\end{tabular}

$\begin{array}{cccc}\text { R-Square } & \text { Coeff Var } & \text { Root MSE } & \text { diff_skw Mean } \\ 0.165814 & 583.9568 & 1.082704 & 0.185408\end{array}$


Table 7-14 Offset Volume Interactions for the Skewness Model

Means with the same letter are not significantly different.

$\begin{array}{lllll}\text { SNK Grouping } & \text { Mean } & \mathrm{N} & \text { off_vol } \\ \text { A } & 0.83695 & 1350 & 41 \\ \text { B } & 0.68626 & 1350 & 42 \\ \text { B } & & & \\ \text { B } & 0.58699 & 2700 & 31 \\ \text { C } & 0.39955 & 2700 & 32 \\ \text { D } & 0.11003 & 1350 & 43 \\ \text { D } & & & \\ \text { D } & 0.02549 & 2700 & 33 \\ \text { D } & & & \\ \text { D } & -0.01599 & 2700 & 22 \\ \text { D } & & & \\ \text { D } & -0.02362 & 1350 & 12 \\ \text { D } & & & \\ \text { D } & -0.03007 & 2700 & 23 \\ \text { D } & & & \\ \text { D } & -0.03465 & 1350 & 13 \\ \text { D } & & & \\ \text { D } & -0.05531 & 2700 & 21 \\ \text { D } & & & \\ \text { D } & -0.05896 & 1350 & 11\end{array}$


Table 7-15 Offset Turn Interaction for the Skewness Model

Means with the same letter are not significantly different.

\begin{tabular}{rllll} 
SNK Grouping & \multicolumn{1}{l}{ Mean } & N & off_turn \\
A & 0.83415 & 1350 & 41 \\
& & & & \\
B & 0.53374 & 1350 & 42 \\
B & & & \\
B & 0.51314 & 2700 & 31 \\
C & 0.36356 & 2700 & 32 \\
C & & & & \\
C & 0.26536 & 1350 & 43 \\
D & 0.13533 & 2700 & 33 \\
D & & & & \\
E D & 0.09503 & 1350 & 11 \\
E D & & & & \\
E D & F & 0.03378 & 2700 & 21 \\
E & F & & & \\
E G & F & -0.03167 & 2700 & 23 \\
G & F & & & \\
G & F & -0.07253 & 1350 & 12 \\
G & F & & & \\
G & F & -0.10348 & 2700 & 22 \\
G & & & & \\
G & -0.13973 & 1350 & 13
\end{tabular}

\subsection{The F' Model}

The F' Model is named after the F statistics testing approach in ANOVA. The philosophy behind this approach is to test for the effect of the shockwave created by the red light facing the platoon at the downstream signal using the detector actuation. The $\mathrm{F}$ test in ANOVA compares the F value obtained by dividing the Mean Square Error (MSE) of the model by the MSE of the error term to test whether the resulting value follows the $\mathrm{F}$ distribution. If the resulting value exceeds the $\mathrm{F}$ value predicted by the $\mathrm{F}$ distribution, 
this is interpreted as a significant existence of the tested factor variance in the model variance (versus the effect of the error term variance alone). Through the use of count and occupancy profile, one can calculate the MSE of each as an estimate of their variance. Equation (7-1) shows how the occupancy relates to vehicle speed and length.

$$
E[o c c]=\frac{1}{I} E\left[\sum_{i=1}^{n} \frac{L_{i}^{v}+L^{d}}{S_{i}^{v}}\right]
$$

where:

I: interval length over which occupancy is calculated (bin width)

$L_{i}^{v}$ : length of vehicle I]

$L^{d}$ : length of detector

$S_{i}^{v}$ : speed of vehicle I

Let's define a variable $x_{i}$ such that:

$$
x_{i}=\frac{L_{i}^{v}+L^{d}}{I^{*} S_{i}^{v}}
$$

The occupancy equation can be written as:

$$
E[o c c]=\operatorname{var}\left(\sum_{i=1}^{N} x_{i}\right)
$$

And since: 


$$
\operatorname{var}(\text { occ })=\operatorname{var}\left(\sum_{i=1}^{N} x_{i}\right)=\operatorname{var}(N) E\left[x^{2}\right]+(E[N])^{2} \operatorname{var}(x)
$$

(summation of variance of random number of random variables) then the F'value defined as the var (occ)/var(count) can be calculated as:

$F^{\prime}$ Value $=\frac{\operatorname{var}(\text { occ })}{\operatorname{var}(\text { count })}=\frac{\operatorname{var}\left(\sum_{i=1}^{N} x_{i}\right)}{\operatorname{var}(N)}$

or alternatively:

$F^{\prime}$ Value $=E\left[x^{2}\right]+\frac{(E[N])^{2}}{\operatorname{var}(N)} \operatorname{var}(x)$

Since:

$$
x_{i}=\frac{L_{i}^{v}+L^{d}}{I * S_{i}^{v}}
$$

Assuming a small variation in vehicle length compared to vehicle speed, as well as no change in the variance of vehicle length in relation to the offset.

$$
x_{i}=\frac{L}{S_{i}^{v}}
$$

where $\mathrm{L}$ is a constant

substituting in Equation (7-6)

$$
F^{\prime} \text { Value }=L^{2} E\left[\frac{1}{S_{i}^{v 2}}\right]+\frac{(E[N])^{2}}{\operatorname{var}(N)} L^{2} \operatorname{var}\left(\frac{1}{S_{i}^{v}}\right)
$$

or alternatively 


$$
F^{\prime} \text { Value }=L^{2}\left[E\left[\frac{1}{S_{i}^{\nu 2}}\right]+\frac{(E[N])^{2}}{\operatorname{var}(N)} \operatorname{var}\left(\frac{1}{S_{i}^{v}}\right)\right]
$$

Equation (7-7) suggests that the F' value increases as the variance of the reciprocal of the speed increases, which can be used to test whether there is an effect of the shockwave in the F' value. Figure 7-8 illustrates the effect of offset on speed variance. Note that when the offset is well designed, vehicle platoons continue through the signal without interruption. When the offset is not performing well, some vehicles experience a delay or even come to a complete stop. This tends to increase the variation of speed in the platoon, a phenomenon that can be used to identify a bad offset.
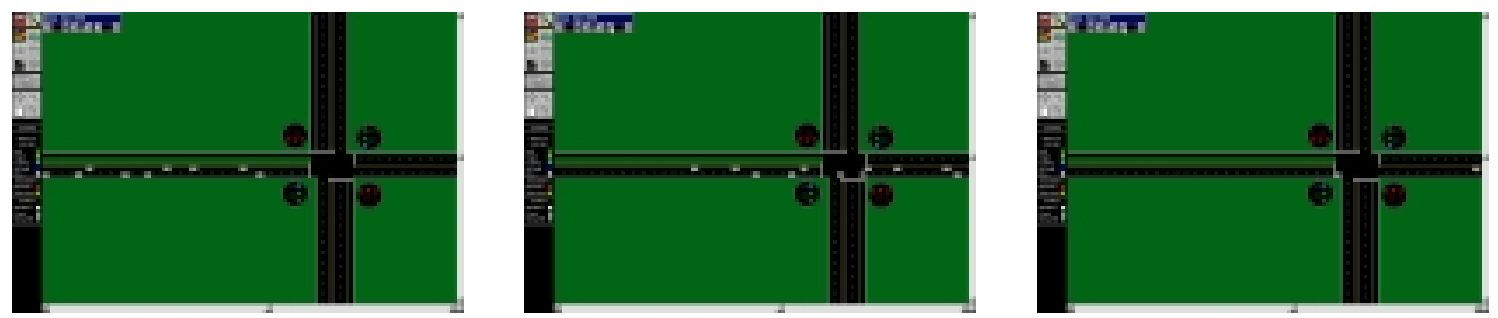

a) Low Speed Variance Due to a Good Offset
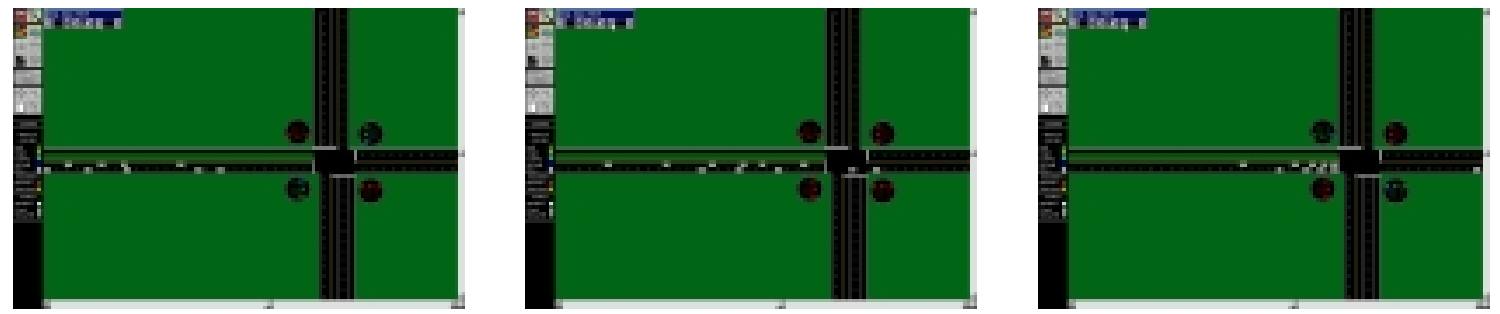

b) High Speed Variance Due to a Bad offset

Figure 7-8 Effect of offset on Speed variance

\subsubsection{ANOVA Results of the F' Model}

An ANOVA was conducted for the F' Model to test for its sensitivity for different parameters and the ANOVA output is attached in Appendix C. Table 7-16 shows the F' 
model ANOVA output. The Table shows an F value higher than that of the Skewness model (7.03), which means that the F' model has higher significance than the skewness model. As can be seen in the output, most of the interaction terms were found to be significant with $\mathrm{P}$ values less than 0.05 . Table $7-17$ shows an interesting output, the interaction between the offset class and the turn percentage. The table shows that most of the offset classes were grouped together regardless of the turn percentage (assigned the same letter under the SNK grouping column). Note that the significant effect of turning percentage was a major drawback in the Skewness model. This result show a promising advantage of the F' model.

\section{Table 7-16 F' Model's ANOVA}

The GLM Procedure

Dependent Variable: fvalue

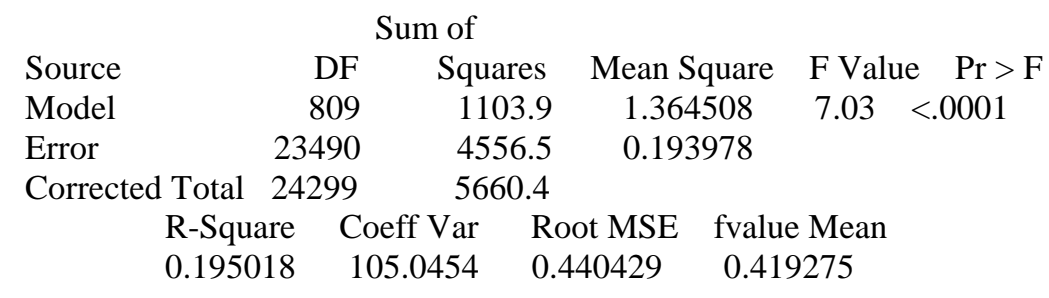


Table 7-17 Offset Turn Interactions of the F' Model

Means with the same letter are not significantly different.

$\begin{array}{lllll}\text { SNK Grouping } & \text { Mean } & \mathrm{N} & \text { off_turn } \\ \text { A } & 0.62917 & 1350 & 41 \\ \text { A } & & & \\ \text { A } & 0.61900 & 1350 & 42 \\ \text { A } & & & \\ \text { B A } & 0.57820 & 1350 & 43 \\ \text { B } & & & \\ \text { B C } & 0.53232 & 2700 & 31 \\ \text { B C } & & & \\ \text { B C } & 0.52248 & 2700 & 32 \\ \text { C } & & & \\ \text { C } & 0.50607 & 2700 & 33 \\ & \text { D } & 0.36017 & 2700 & 23 \\ \text { D } & & & \\ \text { E } & \text { D } & 0.31353 & 1350 & 13 \\ \text { E } & \text { D } & & & \\ \text { E } & \text { D } & 0.31097 & 2700 & 22 \\ \text { E } & & & & \\ \text { E } & 0.28576 & 2700 & 21 \\ & & & & \\ \text { F } & 0.20180 & 1350 & 12 \\ \text { F } & & & \\ \text { F } & 0.16971 & 1350 & 11\end{array}$

\subsection{Discriminant Analysis}

Discriminant analysis was used to develop threshold values by which offsets can be classified into four different groups, namely, level 1 for a good offset, level 2 for 1/6 of a cycle deviation from the good offset, level 3 for $2 / 6$ deviation from the good offset, and level 4 which is $1 / 2$ a cycle away from the good offset. PRO-TRACTS compares the F' values calculated from the occupancy and count profiles to these thresholds to determine the class of the current cycles's offset. This information is used in determining the magnitude of offset shift needed to fine tune the offset. 


\subsubsection{Overview of Discriminant Analysis}

Classificatory discriminant analysis is used to classify observations into known groups on the basis of one or more quantitative variables. The method used can be either parametric, for approximately normal within-class distribution, or non-parametric for a non-normal within class distribution. Discriminant analysis also provides error rates (probability of misclassification) that can be used as a measure of performance.

Unlike cluster analysis, discriminant analysis requires prior knowledge of the observations' classes. Discriminant analysis makes use of the posterior probabilities of observations to determine the groups to which the observations belong, using maximum likelihood rules.

\subsubsection{Offset Warrant Thresholds}

Discriminant analysis was used to develop thresholds to identify offset classes using the data from the previous experiment. The SAS Discrim procedure was used to develop the thresholds that divide different groups of offset classes. The SAS code and sample results are attached in Appendix C. Figure 7-9 shows the linear discriminant functions for different classes of offset. For any observation value of $\mathrm{F}^{\prime}$, the discriminant function with the highest value determines the class to which the observation belongs. The point at which any two lines intersect identifies a threshold value for a higher offset class. For example, Figure 7-9 shows that the offset class 1 has the highest value of discriminant function until the point where offset class 1 line intersects with offset class 6 line. This point defines a threshold beyond which observations are classified into offset 
class 6 . The threshold can be calculated by solving for the intersection point of the two offset class line equations shown in Table 7-18. By solving for $\mathrm{X}$ in the equation:

$-1.958+1.794 \mathrm{X}=-2.373+3.355 \mathrm{X}$, the threshold between class 1 and class 6 was found to be 0.26 . Note that both offset class 2 and offset class 6 denote a $1 / 6$ deviation from the good offset, only in a different direction.

By solving the line equations for the intersection points, the three thresholds (rounded) were found to be $0.25,0.75$, and 1.0 to identify a deviation of $1 / 6,2 / 6$, and $1 / 2$ of the cycle length from the good offset, respectively.

Table 7-18 Linear Discriminant Function Equations

\begin{tabular}{|l|l|l|l|l|l|l|}
\hline Offset Class & 1 & 2 & 3 & 4 & 5 & 6 \\
\hline Constant & -1.958 & -4.415 & -6.629 & -6.411 & -4.998 & -2.373 \\
\hline Variable Coeff & 1.794 & 7.127 & 9.677 & 9.457 & 7.879 & 3.355 \\
\hline
\end{tabular}




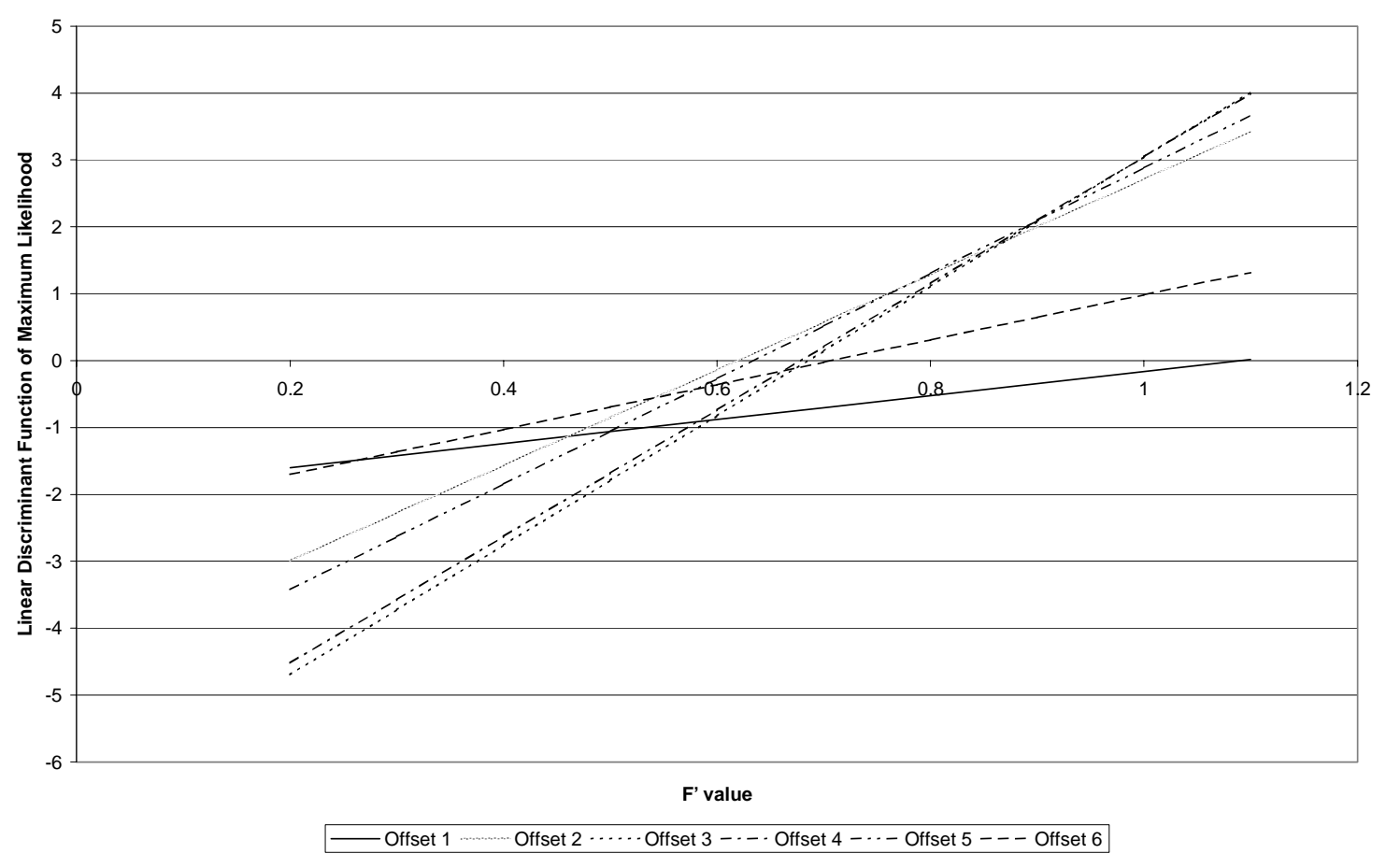

Figure 7-9 Linear Discriminant Functions for Different Offset Classes

\subsection{Chapter's Conclusion}

This chapter introduced the F' model, which captures the shockwave effect by calculating a value related to the speed variance from the occupancy and count profiles. The effects of factors such as traffic volume and the percentage of turning traffic on the performance of offsets were also discussed. Analysis of variance was conducted for both the skewness and the F' models, under different conditions, with the conclusion that the F' model was found to be less sensitive to turning traffic percentage than the skewness model. The skewness model was found to be insensitive to offset changes with low traffic volume, or if the change was relatively small, with no significant difference found 
between class 1 and class 2. Discriminant analysis was used to develop thresholds to which the F' values could be compared, to estimate the performance of the current cycle's offset. These values will be used in the following chapters to activate PROTRACTS and to determine the amount of change that needs to be applied to the current offset. 


\section{CHAPTER 8- CASE STUDY: SR 26, LAFAYETTE, INDIANA}

This chapter discusses the results of the application of PRO-TRACTS to SR26 in Lafayette, Indiana during the a.m. and p.m. peak periods. The studied network consists of seven intersections between Earl Avenue and Creasy Lane, as shown in Figure 8-1. The intersection layout, ring structure, timings, detector configuration, phase parameters, traffic volumes, and turning percentages are listed in Appendix A. Transitioning in offset and cycle length are also discussed at the end of each section.

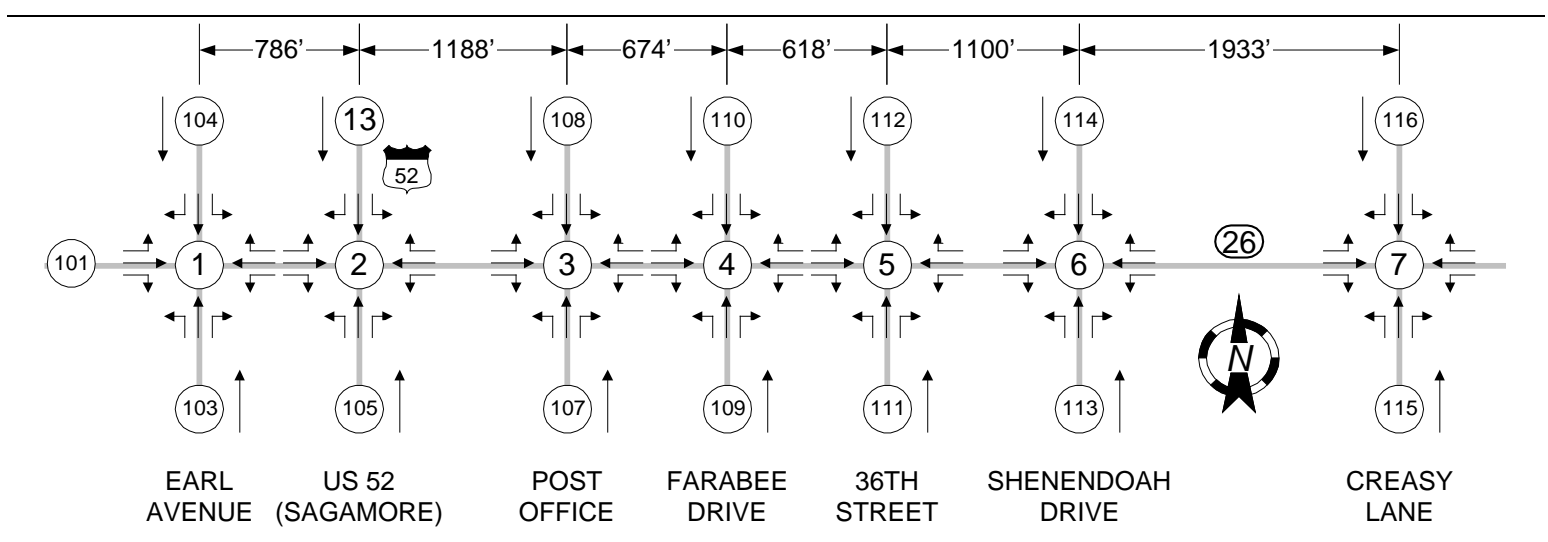

Figure 8-1 SR 26 (South) Network - Earl Avenue to Creasy Lane

\subsection{Phase Timings}

Phase timings were designed with Synchro for both the a.m. and p.m. peak periods. The best cycle length for the a.m. peak $(\mathrm{COS}=111)$ was found to be 75 seconds, 
while the optimum cycle length for the p.m. peak $(\operatorname{COS}=311)$ was found to be 120 seconds. Table 8-1 lists the offsets and phase splits for the seven studied intersections.

Table 8-1 SR 26 (South) Coordination Plans and Splits

\begin{tabular}{|l|l|l|l|l|l|l|l|l|l|l|l|}
\hline INT & COS & CYC & OFF & 1 & 2 & 3 & 4 & 5 & 6 & 7 & 8 \\
\hline 1 & 111 & 75 & 0 & 4 & 30 & 3 & 18 & 3 & 31 & 5 & 16 \\
\hline 1 & 311 & 120 & 0 & 10 & 44 & 3 & 43 & 4 & 51 & 14 & 32 \\
\hline 2 & 111 & 75 & 2 & 13 & 18 & 7 & 17 & 6 & 26 & 9 & 15 \\
\hline 2 & 311 & 120 & 108 & 19 & 33 & 17 & 33 & 15 & 36 & 20 & 30 \\
\hline \hline 3 & 111 & 75 & 40 & 13 & 26 & - & 21 & 8 & 32 & - & 21 \\
\hline 3 & 311 & 120 & 36 & 6 & 82 & - & 18 & 14 & 74 & - & 18 \\
\hline \hline 4 & 111 & 75 & 37 & 9 & 25 & - & 27 & 9 & 25 & 5 & 17 \\
\hline 4 & 311 & 120 & 30 & 14 & 50 & - & 42 & 10 & 54 & 5 & 32 \\
\hline 5 & 111 & 75 & 31 & 3 & 22 & - & 15 & 3 & 22 & - & 15 \\
\hline 5 & 311 & 120 & 38 & 5 & 61 & - & 17 & 9 & 57 & - & 17 \\
\hline 6 & 111 & 75 & 20 & 5 & 28 & 5 & 17 & 6 & 28 & 5 & 17 \\
\hline 6 & 311 & 120 & 23 & 5 & 74 & 5 & 17 & 8 & 71 & 5 & 17 \\
\hline 7 & 111 & 75 & 69 & 11 & 17 & 11 & 17 & 6 & 22 & 13 & 15 \\
\hline 7 & 311 & 120 & 84 & 11 & 34 & 31 & 25 & 14 & 31 & 28 & 28 \\
\hline \hline
\end{tabular}

\subsection{PRO-TRACTS Parameters}

The test of PRO-TRACTS was run with the CORSIM internal control logic through CORSIM's real-time extension DLL. PRO-TRACTS detectors were set 150 feet upstream of each traffic signal. The incremental thresholds used for the activation for PRO-TRACTS were set at $0.25,0.75$, and 1.0 as discussed in Section 7.4.3 in Chapter 7. The algorithm was configured to evaluate the cycle profiles at the end of every cycle. Maximum offset step was set to 35 seconds to minimize the frequency of activating the unpredictable CORSIM's internal cycle transitioning mechanisms. 


\subsection{Simulation Results}

CORSIM simulations were run with varying demand volumes over a four hours period for both a.m. and p.m. peak periods (6-10 a.m. and 2-6 p.m. respectively). The simulations were run with five different random number seeds to account for the stochastic nature of traffic. Table 8-2 shows the entry volume to intersection approaches by the period. These volumes were obtained from actual INDOT turning movement counts and tube counts. Note that the volumes were considerably higher in the p.m. period.

The following three scenarios were studied:

- A base case with the traffic network operating with Synchro's timing plans, except that the offsets were changed such that they provided poor progression for the eastbound traffic. This case was run with normal CORSIM simulation run without activating PRO-TRACTS.

- A scenario similar to the base case, but with PRO-TRACTS running to improve the coordination of eastbound traffic.

- A scenario with the network operating with offsets designed by Synchro, which was included as a benchmark for comparison purposes. 
Table 8-2 SR 26 (South) Entry Volumes per Period (VPH)

\begin{tabular}{|c|c|c|c|c|c|c|c|c|c|c|c|c|c|}
\hline INT & $\begin{array}{l}\text { BEG } \\
\text { END }\end{array}$ & $\begin{array}{l}\text { 7AM } \\
8 \mathrm{AM}\end{array}$ & $\begin{array}{l}8 \mathrm{AM} \\
9 \mathrm{AM}\end{array}$ & $\begin{array}{c}9 \mathrm{AM} \\
10 \mathrm{~A}\end{array}$ & $\begin{array}{l}10 \mathrm{~A} \\
11 \mathrm{~A}\end{array}$ & $\begin{array}{l}11 \mathrm{~A} \\
12 \mathrm{P}\end{array}$ & $\begin{array}{c}12 \mathrm{P} \\
1 \mathrm{PM}\end{array}$ & $\begin{array}{l}1 \mathrm{PM} \\
2 \mathrm{PM}\end{array}$ & $\begin{array}{l}2 \mathrm{PM} \\
3 \mathrm{PM}\end{array}$ & $\begin{array}{l}3 \mathrm{PM} \\
4 \mathrm{PM}\end{array}$ & $\begin{array}{l}4 \mathrm{PM} \\
5 \mathrm{PM}\end{array}$ & $\begin{array}{l}5 \mathrm{PM} \\
6 \mathrm{PM}\end{array}$ & $\begin{array}{l}6 \mathrm{PM} \\
7 \mathrm{PM}\end{array}$ \\
\hline 1 & EB & 311 & 342 & 460 & 530 & 654 & 674 & 563 & 706 & 813 & 705 & 709 & 575 \\
\hline 1 & WB & 590 & 620 & 605 & 551 & 690 & 712 & 692 & 758 & 604 & 695 & 696 & 548 \\
\hline 1 & NB & 406 & 334 & 388 & 351 & 478 & 457 & 382 & 506 & 713 & 433 & 536 & 316 \\
\hline 1 & SB & 292 & 341 & 453 & 429 & 538 & 565 & 437 & 544 & 608 & 552 & 515 & 356 \\
\hline 2 & EB & 425 & 456 & 582 & 665 & 864 & 857 & 669 & 904 & 1050 & 905 & 910 & 715 \\
\hline 2 & WB & 690 & 867 & 785 & 717 & 880 & 889 & 845 & 933 & 788 & 899 & 816 & 729 \\
\hline 2 & NB & 735 & 600 & 676 & 817 & 1060 & 1134 & 1057 & 1254 & 1228 & 1117 & 1251 & 883 \\
\hline 2 & SB & 765 & 615 & 768 & 783 & 1000 & 1063 & 1024 & 1049 & 962 & 909 & 1099 & 784 \\
\hline 3 & EB & 590 & 573 & 700 & 744 & 1060 & 1080 & 901 & 1042 & 1096 & 1003 & 1178 & 912 \\
\hline 3 & WB & 657 & 843 & 746 & 648 & 836 & 820 & 861 & 933 & 842 & 972 & 794 & 788 \\
\hline 3 & NB & 11 & 6 & 20 & 10 & 40 & 120 & 66 & 40 & 12 & 28 & 49 & 31 \\
\hline 3 & SB & 180 & 191 & 186 & 203 & 219 & 207 & 205 & 216 & 155 & 232 & 274 & 220 \\
\hline 4 & EB & 530 & 559 & 685 & 718 & 96 & 1012 & 87 & 979 & 994 & 959 & 1132 & 940 \\
\hline 4 & WB & 743 & 957 & 757 & 669 & 905 & 806 & 88 & 959 & 930 & 1019 & 803 & 777 \\
\hline 4 & NB & 197 & 215 & 212 & 237 & 336 & 304 & 24 & 258 & 38 & 348 & 367 & 231 \\
\hline 4 & SB & 49 & 53 & 106 & 133 & 158 & 194 & 176 & 186 & 130 & 180 & 186 & 174 \\
\hline 5 & EB & 607 & 649 & 738 & 810 & 1095 & 1135 & 956 & 1040 & 1172 & 1134 & 1339 & 1057 \\
\hline 5 & WB & 737 & 948 & 736 & 635 & 869 & 790 & 850 & 933 & 857 & 952 & 778 & 761 \\
\hline 5 & NB & 49 & 30 & 18 & 26 & 32 & 24 & 25 & 77 & 102 & 61 & 28 & 23 \\
\hline 5 & SB & 71 & 169 & 91 & 104 & 141 & 165 & 142 & 107 & 147 & 191 & 174 & 102 \\
\hline 6 & EB & 560 & 66 & 705 & 787 & 1102 & 1150 & 954 & 1048 & 1176 & 1166 & 1374 & 1074 \\
\hline 6 & WB & 768 & 872 & 716 & 599 & 811 & 730 & 799 & 897 & 829 & 890 & 736 & 769 \\
\hline 6 & NB & 59 & 23 & 11 & 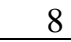 & 24 & 18 & 22 & 116 & 177 & 72 & 51 & 21 \\
\hline 6 & SB & 86 & 55 & 72 & 80 & 73 & 111 & 126 & 122 & 114 & 111 & 125 & 114 \\
\hline 7 & EB & 600 & 655 & 694 & 770 & 1083 & 1123 & 953 & 1122 & 1314 & 1206 & 1392 & 1072 \\
\hline 7 & WB & 860 & 991 & 794 & 599 & 846 & 752 & 824 & 868 & 833 & 732 & 654 & 778 \\
\hline 7 & NB & 635 & 600 & 514 & 572 & 759 & 606 & 830 & 1180 & 1220 & 1555 & 1423 & 1179 \\
\hline 7 & SB & 715 & 641 & 572 & 575 & 799 & 622 & 712 & 726 & 707 & 994 & 1094 & 898 \\
\hline
\end{tabular}

\subsubsection{A.M. Travel Time, Stops and Delay}

Figure 8-2 and Figure 8-3 show the cumulative travel time through the arterial for eastbound and westbound traffic, respectively. The three different lines in the figures represent the results from the three different scenarios discussed above. Figure 8-2 shows that PRO-TRACTS reduced the eastbound travel time by 4\%, approaching the performance of Synchro (6\%). Figure 8-3 shows only a minor improvement of $1 \%$ in westbound cumulative travel time with PRO-TRACTS, while Synchro improved the cumulative travel time by $9 \%$. This result was expected since PRO-TRACTS was only 
configured to improve the eastbound signal coordination. Figure 8-4 and Figure 8-5 show the cumulative number of east and westbound stops respectively. Figure 8-4 shows that PRO-TRACTS reduced the cumulative number of eastbound stops during the four hours of simulations by $10 \%, 9 \%, 13 \%$, and $13 \%$ respectively. Figure $8-5$ shows a minor increase in the cumulative number of westbound stops of $1 \%$ and $2 \%$ during the last two hours. Figure 8-6, which shows the total delay and number of stops on an hour-by-hour basis, indicates a reduction in the total number of stops and system delay of 3\% and $1 \%$, respectively, with PRO-TRACTS during the four hours. These results can be further explained by studying the offset and cycle transitioning graphs discussed in the next section. 


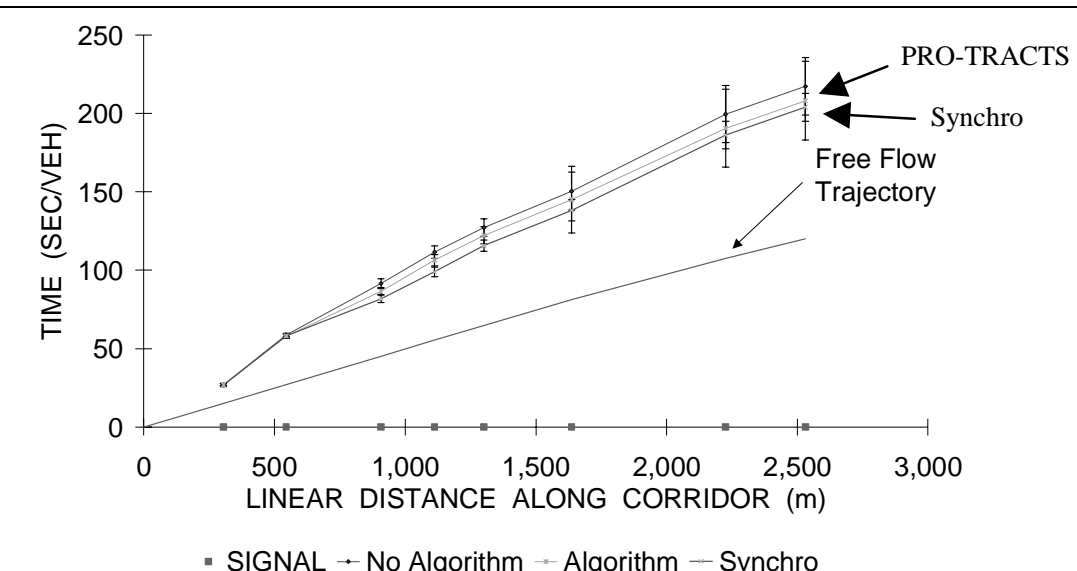

a) First Hour
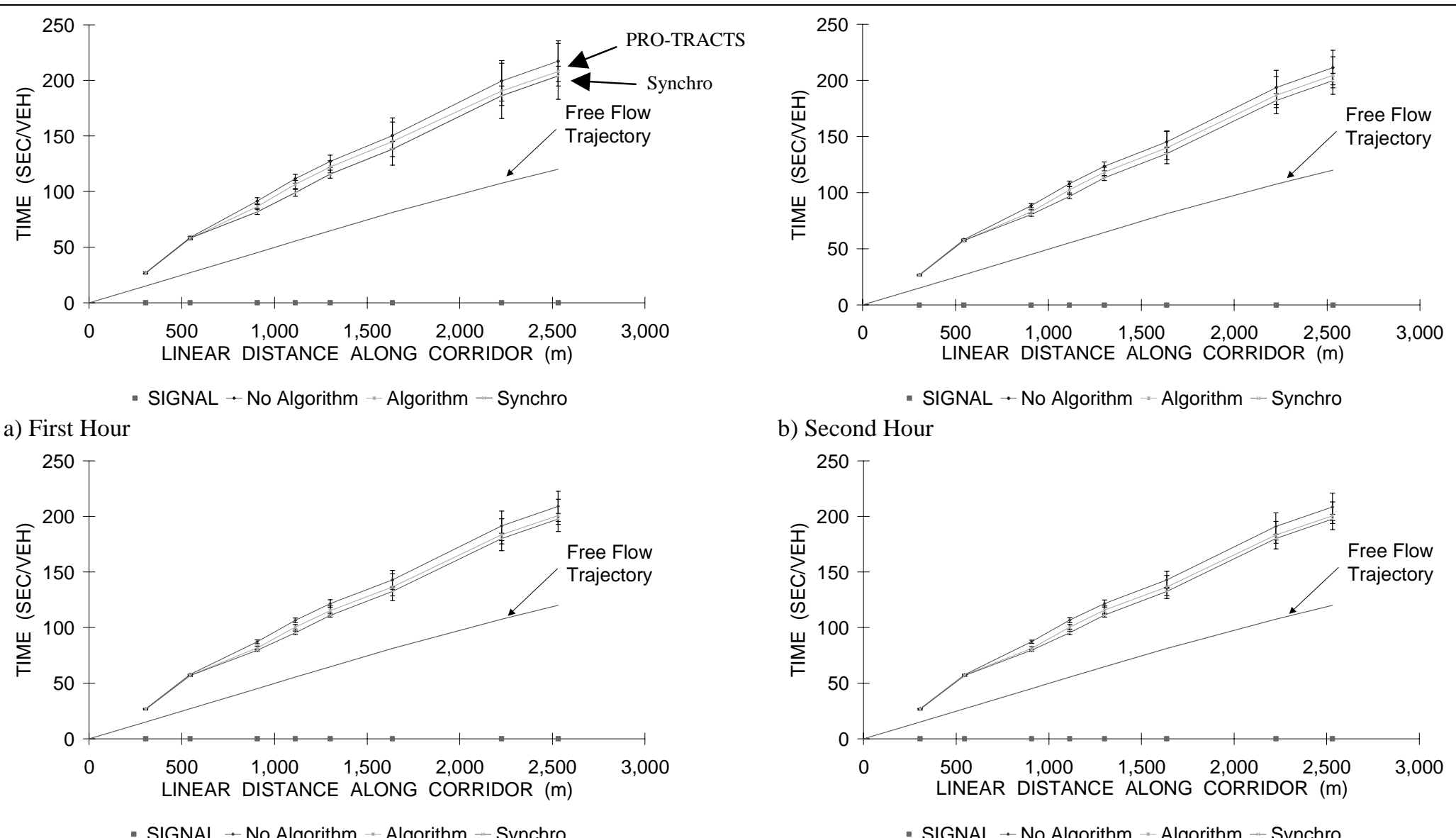

c) Third Hour

b) Second Hour

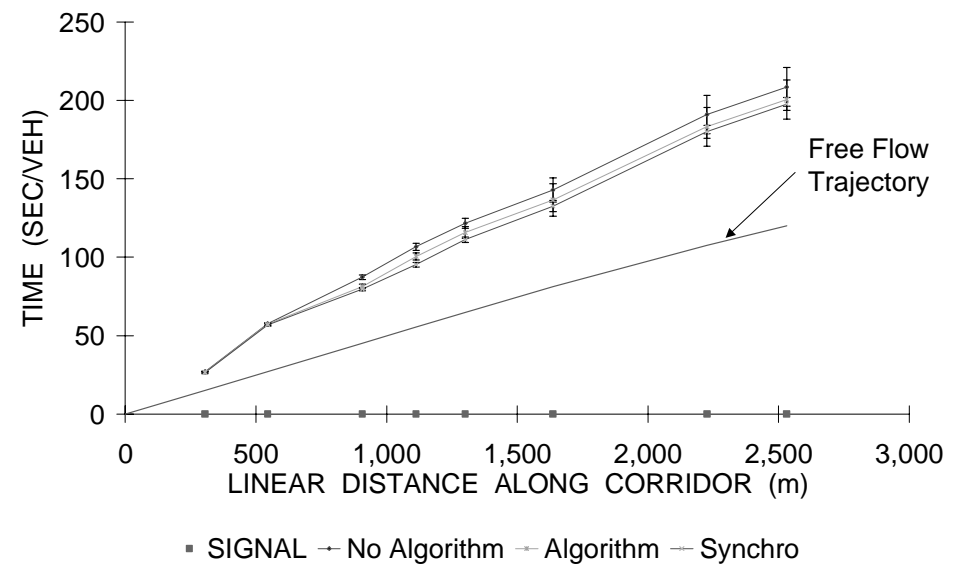

d) Fourth Hour

Figure 8-2 SR 26 (South) East Bound Cumulative Travel Time-A.M. Period 


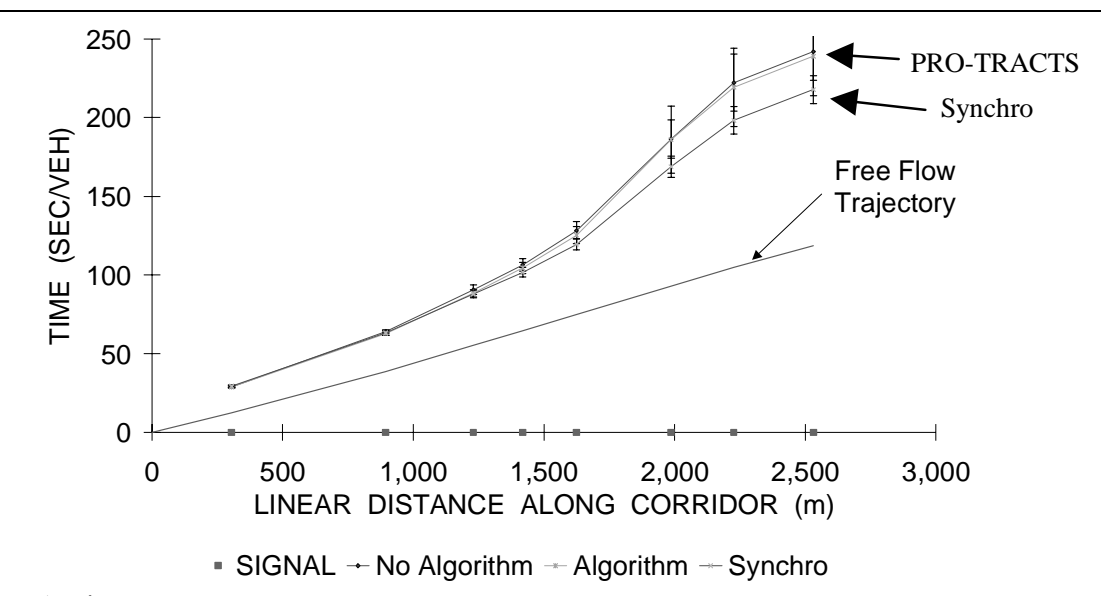

a) First Hour

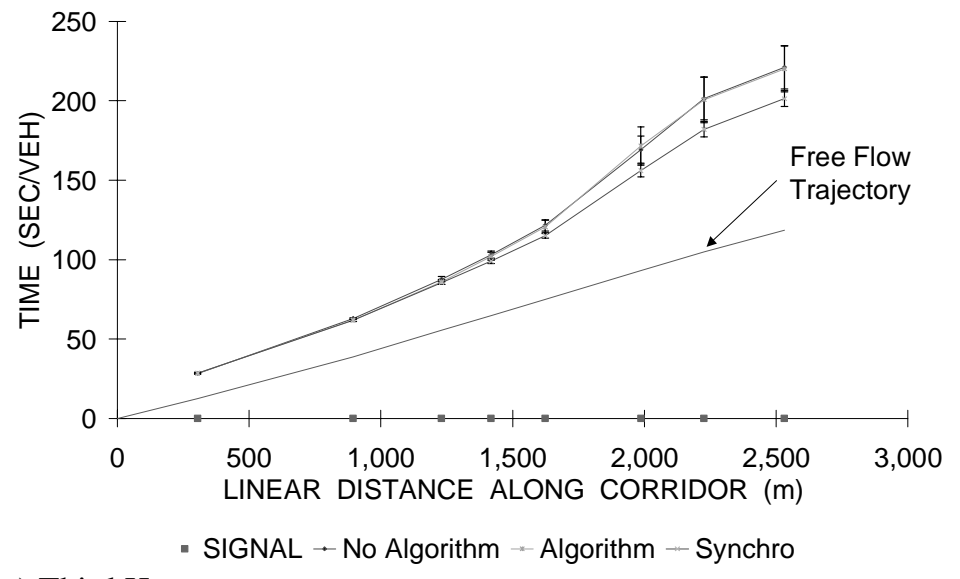

c) Third Hour

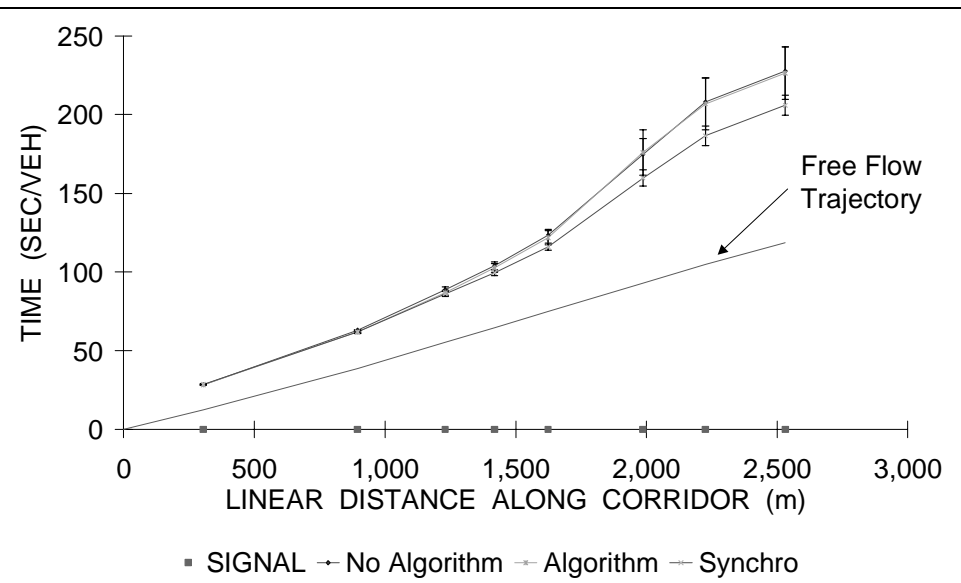

b) Second Hour

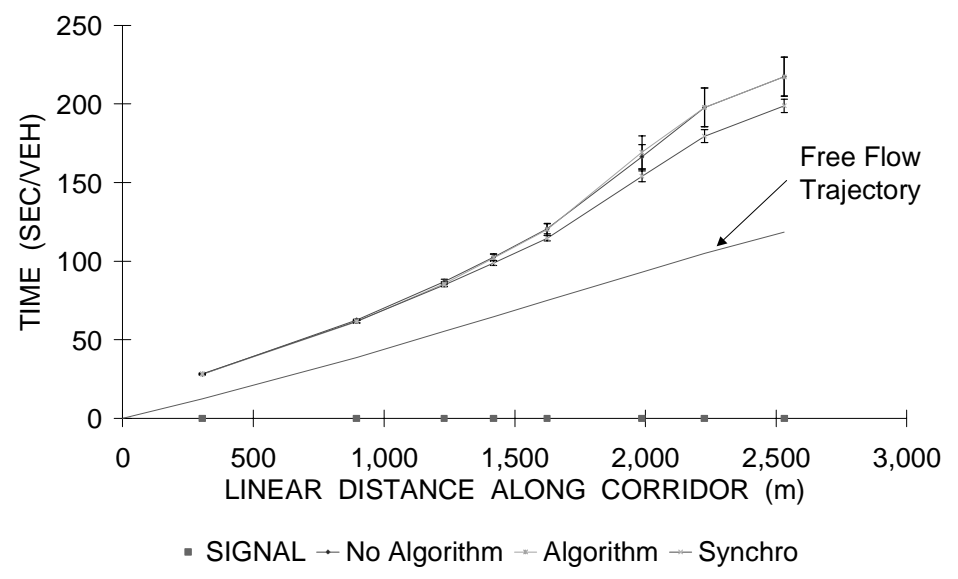

d) Fourth Hour

Figure 8-3 SR 26 (South) West Bound Cumulative Travel Time-A.M. Period 


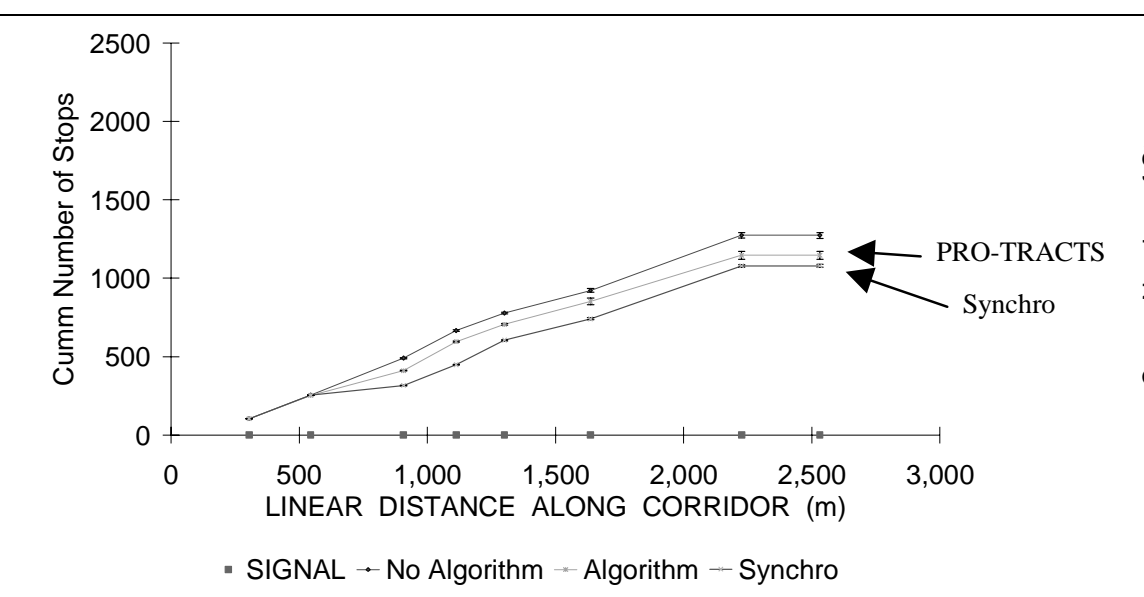

a) First Hour

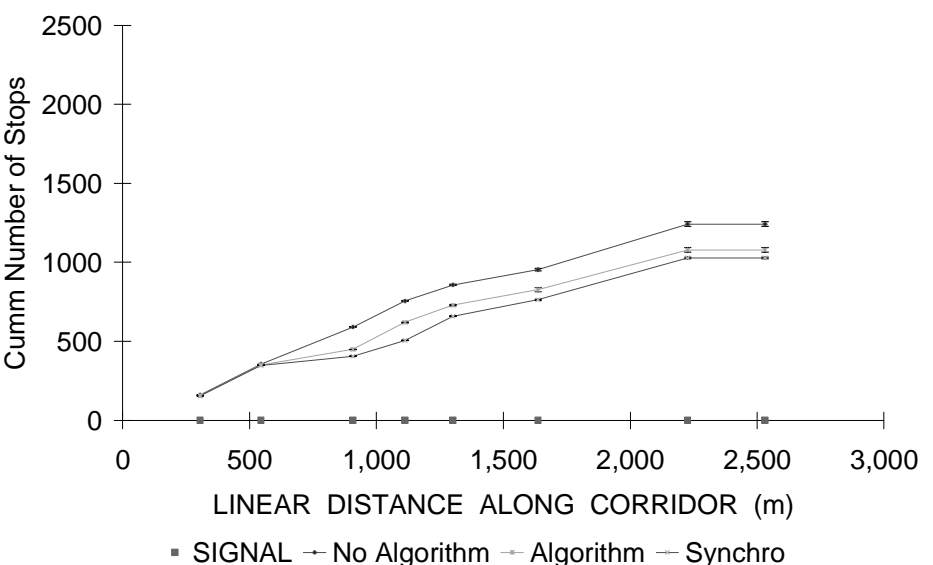

c) Third Hour

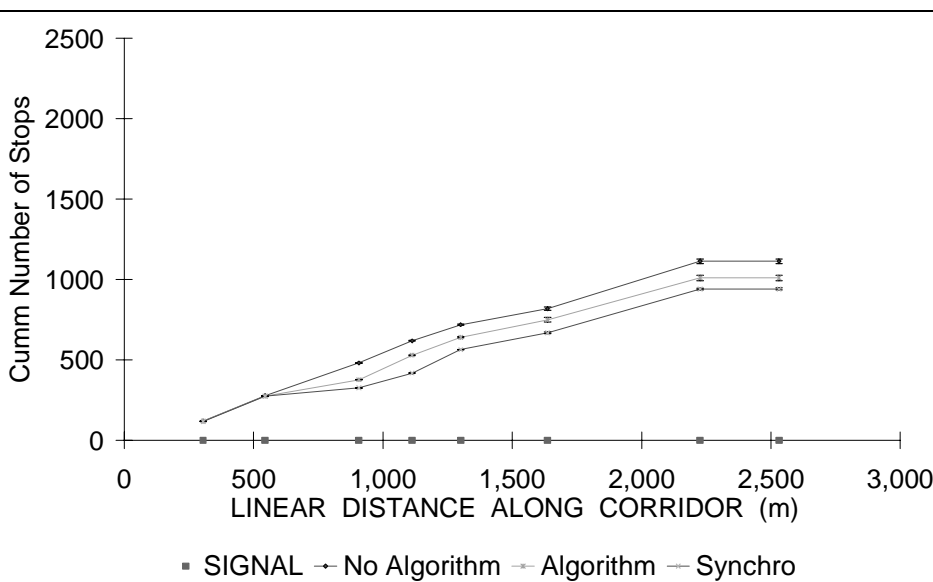

b) Second Hour

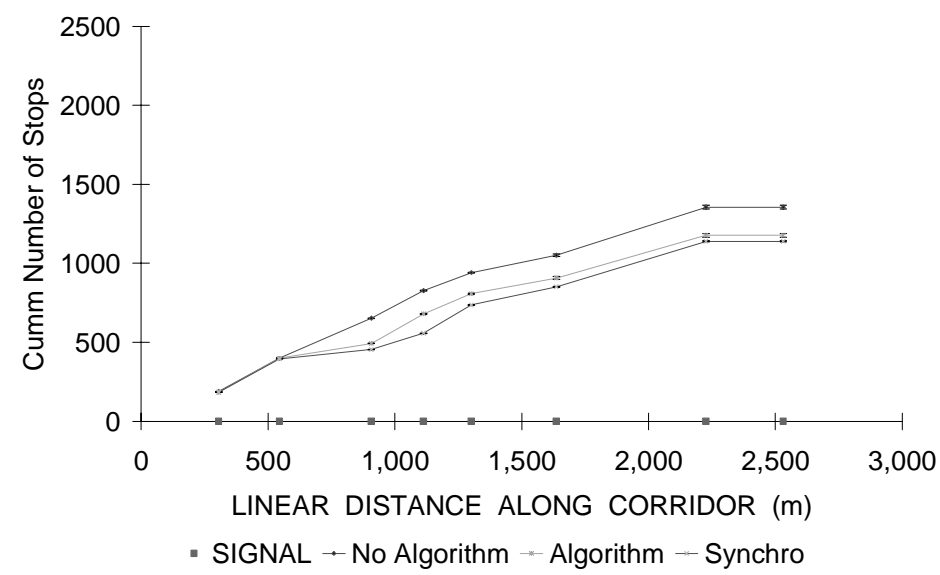

d) Fourth Hour

Figure 8-4 SR 26 (South) East Bound Cumulative Number of Stops-A.M. Period 

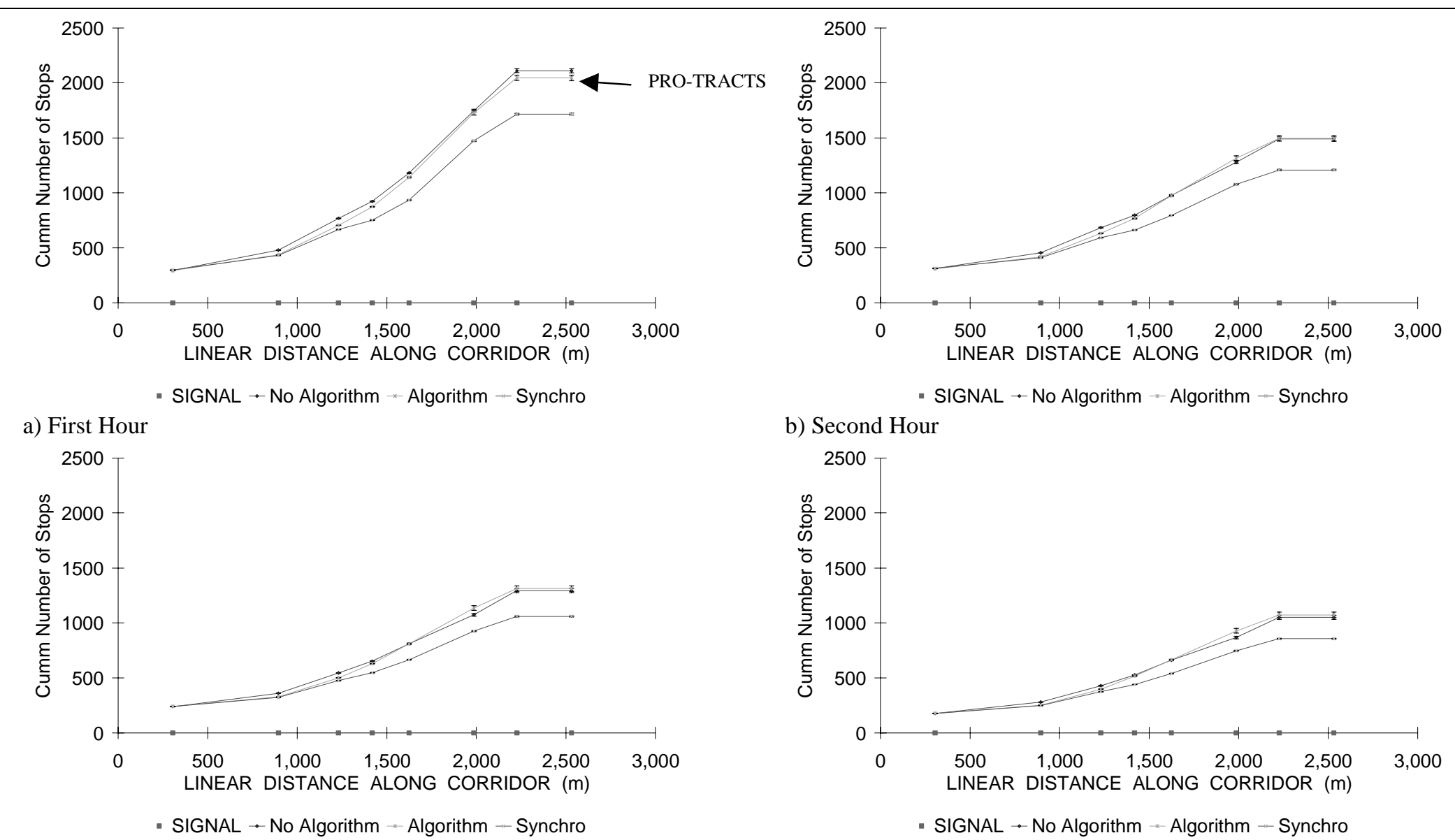

c) Third Hour

b) Second Hour

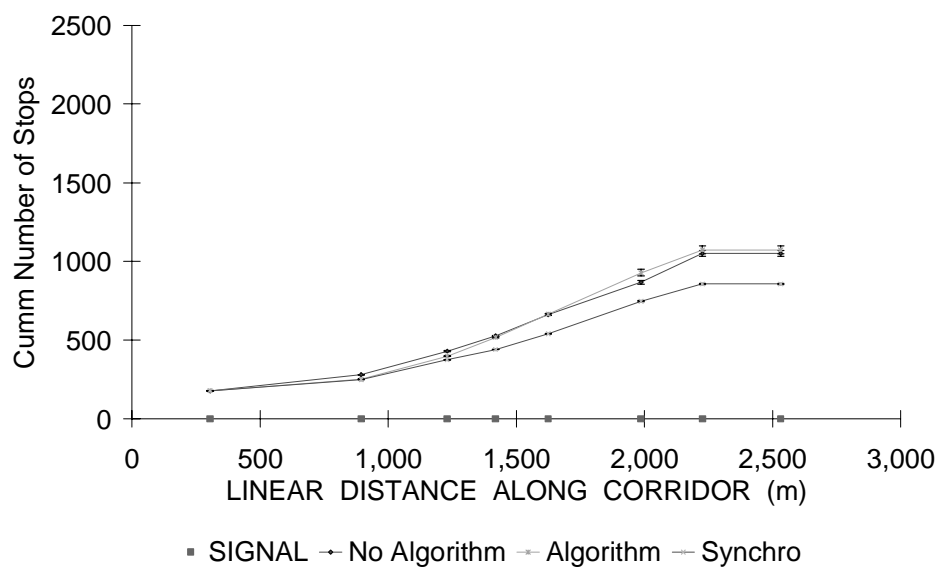

d) Fourth Hour

Figure 8-5 SR 26 (South) West Bound Cumulative Number of Stops-A.M. Period 


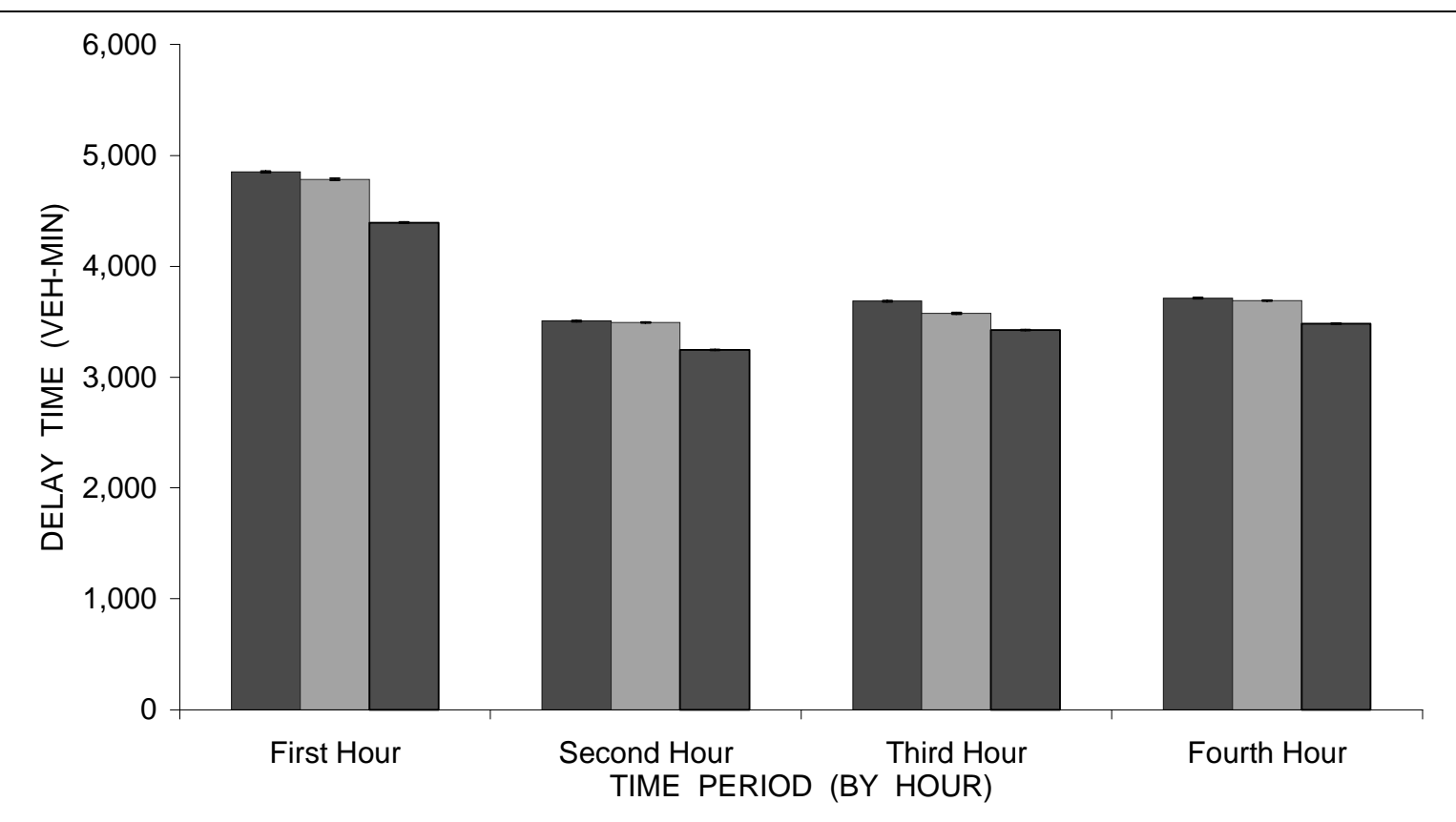

$\square$ No Algorithm $\square$ Algorithm $\square$ Synchro

a) Total Delay Time

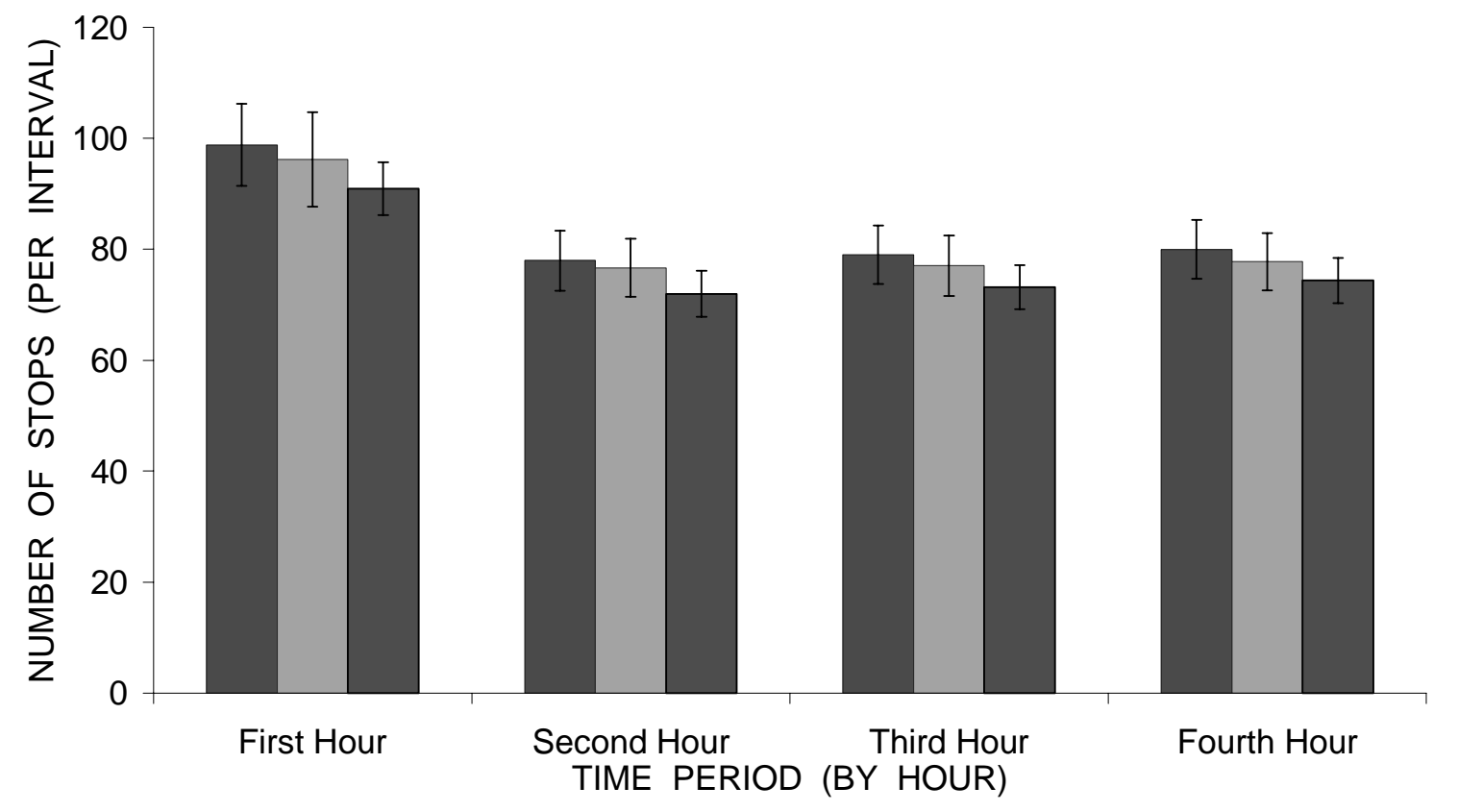

$\square$ No Algorithm $\square$ Algorithm $\square$ Synchro

b) Total Number of Stops

Figure 8-6 SR 26 (South) Total Delay and Number of Stops-A.M. Period 


\subsubsection{A.M. Offset and Cycle Transitioning}

Figure 8-7 shows the transitioning in the offsets and cycle length for each of the seven intersections studied. Figure 8-7-a shows the transitioning in the offset values. An interesting pattern is the continuous decrease in node 6's offset during the course of simulation. Studying Figure 8-7-a more closely, one can observe that node 7's offset (the uppermost line from the left) continues to decrease at a lower rate than node 6's offset. The effect of node 6's offset at node 7's offset is caused by PRO-TRACTS coordination logic that references all offsets to their immediate upstream intersection. When the upstream intersection goes through an offset change, all downstream intersections change their offsets accordingly, such that they do not lose coordination. Other nodes show a fairly stable transitioning throughout the course of simulation.

Instability in offset transitioning can be explained by two factors, the first of which is the arrival type at the intersection. For example, looking at node 6's traffic pattern in Table $8-4$, there is a very high turning percentage of traffic into the eastbound (from north bound right and southbound left). This high percentage translates into a continuous arrival of vehicles at node 7 , both from the through and turn traffic from node 6. This phenomenon negatively affects the benefit of coordinating node 7 with node 6 .

The other factor is the low traffic volume at nodes 3,5 , and 6 side streets that can be observed by studying Table 8-2. Low side street volumes cause side street phases to be skipped, allowing the eastbound traffic to continue through the extended green and arrive with the downstream signal at a different pattern than if the side street phases were not skipped. This situation is illustrated in Figure 8-8 and Figure 8-9. Figure 8-8 shows three 
cycles, where the side street phase is skipped during the first cycle but not during the second cycle for node 5. During the first cycle, the low traffic volume turning from the side street at node 4 (traffic condition A) arrives at node 5 during the red interval and waits for the main street green interval to be released. At that time, the main street through-movement (traffic condition B) arrives and the two traffic streams travel together to reach node 6 at the start of the green. Note that in such a case, the offset at node 6 does not need to be changed. However, during the following cycle, the low traffic volume at node 5 causes a phase skip, allowing the low traffic volume (traffic condition A) turning from the side street to continue through the uninterrupted green and arrive at the downstream node 6 earlier than it did in the previous cycle. When the main street through-movement (traffic condition B) arrives at the downstream node 6, it faces volume A queues, forming a different traffic pattern from that of the previous cycle. If the green window at node 6 was wide enough to fit the new traffic pattern without inducing delays, PRO-TRACTS would shift the offset such that the queues of traffic condition A would pass before the arrival of traffic condition B, as shown in Figure 8-9.

Since PRO-TRACTS is a reactive algorithm, it adjusts the offset such that it adapts to the current cycle's pattern. If the side street phases were not skipped at the next cycle, the offsets would not be appropriate and PRO-TRACTS would adjust them accordingly. This process will cause PRO-TRACTS to fluctuate around the optimum value of the offset without reaching it, which can degrade its performance. The phase skips can be observed by studying Figure 8-7, which shows cycle lengths approaching up to 360 seconds at node 5 . 
Actual Offset Transitioning

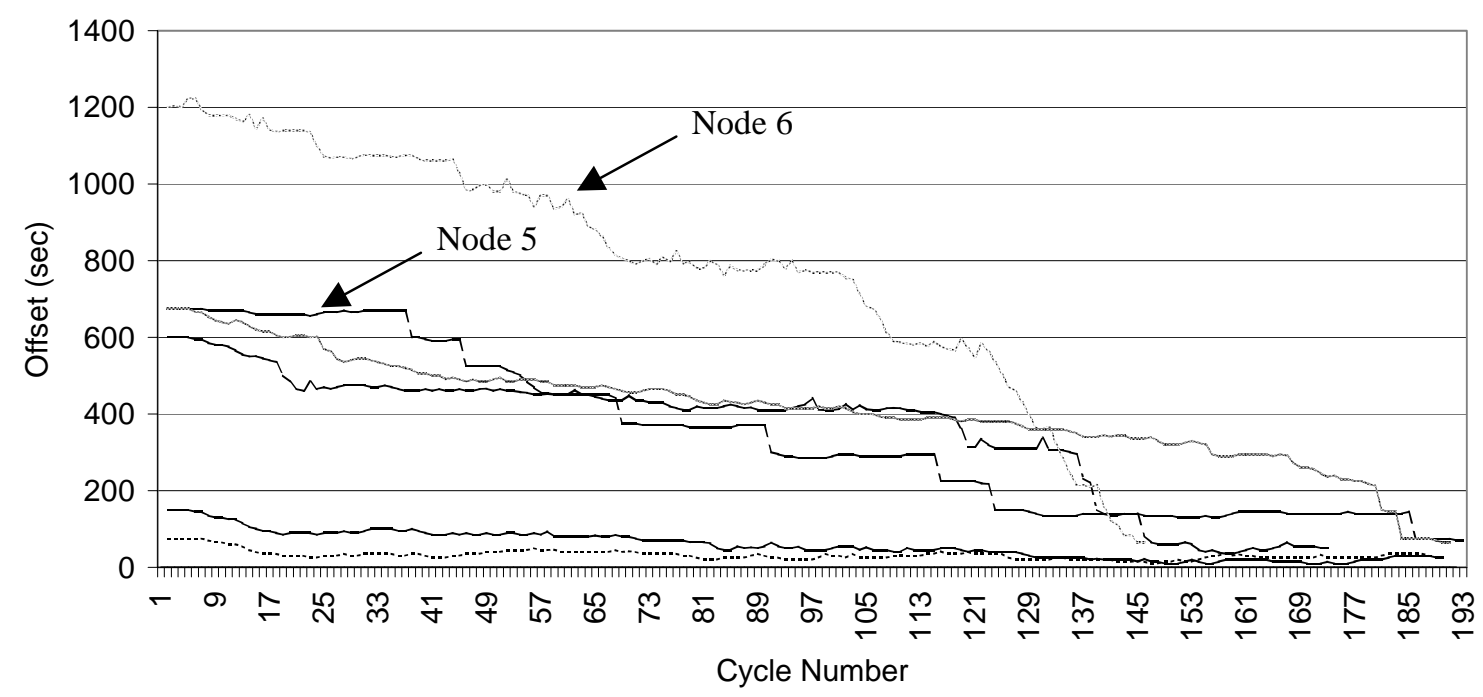

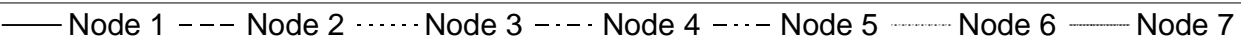

a) Offset Transitioning

\section{Cycle Transitioning}

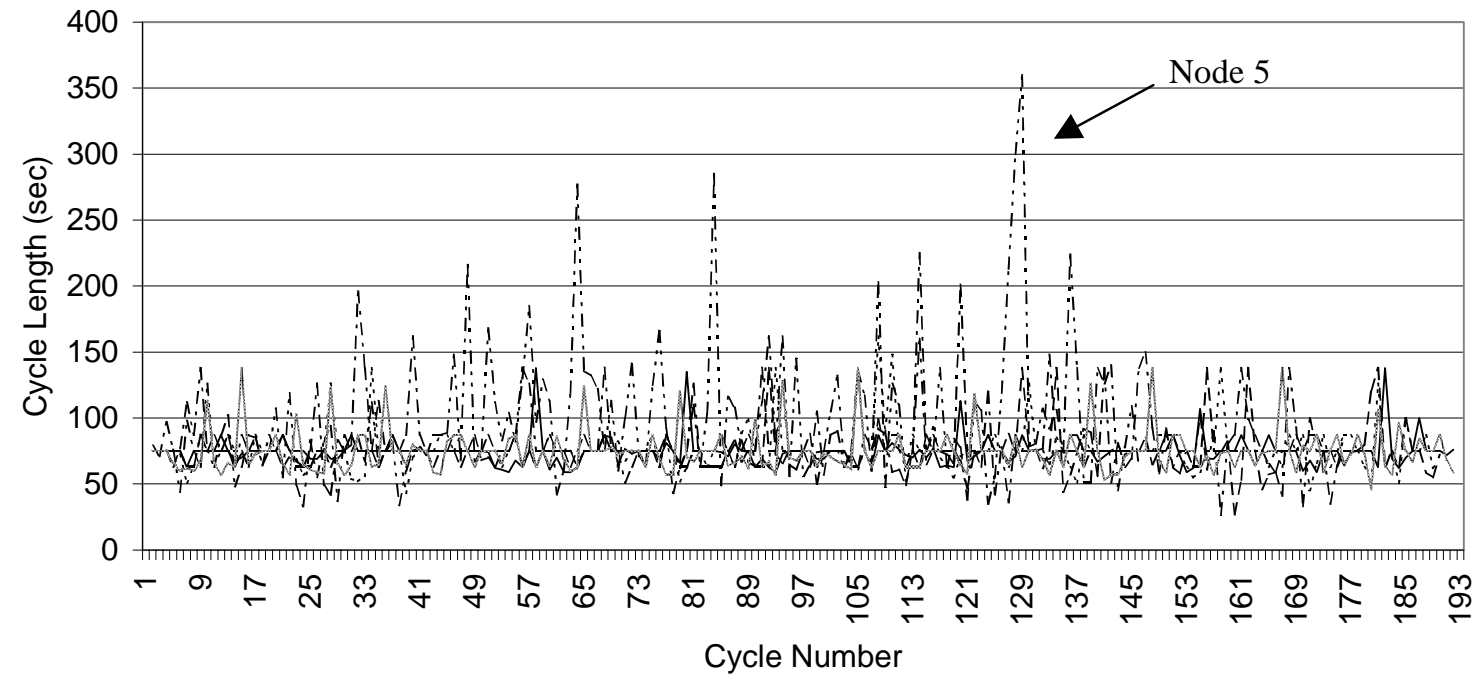

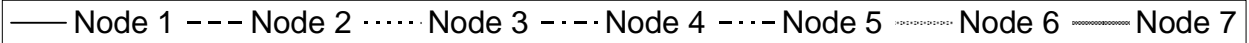

b) Cycle Transitioning

Figure 8-7 SR 26 (South) Offset and Cycle Transitions-A.M. Period 
Table 8-3 SR 26 (South) Turning Movements (Percentages - Table 1 of 2)

\begin{tabular}{|c|c|c|c|c|c|c|c|c|c|c|c|c|c|}
\hline INT & $\begin{array}{l}\text { BEG } \\
\text { END }\end{array}$ & $\begin{array}{l}7 \mathrm{AM} \\
8 \mathrm{AM}\end{array}$ & $\begin{array}{l}8 \mathrm{AM} \\
9 \mathrm{AM}\end{array}$ & $\begin{array}{c}9 \mathrm{AM} \\
10 \mathrm{~A}\end{array}$ & $\begin{array}{l}10 \mathrm{~A} \\
11 \mathrm{~A}\end{array}$ & $\begin{array}{l}11 \mathrm{~A} \\
12 \mathrm{P}\end{array}$ & $\begin{array}{c}12 \mathrm{P} \\
1 \mathrm{PM}\end{array}$ & $\begin{array}{l}1 \mathrm{PM} \\
2 \mathrm{PM}\end{array}$ & $\begin{array}{l}2 \mathrm{PM} \\
3 \mathrm{PM}\end{array}$ & $\begin{array}{l}3 \mathrm{PM} \\
4 \mathrm{PM}\end{array}$ & $\begin{array}{l}4 \mathrm{PM} \\
5 \mathrm{PM}\end{array}$ & $\begin{array}{l}5 \mathrm{PM} \\
6 \mathrm{PM}\end{array}$ & $\begin{array}{l}6 \mathrm{PM} \\
7 \mathrm{PM}\end{array}$ \\
\hline \multirow{12}{*}{ 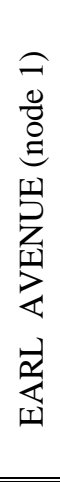 } & EBLT & 13 & 11 & 10 & 10 & 6 & 10 & 9 & 7 & 7 & 5 & 6 & 5 \\
\hline & EBTH & 81 & 82 & 82 & 82 & 85 & 83 & 83 & 84 & 85 & 90 & 87 & 92 \\
\hline & EBRT & 6 & 7 & 8 & 8 & 9 & 7 & 8 & 9 & 8 & 5 & 7 & 3 \\
\hline & WBLT & 13 & 14 & 12 & 14 & 15 & 18 & 14 & 15 & 16 & 16 & 17 & 19 \\
\hline & WBTH & 70 & 68 & 73 & 68 & 67 & 65 & 68 & 69 & 71 & 68 & 70 & 72 \\
\hline & WBRT & 17 & 18 & 15 & 18 & 18 & 17 & 18 & 16 & 13 & 16 & 13 & 9 \\
\hline & NBLT & 5 & 5 & 15 & 10 & 6 & 7 & 7 & 7 & 9 & 6 & 6 & 7 \\
\hline & NBTH & 69 & 73 & 66 & 66 & 69 & 70 & 71 & 67 & 68 & 71 & 69 & 69 \\
\hline & NBRT & 26 & 22 & 19 & 24 & 25 & 23 & 22 & 26 & 23 & 23 & 25 & 24 \\
\hline & SBLT & 23 & 30 & 29 & 34 & 35 & 34 & 27 & 33 & 32 & 31 & 31 & 31 \\
\hline & SBTH & 72 & 65 & 60 & 60 & 60 & 62 & 67 & 59 & 64 & 64 & 65 & 59 \\
\hline & SBRT & 5 & 5 & 11 & 6 & 5 & 4 & 6 & 8 & 4 & 5 & 4 & 10 \\
\hline \multirow{12}{*}{ 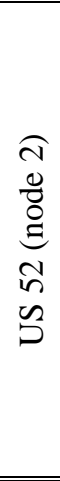 } & EBLT & 19 & 20 & 18 & 16 & 18 & 18 & 19 & 18 & 19 & 16 & 16 & 14 \\
\hline & EBTH & 70 & 67 & 68 & 65 & 62 & 62 & 60 & 62 & 62 & 64 & 69 & 67 \\
\hline & EBRT & 11 & 13 & 14 & 19 & 20 & 20 & 21 & 20 & 19 & 20 & 15 & 19 \\
\hline & WBLT & 20 & 24 & 22 & 27 & 27 & 27 & 26 & 28 & 27 & 24 & 24 & 28 \\
\hline & WBTH & 57 & 54 & 55 & 49 & 48 & 48 & 51 & 50 & 47 & 51 & 55 & 51 \\
\hline & WBRT & 23 & 22 & 23 & 24 & 25 & 25 & 23 & 22 & 26 & 25 & 21 & 21 \\
\hline & NBLT & 9 & 11 & 12 & 12 & 13 & 13 & 15 & 14 & 12 & 13 & 11 & 12 \\
\hline & NBTH & 72 & 65 & 68 & 69 & 63 & 62 & 62 & 66 & 69 & 67 & 67 & 63 \\
\hline & NBRT & 19 & 24 & 20 & 19 & 24 & 25 & 23 & 20 & 19 & 20 & 22 & 25 \\
\hline & SBLT & 20 & 20 & 22 & 20 & 27 & 25 & 25 & 22 & 22 & 22 & 25 & 27 \\
\hline & SBTH & 63 & 66 & 66 & 67 & 60 & 62 & 65 & 67 & 69 & 68 & 65 & 64 \\
\hline & SBRT & 17 & 14 & 12 & 13 & 13 & 13 & 10 & 11 & 9 & 10 & 10 & 9 \\
\hline \multirow{12}{*}{ 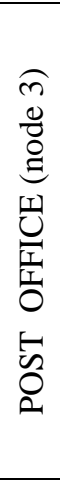 } & EBLT & 19 & 14 & 10 & 11 & 9 & 11 & 15 & 14 & 15 & 14 & 13 & 10 \\
\hline & EBTH & 80 & 85 & 88 & 87 & 84 & 82 & 83 & 83 & 83 & 83 & 84 & 87 \\
\hline & EBRT & 1 & 1 & 2 & 2 & 7 & 7 & 2 & 3 & 2 & 3 & 3 & 3 \\
\hline & WBLT & 1 & 1 & 1 & 1 & 6 & 5 & 2 & 1 & 0 & 2 & 3 & 5 \\
\hline & WBTH & 85 & 88 & 87 & 89 & 83 & 85 & 82 & 85 & 84 & 79 & 81 & 80 \\
\hline & WBRT & 14 & 11 & 12 & 10 & 11 & 10 & 16 & 14 & 16 & 19 & 16 & 15 \\
\hline & NBLT & 55 & 17 & 35 & 80 & 60 & 48 & 42 & 38 & 33 & 32 & 45 & 13 \\
\hline & NBTH & 9 & 0 & 10 & 0 & 0 & 4 & 2 & 4 & 9 & 4 & 10 & 3 \\
\hline & NBRT & 36 & 83 & 55 & 20 & 40 & 48 & 56 & 58 & 58 & 64 & 45 & 84 \\
\hline & SBLT & 30 & 35 & 31 & 34 & 25 & 33 & 43 & 42 & 50 & 47 & 44 & 55 \\
\hline & SBTH & 0 & 0 & 0 & 1 & 1 & 2 & 3 & 0 & 1 & 0 & 1 & 2 \\
\hline & SBRT & 70 & 65 & 69 & 65 & 74 & 65 & 54 & 58 & 49 & 53 & 55 & 43 \\
\hline \multirow{12}{*}{ 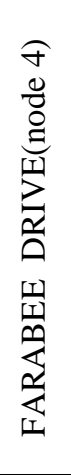 } & EBLT & 3 & 6 & 8 & 8 & 9 & 9 & 8 & 9 & 9 & 7 & 5 & 7 \\
\hline & EBTH & 86 & 85 & 84 & 85 & 85 & 85 & 85 & 85 & 84 & 87 & 90 & 89 \\
\hline & EBRT & 11 & 9 & 8 & 7 & 6 & 6 & 7 & 6 & 7 & 6 & 5 & 4 \\
\hline & WBLT & 18 & 14 & 11 & 12 & 12 & 12 & 13 & 13 & 17 & 12 & 13 & 9 \\
\hline & WBTH & 81 & 82 & 84 & 81 & 81 & 83 & 81 & 81 & 78 & 80 & 80 & 85 \\
\hline & WBRT & 1 & 4 & 5 & 7 & 7 & 5 & 6 & 6 & 5 & 8 & 7 & 6 \\
\hline & NBLT & 17 & 15 & 21 & 17 & 17 & 19 & 23 & 20 & 13 & 19 & 18 & 16 \\
\hline & NBTH & 7 & 8 & 10 & 15 & 12 & 13 & 11 & 18 & 9 & 11 & 8 & 15 \\
\hline & NBRT & 76 & 77 & 69 & 68 & 71 & 68 & 66 & 62 & 78 & 70 & 74 & 69 \\
\hline & SBLT & 4 & 17 & 16 & 29 & 25 & 35 & 29 & 26 & 31 & 31 & 26 & 35 \\
\hline & SBTH & 53 & 34 & 22 & 21 & 16 & 17 & 19 & 18 & 17 & 19 & 28 & 13 \\
\hline & SBRT & 43 & 49 & 62 & 50 & 29 & 48 & 52 & 56 & 52 & 50 & 46 & 52 \\
\hline
\end{tabular}


Table 8-4 SR 26 (South) Turning Movements (Percentages -Table 2 of 2)

\begin{tabular}{|c|c|c|c|c|c|c|c|c|c|c|c|c|c|}
\hline INT & $\begin{array}{l}\text { BEG } \\
\text { END }\end{array}$ & $\begin{array}{l}\text { 7AM } \\
8 \mathrm{AM}\end{array}$ & $\begin{array}{l}\text { 8AM } \\
9 \mathrm{AM}\end{array}$ & $\begin{array}{c}9 \mathrm{AM} \\
10 \mathrm{~A}\end{array}$ & $\begin{array}{l}10 \mathrm{~A} \\
11 \mathrm{~A}\end{array}$ & $\begin{array}{l}11 \mathrm{~A} \\
12 \mathrm{P}\end{array}$ & $\begin{array}{l}12 \mathrm{P} \\
1 \mathrm{PM}\end{array}$ & $\begin{array}{l}1 \mathrm{PM} \\
2 \mathrm{PM}\end{array}$ & $\begin{array}{l}2 \mathrm{PM} \\
3 \mathrm{PM}\end{array}$ & $\begin{array}{l}3 \mathrm{PM} \\
4 \mathrm{PM}\end{array}$ & $\begin{array}{l}4 \mathrm{PM} \\
5 \mathrm{PM}\end{array}$ & $\begin{array}{l}5 \mathrm{PM} \\
6 \mathrm{PM}\end{array}$ & $\begin{array}{l}6 \mathrm{PM} \\
7 \mathrm{PM}\end{array}$ \\
\hline \multirow{12}{*}{ 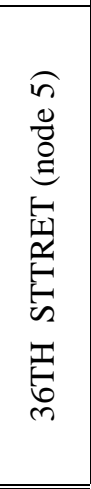 } & EBLT & 13 & 8 & 9 & 7 & 6 & 6 & 6 & 6 & 7 & 6 & 5 & 5 \\
\hline & EBTH & 86 & 90 & 90 & 92 & 94 & 93 & 93 & 94 & 93 & 93 & 94 & 95 \\
\hline & EBRT & 1 & 2 & 1 & 1 & 0 & 1 & 1 & 0 & 0 & 1 & 1 & 0 \\
\hline & WBLT & 1 & 1 & 1 & 1 & 1 & 1 & 1 & 1 & 1 & 0 & 0 & 0 \\
\hline & WBTH & 90 & 89 & 94 & 92 & 93 & 91 & 92 & 92 & 92 & 93 & 93 & 95 \\
\hline & WBRT & 9 & 10 & 5 & 7 & 6 & 8 & 7 & 7 & 7 & 7 & 7 & 5 \\
\hline & NBLT & 90 & 63 & 44 & 65 & 66 & 63 & 56 & 69 & 61 & 69 & 54 & 39 \\
\hline & NBTH & 2 & 17 & 6 & 16 & 6 & 20 & 4 & 13 & 20 & 8 & 25 & 4 \\
\hline & NBRT & 8 & 20 & 50 & 19 & 28 & 17 & 40 & 18 & 19 & 23 & 21 & 57 \\
\hline & SBLT & 48 & 42 & 35 & 35 & 45 & 55 & 39 & 53 & 45 & 51 & 63 & 56 \\
\hline & SBTH & 1 & 2 & 2 & 0 & 1 & 1 & 2 & 2 & 1 & 1 & 0 & 0 \\
\hline & SBRT & 51 & 56 & 63 & 65 & 54 & 44 & 59 & 45 & 54 & 48 & 37 & 44 \\
\hline \multirow{12}{*}{ 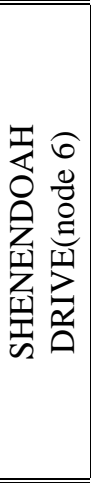 } & & 4 & 4 & 5 & 6 & 5 & 6 & 5 & 5 & 5 & 6 & 6 & 7 \\
\hline & EBTH & 96 & 95 & 94 & 94 & 95 & 93 & 94 & 95 & 95 & 94 & 94 & 93 \\
\hline & EBRT & 0 & 1 & 1 & 0 & 0 & 1 & 1 & 0 & 0 & 0 & 0 & 0 \\
\hline & WBLT & 10 & 3 & 2 & 1 & 2 & 1 & 1 & 2 & 5 & 1 & 1 & 0 \\
\hline & WBTH & 89 & 94 & 94 & 94 & 94 & 94 & 94 & 94 & 91 & 95 & 93 & 92 \\
\hline & WBRT & 1 & 3 & 4 & 5 & 4 & 5 & 5 & 4 & 4 & 4 & 6 & 8 \\
\hline & NBLT & 7 & 22 & 18 & 38 & 13 & 17 & 23 & 9 & 7 & 7 & 12 & 10 \\
\hline & NBTH & 10 & 8 & 9 & 12 & 16 & 16 & 13 & 9 & 6 & 10 & 12 & 9 \\
\hline & NBRT & 83 & 70 & 73 & 50 & 71 & 67 & 64 & 82 & 87 & 83 & 76 & 81 \\
\hline & SBLT & 15 & 20 & 32 & 33 & 27 & 37 & 33 & 25 & 38 & 45 & 49 & 49 \\
\hline & SBTH & 16 & 7 & 1 & 1 & 0 & 2 & 1 & 1 & 1 & 2 & 1 & 0 \\
\hline & SBRT & 69 & 73 & 67 & 66 & 73 & 61 & 66 & 74 & 61 & 53 & 50 & 51 \\
\hline \multirow{12}{*}{ 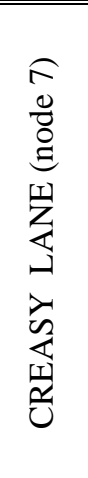 } & & 6 & 8 & 9 & 8 & 7 & 9 & 8 & 8 & 7 & 9 & 7 & 9 \\
\hline & EBTH & 69 & 70 & 63 & 63 & 63 & 61 & 63 & 64 & 67 & 62 & 67 & 65 \\
\hline & EBRT & 25 & 22 & 28 & 29 & 30 & 30 & 29 & 28 & 26 & 29 & 26 & 26 \\
\hline & WBLT & 14 & 13 & 19 & 18 & 21 & 19 & 20 & 20 & 17 & 18 & 18 & 20 \\
\hline & WBTH & 65 & 61 & 59 & 57 & 56 & 59 & 54 & 55 & 57 & 54 & 52 & 51 \\
\hline & WBRT & 21 & 26 & 22 & 25 & 23 & 22 & 26 & 25 & 26 & 28 & 30 & 29 \\
\hline & NBLT & 25 & 35 & 36 & 36 & 35 & 38 & 35 & 30 & 25 & 28 & 24 & 27 \\
\hline & NBTH & 56 & 48 & 46 & 48 & 46 & 51 & 47 & 50 & 55 & 54 & 56 & 52 \\
\hline & NBRT & 19 & 17 & 18 & 16 & 19 & 11 & 18 & 20 & 20 & 18 & 20 & 21 \\
\hline & SBLT & 35 & 40 & 38 & 34 & 42 & 42 & 41 & 37 & 45 & 42 & 43 & 45 \\
\hline & SBTH & 58 & 51 & 51 & 57 & 49 & 49 & 50 & 54 & 48 & 52 & 52 & 49 \\
\hline & SBRT & 7 & 9 & 11 & 9 & 9 & 9 & 9 & 9 & 7 & 6 & 5 & 6 \\
\hline
\end{tabular}




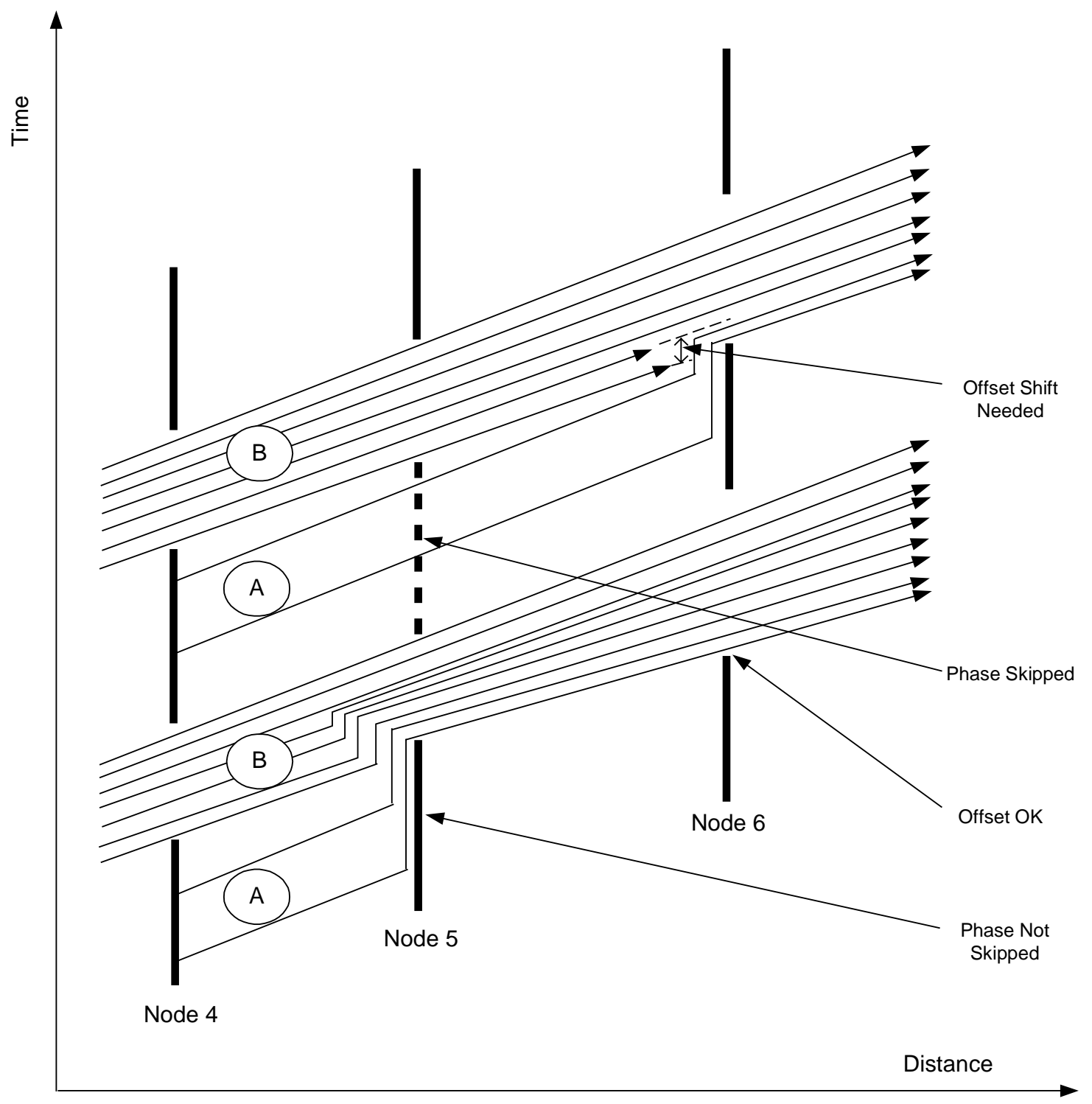

Figure 8-8 Effect of Phase Skips on Progression 


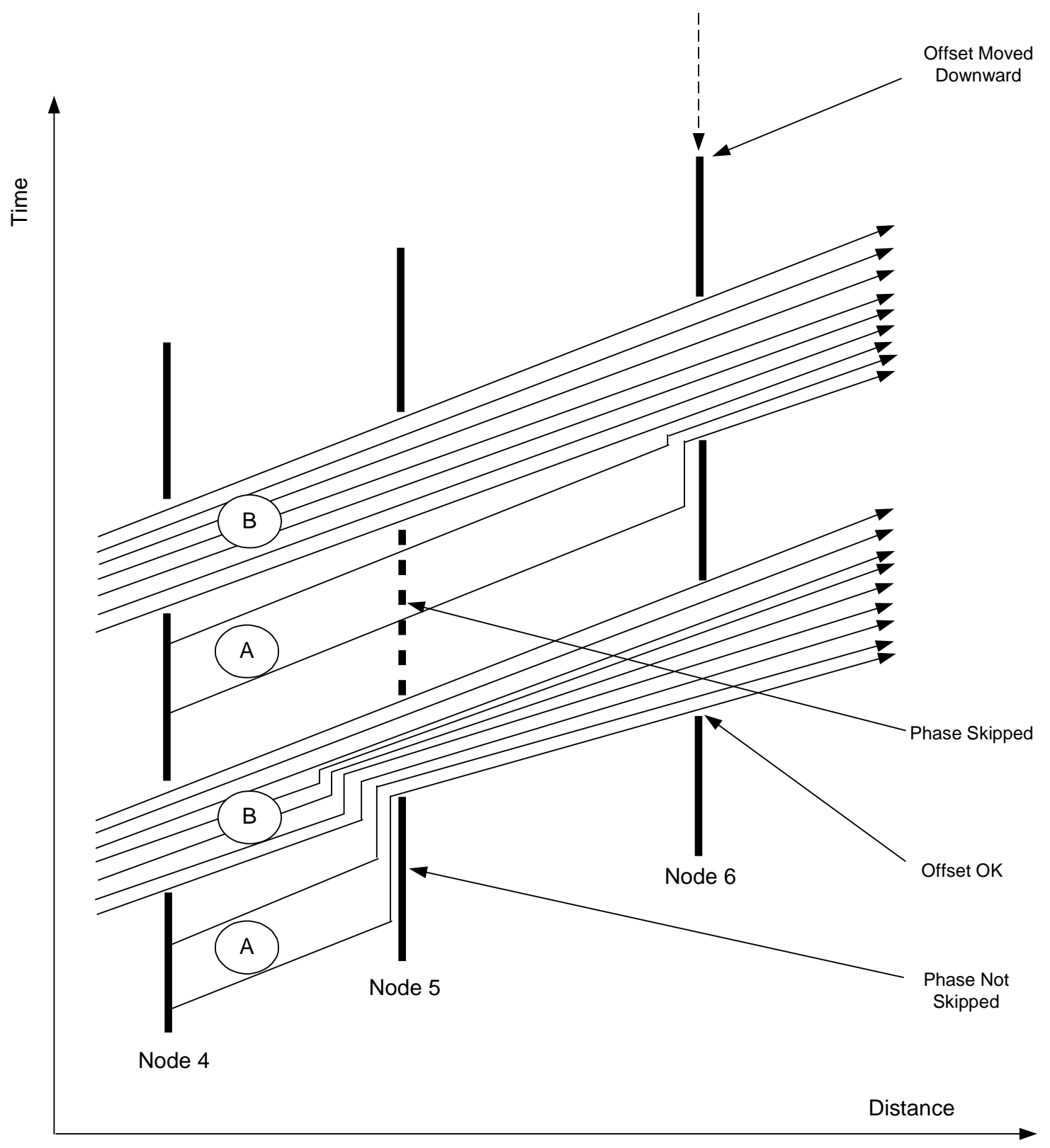

Figure 8-9 Offset Correction for Phase Skips 


\subsubsection{P.M. Travel Time, Stops and Delay}

Figure 8-10 and Figure 8-11 show the cumulative travel time through the arterial for eastbound and westbound, respectively. The figures show a significant reduction of $13 \%, 16 \%, 17 \%$, and $16 \%$ in eastbound cumulative travel time during the four simulation hours and only and increase of $7 \%$ and $8 \%$ in the westbound cumulative travel time during the first hour and the last three hours respectively with PRO-TRACTS, while Synchro plans improved the eastbound travel time by $26 \%, 25 \%, 24 \%$, and $22 \%$ during the four simulation hours respectively and increased the westbound travel time by $3 \%$, $1 \%, 1 \%$, and $2 \%$ during the four simulation hours respectively. Figure 8-12 and Figure 8-13 show the cumulative number of stops on east and west bound respectively. PROTRACTS significantly reduced the cumulative number of stops on the eastbound by $30 \%$, $39 \%, 43 \%$, and $43 \%$ respectively while it only increased the number of stops by $8 \%$ and $6 \%$ for the westbound during the first two hours and the last two hours respectively. These figures illustrate that PRO-TRACTS had higher impacts on the eastbound number of stops, closely approaching Synchro's performance of 50\%, 51\%, 51\%, and $49 \%$ during the hour hours respectively. Figure 8-14 shows the total delay and number of stops on an hour-by-hour basis and indicates a reduction in the total number of stops with PROTRACTS of $7 \%, 9 \%, 12 \%$, and $11 \%$ and an increase in the total delay of $3 \%, 9 \%, 8 \%$, and $2 \%$ during the four hours of simulation. 

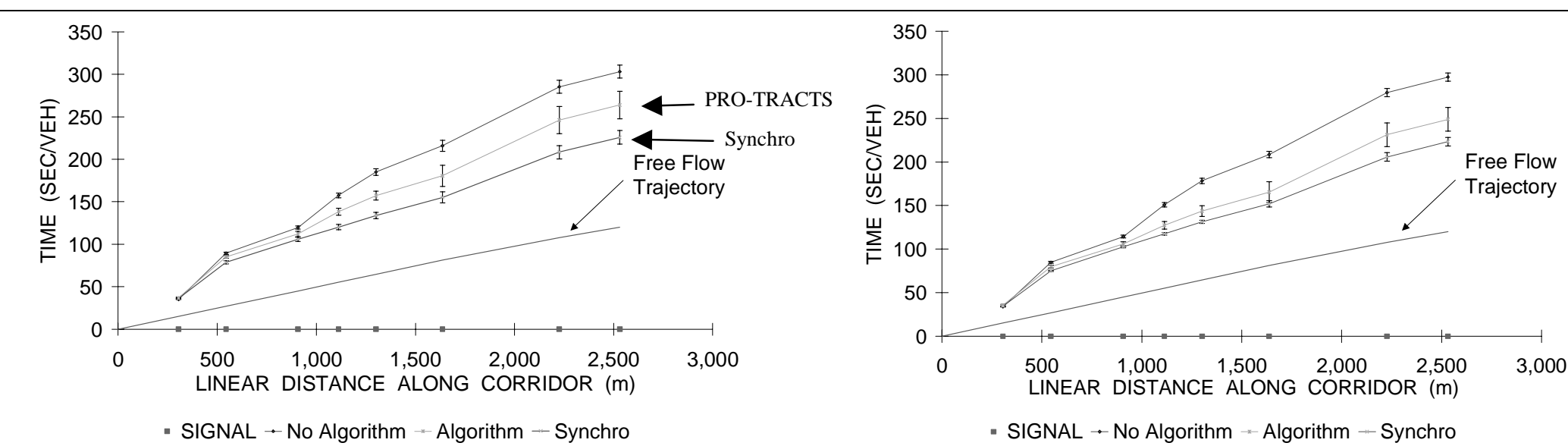

a) First Hour

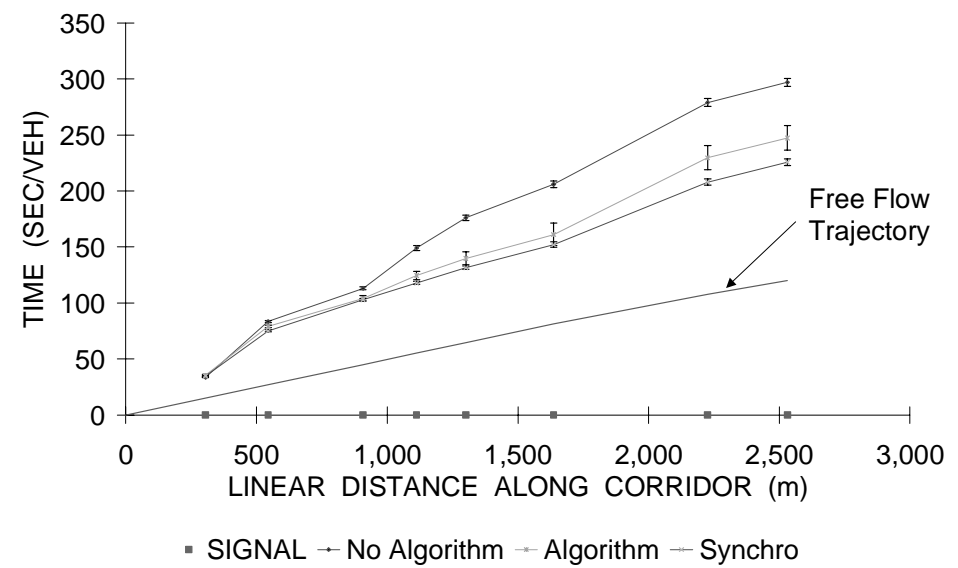

c) Third Hour

b) Second Hour

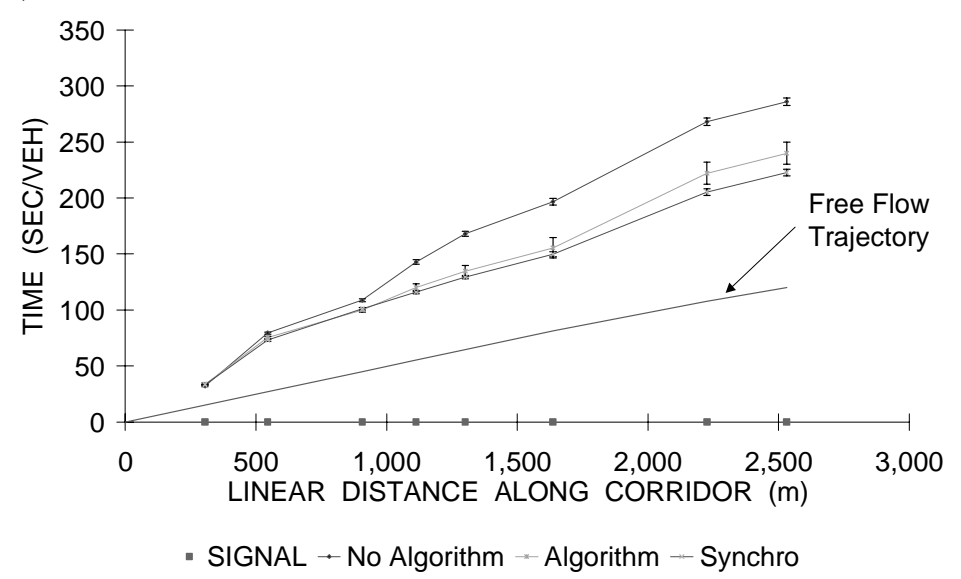

d) Fourth Hour

Figure 8-10 SR 26 (South) East Bound Cumulative Travel Time-P.M. Period 


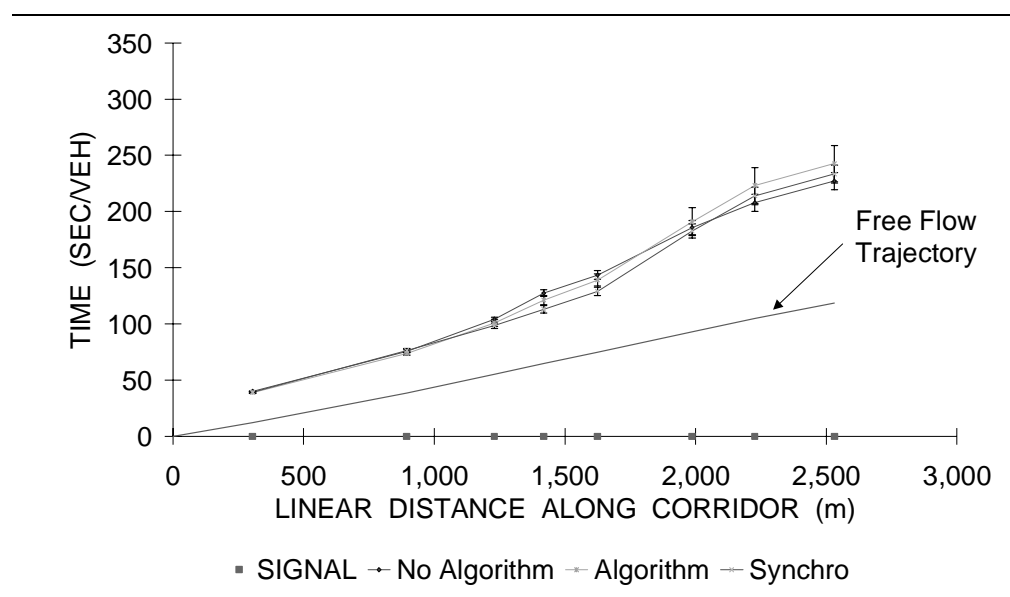

a) First Hour

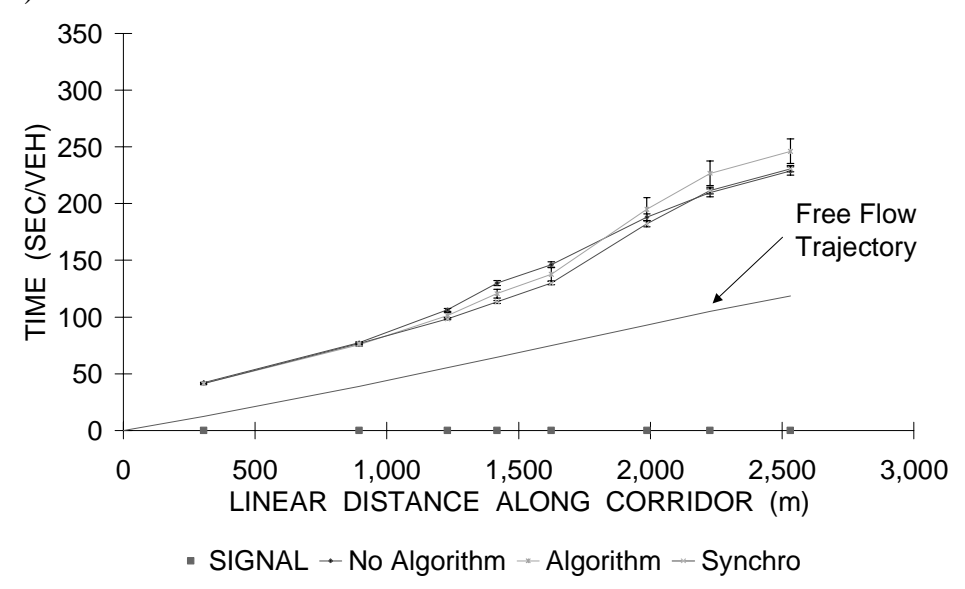

c) Third Hour

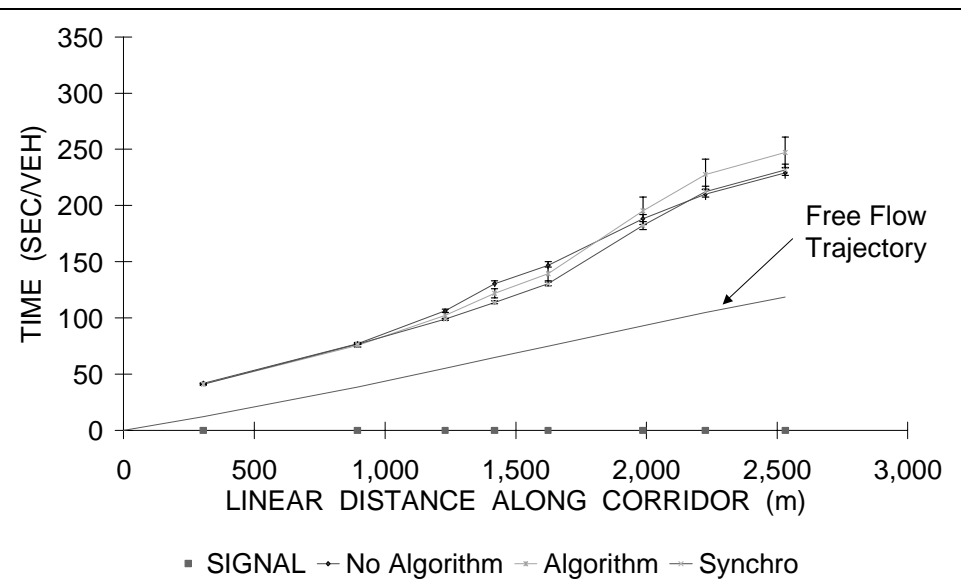

b) Second Hour

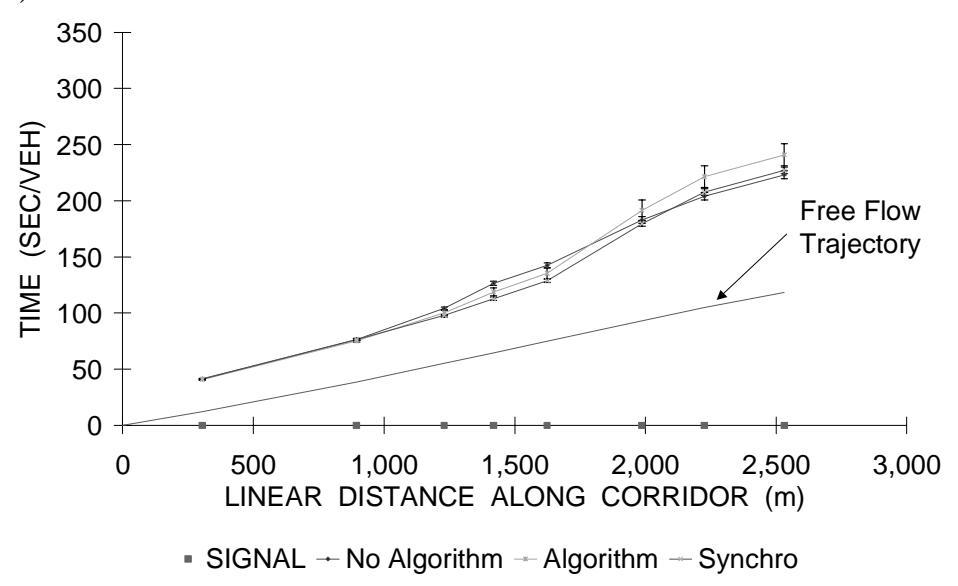

d) Fourth Hour

Figure 8-11 SR 26 (South) West Bound Cumulative Travel Time-P.M. Period 


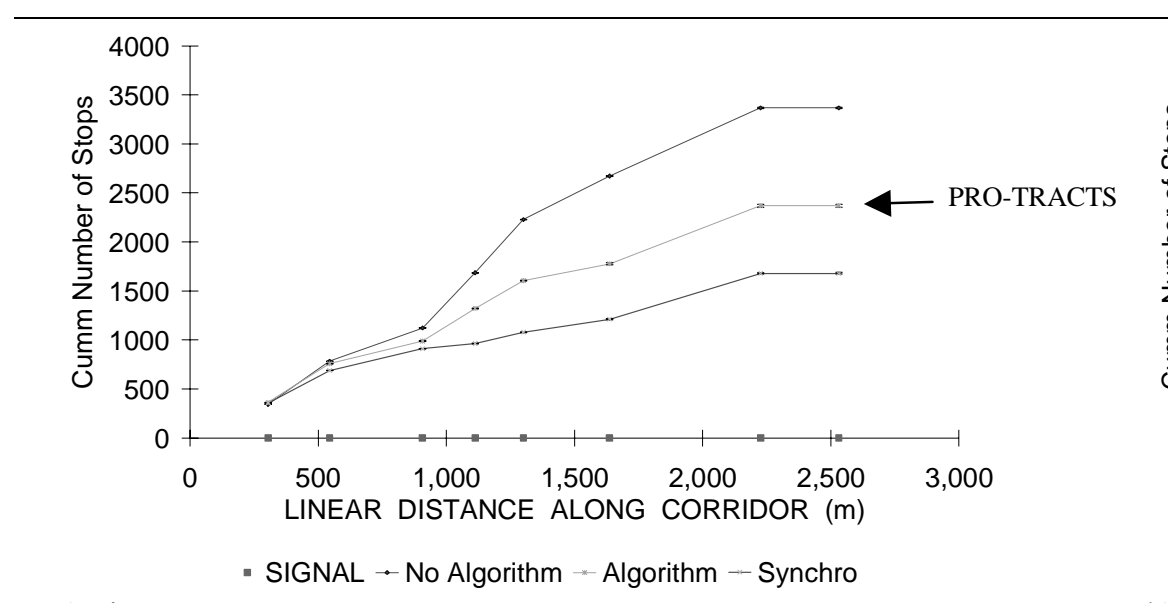

a) First Hour
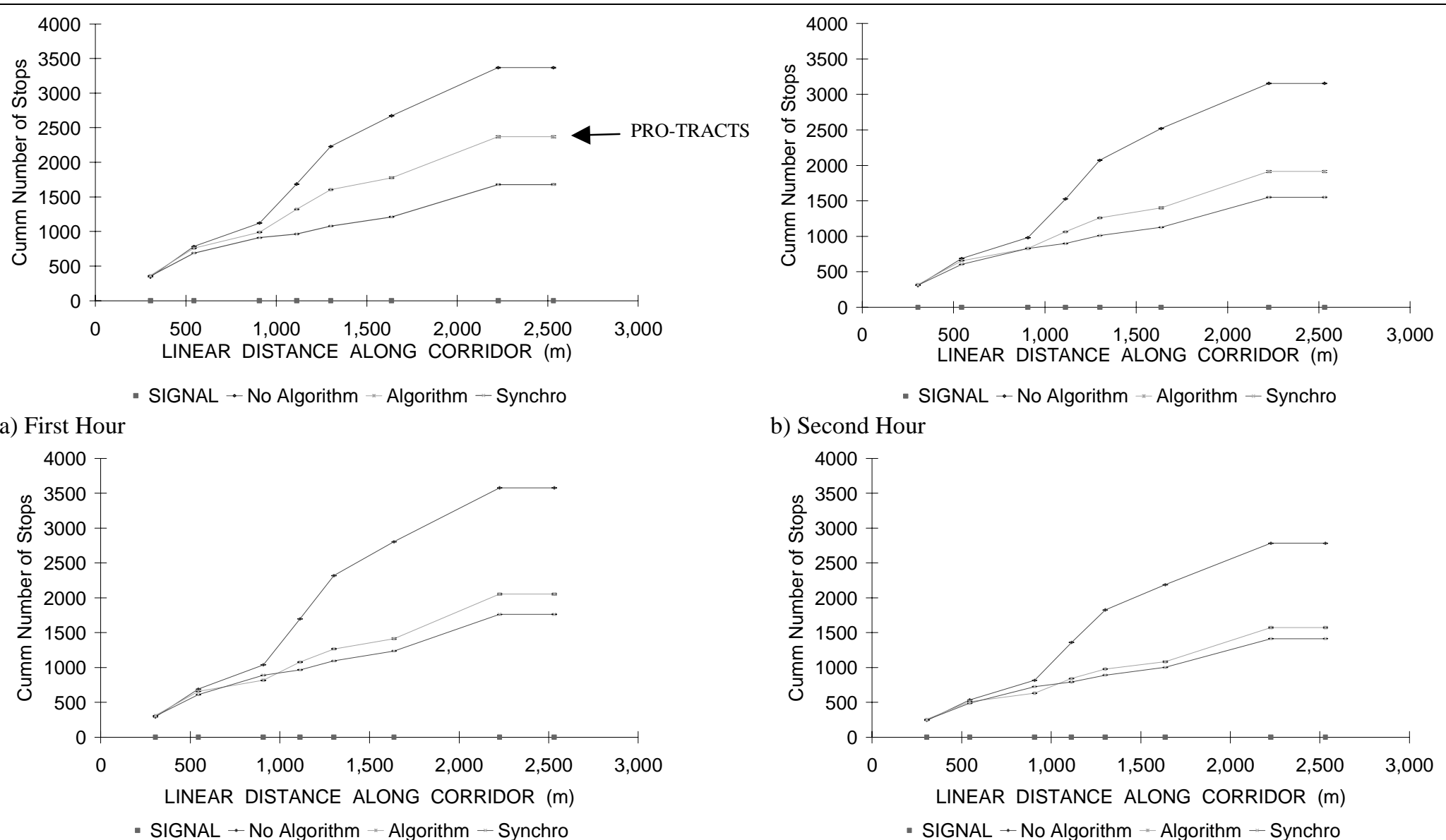

c) Third Hour

b) Second Hour

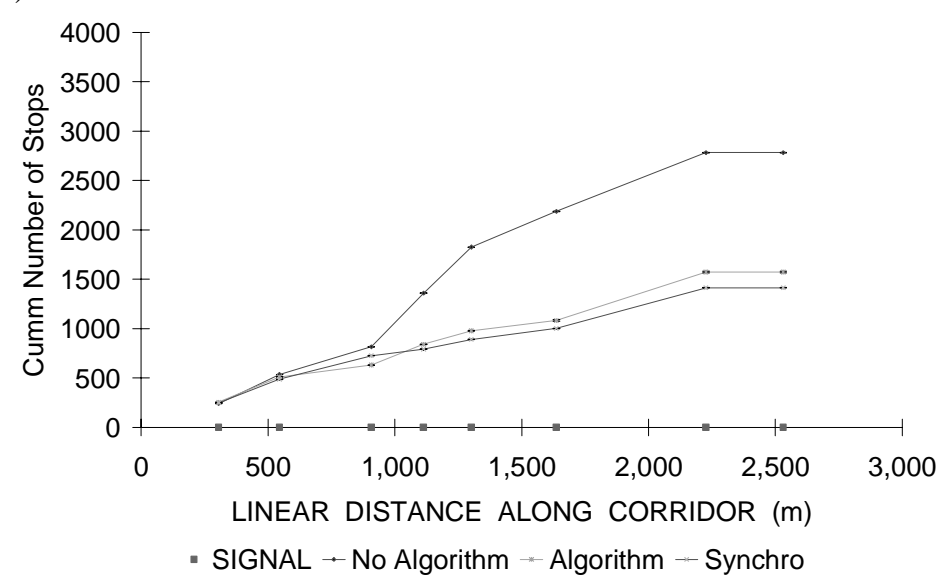

d) Fourth Hour

Figure 8-12 SR 26 (South) East Bound Cumulative Number of Stops-P.M. Period 


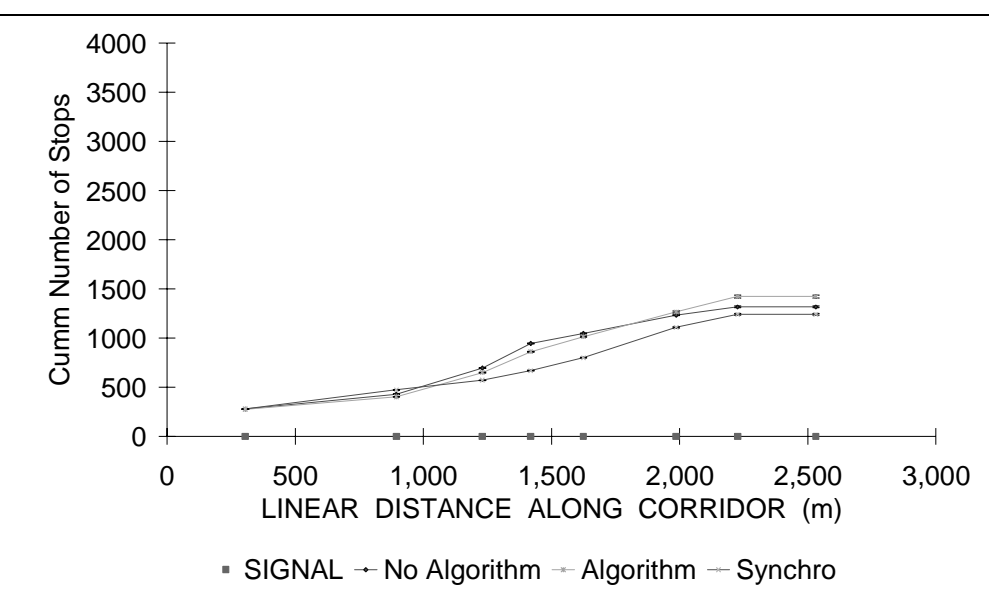

a) First Hour

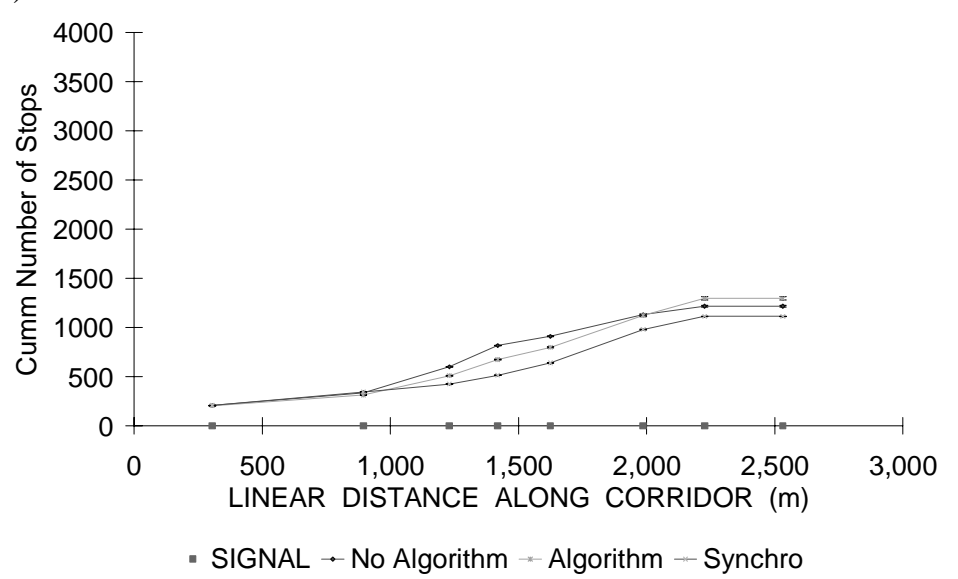

c) Third Hour

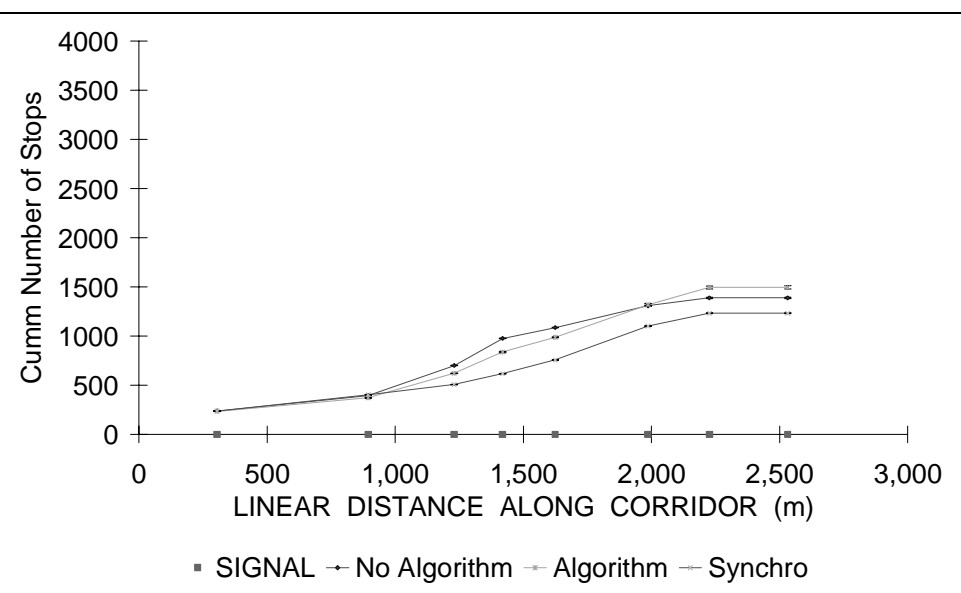

b) Second Hour

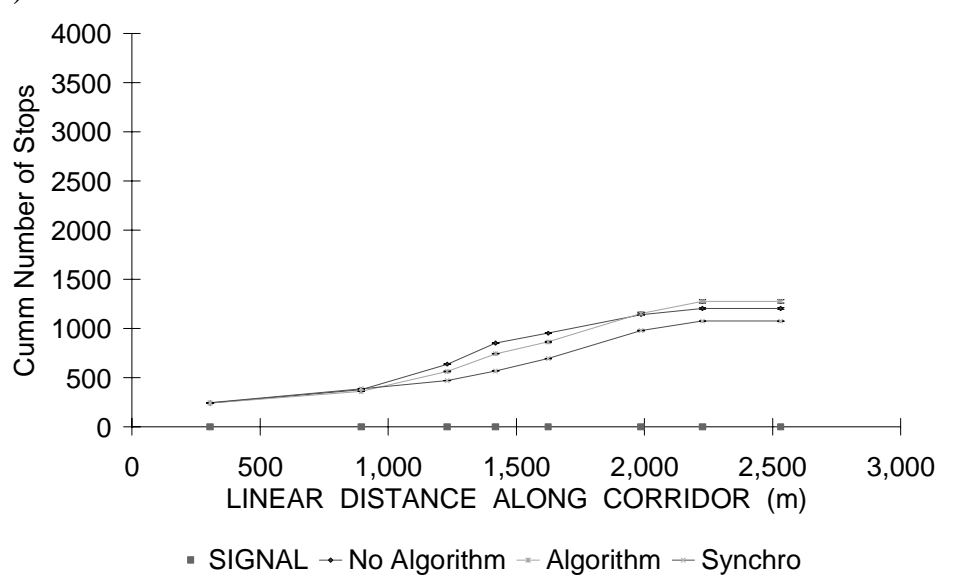

d) Fourth Hour

Figure 8-13 SR 26 (South) West Bound Cumulative Number of Stops-P.M. Period 


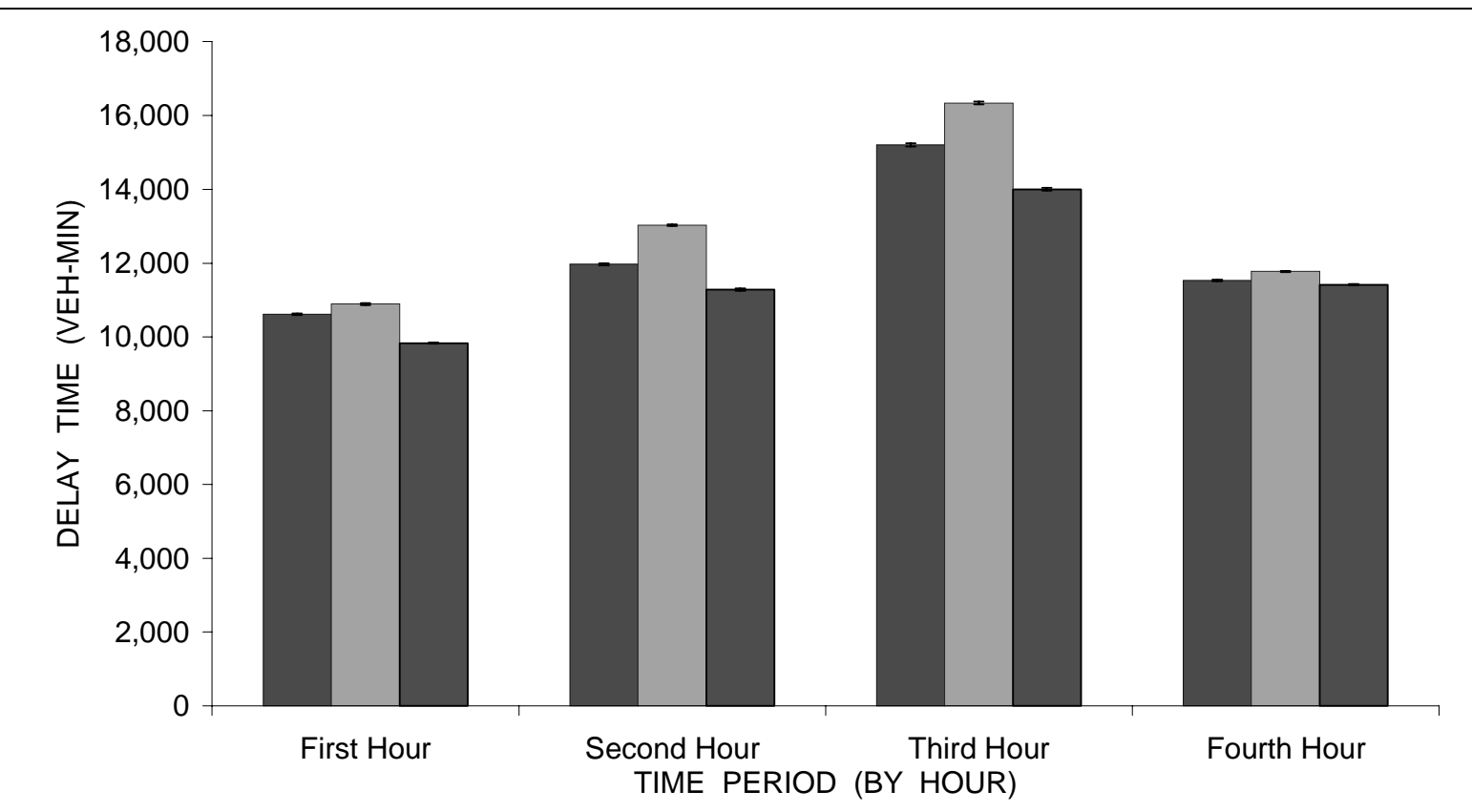

a) Total Delay Time

$\square$ No Algorithm $\square$ Algorithm $\square$ Synchro

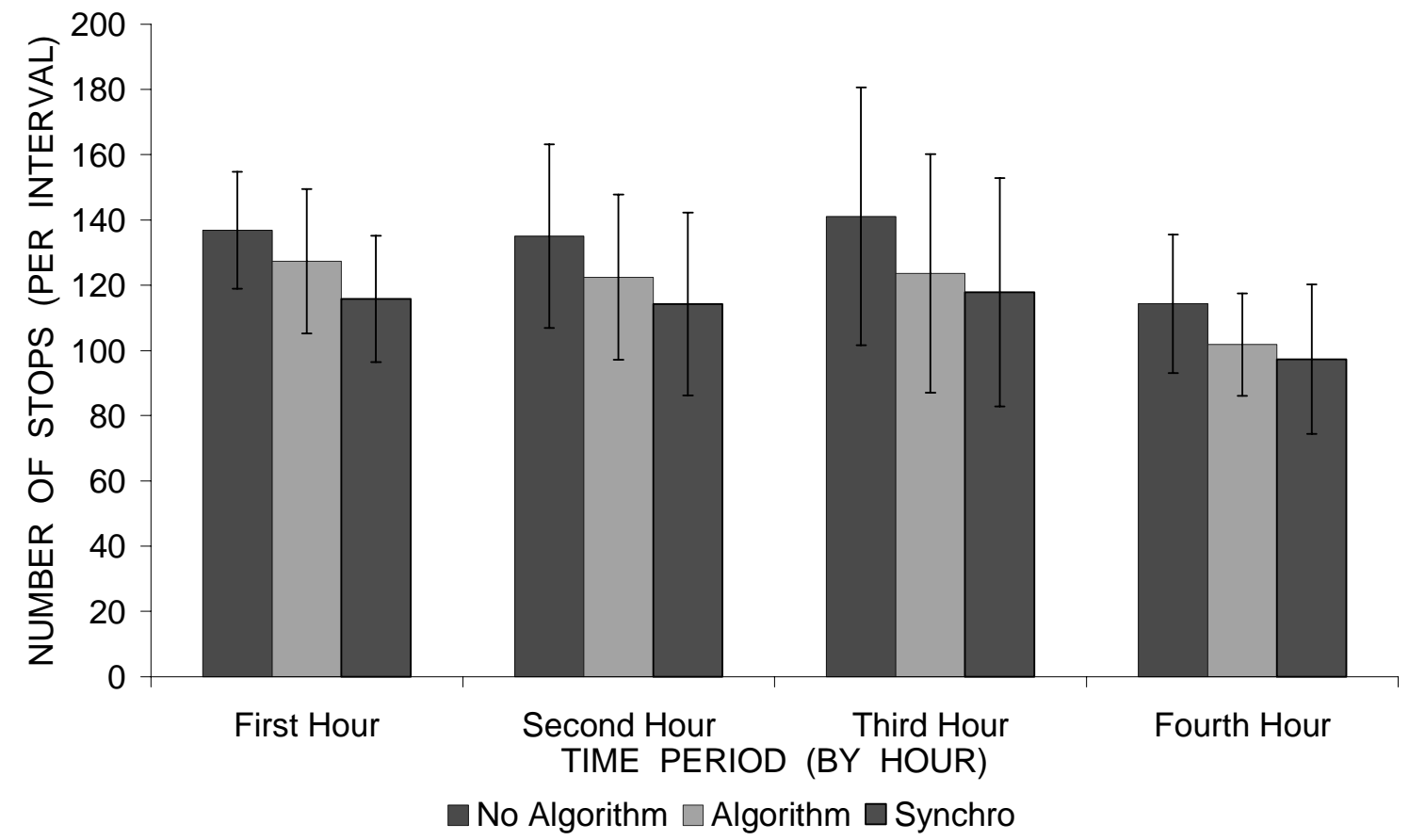

b) Total Number of Stops

Figure 8-14 SR 26 (South) Total Delay and Number of Stops-P.M. Period 


\subsubsection{P.M. Offset and Cycle Transitioning}

Figure 8-15 shows the transitioning in the offsets and cycle length for each of the seven studied intersections. Note that although the offset values continued to decrease during the first three hours of simulation, they became fairly stable during the fourth hour. Figure 8-15-b explains this, whereby somewhat less transitioning in cycle length can be observed during the fourth hour. When comparing Figure 8-15-b with Figure 8-7b, one should note that the p.m. peak cycle length was 120 seconds where the a.m. cycle

length was only 75 seconds. Despite the larger cycle length, Figure 8-15-b shows a maximum cycle of 257 seconds, compared to 360 seconds in the a.m. period.

Table 8-2 shows that the side street volumes increased during the p.m. period, causing less frequency in phase skips. This has an effect on the stability of PROTRACTS since cycle profiles are more reliable in estimating the next cycle profile when few phase skips occur. 
Actual Offset Transitioning

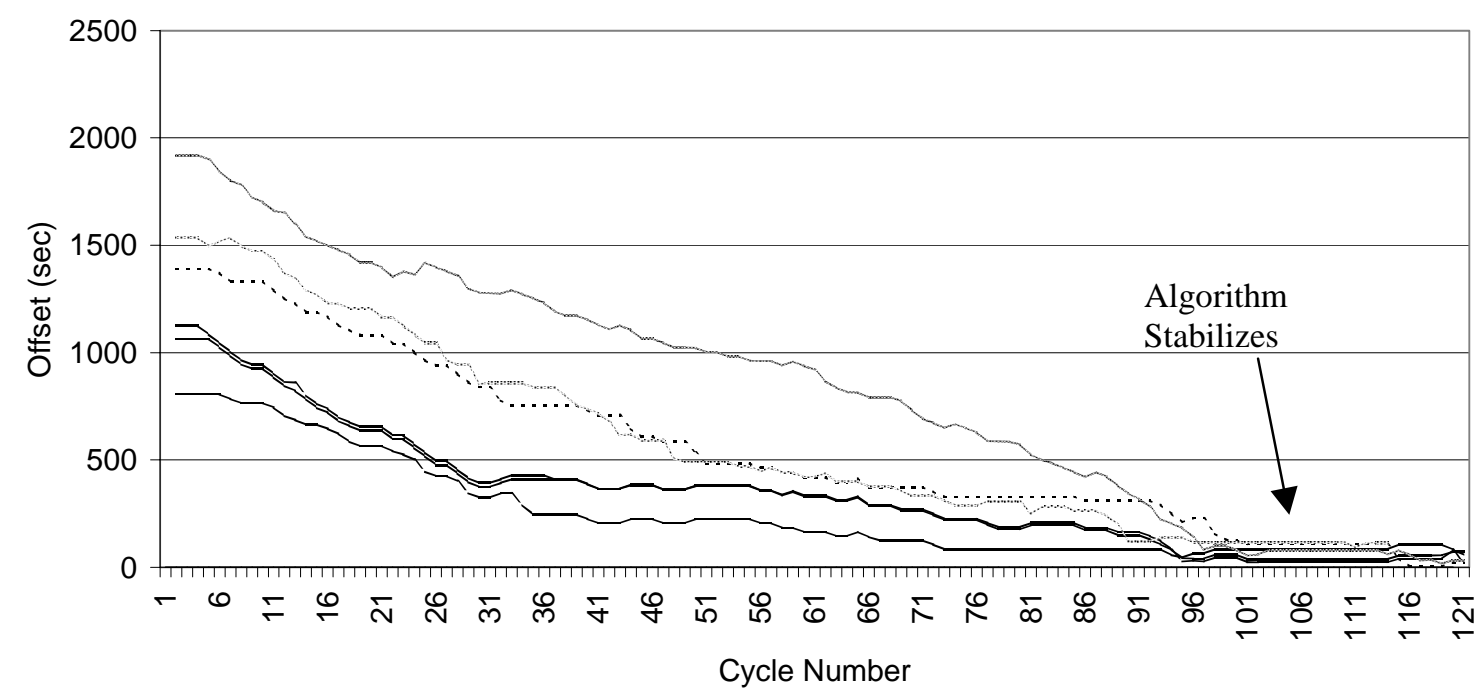

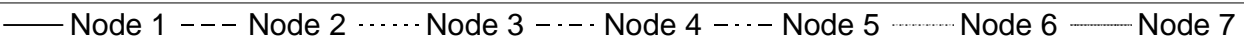

a) Offset Transitioning

\section{Cycle Transitioning}

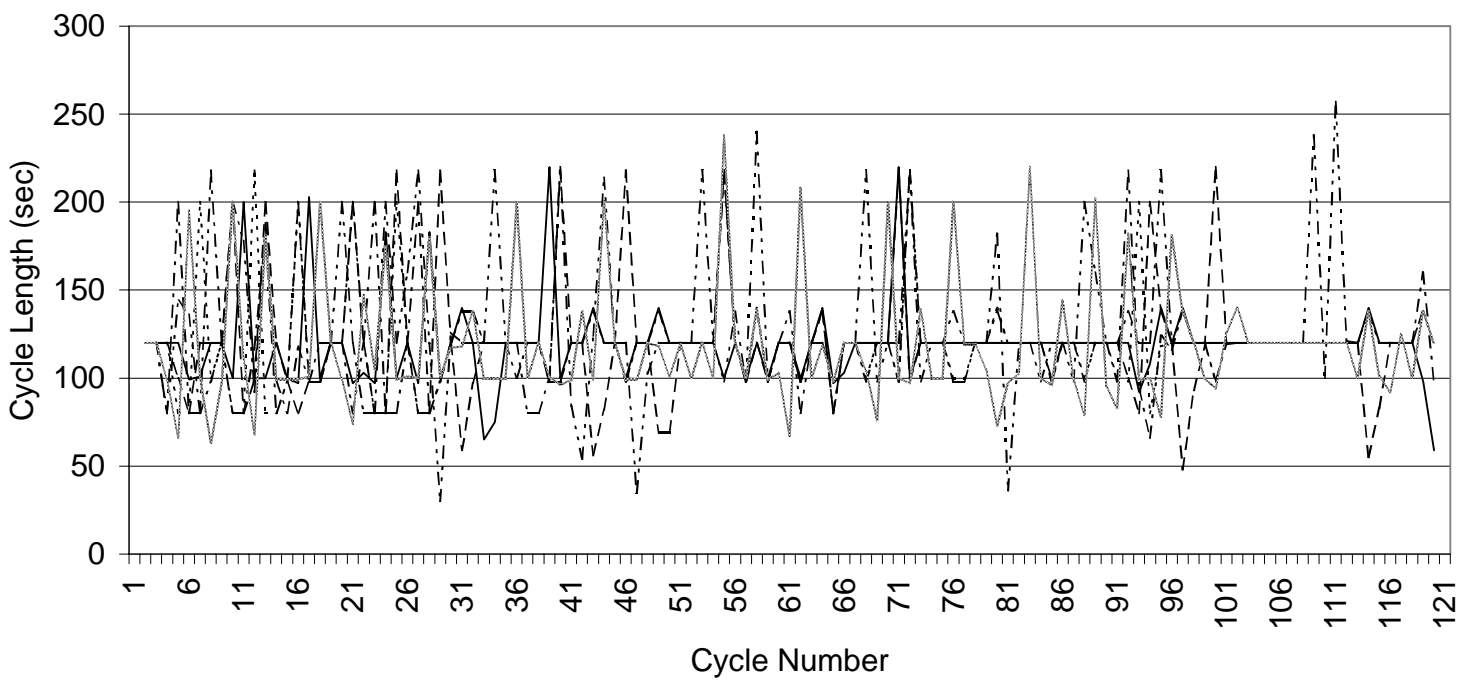

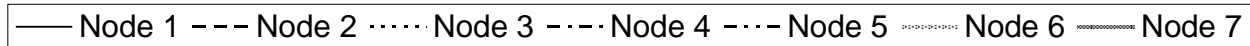

b) Cycle Transitioning

Figure 8-15 SR 26 (South) Offset and Cycle Transitions-P.M. Period 


\subsection{Chapter's Conclusion}

This chapter studied the performance of PRO-TRACTS on the SR 26 network in Lafayette, Indiana. The study showed that, when started with a scenario of offsets that provided poor progression, PRO-TRACTS consistently resulted in an improvement in both the travel time and the number of stops in the arterial direction to which was applied, but without reaching Synchro's performance. It was observed that PROTRACTS had a greater impact in reducing the number of stops, however, PRO-TRACTS caused an increase in total network delay compared to Synchro's timing plans. These results were not surprising since favoring a directional movement is an additional constraint in the system optimization function. Timing plans obtained with Synchro are assumed to be optimum plans that produce minimum delay of the total network. When enforcing a constraint such as favoring a certain traffic movement, the best that can be achieved in the overall system objective function is to keep it constant. However, the overall delay could be expected to degrade unless the original plans were not optimum plans for the current traffic patterns.

An important factor on the performance of PRO-TRACTS was the frequency of phase skips, which is mainly because PRO-TRACTS is a reactive algorithm and therefore assumes that the next cycle's arrivals are comparable to the current cycle's arrivals. With phase skips, the discrepancy in arrival patterns of succeeding cycles causes PROTRACTS' offsets to fluctuate, reducing its efficiency. Subsequent research could take phase skips into consideration. 


\section{CHAPTER 9- HARDWARE-IN-THE-LOOP SIMULATION WITH NTCIP COMPLIANT CONTROLLERS}

Chapters 6 through 8 discussed the integration of PRO-TRACTS with CORSIM's internal control model to provide a preliminary evaluation tool. However, the ultimate objective of the development of PRO-TRACTS is to implement it in the field with real traffic controllers, such that the controllers have the ability of adaptively tuning their offsets in response to changes in traffic patterns. Prior to field implementation of PROTRACTS, two steps were necessary. The first step was to evaluate the performance of PRO-TRACTS with actual controllers to fully understand its effect on the controller transitioning algorithms and to examine whether actual controllers would benefit from PRO-TRACTS applications. The second step was to define an NTCIP object for capturing detector actuations at the controller level to provide efficient low bandwidth communications for field implementation.

This chapter provides an overview of hardware-in-the-loop simulation and illustrates its concept in addition to an overview of NTCIP and how it was integrated with the hardware-in-the-loop simulation. Finally, a section is provided to describe the proposed field implementation and define a new NTCIP object for capturing detector actuation at the controller level. 


\subsection{Overview of Hardware-in-the-Loop Simulation}

\subsubsection{Motivation of Hardware-in-the-Loop Simulation}

As described in Chapter 2, there have been extensive efforts in the development of adaptive control systems over the last few years, both nationally and internationally. Adaptive control systems such as OPAC, RHODES, SCOOT, and SCATS are currently deployed in several cities and regions around the world. Meanwhile, emerging algorithms and several improvements to existing ones are evolving. In this development process, several vendors are implementing unique proprietary features in their signal systems. Most of those features do not exist in traffic evaluation or simulation software. Hence came the need for a method or an environment in which such new and proprietary features could be evaluated prior to deployment, or alternatively what is called Hardwarein-the-loop simulation [Bullock and Catarella, 1998].

Hardware-in-the-loop simulation allows rigorous evaluation of new features in traffic signals and technologies by 1) using both the ability of traffic simulation software to provide stochastic traffic variation and summarizing useful measures of effectiveness (MOEs), and 2) using the new features that are only available in actual traffic controllers.

The concept behind the hardware-in-the-loop simulation is to use the detector actuation provided by the traffic simulators (which also generate the traffic patterns) to stimulate controllers that determine the status of signal indications according to the logic provided by actual control algorithm. The signal indications provided by the traffic controllers are then presented to the traffic simulation software, and with the software

provision of MOEs at the end of the simulation run, the engineer or the analyst can 
evaluate the performance of the actual traffic signal logic or feature and perhaps compare it to other logics.

Hardware-in-the-loop simulation can also be used to train personnel on new hardware features and help them fine tune systems before actual implementation in the field.

\subsubsection{Basic Concepts of Hardware-in-the-Loop Simulation}

There have been several efforts in the United States to integrate microscopic simulation programs with the traffic control hardware in a hardware-in-the-loop environment [Bullock and Catarella, 98; Engelbrecht et al., 99; Huch, 1999]. A typical hardware-in-the-loop architecture is shown in Figure 9-1. The three main components of a hardware-in-the-loop environment are:

1. A controller interface device (CID) (Figure 9-2) that provides the interface between the traffic controller and the computer running the microscopic simulation.

2. A software interface module that provides the interface between the CID and the microscopic simulation program, typically a Dynamic Link Library (DLL).

3. A microscopic simulator that generates and models the traffic and is also used to tabulate MOE's at the end of the simulation.

In this research the traditional hardware-in-the-loop environment is integrated with the NTCIP communication protocol to provide a simulation environment where 
PRO-TRACTS' performance can be evaluated with field controllers, as shown in Figure 9-3. The next section provides an overview of the NTCIP.

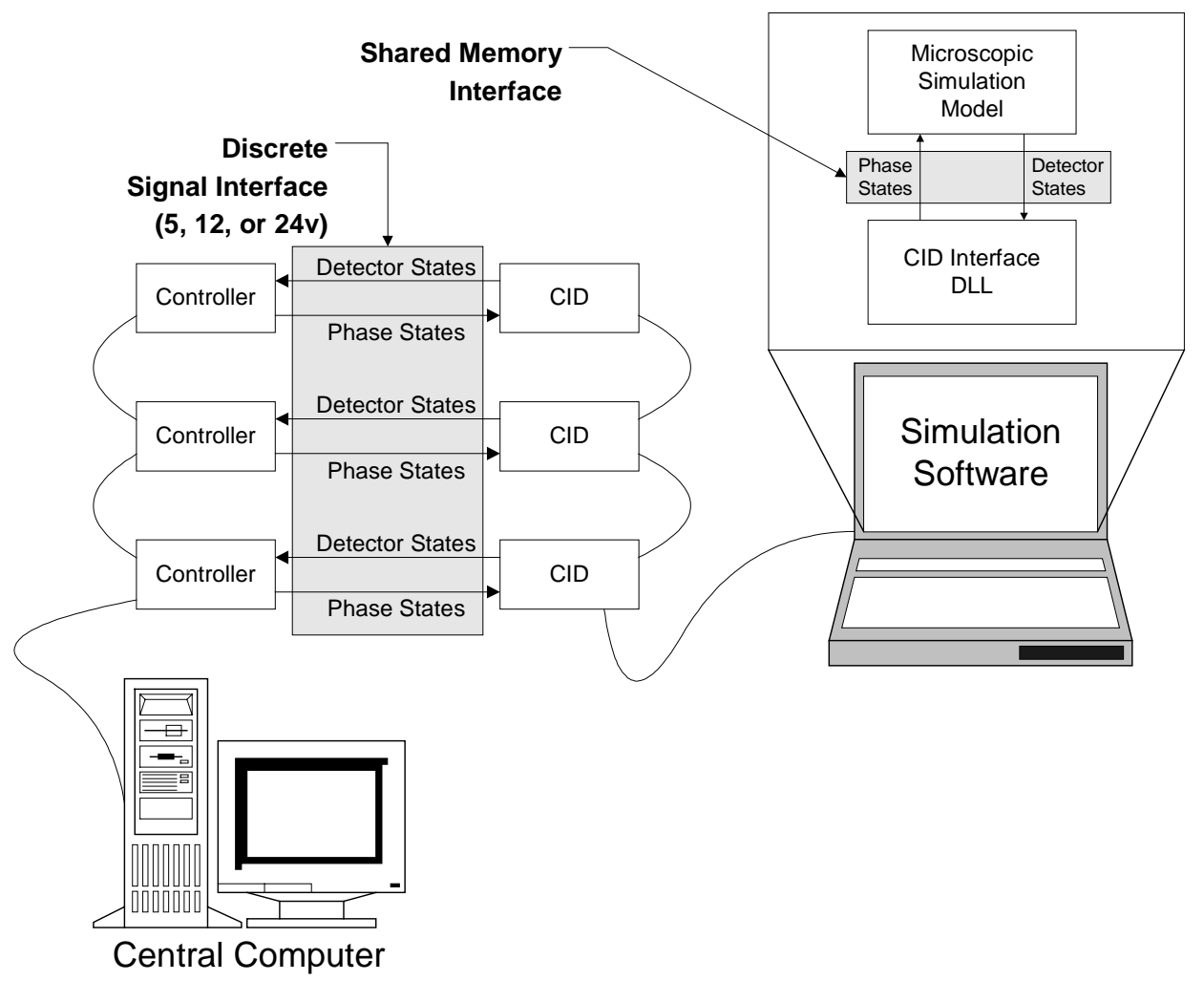

Figure 9-1 Schematic of the Hardware-in-the-Loop Simulation Environment [Source: Bullock and Urbanik 2000]

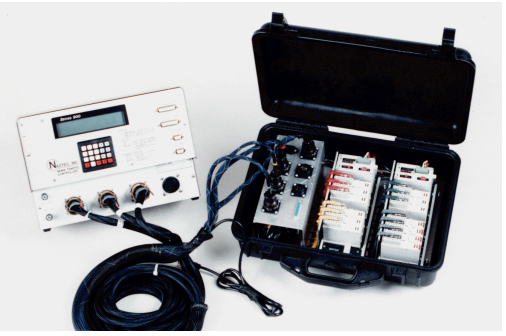

Figure 9-2 Controller Interface Device (CID) 


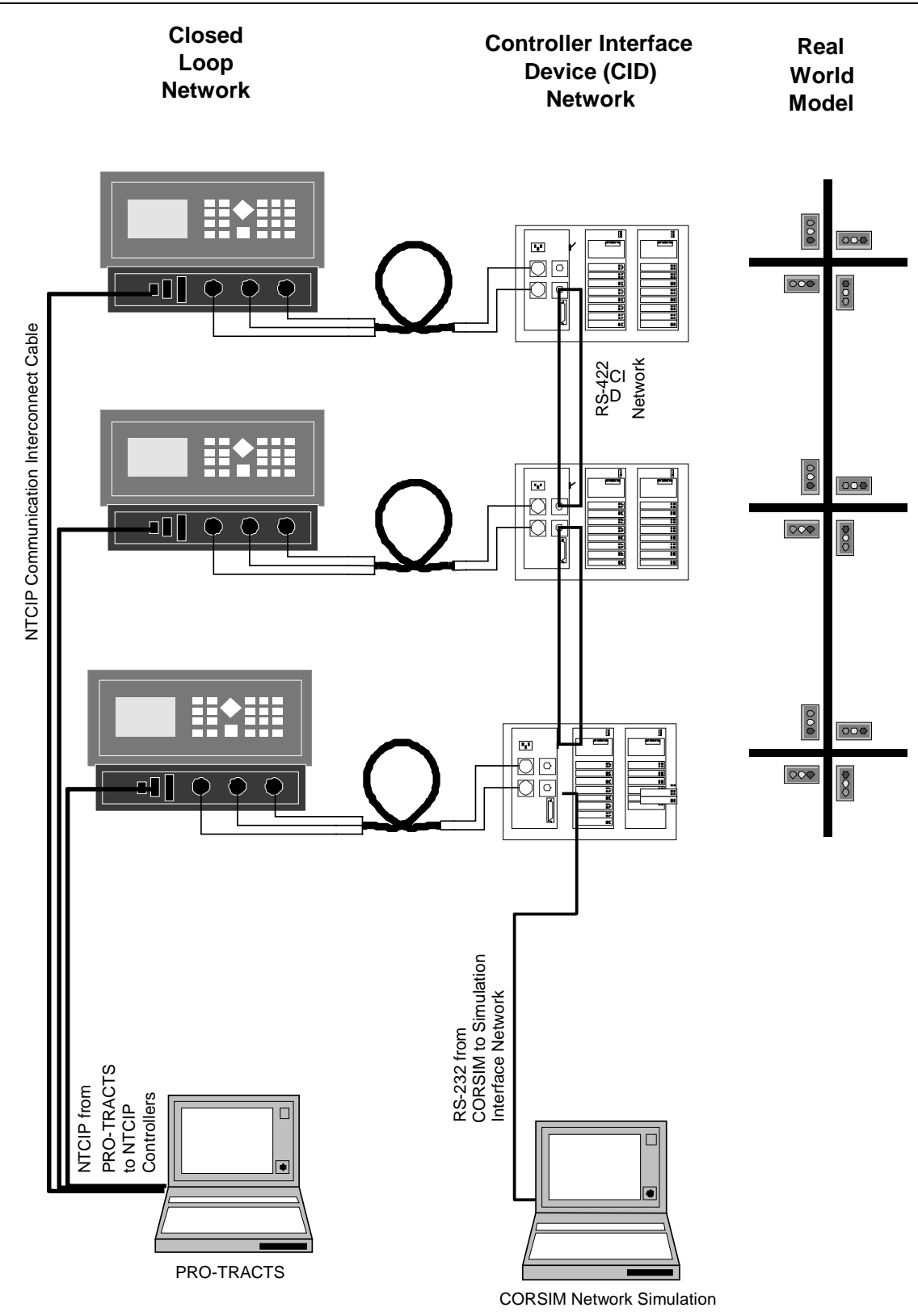

Figure 9-3 Hardware-in-the-Loop Concept 


\subsection{Overview of NTCIP}

The initial TEA 21 legislation [Fischer, 1998] is quite aggressive in specifying the use of standards for ITS projects (Table 9-1 and Table 9-2). However, the NTCIP standard supporting traffic signal control has been evolving at a relatively slow rate. According to the NTCIP guide [NEMA, 1999A], the 1998 interim guidance appeared to soften the standards' requirement to conditions "where applicable," presumably because the standards were continuing to evolve. In 1999, the NTCIP standard suites begin to see their first implementations [NEMA, 1999B; NEMA, 1999C; NEMA, 1999D]. These initial deployments have demonstrated that the standard is viable. However, they have also indicated that many of the interchangeability goals of NTCIP cannot be achieved without very carefully planning the data objects. If these data objects are not precisely defined, essential data objects must be defined by implementing vendors in a proprietary object. Such proprietary objects defeat the interchangeability of an NTCIP system. In the case of traffic signal systems, interchangeability is an extremely important goal in growing a system in an orderly manner over several years where individual phases are competitively bid. 
Table 9-1 TEA21 Requirements for Conformity with National Architecture [Source: Fischer, 1998]

"SEC. 5206. NATIONAL ARCHITECTURE AND STANDARDS.

(a) IN GENERAL.-

(1) DEVELOPMENT, IMPLEMENTATION, AND MAINTENANCE.-Consistent with section 12(d) of the National Technology Transfer and Advancement Act of 1995 (15 U.S.C. 272 note; 110 Stat. 783), the Secretary shall develop, implement, and maintain a national architecture and supporting standards and protocols to promote the widespread use and evaluation of intelligent transportation system technology as a component of the surface transportation systems of the United States.

(2) INTEROPERABILITY AND EFFICIENCY.-To the maximum extent practicable, the national architecture shall promote interoperability among, and efficiency of, intelligent transportation system technologies implemented throughout the United States.

(3) USE OF STANDARDS DEVELOPMENT ORGANIZATIONS.-In carrying out this section, the Secretary may use the services of such standards development organizations as the Secretary determines to be appropriate.

(b) REPORT ON CRITICAL STANDARDS.-Not later than June 1, 1999, the Secretary shall submit a report to the Committee on Environment and Public Works of the Senate and the Committee on Transportation and Infrastructure and the Committee on Science of the House of Representatives identifying which standards are critical to ensuring national interoperability or critical to the development of other standards and specifying the status of the development of each standard identified.

(e) CONFORMITY WITH NATIONAL ARCHITECTURE.- (1) IN GENERAL.-Except as provided in paragraphs (2) and (3), the Secretary shall ensure that intelligent transportation system projects carried out using funds made available from the Highway Trust Fund, including funds made available under this subtitle to deploy intelligent transportation system technologies, conform to the national architecture, applicable standards or provisional standards, and protocols developed under subsection (a)." 
Table 9-2 TEA21 Requirements for NTCIP [Source: NEMA, 1999A]

\begin{abstract}
"The Transportation Equity Act for the 21st Century (known as "TEA-21") requires that federally funded ITS projects "conform" with the National ITS Architecture. As defined in TEA 21, the term "intelligent transportation system" means "electronics, communications, or information processing used singly or in combination to improve the efficiency or safety of a surface transportation system". The National ITS Architecture defines both the functions performed in implementing ITS, and the information flows between transportation subsystems. In its October 2, 1998 report entitled "Interim Guidance on Conformity with the National ITS Architecture and Standards", the US DOT stated "Highway Trust Fund recipients shall take the appropriate actions to ensure that development of the project(s): (a) engages a wide range of stakeholders, (b) enables the appropriate electronic information sharing between shareholders, (c) facilitates future ITS expansion, and (d) considers the use of applicable ITS standards." The terms interchangeability and interoperability are used throughout the TEA-21 legislation, but interoperability is used extensively. While the simple view of NTCIP often focuses on interchangeability, interoperability is actually far more important, for two reasons. First, since the communications infrastructure is usually the most costly element in a new system, using this infrastructure for multiple purposes lowers the overall cost of the system. Second, and more important in terms of the TEA-21 legislation, interoperability suggests the sharing of data, thereby enhancing the operators ability to efficiently manage these transportation systems, even to the extent of sharing data across jurisdictional boundaries."
\end{abstract}

The following sections of this chapter describe a proposed extension to NTCIP to facilitate the implementation of a new algorithm in an open architecture environment that would permit competitive procurement of traffic control hardware and software. The significance of this definition is that it is based upon the NTCIP standard and provides a mechanism for a public agency to precisely define the functions and associated protocol necessary for supporting new systems functions. The resulting specification is directly usable by all vendors who conform to the NTCIP standard. In the past, specifying new system functions was not practical, as competitive procurement would require unique specifications for each possible vendors' proprietary protocol and menu screens.

A communications protocol is a set of standards for how messages are coded and transmitted between electronic devices [NEMA, 1999A]. In order to communicate successfully, the equipment at each end of a data transmission must use the same 
protocol. The use of NTCIP allows future expansion of transportation systems and provides both interchangeability and interoperability. The term interchangeability reflects the ability to use multiple brands of a device on the same communications channel, where the term interoperability reflects the ability to use many different types of devices on the same communications channel [NTCIP TS3.1...TS3.5, 1996].

NTCIP consists of a whole suite of protocols covering the spectrum, from simple point-to-point command/response protocols to quite sophisticated object-oriented techniques. NTCIP supports continuous, automated data transmissions with no human in the loop, but it can also involve a human operator making requests or issuing instructions.

NTCIP provides communications standards for two different types of ITS communications: center-to-center and center-to-field. An example of the center-to-center communication, also called peer-to-peer communication, is communication between two traffic management centers exchanging information. This "center-to-center" communication can involve communication between any number of systems in a balanced, many-to-many network. It is important to note that NTCIP specifies the data objects and precisely defines the data to be communicated, but it does not completely define the functionality of the central or field devices to which it applies.

The center-to-field type of communication includes the communication between traffic signal management systems and field traffic signals controllers, where the center continuously polls each field device in an unbalanced one-to-many network. Another example is a freeway management system communicating with detectors on the freeway. This type of communication is recommended for the field implementation of PRO- 
TRACTS to avoid the complications of peer-to-peer communications and allow for system monitoring at the control center.

\subsubsection{NTCIP Protocols for Center to Field Communications}

Center-to-field applications, such as Simple Transportation Management Protocol (STMP) [NTCIP TS3.3, 1996], and several sets of new standard data elements called "objects," were the first NTCIP standards developed. The initial NTCIP center-to-field protocol used two existing standards - the Simple Network Management Protocol (SNMP) [Stallings, 1995] standard at the application level, and the High-level Data Link Control (HDLC) standard at the subnetwork level.

The SNMP protocol is based on the Internet protocol for managing networking equipment and computers [Stallings, 1995]. It is a simple, but bandwidth-inefficient, protocol for center-to-field applications; SNMP is suitable only for networks with high bandwidth or low volumes of messages. An extension of SNMP, the STMP, uses dynamic composite objects to send center-to-field messages more efficiently. STMP is better suited to networks with low bandwidth and high volumes of messages, including traffic signal systems. Table 9-3 summarizes the services offered by the two protocols and their implementation requirements. 
Table 9-3 SNMP and STMP Comparison [Source: NEMA, 1999A]

\begin{tabular}{|c|c|c|}
\hline \multirow[t]{2}{*}{9.2 .1 .1 .1 .1 .1 .1 .1} & \multicolumn{2}{|c|}{ SNMP and STMP Comparisons } \\
\hline & SNMP & STMP \\
\hline Can send any base object? & Yes & Yes \\
\hline Bandwidth Efficiency - inverse of packet overhead & Worst & $\begin{array}{c}\text { Best } \\
\text { (uses dynamic composite objects) }\end{array}$ \\
\hline Supports routing \& dial-up & Options & Options \\
\hline Message Set & Supported & Limited to 13 \\
\hline Ease of implementation & Easiest & Hardest \\
\hline
\end{tabular}

Different devices with unique addresses can share the same communications line with other devices using the same subnetwork, e.g., a traffic signal and a dynamic message sign. The management system can communicate with only one of the devices on the line or channel at a time, by sending a message addressed to that device. The NTCIP protocols can also broadcast messages to all devices, e.g., a time clock update, but no device can reply to a broadcast message.

\subsubsection{Application Message Bits and Bytes}

The Tag-Length-Value representation method defined in ISO 8825, Basic Encoding Rules (BER) is used to define the actual bits and bytes of an SNMP message. All objects can be expressed as Tag (or Type) of either SEQUENCE, INTEGER, OCTET STRING, or OBJECT IDENTIFIER. The Tag indicates whether the Value component is a number, string (or text), or an identifier. It can also indicate that what follows is a series of data that is expressed as a Tag-Length-Value. The Length component of the object 
represents how many bytes it takes to store the object in memory. The third component of an object is its Value, which is stored in a hexadecimal form [NEMA, 1999A].

An SNMP message is defined as a SEQUENCE and Length of two predefined fields, one of which describes the protocol and the other defines the data carried by the protocol. A data field that follows it describes the operation that is to be performed. Figure 9-4 shows an SNMP message. Note how the SetRequest PDU (protocol data unit) field at the end of a row is expanded in the row below. An expanded field starts with the Tag of the operation, followed by the Length of the data and consists of Values of three Tag-Length-Value fields. From a communications perspective, each of the objects defined in one of the Object Definitions Standards, such as TS 3.4 or TS 3.5, has two components: an identity and a value [NEMA, 1999A].

The fixed overhead of SNMP messages that are used to get or set one or more objects is 26 bytes, as shown in Figure 9-4. 
PMPP Frame Fields

\begin{tabular}{|c|c|c|c|c|}
\hline Flag & Address & Control & Information & $\mathrm{FCS}$ \\
\hline $7 \mathrm{E}$ & $\begin{array}{c}\text { FF } \\
\text { broadcast } \\
\text { address }\end{array}$ & $\begin{array}{l}\text { CO } \\
\text { UI Frame } \\
\text { Poll }=0\end{array}$ & & $\begin{array}{l}x \times \alpha \\
\mathrm{CRC}\end{array}$ \\
\hline
\end{tabular}

Information Field

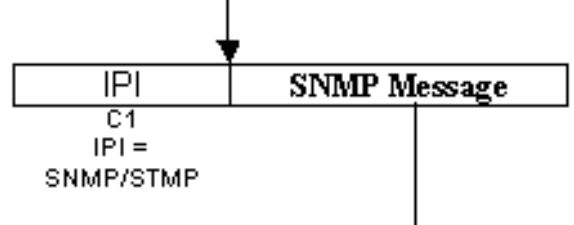

SNMP Message Fields

SEQUENCE

Seq Tag Length of

what follows

$=47$ Bytes

\begin{tabular}{|c|c|c|}
\hline Version & & Comm \\
\hline 020100 & 04 & 06 \\
\hline $\begin{array}{c}\text { Integer Tag } \\
\text { Length Value } \\
\text { Version }=0\end{array}$ & $\begin{array}{c}\text { Octet } \\
\text { String } \\
\text { Tag }\end{array}$ & $\begin{array}{l}\text { Length } \\
=\mathbf{6}\end{array}$ \\
\hline
\end{tabular}

\begin{tabular}{l|l} 
ity Name & SetRequest PDL
\end{tabular}

Name

$=$ "publid"

SetRequest PDU

\begin{tabular}{|c|c|c|c|c|c|}
\hline PDU & Type & Request ID & Error Status & Error Index & Variable Bindings \\
\hline $\begin{array}{c}\text { A3 } \\
\text { PDU Tag }\end{array}$ & $\begin{array}{c}22 \\
\text { Length of } \\
\text { what } \\
\text { follows } \\
=34\end{array}$ & $\begin{array}{c}020100 \\
\text { Integer Tag } \\
\text { Length Value } \\
\text { ID }=0\end{array}$ & $\begin{array}{c}020100 \\
\text { Integer Tag } \\
\text { Length Value } \\
\text { Status = } 0\end{array}$ & $\begin{array}{c}020100 \\
\text { Integer Tag } \\
\text { Length Value } \\
\text { Index }=0\end{array}$ & \\
\hline
\end{tabular}

Variable Bindings

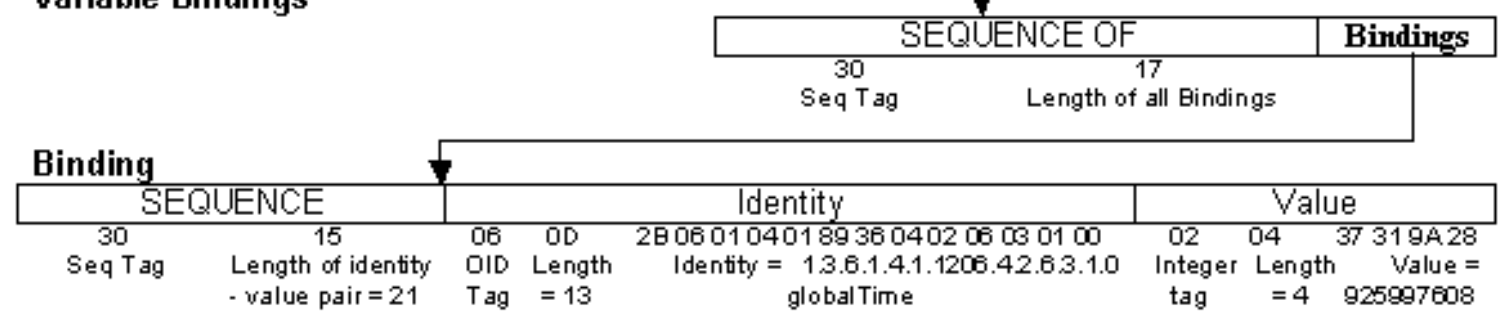

Figure 9-4 SNMP Bits and Bytes [Source: NEMA, 1999A]

The actual bits and bytes of an STMP message are defined using Octet Encoding Rules (OER), as described in NTCIP 1102 - NTCIP Octet Encoding Rules (OER). The STMP message is defined prior to being sent, and hence it is possible to eliminate a number of fields and reduce overhead significantly. In STMP, if the Tag, Length, or Value is known, the component is eliminated. Since all data are expressed as a 
SEQUENCE, all SEQUENCE Tags and SEQUENCE Lengths are eliminated [NEMA, 1999A]. This reduces a lot of overhead as shown in Figure 9-5.

PhPP Frame Fields

Infommation Field

STMP Message Fields and Set Request PDU

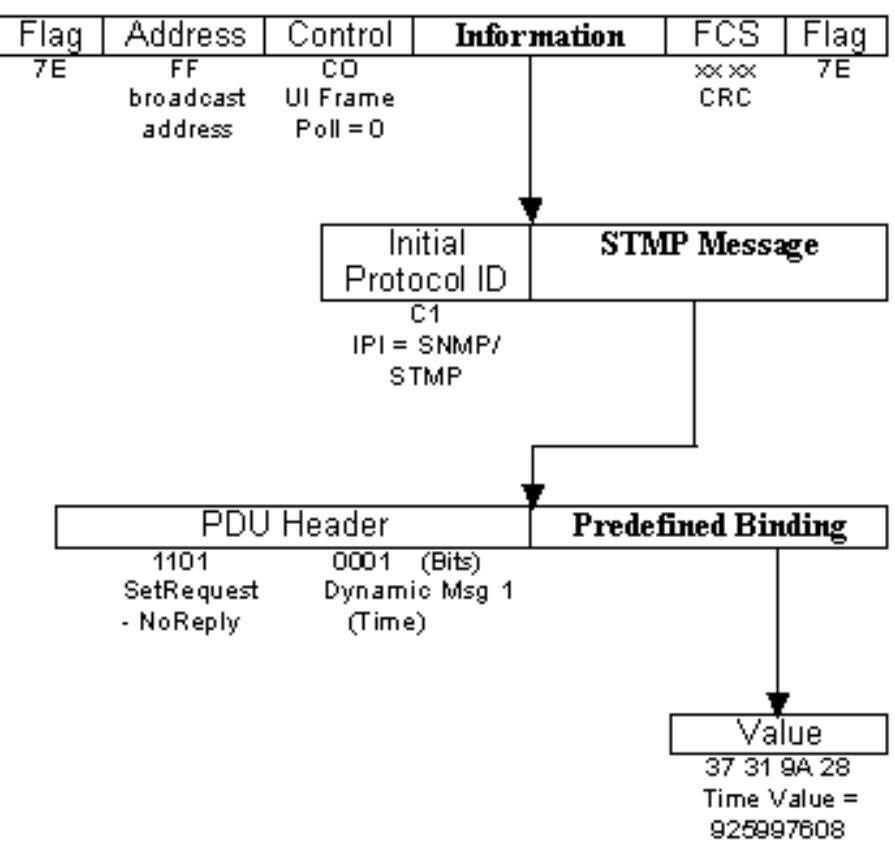

Figure 9-5 STMP Bits and Bytes [Source: NEMA, 1999A]

\subsection{Hardware-in-the-Loop Simulation with NTCIP Offset Download}

Hardware-in-the-loop simulation is used to evaluate PRO-TRACTS' performance

prior to its deployment for safety and efficiency reasons. Using simulation will eliminate the inconveniences to real traffic real traffic of the experimenting with new algorithms, while using the real traffic controllers will provide more realistic results. The schematic 
presented in Figure 9-1 illustrates how PRO-TRACTS was integrated with the traditional hardware-in-the-loop simulation. In this schema, the CORSIM simulation package was used for two objectives: 1) to generate the detector calls caused by the simulated traffic and 2) to gather the output statistics and MOEs. The CIDs shown in Figure 9-2 transfer the detector calls from the CORSIM simulation to the actual controllers and then transfer back the phase indicators from the controllers to the CORSIM simulation. PRO-TRACTS uses the detector actuation from the CORSIM simulation and the phase indications to fine-tune the offsets and send the updated offsets values to the database of a traffic management software, such as ICONS database. The updated offsets are downloaded to the controllers using the SNMP protocol functions provided by Siemen's Gardner Systems ICONS software. 


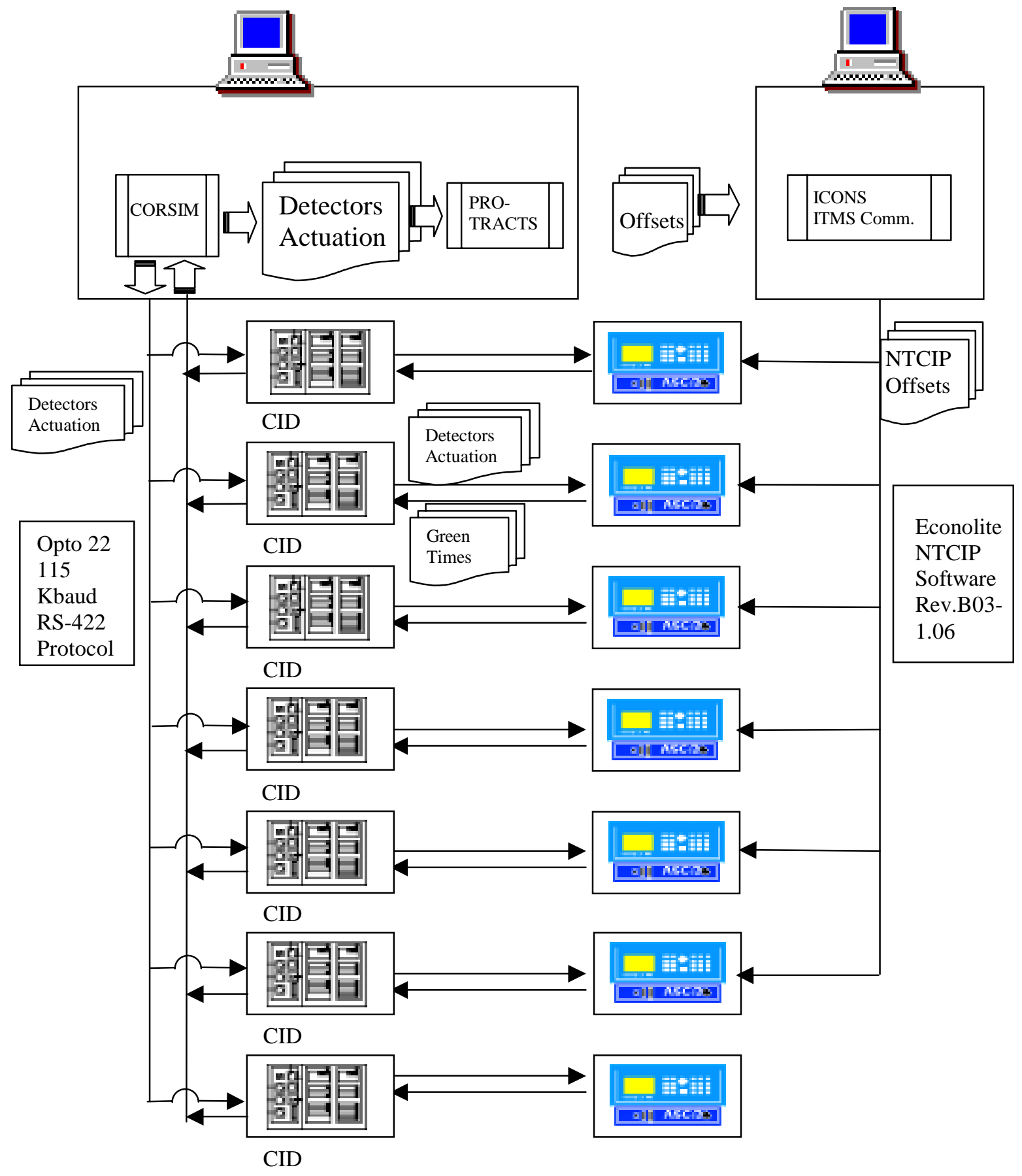

Figure 9-6 Hardware-in-the-Loop with NTCIP Offset Download 


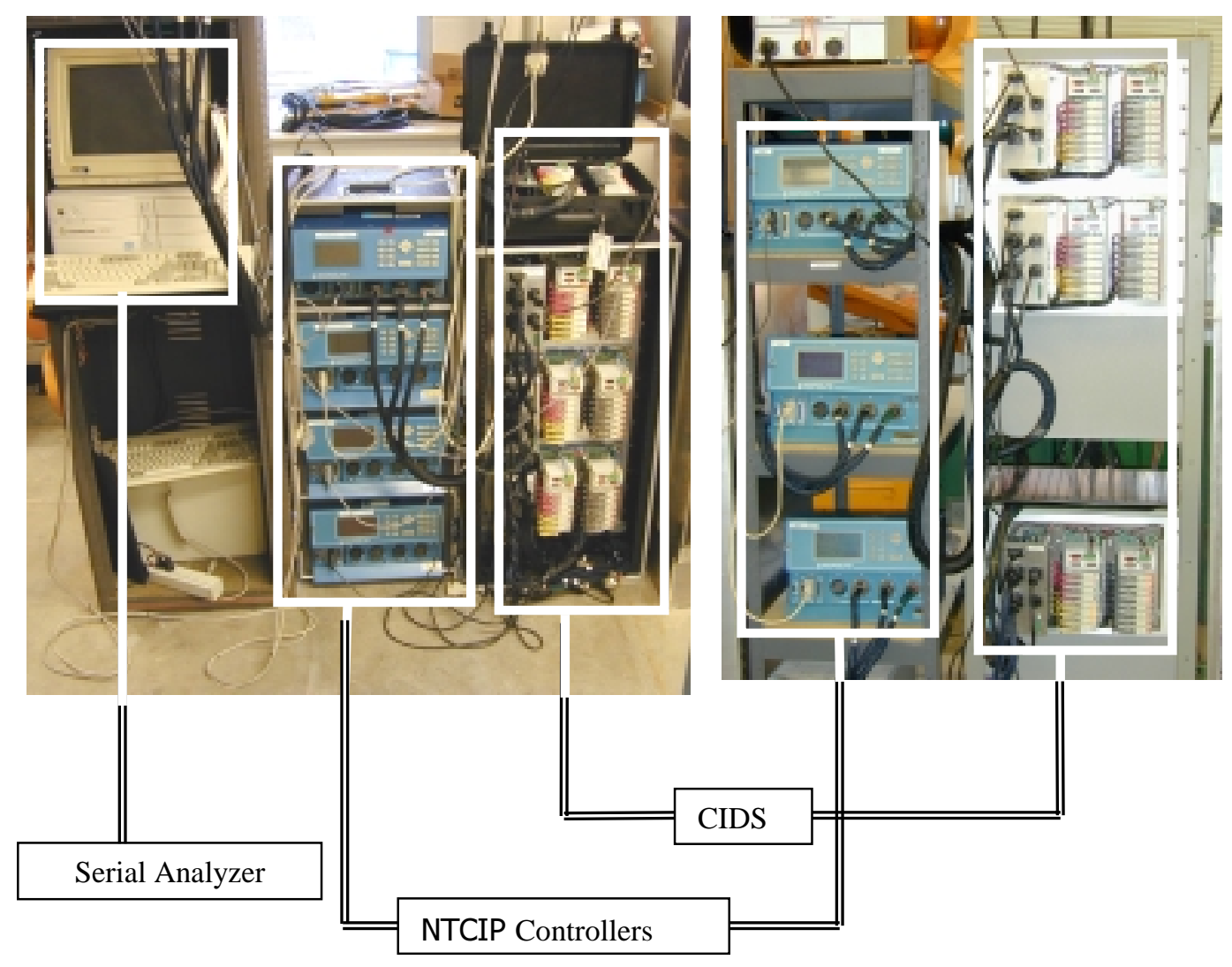

Figure 9-7 Sample of NTCIP Lab Equipment

Figure 9-7 shows the actual lab equipment used for the PRO-TRACTS evaluation. The NTCIP controllers models ASC 2S'S were provided by Econolite. The serial analyzer was used to study the NTCIP messages during the development process. Table 9-4a shows the ASCII code of the message observed from the Data Terminal Equipment (DTE) and Data Communications Equipment (DCE), while Table 9-4b shows the DTE and DCE messages bytes for offset download. The downloaded offset value is shown with a black background in the figure. 
Table 9-4 Example NTCIP message for Download an Offset Change of 10 Seconds to Controller

a) Equivalent ASCII Code for DTE and DCE Message Bytes

\begin{tabular}{|c|c|c|c|c|c|c|c|c|c|c|c|c|c|c|c|c|c|c|c|}
\hline \multicolumn{20}{|c|}{ DTE } \\
\hline$\sim$ & . & . & . & 0 & . &. & 7 & . & . & . & . & . & $a$ & $\mathrm{~d}$ & $\mathrm{~m}$ & $\mathrm{i}$ & $\mathrm{n}$ & $\mathrm{i}$ & $\mathrm{S}$ \\
\hline $\mathrm{T}$ & $\mathrm{r}$ & $\mathrm{a}$ & $\mathrm{t}$ & 0 & $\mathrm{r}$ & . & \# & . & . & . &. & . &. & . & . & . & 0 & . & 0 \\
\hline . & . & . & . &. &. & . & . & . & . & . & 6 & . &. & . & . & . & . &. & . \\
\hline . & . &. & . &. & $\sim$ & & & & & & & & & & & & & & \\
\hline \multicolumn{20}{|c|}{$\mathrm{DCE}$} \\
\hline$\sim$ &. &. & . & 0 & . &. & 7 & . &. & . &. & . & $a$ & $\mathrm{~d}$ & $\mathrm{~m}$ & $\mathrm{i}$ & $\mathrm{n}$ & $\mathrm{i}$ & $\mathrm{S}$ \\
\hline $\mathrm{t}$ & $r$ & $\mathrm{a}$ & $\mathrm{t}$ & o & $r$ & . & $\#$ & . & . & . &. & . & . & . & . & . & 0 & . & 0 \\
\hline . & . &. & . &. & . &. & . & . & . & . & 6 & . &. & . & . &. &. & . & . \\
\hline . & . &. & . &. & $\sim$ & & & & & & & & & & & & & & \\
\hline
\end{tabular}

b) DTE and DCE Message Bytes for Offset Download

DTE

\begin{tabular}{|l|l|l|l|l|l|l|l|l|l|l|l|l|l|l|l|l|l|l|l|}
\hline $7 \mathrm{E}$ & 05 & 33 & $\mathrm{C} 1$ & 30 & 82 & 00 & 37 & 02 & 01 & 00 & 04 & $0 \mathrm{D}$ & 61 & 64 & $6 \mathrm{D}$ & 69 & $6 \mathrm{E}$ & 69 & 73 \\
\hline 74 & 72 & 61 & 74 & $6 \mathrm{~F}$ & 72 & $\mathbf{A 3}$ & 23 & 02 & 01 & 00 & 02 & 01 & 00 & 02 & 01 & 00 & 30 & 18 & 30 \\
\hline 82 & 00 & 14 & 06 & $0 \mathrm{~F}$ & 2B & 06 & 01 & 04 & 01 & 89 & 36 & 03 & 05 & 01 & 04 & 16 & 01 & 04 & 01 \\
\hline 02 & 01 & $0 \mathrm{~A}$ & $\mathbf{9 9}$ & $\mathbf{D 3}$ & $7 \mathrm{E}$ & & & & & & & & & & & & & & \\
\hline
\end{tabular}

DCE

\begin{tabular}{|l|l|l|l|l|l|l|l|l|l|l|l|l|l|l|l|l|l|l|l|}
\hline $7 \mathrm{E}$ & 05 & $\mathbf{1 3}$ & $\mathrm{C} 1$ & 30 & 82 & 00 & 37 & 02 & 01 & 00 & 04 & $0 \mathrm{D}$ & 61 & 64 & $6 \mathrm{D}$ & 69 & $6 \mathrm{E}$ & 69 & 73 \\
\hline 74 & 72 & 61 & 74 & $6 \mathrm{~F}$ & 72 & $\mathrm{~A} 2$ & 23 & 02 & 01 & 00 & 02 & 01 & 00 & 02 & 01 & 00 & 30 & 18 & 30 \\
\hline 82 & 00 & 14 & 06 & 0F & $2 \mathrm{~B}$ & 06 & 01 & 04 & 01 & 89 & 36 & 03 & 05 & 01 & 04 & 16 & 01 & 04 & 01 \\
\hline 02 & 01 & $0 \mathrm{~A}$ & $\mathbf{A 8}$ & $\mathbf{E E}$ & $7 \mathrm{E}$ & & & & & & & & & & & & & & \\
\hline
\end{tabular}

PORT 101

OFFSET $=10$

COLOR CODES:

Black: byte contains offset value

Dark Grey: download ok

Bold: CRC or FCS value 


\subsection{Proposed NTCIP Object}

\subsubsection{Proposed Field Implementation}

In order to implement PRO-TRACTS in the field, a new set of NTCIP objects for capturing detector actuations needed to be defined. The proposed NTCIP object will enable the exchange of the data required to fine-tune the offsets in real-time. Figure 9-8 shows a schematic proposal of the anticipated deployment where PRO-TRACTS is deployed on a system level using center-to-field communication. The circled intersections in the figure are controlled by NTCIP traffic signal controllers that communicate their detectors' actuation to the computer running the ICONS software or its equivalent. The proposed NTCIP object will enable the transfer of the actuation information to PRO-TRACTS through a TCP/IP protocol, and PRO-TRACTS will then calculate the offset adjustment and download the updated offsets to the NTCIP controllers.

The proposed NTCIP object will capture the information from the actuation profiles introduced in Figure 6-2. The proposed object will enable the tabulation of critical detector information at the controller level as well for possible future implementation of the algorithm on the actual controller. Although local implementation is still a possibility, activating PRO-TRACTS at the system level will probably be the

preferred approach to avoid the complication of peer-to-peer communications when implementing the coordination logic discussed in Section 6.3.6. 


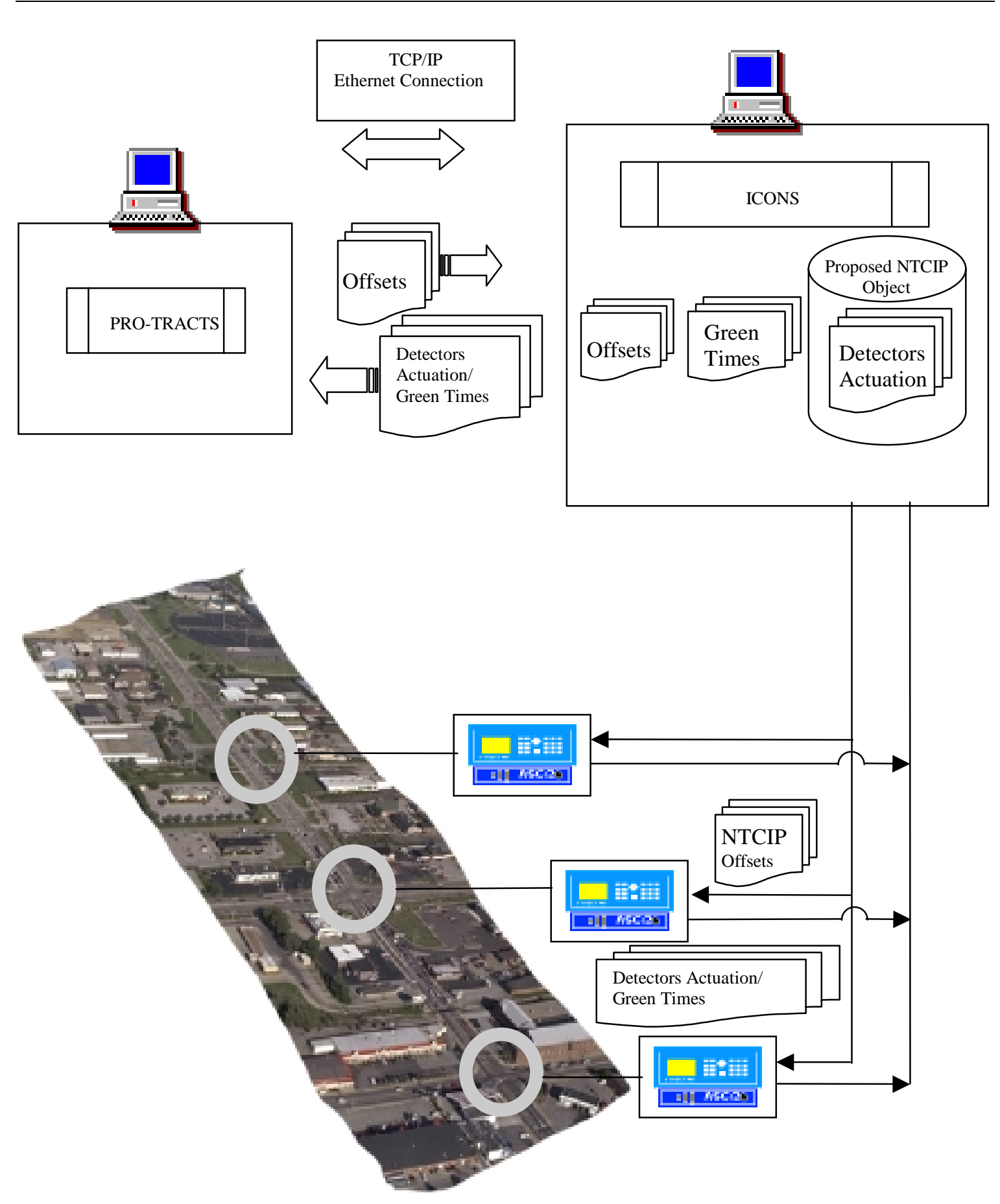

Figure 9-8 Schematic Proposal of PRO-TRACTS Implementation 


\subsubsection{NTCIP Object Definitions for PRO-TRACTS Application Support}

One approach would be to transmit detector information to a central system every time a PRO-TRACTS detector is activated. However, such an approach would consume a tremendous amount of bandwidth. Alternatively, storing and processing detector actuation at the controller level will enable a significantly lower bandwidth demand while using NTCIP. Tabulating the detector information at the controller level also allows the controllers to work independently if necessary. For example, controllers at the coordination system boundaries can "fine-tune" themselves to the arrival pattern if vehicle platoons at the boundaries existed.

This section defines the proposed new NTCIP object for capturing detector actuation at the controller level, patterned on the NTCIP TS 3.5 standards. The attributes of the new object are described and the abstract syntax notation (ASN) for each attribute is provided. The $\mathrm{X}$ label at the beginning of the defined ASN is the node to which the object is attached. Most likely, $\mathrm{X}$ will be 8 in section 2.3 of TS 3.5 object definition hierarchy shown in Table 9-5. In such a case, all "X" labels in the following section shall be replaced by the notation "2.3.8". Table 9-6 shows a table of tables that provides the reader with a link between this chapter's ASN definitions and example data for the new object's attributes. Note that supporting PRO-TRACTS implementation is optional and therefore the PRO-TRACTS detectors object is optional. However, if the controller supports PRO-TRACTS implementation, it must support all of the object attributes of PRO-TRACTS, and therefore all of the attributes are mandatory. 
Table 9-5 TS 3.5 Object Definition Hierarchy

\begin{tabular}{|l|l|l|}
\hline TS 3.5 Section & Object Definition & \\
\hline 2.1 & MIB Header & \\
\hline 2.2 & Phase Parameters & \\
\hline 2.3 & Detector Parameters & \\
\hline & 2.3 .1 & Maximum Vehicle Detectors \\
\hline & 2.3 .2 & Vehicle Detector Parameter Table \\
\hline & 2.3 .3 & Maximum Vehicle Detector Status Group \\
\hline & 2.3 .4 & Vehicle Detector Status Group Table \\
\hline & 2.3 .5 & Volume/Occupancy Report \\
\hline & 2.3 .6 & Maximum Pedestrian Detectors \\
\hline & 2.3 .7 & Pedestrian Detector Parameter Table \\
\hline 2.4 & Unit Parameters & \\
\hline 2.6 & Coordination Parameters & \\
\hline 2.7 & Time Base Parameters & \\
\hline 2.8 & Preempt Parameters & \\
\hline 2.9 & Ring Parameters & \\
\hline 2.10 & Channel Parameters & \\
\hline 2.11 & Overlap Parameters & \\
\hline
\end{tabular}

Table 9-6 Table of Tables for New Object's ASN Definitions and Example Data

\begin{tabular}{|l|l|}
\hline ASN Definition & Example Data \\
\hline Table 9-7 & Table 9-8 \\
\hline Table 9-9 & Table 9-10 \\
\hline Table 9-11, Table 9-12, and Table 9-13 & Table 9-14 \\
\hline Table 9-15 & Table 9-16 \\
\hline Table 9-17 & Figure 9-12 \\
\hline Table 9-18 & Figure 9-13 \\
\hline
\end{tabular}

NTCIP TS 3.5 standards define the detectors' objects for NTCIP compliant controllers. From those detectors, only a limited number need to support PRO-TRACTS implementation. Although current implementation of PRO-TRACTS requires only one detector per device (controller), future work might need more than one detector. For 
example, to account for two-way coordination, a PRO-TRACTS detector will be required on at least two different approaches.

The maximum number of PRO-TRACTS detectors, along with the number of PRO-TRACTS detectors currently activated in the controller is determined by the maxPRO-TRACTSDetectors and activePRO-TRACTSDetectors parameters respectively as described in Table 9-7. Example values of maxPRO-TRACTSDetectors and activePRO-TRACTSDetectors are shown in Table 9-8.

\section{Table 9-7 PRO-TRACTS Detectors Object}

\section{PRO-TRACTS detectors}

PRO-TRACTSDetector OBJECT IDENTIFIER

$::=\{$ detector $\mathrm{X}\}$

-- This node contains the object necessary to support PRO-TRACTS application

X.1 Maximum PRO-TRACTS detectors

maxPRO-TRACTSDetectors OBJECT-TYPE

SYNTAX INTEGER $(0 . .255)$

ACCESS read-only

STATUS mandatory

DESCRIPTION

"The maximum number of PRO-TRACTS detectors supported in this device. This value indicates how many rows are in the physicalChannelsTable object"

\section{X.2 Active PRO-TRACTS detectors}

activePRO-TRACTSDetectors OBJECT-TYPE

SYNTAX INTEGER $(0 . .255)$

ACCESS read-write

STATUS mandatory

DESCRIPTION

"The number of PRO-TRACTS detector in this device. This object value multiplied by the value of cyclesSaved object indicates how many rows are in the occcupancyProfileTable and countProfileTable objects"

Table 9-8 Example of PRO-TRACTS Detectors

\begin{tabular}{|l|l|}
\hline MaxPRO-TRACTSDetectors & 10 \\
\hline ActivePRO-TRACTSDetectors & 3 \\
\hline
\end{tabular}


Since PRO-TRACTS detectors are basically advanced detectors, they possess all of the attributes of the ordinary detectors plus some special attributes. The relationship between PRO-TRACTS detectors and the advanced detectors is specified in the physical channels table (Table 9-9). This table maps PRO-TRACTS detectors to the controller detectors object. Table 9-10 shows an example of a PRO-TRACTS detectors assignment to the controller's detectors of the intersection shown in Figure 9-9. In the figure, controller detector number 2 is declared as the first PRO-TRACTS detector, while controller detector number 10 is declared as the second PRO-TRACTS detector. The third detector is shown to include both detectors 2 and 10.

In general, the PRO-TRACTS algorithm uses just one lane. However, Table 9-8 shows alternate detector configurations that may be considered. 
Table 9-9 PRO-TRACTS Physical Channel Table

\section{X.3 Physical Channels Table}

physicalChannelsTable OBJECT-TYPE

SYNTAX SEQUENCE OF physicalChannelsEntry

ACCESS not-accessible

STATUS mandatory

DESCRIPTION

"A table containing physical channel definitions for PRO-TRACTS detectors"

physicalChannelsEntry OBJECT-TYPE

SYNTAX physicalChannelsEntry

ACCESS not-accessible

STATUS mandatory

DESCRIPTION

"The physical channel address for one of PRO-TRACTS detectors"

INDEX \{PRO-TRACTSDetectorNumber\}

physicalChannelsEntry::= SEQUENCE\{

PRO-TRACTSDetectorNumber INTEGER,

PRO-TRACTSDetectorChannel INTEGER\}

\section{X.3.1 PRO-TRACTS Detector Number}

PRO-TRACTSDetectorNumber OBJECT-TYPE

SYNTAX INTEGER $(0 . .255)$

ACCESS read-only

Status mandatory

DESCRIPTION

"PRO-TRACTS Detector Number for this entry. This value shall not exceed activePROTRACTSDetectors object value"

\section{X.3.2 PRO-TRACTS Detector Channel}

PRO-TRACTSDetectorChannel OBJECT-TYPE

SYNTAX INTEGER $(0 . .255)$

ACCESS read-write

Status mandatory

DESCRIPTION

"PRO-TRACTS detector channel for this entry. This value shall not exceed vehicleDetectorNumber* object value"

* Defined in TS 3.5 section 2.3.2 


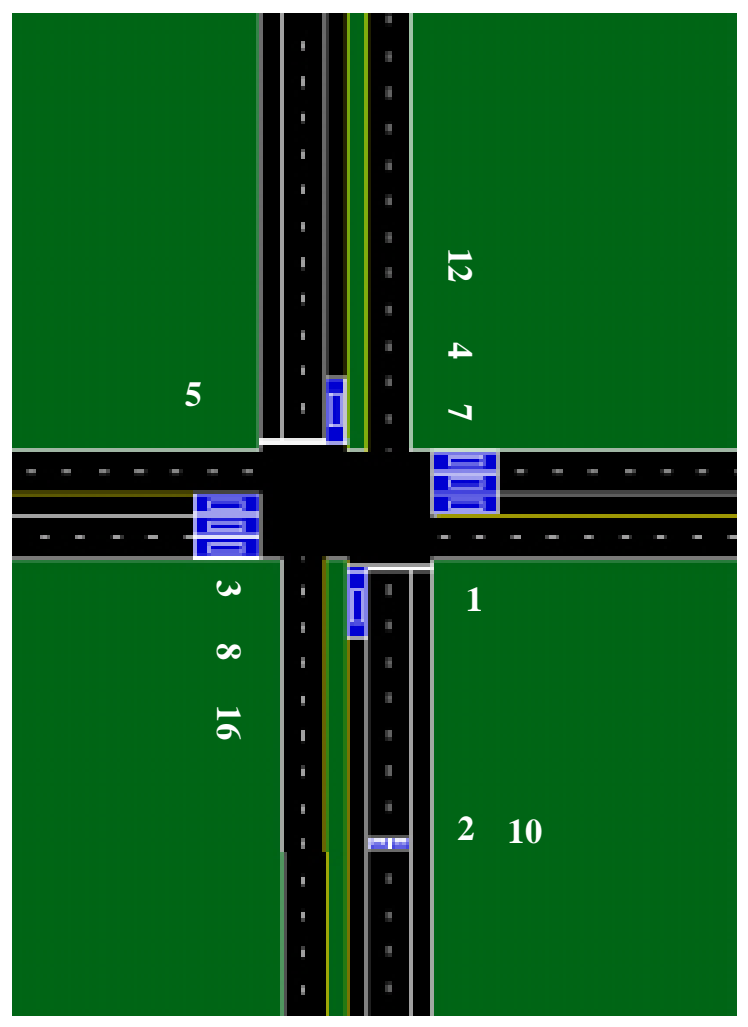

Figure 9-9 PRO-TRACTS Detectors Assignment

Table 9-10 PRO-TRACTS Detectors Assignment

\begin{tabular}{|l|l|l|l|l|l|l|l|l|l|l|l|l|l|l|l|l|}
\hline $\begin{array}{l}\text { PRO-TRACTS } \\
\text { Detectors } \\
(\mathrm{X})\end{array}$ & \multicolumn{9}{|l|}{ Intersection Detectors (2.3) } \\
\cline { 2 - 13 } & 1 & 2 & 3 & 4 & 5 & 6 & 7 & 8 & 9 & 10 & 11 & 12 & 13 & 14 & 15 & 16 \\
\hline 1 & & $\mathrm{X}$ & & & & & & & & & & & & & & \\
\hline 2 & & & & & & & & & & $\mathrm{X}$ & & & & & & \\
\hline 3 & $\mathrm{X}$ & & & & & & & & $\mathrm{X}$ & & & & & & \\
\hline
\end{tabular}

All PRO-TRACTS parameters necessary for the algorithm to function properly are specified in the PRO-TRACTS parameter table (Table 9-14). The ASN definitions for those parameters are defined in Table 9-11 through Table 9-13 and example data are 
shown in Table 9-14. The PRO-TRACTS parameters table lists the thresholds needed for activation of PRO-TRACTS. As discussed in Chapter 7, PRO-TRACTS is activated at three different levels, depending on the value of the calculated F'. The default values for the three F' thresholds are $0.25,0.75$, and 1.0 , respectively. These values were developed and discussed in Section 7.4.3 of Chapter 7. The first level moves the offset by the value of the offsetStep1 parameter; the second level moves the offset by the value of the offsetStep2 parameter; and the third level moves the offset by the value of the offsetStep3 parameter. Although the F' thresholds were developed using increments of $1 / 6$ of the cycle, it is recommended that the offset steps be of smaller values based on the fact that PRO-TRACTS is a reactive algorithm that uses previous cycle's information to adjust the offset. Since the next cycle's information might not be the same, it is better not to commit to high offset shifts. The default values for these parameters are 5 seconds, 2/12, and 3/12 of the cycle length. A small value is recommended for the offsetStep1 parameter so that a higher resolution of offset tuning can be achieved. Table 9-14 shows example default values calculated for a 90-second cycle length.

Once the algorithm is activated, the offset shift direction is determined by calculating the occupancy profile's areas to the left and to the right of the green window and the offset is moved in the direction of the largest area. The summation threshold determines how much the occupancy profile's area in one side of the green window has to exceed the occupancy profile's area at the other side of the green window for the algorithm to move the offset. This threshold is needed for stability purposes. PROTRACTS will not move the offset unless the difference in areas exceeds the threshold. The default value for this parameter is five seconds. 
The binSize parameter determines the duration in seconds over which the occupancy and count values are averaged. Averaging occupancy and count over bin sizes greater than one second helps to filter noise in data. However, averaging over too-large bin sizes causes the algorithm to smooth out detector actuation that may be important in determining the performance of the current offset. The default value for this parameter is five seconds.

The numberOfCycles parameter determines the number of cycles over which the actuation profiles are averaged. It should be noted that averaging the profiles over several cycles introduces stability into the algorithm performance, by averaging out the effect of the noise in the traffic pattern data. However, the algorithm responds slower to the changes in traffic patterns in this case. The default number of the numberOfCycles parameter is one cycle.

The shiftValue parameter specifies the width of the abscissa of the occupancy profile's area to either side of the green window that is used in determining the direction of the offset shift. This is not necessarily the shift in the offset applied by the algorithm, which is only affected by the cycle length and the maxStep parameters.

The updateFrequency parameter determines how frequently PRO-TRACTS evaluates the performance of the current offsets. This parameter should be coupled with the numberOfCycles parameter, such that the algorithm uses the information of the ' $n$ ' previous cycles if it is activated every ' $n$ ' number of cycles. However, this is not mandatory, rather it is an option for the operator of the system. Activating PRO-TARCTS every two or three cycles, instead of every cycle, could be useful to minimize or avoid the 
effects introduced by cycle transitioning. For example, in Figure 9-10-a, the updateFrequency was configured to be 1 . In Figure $9-10-b$, the update frequency was configured to be 5. If cycle transitioning introduces instabilities to the system, it might be beneficial to delay evaluating the performance of the offset until the system stabilizes. However, if the cycle transitioning does not significantly affect the system state, evaluating PRO-TRACTS every few cycles might lead to sluggishness in the algorithm performance by not responding to changes in traffic demand instantaneously. 
Actual Offset Transitioning

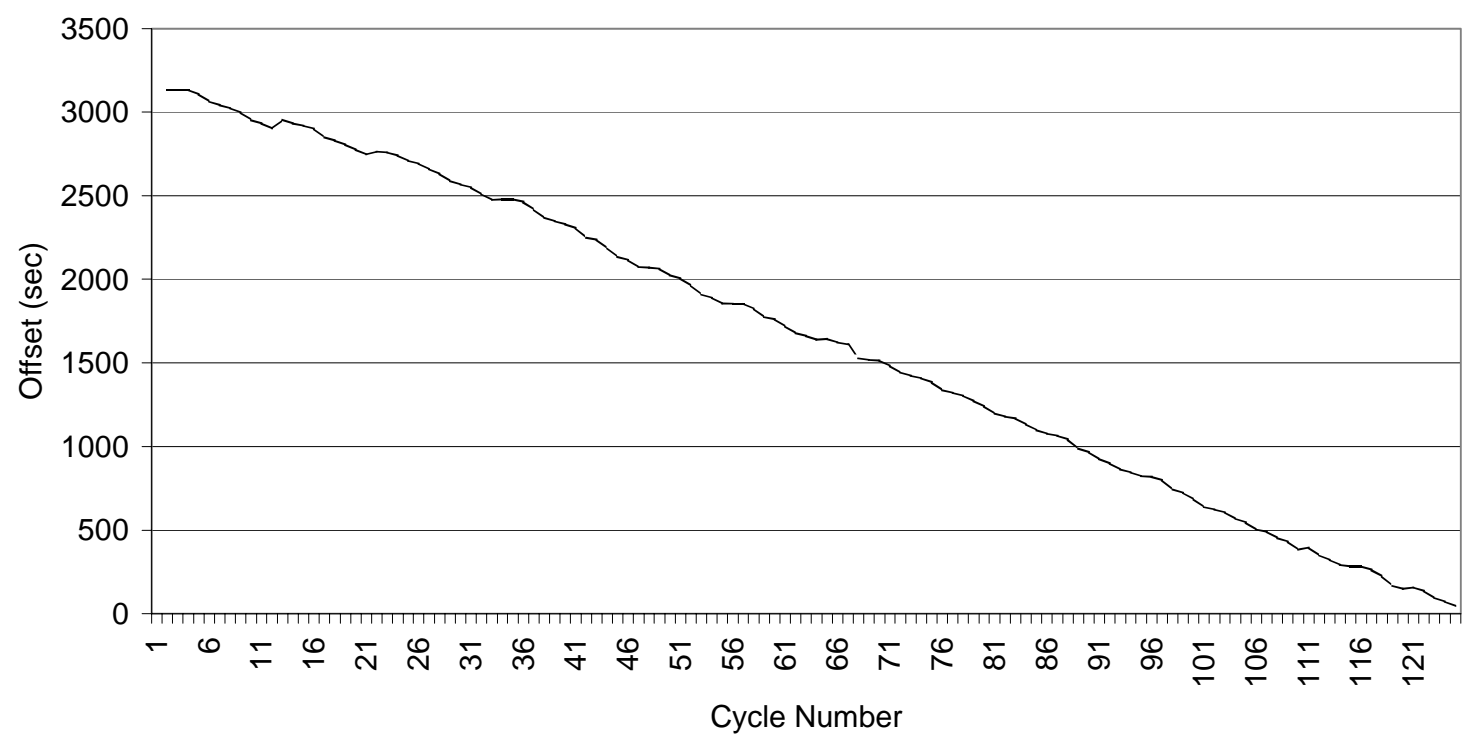

a) PRO-TRACTS Activated Every Cycle

Actual Offset Transitioning

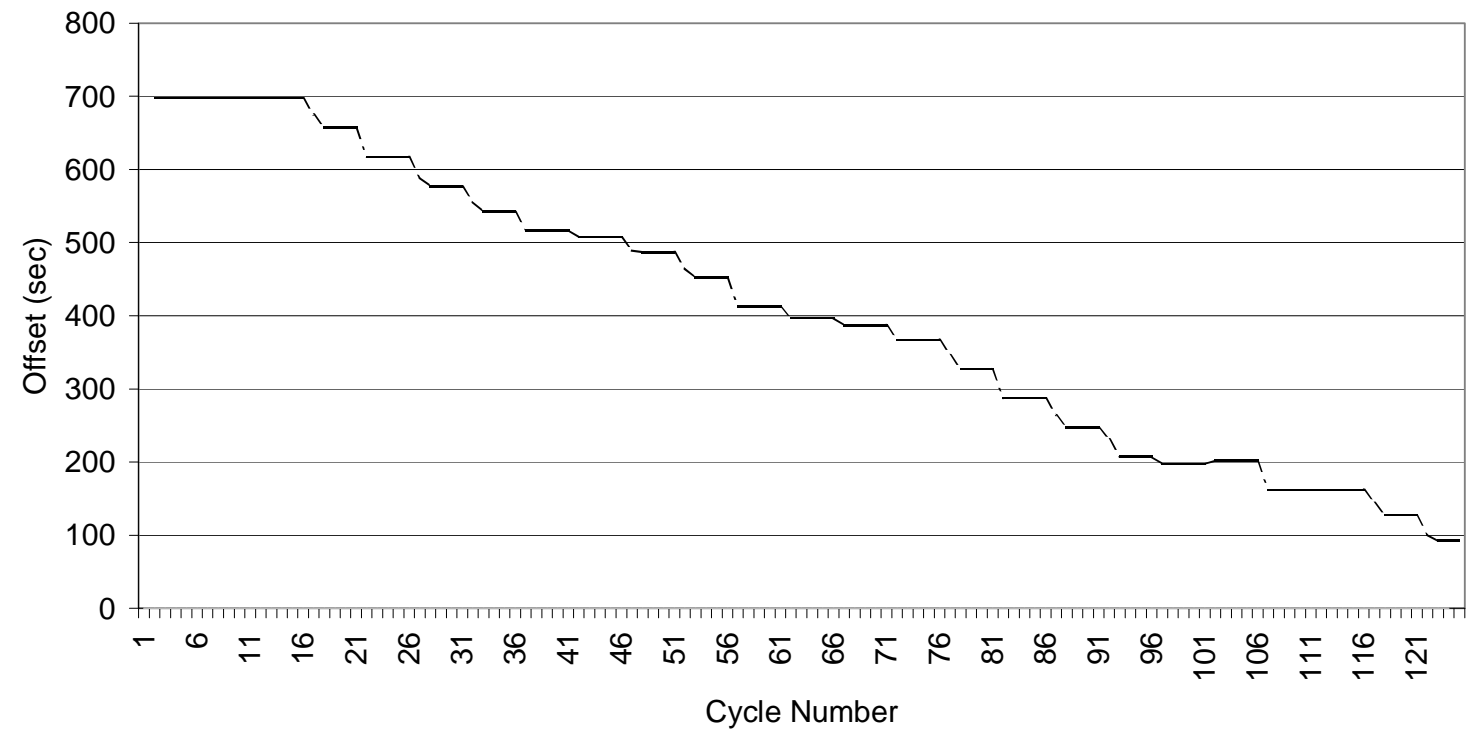

b) PRO-TRACTS Activated Every 5 Cycles

Figure 9-10 Effect of the updateFrequency Parameter on Offset Transitioning 
The maxStep parameter determines the maximum value the offset can be incremented or decremented at the controller. The parameter needs to be set to a maximum value that will not trigger the plans' transitioning algorithms. This parameter is only provided to control the plan transitioning algorithms in the controller. If the transitioning algorithms are not activated or the offset download does not activate them, which could be the case, then this parameter need not be set.

Figure 9-11 shows a conceptual illustration of some of PRO-TRACTS parameters.

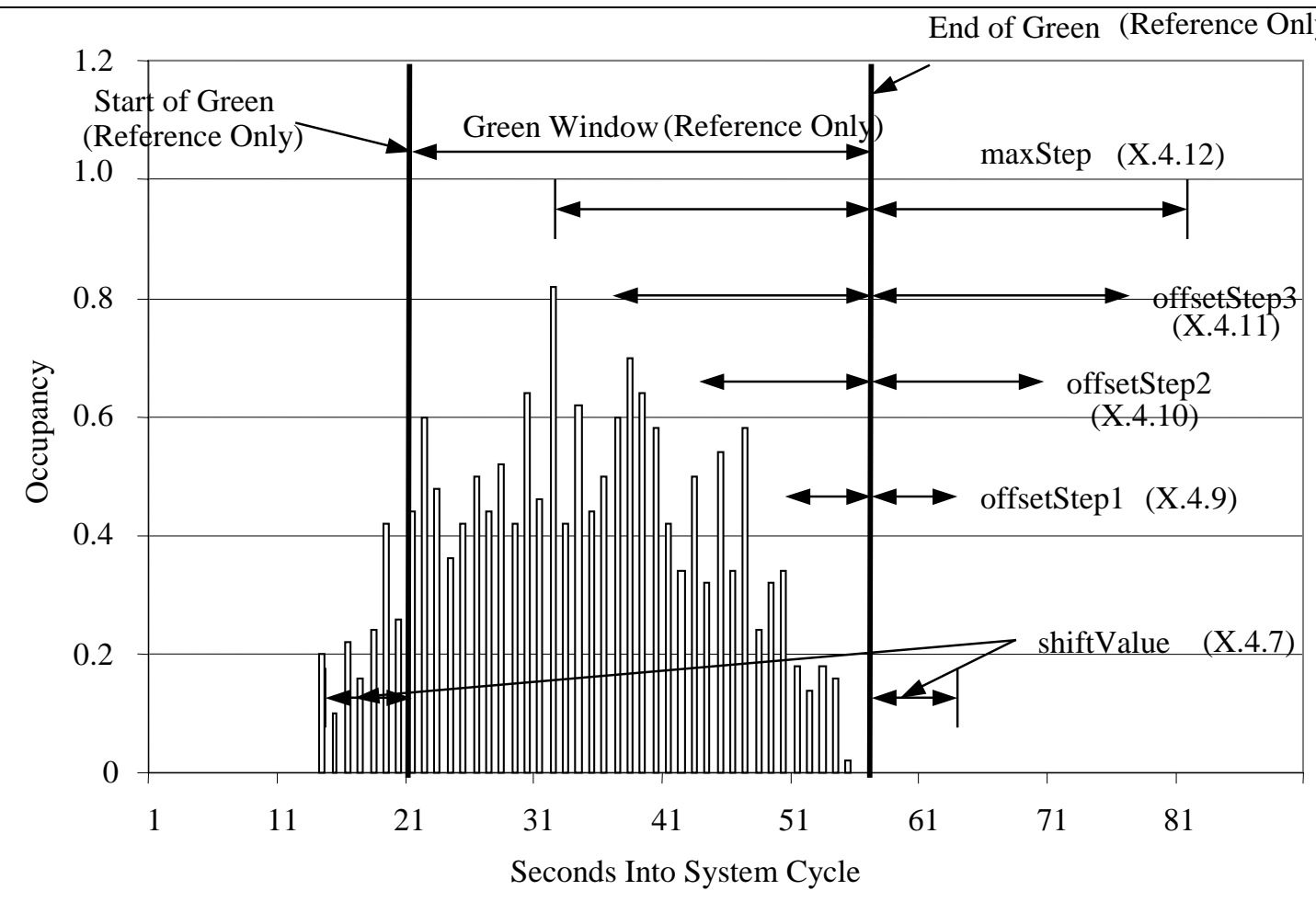

Figure 9-11 PRO-TRACTS Parameters 
Table 9-11 PRO-TRACTS Parameters (table 1 of 3)

\section{X.4 PRO-TRACTS Parameter Table \\ PRO-TRACTSParameters OBJECT-TYPE \\ SYNTAX PRO-TRACTSParameters \\ ACCESS not-accessible \\ STATUS mandatory \\ DESCRIPTION}

"PRO-TRATCS control parameters"

PRO-TRACTSParameters::= SEQUENCE\{

F-Threshold1 INTEGER,

F-Threshold2 INTEGER,

F-Threshold3 INTEGER,

sumThresh INTEGER,

binSize INTEGER,

numberOfCycles INTEGER,

shiftValue INTEGER,

updateFrequency INTEGER,

offsetStep1 INTEGER,

offsetStep2 INTEGER,

offsetStep3 INTEGER,

maxStep INTEGER

\section{X.4.1 F-Threshold1}

F-Threshold1 OBJECT-TYPE

SYNTAX INTEGER $(0 . .255)$

ACCESS read-write

Status mandatory

DESCRIPTION

" F' threshold multiplied by a 100 to invoke PRO-TRACTS and apply an offset change determined by offsetStep 1 parameter. This value should range between 0 and 100 "

\section{X.4.2 F-Threshold2}

F-Threshold2 OBJECT-TYPE

SYNTAX INTEGER $(0 . .255)$

ACCESS read-write

Status mandatory

DESCRIPTION

"F' threshold multiplied by a 100 to invoke PRO-TRACTS and apply an offset change determined by offsetStep 2 parameter. This value should range between 0 and 100 "

\section{X.4.3 F-Threshold3}

F-Threshold3 OBJECT-TYPE

SYNTAX INTEGER $(0 . .255)$

ACCESS read-write

Status mandatory

DESCRIPTION

" F' threshold multiplied by a 100 to invoke PRO-TRACTS and apply an offset change determined by offsetStep3 parameter. This value should range between 0 and 100 ” 
Table 9-12 PRO-TRACTS Parameters (table 2 of 3)

X.4.4 sumThreshold

sumThreshold OBJECT-TYPE

SYNTAX INTEGER $(0 . .255)$

ACCESS read-write

Status mandatory

DESCRIPTION

"Sum threshold. Determines the difference between occupancy profile areas to the right and left of the current green window to invoke PRO-TRACTS. This value should range between 0 and 255 "

X.4.5 Bin Size

binSize OBJECT-TYPE

SYNTAX INTEGER $(0 . .255)$

ACCESS read-write

Status mandatory

DESCRIPTION

"Bin size measured in seconds over which count and occupancy are averaged in each

cycle"

X.4.6 Number of Cycles

numberOfCycles OBJECT-TYPE

SYNTAX INTEGER $(0 . .255)$

ACCESS read-write

Status mandatory

DESCRIPTION

"Number of cycles over which count and occupancy profiles are to be aggregated"

X.4.7 Shift Value

shiftValue OBJECT-TYPE

SYNTAX INTEGER $(0 . .255)$

ACCESS read-write

Status mandatory

DESCRIPTION

"A parameter that specifies how many seconds PRO-TRACTS uses to estimate the potential benefit if offset was moved in either direction"

X.4.8 Update Frequency

updateFrequency OBJECT-TYPE

SYNTAX INTEGER $(0 . .255)$

ACCESS read-write

Status mandatory

DESCRIPTION the offsets"

"A parameter that specifies how often (in cycles) PRO-TRACTS evaluates and updates 
Table 9-13 PRO-TRACTS Parameters (table 3 of 3)

X.4.9 Offset Step1

OffsetStep1 OBJECT-TYPE

SYNTAX INTEGER (0..255)

ACCESS read-write

Status mandatory

DESCRIPTION

"A parameter that specifies by how many seconds PRO-TRACTS increments or decrements the offset once the calculated F' value exceeds F-Threshold1 but not F-Threshold2"

X.4.10 Offset Step2

offsetStep OBJECT-TYPE

SYNTAX INTEGER $(0 . .255)$

ACCESS read-write

Status mandatory

DESCRIPTION

"A parameter that specifies by how many seconds PRO-TRACTS increments or decrements the offset once the calculated F' value exceeds F-Threshold2 but not F-Threshold3"

X.4.11 Offset Step3

offsetStep OBJECT-TYPE

SYNTAX INTEGER $(0 . .255)$

ACCESS read-write

Status mandatory

DESCRIPTION

"A parameter that specifies by how many seconds PRO-TRACTS increments or decrements the offset the calculated F' value exceeds F-Threshold3"

X.4.12 Max Offset Step

maxStep OBJECT-TYPE

SYNTAX INTEGER $(0 . .255)$

ACCESS read-write

Status mandatory

DESCRIPTION

"A parameter that specifies the maximum number of seconds PRO-TRACTS can increment or decrement the offset once offset transitioning is warranted. This value overrides the fraction of the cycle suggested by the F thresholds" 
Table 9-14 Example of PRO-TRACTS Parameters

\begin{tabular}{|l|l|}
\hline PRO-TRACTS Parameter & Value \\
\hline F-Threshold1 & 0.25 \\
\hline F-Threshold2 & 0.75 \\
\hline F-Threshold3 & 1.0 \\
\hline sumThresh & 5 \\
\hline binSize & 5 \\
\hline numberOfCycles & 1 \\
\hline shiftValue & 10 \\
\hline updateFrequency & 1 \\
\hline offsetStep1 & 5 \\
\hline offsetStep2 & 15 \\
\hline offsetStep3 & 30 \\
\hline maxStep & 40 \\
\hline
\end{tabular}

PRO-TRACTS saves the actuation of several previous cycles to use in its evaluation of the offset performance. The number of cycles' actuation needed for evaluation purposes depends on the numberOfCycles and the updateFrequency parameters discussed in the previous section. Although several cycles' actuation could be used, controllers that support PRO-TRACTS implementation should define how many cycles could be stored. The maxCycleSaved parameter determines how many cycles' actuation can be saved in this controller where the cycleSaved parameter determines the actual number of cycles' actuation saved as determined by the user. The ASN for saved cycles attributes is shown in Table 9-15. An example value for cyclesSaved is shown in Table 9-16. 


\section{Table 9-15 PRO-TRACTS Saved Cycles}

X.5 Max Number of Saved Cycles

maxCyclesSaved OBJECT-TYPE

SYNTAX INTEGER $(0 . .255)$

ACCESS read-only

Status mandatory

DESCRIPTION

"Maximum number of cycles over which count and occupancy profiles can be saved"

X.6 Number of Saved Cycles

cyclesSaved OBJECT-TYPE

SYNTAX INTEGER $(0 . .255)$

ACCESS read-write

Status mandatory

DESCRIPTION

"The number of cycles over which latest count and occupancy profiles are saved. This number is not to exceed the value of maxCycleSaved object"

Table 9-16 Example of PRO-TRACTS Saved Cycles

\begin{tabular}{|l|l|}
\hline cyclesSaved & 5 \\
\hline
\end{tabular}

The occupancy table stores the occupancy profiles over all saved cycles and for all PRO-TRACTS detectors. Each entry in the table is the occupancy profile of one PROTRACTS detector over a previous cycle. The table is laid out such that each entry is a PRO-TRACTS detector number, a cycle number, and an occupancy profile. The combination of the PRO-TRACTS detector number and the cycle number form the primary key in the table. The occupancy profile is a sequence of occupancy values averaged over the bin size, as defined earlier.

Figure 9-12 shows a conceptual occupancy profile's table. The ASN for the occupancy profile is shown in Table 9-17. 


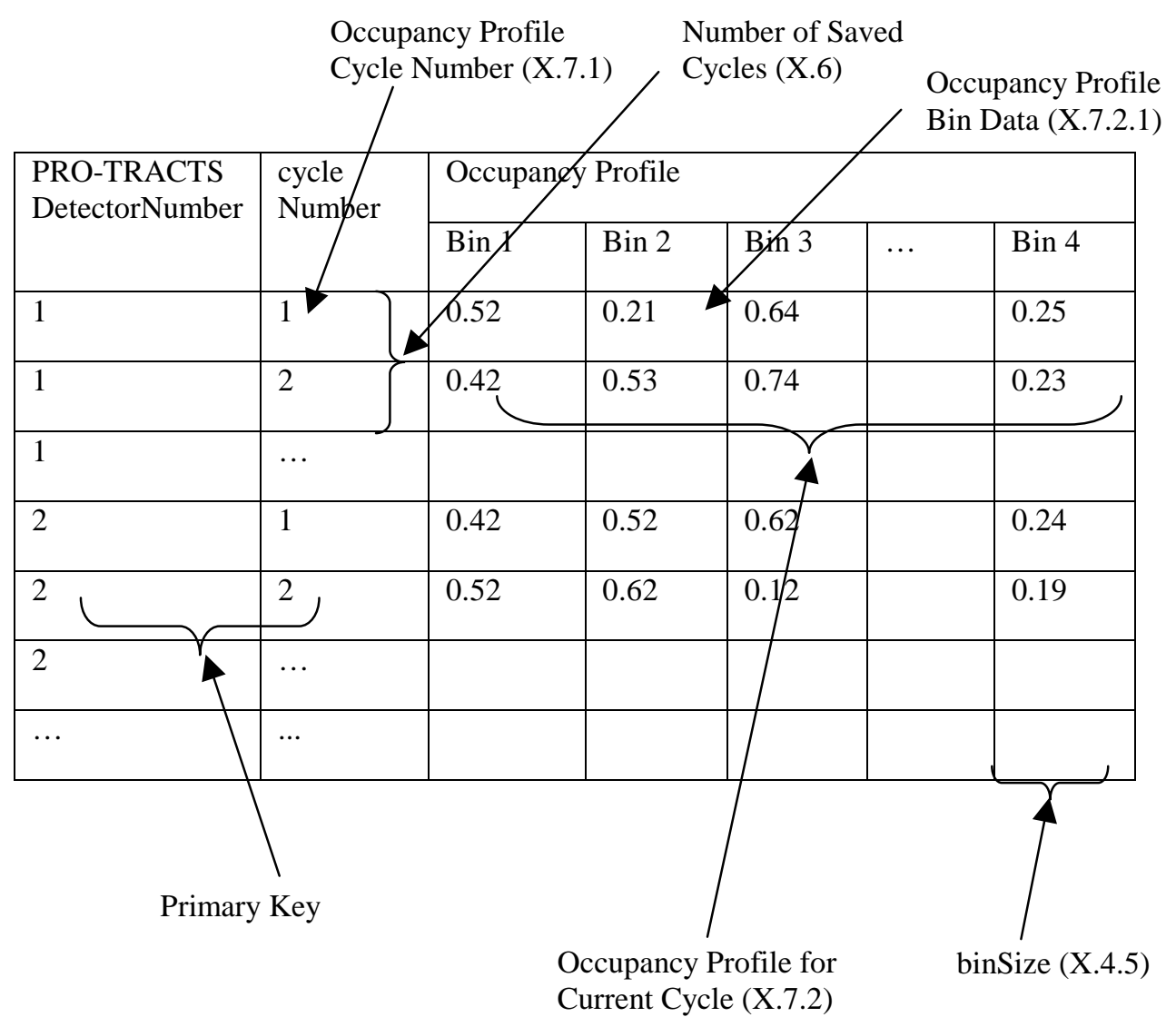

Figure 9-12 Occupancy Profile Table 


\section{Table 9-17 PRO-TRACTS Occupancy Table}

X.7 Occupancy Table occupancyProfileTable OBJECT-TYPE

SYNTAX SEQUENCE OF occupancyProfileEntry

ACCESS not-accessible

STATUS mandatory

DESCRIPTION

"A table containing occupancy profile collected for all PRO-TRACTS detectors over the cycles saved in this unit. The number of rows in this table is equal to the active PRO-

TRACTSDetectors object value multiplied by cyclesSaved object value" occupancyProfileEntry OBJECT-TYPE

SYNTAX occupancyProfileEntry

ACCESS not-accessible

STATUS mandatory

DESCRIPTION

"The occupancy profile data over the bins collected for one of the PRO-TRACTS detectors in the device over one of the saved cycles"

INDEX \{PRO-TRACTSDetectorNumber, cycleNumber\}

occupancyProfileEntry::= SEQUENCE \{

cycleNumber INTEGER,

occupancyProfileBins occupancyProfileBins\}

X.7.1 Occupancy Profile Cycle Number

occupancyCycleNumber OBJECT-TYPE

SYNTAX INTEGER $(0 . .255)$

ACCESS read-only

Status mandatory

DESCRIPTION

"Cycle number for objects in this row. The value shall not exceed the cyclesSaved object value"

\section{X.7.2 Occupancy Profile}

occupancyProfileBins OBJECT-TYPE

SYNTAX occupancyProfileBins

ACCESS not-accessible

STATUS mandatory

DESCRIPTION

"The occupancy profile data over the bins collected for one of the PRO-TRACTS

detectors in the device over occupancyCycleNumber object value"

occupancyProfileBins::= SEQUENCE \{

occupancyBin INTEGER\}

X.7.2.1 Occupancy Profile bin data

occupancyBin OBJECT TYPE

SYNTAX INTEGER $(0 . .255)$

ACCESS read-only

STATUS mandatory

DESCRIPTION

"Average PRO-TRACTS detector occupancy collected over binSize object value. This value should range from 0 to $100 . "$ 
The count table stores the count profiles over all saved cycles and for all PROTRACTS detectors. Similar to the occupancy table, each entry in the table is a PROTRACTS detector number, a cycle number, and a count profile as shown in Figure 9-13. The ASN definition is shown in Table 9-18.

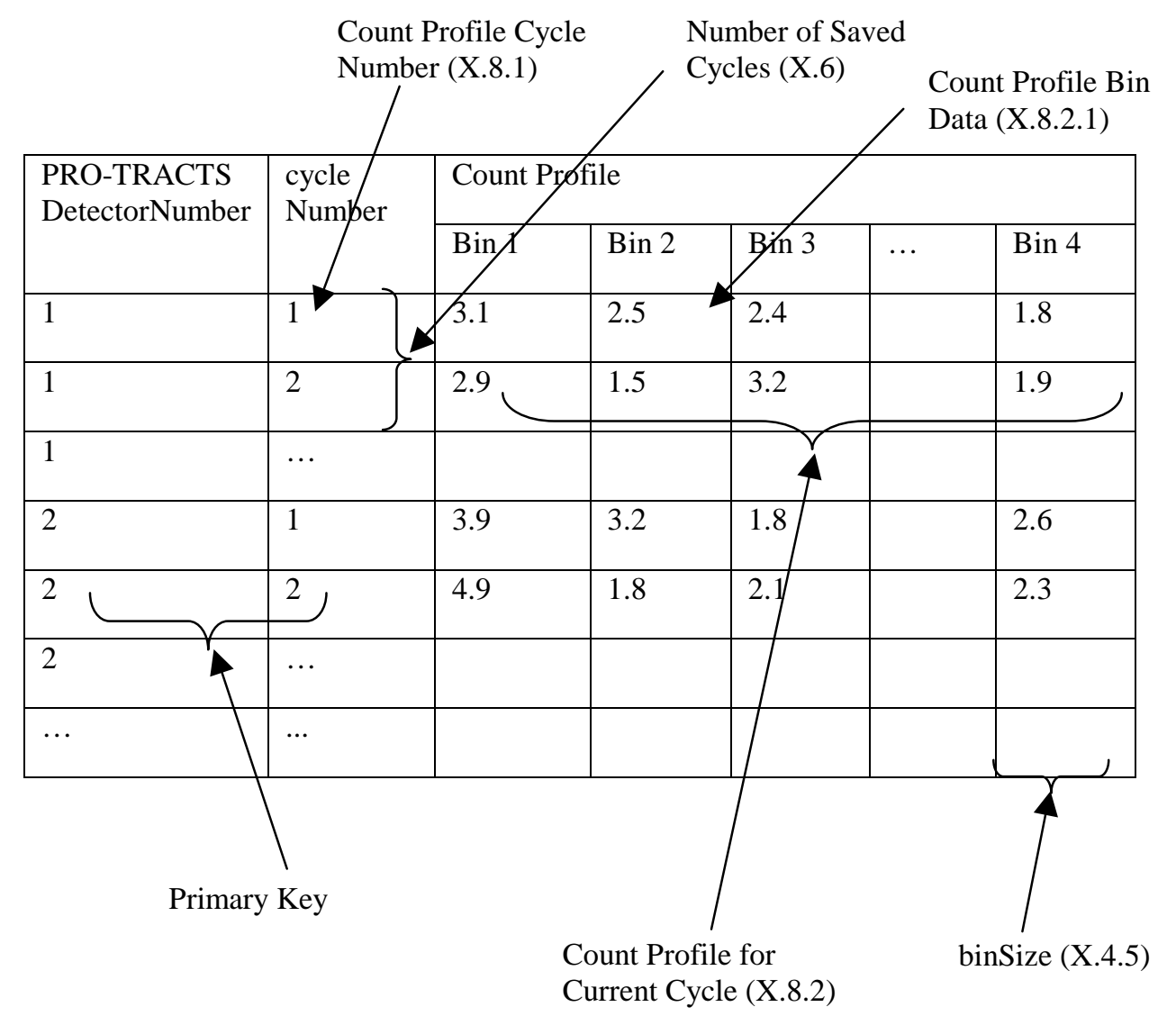

Figure 9-13 Count Profile Table 


\section{Table 9-18 PRO-TRACTS Count Table}

X.8 Count Table

countProfileTable OBJECT-TYPE

SYNTAX SEQUENCE OF countProfileEntry

ACCESS not-accessible

STATUS mandatory

DESCRIPTION

"A table containing count profile collected for all PRO-TRACTS detectors over the cycles saved in this unit. The number of rows in this table is equal to the active PRO-

TRACTSDetectors object value multiplied by cyclesSaved object value"

countProfileEntry OBJECT-TYPE

SYNTAX countProfileEntry

ACCESS not-accessible

STATUS mandatory

DESCRIPTION

"The count profile data over the bins collected for one of the PRO-TRACTS detectors in the device over one of the saved cycles"

INDEX \{PRO-TRACTSDetectorNumber, cycleNumber\}

countProfileEntry::= SEQUENCE\{

cycleNumber INTEGER,

countProfileBins countProfileBins

X.8.1 Count Profile Cycle Number

countCycleNumber OBJECT-TYPE

SYNTAX INTEGER $(0 . .255)$

ACCESS read-only

Status mandatory

DESCRIPTION

"Cycle number for objects in this row. The value shall not exceed the cyclesSaved object value"

\section{X.8.2 count Profile}

countProfileBins OBJECT-TYPE

SYNTAX countProfileBins

ACCESS not-accessible

STATUS mandatory

DESCRIPTION

"The count profile data over the bins collected for one of the PRO-TRACTS detectors in the device over countCycleNumber object value"

countProfileBins::= SEQUENCE \{

countBin INTEGER\}

X.8.2.1 Count Profile bin data

countBin OBJECT TYPE

SYNTAX INTEGER $(0 . .255)$

ACCESS read-only

STATUS mandatory

DESCRIPTION

"Average PRO-TRACTS detector count collected over binSize object value in a scale of 0 to 100 where 100 to 1 vehicle/second" 


\subsubsection{Memory Requirements}

The occupancy and count profile tables are the most demanding PRO-TRACTS parameters in terms of memory. The memory size needed for both tables would obviously depend on the maxCyclesSaved and maxPRO-TRACTSDetectors parameters. A memory requirement estimate is shown in Table 9-19. Note that the calculation takes into account the extreme case of the user selecting a binSize of 1 , which will require 255 bins in the occupancy and count profiles. It was also assumed that each of the occupancyBin and countBin values can be stored in one byte. In the example, one can see that the memory required would be 12,750 bytes plus a few hundred additional bytes to hold the information defined in Table 9-7, Table 9-9, Table 9-11, Table 9-12, Table 9-13 and Table 9-15. Alternatively, if a bin size of five seconds was selected, the memory required would be a more modest 2,550 bytes for the occupancy and count profiles.

Table 9-19 Example Memory Requirements

\begin{tabular}{|l|l|}
\hline maxPRO-TRACTSDetectors & 10 \\
\hline maxCyclesSaved & 5 \\
\hline Occupancy Table & 10 (detectors) $* 5$ (cycles) $* 255($ bins $) * 1($ byte $)=6,375$ Bytes \\
\hline Count Table & 10 (detectors $) * 5($ cycles $) * 255($ bins $) * 1($ byte $)=6,375$ Bytes \\
\hline
\end{tabular}

\subsection{Chapter's Conclusion}

This chapter provided an overview of hardware-in-the-loop simulation and NTCIP communication and described how hardware-in-the-loop simulation is integrated with NTCIP compliant controllers to evaluate PRO-TRACTS performance prior to field 
implementation. Finally, a new NTCIP object was defined for capturing detector actuation at the controller level. These objects were defined in ASN.1 and example data screens were presented. The chapter concluded by showing that the memory demands of new PRO-TRACTS detectors would be on the amount of 3,000 to 13,000 bytes, depending upon the binSize (X.4.5) selected, which are relatively modest memory requirements given the capabilities of most controllers.

Finally, for conceptual simplicity "binSize" was defined instead of the minimum number of bins. In a future deployment, the minimum number of bins may be a better method of specifying the Spacing of the count and occupancy tables. 


\section{CHAPTER 10- CASE STUDY: US31, KOKOMO, INDIANA}

This chapter discusses the results of the application of PRO-TRACTS to US 31 in Kokomo, Indiana both with CORSIM's internal Model and hardware-in-the-loop with the NTCIP controllers described in the previous chapter in section 9.3. The studied network consists of seven intersections in US31 between Lincoln Street and Sycamore Drive, shown in Figure 10-1. The intersection layout, ring structure, timings, detector configuration, phase parameters, traffic volumes, and turning percentages are listed in Appendix B. The impacts of applying PRO-TRACTS during the a.m. and p.m. peak periods will be discussed. The application of the hardware-in-the-loop with NTCIP controllers and offset transitioning will be given special emphasis. 

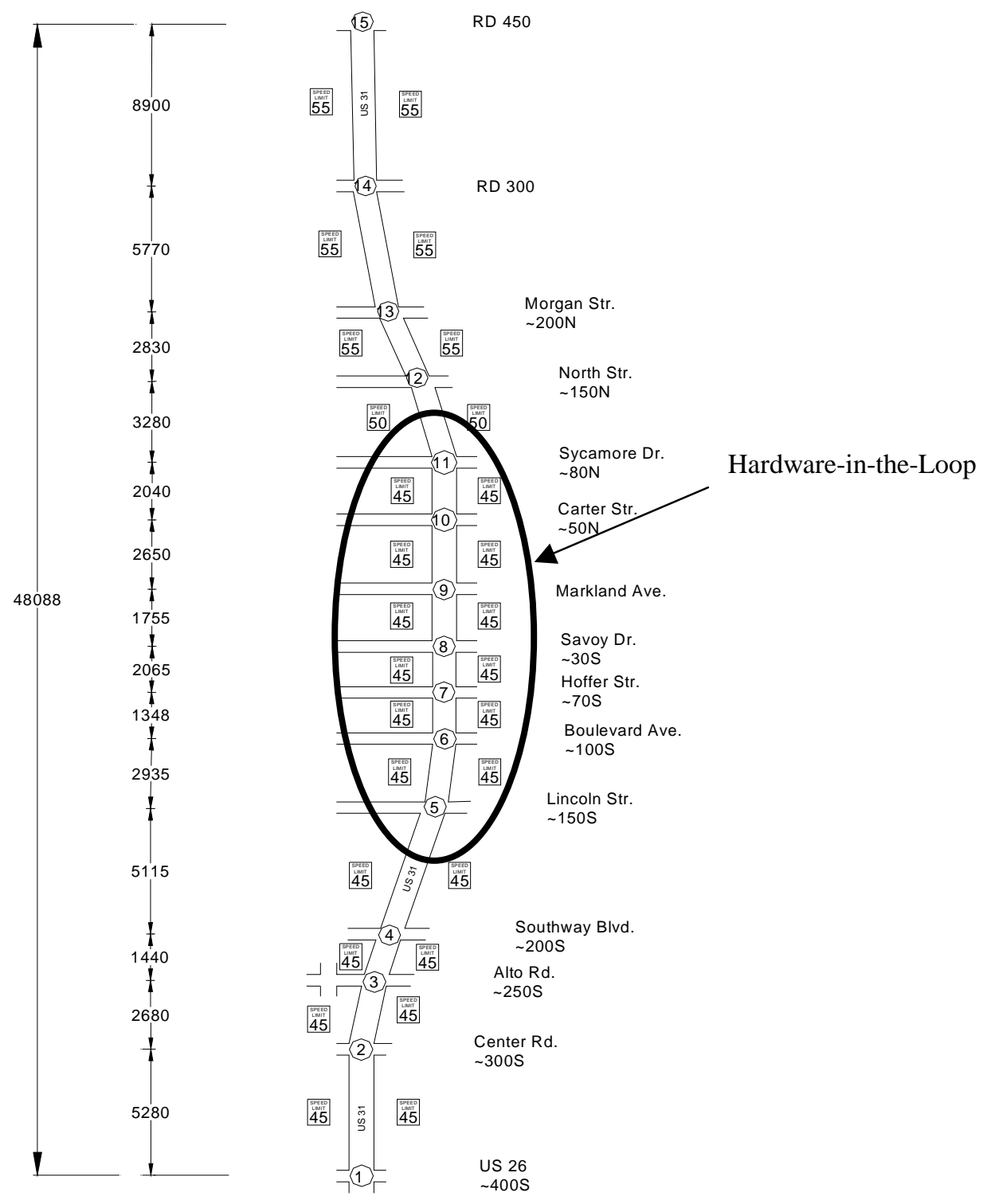

Figure 10-1 US 31 - Lincoln Street and Sycamore Drive 


\subsection{Phase Timings}

All phase timings and parameters were obtained from INDOT. Those timings were designed with Synchro for both the a.m. and p.m. peak periods. Table 10-1 lists the offsets and phase splits for the seven studied intersections.

Table 10-1 US 31 Coordination Plans and Splits

\begin{tabular}{|l|l|l|l|l|l|l|l|l|l|l|l|l|}
\hline & & \multicolumn{3}{l|}{ OFFSET } & & & & & & & & \\
\hline INT & TIME & CYC & COR & CON & 1 & 2 & 3 & 4 & 5 & 6 & 7 & 8 \\
\hline 1 & Am & 92 & 0 & 37 & 15 & 37 & 15 & 19 & 15 & 37 & -- & 34 \\
\hline 1 & Pm & 116 & 0 & 99 & 15 & 64 & 16 & 21 & 23 & 56 & -- & 37 \\
\hline 2 & Am & 92 & 55 & 83 & 14 & 46 & 14 & 18 & 24 & 36 & 14 & 18 \\
\hline 2 & Pm & 116 & 64 & 46 & 14 & 65 & 14 & 23 & 33 & 46 & 14 & 23 \\
\hline 3 & Am & 92 & 13 & 44 & 26 & 31 & -- & 35 & 14 & 43 & 14 & 21 \\
\hline 3 & Pm & 116 & 18 & 112 & 29 & 50 & -- & 37 & 14 & 65 & 14 & 23 \\
\hline 4 & Am & 92 & 3 & 34 & 14 & 43 & 14 & 21 & 14 & 43 & -- & 35 \\
\hline 4 & Pm & 116 & 107 & 90 & 26 & 53 & 15 & 22 & 15 & 64 & -- & 37 \\
\hline 5 & Am & 92 & 10 & 44 & 24 & 33 & 14 & 21 & 17 & 40 & 14 & 21 \\
\hline 5 & Pm & 116 & 67 & 55 & 22 & 54 & 21 & 19 & 17 & 59 & 21 & 19 \\
\hline 6 & Am & 92 & 48 & 80 & 20 & 36 & 15 & 21 & 14 & 42 & 15 & 21 \\
\hline 6 & Pm & 116 & 24 & 10 & 20 & 56 & 16 & 24 & 15 & 61 & 16 & 24 \\
\hline 7 & Am & 92 & 77 & 6 & 18 & 51 & -- & 23 & 16 & 53 & -- & 23 \\
\hline 7 & Pm & 116 & 50 & 25 & 17 & 66 & -- & 33 & 12 & 71 & -- & 33 \\
\hline 8 & Am & 92 & 38 & 47 & 24 & 52 & -- & 16 & 11 & 65 & -- & 16 \\
\hline 8 & Pm & 116 & 12 & 98 & 23 & 70 & -- & 23 & 16 & 77 & -- & 23 \\
\hline 9 & Am & 92 & 68 & 12 & 16 & 36 & 15 & 25 & 14 & 38 & 19 & 21 \\
\hline 9 & Pm & 116 & 68 & 72 & 19 & 39 & 32 & 26 & 15 & 43 & 26 & 32 \\
\hline 10 & Am & 92 & 31 & 52 & 16 & 53 & -- & 23 & 16 & 53 & -- & 23 \\
\hline 10 & Pm & 116 & 28 & 7 & 14 & 68 & -- & 34 & 20 & 62 & -- & 34 \\
\hline 11 & Am & 92 & 83 & 22 & 14 & 43 & 14 & 21 & 14 & 43 & 14 & 21 \\
\hline 11 & Pm & 116 & 11 & 113 & 22 & 53 & 14 & 27 & 14 & 61 & 14 & 27 \\
\hline
\end{tabular}

\subsection{PRO-TRACTS Parameters}

PRO-TRACTS was run with both CORSIM's internal control logic and hardwarein-the-loop in order to 1) to test the NTCIP offset download procedure, and 2) compare and contrast the results obtained with CORSIM's internal model to the results obtained 
with hardware-in-the-loop simulation. PRO-TRACTS detectors were set 150 feet upstream of each traffic signal. The incremental thresholds used for the activation for PRO-TRACTS were set at 0.25, 0.75, and 1.0 as discussed in Chapter 7, Section 7.4.2. The algorithm was set to evaluate the cycle profiles at the end of every cycle. Offsets were set to change by 5,15 , and 35 seconds, respectively, depending on the threshold exceeded.

\subsection{Simulation Results}

CORSIM simulations were run to evaluate the performance of PRO-TRACTS for both a.m. and p.m. peak periods, 6-10 a.m. and 2-6 p.m., respectively, with five different random number seeds. Traffic volumes during the a.m. and p.m. peak periods covered a wide range of traffic demand. Table 10-2 through Table 10-4 show the detailed entry volumes to intersection approaches by the period.

Simulations were conducted with the existing traffic patterns and offsets to study the effect of traffic pattern variation on the performance of PRO-TRACTS. As well as to compare and contrast the results obtained by internal CORSIM's logic and hardware-inthe-loop.PRO_TRACTS was run to coordinate the northbound traffic. 
Table 10-2 US 31 Detailed Traffic Volume (vph Table 1 of 3)

\begin{tabular}{|c|c|c|c|c|c|c|c|c|c|c|c|c|c|}
\hline INT & $\begin{array}{l}\text { BEG } \\
\text { END }\end{array}$ & $\begin{array}{l}7 \mathrm{AM} \\
8 \mathrm{AM}\end{array}$ & $\begin{array}{l}8 \mathrm{AM} \\
9 \mathrm{AM}\end{array}$ & $\begin{array}{c}9 \mathrm{AM} \\
10 \mathrm{~A}\end{array}$ & $\begin{array}{l}10 \mathrm{~A} \\
11 \mathrm{~A}\end{array}$ & $\begin{array}{l}11 \mathrm{~A} \\
12 \mathrm{P}\end{array}$ & $\begin{array}{l}12 \mathrm{P} \\
1 \mathrm{PM}\end{array}$ & $\begin{array}{l}1 \mathrm{PM} \\
2 \mathrm{PM}\end{array}$ & $\begin{array}{l}2 \mathrm{PM} \\
3 \mathrm{PM}\end{array}$ & $\begin{array}{l}3 \mathrm{PM} \\
4 \mathrm{PM}\end{array}$ & $\begin{array}{l}4 \mathrm{PM} \\
5 \mathrm{PM}\end{array}$ & $\begin{array}{l}5 \mathrm{PM} \\
6 \mathrm{PM}\end{array}$ & $\begin{array}{l}6 \mathrm{PM} \\
7 \mathrm{PM}\end{array}$ \\
\hline \multirow{12}{*}{$\begin{array}{l}\text { N } \\
\text { v } \\
\sim\end{array}$} & EBLT & 188 & 184 & 159 & 96 & 97 & 113 & 122 & 122 & 148 & 116 & 127 & 127 \\
\hline & EBTH & 66 & 81 & 72 & 60 & 54 & 57 & 79 & 53 & 78 & 97 & 107 & 116 \\
\hline & EBRT & 98 & 58 & 60 & 46 & 60 & 44 & 47 & 62 & 45 & 61 & 83 & 73 \\
\hline & WBLT & 57 & 70 & 57 & 40 & 36 & 37 & 37 & 31 & 37 & 65 & 52 & 44 \\
\hline & WBTH & 72 & 108 & 101 & 71 & 75 & 70 & 73 & 67 & 90 & 133 & 113 & 114 \\
\hline & WBRT & 56 & 81 & 77 & 73 & 68 & 102 & 77 & 98 & 68 & 83 & 63 & 66 \\
\hline & NBLT & 57 & 100 & 82 & 53 & 53 & 61 & 40 & 64 & 58 & 68 & 86 & 95 \\
\hline & NBTH & 798 & 982 & 762 & 590 & 551 & 521 & 526 & 581 & 631 & 532 & 684 & 726 \\
\hline & NBRT & 45 & 36 & 53 & 37 & 41 & 26 & 28 & 45 & 20 & 54 & 41 & 71 \\
\hline & SBLT & 38 & 59 & 52 & 49 & 51 & 77 & 84 & 78 & 88 & 114 & 95 & 124 \\
\hline & SBTH & 485 & 654 & 533 & 503 & 478 & 540 & 563 & 585 & 757 & 921 & 993 & 948 \\
\hline & SBRT & 41 & 78 & 62 & 68 & 100 & 126 & 115 & 109 & 153 & 230 & 207 & 193 \\
\hline \multirow{12}{*}{ 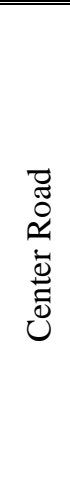 } & EBLT & 44 & 63 & 53 & 66 & 85 & 123 & 130 & 112 & 110 & 86 & 97 & 108 \\
\hline & EBTH & 35 & 79 & 62 & 51 & 87 & 103 & 149 & 125 & 119 & 144 & 153 & 145 \\
\hline & EBRT & 8 & 21 & 27 & 15 & 32 & 43 & 57 & 45 & 46 & 60 & 73 & 47 \\
\hline & WBLT & 59 & 56 & 68 & 55 & 44 & 45 & 62 & 72 & 53 & 93 & 72 & 67 \\
\hline & WBTH & 48 & 78 & 69 & 62 & 54 & 88 & 91 & 94 & 107 & 131 & 82 & 165 \\
\hline & WBRT & 112 & 173 & 199 & 158 & 150 & 227 & 227 & 181 & 231 & 162 & 183 & 175 \\
\hline & NBLT & 26 & 43 & 45 & 53 & 41 & 63 & 99 & 75 & 79 & 58 & 88 & 73 \\
\hline & NBTH & 1042 & 1326 & 1105 & 838 & 776 & 887 & 829 & 833 & 1016 & 787 & 1008 & 916 \\
\hline & NBRT & 49 & 43 & 43 & 31 & 31 & 36 & 40 & 33 & 42 & 33 & 52 & 64 \\
\hline & SBLT & 72 & 135 & 159 & 182 & 218 & 322 & 418 & 256 & 331 & 335 & 364 & 358 \\
\hline & SBTH & 568 & 726 & 606 & 657 & 723 & 790 & 796 & 869 & 1102 & 1315 & 1507 & 1409 \\
\hline & SBRT & 13 & 35 & 42 & 42 & 59 & 104 & 105 & 95 & 104 & 131 & 133 & 116 \\
\hline \multirow{12}{*}{ 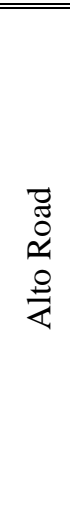 } & EBLT & 16 & 36 & 25 & 40 & 43 & 47 & 55 & 59 & 48 & 41 & 40 & 45 \\
\hline & EBTH & 31 & 46 & 86 & 70 & 105 & 121 & 158 & 113 & 119 & 113 & 160 & 151 \\
\hline & EBRT & 1 & 6 & 4 & 6 & 11 & 10 & 14 & 20 & 18 & 18 & 24 & 20 \\
\hline & WBLT & 35 & 64 & 59 & 48 & 62 & 73 & 92 & 88 & 93 & 99 & 125 & 110 \\
\hline & WBTH & 43 & 68 & 105 & 91 & 91 & 122 & 129 & 121 & 101 & 135 & 135 & 126 \\
\hline & WBRT & 34 & 47 & 43 & 34 & 44 & 48 & 71 & 43 & 46 & 40 & 40 & 57 \\
\hline & NBLT & 121 & 255 & 273 & 275 & 264 & 317 & 386 & 361 & 301 & 362 & 361 & 386 \\
\hline & NBTH & 1093 & 1490 & 1124 & 772 & 718 & 864 & 846 & 720 & 1014 & 860 & 959 & 931 \\
\hline & NBRT & 64 & 99 & 64 & 57 & 55 & 75 & 50 & 25 & 94 & 45 & 83 & 56 \\
\hline & SBLT & 15 & 37 & 59 & 63 & 65 & 110 & 118 & 61 & 52 & 42 & 69 & 131 \\
\hline & SBTH & 425 & 673 & 601 & 536 & 660 & 789 & 877 & 711 & 1138 & 1326 & 1363 & 1203 \\
\hline & SBRT & 6 & 29 & 46 & 58 & 66 & 71 & 76 & 50 & 60 & 48 & 64 & 57 \\
\hline \multirow{12}{*}{$\begin{array}{l}\vec{D} \\
\frac{3}{n} \\
\overrightarrow{3} \\
3 \\
\overline{3} \\
\overline{0} \\
0\end{array}$} & "EBLT & $\begin{array}{lll}117 \\
\end{array}$ & (162 & 1125 & $\begin{array}{ll}109 \\
\end{array}$ & 121 & $\begin{array}{ll}185 \\
\end{array}$ & 197 & $\begin{array}{ll}184 \\
\end{array}$ & $\begin{array}{ll}182 \\
\end{array}$ & 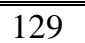 & 185 & $\begin{array}{ll}190 \\
\end{array}$ \\
\hline & EBTH & 36 & 83 & 126 & 114 & 123 & 200 & 200 & 156 & 153 & 227 & 263 & 376 \\
\hline & EBRT & 8 & 3 & 6 & 16 & 22 & 33 & 35 & 36 & 39 & 54 & 56 & 57 \\
\hline & WBLT & 16 & 23 & 31 & 14 & 22 & 55 & 23 & 30 & 29 & 49 & 55 & 63 \\
\hline & WBTH & 63 & 101 & 151 & 121 & 150 & 199 & 205 & 166 & 190 & 231 & 233 & 210 \\
\hline & WBRT & 22 & 45 & 59 & 39 & 44 & 49 & 100 & 96 & 72 & 88 & 50 & 87 \\
\hline & NBLT & 7 & 21 & 37 & 37 & 37 & 52 & 49 & 60 & 66 & 55 & 69 & 70 \\
\hline & NBTH & 1015 & 340 & 1088 & 848 & 761 & 856 & 863 & 800 & 1073 & 869 & 953 & 907 \\
\hline & NBRT & 16 & 36 & 34 & 25 & 10 & 36 & 29 & 33 & 34 & 29 & 44 & 54 \\
\hline & SBLT & 15 & 14 & 36 & 48 & 60 & 122 & 152 & 108 & 107 & 115 & 164 & 164 \\
\hline & SBTH & 408 & 678 & 711 & 666 & 789 & 969 & 894 & 862 & 1098 & 448 & 297 & 465 \\
\hline & SBRT & 33 & 78 & 93 & 63 & 116 & 218 & 122 & 130 & 141 & 175 & 173 & 168 \\
\hline
\end{tabular}


Table 10-3 US 31 Detailed Traffic Volume (vph Table 2 of 3)

\begin{tabular}{|c|c|c|c|c|c|c|c|c|c|c|c|c|c|}
\hline INT & $\begin{array}{l}\text { BEG } \\
\text { END }\end{array}$ & $\begin{array}{l}7 \mathrm{AM} \\
8 \mathrm{AM} \\
\end{array}$ & $\begin{array}{l}8 \mathrm{AM} \\
9 \mathrm{AM} \\
\end{array}$ & $\begin{array}{c}9 \mathrm{AM} \\
10 \mathrm{~A} \\
\end{array}$ & $\begin{array}{l}10 \mathrm{~A} \\
11 \mathrm{~A}\end{array}$ & $\begin{array}{l}11 \mathrm{~A} \\
12 \mathrm{P} \\
\end{array}$ & $\begin{array}{c}12 \mathrm{P} \\
1 \mathrm{PM} \\
\end{array}$ & $\begin{array}{l}1 \mathrm{PM} \\
2 \mathrm{PM}\end{array}$ & $\begin{array}{l}2 \mathrm{PM} \\
3 \mathrm{PM} \\
\end{array}$ & $\begin{array}{l}3 \mathrm{PM} \\
4 \mathrm{PM}\end{array}$ & $\begin{array}{l}4 \mathrm{PM} \\
5 \mathrm{PM} \\
\end{array}$ & $\begin{array}{l}5 \mathrm{PM} \\
6 \mathrm{PM} \\
\end{array}$ & $\begin{array}{l}6 \mathrm{PM} \\
7 \mathrm{PM} \\
\end{array}$ \\
\hline \multirow{12}{*}{ 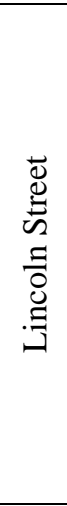 } & EBLT & 56 & 82 & 99 & 113 & 146 & 186 & 204 & 175 & 236 & 256 & 178 & 278 \\
\hline & EBTH & 280 & 339 & 240 & 137 & 164 & 184 & 416 & 281 & 367 & 323 & 296 & 301 \\
\hline & EBRT & 45 & 89 & 63 & 60 & 101 & 78 & 110 & 145 & 162 & 296 & 136 & 235 \\
\hline & WBLT & 41 & 29 & 73 & 55 & 96 & 137 & 121 & 93 & 141 & 241 & 280 & 320 \\
\hline & WBTH & 230 & 182 & 199 & 128 & 213 & 140 & 239 & 166 & 446 & 343 & 395 & 327 \\
\hline & WBRT & 85 & 158 & 124 & 100 & 151 & 253 & 175 & 95 & 191 & 234 & 192 & 146 \\
\hline & NBLT & 252 & 209 & 119 & 122 & 102 & 272 & 114 & 123 & 236 & 122 & 97 & 137 \\
\hline & NBTH & 709 & 1119 & 950 & 868 & 770 & 1016 & 970 & 934 & 1026 & 1056 & 1118 & 1146 \\
\hline & NBRT & 232 & 407 & 210 & 63 & 68 & 41 & 138 & 129 & 106 & 79 & 51 & 90 \\
\hline & SBLT & 140 & 189 & 122 & 94 & 91 & 206 & 219 & 179 & 147 & 127 & 117 & 144 \\
\hline & SBTH & 416 & 698 & 695 & 771 & 763 & 1132 & 934 & 1053 & 1012 & 1237 & 1175 & 1269 \\
\hline & SBRT & 83 & 91 & 53 & 54 & 100 & 83 & 80 & 117 & 183 & 121 & 97 & 99 \\
\hline \multirow{12}{*}{ 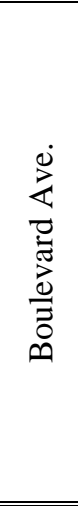 } & EBLT & 58 & 121 & 61 & 42 & 77 & 84 & 107 & 71 & 208 & 180 & 95 & 89 \\
\hline & EBTH & 157 & 237 & 139 & 107 & 110 & 262 & 266 & 205 & 389 & 395 & 239 & 216 \\
\hline & EBRT & 47 & 107 & 119 & 115 & 120 & 236 & 193 & 217 & 216 & 302 & 229 & 259 \\
\hline & WBLT & 86 & 99 & 60 & 57 & 66 & 120 & 87 & 76 & 189 & 118 & 99 & 87 \\
\hline & WBTH & 276 & 293 & 192 & 144 & 207 & 395 & 249 & 170 & 590 & 403 & 338 & 165 \\
\hline & WBRT & 35 & 43 & 38 & 25 & 65 & 176 & 52 & 40 & 92 & 64 & 56 & 34 \\
\hline & NBLT & 80 & 191 & 149 & 164 & 135 & 263 & 225 & 160 & 231 & 211 & 159 & 153 \\
\hline & NBTH & 721 & 985 & 909 & 853 & 1010 & 1306 & 1200 & 1034 & 1217 & 1284 & 1311 & 1214 \\
\hline & NBRT & 97 & 90 & 86 & 47 & 68 & 103 & 36 & 74 & 80 & 80 & 69 & 67 \\
\hline & SBLT & 85 & 68 & 50 & 36 & 44 & 176 & 138 & 95 & 152 & 83 & 61 & 62 \\
\hline & SBTH & 572 & 778 & 823 & 828 & 768 & 988 & 1074 & 963 & 114 & 1173 & 1113 & 1185 \\
\hline & SBRT & 96 & 49 & 58 & 50 & 66 & 102 & 66 & 102 & 77 & 116 & 54 & 55 \\
\hline \multirow{12}{*}{ 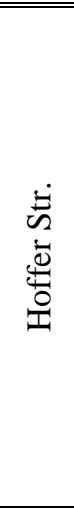 } & BLT & 33 & 52 & 63 & 78 & 119 & 228 & 198 & 235 & 204 & 204 & 197 & 197 \\
\hline & EBTH & 6 & 30 & 22 & 28 & 16 & 37 & 32 & 25 & 26 & 31 & 32 & 32 \\
\hline & EBRT & 57 & 45 & 49 & 73 & 77 & 152 & 115 & 186 & 130 & 146 & 119 & 119 \\
\hline & WBLT & 2 & 7 & 6 & 18 & 15 & 39 & 20 & 18 & 15 & 17 & 13 & 13 \\
\hline & WBTH & 2 & 13 & 15 & 14 & 15 & 34 & 27 & 24 & 35 & 24 & 26 & 26 \\
\hline & WBRT & 5 & 15 & 27 & 28 & 40 & 39 & 38 & 29 & 29 & 27 & 33 & 33 \\
\hline & NBLT & 50 & 95 & 98 & 69 & 56 & 117 & 12 & 138 & 103 & 114 & 110 & 110 \\
\hline & NBTH & 680 & 897 & 813 & 805 & 826 & 1104 & 1095 & 1185 & 1287 & 1245 & 1163 & 1163 \\
\hline & NBRT & 5 & 21 & 17 & 14 & 8 & 15 & 14 & 13 & 15 & 13 & 9 & 9 \\
\hline & SBLT & 12 & 18 & 42 & 37 & 33 & 41 & 42 & 24 & 31 & 24 & 33 & 33 \\
\hline & SBTH & 819 & 761 & 772 & 810 & 779 & 1231 & 1040 & 1256 & 1129 & 1212 & 1162 & 1162 \\
\hline & SBRT & 41 & 65 & 84 & 107 & 99 & 276 & 168 & 213 & 148 & 208 & 151 & 151 \\
\hline \multirow{12}{*}{$\begin{array}{l}\vec{D} \\
\vec{D} \\
\stackrel{\vec{\omega}}{\Delta}\end{array}$} & EBLT & 1 & 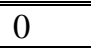 & 7 & 12 & 36 & 89 & 53 & 98 & 76 & 67 & 60 & 83 \\
\hline & EBTH & 0 & 4 & 6 & 6 & 15 & 19 & 15 & 16 & 17 & 17 & 25 & 27 \\
\hline & EBRT & 3 & 5 & 25 & 14 & 60 & 100 & 60 & 88 & 82 & 103 & 86 & 103 \\
\hline & WBLT & 5 & 20 & 17 & 23 & 16 & 12 & 9 & 20 & 25 & 18 & 10 & 14 \\
\hline & WBTH & 7 & 13 & 14 & 12 & 19 & 23 & 16 & 17 & 17 & 25 & 21 & 28 \\
\hline & WBRT & 8 & 24 & 30 & 27 & 26 & 21 & 9 & 25 & 17 & 35 & 33 & 32 \\
\hline & NBLT & 7 & 13 & 32 & 63 & 94 & 218 & 101 & 157 & 121 & 133 & 126 & 148 \\
\hline & NBTH & 741 & 968 & 792 & 865 & 1039 & 1247 & 626 & 1134 & 1161 & 1487 & 1337 & 1306 \\
\hline & NBRT & 6 & 1 & 8 & 4 & 5 & 7 & 3 & 8 & 1 & 12 & 6 & 5 \\
\hline & SBLT & 4 & 12 & 7 & 15 & 24 & 38 & 17 & 25 & 31 & 28 & 66 & 61 \\
\hline & SBTH & 970 & 937 & 973 & 857 & 1085 & 1265 & 646 & 1156 & 1471 & 1291 & 1342 & 1265 \\
\hline & SBRT & 1 & 8 & 3 & 22 & 56 & 93 & 49 & 94 & 89 & 63 & 76 & 89 \\
\hline
\end{tabular}


Table 10-4 US 31 Detailed Traffic Volume (vph Table 3 of 3)

\begin{tabular}{|c|c|c|c|c|c|c|c|c|c|c|c|c|c|}
\hline INT & $\begin{array}{l}\text { BEG } \\
\text { END }\end{array}$ & $\begin{array}{l}\text { 7AM } \\
8 \mathrm{AM}\end{array}$ & $\begin{array}{l}8 \mathrm{AM} \\
9 \mathrm{AM}\end{array}$ & $\begin{array}{c}9 \mathrm{AM} \\
10 \mathrm{~A}\end{array}$ & $\begin{array}{l}10 \mathrm{~A} \\
11 \mathrm{~A}\end{array}$ & $\begin{array}{l}11 \mathrm{~A} \\
12 \mathrm{P}\end{array}$ & $\begin{array}{l}12 \mathrm{P} \\
1 \mathrm{PM}\end{array}$ & $\begin{array}{l}1 \mathrm{PM} \\
2 \mathrm{PM}\end{array}$ & $\begin{array}{l}2 \mathrm{PM} \\
3 \mathrm{PM}\end{array}$ & $\begin{array}{l}3 \mathrm{PM} \\
4 \mathrm{PM}\end{array}$ & $\begin{array}{l}4 \mathrm{PM} \\
5 \mathrm{PM}\end{array}$ & $\begin{array}{l}5 \mathrm{PM} \\
6 \mathrm{PM}\end{array}$ & $\begin{array}{l}6 \mathrm{PM} \\
7 \mathrm{PM}\end{array}$ \\
\hline \multirow{12}{*}{ 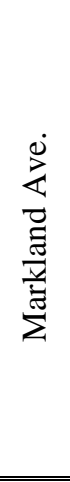 } & EBLT & 55 & 78 & 83 & 105 & 107 & 160 & 188 & 177 & 204 & 298 & 215 & 324 \\
\hline & EBTH & 103 & 164 & 214 & 294 & 390 & 488 & 435 & 440 & 514 & 620 & 567 & 602 \\
\hline & EBRT & 37 & 38 & 47 & 68 & 66 & 130 & 76 & 79 & 73 & 106 & 76 & 107 \\
\hline & WBLT & 88 & 102 & 133 & 154 & 238 & 240 & 241 & 245 & 286 & 376 & 253 & 270 \\
\hline & WBTH & 133 & 258 & 242 & 222 & 351 & 408 & 457 & 382 & 500 & 525 & 467 & 471 \\
\hline & WBRT & 104 & 133 & 135 & 121 & 149 & 215 & 200 & 164 & 220 & 278 & 227 & 246 \\
\hline & NBLT & 55 & 96 & 100 & 90 & 106 & 151 & 159 & 117 & 142 & 189 & 158 & 150 \\
\hline & NBTH & 645 & 689 & 614 & 614 & 651 & 734 & 749 & 688 & 948 & 1067 & 933 & 964 \\
\hline & NBRT & 70 & 116 & 103 & 201 & 171 & 299 & 213 & 259 & 219 & 282 & 256 & 301 \\
\hline & SBLT & 88 & 106 & 132 & 176 & 215 & 238 & 197 & 204 & 231 & 270 & 288 & 258 \\
\hline & SBTH & 726 & 712 & 741 & 598 & 640 & 755 & 644 & 664 & 939 & 986 & 798 & 868 \\
\hline & SBRT & 49 & 83 & 109 & 109 & 110 & 119 & 114 & 87 & 113 & 107 & 92 & 119 \\
\hline \multirow{11}{*}{ 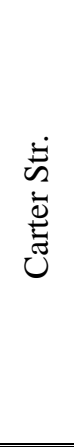 } & EBTH & 22 & 59 & 65 & 41 & 73 & 115 & 81 & 105 & 133 & 151 & 182 & 194 \\
\hline & EBRT & 26 & 38 & 30 & 45 & 50 & 67 & 98 & 58 & 65 & 57 & 71 & 87 \\
\hline & WBLT & 6 & 23 & 23 & 16 & 8 & 22 & 45 & 39 & 27 & 42 & 28 & 32 \\
\hline & WBTH & 24 & 55 & 70 & 45 & 58 & 79 & 125 & 115 & 123 & 111 & 105 & 97 \\
\hline & WBRT & 58 & 89 & 59 & 50 & 40 & 67 & 72 & 96 & 122 & 139 & 124 & 103 \\
\hline & NBLT & 18 & 35 & 27 & 49 & 44 & 100 & 77 & 63 & 72 & 94 & 65 & 56 \\
\hline & NBTH & 731 & 923 & 732 & 730 & 768 & 976 & 994 & 1003 & 1203 & 1428 & 1265 & 1236 \\
\hline & NBRT & 5 & 10 & 13 & 4 & 10 & 25 & 27 & 28 & 15 & 16 & 19 & 28 \\
\hline & SBLT & 52 & 61 & 78 & 33 & 54 & 115 & 79 & 94 & 102 & 132 & 141 & 125 \\
\hline & SBTH & 924 & 1035 & 999 & 851 & 853 & 1092 & 1058 & 1075 & 1141 & 1223 & 1035 & 1150 \\
\hline & SBRT & 15 & 24 & 31 & 23 & 48 & 52 & 31 & 38 & 39 & 45 & 37 & 55 \\
\hline \multirow{11}{*}{ 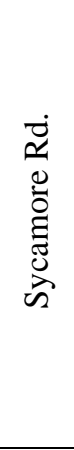 } & EBTH & 27 & 48 & 41 & 36 & 35 & 57 & 61 & 62 & 51 & 115 & 116 & 106 \\
\hline & EBRT & 56 & 51 & 62 & 89 & 83 & 114 & 94 & 102 & 85 & 173 & 124 & 128 \\
\hline & WBLT & 33 & 56 & 105 & 67 & 67 & 73 & 86 & 76 & 59 & 80 & 97 & 106 \\
\hline & WBTH & 53 & 92 & 101 & 54 & 60 & 74 & 65 & 55 & 73 & 102 & 93 & 98 \\
\hline & WBRT & 25 & 26 & 15 & 21 & 18 & 10 & 33 & 46 & 14 & 15 & 20 & 21 \\
\hline & NBLT & 55 & 108 & 120 & 98 & 129 & 148 & 169 & 158 & 205 & 188 & 186 & 133 \\
\hline & NBTH & 946 & 853 & 688 & 622 & 762 & 941 & 922 & 980 & 1430 & 1253 & 1370 & 1174 \\
\hline & NBRT & 46 & 56 & 61 & 36 & 48 & 64 & 55 & 72 & 52 & 74 & 73 & 69 \\
\hline & SBLT & 3 & 15 & 8 & 8 & 10 & 15 & 14 & 10 & 25 & 28 & 25 & 12 \\
\hline & SBTH & 1030 & 927 & 829 & 825 & 847 & 960 & 838 & 921 & 1153 & 1089 & 1113 & 1105 \\
\hline & SBRT & 48 & 58 & 44 & 53 & 55 & 70 & 79 & 65 & 64 & 64 & 69 & 65 \\
\hline
\end{tabular}




\subsubsection{A.M. Travel Time, Stops, and Delay}

\subsubsection{CORSIM’s Internal Model Simulation}

The performance of the algorithm during the a.m. period with the internal model is documented in Figure 10-2 to Figure 10-4. Figure 10-2 shows the northbound cumulative travel time through the arterial, while Figure 10-3 shows the southbound cumulative travel time. Both figures were obtained with CORSIM's internal logic simulation. The two lines in the figure represent the results from the case with Synchro timing plans and the case with PRO-TRACTS running for the north bound direction. Figure 10-2 shows a reduction of $7 \%, 4 \%$, and $3 \%$ in the northbound travel time during the first hour, second hour, and last two hours, respectively. Figure 10-3 shows a reduction of $2 \%$ in southbound travel time during the first hour of simulation and an increase of $1 \%$ during the last two hours of simulation. The total system delay shown in Figure 10-4 decreased by $28 \%$ during the first hour and then increased by $16 \%, 65 \%$, and $10 \%$ during the last three hours respectively. The total number of stops decreased by $27 \%$ during the first hour and increased by $2 \%, 17 \%$, and $4 \%$ during the last three hours. These results show that PRO-TRACTS performed better than Synchro's plans during the first hour only. It should be emphasized that these results were obtained using CORSIM's internal simulation model, which has a very primitive offset transitioning algorithm. Subsequent sections will discuss the significance of the offset transitioning algorithm. However, the impact of just changing the offset can have on a system is illustrated in the third hour in Figure 10-4. The delay with the offset seeking increased by $65 \%$. 

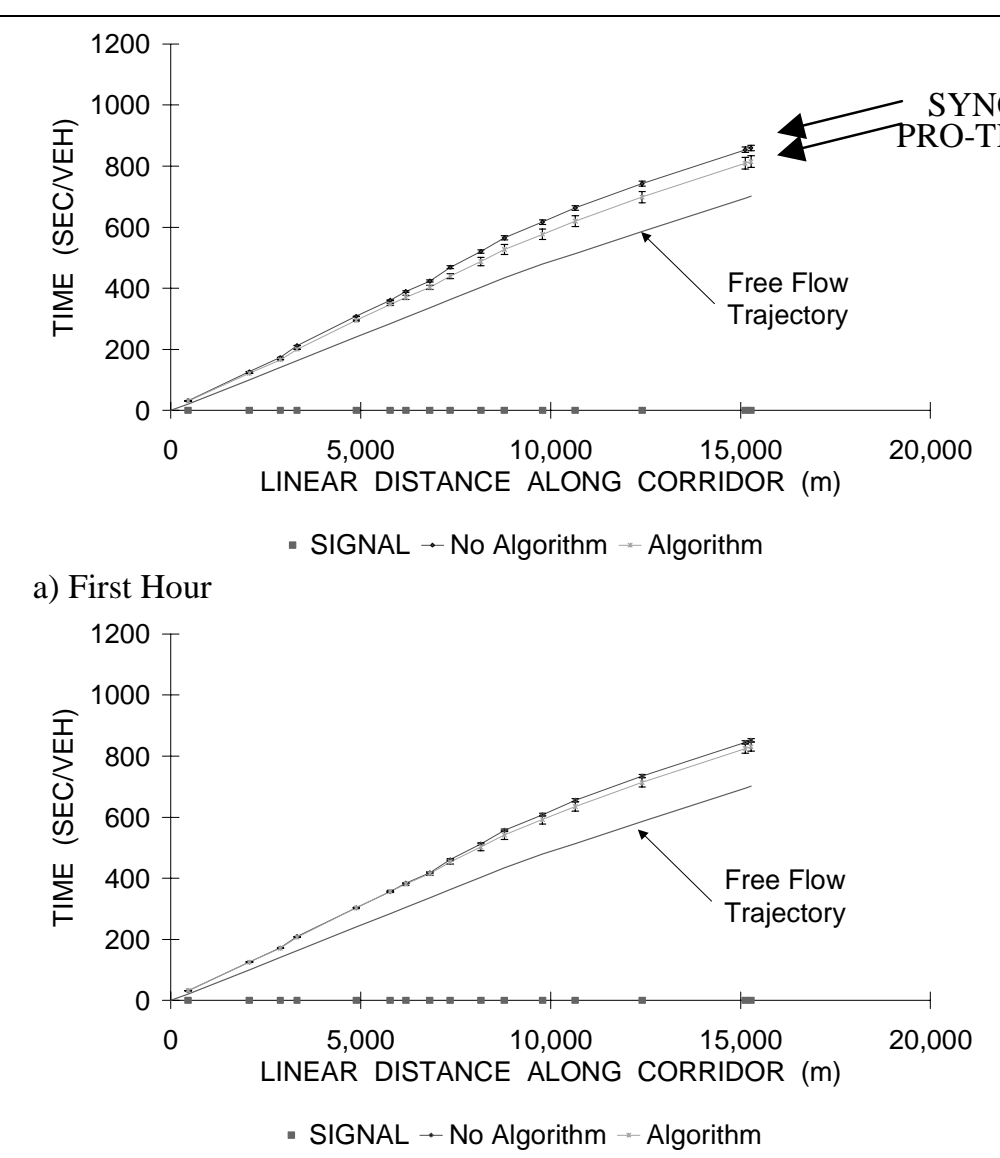

c) Third Hour

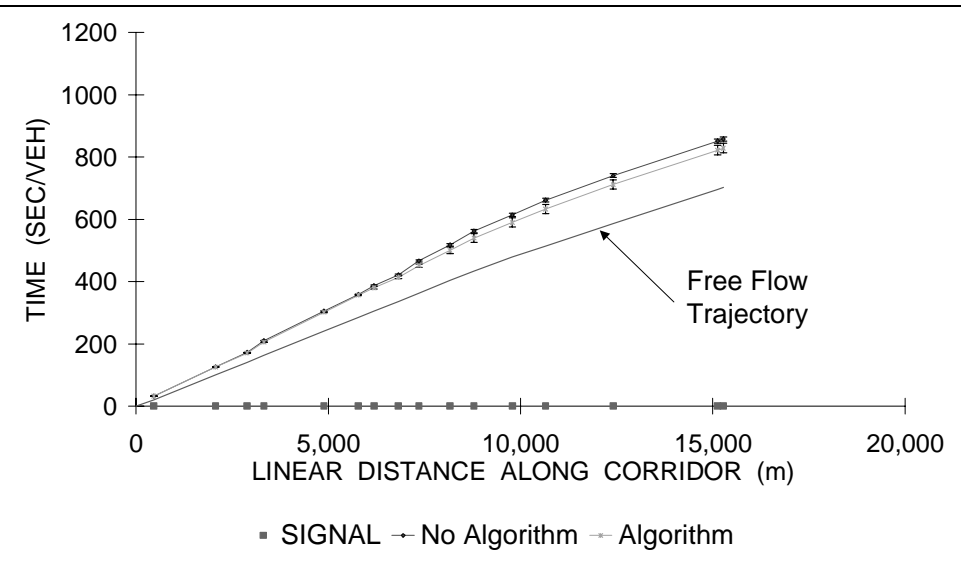

b) Second Hour

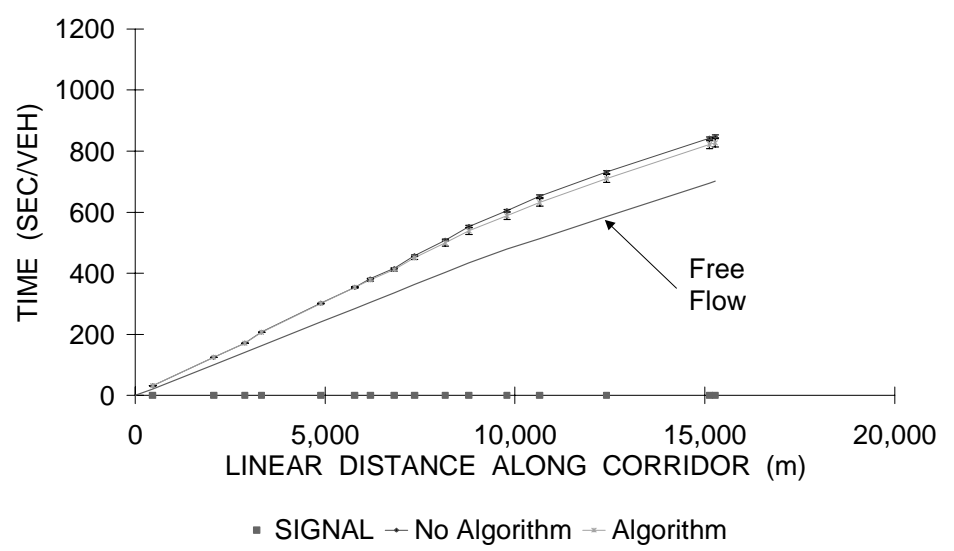

d) Fourth Hour

Figure 10-2 US 31 North Bound Cumulative Travel Time-A.M. Period 

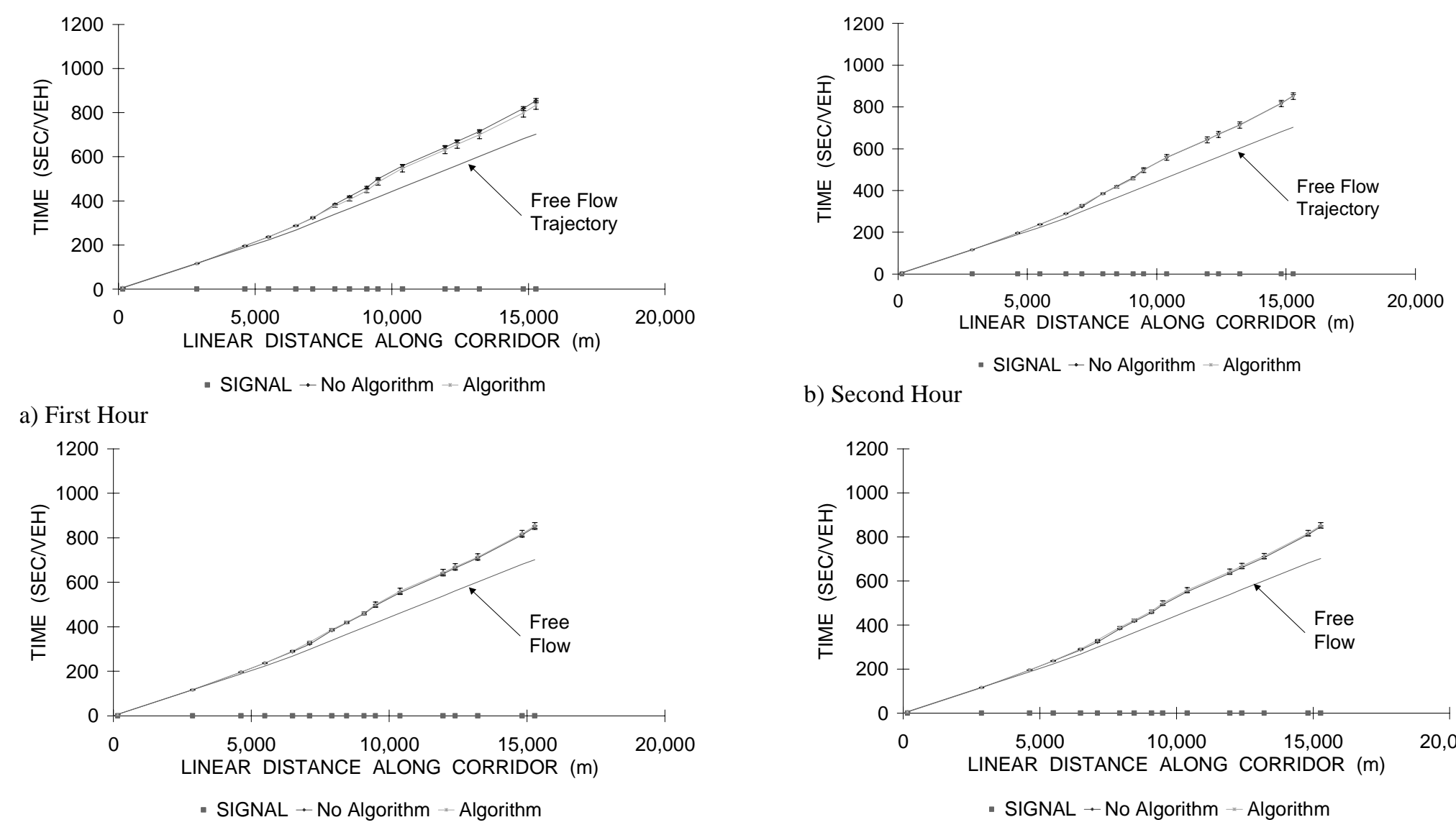

b) Second Hour

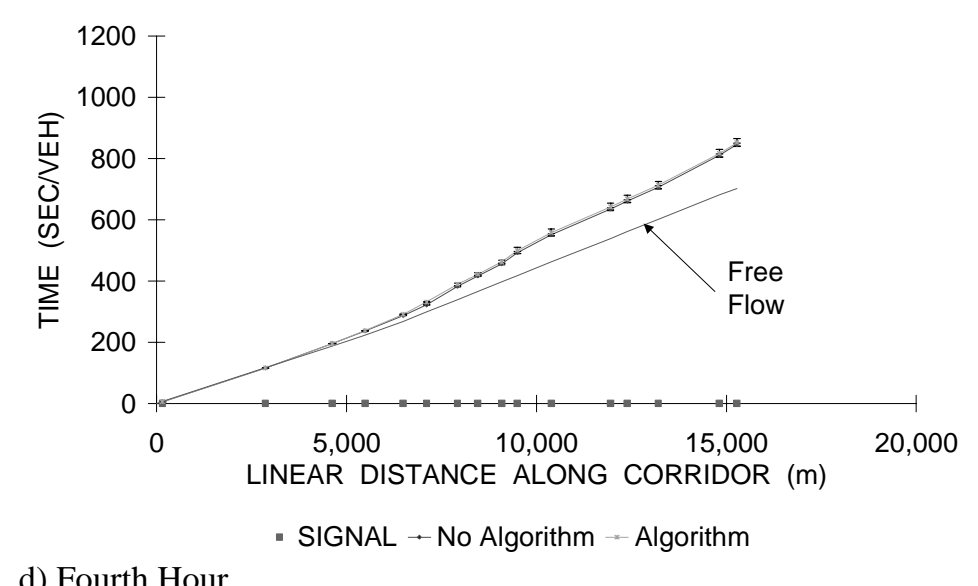

Figure 10-3 US 31 South Bound Cumulative Travel Time-A.M. Period 


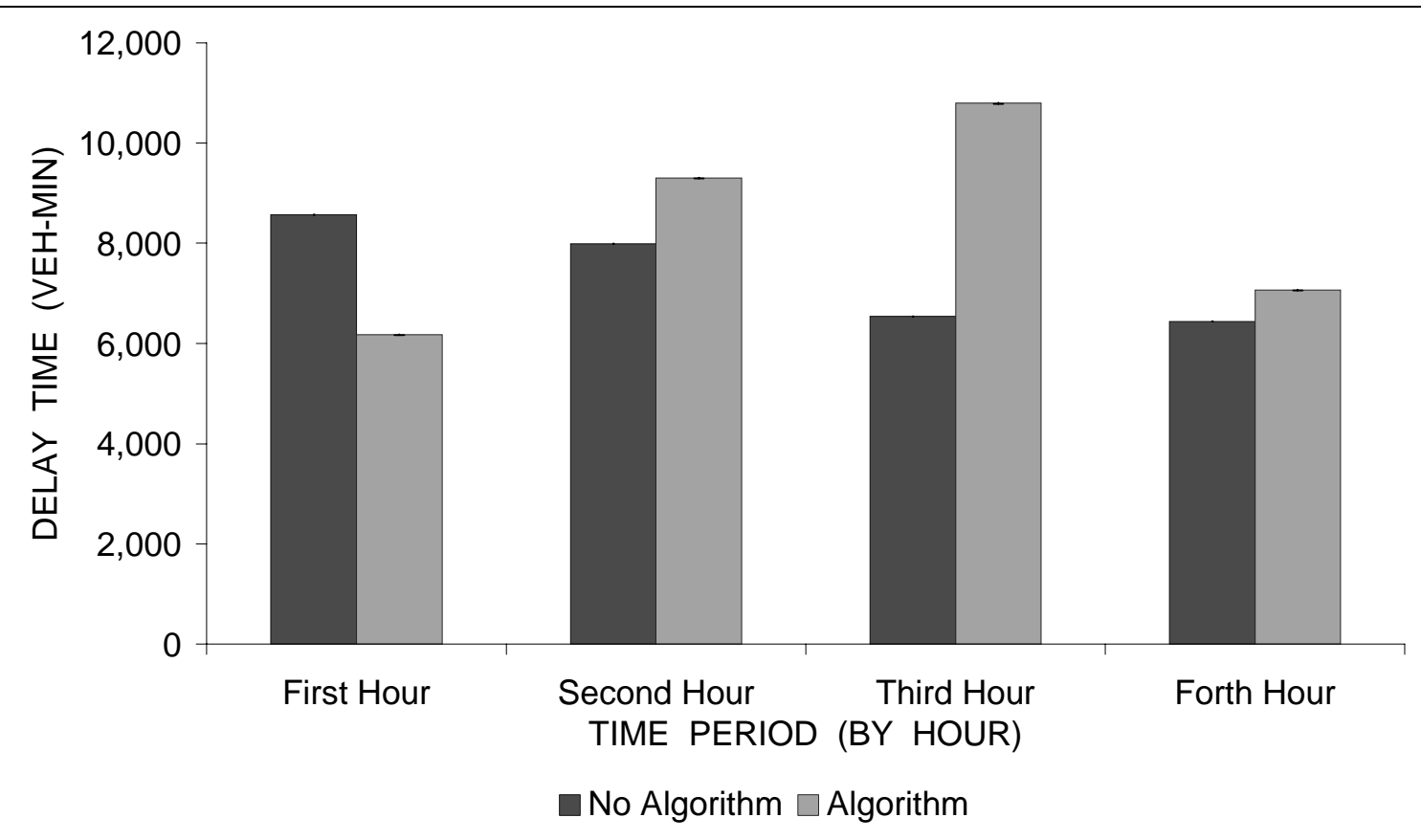

a) Total Delay Time

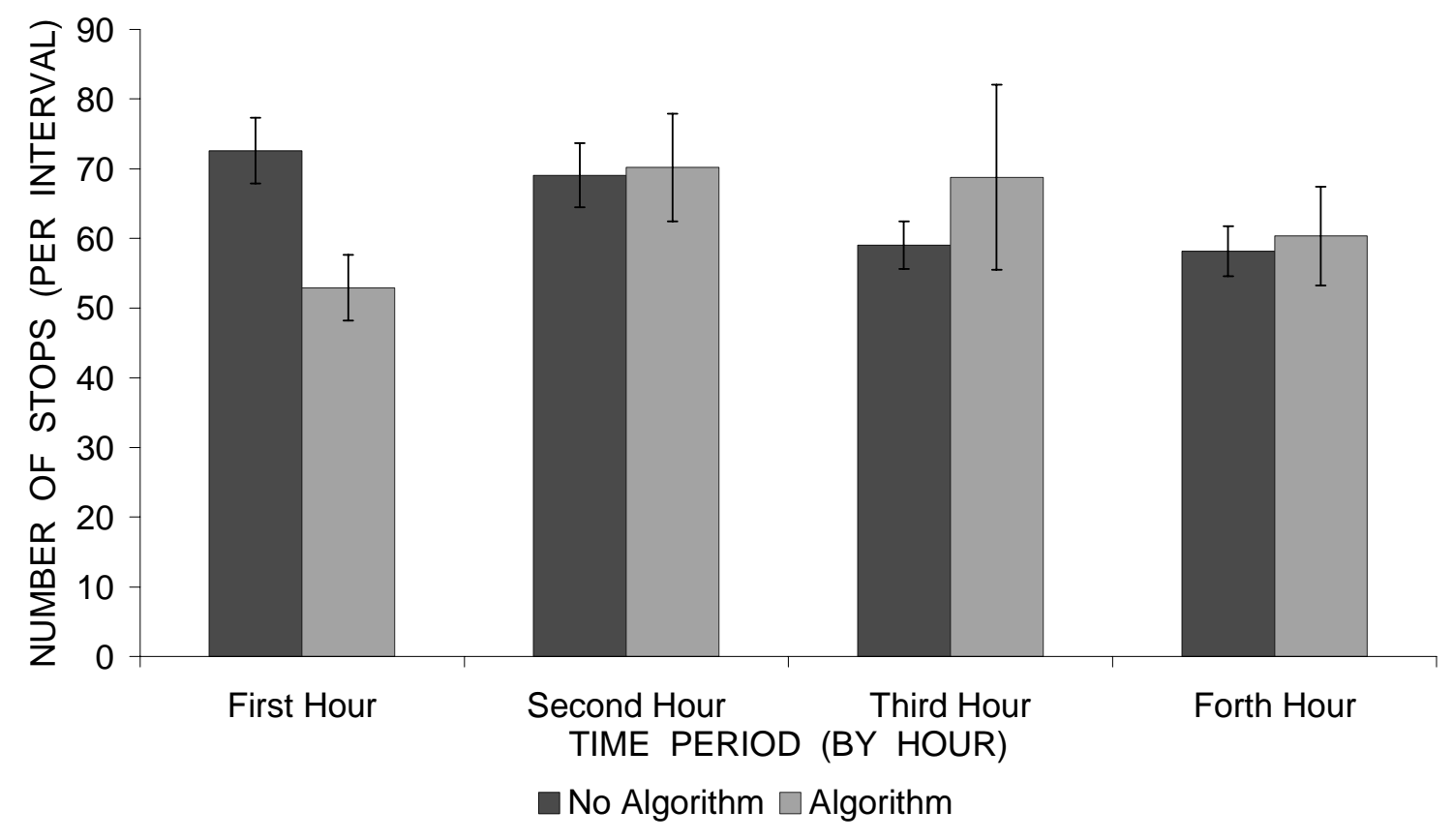

b) Total Number of Stops

Figure 10-4 US 31 Total Delay and Number of Stops-A.M. Period 


\subsubsection{Hardware-in-the-Loop Simulation}

The performance of the algorithm with hardware-in-the-loop simulation during

the a.m. period is shown in Figure 10-5 through Figure 10-7. Hardware-in-the-loop simulation did not show a difference in travel time during the four hours of simulation, as shown in Figure 10-5 and Figure 10-6. Nevertheless, hardware-in-the-loop simulation resulted in a reduction of $1 \%$ in cumulative travel time during the first hour, followed by $2 \%$ during the last three hours respectively. The overall system delay averaged to zero, with a decrease of $1 \%$ and $2 \%$ during the first and last hours and about $1 \%$ and $3 \%$ during the second and third hours respectively. 

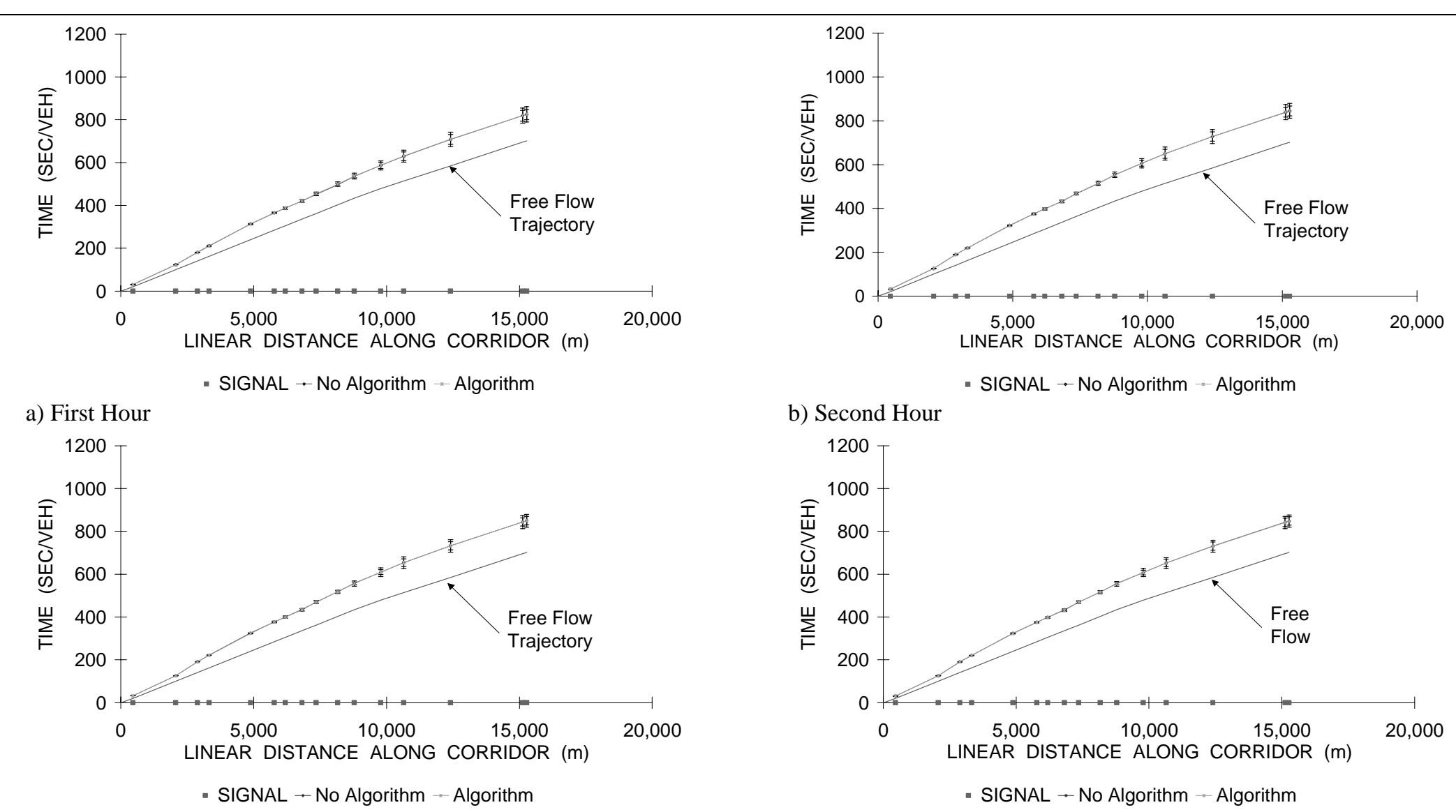

c) Third Hour

d) Fourth Hour

Figure 10-5 US 31 North Bound Cumulative Travel time-A.M. Period with Hardware-in-the-loop 

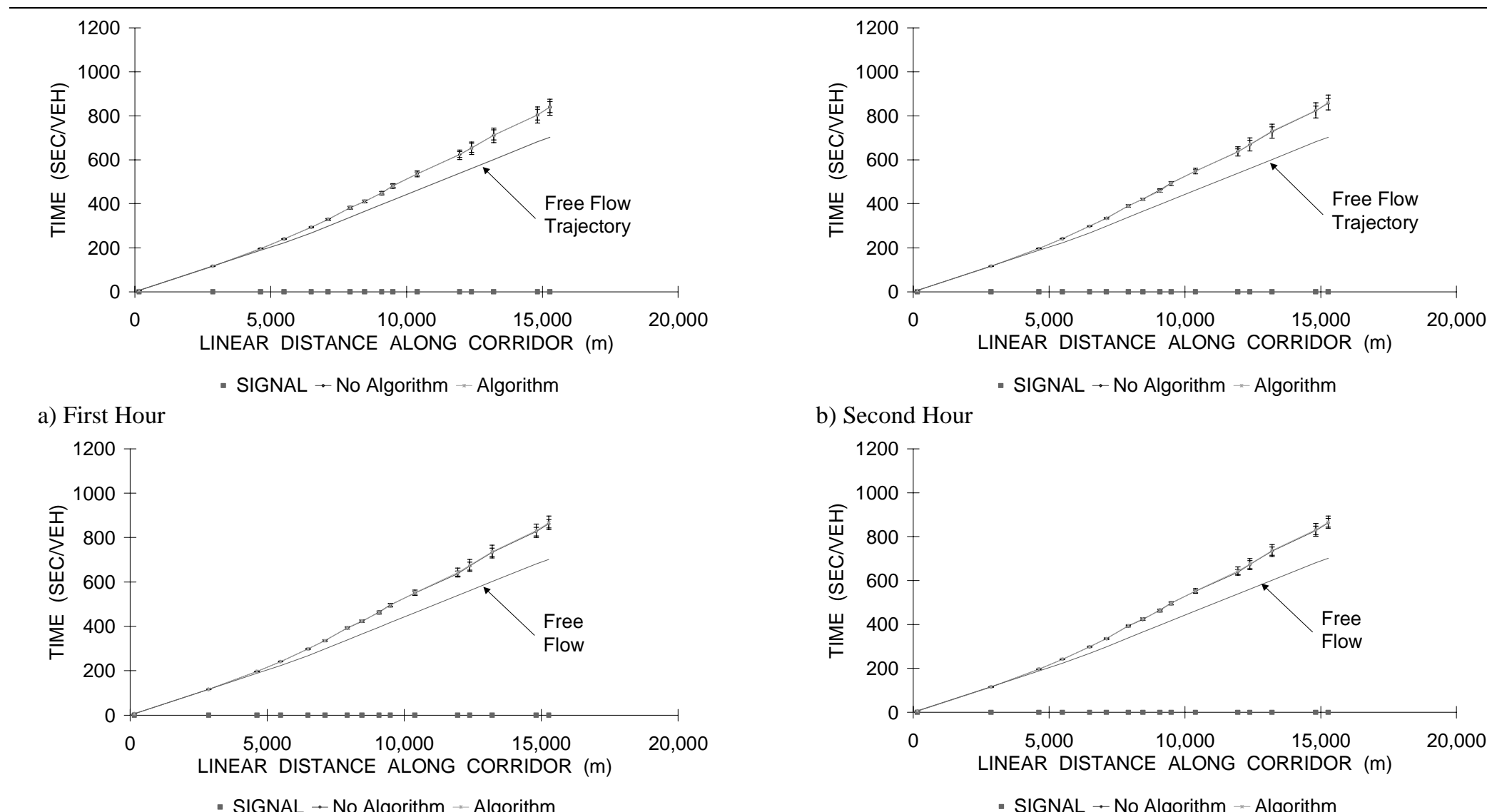

b) Second Hour

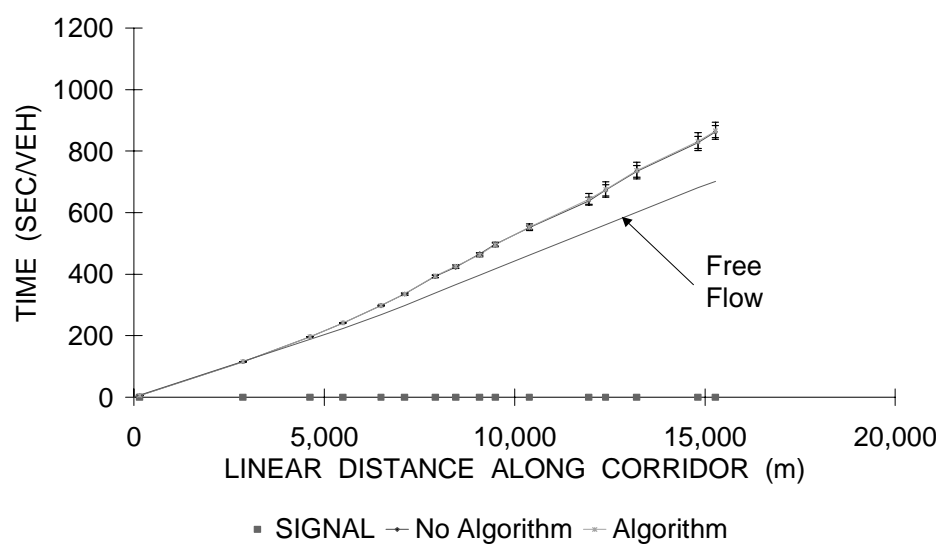

d) Fourth Hour

c) Third Hour

Figure 10-6 US 31 South Bound Cumulative Travel time-A.M. Period with Hardware-in-the-loop 


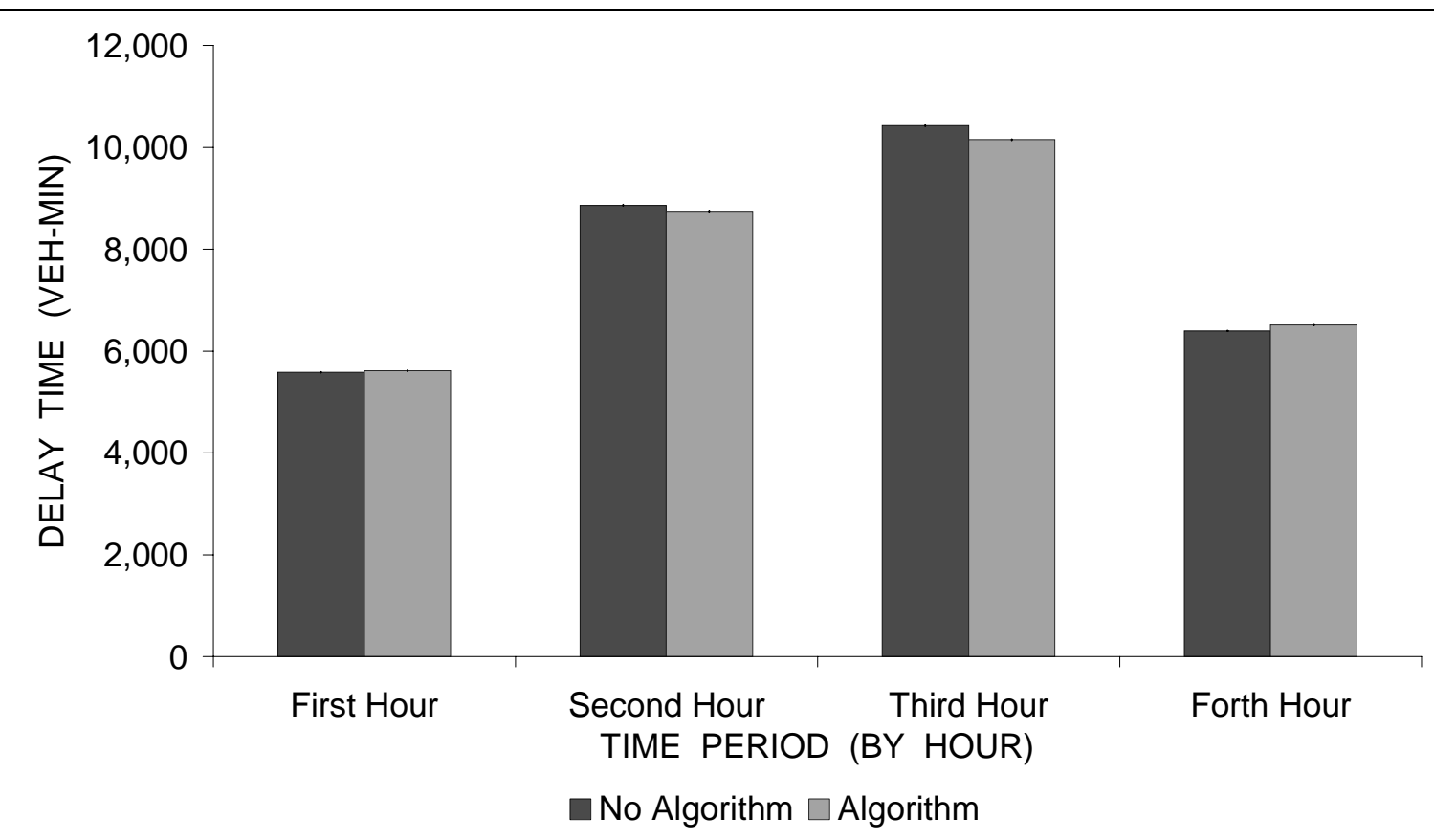

a) Total Delay Time

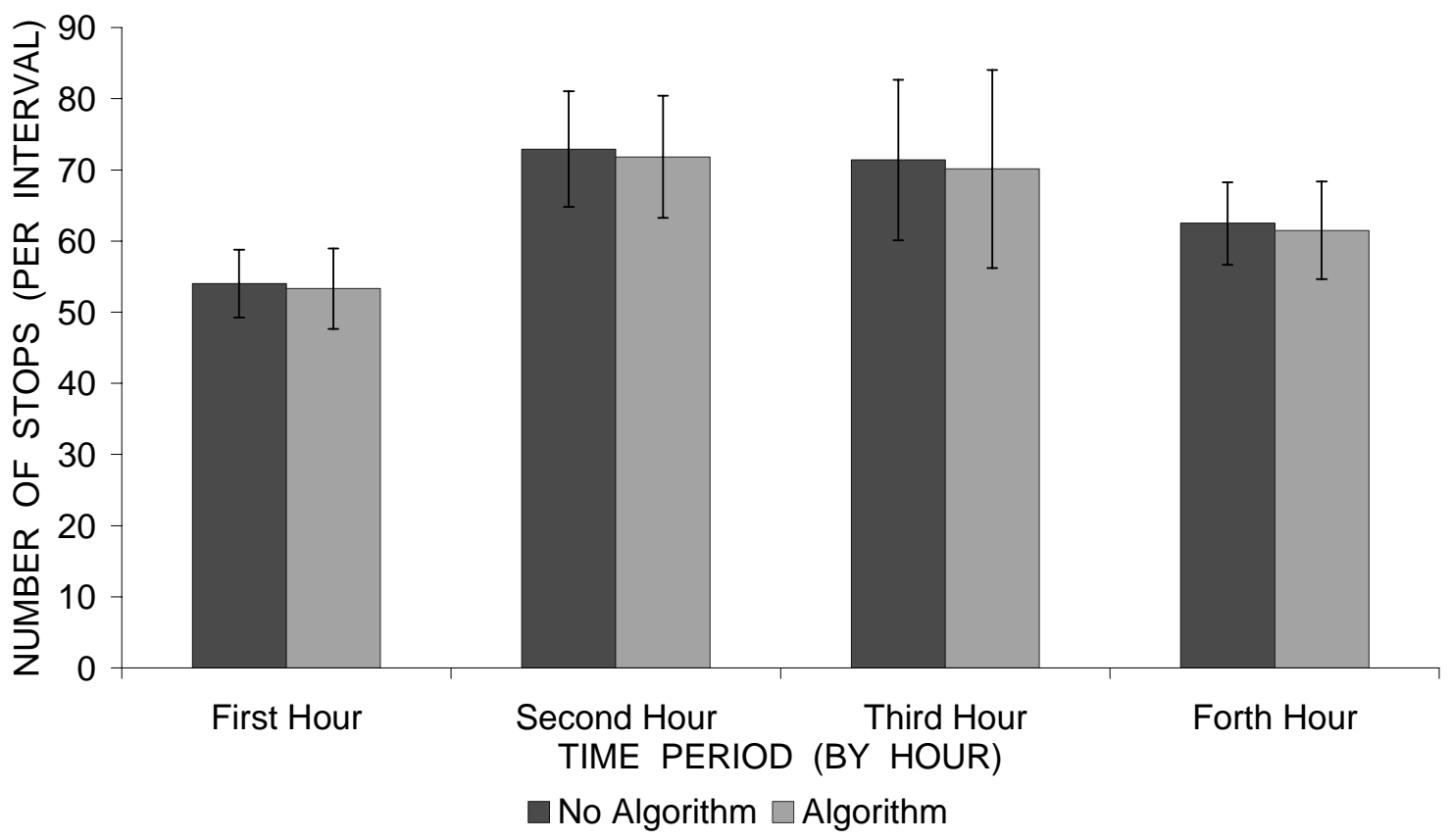

b) Total Number of Stops

Figure 10-7 US 31 Total Delay and Number of Stops- A.M. Period with Hardware-in-theLoop 
10.3.2 A.M. Offset and Cycle Transitioning

Figure 10-8 shows the transitioning in the offsets and cycle length for each of the seven studied intersections evaluated using CORSIM's internal model. Figure 10-8-a shows the transitioning in the offset values with the internal model's run. Figure 10-8-a shows a stable transitioning during the first hour, with a continuous decrease in offset during the remainder of the simulation. Figure 10-9 shows the transitioning for each of the seven studied intersections, evaluated using hardware-in-the-loop. Unlike the CORSIM internal model's offset transitioning, hardware-in-the-loop simulation showed a more stable transitioning because it uses the actual offset transitioning provided by Econolite controllers. An interesting pattern that can be observed in Figure 10-9-a is the cyclic offset transitioning at node 9 instead of the continuous decrease in offset that the node went through in Figure 10-8-a. Figure 10-8-b shows a higher fluctuation in the cycle length with CORSIM's internal model than with the Econolite controller depicted in Figure 10-9-b. 


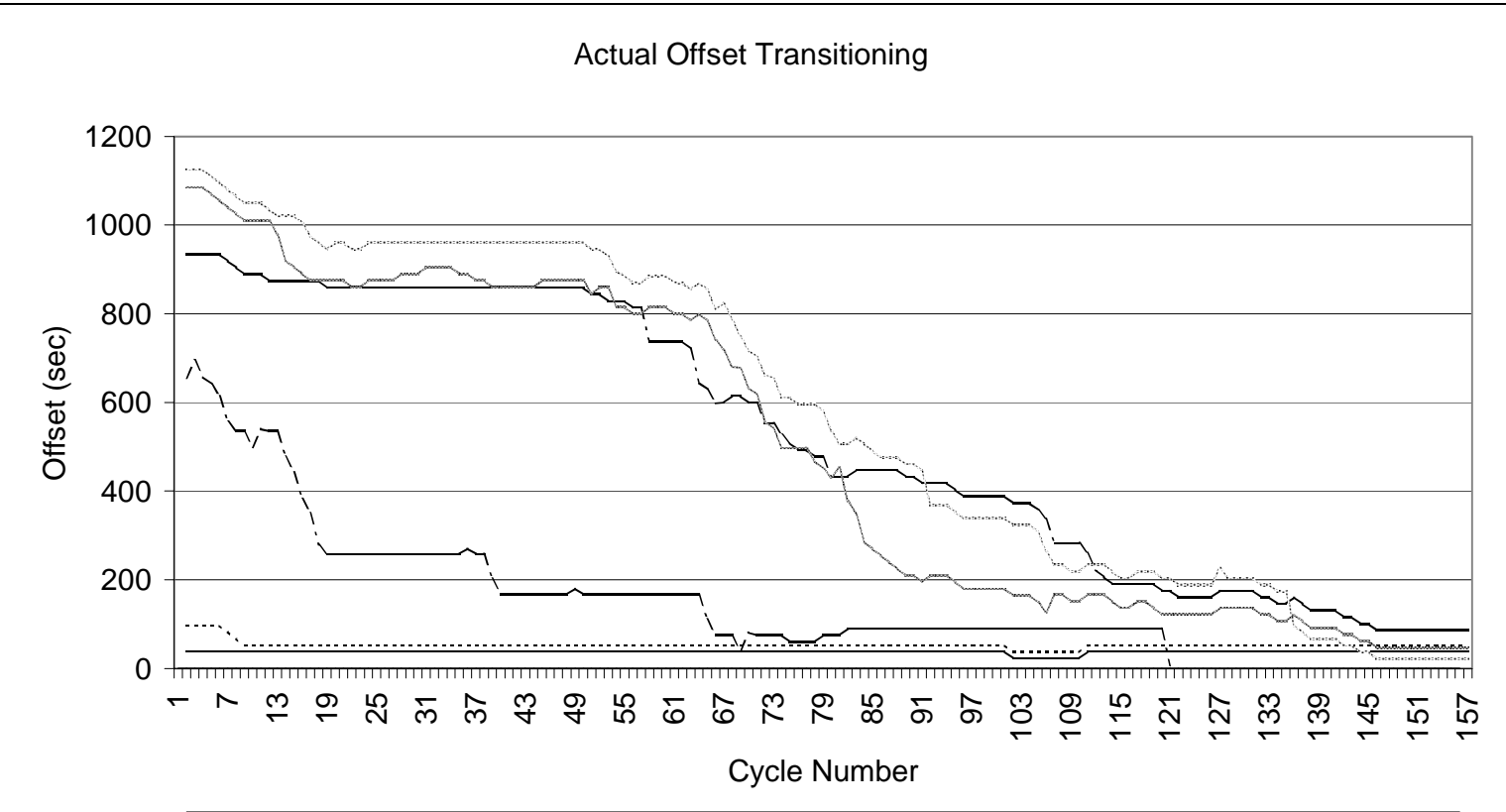

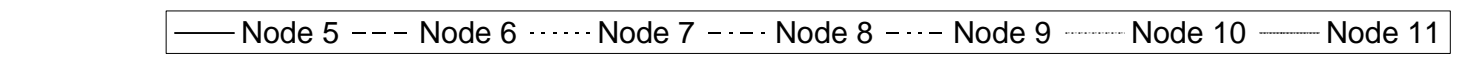

a) Offset Transitioning

Cycle Transitioning

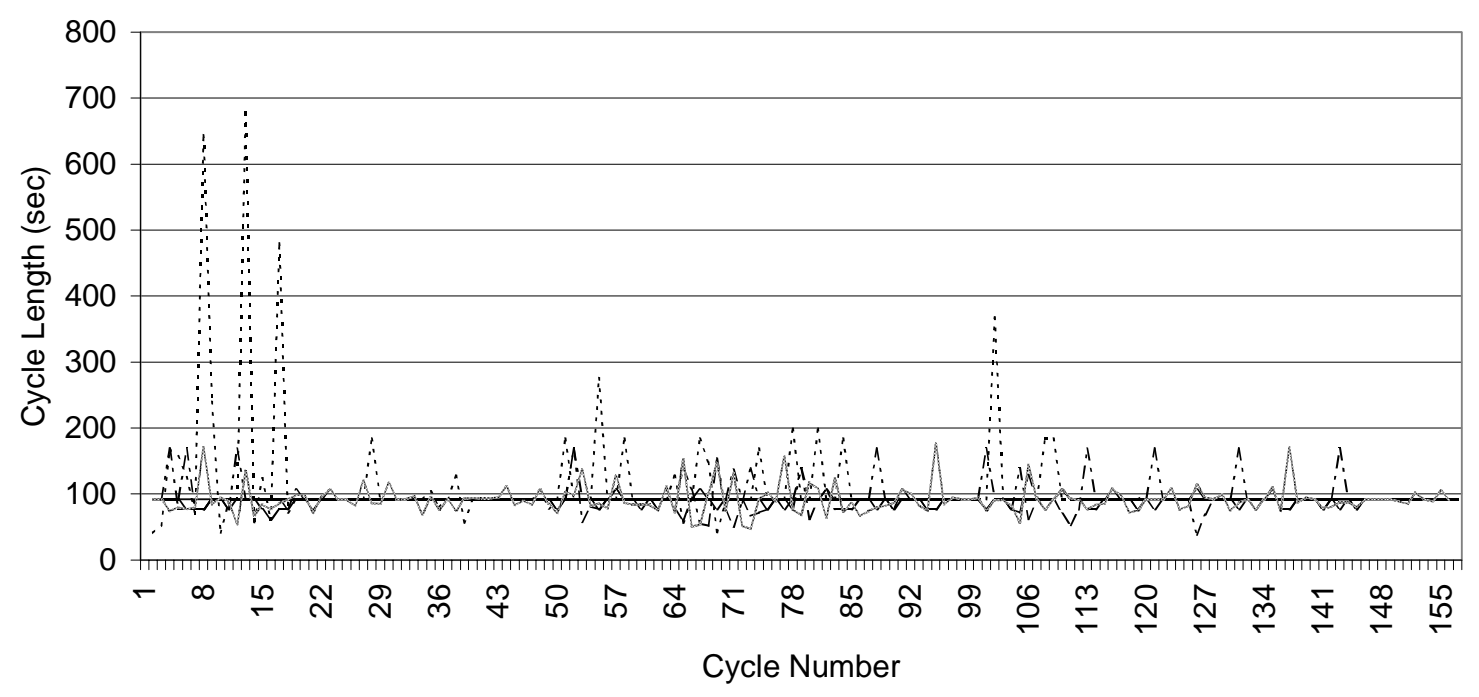

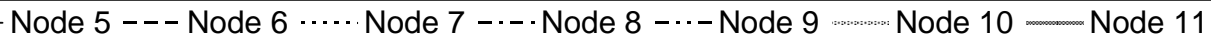

b) Cycle Transitioning

Figure 10-8 US 31 Offset and Cycle Transitions-A.M. Period, Internal CORSIM Model 


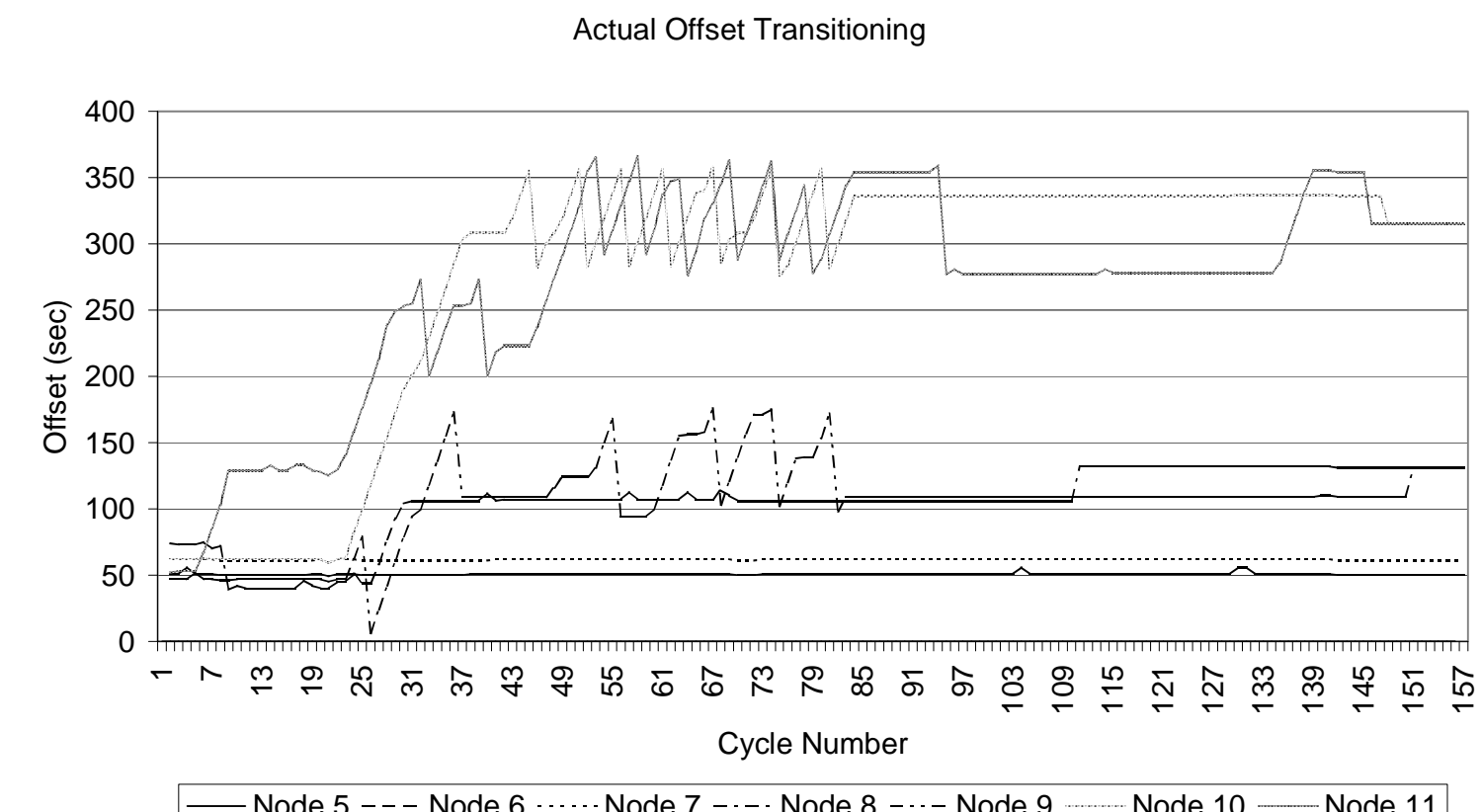

a) Offset Transitioning

\section{Cycle Transitioning}

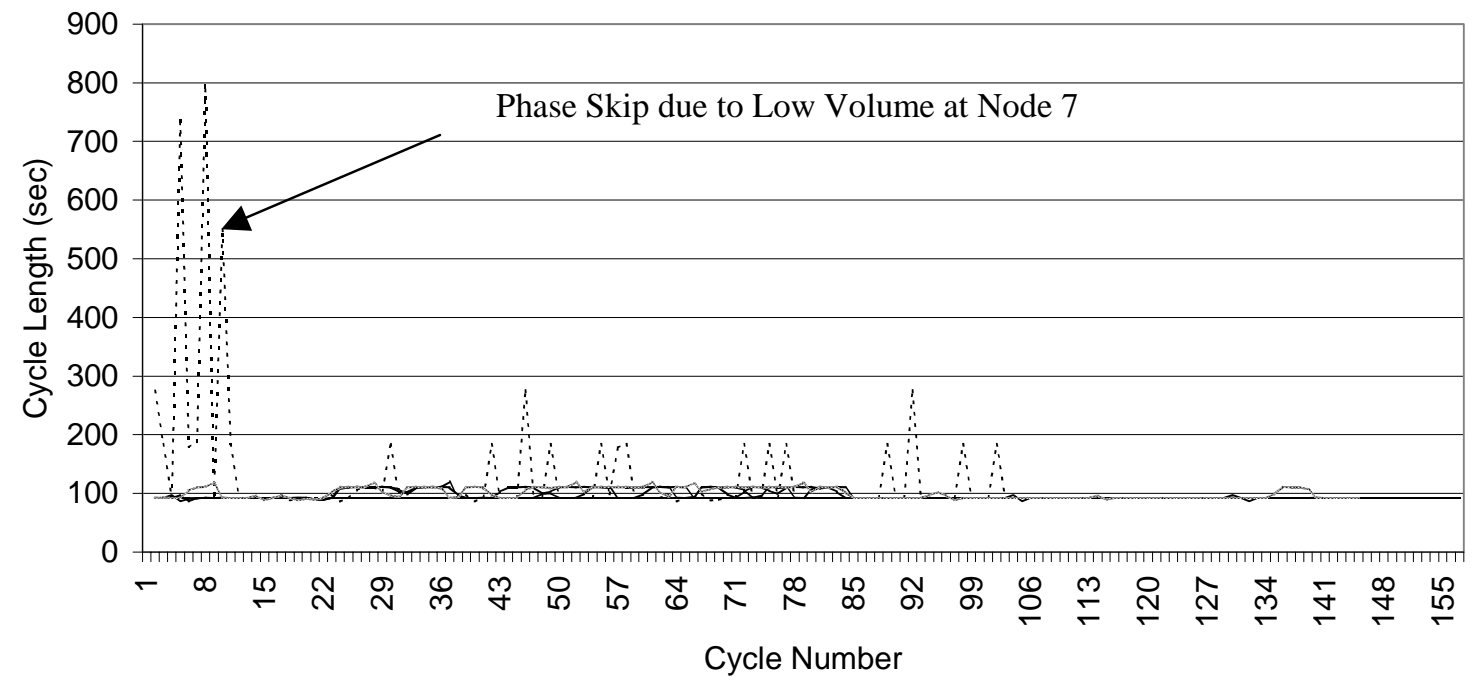

— Node 5 ---Node $6 \cdots$ Node 7 --- Node 8 -.-Node $9 \cdots$ Node $10-$ Node 11

b) Cycle Transitioning

Figure 10-9 US 31 Offset and Cycle Transitions-A.M. Period with Hardware-in-the-Loop 


\subsubsection{P.M. Travel Time, Stops, and Delay}

Figure 10-10 and Figure 10-11 show the cumulative travel time for northbound and southbound, respectively, with the internal model's simulation. Figure 10-10 shows a reduction of $1 \%, 2 \%, 2 \%$, and $3 \%$ in northbound cumulative travel time, while Figure 10-11 shows an increase of $12 \%$ during the four hours of simulation. The overall system delay increased by about $14 \%$ during the four hours of simulation, as shown in Figure 10-12. The figure also shows an increase in the total number of stops of $4 \%$ during the first three hours and $2 \%$ during the last hour.

Figure 10-13 to Figure 10-15 show the performance of the algorithm during the p.m. period for the hardware-in-the-loop simulation. Hardware-in-the-loop performed much better than the internal model during the p.m. period. The reduction in cumulative travel time was 7\% during the four hours as shown in Figure 10-13 while the increase in travel time for the south bound stayed at $2 \%$ during the first three hours and increased during the last hour to $6 \%$.

The overall system delay was reduced by $10 \%, 14 \%, 11 \%$ and $9 \%$ respectively as shown in Figure 10-15. The total number of stops was reduced by $4 \%, 2 \%, 1 \%$ and $11 \%$ respectively.

Note that Figure 10-10 and Figure 10-13 show larger error bars at the nodes outside of the hardware-in-the-loop system in Figure 10-11 and Figure 10-13. The offset values at these nodes remained fixed and were not adjusted to reference the upstream node's offset during the simulation because only seven controllers were available in the lab. 

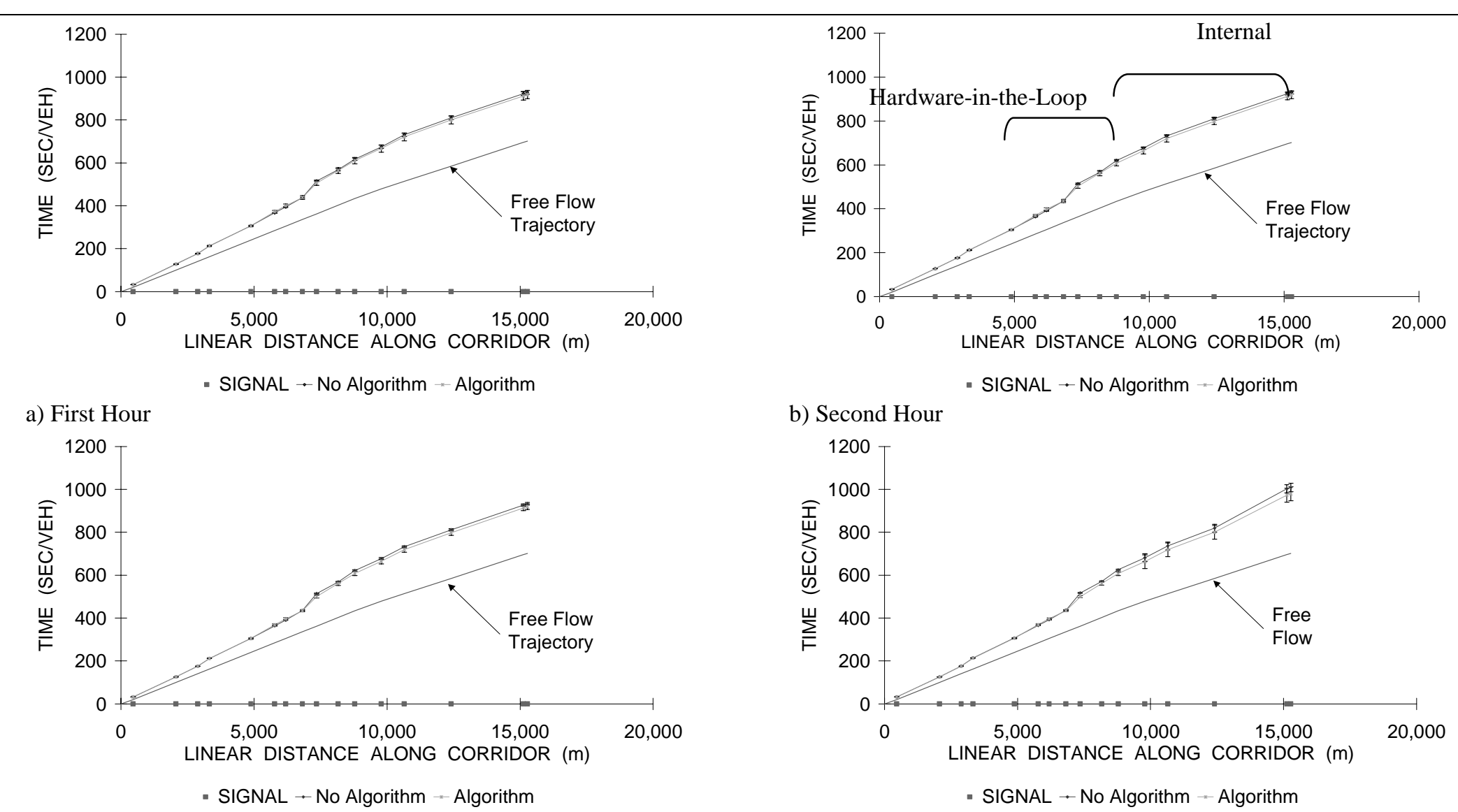

c) Third Hour

b) Second Hour

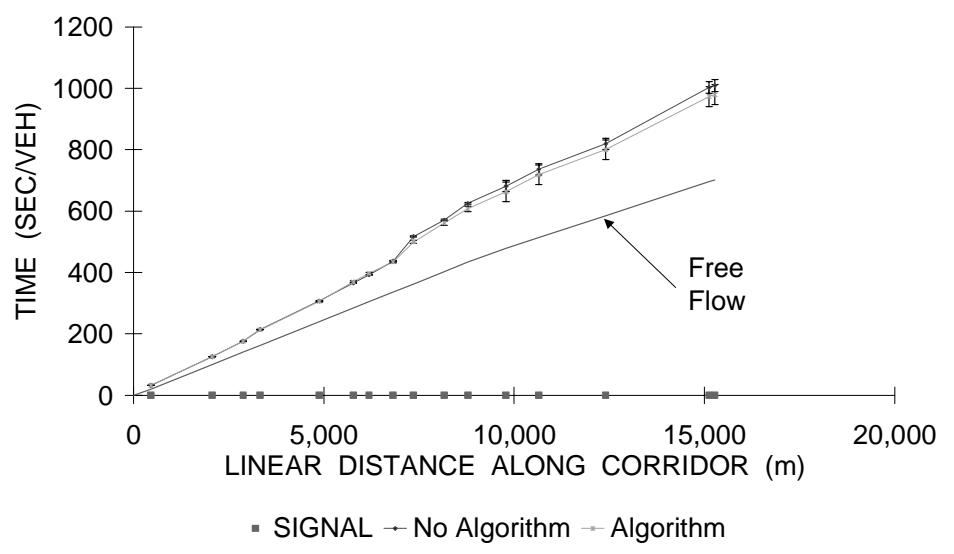

d) Fourth Hour

Figure 10-10 US 31 North Bound Cumulative Travel Time-P.M. Period, Internal Model 

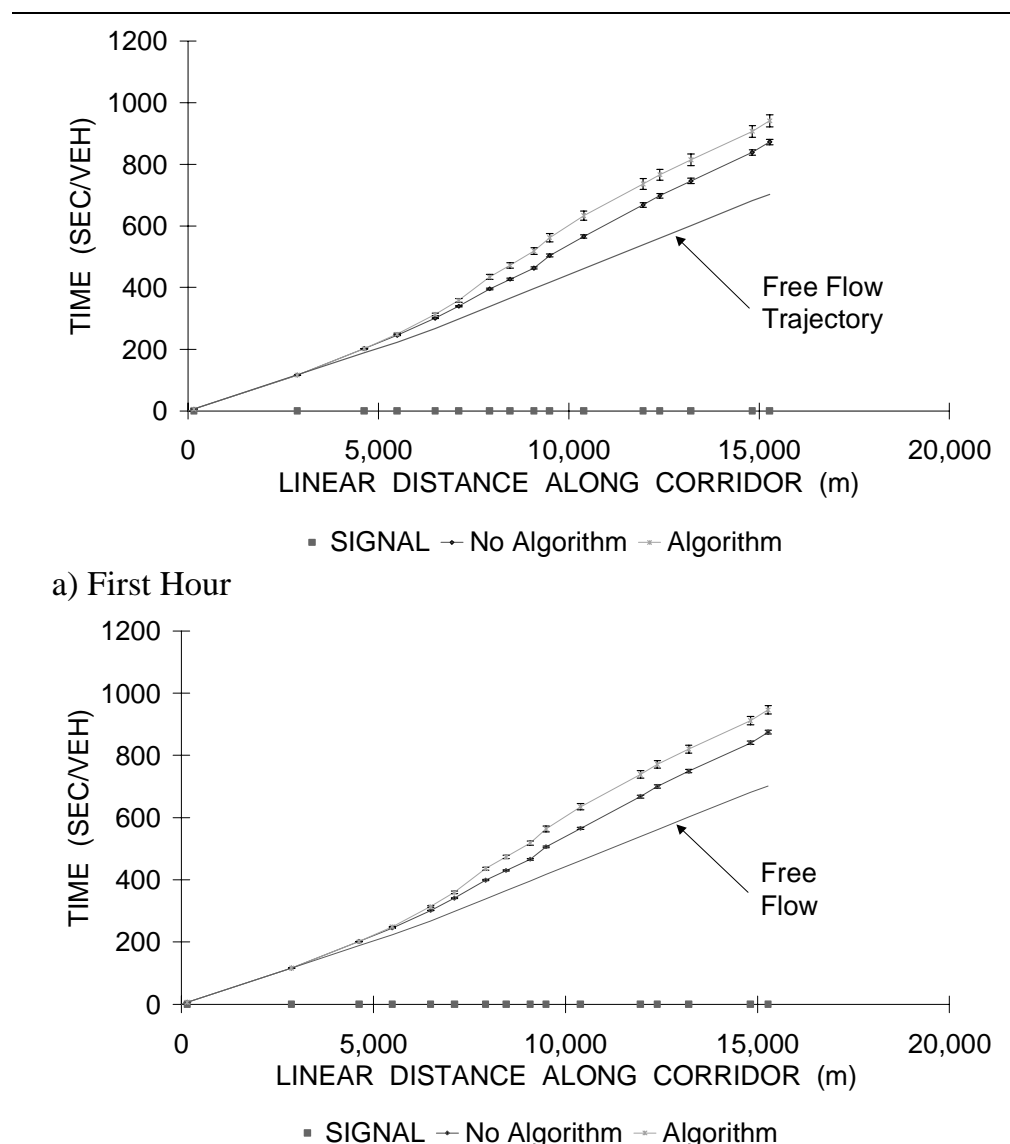

c) Third Hour

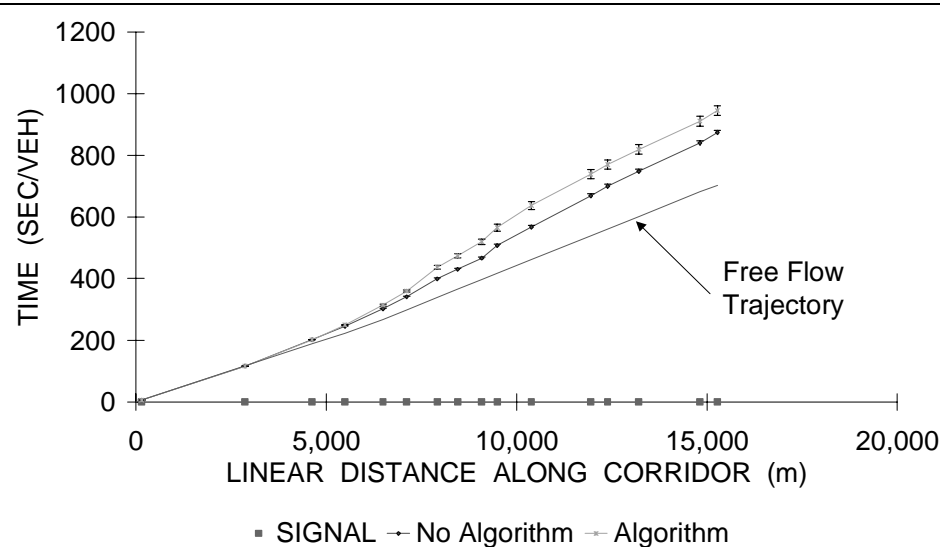

b) Second Hour

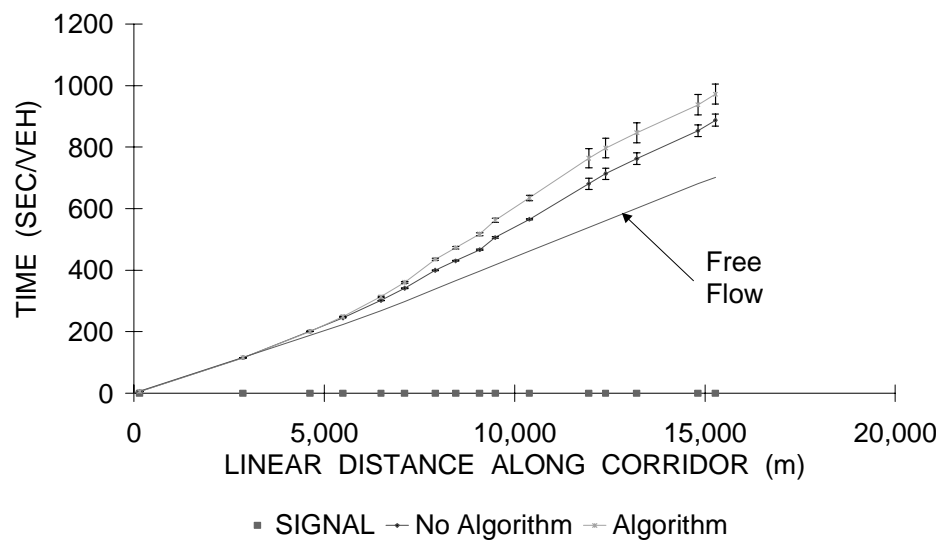

d) Fourth Hour

Figure 10-11 US 31 South Bound Cumulative Travel Time-P.M. Period, Internal Model 


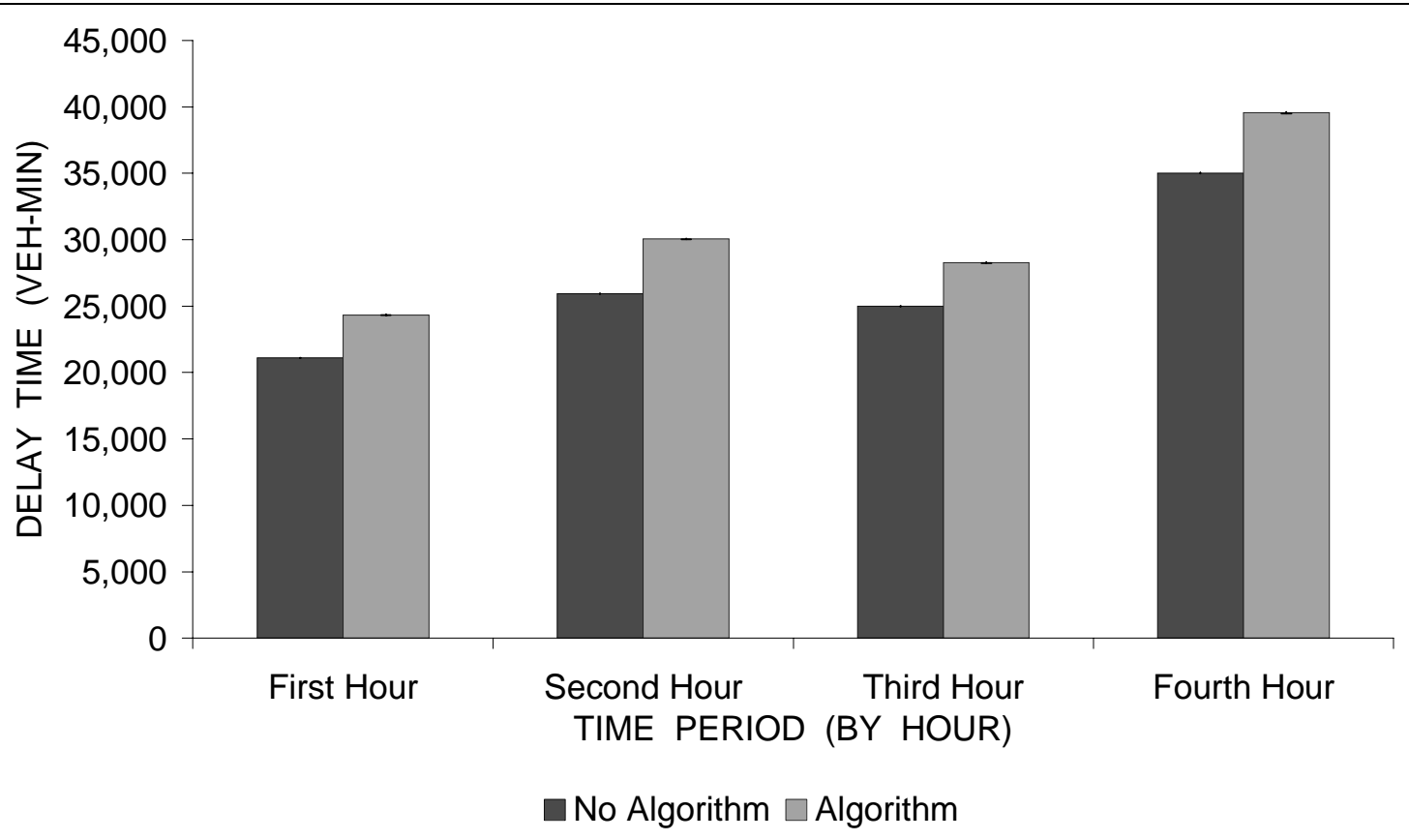

a) Total Delay Time

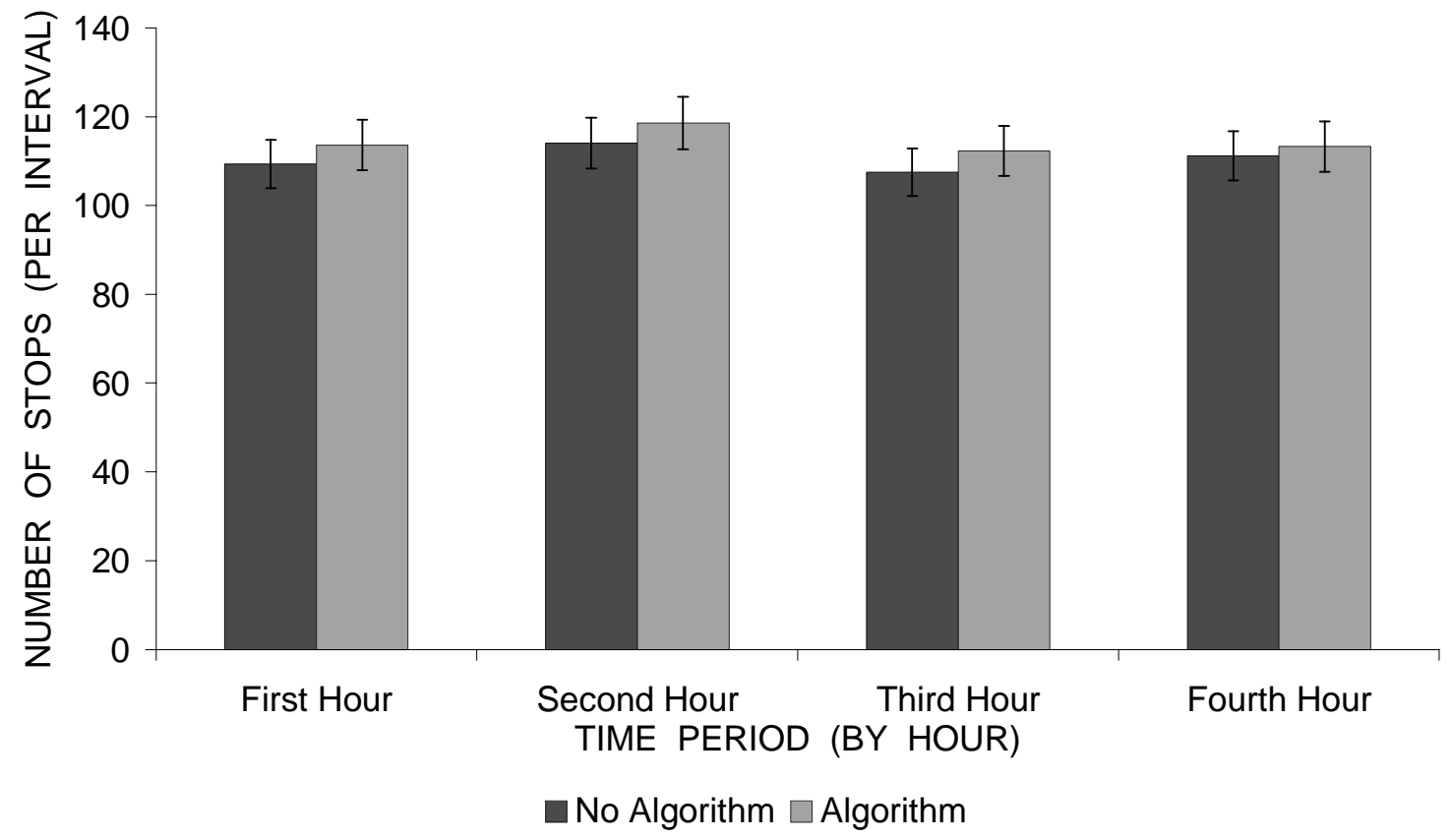

b) Total Number of Stops

Figure 10-12 US 31 Total Delay and Number of Stops-P.M. Period, Internal Model 

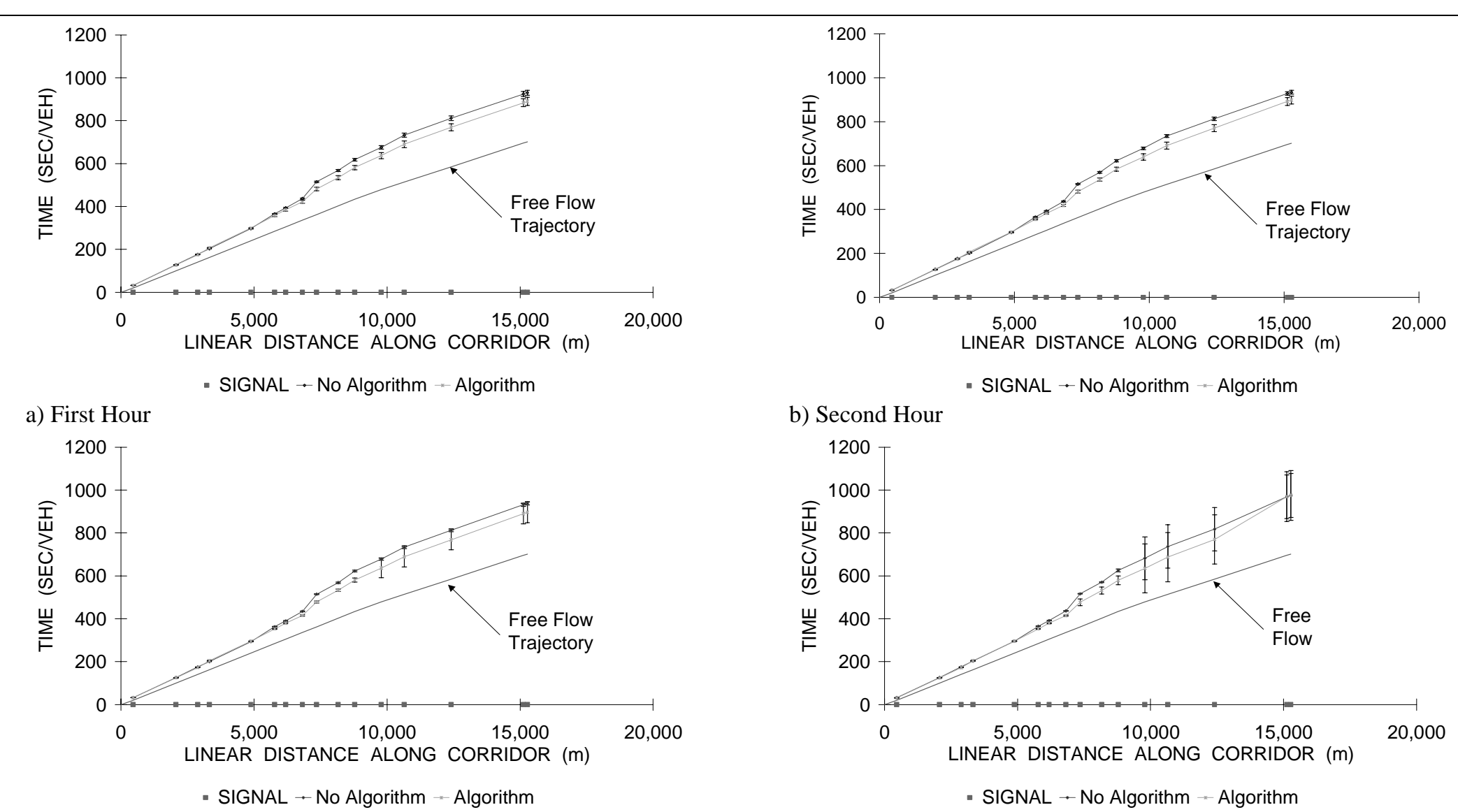

b) Second Hour

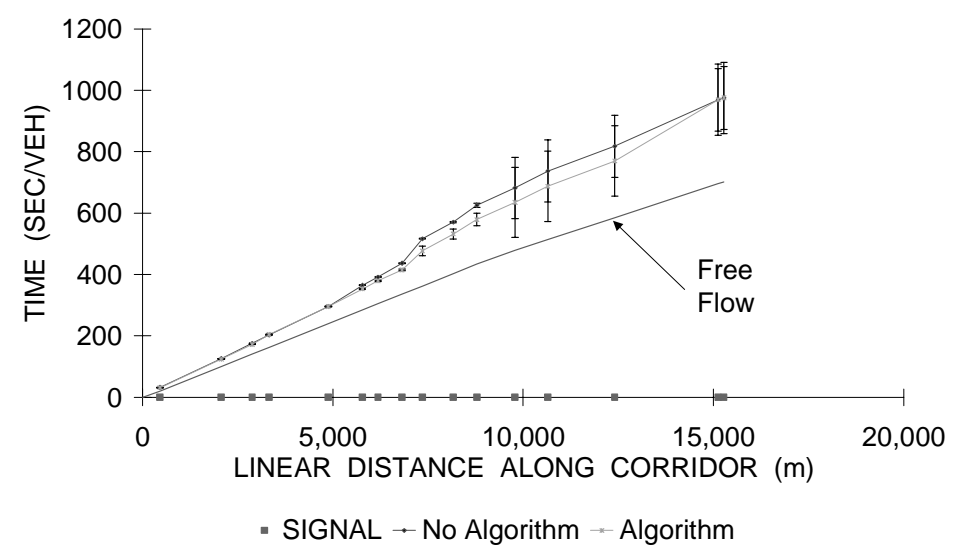

c) Third Hour

d) Fourth Hour

Figure 10-13 US 31 North Bound Cumulative Travel time-P.M. Period with Hardware-in-the-loop 

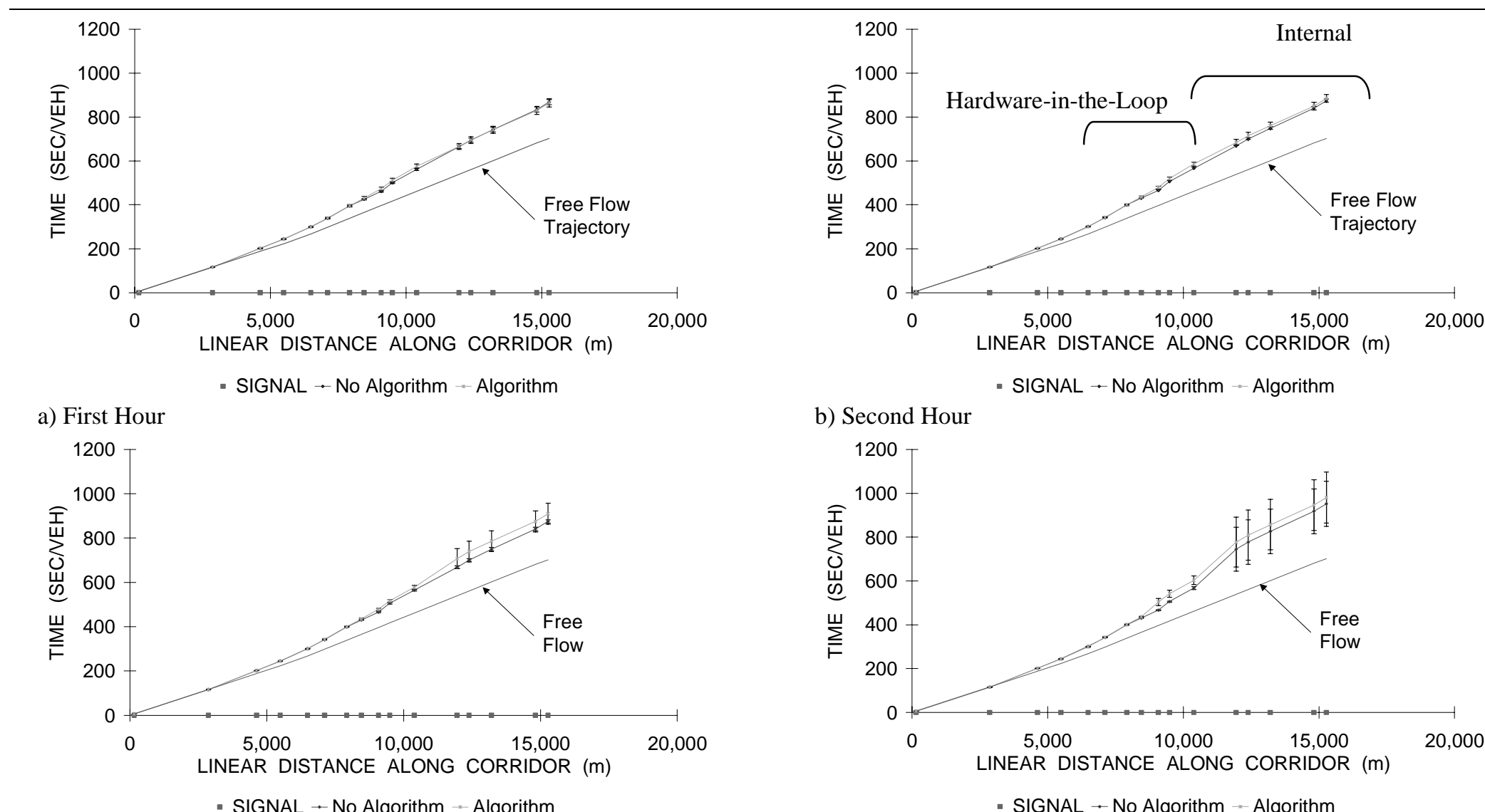

b) Second Hour

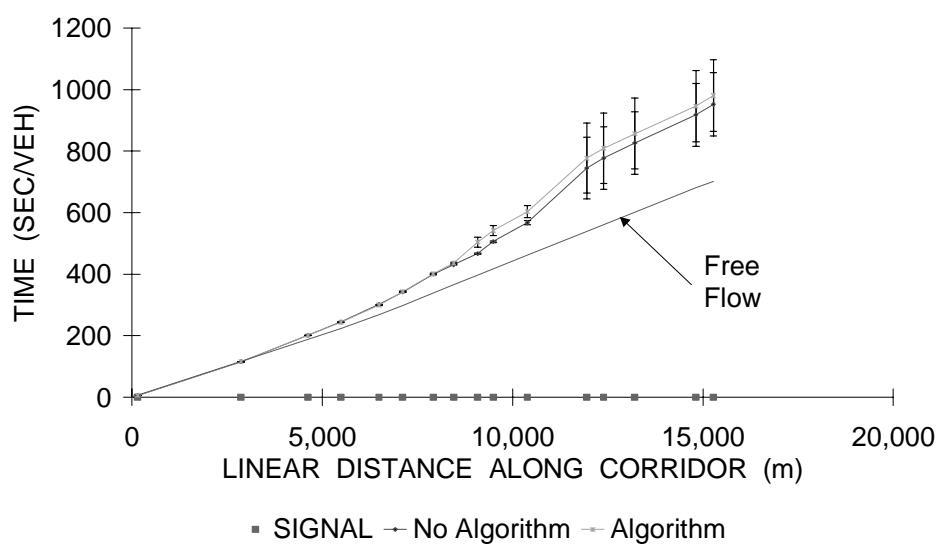

c) Third Hour

d) Fourth Hour

Figure 10-14 US 31 South Bound Cumulative Travel time-P.M. Period with Hardware-in-the-loop 


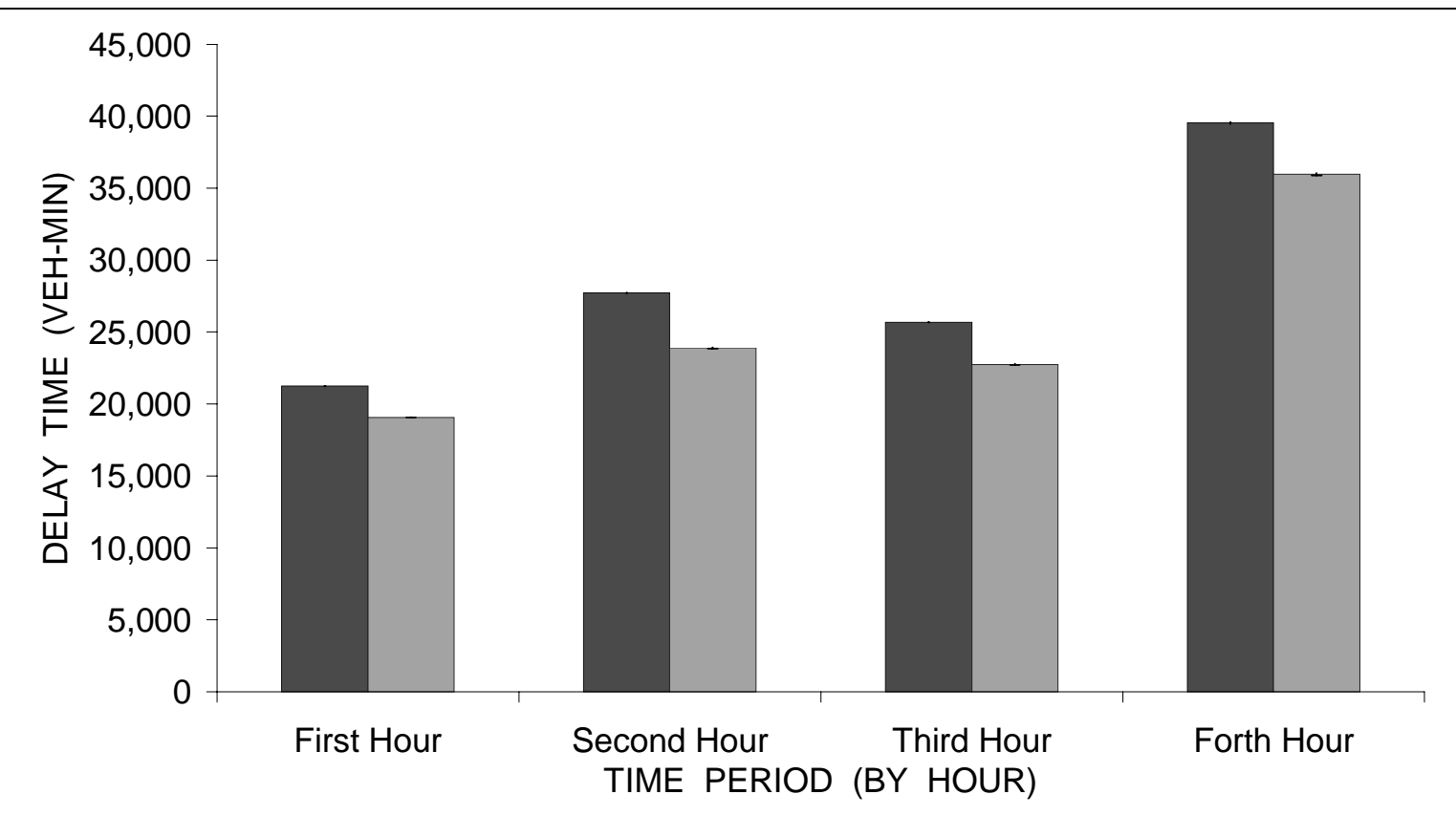

$\square$ No Algorithm $\square$ Algorithm

a) Total Delay Time

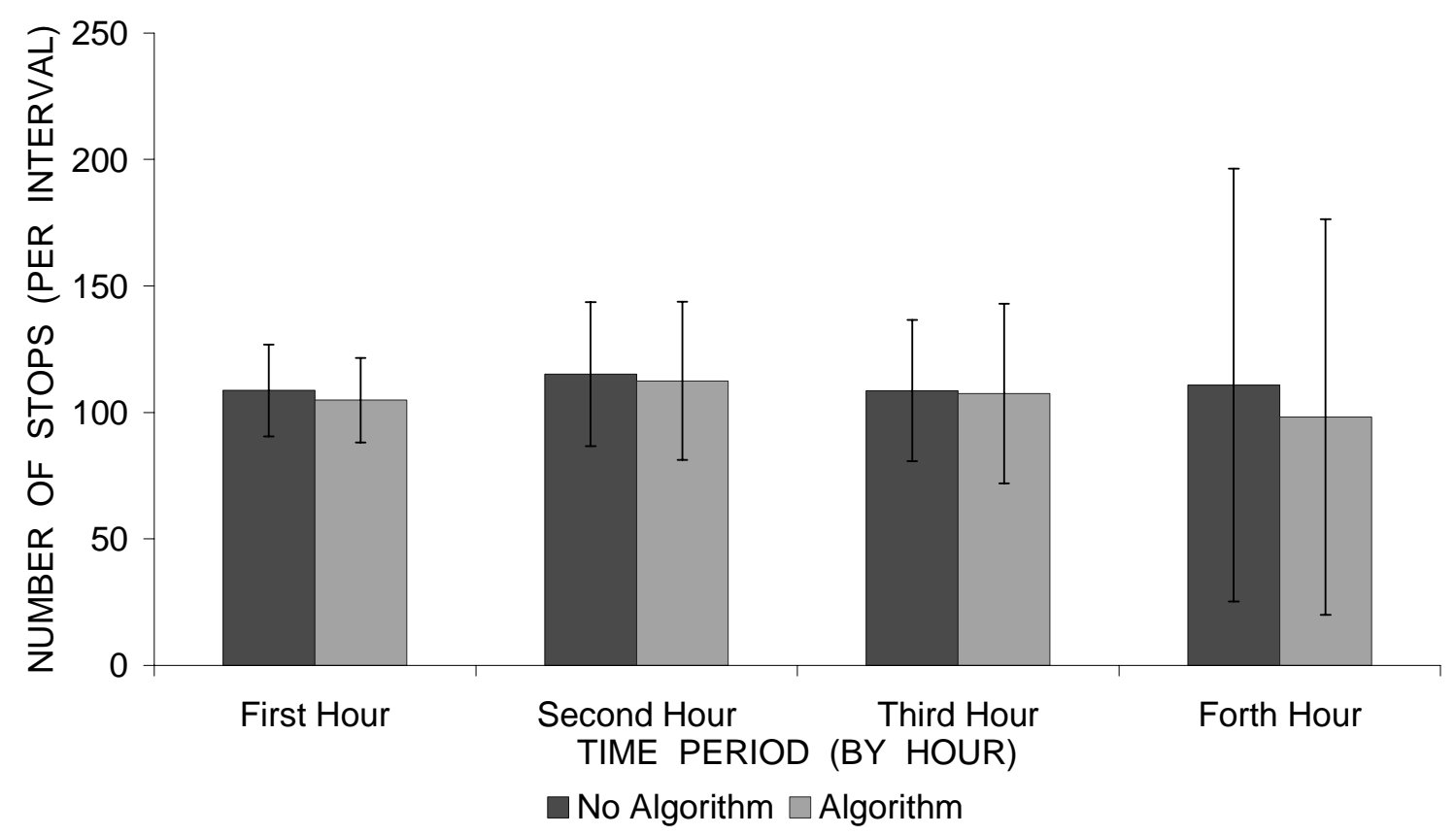

b) Total Number of Stops

Figure 10-15 US 31 Total Delay and Number of Stops-P.M. Period with Hardware-in-theLoop 


\subsubsection{P.M. Offset and Cycle Transitioning}

Figure 10-16 and Figure 10-17 show the transitioning in the offsets and cycle length for each of the seven studied intersections for CORSIM's internal model's run and the hardware-in-the-loop simulation respectively. Note that the offset transitioning patterns are different in the two cases. Looking more closely, it can be seen that nodes 6 , 7, and 8 had almost the same transitioning pattern in both figures, while nodes 9,10 , and 11 transitioned quite differently. Figure 10-18 provides an insight into the reason for this phenomenon, whereby it shows the main street green during a normal simulation run (without PRO-TRACTS). Note the cyclic pattern of early-return-to-green at node 9 compared to node 8 . Figure 10-16-b and Figure 10-17-b also show a more stable cycle transitioning with the hardware-in-the-loop simulation. 


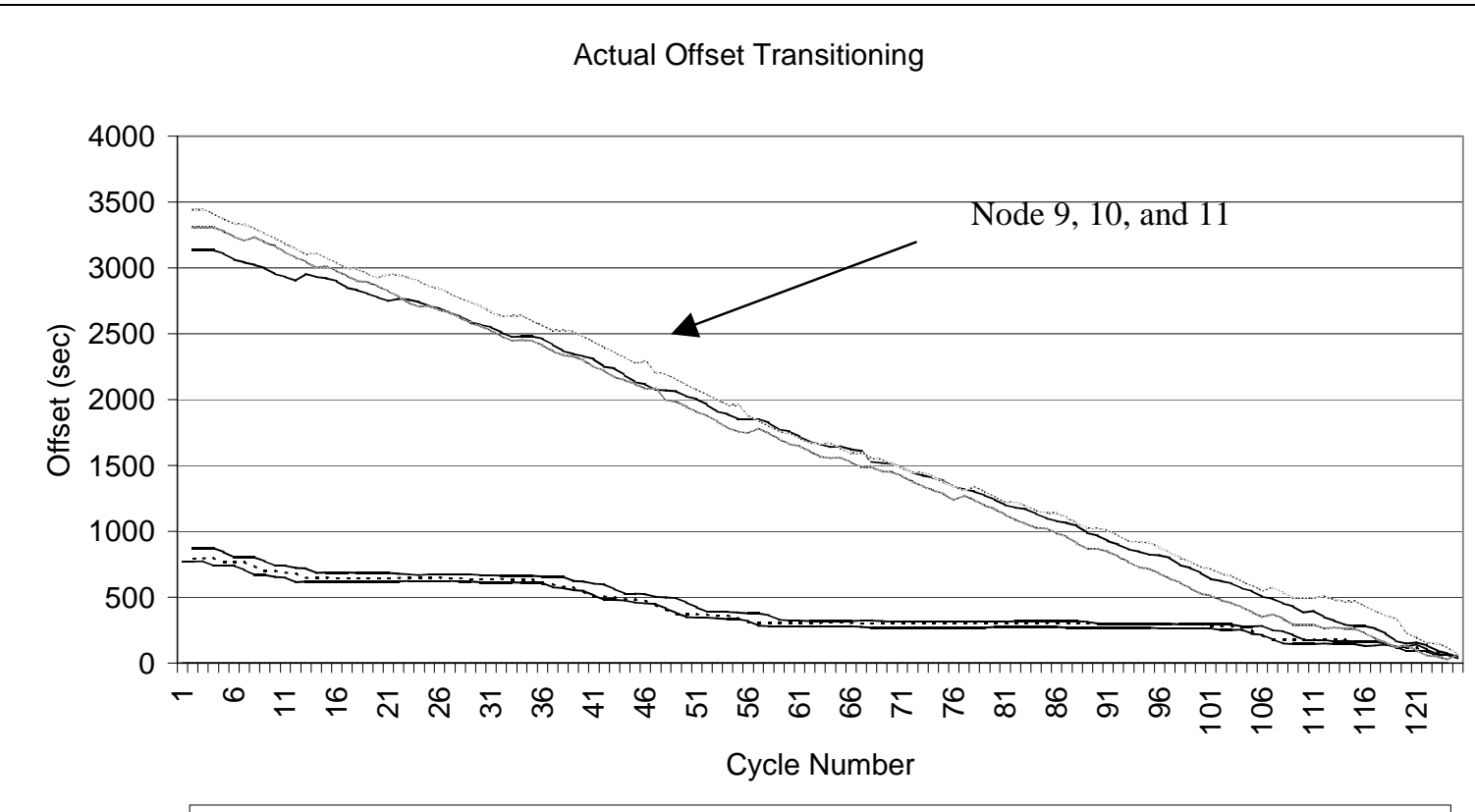

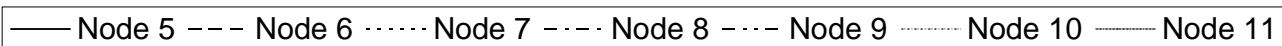

a) Offset Transitioning

Cycle Transitioning

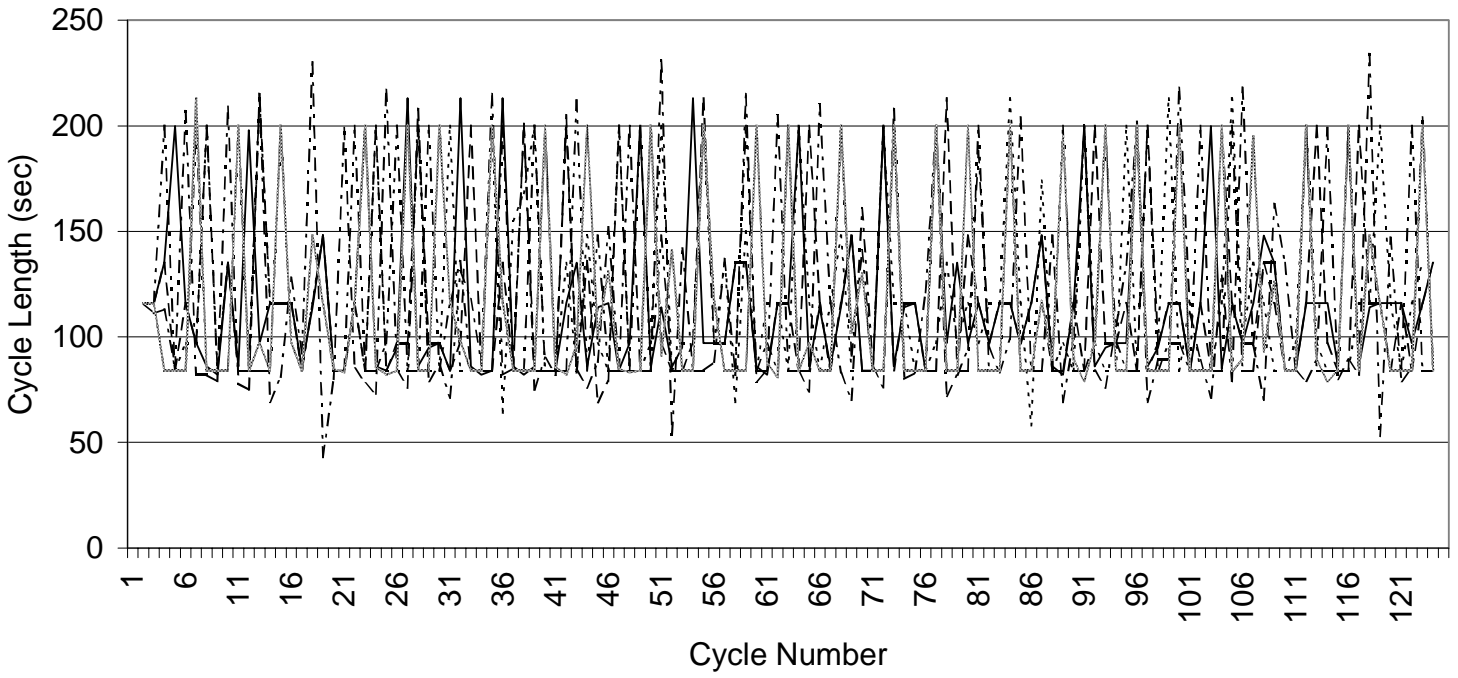

— Node 5 ---Node $6 \cdots$ Node 7 -.-Node 8 -... Node $9 \cdots$ Node $10 \longrightarrow$ Node 11

b) Cycle Transitioning

Figure 10-16 US 31 Offset and Cycle Transitions-P.M. Period 


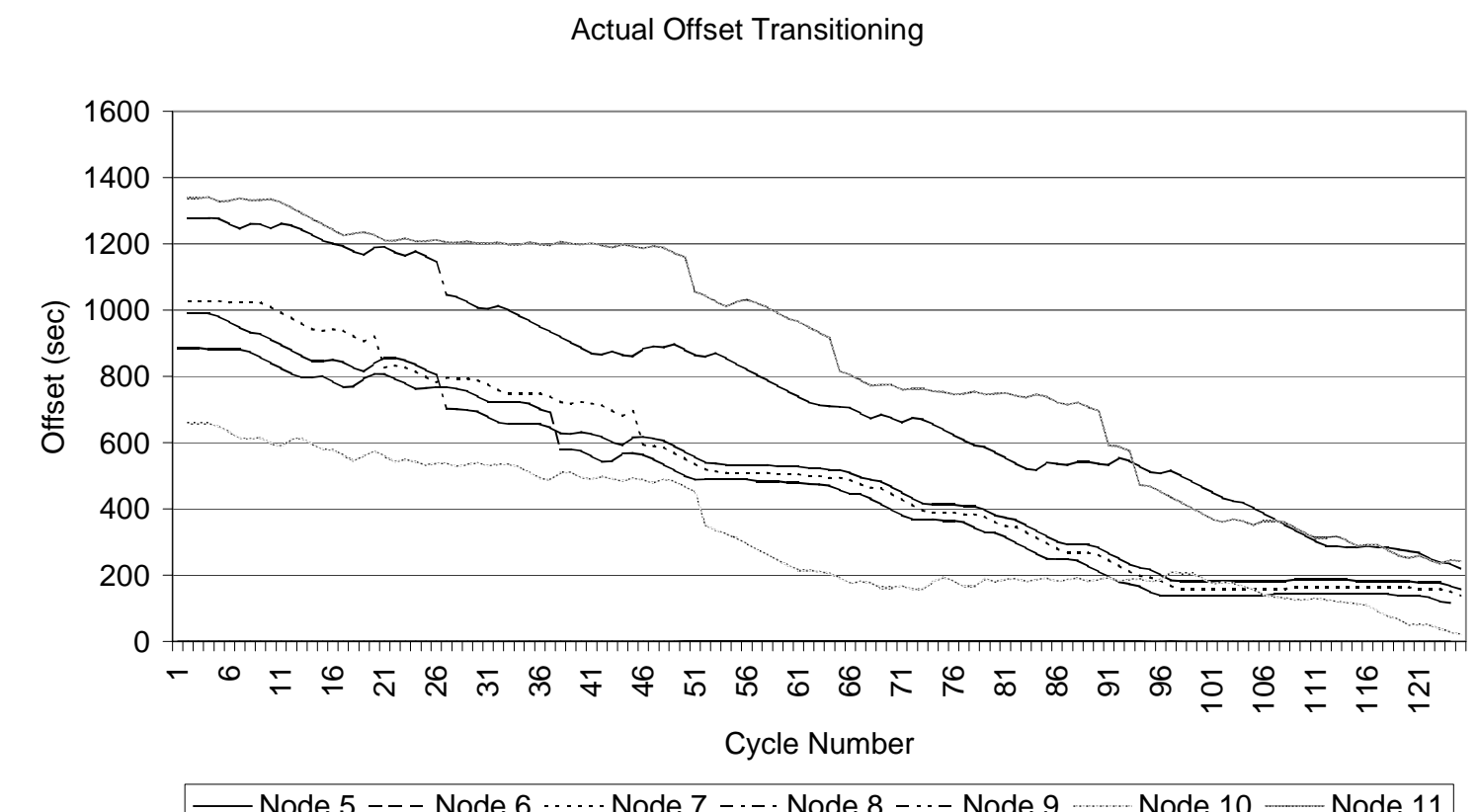

a) Offset Transitioning

\section{Cycle Transitioning}

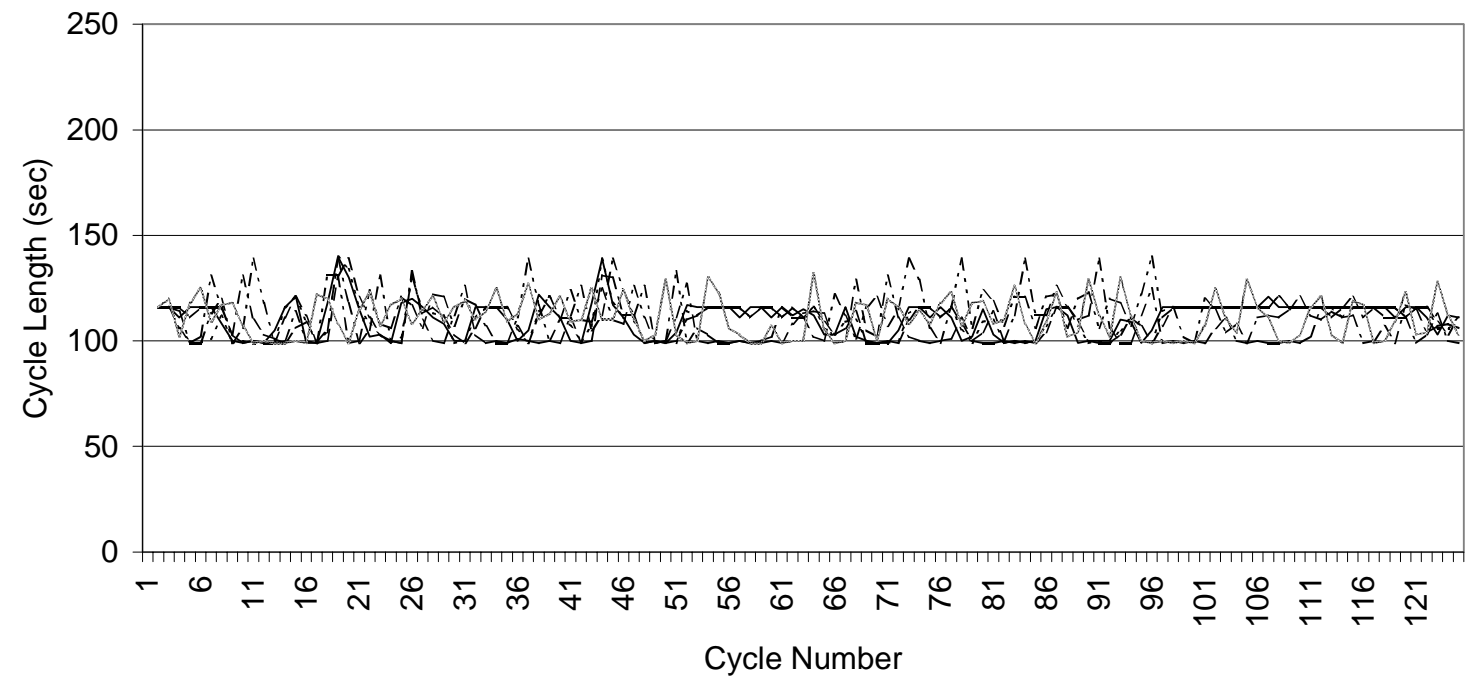

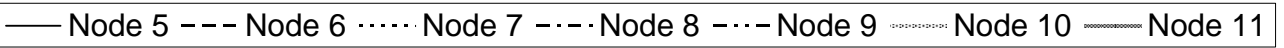

b) Cycle Transitioning

Figure 10-17 US 31 Offset and Cycle Transitions-P.M. Period with Hardware-in-theLoop 
Main Street Green

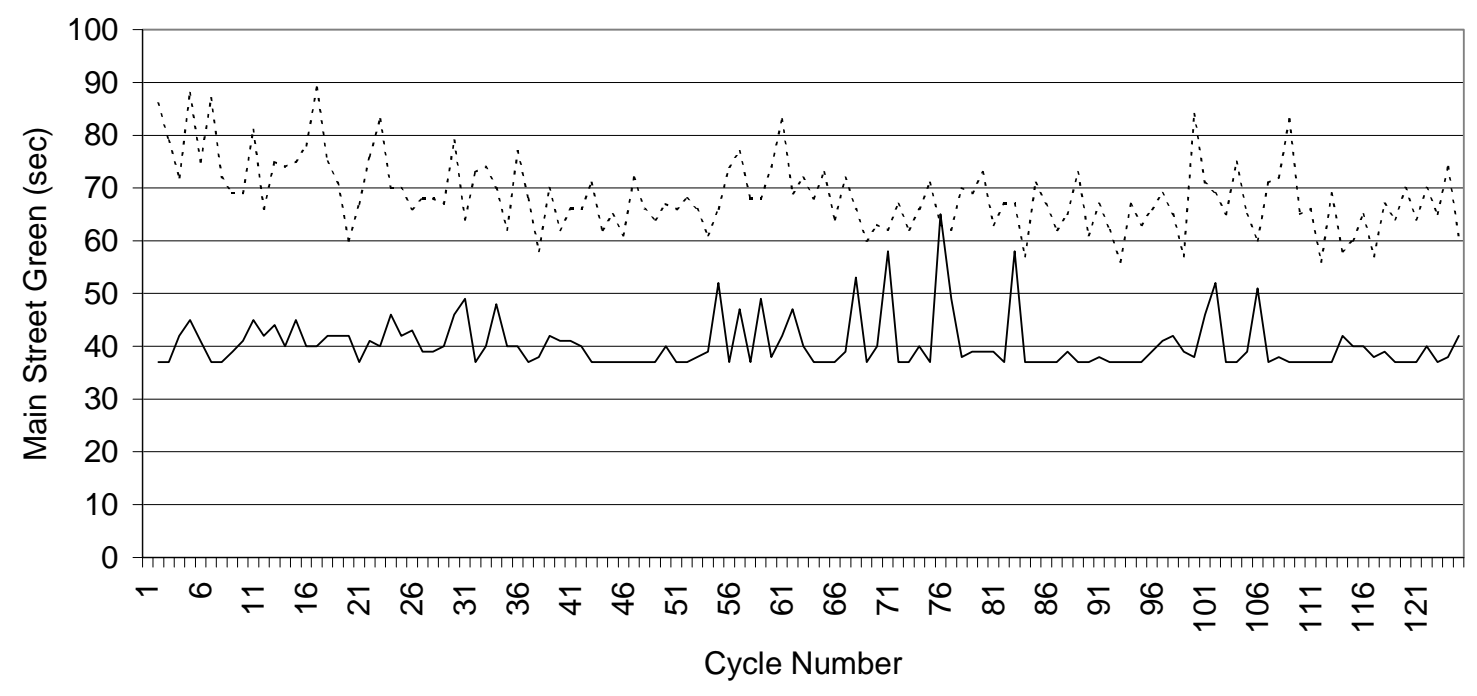

- Node $8 \cdots$ Node 9

Figure 10-18 Main Street Green at Nodes 8 and 9

Some spill back and lane blockage were observed when looking at CORSIM's animation file, especially at node 9 which explains in part the cyclic early return to green pattern, these conditions are shown in Figure 10-19. 


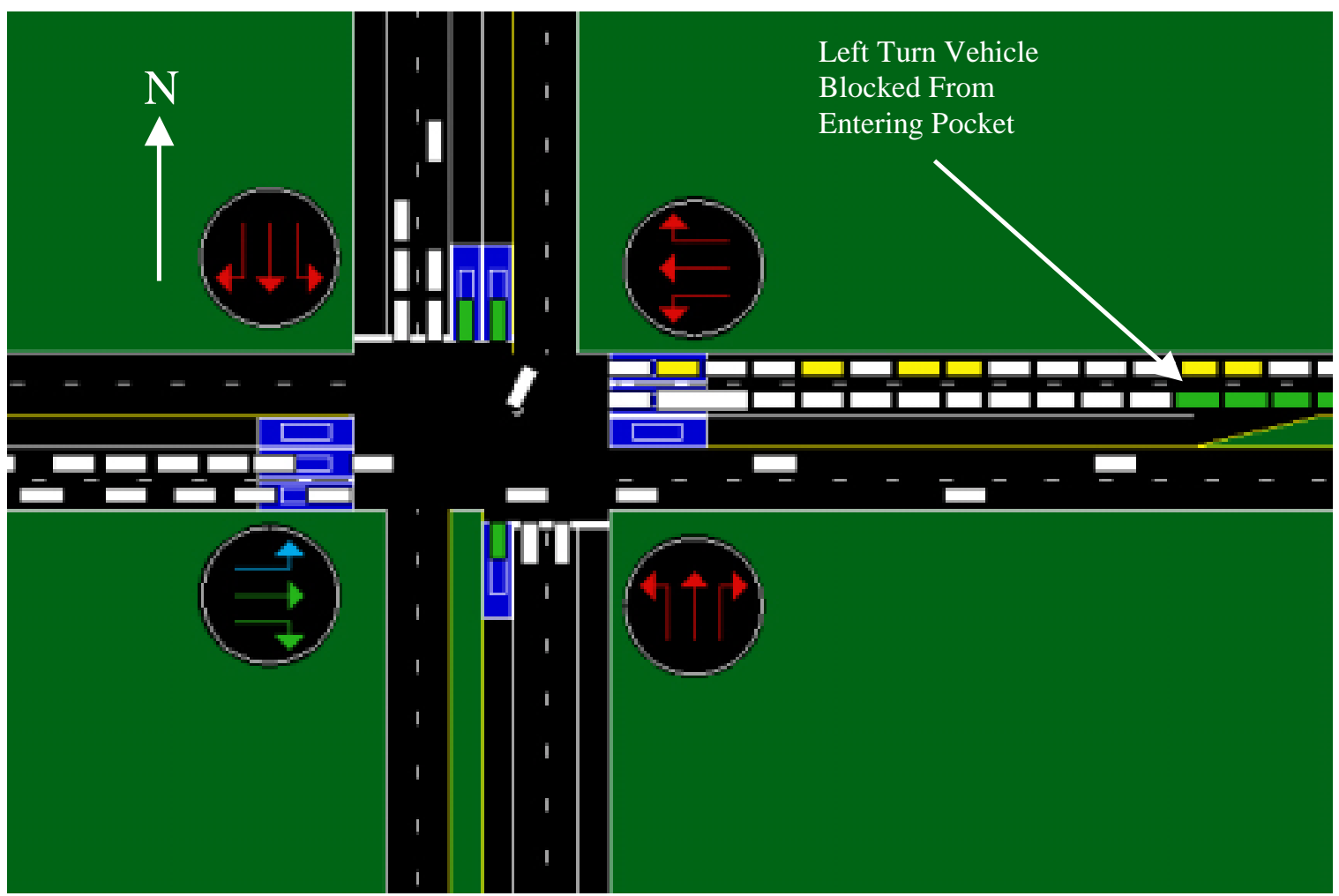

a) Left Lane Blockage at Node 9

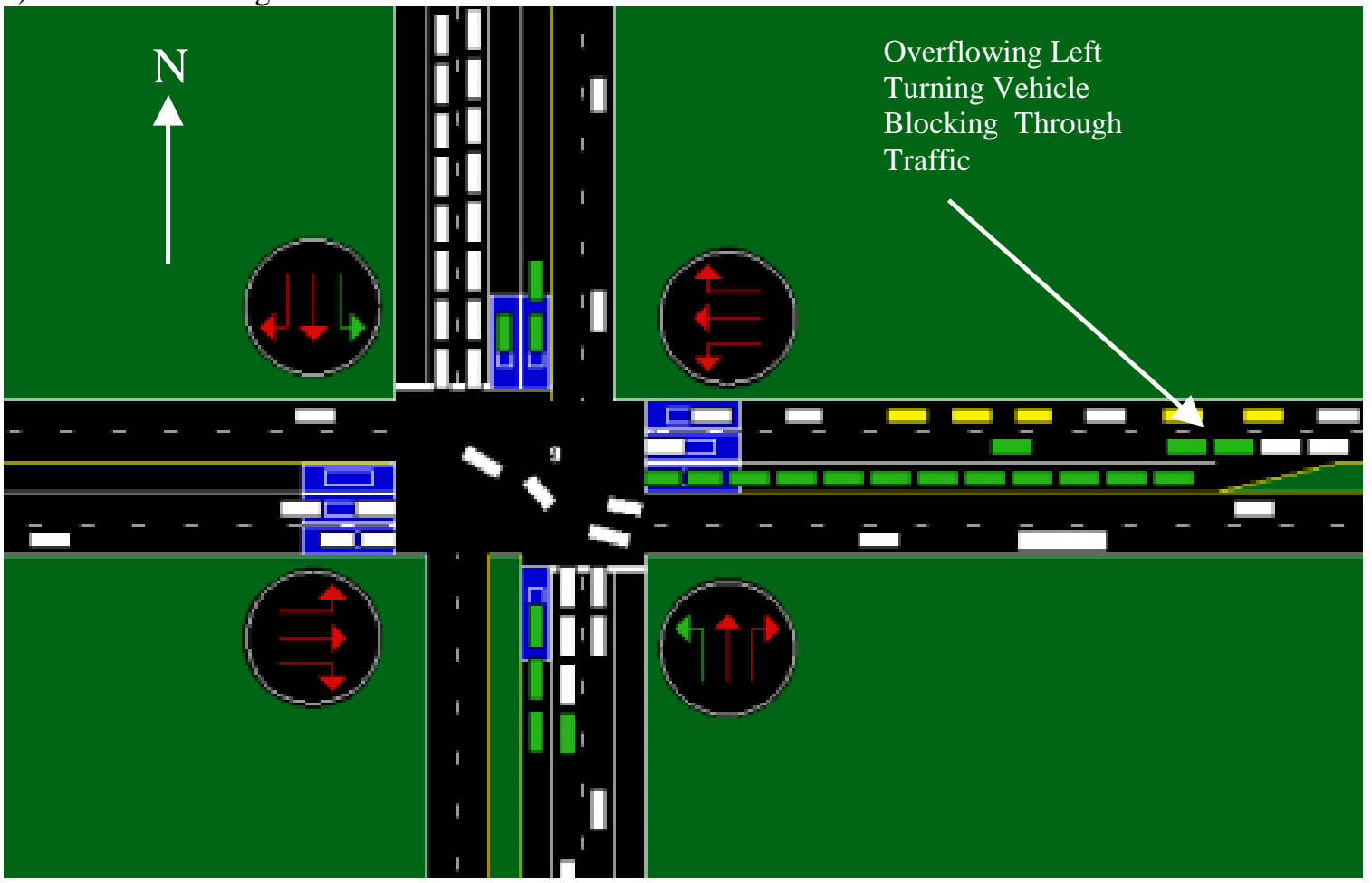

b) Through Lane Blockage at Node 9

Figure 10-19 Lane Blockage at Node 9 


\subsection{Chapter's Conclusion}

This chapter studied the adaptation of PRO-TRACTS to changes in traffic patterns of US31 in Kokomo, Indiana. Simulations were run with both CORSIM's internal model and hardware-in-the-loop. The study found that PRO-TRACTS consistently resulted in an improvement in travel time in the arterial direction to which it was applied.

Although PRO-TRACTS running with CORSIM's internal model showed some reduction in travel time, the internal transitioning mechanism in CORSIM had a negative effect on PRO-TRACTS stability. On the other hand, the hardware-in-the-loop simulation was found to be more stable than CORSIM's internal model. The hardware-in-the-loop simulation was also found to be more robust than CORSIM's internal model for unstable on oscillatory traffic patterns. The benefits averaged to $7 \%$ savings in travel time for the northbound direction over Synchro's timing plans with an average saving of $11 \%$ in the total network delay. 


\section{CHAPTER 11- CONCLUSION AND FUTURE RESEARCH}

Over the last few decades, several efforts have been made in the development of adaptive and responsive traffic control systems that can account for short and long-term changes in traffic patterns in their real-time optimization of traffic network. However, adaptive control systems typically require an extensive input of system parameters and weighting factors for favoring individual traffic movements, plus a large number of vehicle detectors to collect movement-specific traffic data.

In a parallel track, coordinated-actuated systems continued to be deployed in arterial systems to provide efficient operation with their ability to respond to cycle-bycycle variations in traffic demand, while still being able to provide progression for the arterial movement. Traffic signals with a variable actuated-phases demand operating under actuated logic, save a significant amount of delay in arterial systems when compared to fixed-time systems. However, since only the coordinated phase's end of green is deterministic under actuated operations, the same logic that provides efficient operation of intersections also creates what is know as the early-return-to-green phenomenon, a phenomenon that is caused by the stochastic nature of traffic flow on individual intersections.

An adaptive real-time offset transitioning algorithm (PRO-TRACTS) was developed in this research. PRO-TRACTS can be viewed as an integrated optimization approach designed to work with traditional coordinated-actuated systems and adds to the 
controllers the ability to adaptively change their offsets in response to changes in traffic patterns. This algorithm provides an intermediate solution between traditional coordinated-actuated control systems and adaptive control systems.

\subsection{Theoretical Contribution}

The report introduced a new metric by which offset performance can be evaluated. The new metric, namely the F' model, makes use of the fact that bad offsets at downstream intersections generate backward shockwaves that can extend to the upstream detector location. Figure 5-3 and Figure 5-4 illustrate this concept. The metric developed in this research tests for the significance of the presence of shockwaves by calculating a proxy to the variance of the reciprocal of the speed at the upstream detector. The philosophy behind this procedure is that a large variance in speed suggests that a shockwave has a significant effect on the traffic flow. This procedure is modeled after the analysis of variance F distribution testing. Discriminant analysis is used in Chapter 7 to develop thresholds to which the F' value is compared to determine the amount of offset adjustment needed.

The algorithm uses a unique cycle-based tabulation of occupancy and count profiles at the upstream detector to calculate the F' value. The effect of shockwaves generated due to the offset on the occupancy and count profile can be clearly seen in Figure 6-2 and Figure 6-6. 


\subsection{Deployment Contribution}

A new NTCIP object for capturing a cycle-based detector actuation profile at the controller level was defined in this report (Figure 9-12 and Figure 9-13). The defined object will enable the implementation of PRO-TRACTS in the field by allowing real-time exchange of the data required to fine-tune the offsets.

\subsection{Case Studies}

Two simulation case studies were conducted on two traffic networks: SR 26 in Lafayette, Indiana and US31 in Kokomo, Indiana. The studies showed that for a network with poor or sub-optimal offsets, PRO-TRACTS consistently resulted in an improvement in both the travel time and the number of stops in the arterial direction to which it was applied, with a greater impact in reducing the number of stops. In the SR26 case study, PRO-TRACTS achieved a performance level close to that of Synchro's timing plans, while it achieved a better performance than Synchro's plans in the US 31 case study. The magnitude of such an improvement depends on the existing performance of the network

offsets. Obviously, if there is only minor room for improvement, one can only expect that much. On the other hand, when starting with a system with poor offsets, computer simulations revealed up to $16 \%$ savings in the total travel time and up to $43 \%$ savings in the total number of stops for the coordinated movement.

\subsection{Limitations and Future Research}

It was found that in some situations, PRO-TRACTS caused an increase in total network delay compared to system-optimum timings when activated with optimum 
timing plans. These results were not surprising since favoring a directional movement is an additional constraint in the system optimization function. When enforcing a constraint such as favoring a certain traffic movement, the best that can be achieved in the overall system objective function is to keep it constant. However, if the current timing plans were not the system optimum plans, PRO-TRACTS can result in a reduction in total system delay as well.

Adjusting offsets in real-time caused some stability problems when PROTRACTS was run with CORSIM's internal control model. The hardware-in-the-loop simulations of the US 31 traffic network in Kokomo, Indiana revealed that field traffic controllers are more stable than CORSIM's internal model in responding to real-time offset adjustment. For example, compare Figure 10-16 and Figure 10-17. Table 11-1 shows a summary of MOEs, comparing the results obtained with the internal model and the hardware-in-the-loop simulations. The table shows better performance obtained with the hardware-in-the-loop simulation, which provides a more realistic results. 
Table 11-1 Summary of PRO-TRACTS Improvements Using Internal and Hardware-inthe-Loop Simulation on US 31

\begin{tabular}{|c|c|c|c|c|c|}
\hline \multirow[t]{2}{*}{ MOE } & \multirow[t]{2}{*}{ Hour } & \multicolumn{2}{|l|}{ A.M. Period } & \multicolumn{2}{|l|}{ P.M. Period } \\
\hline & & $\begin{array}{l}\text { Hardware- } \\
\text { in-the-Loop }\end{array}$ & $\begin{array}{l}\text { Internal } \\
\text { Model } \\
\text { Simulation }\end{array}$ & $\begin{array}{l}\text { Hardware- } \\
\text { in-the-Loop }\end{array}$ & $\begin{array}{l}\text { Internal } \\
\text { Model } \\
\text { Simulation }\end{array}$ \\
\hline \multirow{4}{*}{$\begin{array}{l}\text { NB (PRO-TRACTS) } \\
\text { Cumulative Travel } \\
\text { Time in Comparison } \\
\text { to Synchro's Plan }\end{array}$} & 1 & $0 \%$ & $7 \%$ & $6 \%$ & $1 \%$ \\
\hline & 2 & $0 \%$ & $4 \%$ & $6 \%$ & $2 \%$ \\
\hline & 3 & $0 \%$ & $3 \%$ & $7 \%$ & $2 \%$ \\
\hline & 4 & $0 \%$ & $3 \%$ & $7 \%$ & $3 \%$ \\
\hline \multirow{4}{*}{$\begin{array}{l}\text { SB Cumulative Travel } \\
\text { Time in Comparison } \\
\text { to Synchro's Plan }\end{array}$} & 1 & $0 \%$ & $2 \%$ & $-2 \%$ & $-12 \%$ \\
\hline & 2 & $0 \%$ & $0 \%$ & $-3 \%$ & $-12 \%$ \\
\hline & 3 & $0 \%$ & $-1 \%$ & $-2 \%$ & $-12 \%$ \\
\hline & 4 & $0 \%$ & $-1 \%$ & $-6 \%$ & $-12 \%$ \\
\hline \multirow{4}{*}{$\begin{array}{l}\text { Total System Delay in } \\
\text { Comparison to } \\
\text { Synchro's Plan }\end{array}$} & 1 & $-1 \%$ & $28 \%$ & $10 \%$ & $-15 \%$ \\
\hline & 2 & $1 \%$ & $-16 \%$ & $14 \%$ & $-16 \%$ \\
\hline & 3 & $3 \%$ & $-65 \%$ & $11 \%$ & $-13 \%$ \\
\hline & 4 & $-2 \%$ & $-10 \%$ & $9 \%$ & $-13 \%$ \\
\hline \multirow{4}{*}{$\begin{array}{l}\text { Total Number of Stops } \\
\text { in Comparison to } \\
\text { Synchro's Plan }\end{array}$} & 1 & $1 \%$ & $27 \%$ & $4 \%$ & $-4 \%$ \\
\hline & 2 & $2 \%$ & $-2 \%$ & $2 \%$ & $-4 \%$ \\
\hline & 3 & $2 \%$ & $-17 \%$ & $1 \%$ & $-2 \%$ \\
\hline & 4 & $2 \%$ & $-4 \%$ & $11 \%$ & $-2 \%$ \\
\hline
\end{tabular}

The platooning phenomenon has an effect on all coordination schemes since platoon dispersion can make it impossible to fit the whole platoon within a downstream green window (Figure 7-4). The platooning phenomenon was found to have significant impacts on the performance of PRO-TRACTS. When the traffic turning from the side streets is a low percentage compared to the arterial's through-movement, a good coordination scheme would align the arterial through-movement with the green window, leaving the turning traffic from the side streets to face the red window. This situation causes only a weak shockwave resulting from the turning traffic (Figure 5-4) compared to the strong shockwave that results from a bad offset that align the platoon with the red window (Figure 5-3). However, if the side street traffic volume approaches that of the 
arterial's through, even the turning traffic from the side streets will generate a strong shockwave (Figure 5-6) that could cause PRO-TRACTS activation.

The ideal location for a PRO-TRACTS detector is between the termination of the shockwave generated by the side street turning traffic and the shockwave generated by the arterial's traffic (Figure 7-6). The algorithm is best suited for arterials with primarily through-traffic since a wider separation is provided between the two shockwaves.

Another factor that affected the stability of PRO-TRACTS was the frequency of phase skips and oscillatory traffic patterns caused by certain situations, such as spillbacks or lane blockages. Since PRO-TRACTS is a reactive algorithm, it assumes that the next cycle's arrivals are comparable to the current cycle's arrivals. With phase skips (Figure 8-8), the discrepancy in arrival patterns of succeeding cycles causes PRO-TRACTS offsets to fluctuate, reducing its efficiency. Future research should address this issue.

The case studies, summarized in Table 11-1, investigated in this research found that PRO-TRACTS has always caused an improvement in the direction to which it was applied. However, the impact on the other direction was typically an increase in the travel time. Although coordination usually takes place during the peak period when traffic is typically at its highest in one direction, there might be situations when the two direction of traffic are almost equal, in which a case, it is difficult to decide which direction to coordinate. Future research should also consider this situation by expanding the algorithm to work in two directions. 


\section{LIST OF REFERENCES}

Andrews, C. M., and S. M. Elahi. "Evaluation of New Jersey Route 18 OPAC/MIST Traffic Control System.” Presented at the 76th Annual Meeting of the Transportation Research Board, Washington, DC, January 1997.

Avery, D. J. Jr., and Kenneth G. "Method for Reviewing and Assessing PASSER II and TRANSYT 7-F Signal Timing Optimization outputs." Proceedings of 4th International Conference on Microcomputers in Transportation. American Society of Civil Engineers, New York, NY, 1993, pp. 297-308.

Baass, Karsten G. and Serge Lefebvre. "Analysis of Platoon Dispersion with Respect to Traffic Volume." Transportation Research Record 1194, Transportation Research Board, National Research Council, Washington, DC, 1988, pp. 64-76.

Black, John R. "Richardson, Texas Advanced Traffic Management System.” http://www.naztec.com/richatms/richatms.htm. Accessed 1998.

Bullock, D. and A. Catarella. "A Real-Time Simulation Environment for Evaluating Traffic Signal Systems." Transportation Research Record, \#1634, National Research Council, Washington, DC, 1998, pp. 130-135.

Bullock, D and T Urbanik. "Traffic Signal Systems: Addressing Diverse Technologies and Complex User Needs." Transportation in the New Millennium. National Research Council, Washington DC, 2000.

Castle, David E., and John W. Bonniville. "Platoon Dispersion over Long Road Links." Transportation Research Record 1021, Transportation Research Board, National Research Council, Washington, DC, 1985, pp. 36-44.

Chang, E. C. P. and Joseph Koothrappally. "Field Verification of Coordinated Actuated Control." Transportation Research Record 1456. Transportation Research Board, National Research Council, Washington DC, 1994, pp. 83-90.

Chang, E. C. P. "Guidelines for Actuated Controllers in Coordinated Systems." Transportation Research Record 1554, Transportation Research Board, National Research Council, Washington DC, 1996, pp. 61-73. 
Collins, J.F., and P. Gower. "Dispersion of Traffic Platoons on A4 in Hounslow." TRRL Report SR 29UC, U.K. Transport and Road Research Laboratory, Crowthorne, Berkshire, England, 1974.

Denney, Richard W. "Traffic Platoon Dispersion Modelling." Journal of Transportation Engineering, American Association of Civil Engineers, New York, NY, March 1989.

Econolite Control Products. Advanced System Controller ASC/2 Programming Manual. Anaheim, CA, 1996.

Engelbrecht, R., C. Poe, and K. Balke. "Development of a Distributed Hardware-In-TheLoop Simulation System for Transportation Networks," Transportation Research Board Annual Meeting, National Research Council, Washington, DC, 1999, Preprint \#990599.

Federal Highway Administration. Transportation Planning and ITS: Putting Pieces Together. Sarah J. Siwek \& Associates, United States Department of Transportation, Public Technologies INC, Washington DC, 1996.

Ficklin, N.C. "For and Against Semi-actuated Signals." Traffic Engineering, March 1973.

Fischer. J. W., "The Transportation Equity Act for the 21st Century (TEA21) and the Federal Budget", CRS Report 98-749 E, September 4, 1998.

Gartner, Nathan H. "OPAC: A Demand-Responsive Strategy for Traffic Signal Control." Transportation Research Record 906. Transportation Research Board, National Research Council, Washington DC, 1983.

Gartner, Nathan H., Philip J. Tarnoff and Christina M. Andrews. "Evaluation of Optimized Policies for Adaptive Control Strategy." Transportation Research Record 1324. TRB, National Research Council, Washington DC, 1991.

Gartner, Nathan H., Chronis Stamatiadis, and Phillip J. Tarnoff. "Development of Advanced Traffic Signal Control Strategies for Intelligent Transportation Systems: Mulitlevel Design." Transportation Research Record 1494, Transportation Research Board, National Research Council, Washington DC, 1995.

Gordon, Robert 1., R.A. Reiss, H. Haenel, E.R. Case, R.L. French, A. Mohaddes, and Wolcott. Traffic Control Systems Handbook. FHWA, United States Department of Transportation, Washington DC, 1996.

Head, K. Larry, Pitu B. Mirchandani and Dennis Sheppard. "Hierarchical Framework for Real-Time Traffic Control.” Transportation Research Record 1360, Transportation Research Board, National Research Council, Washington DC, 1992. 
Head, K. Larry. "Event-Based Short-Term Traffic Flow Prediction Model." Transportation Research Record 1510, Transportation Research Board, National Research Council, Washington DC, 1995.

Head, K. Larry, Pitu B. Mirchandani, and Steve Shelby. "The RHODES Prototype: A Description and Some Results." Proceedings from the $77^{\text {th }}$ Annual Meeting of the Transportation Research Board. Transportation Research Board, Washington DC, 1998.

"Highway Capacity Manual." Transportation Research Board Special Report 209, $3^{\text {rd }}$ Edition, TRB, Washington, D.C., 1997.

Hunt, P.B., D.I. Robertson, R.D. Bretherton, and R.I. Winton. "SCOOT- A Traffic Method of Coordinating Signals." Laboratory Report No. LR 1014. Transportation and Road Research, Crowthorne, Bershire, England, 1981.

Husch, David. SimTraffic 1.0 User Guide. Trafficware, Trafficware, Berkely, CA. 1998. Husch, David. Synchro 3.2 User Guide. Trafficware, Trafficware, Berkely, CA. 1998.

Husch, D, SimTraffic CI Supplemental Guide, Trafficware, Berkely, CA. 1999.

ITS National Architecture, Federal Highway Administration, 1998.

Jovanis, P.P. and J.A. Gregor. "Coordination of Actuated Arterial Traffic Signal Systems." Journal of Transportation Engineering, Volume 112, Number 4. American Society of Civil Engineers, New York, NY, July 1986.

Kaman Sciences Corporation. TSIS User's Guide, Version 4.01. Colorado Springs, CO, 1997A.

Kaman Sciences Corporation. CORSIM User' s Manual, Version 1.03. Colorado Springs, CO, 1997B.

Kell, J.H., and I.J. Fullerton. Manual of Traffic Signal Design. Institute of Transportation Engineers, Prentice Hall, Englewood Cliffs, NJ, 1991.

Kuhne, R., J. Palen, C. Gardner, S. Ritchie. "Loop-based Travel Time Measurement." Applications of Advanced Technologies in Transportation, American Society of Civil Engineers, Newport Beach, CA, 1998, pp. 175-182.

Lam, J.K. "Studies of a Platoon Dispersion Model and Its Practical Applications." Seventh International Symposium on Transportation and Traffic Theory, Institute of Systems Science Research, Kyoto, Japan, 1977. 
Lighthill, M.J. and G.B. Whitham "On Kinematic Waves. II. A Theory of Traffic Flow on Long Crowded Roads." Proceedings of the Royal Society of London, Series A, Mathematical and Physical Sciences, Volume 229, Issue 1178, May 10, 1955, pages 317345 .

Manar, Abdelaziz, and Karsten G. Baass. "Traffic Platoon Dispersion Modeling on Arterial Streets." Transportation Research Record 1566, Transportation Research Board, National Research Council, Washington, DC, November 1996, pp. 49-53.

May, Adolf D. Traffic Flow Fundamentals. Englewood Cliffs, N.J. : Prentice Hall, 1990.

McCoy, Patrick T., Elizabeth A., Balderson, Richard T. Hsueh, and Abbas K. Mohaddes "Calibration of TRANSYT Platoon Dispersion Model for Passenger Cars Under LowFriction Traffic Flow Conditions." Transportation Research Record 905, Transportation Research Board, National Research Council, Washington, DC, 1983, pp. 48-52.

Grace, Muriel J. and Renfrey B. Potts. (12 1964), A Theory of the Diffusion of Traffic Platoons, Opns Res., 255-275.

National Electrical Manufacturers Association. National Transportation Communications for ITS Protocol -the NTCIP Guide. NTCIP 9001 V02.06 (Draft), Washington, D.C., 1999A.

National Electrical Manufacturers Association. National Transportation Communications for ITS Protocol - Virginia DOT Statewide VMS Project. NTCIP 9002 V01.04, Washington, DC, 1999B.

National Electrical Manufacturers Association. National Transportation Communications for ITS Protocol - Washington State DOT NTCIP VMS Software Upgrade. NTCIP 9003 V01.04, Washington, DC, 1999C.

National Electrical Manufacturers Association. National Transportation Communications for ITS Protocol - City of Phoenix, Arizona Phoenix Advanced Transportation Management System. NTCIP 9004 V01.05 (Draft), Washington, DC, 1999D.

National Electrical Manufacturers Association. National Transportation Communications for ITS Protocol - Overview. Standards Publication No. TS3.1-1996, Washington, DC, 1996.

National Electrical Manufacturers Association. National Transportation Communications for ITS Protocol - Simple Transportation Management Framework. Standards Publication No. TS3.2-1996, Washington, DC, 1996. 
National Electrical Manufacturers Association. National Transportation Communications for ITS Protocol - Class B Profile. Standards Publication No. TS3.3- 1996, Washington, DC, 1996.

National Electrical Manufacturers Association. National Transportation Communications for ITS Protocol Global Object Definitions. Standards Publication No. TS3.4-1996, Washington, DC, 1996.

National Electrical Manufacturers Association. National Transportation Communications for ITS Protocol Object Definitions for Actuated Traffic Signal Controller Units. Standards Publication No. TS3.5-1996, Washington, DC, 1996.

National Electrical Manufacturers Association. Standards Publication No. TS 1, Washington, DC, 1989.

National Electrical Manufacturers Association. Standards Publication No. TS 2, Washington, DC, 1992.

Nelson, Eric J. "Quantitative Evaluation of Closed-Loop Signal Systems" Master's Thesis, Purdue University, West Lafayette, IN, 2000.

Pacey, G.M., "The Progress of a Bunch of Vehicles Released from a Traffic Signal." Research Note No. RN/2665/GMP, Road Research Laboratory, Crowthorne, Berkshire, England, 1956.

Quinlan, T. Evaluation of Computer Hardware and High-Level Language Software for Field Traffic Control. Technical Report, California Department of Transportation, Sacramento, CA, 1989.

Robertson, D.I., TRANSYT: “A Traffic Network Study Tool.” Report No. LR253. Transport and Road Research Laboratory, United Kingdom, 1969.

Rouphail, Nagui, Andrzej Tarko, and Jing Li. "Traffic Flow at Signalized Intersections." Traffic Flow Theory: A State-of-the-Art Report, Transportation Research Board, National Research Council, Washington DC, 1992.

Seddon, P.A., "Another Look at Platoon Dispersion - 3: The Recurrence Relationship." Traffic Engineering and Control, February 1972, pp. 442-444.

Shoup, G. "Traffic Signal System Offset Tuning Procedure Using Travel Time Data." Master's Thesis, Purdue University, West Lafayette, IN, 1998.

Shoup, G. and D. Bullock, "A Dynamic Offset Tuning Procedure Using Real-Time Travel Data," Transportation Research Record \#1683, Transportation Research Board, National Research Council, Washington, DC, 1999, pp. 84-94. 
Shoup, G. and D. Bullock, "Performance Evaluation of Coordinated-Actuated Traffic Signal Systems." Proceedings of the 1999 ITE Conference, August 1-4, 1999.

Skabardonis, A. "Determination of Timings in Signal Systems with Traffic-Actuated Controllers. Transportation Research Record 1554, Transportation Research Board, National Research Council, Washington, DC, 1996, pp. 18-26.

Stallings, William. "SNMP, SNMPv2, and CMIP: The Practical Guide to Netwoerk Management Standards." Addision Wesley Publishing Company, Inc., Reading, MA, 1995.

Stewart, J. Allan, Katrin Lepik, and M. Van Aerde. "Benefit Sensitivities of Adaptive Traffic Control Strategies at Isolated Traffic Signals." Proceedings from the 77th Annual Meeting of the Transportation Research Board. Transportation Research Board, Washington DC, 1998.

Tompos, Attila. "Real-Time Corridor Simulation Volume Sensitivity Analysis." Master's Project, Purdue University, West Lafayette, IN, 2000.

"Traffic Controller Assemblies with NTCIP Requirements," TS 2-1998, National, Electrical Manufacturer Association.

Transportation Infrastructure, Benefits of Traffic Control Signal Systems Are Not Being Fully Realized. GAO/RCED-94-105, Report to the Chairman, Committee on Energy and Commerce, House of Representatives, United States General Accounting Office, Washington, DC, March 1994.

Wasson, J. M. Abbas, D. Bullock, A. Rhodes, and C. Zhu, "Reconciled Platoon Accommodations at Traffic Signals." FHWA/IN/JTRP-99/01. Joint Transportation Research Program, Purdue University, WL, IN, December 1999.

Wolkomir, R. "A High-Tech Attack on Traffic Jams Helps Motorists Go with the Flow." Smithsonian, Vol. 17, No. 1, April, 1986, pp. 40-51. 
Appendix A- SR 26 Study Network and Controller Parameters 


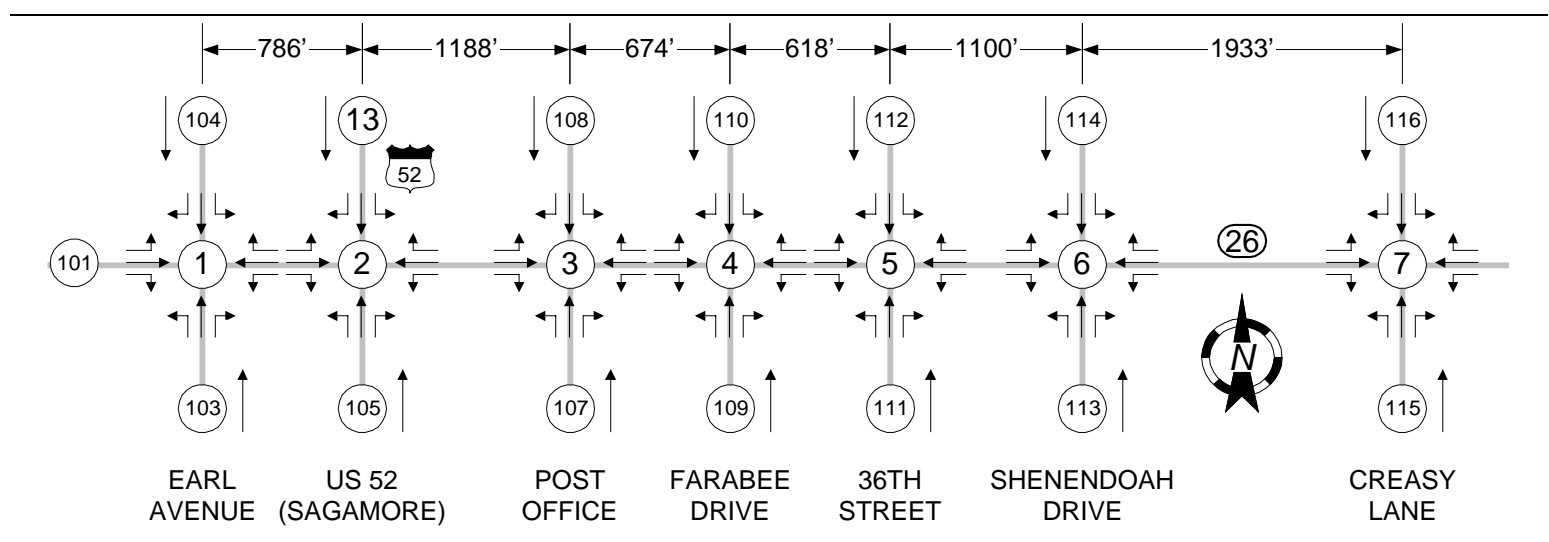

Figure A- 1 SR 26 (South) Network - Earl Avenue to Creasy Lane 

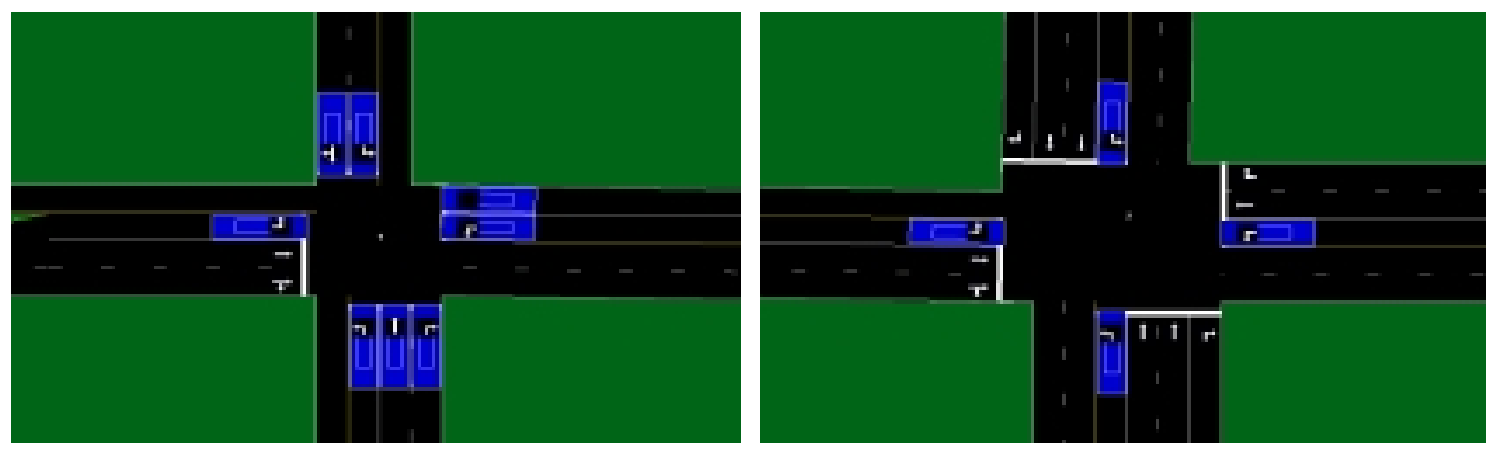

a) Node 1: SR 26 \& Earl Avenue

b) Node 2: SR 26 \& US 52 (Sagamore)
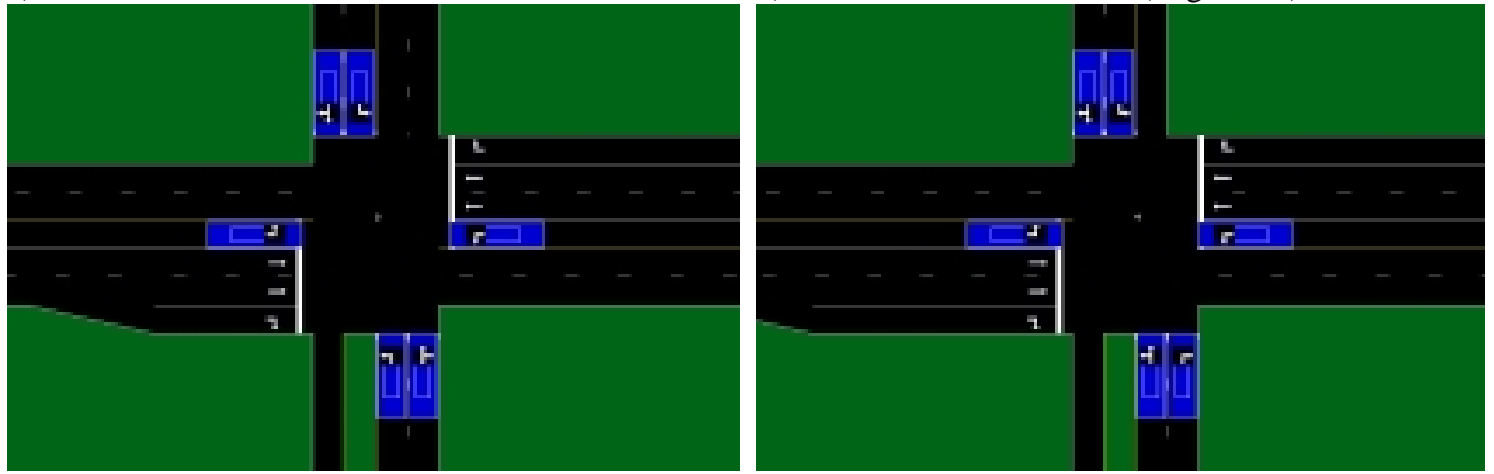

c) Node 3: SR 26 \& Post Office

d) Node 4: SR 26 \& Farabee Drive
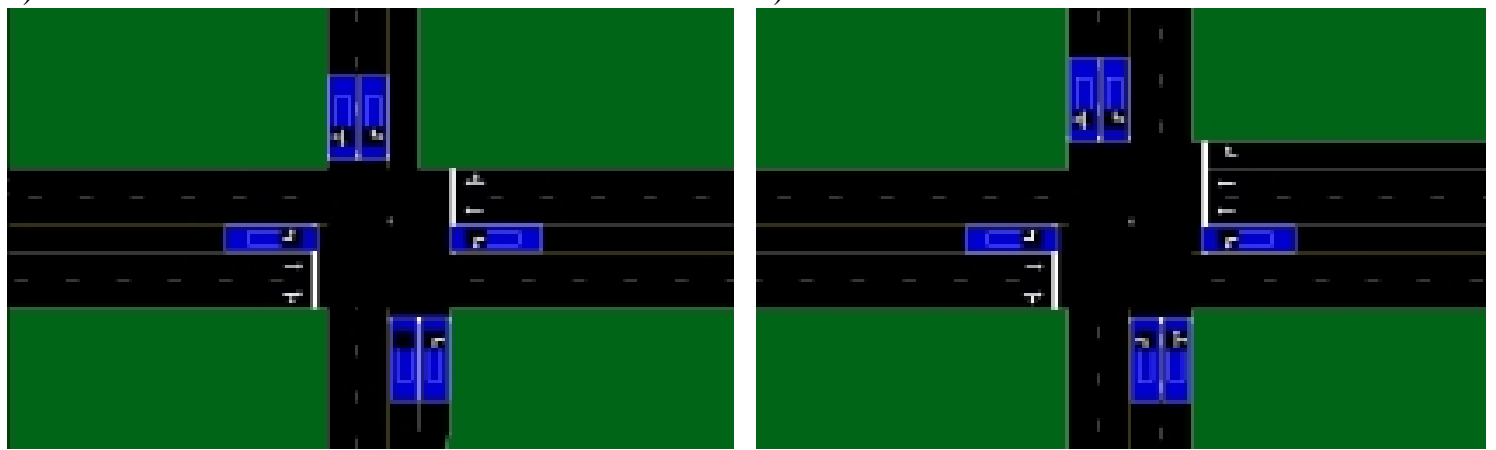

e) Node 5: SR $26 \& 36^{\text {th }}$ Street

f) Node 6: SR 26 \& Shenendoah Drive

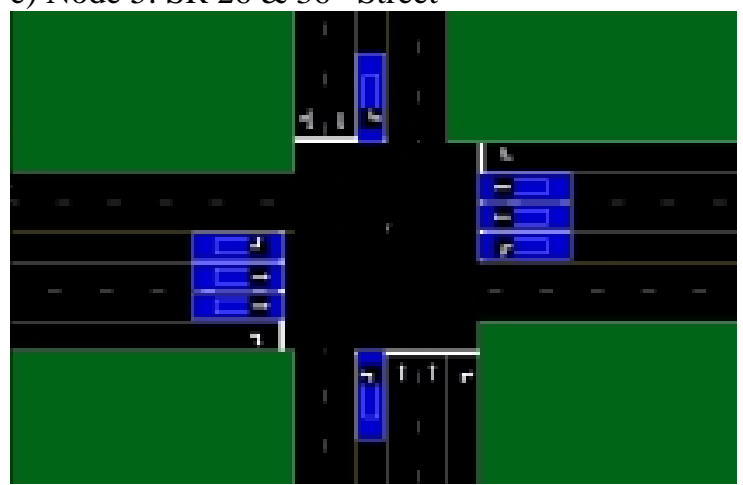

g) Node 7: SR 26 \& Creasy Lane

Figure A- 2 SR 26 Geometries 


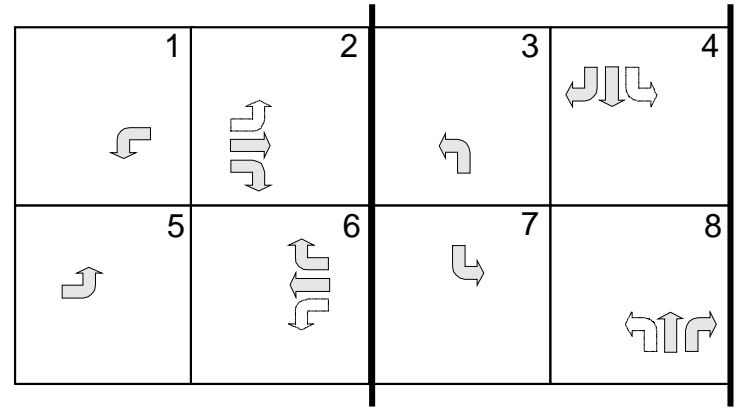

a) Node 1: SR 26 \& Earl Avenue

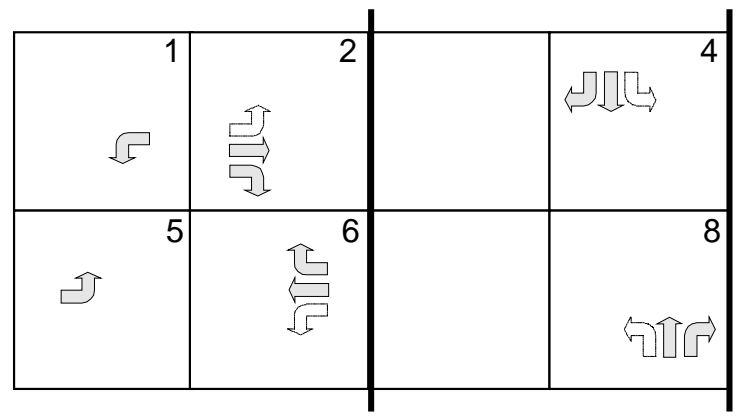

c) Node 3: SR $26 \&$ Post Office

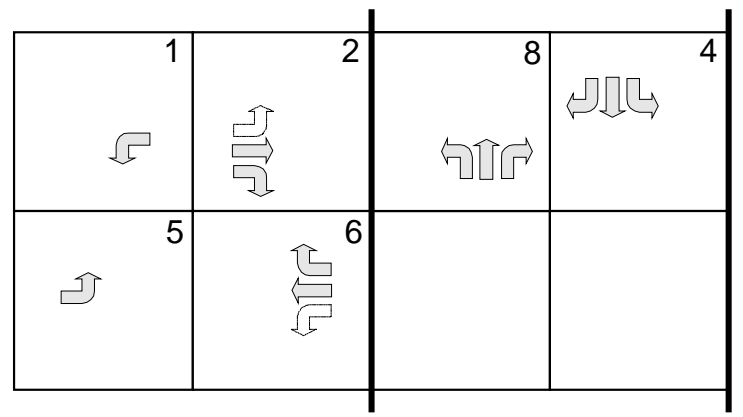

e) Node 5: SR $26 \& 36^{\text {th }}$ Street

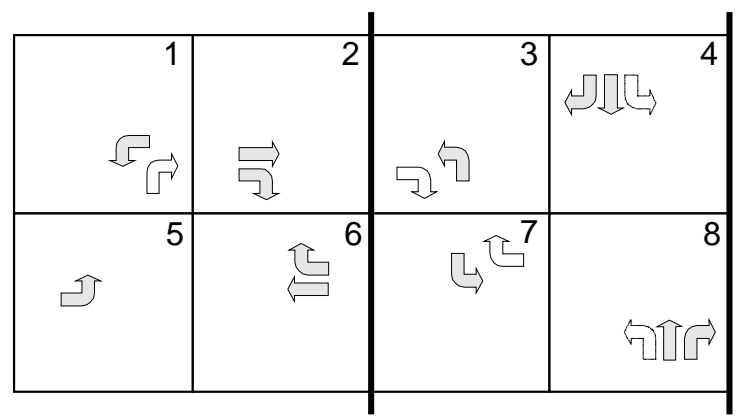

g) Node 7: SR 26 \& Creasy Lane

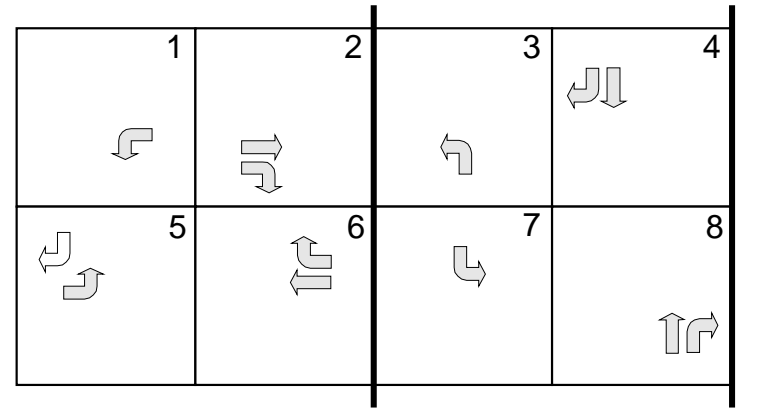

b) Node 2: SR 26 \& US 52 (Sagamore)

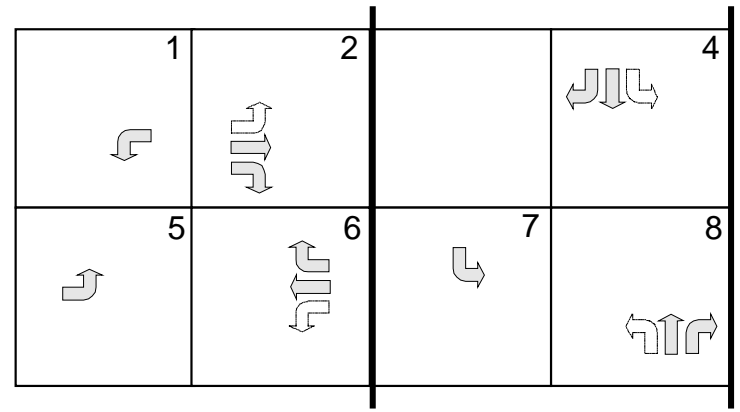

d) Node 4: SR 26 \& Farabee Drive

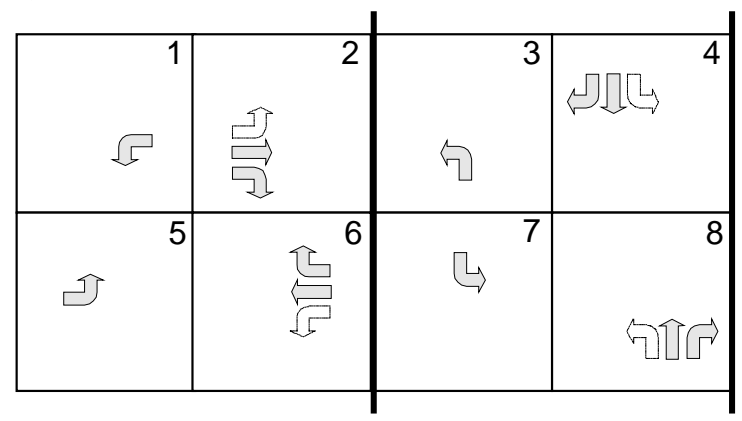

f) Node 6: SR $26 \&$ Shenendoah Drive

Figure A- 3 Intersection Ring Structures for SR 26 


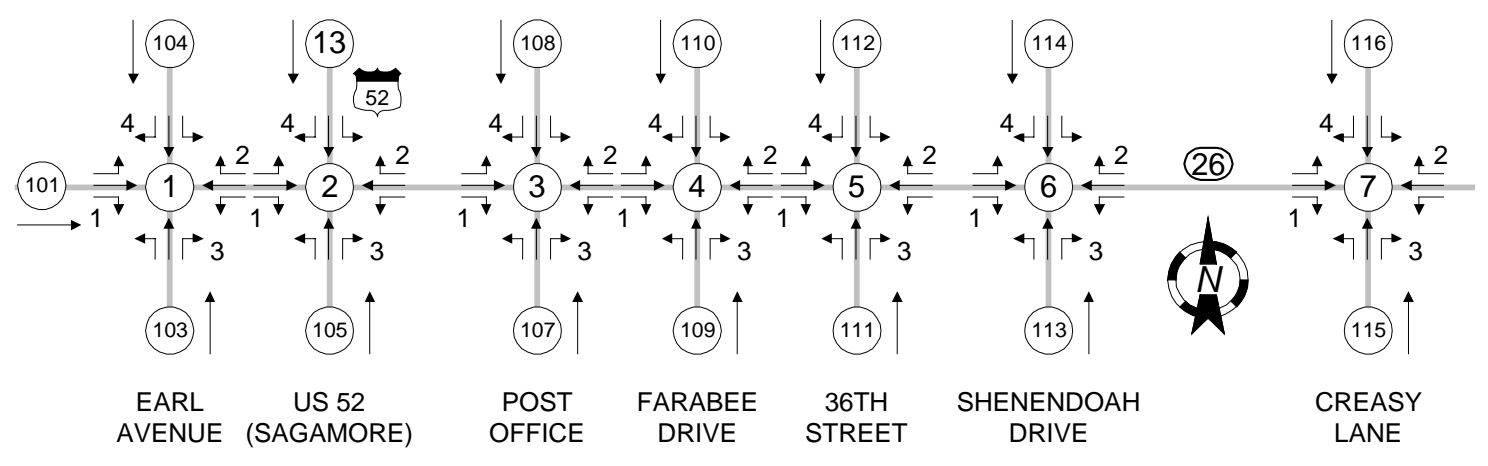

Figure A- 4 SR 26 (South) Approach Labels 


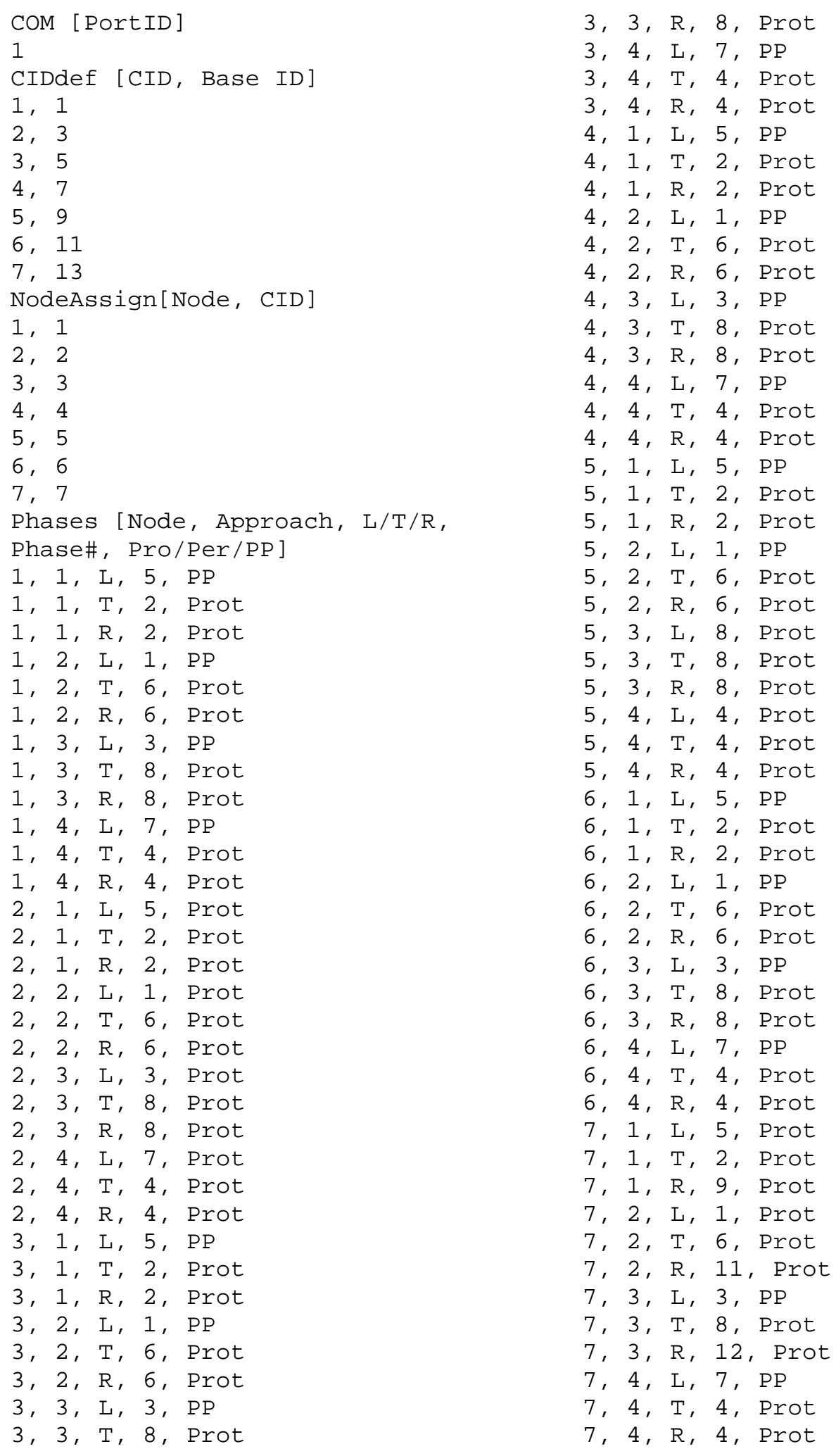

Figure A- 5 External Control File (CID) for Phasing 
Table A- 1 Intersection Pocket Lengths and Detector Locations

\begin{tabular}{|c|c|c|c|c|c|c|c|c|}
\hline \multicolumn{2}{|c|}{$\begin{array}{c}\text { SR } 26 \text { (SOUTH } \\
\text { ST) }\end{array}$} & \multicolumn{2}{|c|}{$\begin{array}{c}\text { POCKET } \\
\text { LENGTHS (FT) }\end{array}$} & \multicolumn{3}{|c|}{$\begin{array}{l}\text { STOP BAR DETECTOR } \\
\text { LENGTHS (FT) }\end{array}$} & \multicolumn{2}{|c|}{$\begin{array}{c}\text { RACEWAY } \\
\text { DETECTORS (FT) }\end{array}$} \\
\hline INT & MOVE & LEFT & RIGHT & LEFT & THRU & RIGHT & THRU & DIST \\
\hline 1 & EB & 100 & -- & 36 & -- & -- & 6 & 300 \\
\hline 1 & WB & 150 & -- & 36 & 36 & -- & 6 & 300 \\
\hline 1 & NB & 70 & -- & 36 & 36 & 36 & -- & -- \\
\hline 1 & SB & -- & -- & 36 & 36 & -- & -- & -- \\
\hline 2 & EB & 205 & -- & 36 & -- & -- & 6 & 300 \\
\hline 2 & WB & 550 & -- & 36 & -- & -- & 6 & 300 \\
\hline 2 & NB & 390 & 305 & 36 & -- & -- & 6 & 300 \\
\hline 2 & SB & 380 & 275 & 36 & -- & -- & 6 & 300 \\
\hline 3 & EB & 315 & 55 & 36 & -- & -- & 6 & 300 \\
\hline 3 & WB & 200 & 140 & 36 & -- & -- & 6 & 300 \\
\hline 3 & NB & -- & -- & -- & 36 & -- & -- & -- \\
\hline 3 & SB & -- & -- & -- & 36 & -- & -- & -- \\
\hline 4 & EB & 180 & 95 & 36 & -- & -- & 6 & 300 \\
\hline 4 & WB & 200 & 140 & 36 & -- & -- & 6 & 300 \\
\hline 4 & NB & -- & -- & & 36 & 36 & -- & -- \\
\hline 4 & SB & -- & -- & 36 & 36 & -- & -- & -- \\
\hline 5 & EB & 140 & -- & 36 & -- & -- & 6 & 300 \\
\hline 5 & WB & 110 & -- & 36 & -- & -- & 6 & 300 \\
\hline 5 & NB & 50 & -- & -- & 36 & 36 & -- & -- \\
\hline 5 & SB & -- & -- & -- & 36 & -- & -- & -- \\
\hline 6 & EB & 200 & -- & 36 & -- & -- & 6 & 300 \\
\hline 6 & WB & 250 & 200 & 36 & -- & -- & 6 & 300 \\
\hline 6 & NB & -- & -- & 36 & 36 & -- & -- & -- \\
\hline 6 & SB & -- & -- & 36 & 36 & -- & -- & -- \\
\hline 7 & EB & 200 & 170 & 36 & -- & -- & 6 & 300 \\
\hline 7 & WB & 180 & 160 & 36 & -- & -- & 6 & 300 \\
\hline 7 & NB & 350 & 190 & 36 & -- & -- & -- & -- \\
\hline 7 & SB & 500 & -- & 36 & -- & -- & -- & -- \\
\hline
\end{tabular}


Table A- 2 Intersection Detector Configuration (CID Logic Only)

\begin{tabular}{|c|c|c|c|c|}
\hline INT & $\begin{array}{c}\text { MOVE- } \\
\text { MENT }\end{array}$ & $\begin{array}{c}\text { DET } \\
\text { NUM }\end{array}$ & $\begin{array}{c}\text { STAT } \\
\text { ID }\end{array}$ & $\begin{array}{c}\text { PUL } \\
\text { PRS }\end{array}$ \\
\hline 1 & EBLT & 5 & 121 & PRS \\
\hline 1 & EBTH & 2 & 118 & PRS \\
\hline 1 & WBLT & 1 & 117 & PRS \\
\hline 1 & WBTH & 6 & 122 & PRS \\
\hline 1 & NBLT & 3 & 119 & PRS \\
\hline 1 & NBTH & 8 & 124 & PRS \\
\hline 1 & NBRT & 16 & 132 & PRS \\
\hline 1 & SBTH & 7 & 123 & PRS \\
\hline 1 & SBTH & 4 & 120 & PRS \\
\hline
\end{tabular}

\begin{tabular}{|c|c|c|c|c|}
\hline INT & $\begin{array}{c}\text { MOVE- } \\
\text { MENT }\end{array}$ & $\begin{array}{c}\text { DET } \\
\text { NUM }\end{array}$ & $\begin{array}{c}\text { STAT } \\
\text { ID }\end{array}$ & $\begin{array}{c}\text { PUL } \\
\text { PRS }\end{array}$ \\
\hline 2 & EBLT & 5 & 205 & PRS \\
\hline 2 & EBTH & 2 & 202 & PRS \\
\hline 2 & WBLT & 1 & 201 & PRS \\
\hline 2 & WBTH & 6 & 206 & PRS \\
\hline 2 & WBRT & 14 & 214 & PRS \\
\hline 2 & NBLT & 3 & 203 & PRS \\
\hline 2 & NBTH & 8 & 208 & PRS \\
\hline 2 & SBLT & 7 & 207 & PRS \\
\hline 2 & SBTH & 4 & 204 & PRS \\
\hline
\end{tabular}

\begin{tabular}{|c|c|c|c|c|}
\hline INT & $\begin{array}{c}\text { MOVE- } \\
\text { MENT }\end{array}$ & $\begin{array}{c}\text { DET } \\
\text { NUM }\end{array}$ & $\begin{array}{c}\text { STAT } \\
\text { ID }\end{array}$ & $\begin{array}{c}\text { PUL } \\
\text { PRS }\end{array}$ \\
\hline 3 & EBLT & 5 & 305 & PRS \\
\hline 3 & EBTH & 2 & 302 & PRS \\
\hline 3 & WBLT & 1 & 301 & PRS \\
\hline 3 & WBTH & 6 & 306 & PRS \\
\hline 3 & NBTH & 8 & 308 & PRS \\
\hline 3 & NBTH & 16 & 316 & PRS \\
\hline 3 & SBTH & 4 & 304 & PRS \\
\hline 3 & SBTH & 12 & 312 & PRS \\
\hline
\end{tabular}

\begin{tabular}{|c|c|c|c|c|}
\hline INT & $\begin{array}{c}\text { MOVE- } \\
\text { MENT }\end{array}$ & $\begin{array}{c}\text { DET } \\
\text { NUM }\end{array}$ & $\begin{array}{c}\text { STAT } \\
\text { ID }\end{array}$ & $\begin{array}{c}\text { PUL } \\
\text { PRS }\end{array}$ \\
\hline 4 & EBLT & 5 & 405 & PRS \\
\hline 4 & EBTH & 2 & 402 & PRS \\
\hline 4 & WBLT & 1 & 401 & PRS \\
\hline 4 & WBTH & 6 & 406 & PRS \\
\hline 4 & NBTH & 8 & 408 & PRS \\
\hline 4 & NBRT & 16 & 416 & PRS \\
\hline 4 & SBLT & 7 & 407 & PRS \\
\hline 4 & SBTH & 4 & 404 & PRS \\
\hline
\end{tabular}

\begin{tabular}{|c|c|c|c|c|}
\hline INT & $\begin{array}{c}\text { MOVE- } \\
\text { MENT }\end{array}$ & $\begin{array}{c}\text { DET } \\
\text { NUM }\end{array}$ & $\begin{array}{c}\text { STAT } \\
\text { ID }\end{array}$ & $\begin{array}{c}\text { PUL } \\
\text { PRS }\end{array}$ \\
\hline 5 & EBLT & 5 & 505 & PRS \\
\hline 5 & EBTH & 2 & 502 & PRS \\
\hline 5 & WBLT & 1 & 501 & PRS \\
\hline 5 & WBTH & 6 & 506 & PRS \\
\hline 5 & NBTH & 8 & 508 & PRS \\
\hline 5 & NBRT & 16 & 516 & PRS \\
\hline 5 & SBTH & 4 & 504 & PRS \\
\hline 5 & SBTH & 12 & 512 & PRS \\
\hline
\end{tabular}

\begin{tabular}{|c|c|c|c|c|}
\hline INT & $\begin{array}{c}\text { MOVE- } \\
\text { MENT }\end{array}$ & $\begin{array}{c}\text { DET } \\
\text { NUM }\end{array}$ & $\begin{array}{c}\text { STAT } \\
\text { ID }\end{array}$ & $\begin{array}{c}\text { PUL } \\
\text { PRS }\end{array}$ \\
\hline 6 & EBLT & 5 & 605 & PRS \\
\hline 6 & EBTH & 2 & 602 & PRS \\
\hline 6 & WBLT & 1 & 601 & PRS \\
\hline 6 & WBTH & 6 & 606 & PRS \\
\hline 6 & NBLT & 3 & 603 & PRS \\
\hline 6 & NBTH & 8 & 608 & PRS \\
\hline 6 & SBLT & 7 & 607 & PRS \\
\hline 6 & SBTH & 4 & 604 & PRS \\
\hline
\end{tabular}

\begin{tabular}{|c|c|c|c|c|}
\hline INT & $\begin{array}{c}\text { MOVE- } \\
\text { MENT }\end{array}$ & $\begin{array}{c}\text { DET } \\
\text { NUM }\end{array}$ & $\begin{array}{c}\text { STAT } \\
\text { ID }\end{array}$ & $\begin{array}{c}\text { PUL } \\
\text { PRS }\end{array}$ \\
\hline 7 & EBLT & 5 & 705 & PRS \\
\hline 7 & EBTH & 2 & 702 & PRS \\
\hline 7 & WBLT & 1 & 701 & PRS \\
\hline 7 & WBTH & 6 & 706 & PRS \\
\hline 7 & NBLT & 3 & 703 & PRS \\
\hline 7 & NBTH & 8 & 708 & PRS \\
\hline 7 & SBLT & 7 & 707 & PRS \\
\hline 7 & SBTH & 4 & 704 & PRS \\
\hline
\end{tabular}


Table A- 3 General Phase Parameters (SR 26 - South)

\begin{tabular}{|c|c|c|c|c|c|c|c|c|c|}
\hline IN & DESCRIPTION (SEC) & 1 & 2 & 3 & 4 & 5 & 6 & 7 & 8 \\
\hline 1 & MINIMUM GREEN & 4 & 15 & 4 & 8 & 4 & 15 & 4 & 8 \\
\hline 1 & VEHICLE EXTENSION & 1.5 & 3 & 1.5 & 2 & 1.5 & 3 & 1.5 & 2 \\
\hline 1 & MAXIMUM GREEN & 20 & 40 & 20 & 30 & 20 & 35 & 20 & 30 \\
\hline 1 & YELLOW CLEARANCE & 3.5 & 3.5 & 3.5 & 3.5 & 3.5 & 3.5 & 3.5 & 3.5 \\
\hline 1 & RED CLEARANCE & 1.5 & 2.0 & 1.5 & 1.5 & 1.5 & 2.0 & 1.5 & 1.5 \\
\hline \multirow[t]{2}{*}{1} & MINIMUM GAP TIME & 1.5 & 3 & 1.5 & 2 & 1.5 & 3 & 1.5 & 2 \\
\hline & MINIMUM GREEN & & & & & & & & \\
\hline 2 & VEHICLE EXTENSION & 1.5 & 3.5 & 1.5 & 4 & 1.5 & 3.5 & 2 & 3.5 \\
\hline 2 & MAXIMUM GREEN & 50 & 90 & 40 & 60 & 50 & 90 & 60 & 60 \\
\hline 2 & YELLOW CLEARANCE & 3.5 & 3.5 & 3.5 & 3.5 & 3.5 & 3.5 & 3.5 & 3.5 \\
\hline 2 & RED CLEARANCE & 1.5 & 2.0 & 1.5 & 2.0 & 1.5 & 2.0 & 1.5 & 2.0 \\
\hline \multirow[t]{2}{*}{2} & MINIMUM GAP TIME & 1.5 & 3.5 & 1.5 & 4 & 1.5 & 3.5 & 2 & 3.5 \\
\hline & MINIMUM GREEN & & & & & & & & \\
\hline 3 & VEHICLE EXTENSION & 1.5 & 4 & - & 3 & 1.5 & 4 & - & 3.0 \\
\hline 3 & MAXIMUM GREEN & 40 & 70 & - & 40 & 40 & 70 & - & 40 \\
\hline 3 & YELLOW CLEARANCE & 3.5 & 3.5 & 3.5 & 3.5 & 3.5 & 3.5 & 3.5 & 3.5 \\
\hline 3 & RED CLEARANCE & 1.5 & 2.0 & - & 1.5 & 1.5 & 2.0 & - & 1.5 \\
\hline \multirow[t]{2}{*}{3} & MINIMUM GAP TIME & 1.5 & 4 & - & 3 & 1.5 & 4 & - & 3.0 \\
\hline & MINIMUM GREEN & & & & & & & & \\
\hline 4 & VEHICLE EXTENSION & 1.5 & 3.5 & - & 2 & 1.5 & 3.5 & 1.5 & 2 \\
\hline 4 & MAXIMUM GREEN & 40 & 70 & - & 70 & 50 & 70 & 40 & 70 \\
\hline 4 & YELLOW CLEARANCE & 3.5 & 3.5 & 3.5 & 3.5 & 3.5 & 3.5 & 3.5 & 3.5 \\
\hline 4 & RED CLEARANCE & 1.5 & 2.0 & - & 1.5 & 1.5 & 2.0 & 1.5 & 1.5 \\
\hline \multirow[t]{2}{*}{4} & MINIMUM GAP TIME & 1.5 & 3.5 & - & 2 & 1.5 & 3.5 & 1.5 & 2 \\
\hline & MINIMUM GREEN & & & & & & & & \\
\hline 5 & VEHICLE EXTENSION & 2 & 4.5 & 2 & 2.5 & 2 & 4.5 & 0 & 0 \\
\hline 5 & MAXIMUM GREEN & 40 & 70 & 60 & 60 & 40 & 70 & 0 & 0 \\
\hline 5 & YELLOW CLEARANCE & 3.5 & 3.5 & 3.5 & 3.5 & 3.5 & 3.5 & 0 & 0 \\
\hline 5 & RED CLEARANCE & 1.5 & 2.0 & 1.5 & 1.5 & 1.5 & 2.0 & 0 & 0 \\
\hline \multirow[t]{2}{*}{5} & MINIMUM GAP TIME & 2 & 4.5 & 2 & 2.5 & 2 & 4.5 & 0 & 0 \\
\hline & MINIMUM GREEN & & & & & & & & \\
\hline 6 & VEHICLE EXTENSION & 2.5 & 5.0 & 2.5 & 5.0 & 2.5 & 5.0 & 2.5 & 5.0 \\
\hline 6 & MAXIMUM GREEN & 35 & 80 & 35 & 45 & 35 & 80 & 35 & 45 \\
\hline 6 & YELLOW CLEARANCE & 3.5 & 3.5 & 3.5 & 3.5 & 3.5 & 3.5 & 3.5 & 3.5 \\
\hline 6 & RED CLEARANCE & 1.5 & 2.0 & 1.5 & 1.5 & 1.5 & 2.0 & 1.5 & 1.5 \\
\hline \multirow[t]{2}{*}{6} & MINIMUM GAP TIME & 2.5 & 5.0 & 2.5 & 5.0 & 2.5 & 5.0 & 2.5 & 5.0 \\
\hline & MINIMUM GREEN & & & & & & & & \\
\hline 7 & VEHICLE EXTENSION & 1.5 & 5 & 1.5 & 3.5 & 1.5 & 5 & 1.5 & 3.5 \\
\hline 7 & MAXIMUM GREEN & 40 & 40 & 35 & 60 & 40 & 40 & 45 & 15 \\
\hline 7 & YELLOW CLEARANCE & 3.5 & 3.5 & 3.5 & 3.5 & 3.5 & 3.5 & 3.5 & 3.5 \\
\hline 7 & RED CLEARANCE & 1.5 & 2 & 1.5 & 2 & 1.5 & 2 & 1.5 & 2 \\
\hline 7 & MINIMUM GAP TIME & 1.5 & 5 & 1.5 & 3.5 & 1.5 & 5 & 1.5 & 3.5 \\
\hline
\end{tabular}


Table A- 4 SR 26 (South) Coordination Plans and Splits

\begin{tabular}{|l|l|l|l|l|l|l|l|l|l|l|l|}
\hline INT & COS & CYC & OFF & 1 & 2 & 3 & 4 & 5 & 6 & 7 & 8 \\
\hline 1 & 111 & 75 & 0 & 4 & 30 & 3 & 18 & 3 & 31 & 5 & 16 \\
\hline 1 & 211 & 120 & 0 & 8 & 51 & 3 & 38 & 3 & 56 & 13 & 28 \\
\hline 1 & 311 & 120 & 0 & 10 & 44 & 3 & 43 & 4 & 51 & 14 & 32 \\
\hline \hline & & & & & & & & & & & \\
\hline 2 & 211 & 95 & 114 & 21 & 30 & 18 & 33 & 14 & 36 & 22 & 29 \\
\hline 2 & 311 & 120 & 108 & 19 & 33 & 17 & 33 & 15 & 36 & 20 & 30 \\
\hline \hline & & & & & & & & & & & \\
\hline 3 & 211 & 95 & 37 & 6 & 80 & - & 20 & 13 & 73 & - & 20 \\
\hline 3 & 311 & 120 & 36 & 6 & 82 & - & 18 & 14 & 74 & - & 18 \\
\hline \hline & & & & & & & & & & & \\
\hline 4 & 211 & 95 & 51 & 13 & 57 & - & 36 & 11 & 59 & 5 & 26 \\
\hline 4 & 311 & 120 & 30 & 14 & 50 & - & 42 & 10 & 54 & 5 & 32 \\
\hline 5 & 211 & 95 & 41 & 5 & 61 & - & 17 & 9 & 57 & - & 17 \\
\hline 5 & 311 & 120 & 38 & 5 & 61 & - & 17 & 9 & 57 & - & 17 \\
\hline \hline & & & & & & & & & & & \\
\hline 6 & 211 & 95 & 54 & 5 & 74 & 5 & 17 & 8 & 71 & 5 & 17 \\
\hline 6 & 311 & 120 & 23 & 5 & 74 & 5 & 17 & 8 & 71 & 5 & 17 \\
\hline \hline & & & & & & & & & & & \\
\hline 7 & 211 & 95 & 95 & 19 & 34 & 26 & 22 & 12 & 41 & 28 & 20 \\
\hline 7 & 311 & 120 & 84 & 11 & 34 & 31 & 25 & 14 & 31 & 28 & 28 \\
\hline \hline
\end{tabular}

NOTE: Offsets are referenced from the beginning of the first coordinated phase. COS 111 represents timing plan for morning peak, COS 211 for mid-day, and COS 311 for after-noon peak traffic 
Table A- 5 Entry Volumes per Period (VPH)

\begin{tabular}{|c|c|c|c|c|c|c|c|c|c|c|c|c|c|}
\hline INT & $\begin{array}{l}\text { BEG } \\
\text { END }\end{array}$ & $\begin{array}{l}7 \mathrm{AM} \\
8 \mathrm{AM}\end{array}$ & $\begin{array}{l}8 \mathrm{AM} \\
9 \mathrm{AM}\end{array}$ & $\begin{array}{c}9 \mathrm{AM} \\
10 \mathrm{~A}\end{array}$ & $\begin{array}{l}10 \mathrm{~A} \\
11 \mathrm{~A}\end{array}$ & $\begin{array}{l}11 \mathrm{~A} \\
12 \mathrm{P}\end{array}$ & $\begin{array}{c}12 \mathrm{P} \\
1 \mathrm{PM}\end{array}$ & $\begin{array}{l}1 \mathrm{PM} \\
2 \mathrm{PM}\end{array}$ & $\begin{array}{l}\text { 2PM } \\
3 \mathrm{PM}\end{array}$ & $\begin{array}{l}3 \mathrm{PM} \\
4 \mathrm{PM}\end{array}$ & $\begin{array}{l}4 \mathrm{PM} \\
5 \mathrm{PM}\end{array}$ & $\begin{array}{l}5 \mathrm{PM} \\
6 \mathrm{PM}\end{array}$ & $\begin{array}{l}6 \mathrm{PM} \\
7 \mathrm{PM}\end{array}$ \\
\hline 1 & EB & 311 & 342 & 460 & 30 & 654 & 674 & 563 & 706 & 813 & 705 & 709 & 575 \\
\hline 1 & WB & 59 & 620 & & & 69 & 71 & 69 & 7 & & 6 & 696 & 548 \\
\hline 1 & NB & 40 & 334 & 18 & 35 & 47 & 45 & 38 & 50 & 71 & 43 & & 316 \\
\hline 1 & SB & 29 & 341 & & 42 & 53 & 56 & 43 & 54 & 60 & 55 & 5 & 356 \\
\hline 2 & EB & 42 & & & & & & $6 c$ & 904 & 10 & & & 715 \\
\hline 2 & WB & 6 & & & & & & 8 & & & & & 29 \\
\hline 2 & NB & & 600 & & 81 & 1060 & 134 & $05 /$ & 1254 & 22 & 11 & 2 & 83 \\
\hline 2 & SB & 7 & 615 & 768 & 78 & 00 & 063 & 1024 & 1049 & 962 & 905 & 099 & 784 \\
\hline 3 & EB & 590 & 57 & 70 & 744 & 1060 & 1080 & 901 & 1042 & 1096 & 100 & 1178 & 912 \\
\hline 3 & WB & 657 & 843 & 746 & 648 & 836 & 82 & 861 & 933 & 84 & 97 & 794 & 788 \\
\hline 3 & NB & 1 & 6 & 20 & 10 & 4 & 12 & 66 & 40 & 1 & 2 & 49 & 21 \\
\hline 3 & SB & 18 & 101 & & 2 & 21 & 20 & 205 & 21 & 15 & 23 & 274 & 220 \\
\hline 4 & EB & & & & & & 10 & & & & 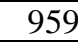 & 11 & 440 \\
\hline 4 & WB & & & & & 9 & & 880 & & & 10 & $J$ & 777 \\
\hline 4 & NB & 197 & 21 & 2 & 2. & 33 & 30 & 24 & 25 & & 3 & 367 & 231 \\
\hline 4 & SB & 4 & 5 & 10 & 13 & 15 & 19 & 176 & 186 & & 18 & 186 & 174 \\
\hline 5 & EB & 60 & 6 & 73 & 81 & 1095 & 113 & 956 & 1040 & 1172 & 11. & 1339 & 1057 \\
\hline 5 & WB & 737 & 94 & 73 & 63 & 86 & 79 & 850 & 93 & 8 & 95 & 778 & 761 \\
\hline 5 & NB & & & & & 3 & 2 & 25 & 7 & & 6 & 28 & 2 \\
\hline 5 & SB & & & & & & & +2 & 11 & & 1 & 174 & 102 \\
\hline 6 & & & & & & & & 4 & 10 & & & 1374 & 1074 \\
\hline 6 & WB & 76 & & 7 & 59 & & 73 & 799 & & & 8 & 736 & 769 \\
\hline 6 & NB & $F$ & & & 8 & 2 & 1 & 22 & 11 & & 2 & 51 & 2 \\
\hline 6 & SB & & & & & 73 & & 126 & 122 & 114 & 111 & 125 & 114 \\
\hline 7 & EB & 0 & & 6 & 77 & 1083 & 11 & 953 & 1122 & 1314 & 1206 & 1392 & 1072 \\
\hline 7 & WB & 86 & & 7 & 59 & 8 & & 824 & 868 & 8 & 732 & 654 & 77 \\
\hline 7 & NB & 63 & 600 & 512 & 572 & 759 & 00 & 830 & 1180 & 1220 & 1555 & 1423 & 117 \\
\hline 1 & SB & 715 & 641 & 572 & 575 & 799 & 622 & 712 & 726 & 707 & 994 & 1094 & \\
\hline
\end{tabular}


Table A- 6 Turning Movements (Percentages - Table 1 of 2)

\begin{tabular}{|c|c|c|c|c|c|c|c|c|c|c|c|c|c|}
\hline INT & $\begin{array}{l}\text { BEG } \\
\text { END }\end{array}$ & $\begin{array}{l}7 \mathrm{~A} \\
\mathrm{M} \\
8 \mathrm{~A} \\
\mathrm{M} \\
\end{array}$ & $\begin{array}{c}8 \mathrm{~A} \\
\mathrm{M} \\
9 \mathrm{~A} \\
\mathrm{M}\end{array}$ & $\begin{array}{c}9 \mathrm{~A} \\
\mathrm{M} \\
10 \mathrm{~A}\end{array}$ & $\begin{array}{l}10 \mathrm{~A} \\
11 \mathrm{~A}\end{array}$ & $\begin{array}{l}11 \mathrm{~A} \\
12 \mathrm{P}\end{array}$ & $\begin{array}{c}12 \mathrm{P} \\
1 \mathrm{P} \\
\mathrm{M}\end{array}$ & $\begin{array}{l}1 \mathrm{PM} \\
2 \mathrm{PM}\end{array}$ & $\begin{array}{l}2 \mathrm{P} \\
\mathrm{M} \\
3 \mathrm{P} \\
\mathrm{M}\end{array}$ & $\begin{array}{l}3 \mathrm{P} \\
\mathrm{M} \\
4 \mathrm{P} \\
\mathrm{M}\end{array}$ & $\begin{array}{l}4 \mathrm{P} \\
\mathrm{M} \\
5 \mathrm{P} \\
\mathrm{M}\end{array}$ & $\begin{array}{l}5 \mathrm{P} \\
\mathrm{M} \\
6 \mathrm{P} \\
\mathrm{M}\end{array}$ & $\begin{array}{l}6 \mathrm{P} \\
\mathrm{M} \\
7 \mathrm{P} \\
\mathrm{M}\end{array}$ \\
\hline \multirow{12}{*}{ 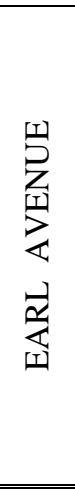 } & EBLT & 13 & 11 & 10 & 10 & 6 & 10 & 9 & 7 & 7 & 5 & 6 & 5 \\
\hline & EBTH & 81 & 82 & 82 & 82 & 85 & 83 & 83 & 84 & 85 & 90 & 87 & 92 \\
\hline & EBRT & 6 & 7 & 8 & 8 & 9 & 7 & 8 & 9 & 8 & 5 & 7 & 3 \\
\hline & WBLT & 13 & 14 & 12 & 14 & 15 & 18 & 14 & 15 & 16 & 16 & 17 & 19 \\
\hline & WBTH & 70 & 68 & 73 & 68 & 67 & 65 & 68 & 69 & 71 & 68 & 70 & 72 \\
\hline & WBRT & 17 & 18 & 15 & 18 & 18 & 17 & 18 & 16 & 13 & 16 & 13 & 9 \\
\hline & NBLT & 5 & 5 & 15 & 10 & 6 & 7 & 7 & 7 & 9 & 6 & 6 & 7 \\
\hline & NBTH & 69 & 73 & 66 & 66 & 69 & 70 & 71 & 67 & 68 & 71 & 69 & 69 \\
\hline & NBRT & 26 & 22 & 19 & 24 & 25 & 23 & 22 & 26 & 23 & 23 & 25 & 24 \\
\hline & SBLT & 23 & 30 & 29 & 34 & 35 & 34 & 27 & 33 & 32 & 31 & 31 & 31 \\
\hline & SBTH & 72 & 65 & 60 & 60 & 60 & 62 & 67 & 59 & 64 & 64 & 65 & 59 \\
\hline & SBRT & 5 & 5 & 11 & 6 & 5 & 4 & 6 & 8 & 4 & 5 & 4 & 10 \\
\hline \multirow{11}{*}{ 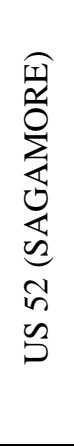 } & EBTH & 70 & 67 & 68 & 65 & 62 & 62 & 60 & 62 & 62 & 64 & 69 & 67 \\
\hline & EBRT & 11 & 13 & 14 & 19 & 20 & 20 & 21 & 20 & 19 & 20 & 15 & 19 \\
\hline & WBLT & 20 & 24 & 22 & 27 & 27 & 27 & 26 & 28 & 27 & 24 & 24 & 28 \\
\hline & WBTH & 57 & 54 & 55 & 49 & 48 & 48 & 51 & 50 & 47 & 51 & 55 & 51 \\
\hline & WBRT & 23 & 22 & 23 & 24 & 25 & 25 & 23 & 22 & 26 & 25 & 21 & 21 \\
\hline & NBLT & 9 & 11 & 12 & 12 & 13 & 13 & 15 & 14 & 12 & 13 & 11 & 12 \\
\hline & NBTH & 72 & 65 & 68 & 69 & 63 & 62 & 62 & 66 & 69 & 67 & 67 & 63 \\
\hline & NBRT & 19 & 24 & 20 & 19 & 24 & 25 & 23 & 20 & 19 & 20 & 22 & 25 \\
\hline & SBLT & 20 & 20 & 22 & 20 & 27 & 25 & 25 & 22 & 22 & 22 & 25 & 27 \\
\hline & SBTH & 63 & 66 & 66 & 67 & 60 & 62 & 65 & 67 & 69 & 68 & 65 & 64 \\
\hline & SBRT & 17 & 14 & 12 & 13 & 13 & 13 & 10 & 11 & 9 & 10 & 10 & 9 \\
\hline \multirow{11}{*}{ 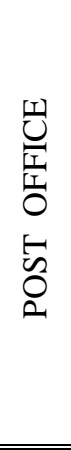 } & EBTH & 80 & 85 & 88 & 87 & 84 & 82 & 83 & 83 & 83 & 83 & 84 & 87 \\
\hline & EBRT & 1 & 1 & 2 & 2 & 7 & 7 & 2 & 3 & 2 & 3 & 3 & 3 \\
\hline & WBLT & 1 & 1 & 1 & 1 & 6 & 5 & 2 & 1 & 0 & 2 & 3 & 5 \\
\hline & WBTH & 85 & 88 & 87 & 89 & 83 & 85 & 82 & 85 & 84 & 79 & 81 & 80 \\
\hline & WBRT & 14 & 11 & 12 & 10 & 11 & 10 & 16 & 14 & 16 & 19 & 16 & 15 \\
\hline & NBLT & 55 & 17 & 35 & 80 & 60 & 48 & 42 & 38 & 33 & 32 & 45 & 13 \\
\hline & NBTH & 9 & 0 & 10 & 0 & 0 & 4 & 2 & 4 & 9 & 4 & 10 & 3 \\
\hline & NBRT & 36 & 83 & 55 & 20 & 40 & 48 & 56 & 58 & 58 & 64 & 45 & 84 \\
\hline & SBLT & 30 & 35 & 31 & 34 & 25 & 33 & 43 & 42 & 50 & 47 & 44 & 55 \\
\hline & SBTH & 0 & 0 & 0 & 1 & 1 & 2 & 3 & 0 & 1 & 0 & 1 & 2 \\
\hline & SBRT & 70 & 65 & 69 & 65 & 74 & 65 & 54 & 58 & 49 & 53 & 55 & 43 \\
\hline \multirow{11}{*}{ 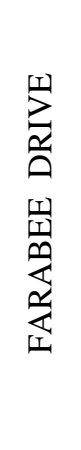 } & EBTH & 86 & 85 & 84 & 85 & 85 & 85 & 85 & 85 & 84 & 87 & 90 & 89 \\
\hline & EBRT & 11 & 9 & 8 & 7 & 6 & 6 & 7 & 6 & 7 & 6 & 5 & 4 \\
\hline & WBLT & 18 & 14 & 11 & 12 & 12 & 12 & 13 & 13 & 17 & 12 & 13 & 9 \\
\hline & WBTH & 81 & 82 & 84 & 81 & 81 & 83 & 81 & 81 & 78 & 80 & 80 & 85 \\
\hline & WBRT & 1 & 4 & 5 & 7 & 7 & 5 & 6 & 6 & 5 & 8 & 7 & 6 \\
\hline & NBLT & 17 & 15 & 21 & 17 & 17 & 19 & 23 & 20 & 13 & 19 & 18 & 16 \\
\hline & NBTH & 7 & 8 & 10 & 15 & 12 & 13 & 11 & 18 & 9 & 11 & 8 & 15 \\
\hline & NBRT & 76 & 77 & 69 & 68 & 71 & 68 & 66 & 62 & 78 & 70 & 74 & 69 \\
\hline & SBLT & 4 & 17 & 16 & 29 & 25 & 35 & 29 & 26 & 31 & 31 & 26 & 35 \\
\hline & SBTH & 53 & 34 & 22 & 21 & 16 & 17 & 19 & 18 & 17 & 19 & 28 & 13 \\
\hline & SBRT & 43 & 49 & 62 & 50 & 29 & 48 & 52 & 56 & 52 & 50 & 46 & 52 \\
\hline
\end{tabular}


Table A- 7 Turning Movements (Percentages -Table 2 of 2)

\begin{tabular}{|c|c|c|c|c|c|c|c|c|c|c|c|c|c|}
\hline INT & $\begin{array}{l}\text { BEG } \\
\text { END }\end{array}$ & $\begin{array}{c}7 \mathrm{~A} \\
\mathrm{M} \\
8 \mathrm{~A} \\
\mathrm{M}\end{array}$ & $\begin{array}{c}8 \mathrm{~A} \\
\mathrm{M} \\
9 \mathrm{~A} \\
\mathrm{M}\end{array}$ & $\begin{array}{c}9 \mathrm{~A} \\
\mathrm{M} \\
10 \mathrm{~A}\end{array}$ & $\begin{array}{l}10 \mathrm{~A} \\
11 \mathrm{~A}\end{array}$ & $\begin{array}{l}11 \mathrm{~A} \\
12 \mathrm{P}\end{array}$ & $\begin{array}{c}12 \mathrm{P} \\
1 \mathrm{P} \\
\mathrm{M}\end{array}$ & $\begin{array}{l}1 \mathrm{P} \\
\mathrm{M} \\
2 \mathrm{P} \\
\mathrm{M}\end{array}$ & $\begin{array}{l}2 \mathrm{P} \\
\mathrm{M} \\
3 \mathrm{P} \\
\mathrm{M}\end{array}$ & $\begin{array}{l}3 \mathrm{P} \\
\mathrm{M} \\
4 \mathrm{P} \\
\mathrm{M}\end{array}$ & $\begin{array}{l}4 \mathrm{P} \\
\mathrm{M} \\
5 \mathrm{P} \\
\mathrm{M}\end{array}$ & $\begin{array}{l}5 \mathrm{P} \\
\mathrm{M} \\
6 \mathrm{P} \\
\mathrm{M}\end{array}$ & $\begin{array}{l}6 \mathrm{P} \\
\mathrm{M} \\
7 \mathrm{P} \\
\mathrm{M}\end{array}$ \\
\hline \multirow{12}{*}{ 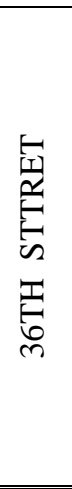 } & EBLT & 13 & 8 & 9 & 7 & 6 & 6 & 6 & 6 & 7 & 6 & 5 & 5 \\
\hline & EBTH & 86 & 90 & 90 & 92 & 94 & 93 & 93 & 94 & 93 & 93 & 94 & 95 \\
\hline & EBRT & 1 & 2 & 1 & 1 & 0 & 1 & 1 & 0 & 0 & 1 & 1 & 0 \\
\hline & WBLT & 1 & 1 & 1 & 1 & 1 & 1 & 1 & 1 & 1 & 0 & 0 & 0 \\
\hline & WBTH & 90 & 89 & 94 & 92 & 93 & 91 & 92 & 92 & 92 & 93 & 93 & 95 \\
\hline & WBRT & 9 & 10 & 5 & 7 & 6 & 8 & 7 & 7 & 7 & 7 & 7 & 5 \\
\hline & NBLT & 90 & 63 & 44 & 65 & 66 & 63 & 56 & 69 & 61 & 69 & 54 & 39 \\
\hline & NBTH & 2 & 17 & 6 & 16 & 6 & 20 & 4 & 13 & 20 & 8 & 25 & 4 \\
\hline & NBRT & 8 & 20 & 50 & 19 & 28 & 17 & 40 & 18 & 19 & 23 & 21 & 57 \\
\hline & SBLT & 48 & 42 & 35 & 35 & 45 & 55 & 39 & 53 & 45 & 51 & 63 & 56 \\
\hline & SBTH & 1 & 2 & 2 & 0 & 1 & 1 & 2 & 2 & 1 & 1 & 0 & 0 \\
\hline & SBRT & 51 & 56 & 63 & 65 & 54 & 44 & 59 & 45 & 54 & 48 & 37 & 44 \\
\hline \multirow{11}{*}{ 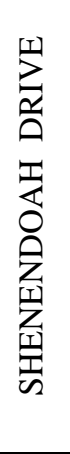 } & EBTH & 96 & 95 & 94 & 94 & 95 & 93 & 94 & 95 & 95 & 94 & 94 & 93 \\
\hline & EBRT & 0 & 1 & 1 & 0 & 0 & 1 & 1 & 0 & 0 & 0 & 0 & 0 \\
\hline & WBLT & 10 & 3 & 2 & 1 & 2 & 1 & 1 & 2 & 5 & 1 & 1 & 0 \\
\hline & WBTH & 89 & 94 & 94 & 94 & 94 & 94 & 94 & 94 & 91 & 95 & 93 & 92 \\
\hline & WBRT & 1 & 3 & 4 & 5 & 4 & 5 & 5 & 4 & 4 & 4 & 6 & 8 \\
\hline & NBLT & 7 & 22 & 18 & 38 & 13 & 17 & 23 & 9 & 7 & 7 & 12 & 10 \\
\hline & NBTH & 10 & 8 & 9 & 12 & 16 & 16 & 13 & 9 & 6 & 10 & 12 & 9 \\
\hline & NBRT & 83 & 70 & 73 & 50 & 71 & 67 & 64 & 82 & 87 & 83 & 76 & 81 \\
\hline & SBLT & 15 & 20 & 32 & 33 & 27 & 37 & 33 & 25 & 38 & 45 & 49 & 49 \\
\hline & SBTH & 16 & 7 & 1 & 1 & 0 & 2 & 1 & 1 & 1 & 2 & 1 & 0 \\
\hline & SBRT & 69 & 73 & 67 & 66 & 73 & 61 & 66 & 74 & 61 & 53 & 50 & 51 \\
\hline \multirow{11}{*}{ 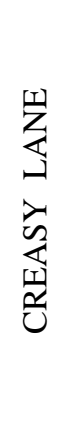 } & EBTH & 69 & 70 & 63 & 63 & 63 & 61 & 63 & 64 & 67 & 62 & 67 & 65 \\
\hline & EBRT & 25 & 22 & 28 & 29 & 30 & 30 & 29 & 28 & 26 & 29 & 26 & 26 \\
\hline & WBLT & 14 & 13 & 19 & 18 & 21 & 19 & 20 & 20 & 17 & 18 & 18 & 20 \\
\hline & WBTH & 65 & 61 & 59 & 57 & 56 & 59 & 54 & 55 & 57 & 54 & 52 & 51 \\
\hline & WBRT & 21 & 26 & 22 & 25 & 23 & 22 & 26 & 25 & 26 & 28 & 30 & 29 \\
\hline & NBLT & 25 & 35 & 36 & 36 & 35 & 38 & 35 & 30 & 25 & 28 & 24 & 27 \\
\hline & NBTH & 56 & 48 & 46 & 48 & 46 & 51 & 47 & 50 & 55 & 54 & 56 & 52 \\
\hline & NBRT & 19 & 17 & 18 & 16 & 19 & 11 & 18 & 20 & 20 & 18 & 20 & 21 \\
\hline & SBLT & 35 & 40 & 38 & 34 & 42 & 42 & 41 & 37 & 45 & 42 & 43 & 45 \\
\hline & SBTH & 58 & 51 & 51 & 57 & 49 & 49 & 50 & 54 & 48 & 52 & 52 & 49 \\
\hline & $\begin{array}{l}\text { SBRT } \\
\end{array}$ & 7 & 9 & 11 & 9 & 9 & 9 & 9 & 9 & 7 & 6 & 5 & 6 \\
\hline
\end{tabular}


APPENDIX B- US 31 NETWORK and CONTROLLER PARAMETERS 

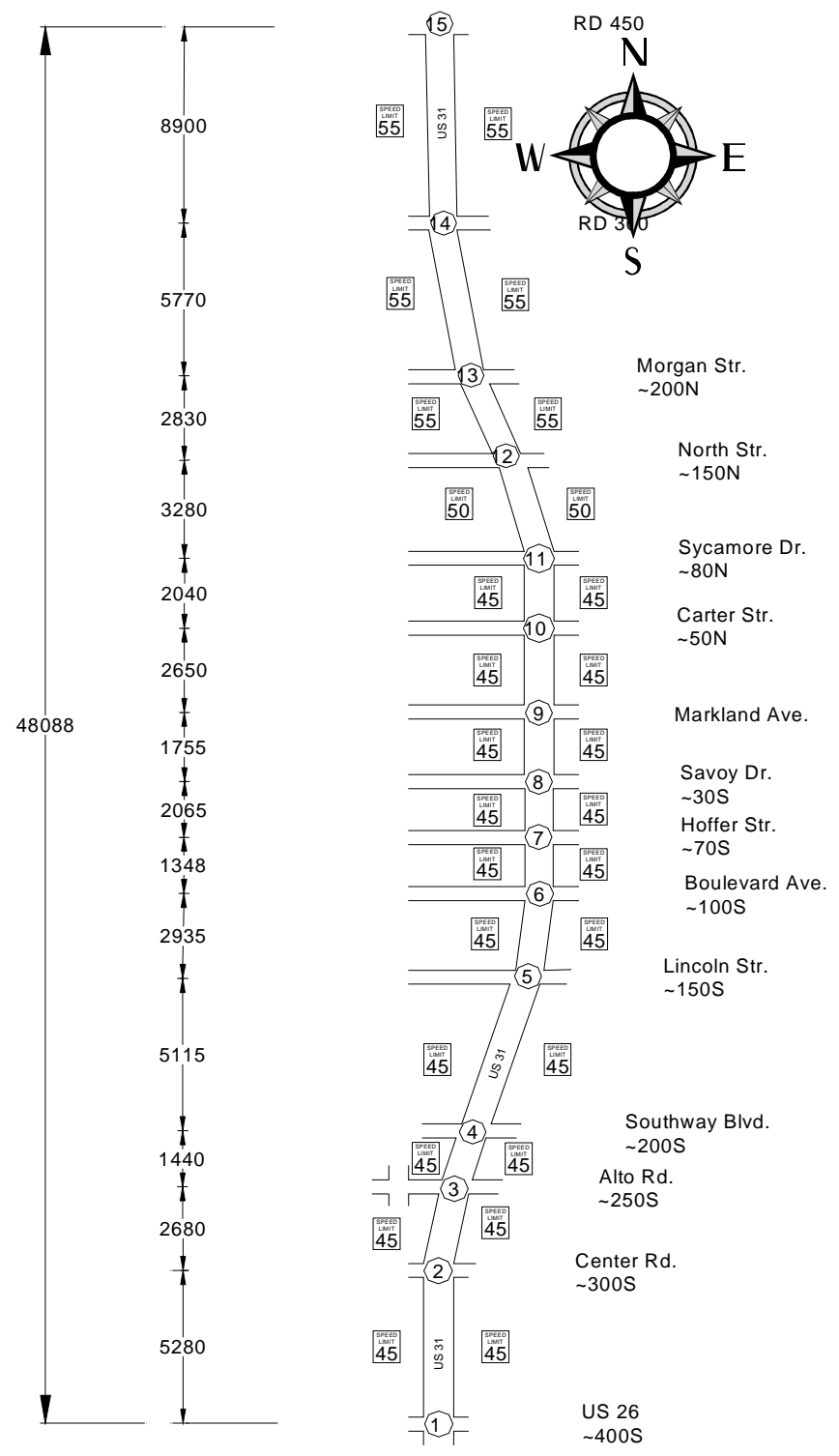

Figure B- 1 US 31 Network - US 26 to Road 450 

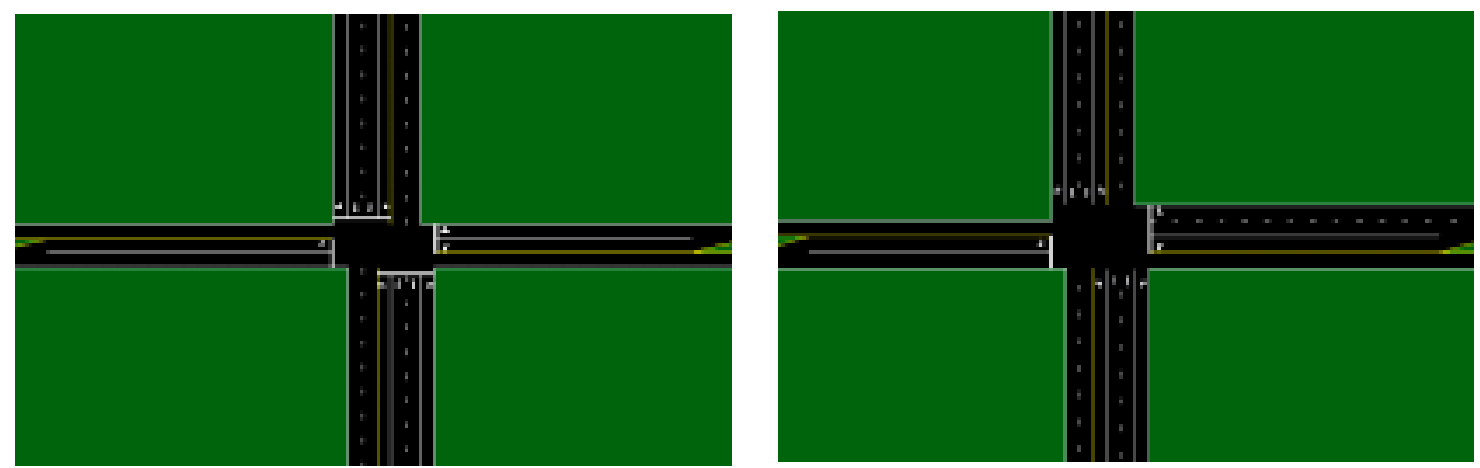

a) Node 1: US $31 \&$ US 26

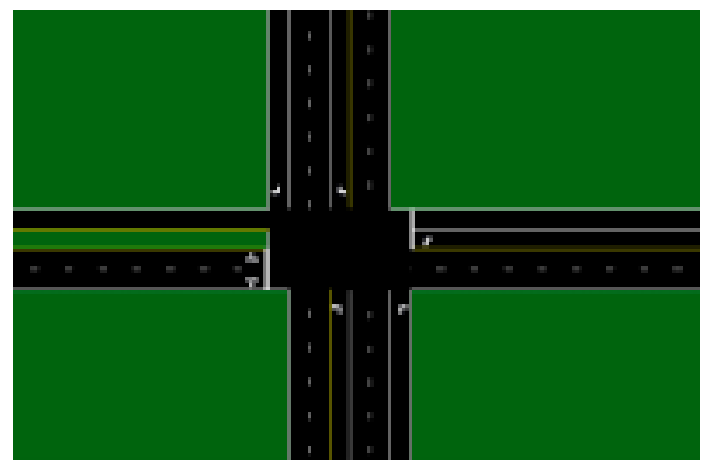

b) Node 2: US 31 \& Center Road

c) Node 3: US 31 \& Alto Road
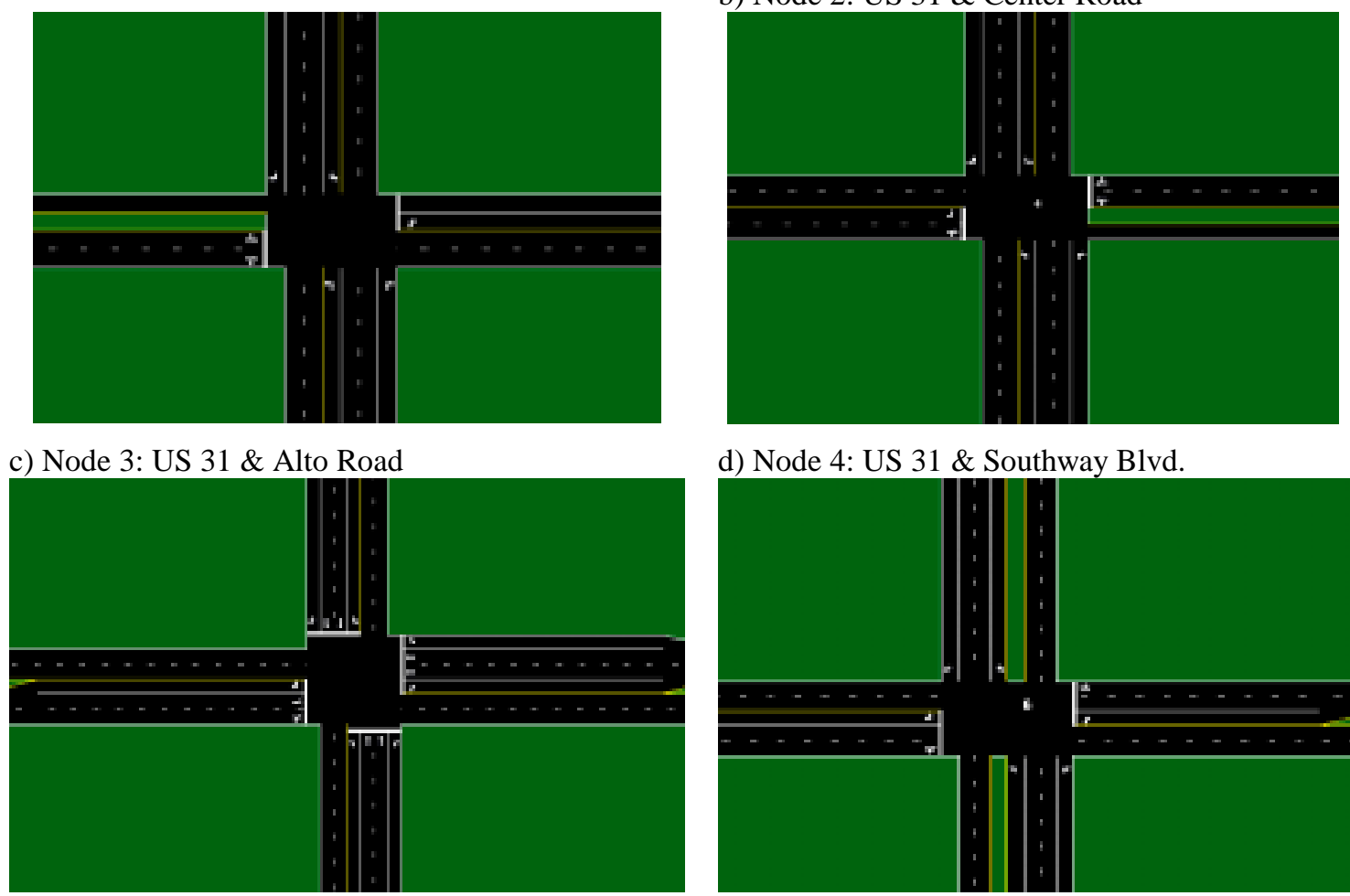

d) Node 4: US 31 \& Southway Blvd.

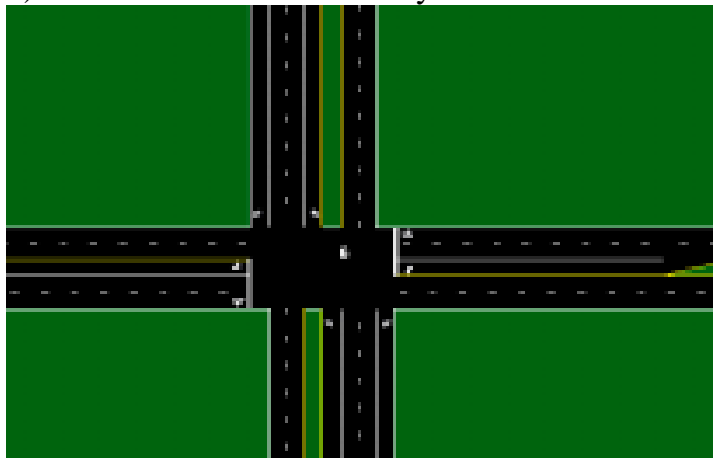

e) Node 5: US $31 \&$ Lincoln

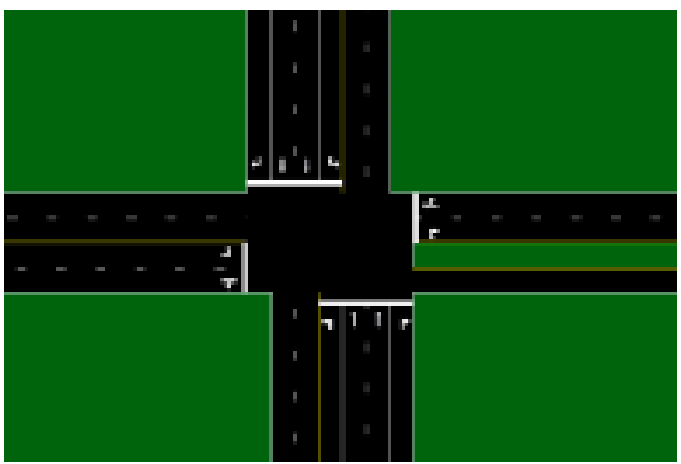

f) Node 6: US $31 \&$ Boulevard Ave.

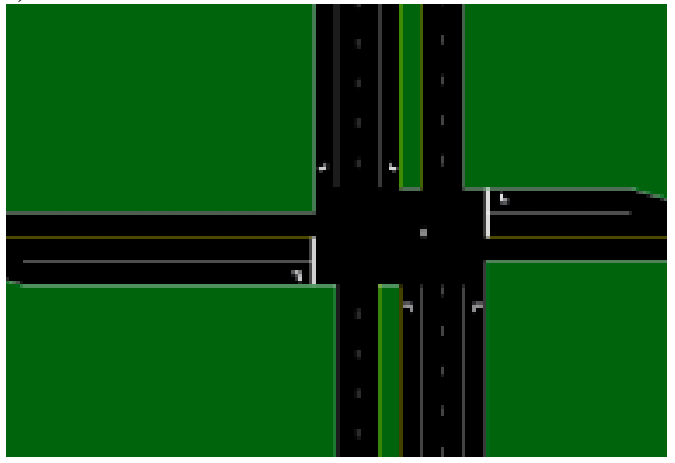

g) Node 7: US $31 \&$ Hoffer Str.

h) Node 8: US 31 \& Savoy Dr.

Figure B- 2 US 31 Geometries 


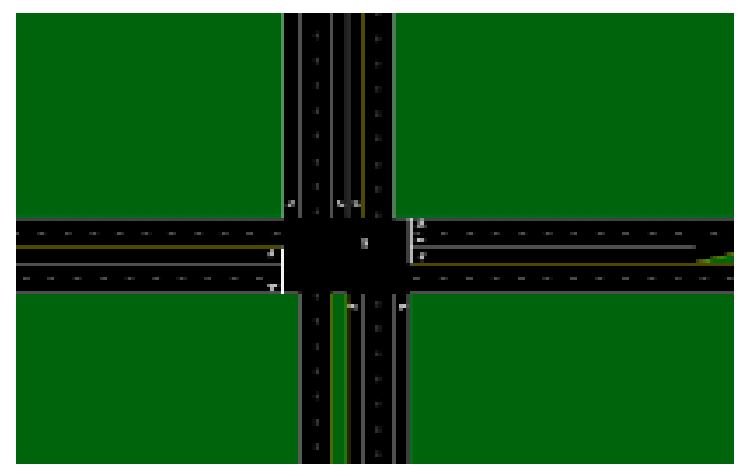

a) Node 9: US 31 \& Markland Ave

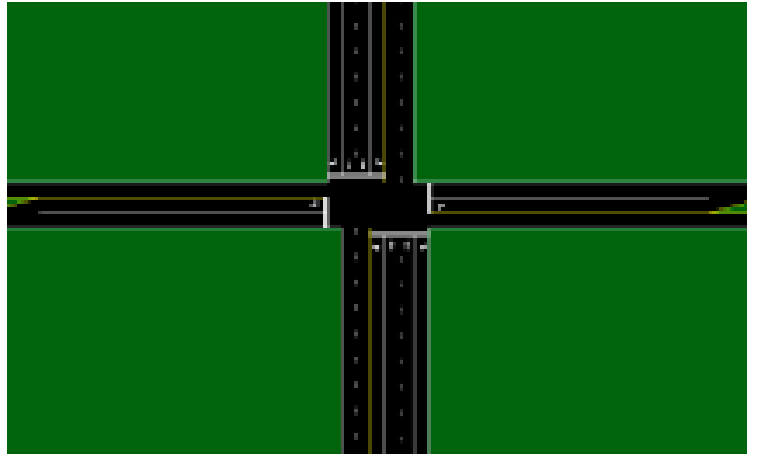

c) Node 11: US 31 \& Sycamore Dr.

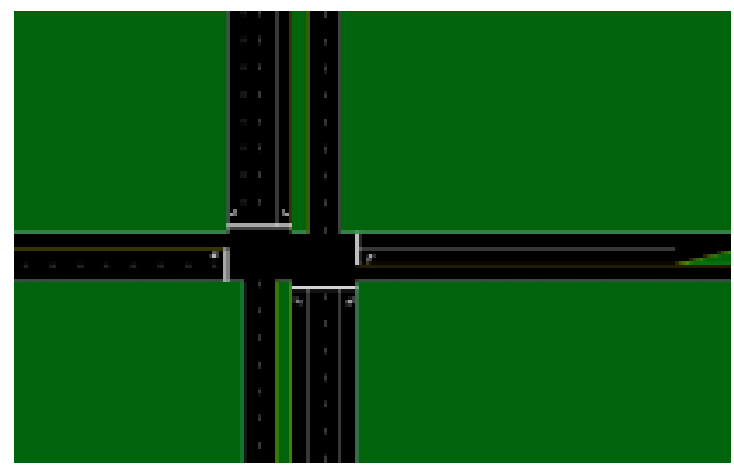

e) Node 13: US $31 \&$ Morgan St.

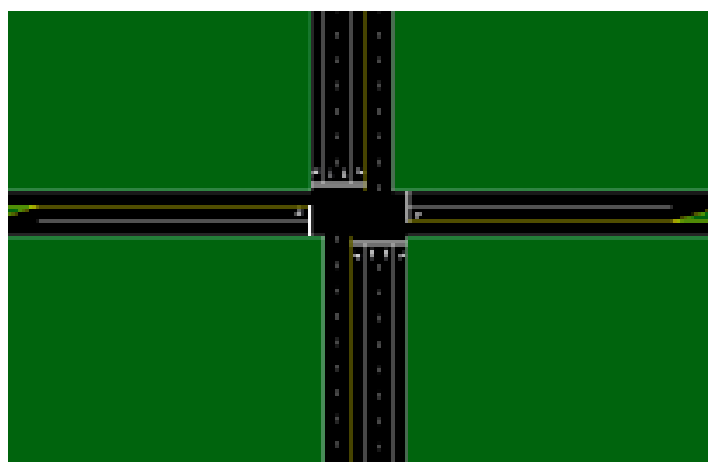

b) Node 10: US 31 \& Carter Str.

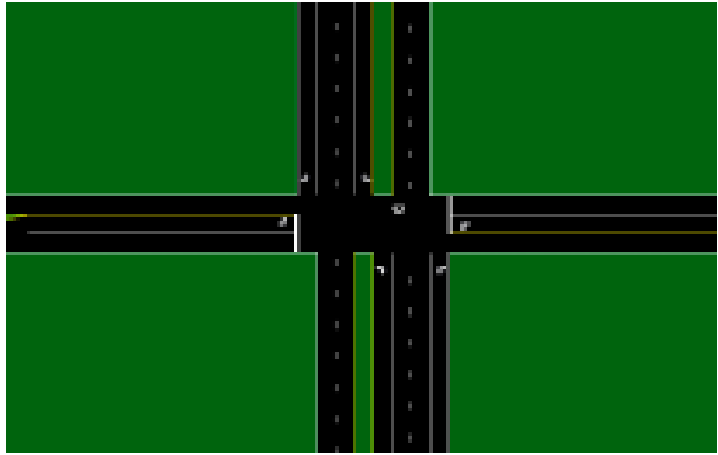

d) Node 12 : US $31 \&$ North Str.

Figure B- 3 US 31 Geometries-Continued 


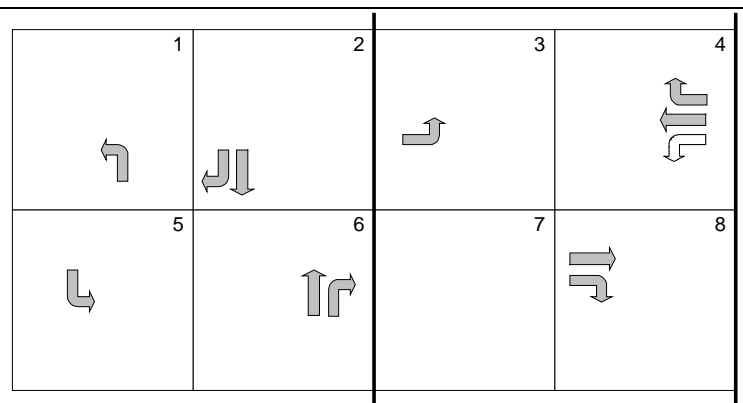

a) Node 1: US $31 \&$ US 26

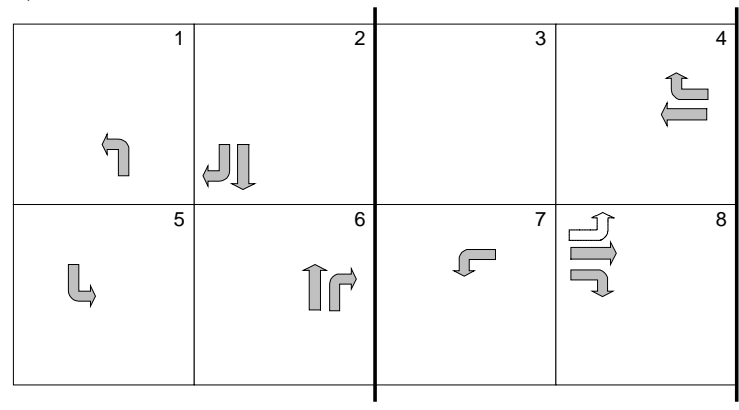

c) Node 3: US 31 \& Alto Road

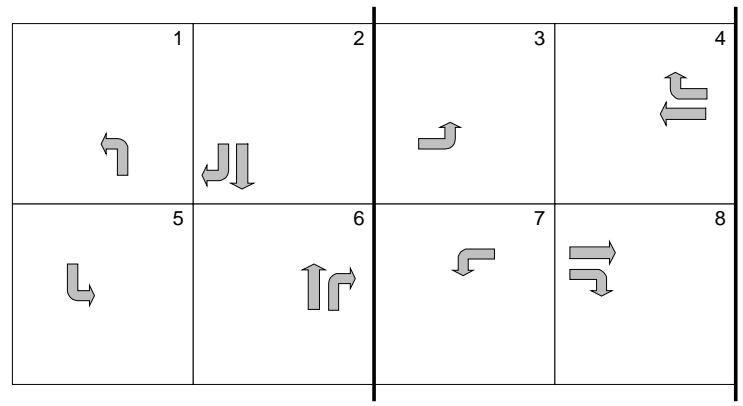

e) Node 5: US $31 \&$ Lincoln

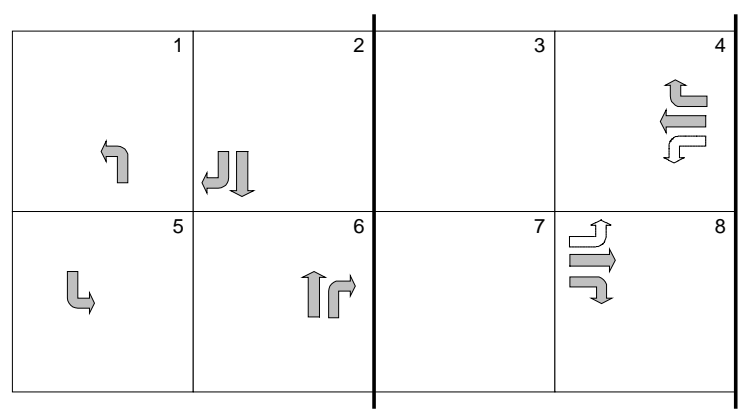

g) Node 7: US $31 \&$ Hoffer Str.

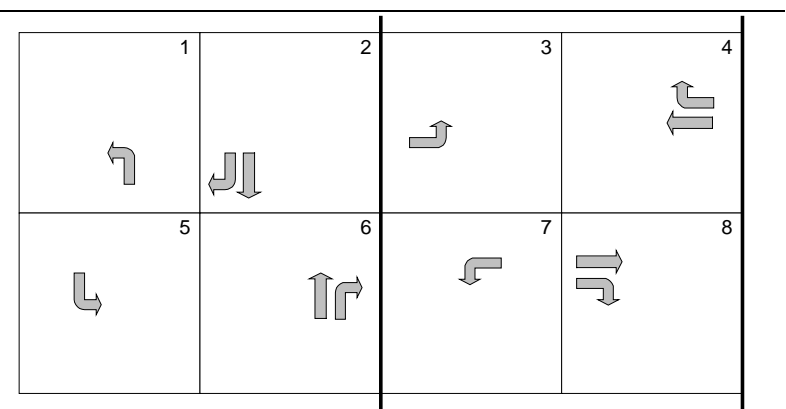

b) Node 2: US $31 \&$ Center Road

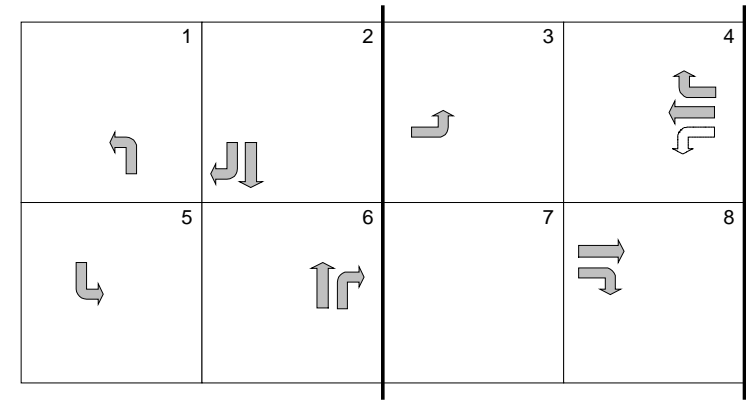

d) Node 4: US 31 \& Southway Blvd.

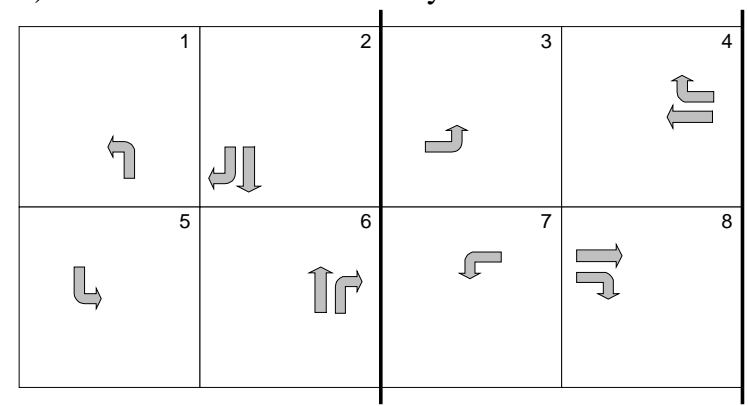

f) Node 6: US $31 \&$ Boulevard Ave.

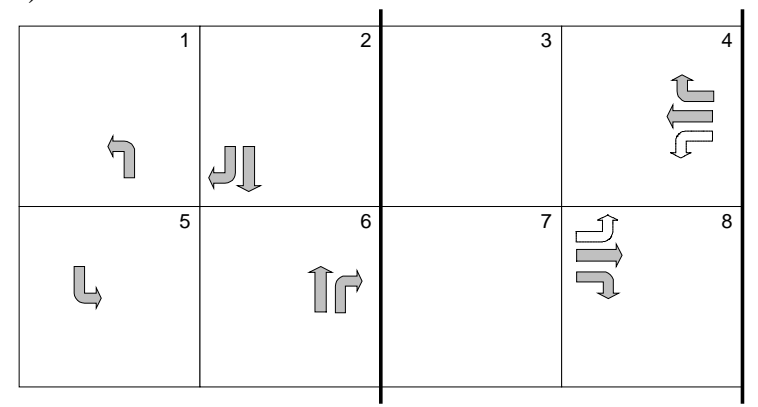

h) Node 8: US $31 \&$ Savoy Dr.

Figure B- 4 Intersection Ring Structures for US 31 


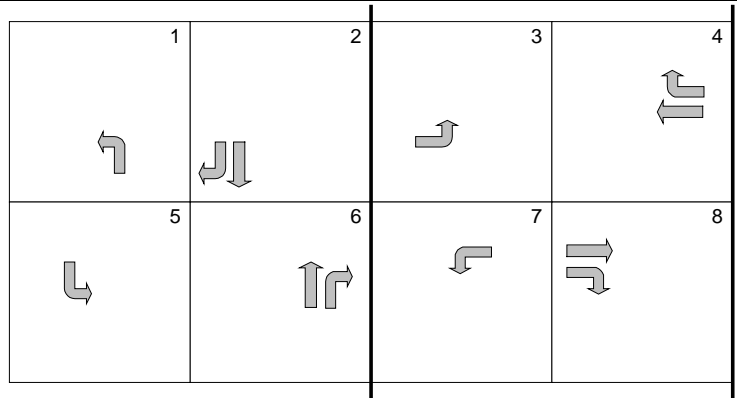

a) Node 9: US 31 \& Markland Ave

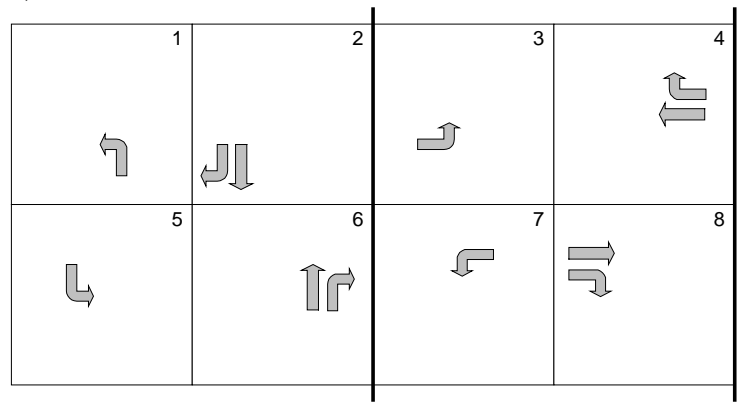

c) Node 11: US $31 \&$ Sycamore Dr.

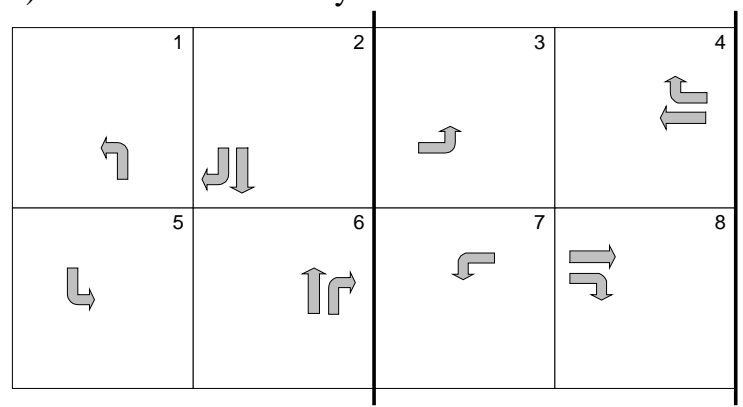

e) Node 13: US $31 \&$ Morgan St.

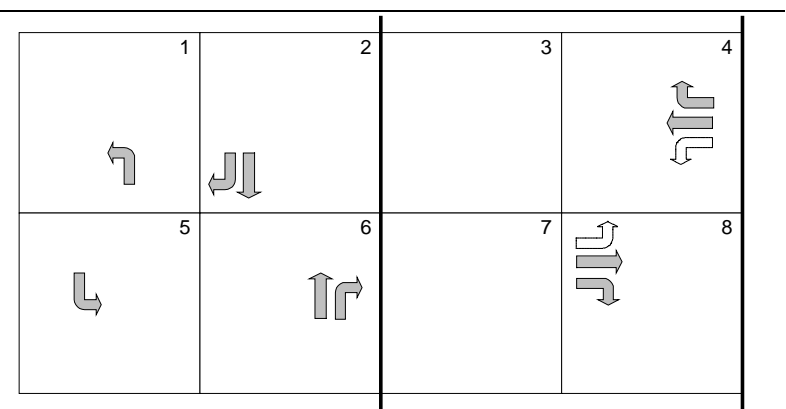

b) Node 10: US $31 \&$ Carter Str.

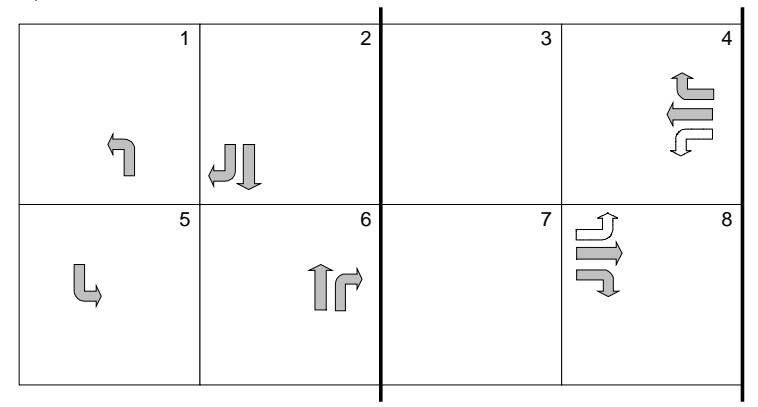

d) Node 12 : US $31 \&$ North Str.

Figure B- 5 Intersection Ring Structures for US 31-Continued 


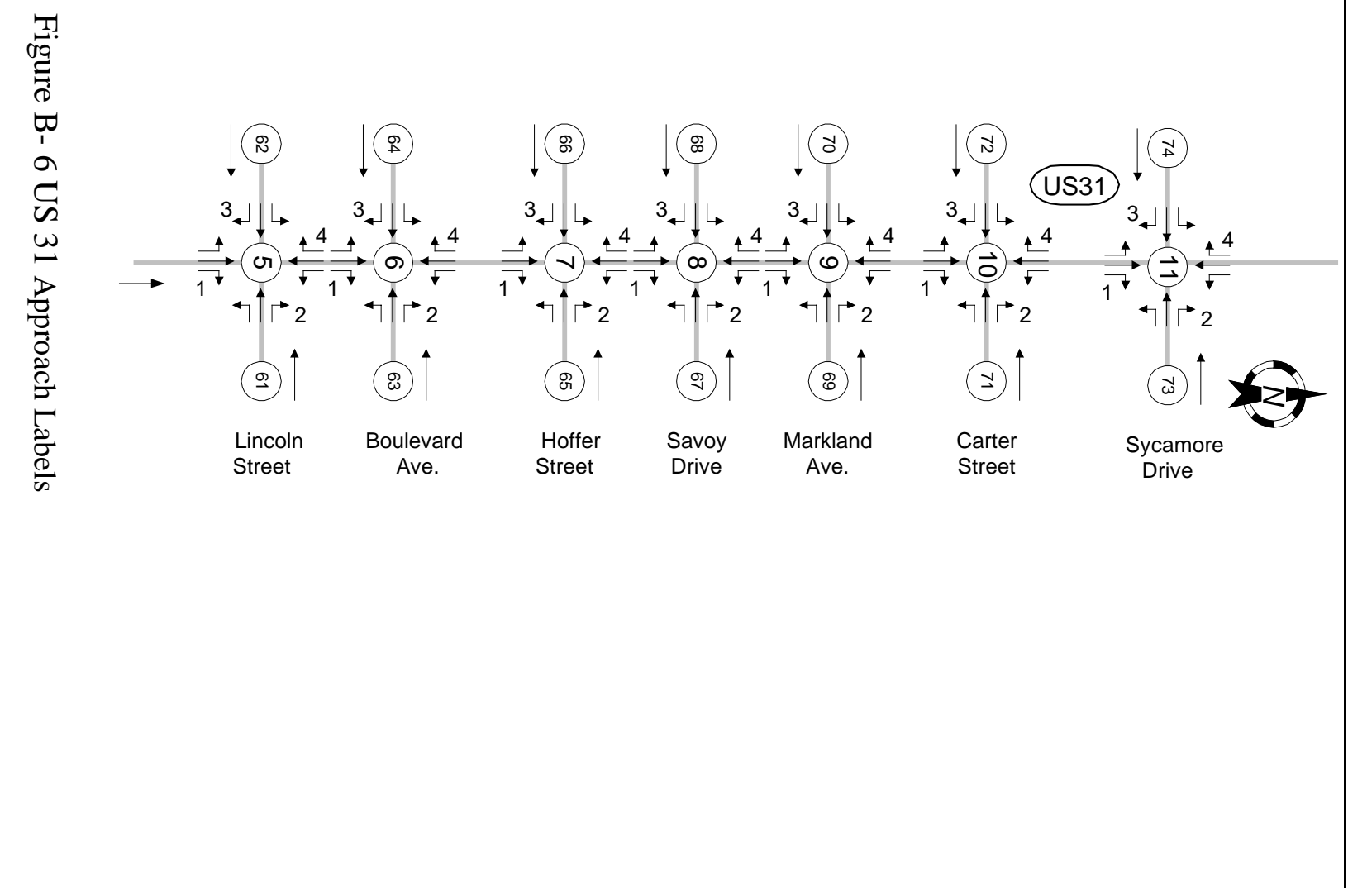




\begin{tabular}{|c|c|}
\hline COM [Portid] & $7,4, \mathrm{~T}, 2, \operatorname{Prot}$ \\
\hline 1 & 7, 4, R, 2, Prot \\
\hline CIDdef [CID, Base ID] & $8,1, L, 1, \mathrm{PP}$ \\
\hline 1,1 & $8,1, \mathrm{~T}, 6, \operatorname{Prot}$ \\
\hline 2,3 & $8,1, R, 6$, Prot \\
\hline 3,5 & $8,2, \mathrm{~L}, 4, \mathrm{PP}$ \\
\hline 4,7 & $8,2, \mathrm{~T}, 4, \operatorname{Prot}$ \\
\hline 5,9 & $8,2, R, 4, \operatorname{Prot}$ \\
\hline 6,11 & $8,3, \mathrm{~L}, 8, \mathrm{PP}$ \\
\hline 7,13 & $8,3, \mathrm{~T}, 8, \operatorname{Prot}$ \\
\hline NodeAssign [Node, CID] & $8,3, \mathrm{R}, 8, \operatorname{Prot}$ \\
\hline 5,1 & $8,4, \mathrm{~L}, 5, \mathrm{PP}$ \\
\hline 6,2 & $8,4, \mathrm{~T}, 2$, Prot \\
\hline 7,3 & $8,4, R, 2, \operatorname{Prot}$ \\
\hline 8,4 & $9,1, \mathrm{~L}, 1, \mathrm{PP}$ \\
\hline 9,5 & 9, 1, T, 6, Prot \\
\hline 10,6 & $9,1, R, 6, \operatorname{Prot}$ \\
\hline 11,7 & $9,2, \mathrm{~L}, 7, \mathrm{PP}$ \\
\hline Phases [Node, Approach, L/T/R, & $9,2, \mathrm{~T}, 4, \operatorname{Prot}$ \\
\hline Phase\#, Pro/Per/PP] & $9,2, R, 4, \operatorname{Prot}$ \\
\hline $5,1, \mathrm{~L}, 1, \mathrm{PP}$ & $9,3, \mathrm{~L}, 3, \mathrm{PP}$ \\
\hline $5,1, \mathrm{~T}, 6$, Prot & 9, 3, $\mathrm{T}, 8$, Prot \\
\hline 5, 1, R, 6, Prot & $9,3, \mathrm{R}, 8, \operatorname{Prot}$ \\
\hline $5,2, \mathrm{~L}, 7, \mathrm{PP}$ & $9,4, \mathrm{~L}, 5, \mathrm{PP}$ \\
\hline $5,2, \mathrm{~T}, 4$, Prot & $9,4, \mathrm{~T}, 2$, Prot \\
\hline $5,2, R, 4$, Prot & $9,4, R, 2$, Prot \\
\hline $5,3, L, 3, P P$ & $10,1, L, 1, \mathrm{PP}$ \\
\hline $5,3, \mathrm{~T}, 8, \operatorname{Prot}$ & 10, 1, T, 6, Prot \\
\hline $5,3, \mathrm{R}, 8$, Prot & 10, 1, R, 6, Prot \\
\hline $5,4, \mathrm{~L}, 5, \mathrm{PP}$ & $10,2, L, 4, \mathrm{PP}$ \\
\hline $5,4, \mathrm{~T}, 2, \operatorname{Prot}$ & $10,2, \mathrm{~T}, 4, \operatorname{Prot}$ \\
\hline $5,4, \mathrm{R}, 2$, Prot & $10,2, R, 4$, Prot \\
\hline $6,1, L, 1, \mathrm{PP}$ & $10,3, L, 8, \mathrm{PP}$ \\
\hline 6, 1, T, 6, Prot & $10,3, \mathrm{~T}, 8$, Prot \\
\hline 6, 1, R, 6, Prot & 10, 3, R, 8, Prot \\
\hline $6,2, L, 7, \mathrm{PP}$ & $10,4, L, 5, \mathrm{PP}$ \\
\hline $6,2, \mathrm{~T}, 4, \operatorname{Prot}$ & 10, 4, T, 2, Prot \\
\hline $6,2, \mathrm{R}, 4, \operatorname{Prot}$ & $10,4, R, 2$, Prot \\
\hline $6,3, L, 3, \mathrm{PP}$ & $11,1, \mathrm{~L}, 1, \mathrm{PP}$ \\
\hline $6,3, \mathrm{~T}, 8$, Prot & 11, 1, T, 6, Prot \\
\hline $6,3, \mathrm{R}, 8, \operatorname{Prot}$ & 11, 1, R, 6, Prot \\
\hline $6,4, \mathrm{~L}, 5, \mathrm{PP}$ & $11,2, L, 7, P P$ \\
\hline $6,4, T, 2$, Prot & $11,2, T, 4$, Prot \\
\hline $6,4, \mathrm{R}, 2$, Prot & $11,2, \mathrm{R}, 4$, Prot \\
\hline $7,1, L, 1, \mathrm{PP}$ & $11,3, L, 3, P P$ \\
\hline 7, 1, T, 6, Prot & $11,3, \mathrm{~T}, 8, \operatorname{Prot}$ \\
\hline 7, 1, R, 6, Prot & $11,3, \mathrm{R}, 8$, Prot \\
\hline $7,2, L, 4, P P$ & $11,4, L, 5, P P$ \\
\hline 7, 2, T, 4, Prot & 11, 4, T, 2, Prot \\
\hline $7,2, \mathrm{R}, 4$, Prot & $11,4, R, 2$, Prot \\
\hline $7,3, \mathrm{~L}, 8, \mathrm{PP}$ & \\
\hline $7,3, \mathrm{~T}, 8, \operatorname{Prot}$ & \\
\hline $7,3, \mathrm{R}, 8, \operatorname{Prot}$ & \\
\hline $7,4, \mathrm{~L}, 5, \mathrm{PP}$ & \\
\hline
\end{tabular}

Figure B- 7 External Control File (CID) for Phasing 
Table B- 1 Intersection Pocket Lengths and Detector Locations (Table 1 of 2)

\begin{tabular}{|c|c|c|c|c|c|c|}
\hline \multicolumn{2}{|c|}{$\begin{array}{c}\text { SR } 26 \\
\text { (SOUTH ST) }\end{array}$} & \multicolumn{2}{|c|}{$\begin{array}{c}\text { POCKET } \\
\text { LENGTHS (FT) }\end{array}$} & \multicolumn{3}{|c|}{$\begin{array}{c}\text { STOP BAR DETECTOR } \\
\text { LENGTHS (FT) }\end{array}$} \\
\hline INT & MOVE & LEFT & RIGHT & LEFT & THRU & RIGHT \\
\hline 1 & NB & 600 & 600 & 36 & -- & -- \\
\hline 1 & SB & 600 & 600 & 36 & -- & -- \\
\hline 1 & EB & 265 & - & 36 & 36 & -- \\
\hline 1 & WB & 230 & - & 36 & 36 & -- \\
\hline 2 & NB & 220 & 500 & 36 & -- & -- \\
\hline 2 & SB & 355 & 468 & 36 & -- & -- \\
\hline 2 & EB & 125 & $\mathrm{~T}$ w/ R & 36 & 36 & -- \\
\hline 2 & WB & 125 & PREV & 36 & 36 & -- \\
\hline 3 & NB & 755 & 755 & 36 & -- & -- \\
\hline 3 & SB & 525 & 700 & 36 & -- & -- \\
\hline 3 & EB & $\mathrm{T}$ w/ L & $\mathrm{T}$ w/ R & -- & 36 & -- \\
\hline 3 & WB & 515 & $\mathrm{~T}$ w/ R & -- & 36 & -- \\
\hline 4 & NB & 550 & 755 & 36 & -- & -- \\
\hline 4 & SB & 570 & 570 & 36 & -- & -- \\
\hline 4 & EB & PREV & $\mathrm{T} \mathrm{w} / \mathrm{R}$ & & 36 & -- \\
\hline 4 & WB & $\mathrm{T}$ w/ L & $\mathrm{T}$ w/ R & 36 & 36 & -- \\
\hline 5 & NB & 670 & 890 & 36 & -- & -- \\
\hline 5 & SB & 670 & 670 & 36 & -- & -- \\
\hline 5 & EB & 155 & $\mathrm{~T} \mathrm{w} / \mathrm{R}$ & -- & 36 & -- \\
\hline 5 & WB & 310 & 310 & -- & 36 & -- \\
\hline 6 & NB & 680 & 680 & 36 & -- & -- \\
\hline 6 & SB & 680 & 680 & 36 & -- & -- \\
\hline 6 & EB & 400 & $\mathrm{~T} \mathrm{w} / \mathrm{R}$ & 36 & 36 & -- \\
\hline 6 & WB & 190 & $\mathrm{~T} \mathrm{w/R}$ & 36 & 36 & -- \\
\hline 7 & NB & 665 & 665 & 36 & -- & -- \\
\hline 7 & SB & 450 & 450 & 36 & -- & -- \\
\hline 7 & EB & PREV & $\mathrm{T} \mathrm{w} / \mathrm{R}$ & 36 & 36 & -- \\
\hline 7 & WB & PREV & $\mathrm{T} \mathrm{w} / \mathrm{R}$ & 36 & 36 & -- \\
\hline
\end{tabular}

NOTE: "PREV" DENOTES THAT THE TURNING BAY BEGINS AT THE PREVIOUS

INTERSECTION. $\mathrm{T}$ w/ $\mathrm{L}$ : left turn lane is shared with through. $\mathrm{T}$ w/ $\mathrm{R}:$ right turn is shared with through 
Table B- 2 Intersection Pocket Lengths and Detector Locations (Table 2 of 2)

\begin{tabular}{|c|c|c|c|c|c|c|}
\hline \multicolumn{2}{|c|}{ US 31} & \multicolumn{2}{|c|}{$\begin{array}{c}\text { POCKET } \\
\text { LENGTHS (FT) }\end{array}$} & \multicolumn{3}{|c|}{$\begin{array}{c}\text { STOP BAR DETECTOR } \\
\text { LENGTHS (FT) }\end{array}$} \\
\hline INT & MOVE & LEFT & RIGHT & LEFT & THRU & RIGHT \\
\hline 8 & NB & 665 & 430 & 36 & -- & -- \\
\hline 8 & SB & 450 & 670 & 36 & -- & -- \\
\hline 8 & EB & $\mathrm{T} \mathrm{w} / \mathrm{L}$ & 180 & 36 & 36 & -- \\
\hline 8 & WB & $\mathrm{T} \mathrm{w/L}$ & 85 & 36 & 36 & -- \\
\hline 9 & NB & 700 & 700 & 36 & -- & -- \\
\hline 9 & SB & $700 \&$ & 700 & 36 & -- & -- \\
\hline 9 & EB & 565 & $\mathrm{~T} \mathrm{w} / \mathrm{R}$ & 36 & 36 & -- \\
\hline 9 & WB & 230 & PREV & 36 & 36 & -- \\
\hline 10 & NB & 450 & 450 & 36 & -- & -- \\
\hline 10 & SB & 450 & 185 & 36 & -- & -- \\
\hline 10 & EB & 435 & $\mathrm{~T} \mathrm{w} / \mathrm{R}$ & -- & 36 & -- \\
\hline 10 & WB & 130 & $\mathrm{~T} \mathrm{w} / \mathrm{R}$ & -- & 36 & -- \\
\hline 11 & NB & 550 & 255 & 36 & -- & -- \\
\hline 11 & SB & 500 & 500 & 36 & -- & -- \\
\hline 11 & EB & 380 & $\mathrm{~T} \mathrm{w} / \mathrm{R}$ & & 36 & -- \\
\hline 11 & WB & 140 & $\mathrm{~T} \mathrm{w} / \mathrm{R}$ & 36 & 36 & -- \\
\hline 12 & NB & 550 & 595 & 36 & -- & -- \\
\hline 12 & SB & 550 & 600 & 36 & -- & -- \\
\hline 12 & EB & 190 & $\mathrm{~T} \mathrm{w} / \mathrm{R}$ & -- & 36 & -- \\
\hline 12 & WB & 225 & $\mathrm{~T} \mathrm{w} / \mathrm{R}$ & -- & 36 & -- \\
\hline 13 & NB & 680 & 680 & 36 & -- & -- \\
\hline 13 & SB & 470 & PREV & 36 & -- & -- \\
\hline 13 & EB & PREV & $\mathrm{T} \mathrm{w} / \mathrm{R}$ & 36 & 36 & -- \\
\hline 13 & WB & 250 & $\mathrm{~T} \mathrm{w} / \mathrm{R}$ & 36 & 36 & -- \\
\hline 14 & NB & 550 & 550 & 36 & -- & -- \\
\hline 14 & SB & 550 & 550 & 36 & -- & -- \\
\hline 14 & EB & 120 & 250 & 36 & -- & -- \\
\hline 14 & WB & 280 & 180 & 36 & -- & -- \\
\hline 15 & NB & 550 & 550 & 36 & -- & -- \\
\hline 15 & SB & 550 & 550 & 36 & -- & -- \\
\hline 15 & EB & 150 & 200 & 36 & -- & -- \\
\hline 15 & WB & 250 & 200 & 36 & -- & -- \\
\hline
\end{tabular}

NOTE: "PREV” DENOTES THAT THE TURNING BAY BEGINS AT THE PREVIOUS INTERSECTION. T w/ L : left turn lane is 
Table B- 3 Intersection Detector Configuration (CID Logic Only)

\begin{tabular}{|c|c|c|c|c|}
\hline INT & $\begin{array}{c}\text { MOVE- } \\
\text { MENT }\end{array}$ & $\begin{array}{c}\text { DET } \\
\text { NUM }\end{array}$ & $\begin{array}{c}\text { STAT } \\
\text { ID }\end{array}$ & $\begin{array}{c}\text { PUL } \\
\text { PRS }\end{array}$ \\
\hline 5 & EBLT & 6 & 106 & PRS \\
\hline 5 & EBTH & 7 & 107 & PRS \\
\hline 5 & EBRT & 8 & 108 & PRS \\
\hline 5 & WBLT & 3 & 103 & PRS \\
\hline 5 & WBTH & 4 & 104 & PRS \\
\hline 5 & WBRT & 5 & 105 & PRS \\
\hline 5 & NBLT & 1 & 101 & PRS \\
\hline 5 & SBLT & 2 & 102 & PRS \\
\hline
\end{tabular}

\begin{tabular}{|c|c|c|c|c|}
\hline INT & $\begin{array}{c}\text { MOVE- } \\
\text { MENT }\end{array}$ & $\begin{array}{c}\text { DET } \\
\text { NUM }\end{array}$ & $\begin{array}{c}\text { STAT } \\
\text { ID }\end{array}$ & $\begin{array}{c}\text { PUL } \\
\text { PRS }\end{array}$ \\
\hline 6 & EBLT & 3 & 203 & PRS \\
\hline 6 & EBTH & 8 & 208 & PRS \\
\hline 6 & EBRT & 16 & 216 & PRS \\
\hline 6 & WBLT & 7 & 207 & PRS \\
\hline 6 & WBTH & 4 & 204 & PRS \\
\hline 6 & WBRT & 12 & 212 & PRS \\
\hline 6 & NBLT & 1 & 201 & PRS \\
\hline 6 & SBLT & 5 & 205 & PRS \\
\hline
\end{tabular}

\begin{tabular}{|c|c|c|c|c|}
\hline INT & $\begin{array}{c}\text { MOVE- } \\
\text { MENT }\end{array}$ & $\begin{array}{c}\text { DET } \\
\text { NUM }\end{array}$ & $\begin{array}{c}\text { STAT } \\
\text { ID }\end{array}$ & $\begin{array}{c}\text { PUL } \\
\text { PRS }\end{array}$ \\
\hline 7 & EBLT & -- & -- & PRS \\
\hline 7 & EBTH & 8 & 308 & PRS \\
\hline 7 & EBRT & 16 & 316 & PRS \\
\hline 7 & WBLT & -- & -- & PRS \\
\hline 7 & WBTH & 4 & 304 & PRS \\
\hline 7 & WBRT & 12 & 312 & PRS \\
\hline 7 & NBLT & 1 & 301 & PRS \\
\hline 7 & SBLT & 5 & 305 & PRS \\
\hline
\end{tabular}

\begin{tabular}{|c|c|c|c|c|}
\hline INT & $\begin{array}{c}\text { MOVE- } \\
\text { MENT }\end{array}$ & $\begin{array}{c}\text { DET } \\
\text { NUM }\end{array}$ & $\begin{array}{c}\text { STAT } \\
\text { ID }\end{array}$ & $\begin{array}{c}\text { PUL } \\
\text { PRS }\end{array}$ \\
\hline 8 & EBLT & -- & -- & PRS \\
\hline 8 & EBTH & 8 & 408 & PRS \\
\hline 8 & EBRT & 16 & 416 & PRS \\
\hline 8 & WBLT & -- & -- & PRS \\
\hline 8 & WBTH & 4 & 404 & PRS \\
\hline 8 & WBRT & 12 & 412 & PRS \\
\hline 8 & NBLT & 1 & 401 & PRS \\
\hline 8 & SBLT & 5 & 405 & PRS \\
\hline
\end{tabular}

\begin{tabular}{|c|c|c|c|c|}
\hline INT & $\begin{array}{c}\text { MOVE- } \\
\text { MENT }\end{array}$ & $\begin{array}{c}\text { DET } \\
\text { NUM }\end{array}$ & $\begin{array}{c}\text { STAT } \\
\text { ID }\end{array}$ & $\begin{array}{c}\text { PUL } \\
\text { PRS }\end{array}$ \\
\hline 9 & EBLT & 3 & 503 & PRS \\
\hline 9 & EBTH & 8 & 508 & PRS \\
\hline 9 & EBRT & 16 & 516 & PRS \\
\hline 9 & WBLT & 7 & 507 & PRS \\
\hline 9 & WBTH & 4 & 504 & PRS \\
\hline 9 & WBRT & 12 & 512 & PRS \\
\hline 9 & NBLT & 1 & 501 & PRS \\
\hline 9 & SBLT & 5 & 505 & PRS \\
\hline
\end{tabular}

\begin{tabular}{|c|c|c|c|c|}
\hline INT & $\begin{array}{c}\text { MOVE- } \\
\text { MENT }\end{array}$ & $\begin{array}{c}\text { DET } \\
\text { NUM }\end{array}$ & $\begin{array}{c}\text { STAT } \\
\text { ID }\end{array}$ & $\begin{array}{c}\text { PUL } \\
\text { PRS }\end{array}$ \\
\hline 10 & EBLT & -- & -- & PRS \\
\hline 10 & EBTH & 8 & 608 & PRS \\
\hline 10 & EBRT & 16 & 616 & PRS \\
\hline 10 & WBLT & -- & -- & PRS \\
\hline 10 & WBTH & 4 & 604 & PRS \\
\hline 10 & WBRT & 12 & 612 & PRS \\
\hline 10 & NBLT & 1 & 601 & PRS \\
\hline 10 & SBLT & 5 & 605 & PRS \\
\hline
\end{tabular}

\begin{tabular}{|c|c|c|c|c|}
\hline INT & $\begin{array}{c}\text { MOVE- } \\
\text { MENT }\end{array}$ & $\begin{array}{c}\text { DET } \\
\text { NUM }\end{array}$ & $\begin{array}{c}\text { STAT } \\
\text { ID }\end{array}$ & $\begin{array}{c}\text { PUL } \\
\text { PRS }\end{array}$ \\
\hline 11 & EBLT & 3 & 703 & PRS \\
\hline 11 & EBTH & 8 & 708 & PRS \\
\hline 11 & EBRT & 16 & 716 & PRS \\
\hline 11 & WBLT & 7 & 707 & PRS \\
\hline 11 & WBTH & 4 & 704 & PRS \\
\hline 11 & WBRT & 12 & 712 & PRS \\
\hline 11 & NBLT & 1 & 701 & PRS \\
\hline 11 & SBLT & 5 & 705 & PRS \\
\hline
\end{tabular}


Table B- 4 General Phase Parameters (US 31)

\begin{tabular}{|c|c|c|c|c|c|c|c|c|c|}
\hline INT & DESCRIPTION (SEC) & 1 & 2 & 3 & 4 & 5 & 6 & 7 & 8 \\
\hline 1 & MINIMUM GREEN & 5 & 10 & 5 & 10 & 5 & 10 & 5 & 10 \\
\hline 1 & VEHICLE EXTENSION & 2 & 2 & 2 & 2 & 2 & 2 & 2 & 2 \\
\hline 1 & MAXIMUM GREEN & 99 & 99 & 99 & 99 & 99 & 99 & 99 & 99 \\
\hline 1 & YELLOW CLEARANCE & 3.5 & 3.5 & 3.5 & 3.5 & 3.5 & 3.5 & 3.5 & 3.5 \\
\hline 1 & RED CLEARANCE & 1.5 & 1.5 & 1.5 & 1.5 & 1.5 & 1.5 & 1.5 & 1.5 \\
\hline \multirow[t]{2}{*}{1} & MINIMUM GAP TIME & 2 & 2 & 2 & 2 & 2 & 2 & 2 & 2 \\
\hline & MINIMUM GREEN & & & & & & & & \\
\hline 2 & VEHICLE EXTENSION & 2 & 2 & 2 & 2 & 2 & 2 & 2 & 2 \\
\hline 2 & MAXIMUM GREEN & 99 & 99 & 99 & 99 & 99 & 99 & 99 & 99 \\
\hline 2 & YELLOW CLEARANCE & 3.5 & 3.5 & 3.5 & 3.5 & 3.5 & 3.5 & 3.5 & 3.5 \\
\hline 2 & RED CLEARANCE & 1.5 & 1.5 & 1.5 & 1.5 & 1.5 & 1.5 & 1.5 & 1.5 \\
\hline \multirow[t]{2}{*}{2} & MINIMUM GAP TIME & 2 & 2 & 2 & 2 & 2 & 2 & 2 & 2 \\
\hline & MINIMUM GREEN & & & & & & & & \\
\hline 3 & VEHICLE EXTENSION & 2 & 2 & 2 & 2 & 2 & 2 & 2 & 2 \\
\hline 3 & MAXIMUM GREEN & 99 & 99 & 99 & 99 & 99 & 99 & 99 & 99 \\
\hline 3 & YELLOW CLEARANCE & 3.5 & 3.5 & 3.5 & 3.5 & 3.5 & 3.5 & 3.5 & 3.5 \\
\hline 3 & RED CLEARANCE & 1.5 & 1.5 & 1.5 & 1.5 & 1.5 & 1.5 & 1.5 & 1.5 \\
\hline \multirow[t]{2}{*}{3} & MINIMUM GAP TIME & 2 & 2 & 2 & 2 & 2 & 2 & 2 & 2 \\
\hline & MINIMUM GREEN & & & & & & & & \\
\hline 4 & VEHICLE EXTENSION & 2 & 2 & 2 & 2 & 2 & 2 & 2 & 2 \\
\hline 4 & MAXIMUM GREEN & 99 & 99 & 99 & 99 & 99 & 99 & 99 & 99 \\
\hline 4 & YELLOW CLEARANCE & 3.5 & 3.5 & 3.5 & 3.5 & 3.5 & 3.5 & 3.5 & 3.5 \\
\hline 4 & RED CLEARANCE & 1.5 & 1.5 & 1.5 & 1.5 & 1.5 & 1.5 & 1.5 & 1.5 \\
\hline \multirow[t]{2}{*}{4} & MINIMUM GAP TIME & 2 & 2 & 2 & 2 & 2 & 2 & 2 & 2 \\
\hline & MINIMUM GREEN & & & & & & & & \\
\hline 5 & VEHICLE EXTENSION & 2 & 2 & 2 & 2 & 2 & 2 & 2 & 2 \\
\hline 5 & MAXIMUM GREEN & 99 & 99 & 99 & 99 & 99 & 99 & 99 & 99 \\
\hline 5 & YELLOW CLEARANCE & 3.5 & 3.5 & 3.5 & 3.5 & 3.5 & 3.5 & 3.5 & 3.5 \\
\hline 5 & RED CLEARANCE & 1.5 & 1.5 & 1.5 & 1.5 & 1.5 & 1.5 & 1.5 & 1.5 \\
\hline \multirow[t]{2}{*}{5} & MINIMUM GAP TIME & 2 & 2 & 2 & 2 & 2 & 2 & 2 & 2 \\
\hline & MINIMUM GREEN & & & & & & & & \\
\hline 6 & VEHICLE EXTENSION & 2 & 2 & 2 & 2 & 2 & 2 & 2 & 2 \\
\hline 6 & MAXIMUM GREEN & 99 & 99 & 99 & 99 & 99 & 99 & 99 & 99 \\
\hline 6 & YELLOW CLEARANCE & 3.5 & 3.5 & 3.5 & 3.5 & 3.5 & 3.5 & 3.5 & 3.5 \\
\hline 6 & RED CLEARANCE & 1.5 & 1.5 & 1.5 & 1.5 & 1.5 & 1.5 & 1.5 & 1.5 \\
\hline \multirow[t]{2}{*}{6} & MINIMUM GAP TIME & 2 & 2 & 2 & 2 & 2 & 2 & 2 & 2 \\
\hline & MINIMUM GREEN & & & & & & & & \\
\hline 7 & VEHICLE EXTENSION & 2 & 2 & 2 & 2 & 2 & 2 & 2 & 2 \\
\hline 7 & MAXIMUM GREEN & 99 & 99 & 99 & 99 & 99 & 99 & 99 & 99 \\
\hline 7 & YELLOW CLEARANCE & 3.5 & 3.5 & 3.5 & 3.5 & 3.5 & 3.5 & 3.5 & 3.5 \\
\hline 7 & RED CLEARANCE & 1.5 & 1.5 & 1.5 & 1.5 & 1.5 & 1.5 & 1.5 & 1.5 \\
\hline 7 & MINIMUM GAP TIME & 2 & 2 & 2 & 2 & 2 & 2 & 2 & 2 \\
\hline
\end{tabular}


Table B- 5 US 31 Coordination Plans and Splits

\begin{tabular}{|c|c|c|c|c|c|c|c|c|c|c|c|c|}
\hline & & & \multicolumn{2}{|c|}{ OFFSET } & & & & & & & & \\
\hline INT & TIME & CYC & COR & $\mathrm{CON}$ & 1 & 2 & 3 & 4 & 5 & 6 & 7 & 8 \\
\hline 1 & $\mathrm{Am}$ & 92 & 0 & 37 & 15 & 37 & 15 & 19 & 15 & 37 & -- & 34 \\
\hline 1 & $\mathrm{Pm}$ & 116 & 0 & 99 & 15 & 64 & 16 & 21 & 23 & 56 & -- & 37 \\
\hline 2 & $\mathrm{Pm}$ & 116 & 64 & 46 & 14 & 65 & 14 & 23 & 33 & 46 & 14 & 23 \\
\hline 3 & $\mathrm{Pm}$ & 116 & 18 & 112 & 29 & 50 & -- & 37 & 14 & 65 & 14 & 23 \\
\hline 4 & $\mathrm{Pm}$ & 116 & 107 & 90 & 26 & 53 & 15 & 22 & 15 & 64 & -- & 37 \\
\hline 5 & $\mathrm{Pm}$ & 116 & 67 & 55 & 22 & 54 & 21 & 19 & 17 & 59 & 21 & 19 \\
\hline 6 & $\mathrm{Pm}$ & 116 & 24 & 10 & 20 & 56 & 16 & 24 & 15 & 61 & 16 & 24 \\
\hline 7 & $\mathrm{Pm}$ & 116 & 50 & 25 & 17 & 66 & -- & 33 & 12 & 71 & -- & 33 \\
\hline 8 & $\mathrm{Pm}$ & 116 & 12 & 98 & 23 & 70 & -- & 23 & 16 & 77 & -- & 23 \\
\hline 9 & $\mathrm{Pm}$ & 116 & 68 & 72 & 19 & 39 & 32 & 26 & 15 & 43 & 26 & 32 \\
\hline 10 & $\mathrm{Pm}$ & 116 & 28 & 7 & 14 & 68 & -- & 34 & 20 & 62 & -- & 34 \\
\hline 11 & $\mathrm{Pm}$ & 116 & 11 & 113 & 22 & 53 & 14 & 27 & 14 & 61 & 14 & 27 \\
\hline
\end{tabular}


Table B- 6 Detailed Traffic Volume (vph - Table 1 of 3)

\begin{tabular}{|c|c|c|c|c|c|c|c|c|c|c|c|c|c|}
\hline INT & $\begin{array}{l}\text { BEG } \\
\text { END }\end{array}$ & $\begin{array}{l}7 \mathrm{AM} \\
8 \mathrm{AM}\end{array}$ & $\begin{array}{l}8 \mathrm{AM} \\
9 \mathrm{AM}\end{array}$ & $\begin{array}{c}9 \mathrm{AM} \\
10 \mathrm{~A}\end{array}$ & $\begin{array}{l}10 \mathrm{~A} \\
11 \mathrm{~A}\end{array}$ & $\begin{array}{l}11 \mathrm{~A} \\
12 \mathrm{P}\end{array}$ & $\begin{array}{c}12 \mathrm{P} \\
1 \mathrm{PM}\end{array}$ & $\begin{array}{l}1 \mathrm{PM} \\
2 \mathrm{PM}\end{array}$ & $\begin{array}{l}2 \mathrm{PM} \\
3 \mathrm{PM}\end{array}$ & $\begin{array}{l}3 \mathrm{PM} \\
4 \mathrm{PM}\end{array}$ & $\begin{array}{l}4 \mathrm{PM} \\
5 \mathrm{PM}\end{array}$ & $\begin{array}{l}5 \mathrm{PM} \\
6 \mathrm{PM}\end{array}$ & $\begin{array}{l}6 \mathrm{PM} \\
7 \mathrm{PM}\end{array}$ \\
\hline \multirow{12}{*}{$\begin{array}{l}\text { N } \\
\text { s. } \\
w\end{array}$} & EBLT & 188 & 184 & 159 & 96 & 97 & 113 & 122 & 122 & 148 & 116 & 127 & 127 \\
\hline & EBTH & 66 & 81 & 72 & 60 & 54 & 57 & 79 & 53 & 78 & 97 & 107 & 116 \\
\hline & EBRT & 98 & 58 & 60 & 46 & 60 & 44 & 47 & 62 & 45 & 61 & 83 & 73 \\
\hline & WBLT & 57 & 70 & 57 & 40 & 36 & 37 & 37 & 31 & 37 & 65 & 52 & 44 \\
\hline & WBTH & 72 & 108 & 101 & 71 & 75 & 70 & 73 & 67 & 90 & 133 & 113 & 114 \\
\hline & WBRT & 56 & 81 & 77 & 73 & 68 & 102 & 77 & 98 & 68 & 83 & 63 & 66 \\
\hline & NBLT & 57 & 100 & 82 & 53 & 53 & 61 & 40 & 64 & 58 & 68 & 86 & 95 \\
\hline & NBTH & 798 & 982 & 762 & 590 & 551 & 521 & 526 & 581 & 631 & 532 & 684 & 726 \\
\hline & NBRT & 45 & 36 & 53 & 37 & 41 & 26 & 28 & 45 & 20 & 54 & 41 & 71 \\
\hline & SBLT & 38 & 59 & 52 & 49 & 51 & 77 & 84 & 78 & 88 & 114 & 95 & 124 \\
\hline & SBTH & 485 & 654 & 533 & 503 & 478 & 540 & 563 & 585 & 757 & 921 & 993 & 948 \\
\hline & SBRT & 41 & 78 & 62 & 68 & 100 & 126 & 115 & 109 & 153 & 230 & 207 & 193 \\
\hline \multirow{11}{*}{ 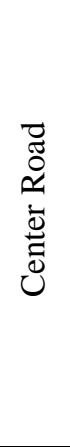 } & EBTH & 35 & 79 & 62 & 51 & 87 & 103 & 149 & 125 & 119 & 144 & 153 & 145 \\
\hline & EBRT & 8 & 21 & 27 & 15 & 32 & 43 & 57 & 45 & 46 & 60 & 73 & 47 \\
\hline & WBLT & 59 & 56 & 68 & 55 & 44 & 45 & 62 & 72 & 53 & 93 & 72 & 67 \\
\hline & WBTH & 48 & 78 & 69 & 62 & 54 & 88 & 91 & 94 & 107 & 131 & 82 & 165 \\
\hline & WBRT & 112 & 173 & 199 & 158 & 150 & 227 & 227 & 181 & 231 & 162 & 183 & 175 \\
\hline & NBLT & 26 & 43 & 45 & 53 & 41 & 63 & 99 & 75 & 79 & 58 & 88 & 73 \\
\hline & NBTH & 1042 & 1326 & 1105 & 838 & 776 & 887 & 829 & 833 & 1016 & 787 & 1008 & 916 \\
\hline & NBRT & 49 & 43 & 43 & 31 & 31 & 36 & 40 & 33 & 42 & 33 & 52 & 64 \\
\hline & SBLT & 72 & 135 & 159 & 182 & 218 & 322 & 418 & 256 & 331 & 335 & 364 & 358 \\
\hline & SBTH & 568 & 726 & 606 & 657 & 723 & 790 & 796 & 869 & 1102 & 1315 & 1507 & 1409 \\
\hline & SBRT & 13 & 35 & 42 & 42 & 59 & 104 & 105 & 95 & 104 & 131 & 133 & 116 \\
\hline \multirow{11}{*}{$\begin{array}{l}\vec{Z} \\
\mathscr{0} \\
\stackrel{2}{2} \\
\stackrel{ᄋ}{Z}\end{array}$} & EBTH & 31 & 46 & 86 & 70 & 105 & 121 & 158 & 113 & 119 & 113 & 160 & 151 \\
\hline & EBRT & 1 & 6 & 4 & 6 & 11 & 10 & 14 & 20 & 18 & 18 & 24 & 20 \\
\hline & WBLT & 35 & 64 & 59 & 48 & 62 & 73 & 92 & 88 & 93 & 99 & 125 & 110 \\
\hline & WBTH & 43 & 68 & 105 & 91 & 91 & 122 & 129 & 121 & 101 & 135 & 135 & 126 \\
\hline & WBRT & 34 & 47 & 43 & 34 & 44 & 48 & 71 & 43 & 46 & 40 & 40 & 57 \\
\hline & NBLT & 121 & 255 & 273 & 275 & 264 & 317 & 386 & 361 & 301 & 362 & 361 & 386 \\
\hline & NBTH & 1093 & 1490 & 1124 & 772 & 718 & 864 & 846 & 720 & 1014 & 860 & 959 & 931 \\
\hline & NBRT & 64 & 99 & 64 & 57 & 55 & 75 & 50 & 25 & 94 & 45 & 83 & 56 \\
\hline & SBLT & 15 & 37 & 59 & 63 & 65 & 110 & 118 & 61 & 52 & 42 & 69 & 131 \\
\hline & SBTH & 425 & 673 & 601 & 536 & 660 & 789 & 877 & 711 & 1138 & 1326 & 1363 & 1203 \\
\hline & SBRT & 6 & 29 & 46 & 58 & 66 & 71 & 76 & 50 & 60 & 48 & 64 & 57 \\
\hline \multirow{11}{*}{ 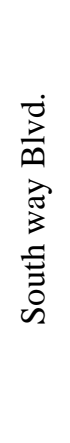 } & EBTH & 36 & 83 & 126 & 114 & 123 & 200 & 200 & 156 & 153 & 227 & 263 & 376 \\
\hline & EBRT & 8 & 3 & 6 & 16 & 22 & 33 & 35 & 36 & 39 & 54 & 56 & 57 \\
\hline & WBLT & 16 & 23 & 31 & 14 & 22 & 55 & 23 & 30 & 29 & 49 & 55 & 63 \\
\hline & WBTH & 63 & 101 & 151 & 121 & 150 & 199 & 205 & 166 & 190 & 231 & 233 & 210 \\
\hline & WBRT & 22 & 45 & 59 & 39 & 44 & 49 & 100 & 96 & 72 & 88 & 50 & 87 \\
\hline & NBLT & 7 & 21 & 37 & 37 & 37 & 52 & 49 & 60 & 66 & 55 & 69 & 70 \\
\hline & NBTH & 1015 & 340 & 1088 & 848 & 761 & 856 & 863 & 800 & 1073 & 869 & 953 & 907 \\
\hline & NBRT & 16 & 36 & 34 & 25 & 10 & 36 & 29 & 33 & 34 & 29 & 44 & 54 \\
\hline & SBLT & 15 & 14 & 36 & 48 & 60 & 122 & 152 & 108 & 107 & 115 & 164 & 164 \\
\hline & SBTH & 408 & 678 & 711 & 666 & 789 & 969 & 894 & 862 & 1098 & 448 & 297 & 465 \\
\hline & SBRT & 33 & 78 & 93 & 63 & 116 & 218 & 122 & 130 & 141 & 175 & 173 & 168 \\
\hline
\end{tabular}


Table B- 7 Detailed Traffic Volume (vph - Table 2 of 3)

\begin{tabular}{|c|c|c|c|c|c|c|c|c|c|c|c|c|c|}
\hline INT & $\begin{array}{l}\text { BEG } \\
\text { END }\end{array}$ & $\begin{array}{l}\text { 7AM } \\
8 \mathrm{AM}\end{array}$ & $\begin{array}{l}8 \mathrm{AM} \\
9 \mathrm{AM}\end{array}$ & $\begin{array}{c}9 \mathrm{AM} \\
10 \mathrm{~A}\end{array}$ & $\begin{array}{l}10 \mathrm{~A} \\
11 \mathrm{~A}\end{array}$ & $\begin{array}{l}11 \mathrm{~A} \\
12 \mathrm{P}\end{array}$ & $\begin{array}{c}12 \mathrm{P} \\
1 \mathrm{PM}\end{array}$ & $\begin{array}{l}1 \mathrm{PM} \\
2 \mathrm{PM}\end{array}$ & $\begin{array}{l}2 \mathrm{PM} \\
3 \mathrm{PM}\end{array}$ & $\begin{array}{l}3 \mathrm{PM} \\
4 \mathrm{PM}\end{array}$ & $\begin{array}{l}4 \mathrm{PM} \\
5 \mathrm{PM}\end{array}$ & $\begin{array}{l}\text { PPM } \\
6 \mathrm{PM}\end{array}$ & $\begin{array}{l}6 \mathrm{PM} \\
7 \mathrm{PM}\end{array}$ \\
\hline \multirow{12}{*}{ 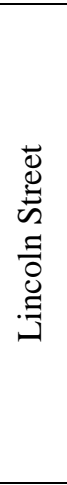 } & EBLT & 56 & 82 & 99 & 113 & 146 & 186 & 204 & 175 & 236 & 256 & 178 & 278 \\
\hline & EBTH & 280 & 339 & 240 & 137 & 164 & 184 & 416 & 281 & 367 & 323 & 296 & 301 \\
\hline & EBRT & 45 & 89 & 63 & 60 & 101 & 78 & 110 & 145 & 162 & 296 & 136 & 235 \\
\hline & WBLT & 41 & 29 & 73 & 55 & 96 & 137 & 121 & 93 & 141 & 241 & 280 & 320 \\
\hline & WBTH & 230 & 182 & 199 & 128 & 213 & 140 & 239 & 166 & 446 & 343 & 395 & 327 \\
\hline & WBRT & 85 & 158 & 124 & 100 & 151 & 253 & 175 & 95 & 191 & 234 & 192 & 146 \\
\hline & NBLT & 252 & 209 & 119 & 122 & 102 & 272 & 114 & 123 & 236 & 122 & 97 & 137 \\
\hline & NBTH & 709 & 1119 & 950 & 868 & 770 & 1016 & 970 & 934 & 1026 & 1056 & 1118 & 1146 \\
\hline & NBRT & 232 & 407 & 210 & 63 & 68 & 41 & 138 & 129 & 106 & 79 & 51 & 90 \\
\hline & SBLT & 140 & 189 & 122 & 94 & 91 & 206 & 219 & 179 & 147 & 127 & 117 & 144 \\
\hline & SBTH & 416 & 698 & 695 & 771 & 763 & 1132 & 934 & 1053 & 1012 & 1237 & 1175 & 1269 \\
\hline & SBRT & 83 & 91 & 53 & 54 & 100 & 83 & 80 & 117 & 183 & 121 & 97 & 99 \\
\hline \multirow{11}{*}{ 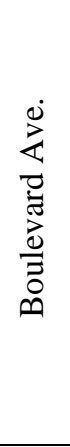 } & EBTH & 157 & 237 & 139 & 107 & 110 & 262 & 266 & 205 & 389 & 395 & 239 & 216 \\
\hline & EBRT & 47 & 107 & 119 & 115 & 120 & 236 & 193 & 217 & 216 & 302 & 229 & 259 \\
\hline & WBLT & 86 & 99 & 60 & 57 & 66 & 120 & 87 & 76 & 189 & 118 & 99 & 87 \\
\hline & WBTH & 276 & 293 & 192 & 144 & 207 & 395 & 249 & 170 & 590 & 403 & 338 & 165 \\
\hline & WBRT & 35 & 43 & 38 & 25 & 65 & 176 & 52 & 40 & 92 & 64 & 56 & 34 \\
\hline & NBLT & 80 & 191 & 149 & 164 & 135 & 263 & 225 & 160 & 231 & 211 & 159 & 153 \\
\hline & NBTH & 721 & 985 & 909 & 853 & 1010 & 1306 & 1200 & 1034 & 1217 & 1284 & 1311 & 1214 \\
\hline & NBRT & 97 & 90 & 86 & 47 & 68 & 103 & 36 & 74 & 80 & 80 & 69 & 67 \\
\hline & SBLT & 85 & 68 & 50 & 36 & 44 & 176 & 138 & 95 & 152 & 83 & 61 & 62 \\
\hline & SBTH & 572 & 778 & 823 & 828 & 768 & 988 & 1074 & 963 & 1145 & 1173 & 1113 & 1185 \\
\hline & SBRT & 96 & 49 & 58 & 50 & 66 & 102 & 66 & 102 & 77 & 116 & 54 & 55 \\
\hline \multirow{11}{*}{ 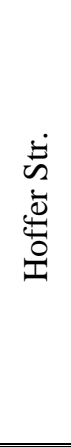 } & EBTH & 6 & 30 & 22 & 28 & 16 & 37 & 32 & 25 & 26 & 31 & 32 & 32 \\
\hline & EBRT & 57 & 45 & 49 & 73 & 77 & 152 & 115 & 186 & 130 & 146 & 119 & 119 \\
\hline & WBLT & 2 & 7 & 6 & 18 & 15 & 39 & 20 & 18 & 15 & 17 & 13 & 13 \\
\hline & WBTH & 2 & 13 & 15 & 14 & 15 & 34 & 27 & 24 & 35 & 24 & 26 & 26 \\
\hline & WBRT & 5 & 15 & 27 & 28 & 40 & 39 & 38 & 29 & 29 & 27 & 33 & 33 \\
\hline & NBLT & 50 & 95 & 98 & 69 & 56 & 117 & 121 & 138 & 103 & 114 & 110 & 110 \\
\hline & NBTH & 680 & 897 & 813 & 805 & 826 & 1104 & 1095 & 1185 & 1287 & 1245 & 1163 & 1163 \\
\hline & NBRT & 5 & 21 & 17 & 14 & 8 & 15 & 14 & 13 & 15 & 13 & 9 & 9 \\
\hline & SBLT & 12 & 18 & 42 & 37 & 33 & 41 & 42 & 24 & 31 & 24 & 33 & 33 \\
\hline & SBTH & 819 & 761 & 772 & 810 & 779 & 1231 & 1040 & 1256 & 1129 & 1212 & 1162 & 1162 \\
\hline & SBRT & 41 & 65 & 84 & 107 & 99 & 276 & 168 & 213 & 148 & 208 & 151 & 151 \\
\hline \multirow{11}{*}{$\begin{array}{l}\dot{\vec{D}} \\
\overrightarrow{0} \\
\overrightarrow{\tilde{D}}\end{array}$} & EBTH & 0 & 4 & 6 & 6 & 15 & 19 & 15 & 16 & 17 & 17 & 25 & 27 \\
\hline & EBRT & 3 & 5 & 25 & 14 & 60 & 100 & 60 & 88 & 82 & 103 & 86 & 103 \\
\hline & WBLT & 5 & 20 & 17 & 23 & 16 & 12 & 9 & 20 & 25 & 18 & 10 & 14 \\
\hline & WBTH & 7 & 13 & 14 & 12 & 19 & 23 & 16 & 17 & 17 & 25 & 21 & 28 \\
\hline & WBRT & 8 & 24 & 30 & 27 & 26 & 21 & 9 & 25 & 17 & 35 & 33 & 32 \\
\hline & NBLT & 7 & 13 & 32 & 63 & 94 & 218 & 101 & 157 & 121 & 133 & 126 & 148 \\
\hline & NBTH & 741 & 968 & 792 & 865 & 1039 & 1247 & 626 & 1134 & 1161 & 1487 & 1337 & 1306 \\
\hline & NBRT & 6 & 1 & 8 & 4 & 5 & 7 & 3 & 8 & 1 & 12 & 6 & 5 \\
\hline & SBLT & 4 & 12 & 7 & 15 & 24 & 38 & 17 & 25 & 31 & 28 & 66 & 61 \\
\hline & SBTH & 970 & 937 & 973 & 857 & 1085 & 1265 & 646 & 1156 & 1471 & 1291 & 1342 & 1265 \\
\hline & SBRT & 1 & 8 & 3 & 22 & 56 & 93 & 49 & 94 & 89 & 63 & 76 & 89 \\
\hline
\end{tabular}


Table B- 8 Detailed Traffic Volume (vph - Table 3 of 3)

\begin{tabular}{|c|c|c|c|c|c|c|c|c|c|c|c|c|c|}
\hline INT & $\begin{array}{l}\text { BEG } \\
\text { END }\end{array}$ & $\begin{array}{l}\text { 7AM } \\
8 \mathrm{AM}\end{array}$ & $\begin{array}{l}8 \mathrm{AM} \\
9 \mathrm{AM}\end{array}$ & $\begin{array}{c}9 \mathrm{AM} \\
10 \mathrm{~A}\end{array}$ & $\begin{array}{l}10 \mathrm{~A} \\
11 \mathrm{~A}\end{array}$ & $\begin{array}{l}11 \mathrm{~A} \\
12 \mathrm{P}\end{array}$ & $\begin{array}{c}12 \mathrm{P} \\
1 \mathrm{PM}\end{array}$ & $\begin{array}{l}1 \mathrm{PM} \\
2 \mathrm{PM}\end{array}$ & $\begin{array}{l}2 \mathrm{PM} \\
3 \mathrm{PM}\end{array}$ & $\begin{array}{l}3 \mathrm{PM} \\
4 \mathrm{PM}\end{array}$ & $\begin{array}{l}4 \mathrm{PM} \\
5 \mathrm{PM}\end{array}$ & $\begin{array}{l}5 \mathrm{PM} \\
6 \mathrm{PM}\end{array}$ & $\begin{array}{l}6 \mathrm{PM} \\
7 \mathrm{PM}\end{array}$ \\
\hline \multirow{12}{*}{ 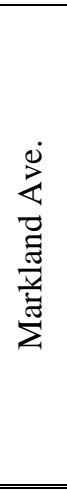 } & EBLT & 55 & 78 & 83 & 105 & 107 & 160 & 188 & 177 & 204 & 298 & 215 & 324 \\
\hline & EBTH & 103 & 164 & 214 & 294 & 390 & 488 & 435 & 440 & 514 & 620 & 567 & 602 \\
\hline & EBRT & 37 & 38 & 47 & 68 & 66 & 130 & 76 & 79 & 73 & 106 & 76 & 107 \\
\hline & WBLT & 88 & 102 & 133 & 154 & 238 & 240 & 241 & 245 & 286 & 376 & 253 & 270 \\
\hline & WBTH & 133 & 258 & 242 & 222 & 351 & 408 & 457 & 382 & 500 & 525 & 467 & 471 \\
\hline & WBRT & 104 & 133 & 135 & 121 & 149 & 215 & 200 & 164 & 220 & 278 & 227 & 246 \\
\hline & NBLT & 55 & 96 & 100 & 90 & 106 & 151 & 159 & 117 & 142 & 189 & 158 & 150 \\
\hline & NBTH & 645 & 689 & 614 & 614 & 651 & 734 & 749 & 688 & 948 & 1067 & 933 & 964 \\
\hline & NBRT & 70 & 116 & 103 & 201 & 171 & 299 & 213 & 259 & 219 & 282 & 256 & 301 \\
\hline & SBLT & 88 & 106 & 132 & 176 & 215 & 238 & 197 & 204 & 231 & 270 & 288 & 258 \\
\hline & SBTH & 726 & 712 & 741 & 598 & 640 & 755 & 644 & 664 & 939 & 986 & 798 & 868 \\
\hline & SBRT & 49 & 83 & 109 & 109 & 110 & 119 & 114 & 87 & 113 & 107 & 92 & 119 \\
\hline \multirow{11}{*}{ 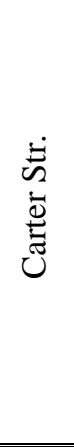 } & EBTH & 22 & 59 & 65 & 41 & 73 & 115 & 81 & 105 & 133 & 151 & 182 & 194 \\
\hline & EBRT & 26 & 38 & 30 & 45 & 50 & 67 & 98 & 58 & 65 & 57 & 71 & 87 \\
\hline & WBLT & 6 & 23 & 23 & 16 & 8 & 22 & 45 & 39 & 27 & 42 & 28 & 32 \\
\hline & WBTH & 24 & 55 & 70 & 45 & 58 & 79 & 125 & 115 & 123 & 111 & 105 & 97 \\
\hline & WBRT & 58 & 89 & 59 & 50 & 40 & 67 & 72 & 96 & 122 & 139 & 124 & 103 \\
\hline & NBLT & 18 & 35 & 27 & 49 & 44 & 100 & 77 & 63 & 72 & 94 & 65 & 56 \\
\hline & NBTH & 731 & 923 & 732 & 730 & 768 & 976 & 994 & 1003 & 1203 & 1428 & 1265 & 1236 \\
\hline & NBRT & 5 & 10 & 13 & 4 & 10 & 25 & 27 & 28 & 15 & 16 & 19 & 28 \\
\hline & SBLT & 52 & 61 & 78 & 33 & 54 & 115 & 79 & 94 & 102 & 132 & 141 & 125 \\
\hline & SBTH & 924 & 1035 & 999 & 851 & 853 & 1092 & 1058 & 1075 & 1141 & 1223 & 1035 & 1150 \\
\hline & SBRT & 15 & 24 & 31 & 23 & 48 & 52 & 31 & 38 & 39 & 45 & 37 & 55 \\
\hline \multirow{11}{*}{ 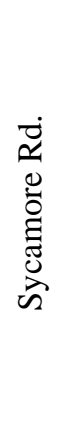 } & EBTH & 27 & 48 & 41 & 36 & 35 & 57 & 61 & 62 & 51 & 115 & 116 & 106 \\
\hline & EBRT & 56 & 51 & 62 & 89 & 83 & 114 & 94 & 102 & 85 & 173 & 124 & 128 \\
\hline & WBLT & 33 & 56 & 105 & 67 & 67 & 73 & 86 & 76 & 59 & 80 & 97 & 106 \\
\hline & WBTH & 53 & 92 & 101 & 54 & 60 & 74 & 65 & 55 & 73 & 102 & 93 & 98 \\
\hline & WBRT & 25 & 26 & 15 & 21 & 18 & 10 & 33 & 46 & 14 & 15 & 20 & 21 \\
\hline & NBLT & 55 & 108 & 120 & 98 & 129 & 148 & 169 & 158 & 205 & 188 & 186 & 133 \\
\hline & NBTH & 946 & 853 & 688 & 622 & 762 & 941 & 922 & 980 & 1430 & 1253 & 1370 & 1174 \\
\hline & NBRT & 46 & 56 & 61 & 36 & 48 & 64 & 55 & 72 & 52 & 74 & 73 & 69 \\
\hline & SBLT & 3 & 15 & 8 & 8 & 10 & 15 & 14 & 10 & 25 & 28 & 25 & 12 \\
\hline & SBTH & 1030 & 927 & 829 & 825 & 847 & 960 & 838 & 921 & 1153 & 1089 & 1113 & 1105 \\
\hline & $\begin{array}{l}\text { SBRT } \\
\end{array}$ & 48 & 58 & 44 & 53 & 55 & 70 & 79 & 65 & 64 & 64 & 69 & 65 \\
\hline
\end{tabular}


APPENDIX C- SAS CODE AND RESULTS 
data offsets;

INFILE 'offsets.inp';

input length 1-6 offset 7-12 cycle 13-18 split 19-24 turn 25-30 vol 31-36 det_loc 37-42 cnt_skw 43-50 occ_skw 51-57;

diff_skw=ocC_skw-cnt_skw;

IF offset=5 THEN offset $2=3$;

ELSE IF offset $=6$ THEN offset $2=2$;

ELSE offset $2=$ offset;

run;

data repli;

INFILE 'rep.inp';

input rep 1-1;

data all;

merge offsets repli;

PROC GLM DATA=all:

class length cycle split vol turn rep;

model diff $\mathrm{skw}=$

length Cycle split(cycle) vol(split) turn

length*cycle length*split(cycle) length*vol(split) length*turn

cycle*vol (split) cycle*turn turn*split(cycle) turn*vol (split)

rep(length cycle split vol turn)

offset 2

offset $2 *$ length offset $2{ }^{*}$ Cycle offset $2 * \operatorname{split}($ cycle) offset $2 *$ vol (split) offset $2 * t u r n$

offset ${ }^{*}$ rep (length cycle split vol turn)

det_loc

det_loc*length det_loc*Cycle det_loc*split(cycle) det_loc*vol(split) det_loc*turn

det_loc*rep(length cycle split vol turn)

offset $2 *$ det_loc 
offset $2 *$ det_loc*rep (length cycle split vol turn);

RANDOM rep(length cycle split vol turn) offset2*rep(length cycle split vol turn) det_loc*rep(length cycle split vol turn) offset2*det_loc*rep(length cycle split vol turn)

/test;

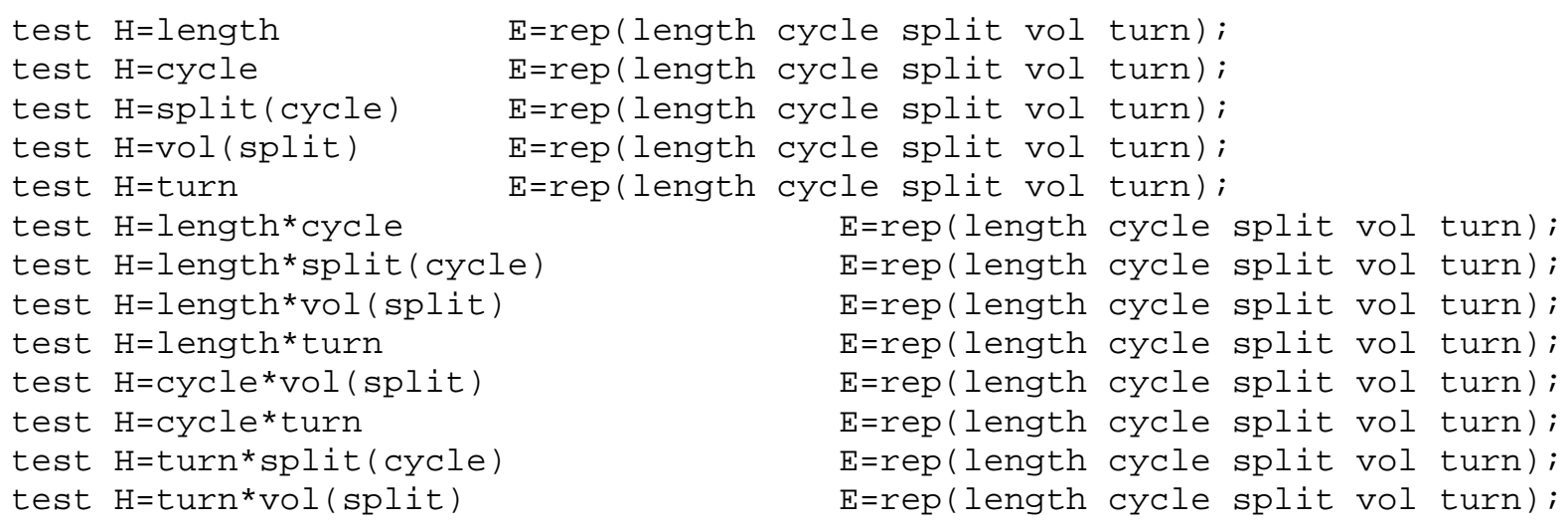

test $\mathrm{H}=$ offset 2

$\mathrm{E}=$ offset 2 *ep (length cycle split vol turn); test $\mathrm{H}=$ offset 2 *length

$\mathrm{E}=$ offset ${ }^{*}$ rep (length cycle split vol turn); test $\mathrm{H}=$ offset $2{ }^{*} \mathrm{Cycle}$

$\mathrm{E}=$ offset 2 *ep (length cycle split vol turn); test $\mathrm{H}=$ offset $2 * \operatorname{split}$ (cycle)

$\mathrm{E}=$ offset 2 * rep (length cycle split vol turn); test $\mathrm{H}=\mathrm{offset} 2^{*} \mathrm{Vol}$ (split)

$\mathrm{E}=$ offset ${ }^{*}$ rep (length cycle split vol turn); test $\mathrm{H}=\mathrm{offset} 2 *$ turn

$\mathrm{E}=$ offset 2 *ep (length cycle split vol turn);

test $\mathrm{H}=\operatorname{det} \mathrm{loc}$

$\mathrm{E}=$ det_loc*rep (length cycle split vol turn); test $\mathrm{H}=$ det_loc*length

$\mathrm{E}=$ det_loc*rep (length cycle split vol turn); 
test $\mathrm{H}=$ det_loc*Cycle

$\mathrm{E}=$ det_loc*rep (length cycle split vol turn)

test $\mathrm{H}=$ det_loc*split (cycle)

$\mathrm{E}=$ det_loc*rep (length cycle split vol turn);

test H=det_loc*vol (split)

$\mathrm{E}=$ det_loc*rep (length cycle split vol turn);

test $\mathrm{H}=$ det loc*turn

$\mathrm{E}=$ det_loc*rep (length cycle split vol turn);

test $\mathrm{H}=\mathrm{offset} 2 *$ det_loc

$\mathrm{E}=$ offset ${ }^{*}$ det_loc*rep (length cycle split vol turn);

output out=resplot p=pred_off r=resid;

RUN;

PROC plot data=resplot;

plot pred_off*resid;

run; 


\section{F' Model GLM}

15:43 Sunday, March 18, 20011

Class
length
cycle
split
vol
turn
offset 2
rep
len_vol
cyc_vol
spl_turn
vol_turn
off_len
off_cyc

The SAS System

\section{The GLM Procedure}

Class Level Information

Levels Values

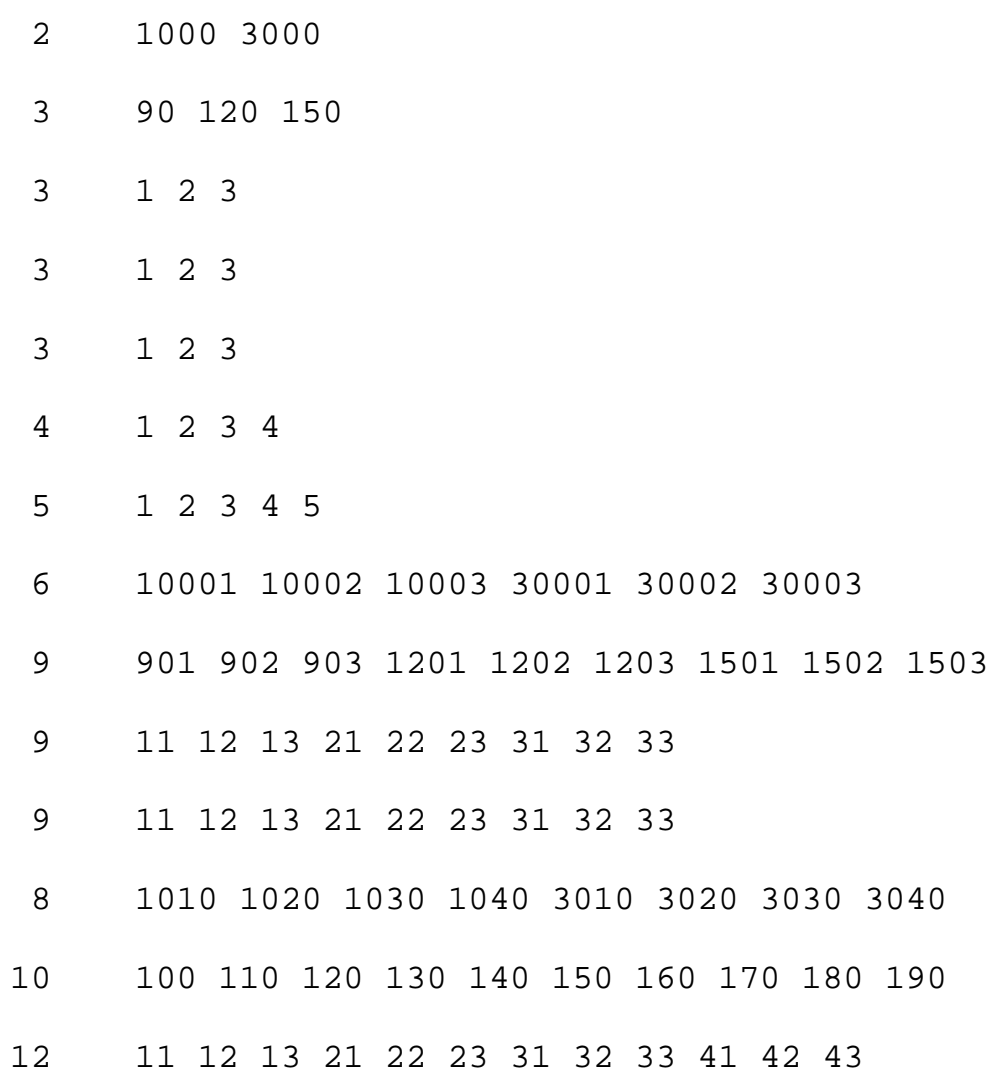




off_vol
off_turn
det_len
det_vol
det_turn

$\overline{1} 5: 43$ Sunday, March 18, 2001

2

Dependent Variable: fvalue

\section{$>\mathrm{F}$}

$<.0001$

Source $\begin{array}{lllllllllllll}12 & 11 & 12 & 13 & 21 & 22 & 23 & 31 & 32 & 33 & 41 & 42 & 43\end{array}$

$\begin{array}{lllllllllllll}12 & 11 & 12 & 13 & 21 & 22 & 23 & 31 & 32 & 33 & 41 & 42 & 43\end{array}$

$10 \quad 1010 \quad 10201030 \quad 1040 \quad 1050 \quad 3010 \quad 3020 \quad 3030 \quad 3040 \quad 3050$

$\begin{array}{llllllllllllllll}15 & 11 & 12 & 13 & 21 & 22 & 23 & 31 & 32 & 33 & 41 & 42 & 43 & 51 & 52 & 53\end{array}$

15
Number of observations 24300
The SAS System

The GLM Procedure

$\begin{array}{rrrrr}\text { DF } & \begin{array}{r}\text { Sum of } \\ \text { Squares }\end{array} & \text { Mean Square } & \text { F Value } & \text { Pr } \\ 3239 & 2156.780494 & 0.665879 & 4.00 \\ 21060 & 3503.649887 & 0.166365 & \\ 24299 & 5660.430381 & & \end{array}$

$\begin{array}{lrrr}\text { R-Square } & \text { Coeff Var } & \text { Root MSE } & \text { fvalue Mean } \\ 0.381028 & 97.28191 & 0.407879 & 0.419275\end{array}$

$\mathrm{DF}$

Type I SS

Mean Square

F Value

$\operatorname{Pr}$ 


$$
\begin{aligned}
& <.0001 \\
& <.0001
\end{aligned}
$$

$>\mathrm{F}$

$<.0001$

$\overline{1} 5: 43$ Sunday, March 18, 20013

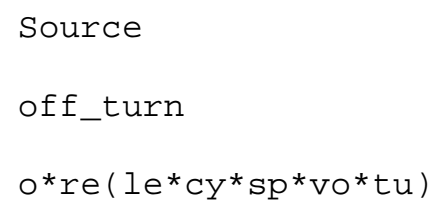

$\overline{15}: 43$ Sunday, March 18, 2001

\begin{abstract}
off_turn
$o * r e\left(1 e^{*} \mathrm{cy}{ }^{\star} \mathrm{sp} \mathrm{*}^{*} \mathrm{v}\right.$ *tu $)$
\end{abstract}

Source

off_turn

o*re $\left(1 e^{\star} \mathrm{Cy}{ }^{\star} \mathrm{sp} \mathrm{N}^{\star} \mathrm{v}\right.$ *tu $)$

$\begin{array}{rrrr}11 & 483.287859 & 43.935260 & 264.09 \\ 3228 & 1673.492635 & 0.518430 & 3.12\end{array}$

$\mathrm{DF}$

Type III SS

Mean Square

F Value

Pr

$\begin{array}{rrcc}0 & 0.000000 & . & . \\ 3228 & 1673.492635 & 0.518430 & 3.12\end{array}$

The SAS system

The GLM Procedure

Type III Expected Mean Square

0

$\operatorname{Var}($ Error $)+7.5 \operatorname{Var}\left(o^{*} r e\left(1 e^{*} \mathrm{cy}{ }^{\star} \mathrm{sp}{ }^{\star} \operatorname{Vo*tu}\right)\right)$

The SAS system

The GLM Procedure

Student-Newman-Keuls Test for fvalue

NOTE: This test controls the Type I experimentwise error rate under the complete null hypothesis but not under partial null

hypotheses.

$\begin{array}{lr}\text { Alpha } & 0.05 \\ \text { Error Degrees of Freedom } & 3228 \\ \text { Error Mean Square } & 0.51843\end{array}$


NOTE: Cell sizes are not equal.

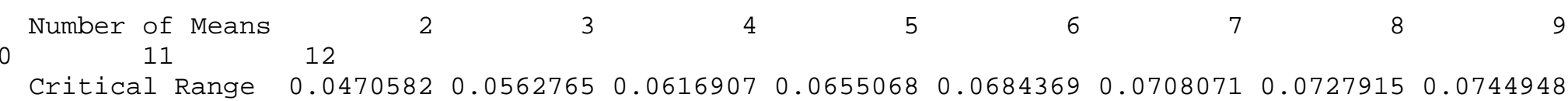
0.07598420 .07730590 .0784925

Means with the same letter are not significantly different.

\begin{tabular}{|c|c|c|c|c|}
\hline SNK Grou & $\mathrm{ng}$ & Mean & $\mathrm{N}$ & off_turn \\
\hline & A & 0.62917 & 1350 & 41 \\
\hline & A & & & \\
\hline & A & 0.61900 & 1350 & 42 \\
\hline & A & & & \\
\hline B & A & 0.57820 & 1350 & 43 \\
\hline B & & & & \\
\hline B & $\mathrm{C}$ & 0.53232 & 2700 & 31 \\
\hline B & $\mathrm{C}$ & & & \\
\hline B & $\mathrm{C}$ & 0.52248 & 2700 & 32 \\
\hline & $\mathrm{C}$ & & & \\
\hline & $\mathrm{C}$ & 0.50607 & 2700 & 33 \\
\hline & $\mathrm{D}$ & 0.36017 & 2700 & 23 \\
\hline & $\mathrm{D}$ & & & \\
\hline $\mathrm{E}$ & $\mathrm{D}$ & 0.31353 & 1350 & 13 \\
\hline $\mathrm{E}$ & $\mathrm{D}$ & & & \\
\hline $\mathrm{E}$ & $\mathrm{D}$ & 0.31097 & 2700 & 22 \\
\hline $\mathrm{E}$ & & & & \\
\hline $\mathrm{E}$ & & 0.28576 & 2700 & 21 \\
\hline & F & 0.20180 & 1350 & 12 \\
\hline & $\mathrm{F}$ & & & \\
\hline
\end{tabular}


The GLM Procedure

\section{Class Level Information}

\begin{tabular}{|c|c|c|}
\hline Class & Levels & Values \\
\hline length & 2 & 10003000 \\
\hline cycle & 3 & 90120150 \\
\hline split & 3 & 123 \\
\hline vol & 3 & 123 \\
\hline turn & 3 & 123 \\
\hline rep & 5 & 12345 \\
\hline len_vol & 6 & 100011000210003300013000230003 \\
\hline cyc_vol & 9 & $901902903 \quad 1201 \quad 1202 \quad 1203 \quad 1501 \quad 1502 \quad 1503$ \\
\hline spl_turn & 9 & $\begin{array}{lllllllll}11 & 12 & 13 & 21 & 22 & 23 & 31 & 32 & 33\end{array}$ \\
\hline vol_turn & 9 & $\begin{array}{lllllllll}11 & 12 & 13 & 21 & 22 & 23 & 31 & 32 & 33\end{array}$ \\
\hline off_len & 8 & 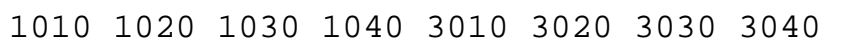 \\
\hline off_cyc & 10 & $\begin{array}{llllllllll}100 & 110 & 120 & 130 & 140 & 150 & 160 & 170 & 180 & 190\end{array}$ \\
\hline off_spl & 12 & $\begin{array}{llllllllllll}11 & 12 & 13 & 21 & 22 & 23 & 31 & 32 & 33 & 41 & 42 & 43\end{array}$ \\
\hline off_vol & 12 & $\begin{array}{llllllllllll}11 & 12 & 13 & 21 & 22 & 23 & 31 & 32 & 33 & 41 & 42 & 43\end{array}$ \\
\hline
\end{tabular}




$\begin{array}{lcccccccccccccccccc}\text { off_turn } & 12 & 11 & 12 & 13 & 21 & 22 & 23 & 31 & 32 & 33 & 41 & 42 & 43 & & & \\ \text { det_len } & 10 & 1010 & 1020 & 1030 & 1040 & 1050 & 3010 & 3020 & 3030 & 3040 & 3050 \\ \text { det_vol } & 15 & 11 & 12 & 13 & 21 & 22 & 23 & 31 & 32 & 33 & 41 & 42 & 43 & 51 & 52 & 53 & \\ \text { det_turn } & 15 & 11 & 12 & 13 & 21 & 22 & 23 & 31 & 32 & 33 & 41 & 42 & 43 & 51 & 52 & 53\end{array}$

Saturday, March 17, 20012

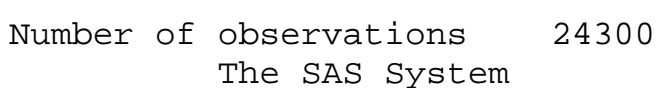

The GLM Procedure

Dependent Variable: fvalue

$\begin{array}{ll}>\mathrm{F} & \text { Source } \\ <.0001 & \text { Model }\end{array}$

Error

Corrected Total

Source

$>F$

$$
\begin{gathered}
\text { R-Square } \\
0.195018
\end{gathered}
$$

\begin{tabular}{|c|c|c|c|c|c|c|c|c|}
\hline \multicolumn{9}{|c|}{ Sum of } \\
\hline & 809 & 110 & 260 & & .364508 & & 7.03 & \\
\hline & 23490 & 455 & 121 & & .193978 & & & \\
\hline & 24299 & 566 & 381 & & & & & \\
\hline R-Square & Coeff & Var & Root & MSE & fvalue & Mean & & \\
\hline 0.195018 & 105.0 & 0454 & 0.44 & 0429 & 0.41 & 19275 & & \\
\hline
\end{tabular}

$\mathrm{DF}$

Type I SS

Mean Square

F Value

Pr 


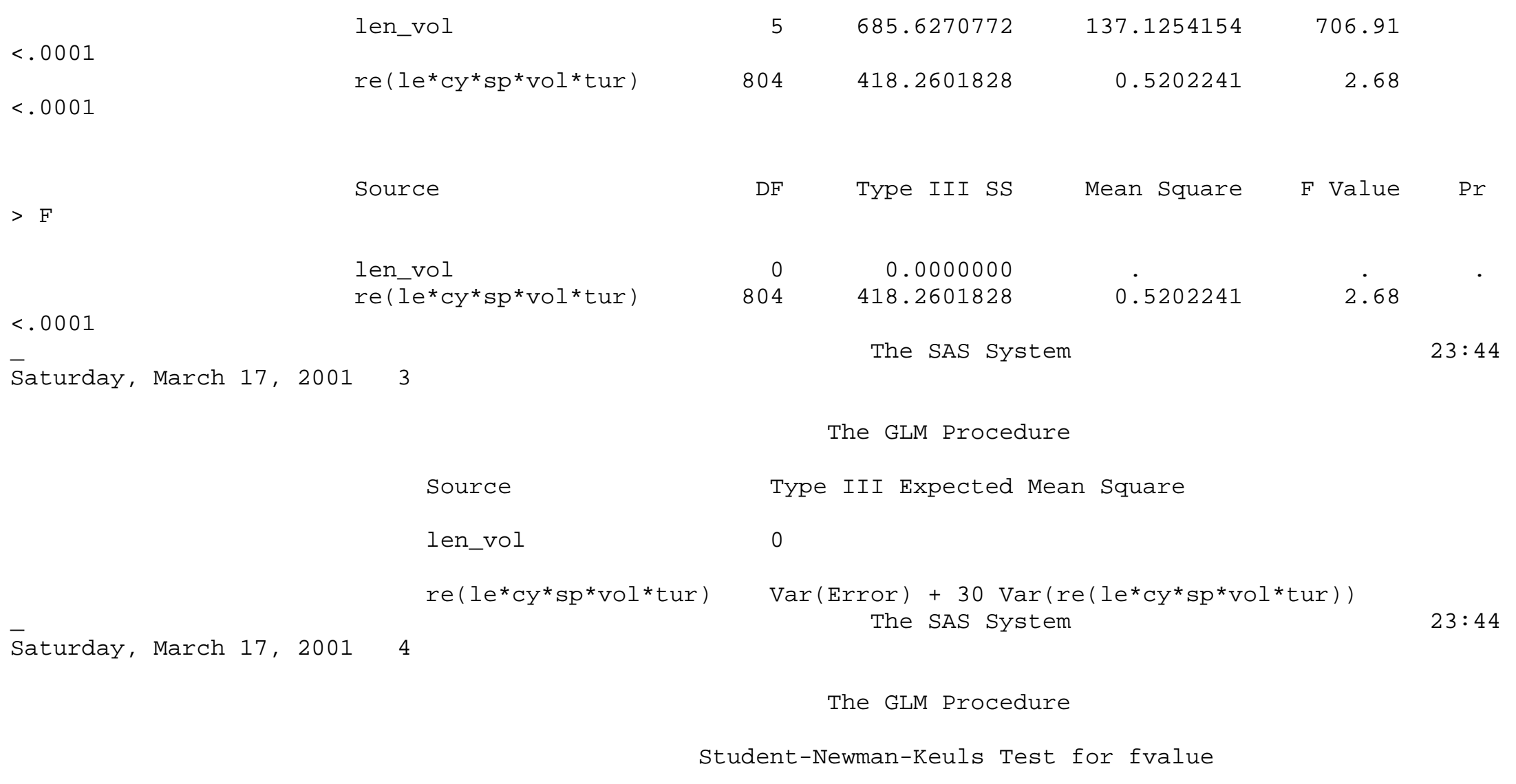

NOTE: This test controls the Type I experimentwise error rate under the complete null hypothesis but not under partial null

hypotheses.

$\begin{array}{lr}\text { Alpha } & 0.05 \\ \text { Error Degrees of Freedom } & 804 \\ \text { Error Mean Square } & 0.520224\end{array}$




\section{6}

0.0457862

Saturday, March 17, 2001
Number of Means

Critical Range
0.0314619

0.037635

0.0412639

4

Means with the same letter are not significantly different.

$\begin{array}{rcrc}\text { SNK Grouping } & \text { Mean } & \text { N } & \text { len_vol } \\ \text { A } & 0.60692 & 4050 & 30001 \\ \text { A } & 0.60310 & 4050 & 10001 \\ \text { A } & 0.46659 & 4050 & 30002 \\ \text { B } & 0.44198 & 4050 & 10002 \\ \text { B } & 0.20465 & 4050 & 30003 \\ \text { B } & 0.19241 \quad 4050 & 10003 \\ \text { C } & \text { The SAS System } \\ \text { C } & 0 & \end{array}$

The GLM Procedure

Class Level Information

$\begin{array}{lcl}\text { Class } & \text { Levels } & \text { Values } \\ \text { length } & 2 & 10003000 \\ \text { cycle } & 3 & 90120150 \\ \text { split } & 3 & 123\end{array}$




vol
turn
rep
len_vol
cyc_vol
spl_turn
vol_turn
off_len
off_cyc
off_spl
off_vol
off_turn
det_len
det_vol
det_turn

Saturday, March 17, 2001

6

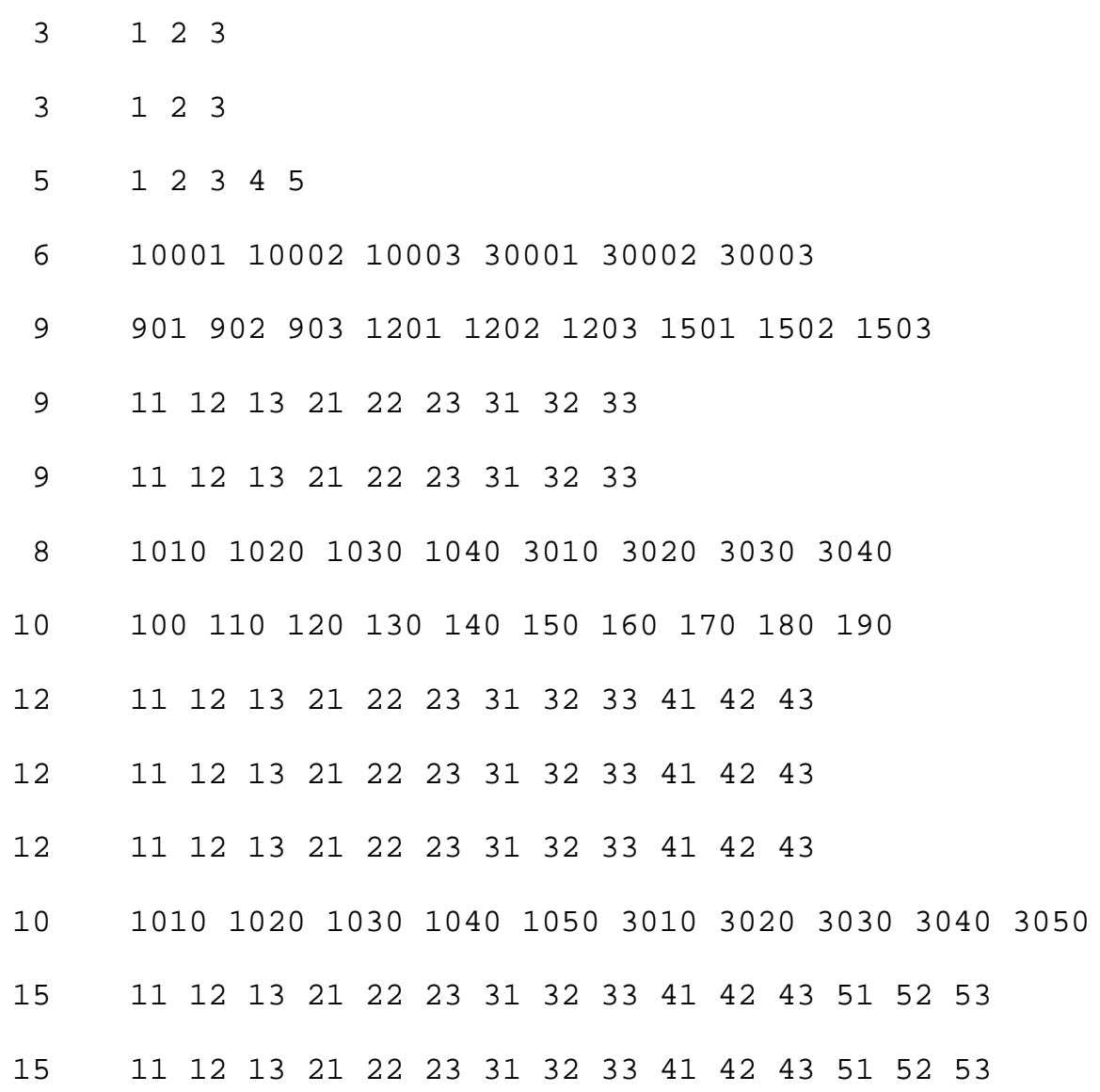

Dependent Variable: fvalue 


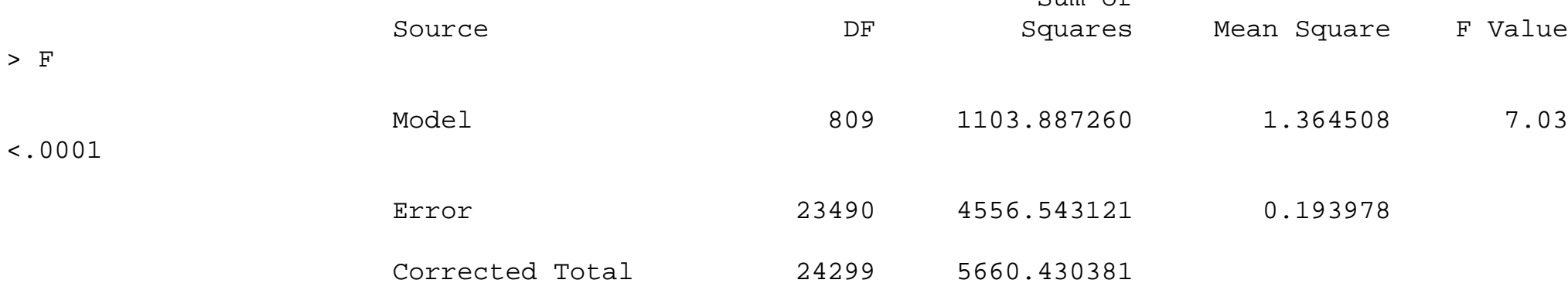

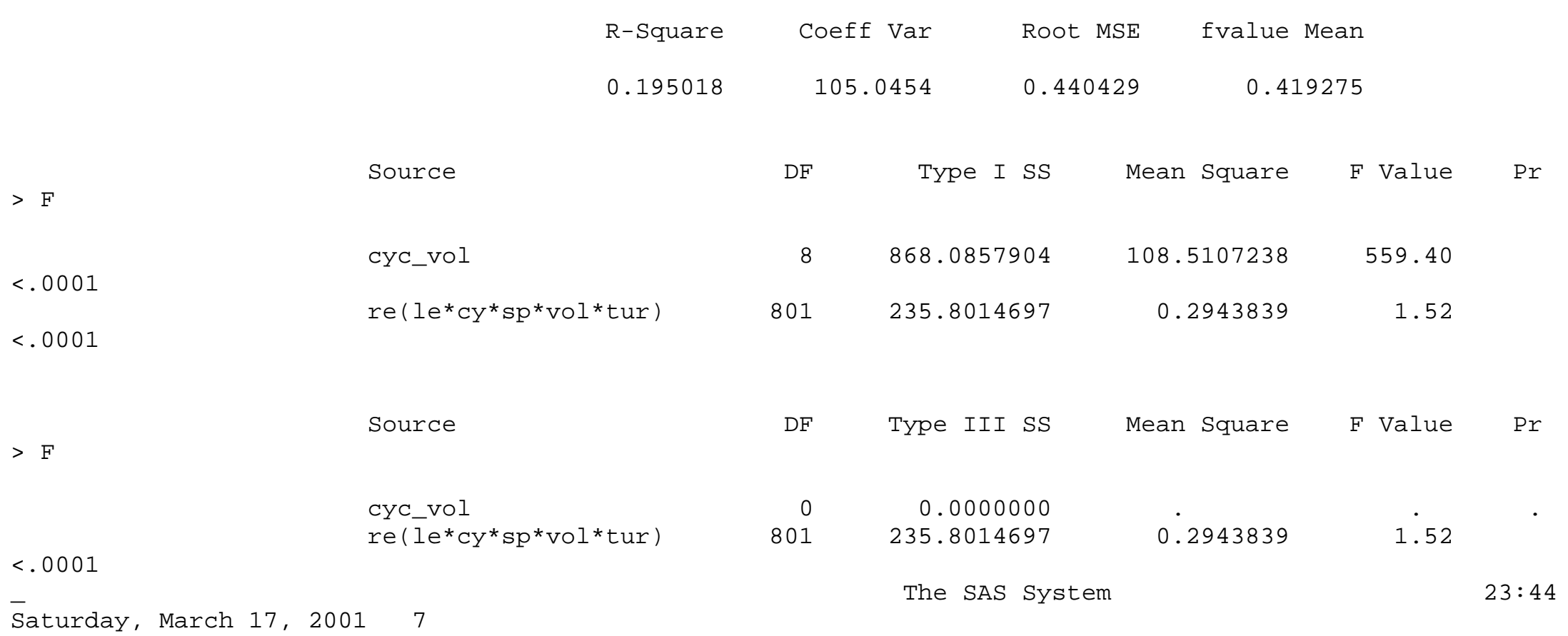

The GLM Procedure 
Source

cyc_vol

$r e\left(1 e^{*} \mathrm{Cy}^{\star} \mathrm{sp}^{\star} \operatorname{vol}{ }^{*} \operatorname{tur}\right)$

Saturday, March 17, 2001

8

Type III Expected Mean Square

0

$\operatorname{Var}(E r r o r)+30 \operatorname{Var}\left(r e\left(1 e^{*} \mathrm{Cy}^{*} \mathrm{sp}{ }^{*} \operatorname{Vol}{ }^{*} \operatorname{tur}\right)\right)$

The SAS system

$23: 44$

The GLM Procedure

Student-Newman-Keuls Test for fvalue

NOTE: This test controls the Type I experimentwise error rate under the complete null hypothesis but not under partial null

\section{hypotheses.}

$\begin{array}{lr}\text { Alpha } & 0.05 \\ \text { Error Degrees of Freedom } & 801 \\ \text { Error Mean Square } & 0.294384\end{array}$

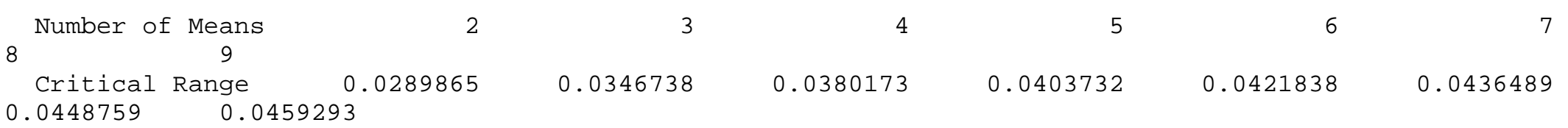

Means with the same letter are not significantly different.

$\begin{array}{rrrr}\text { SNK Grouping } & \text { Mean } & \text { N } & \text { cyc_vol } \\ \text { A } & 0.69470 & 2700 & 1501 \\ \text { B } & 0.62149 & 2700 & 1201 \\ \text { B } & & & \\ \text { B } & 0.60733 & 2700 & 1502\end{array}$




\begin{tabular}{llll} 
C & 0.49885 & 2700 & 901 \\
D & 0.45603 & 2700 & 1202 \\
E & 0.29950 & 2700 & 902 \\
F & 0.22581 & 2700 & 1503 \\
G & 0.19080 & 2700 & 1203 \\
G & 0.17897 & 2700 & 903 \\
G & \multicolumn{2}{c}{ The SAS System } \\
& \multicolumn{3}{c}{ S S }
\end{tabular}

The GLM Procedure

\section{Class Level Information}

Class
length
cycle
split
vol
turn
rep
len_vol
cyc_vol
spl_turn

Levels Values

$21000 \quad 3000$

$390 \quad 120 \quad 150$

$\begin{array}{llll}3 & 1 & 2 & 3\end{array}$

$3 \quad 123$

$\begin{array}{llll}3 & 1 & 2 & 3\end{array}$

$\begin{array}{llllll}5 & 1 & 2 & 3 & 4 & 5\end{array}$

$6 \quad 100011000210003300013000230003$

$999019029031201 \quad 1202 \quad 12031501 \quad 1502 \quad 1503$

$\begin{array}{llllllllll}9 & 11 & 12 & 13 & 21 & 22 & 23 & 31 & 32 & 33\end{array}$ 


$$
\begin{aligned}
& \text { vol_turn } \\
& \text { off_len } \\
& \text { off_cyc } \\
& \text { off_spl } \\
& \text { off_vol } \\
& \text { off_turn } \\
& \text { det_len } \\
& \text { det_vol } \\
& \text { det_turn }
\end{aligned}
$$

Saturday, March 17, 2001

10

Dependent Variable: fvalue

$\begin{array}{ll}>\mathrm{F} & \text { Source } \\ <.0001 & \text { Model }\end{array}$

Error

Corrected Total $\begin{array}{llllllllll}9 & 11 & 12 & 13 & 21 & 22 & 23 & 31 & 32 & 33\end{array}$

$8 \quad 1010 \quad 10201030 \quad 1040 \quad 3010 \quad 3020 \quad 3030 \quad 3040$

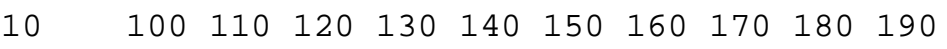

\begin{tabular}{|c|c|c|c|c|}
\hline $\mathrm{DF}$ & $\begin{array}{r}\text { Sum of } \\
\text { Squares }\end{array}$ & Mean Square & $\mathrm{F}$ & Value \\
\hline 9 & 1103.887260 & 1.364508 & & 7.03 \\
\hline & 4556.543121 & 0.193978 & & \\
\hline & 5660.430381 & & & \\
\hline
\end{tabular}

$\begin{array}{lllllllllllll}12 & 11 & 12 & 13 & 21 & 22 & 23 & 31 & 32 & 33 & 41 & 42 & 43\end{array}$

$\begin{array}{lllllllllllll}12 & 11 & 12 & 13 & 21 & 22 & 23 & 31 & 32 & 33 & 41 & 42 & 43\end{array}$

$\begin{array}{lllllllllllll}12 & 11 & 12 & 13 & 21 & 22 & 23 & 31 & 32 & 33 & 41 & 42 & 43\end{array}$

$10101010201030 \quad 1040 \quad 1050 \quad 3010 \quad 3020 \quad 3030 \quad 3040 \quad 3050$

$\begin{array}{llllllllllllllll}15 & 11 & 12 & 13 & 21 & 22 & 23 & 31 & 32 & 33 & 41 & 42 & 43 & 51 & 52 & 53\end{array}$

$\begin{array}{llllllllllllllll}15 & 11 & 12 & 13 & 21 & 22 & 23 & 31 & 32 & 33 & 41 & 42 & 43 & 51 & 52 & 53\end{array}$

The GLM Procedure 


$\begin{array}{rrrr}\text { R-Square } & \text { Coeff Var } & \text { Root MSE fvalue Mean } \\ 0.195018 & 105.0454 & 0.440429 & 0.419275\end{array}$

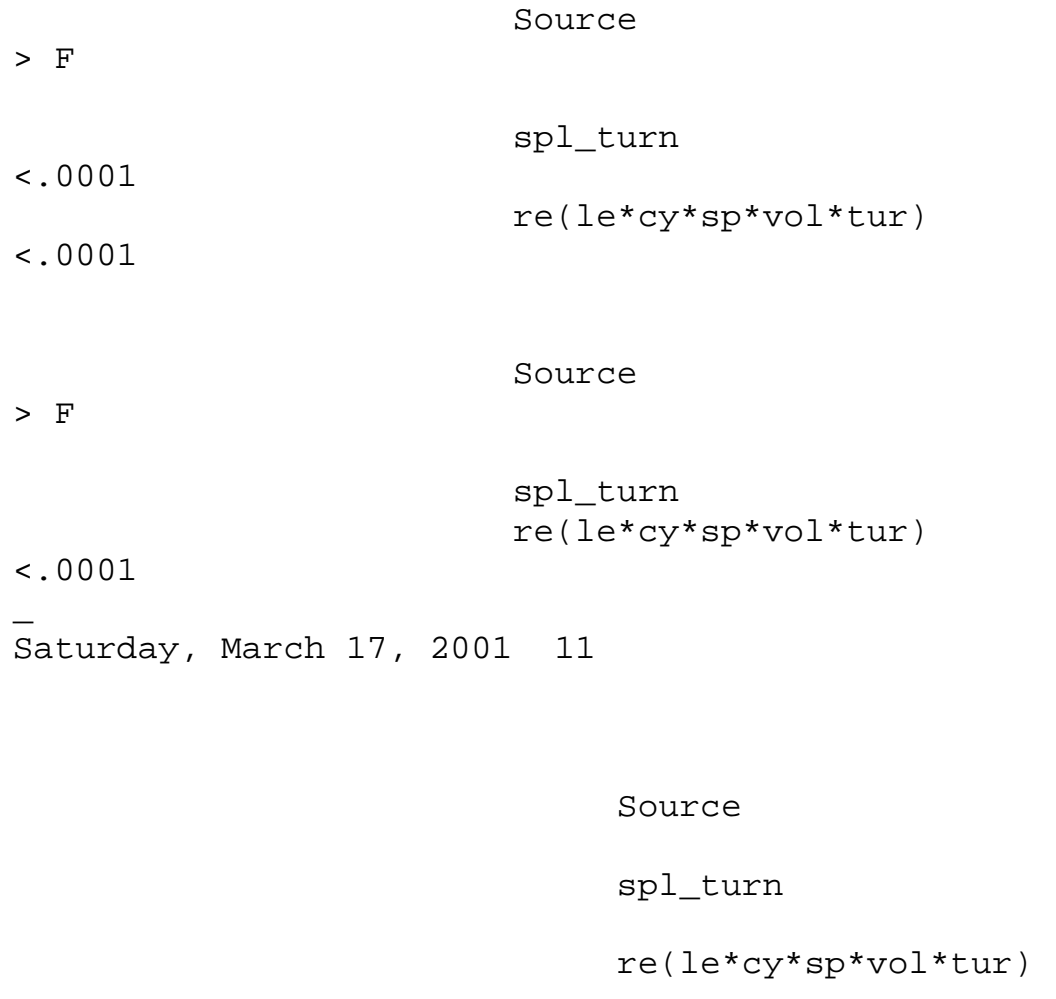

Saturday, March 17, 200112

$\begin{array}{rrrrr}\text { DF } & \text { Type I SS } & \text { Mean Square } & \text { F Value } & \text { Pr } \\ 8 & 61.487298 & 7.685912 & 39.62 & \\ 801 & 1042.399962 & 1.301373 & 6.71 & \\ & & & & \\ \text { DF } & \text { Type III SS } & \text { Mean Square } & \text { F Value } & \text { Pr } \\ & & & & \\ 801 & \begin{aligned} 0.000000 \\ \text { 1042.399962 }\end{aligned} & 1.301373 & 6.71 & \\ & \text { The SAS System } & & & 23: 44\end{array}$

The GLM Procedure

Type III Expected Mean Square

0

$\operatorname{Var}($ Error $)+30 \operatorname{Var}\left(r e\left(1 e^{*} \mathrm{Cy}{ }^{\star} \mathrm{sp}{ }^{\star} \operatorname{Vol}{ }^{*} \operatorname{tur}\right)\right)$ The SAS system

The GLM Procedure

Student-Newman-Keuls Test for fvalue 
NOTE: This test controls the Type I experimentwise error rate under the complete null hypothesis but not under partial null

hypotheses.

\begin{tabular}{|c|c|c|c|c|c|c|}
\hline & & & $\begin{array}{l}\text { Alpha } \\
\text { Error Degr } \\
\text { Error Mean }\end{array}$ & $\begin{array}{l}\text { ees of Freedom } \\
\text { Square }\end{array}$ & $\begin{array}{r}0.05 \\
801 \\
1373\end{array}$ & \\
\hline $8 \begin{array}{c}\text { Number of } \begin{array}{c}\text { Means } \\
9\end{array} \\
9\end{array}$ & 2 & 3 & 4 & 5 & 6 & 7 \\
\hline $\begin{array}{rr}\text { Critical Range } \\
0.0943533 & 0 .\end{array}$ & $\begin{array}{l}0.0609451 \\
58\end{array}$ & 0.0729031 & 0.0799327 & 0.0848862 & 0.0886931 & 0.0917735 \\
\hline
\end{tabular}

Means with the same letter are not significantly different.

$\begin{array}{rrrr}\text { SNK Grouping } & \text { Mean } & \text { N } & \text { spl_turn } \\ \text { A } & 0.48735 & 2700 & 13 \\ \text { A } & & & \\ \text { A } & 0.46341 & 2700 & 23 \\ \text { A } & & & \\ \text { A } & 0.45426 & 2700 & 12 \\ \text { A } & & & \\ \text { A } & 0.44226 & 2700 & 11 \\ \text { A } & 0.44161 & 2700 & 22 \\ \text { A } & 0.42945 & 2700 & 21 \\ \text { A } & 0.36135 & 2700 & 33 \\ \text { A } & 0.34798 & 2700 & 32 \\ \text { B } & 0 & & \\ \text { B } & & \end{array}$


The GLM Procedure

Class Level Information

Class
length
cycle
split
vol
turn
rep
len_vol
cyc_vol
spl_turn
vol_turn
off_len
off_cyc
off_spl
off_vol

Levels Values

210003000

390120150

$\begin{array}{llll}3 & 1 & 2 & 3\end{array}$

$\begin{array}{llll}3 & 1 & 2 & 3\end{array}$

$3 \quad 123$

$\begin{array}{llllll}5 & 1 & 2 & 3 & 4 & 5\end{array}$

6100011000210003300013000230003

$9990190290312011202 \quad 12031501 \quad 1502 \quad 1503$

$\begin{array}{llllllllll}9 & 11 & 12 & 13 & 21 & 22 & 23 & 31 & 32 & 33\end{array}$

$\begin{array}{lllllllllll}9 & 11 & 12 & 13 & 21 & 22 & 23 & 31 & 32 & 33\end{array}$

$8 \quad 1010102010301040 \quad 3010 \quad 302030303040$

$10100110120130140 \quad 150 \quad 160 \quad 170 \quad 180190$

$\begin{array}{lllllllllllll}12 & 11 & 12 & 13 & 21 & 22 & 23 & 31 & 32 & 33 & 41 & 42 & 43\end{array}$

$\begin{array}{lllllllllllll}12 & 11 & 12 & 13 & 21 & 22 & 23 & 31 & 32 & 33 & 41 & 42 & 43\end{array}$ 


$$
\begin{aligned}
& \text { off_turn } \\
& \text { det_len } \\
& \text { det_vol } \\
& \text { det_turn }
\end{aligned}
$$

Saturday, March 17, 2001 14

14

Dependent Variable: fvalue

$\begin{array}{ll}>\mathrm{F} & \text { Source } \\ <.0001 & \text { Model } \\ & \text { Error } \\ & \text { Corrected Total }\end{array}$

Corrected Total

Source $\begin{array}{lllllllllllll}12 & 11 & 12 & 13 & 21 & 22 & 23 & 31 & 32 & 33 & 41 & 42 & 43\end{array}$

$10 \quad 1010 \quad 1020 \quad 1030 \quad 1040 \quad 1050 \quad 3010 \quad 3020 \quad 3030 \quad 3040 \quad 3050$

$\begin{array}{llllllllllllllll}15 & 11 & 12 & 13 & 21 & 22 & 23 & 31 & 32 & 33 & 41 & 42 & 43 & 51 & 52 & 53\end{array}$

15

Number of observations

24300

The SAS system

$23: 44$

The GLM Procedure

Sum of

$\mathrm{DF}$

Squares

Mean Square

F Value

Pr

809

1103.887260

1.364508

7.03

23490

4556.543121

0.193978

24299

5660.430381

$\begin{array}{lrrr}\text { R-Square } & \text { Coeff Var } & \text { Root MSE } & \text { fvalue Mean } \\ 0.195018 & 105.0454 & 0.440429 & 0.419275\end{array}$

DF

Type I SS

Mean Square

F Value

Pr 


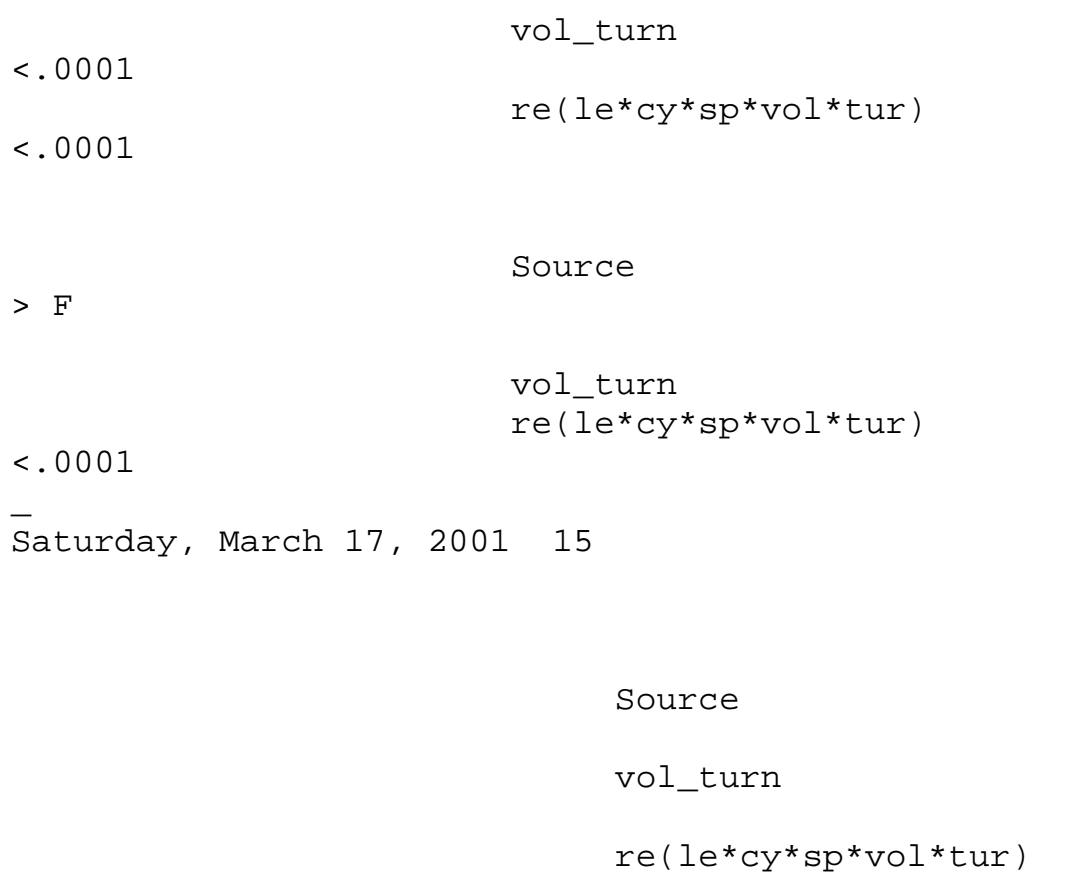

Saturday, March 17, 200116

$\begin{array}{rrrrr}8 & 712.6528282 & 89.0816035 & 459.24 & \\ 801 & 391.2344318 & 0.4884325 & 2.52 & \\ & & & & \\ \text { DF } & \text { Type III SS } & \text { Mean Square } & \text { F Value } & \text { Pr } \\ & & & & \\ 801 & 0.0000000 & . & . & \\ & 391.2344318 & 0.4884325 & 2.52 & \\ & \text { The SAS System } & & & 23: 44\end{array}$

The GLM Procedure

Type III Expected Mean Square

0

$\operatorname{Var}(E r r o r)+30 \operatorname{Var}\left(r e\left(1 e^{*} \mathrm{Cy}^{*} \mathrm{sp}^{\star} \operatorname{Vol}{ }^{*} \mathrm{tur}\right)\right)$

The SAS system

$23: 44$

The GLM Procedure

Student-Newman-Keuls Test for fvalue

NOTE: This test controls the Type I experimentwise error rate under the complete null hypothesis but not under partial null

hypotheses.

$\begin{array}{lr}\text { Alpha } & 0.05 \\ \text { Error Degrees of Freedom } & 801 \\ \text { Error Mean Square } & 0.488432\end{array}$


Number of Means

8

Critical Range 0.0373371

$0.0578041 \quad 0.0591609$

Saturday, March 17, 200117
0.0446629

0.0489695

0.0520042

0.0543365

0.0562236

Means with the same letter are not significantly different.

$\begin{array}{cccc}\text { SNK Grouping } & \text { Mean } & \text { N } & \text { vol_turn } \\ \text { A } & 0.68195 & 2700 & 13 \\ \text { B } & 0.59077 & 2700 & 12 \\ \text { C } & 0.54232 & 2700 & 11 \\ \text { D } & 0.46244 & 2700 & 21 \\ \text { D } & 0.45541 & 2700 & 22 \\ \text { D } & 0.44500 & 2700 & 23 \\ \text { D } & & & \\ \text { D } & 0.21275 & 2700 & 31 \\ \text { E } & 0.19768 & 2700 & 32 \\ \text { E } & 0.18516 & 2700 & 33 \\ \text { E } & \text { The SAS System } & \end{array}$

The GLM Procedure

Class Level Information

Class Levels Values 


length
cycle
split
vol
turn
offset2
rep
len_vol
cyc_vol
spl_turn
vol_turn
off_len
off_cyc
off_spl
off_vol
off_turn
det_len
det_vol
det_turn


Saturday, March 17, 200118

Dependent Variable: fvalue

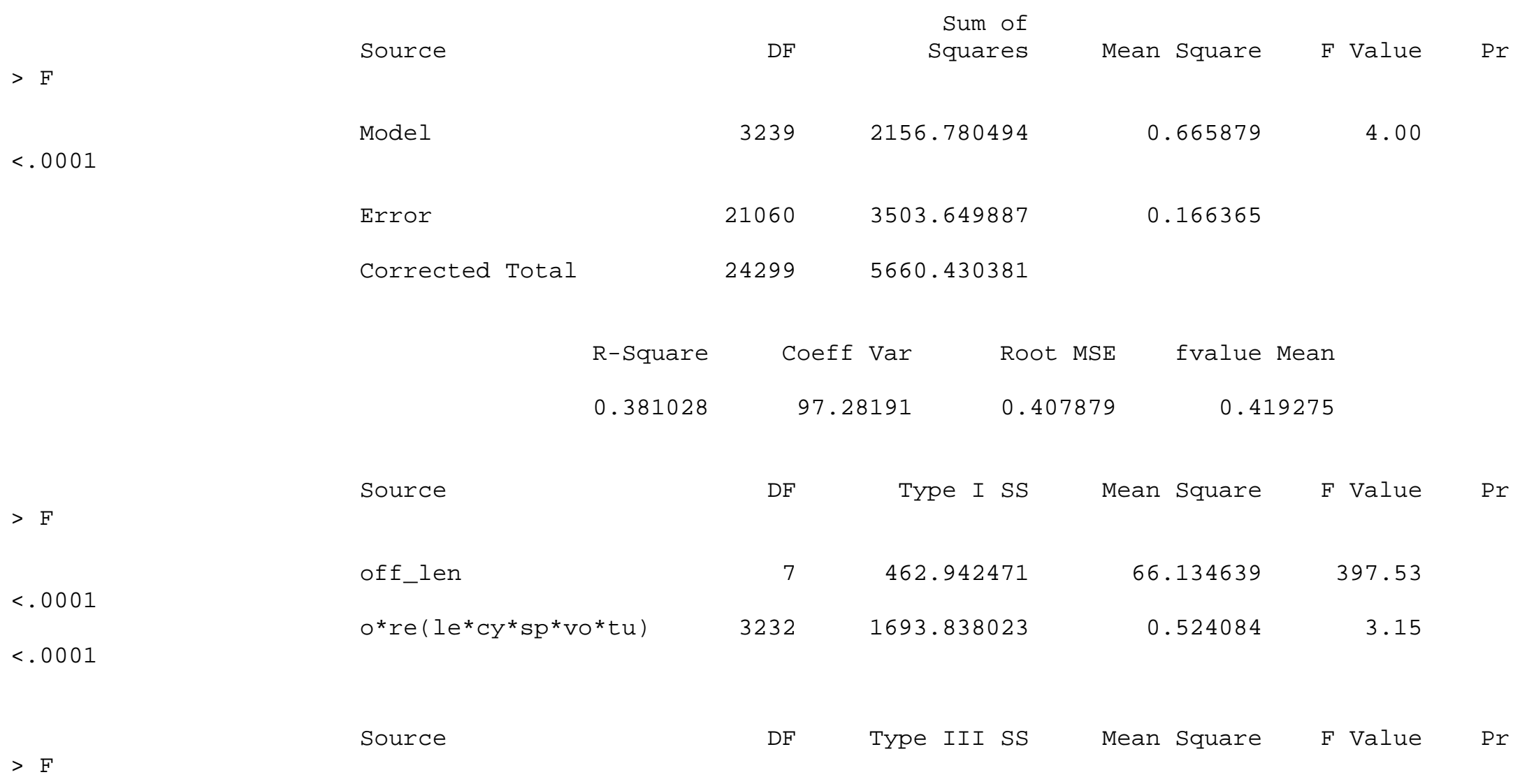




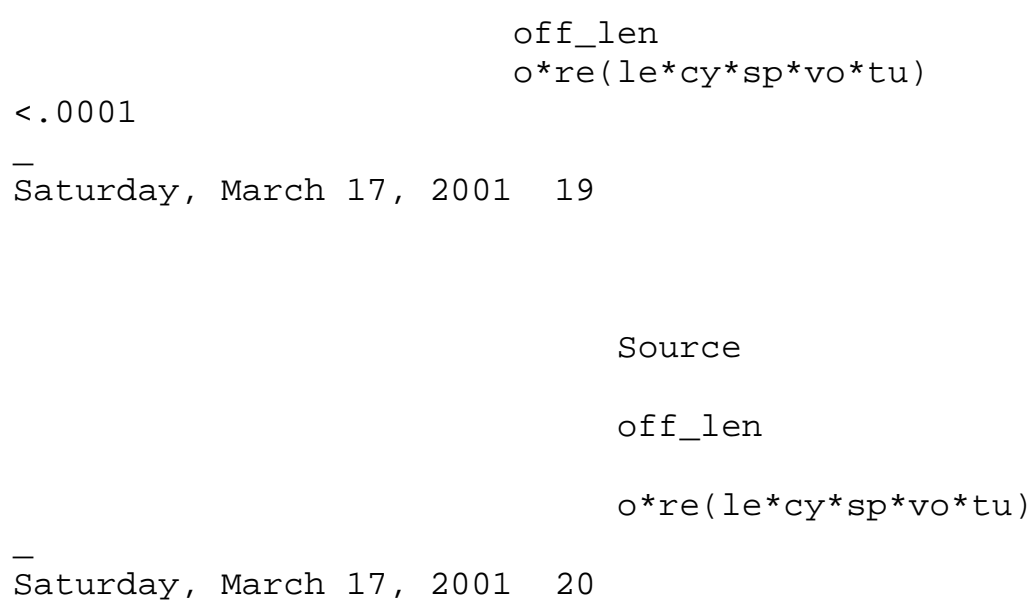

Type III Expected Mean Square

0

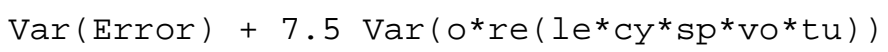

The SAS System

$23: 44$

The GLM Procedure

Student-Newman-Keuls Test for fvalue

NOTE: This test controls the Type I experimentwise error rate under the complete null hypothesis but not under partial null

hypotheses.

$\begin{array}{lr}\text { Alpha } & 0.05 \\ \text { Error Degrees of Freedom } & 3232 \\ \text { Error Mean Square } & 0.524084 \\ \text { Harmonic Mean of Cell Sizes } & 2700\end{array}$

NOTE: Cell sizes are not equal.

Number of Means

$$
8
$$

7

0.058128

3 
Means with the same letter are not significantly different.

$\begin{array}{cccc}\text { SNK Grouping } & \text { Mean } & \text { N } & \text { off_len } \\ \text { A } & 0.63703 & 2025 & 3040 \\ \text { B } & 0.58055 & 2025 & 1040 \\ \text { C } & 0.53494 & 4050 & 3030 \\ \text { C } & 0.50564 & 4050 & 1030 \\ \text { C } & 0.32832 & 4050 & 1020 \\ \text { D } & 0.30961 & 4050 & 3020 \\ \text { D } & 0.23018 & 2025 & 3010 \\ \text { D } & 0.22651 & 2025 & 1010 \\ \text { E } & \text { The SAS System } & \end{array}$

\section{The GLM Procedure}

Class Level Information

$\begin{array}{lcl}\text { Class } & \text { Levels } & \text { Values } \\ \text { length } & 2 & 10003000 \\ \text { cycle } & 3 & 90120150 \\ \text { split } & 3 & 123 \\ \text { vol } & 3 & 123\end{array}$




turn
offset2
rep
len_vol
cyc_vol
spl_turn
vol_turn
off_len
off_cyc
off_spl
off_vol
off_turn
det_len
det_vol
det_turn

Saturday, March 17, 2001

22

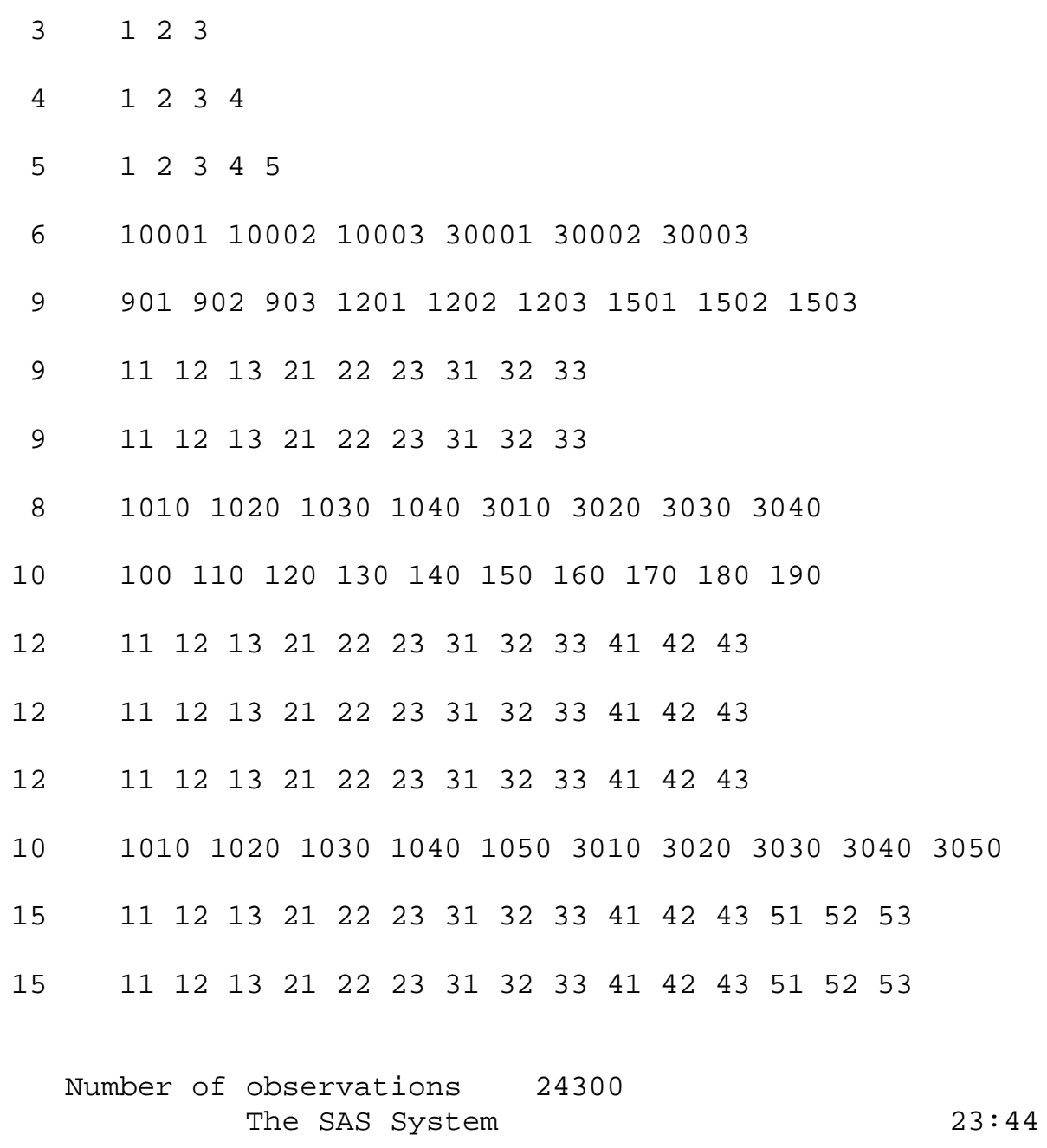




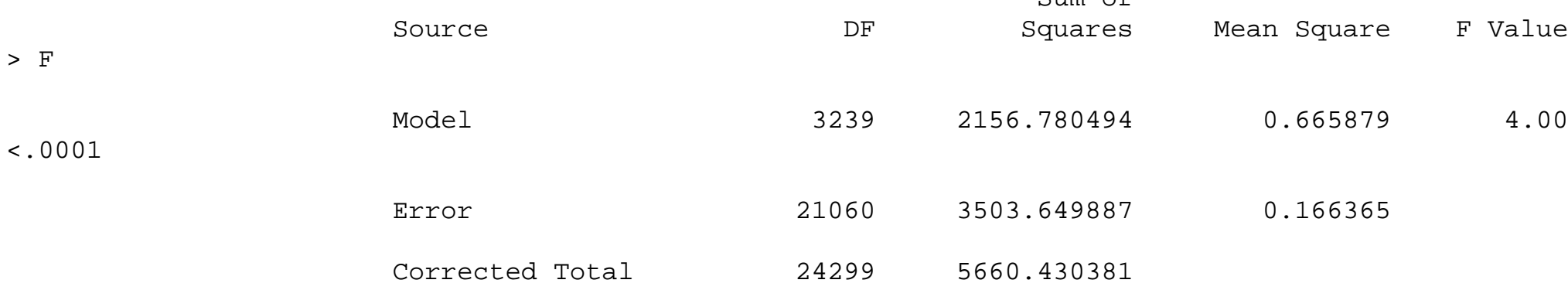

$\begin{array}{lrrr}\text { R-Square } & \text { Coeff Var } & \text { Root MSE } & \text { fvalue Mean } \\ 0.381028 & 97.28191 & 0.407879 & 0.419275\end{array}$

$>F$

$<.0001$

$<.0001$

$>\mathrm{F}$

$<.0001$

Saturday, March 17, 200123
Source

off_cyc

$o^{*} r e\left(l e^{*} \mathrm{Cy}^{*} \mathrm{sp}{ }^{\star} \mathrm{Vo}{ }^{\star} t u\right)$

Source

off_cyc

$o^{*} r e\left(l e^{*} \mathrm{Cy}^{\star} \mathrm{sp}^{*} \mathrm{Vo}^{*} \mathrm{tu}\right)$
$\mathrm{DF}$

3230

3230

$\mathrm{DF}$

1627.208769

The SAS system

Mean Square

F Value

$\operatorname{Pr}$

$\begin{array}{rrrr}9 & 529.571726 & 58.841303 & 353.69 \\ 30 & 1627.208769 & 0.503780 & 3.03\end{array}$

Type III SS

Mean Square

F Value

Pr

The GLM Procedure

Pr

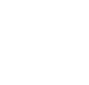


Source

off_cyc

$o * r e\left(1 e^{\star} \mathrm{cy}{ }^{\star} \mathrm{sp}{ }^{\star}\right.$ vo*tu $)$

Saturday, March 17, 2001

24

Type III Expected Mean Square

0

$\operatorname{Var}($ Error $)+7.4985 \operatorname{Var}\left(o^{*} r e\left(1 e^{*} \mathrm{Cy}{ }^{*} \mathrm{sp}{ }^{*} \mathrm{Vo}^{*} \mathrm{tu}\right)\right)$

The SAS system

$23: 44$

The GLM Procedure

Student-Newman-Keuls Test for fvalue

NOTE: This test controls the Type I experimentwise error rate under the complete null hypothesis but not under partial null

\section{hypotheses.}

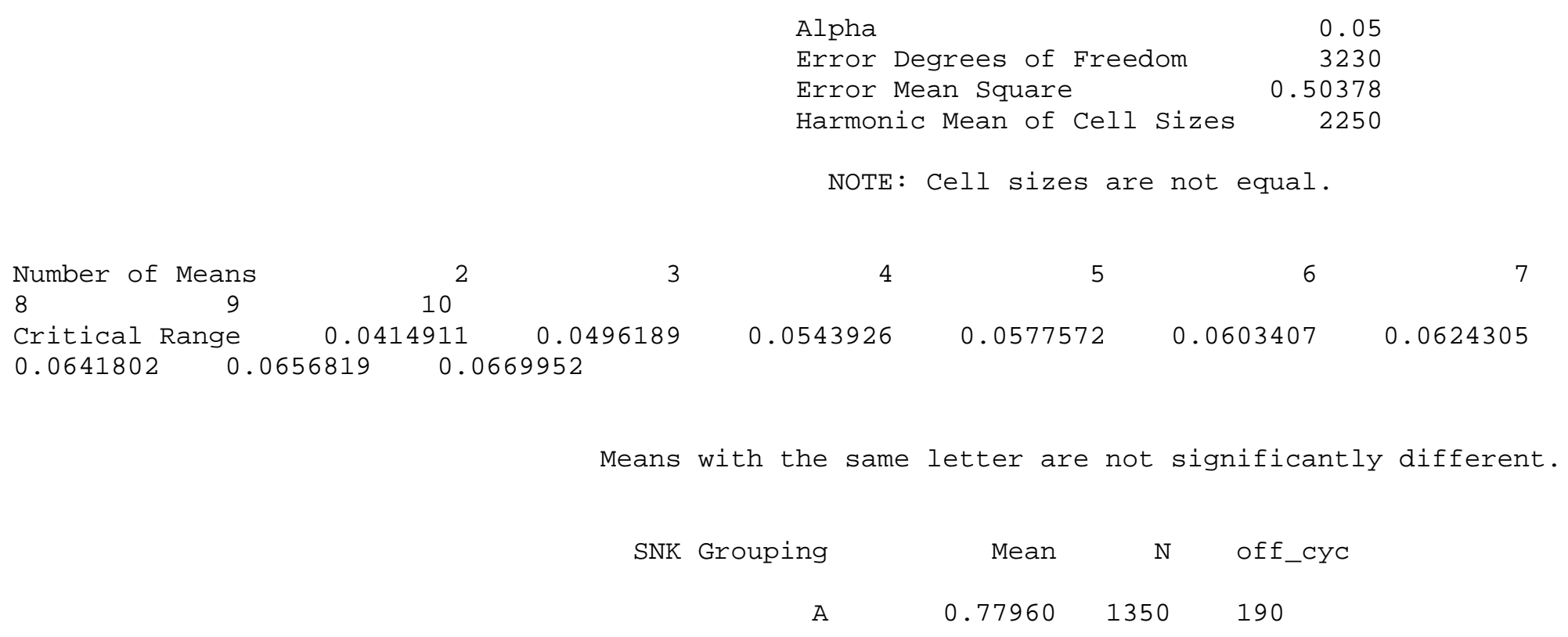


Saturday, March 17, 200125

$\begin{array}{llll}\text { B } & 0.64960 & 2700 & 180 \\ \text { C } & 0.53029 & 2700 & 150 \\ \text { D } & 0.43633 & 2700 & 160 \\ \text { E } & 0.38098 & 2700 & 120 \\ \text { E } & 0.36523 & 2700 & 170 \\ \text { E } & 0.32049 & 2700 & 130 \\ \text { F } & 0.31465 & 2700 & 140 \\ \text { F } & & & \\ \text { F } & 0.27701 & 2700 & 110 \\ \text { F } & 0.21816 & 1350 & 100 \\ \text { F } & \text { The SAS System } & \\ \text { G } & & & \end{array}$

The GLM Procedure

Class Level Information

Class
length
cycle
split
vol
turn
offset2

Levels Values

$2 \quad 1000 \quad 3000$

$390 \quad 120 \quad 150$

$3 \quad 123$

$\begin{array}{llll}3 & 1 & 2 & 3\end{array}$

$\begin{array}{llll}3 & 1 & 2 & 3\end{array}$

$\begin{array}{lllll}4 & 1 & 2 & 3 & 4\end{array}$ 


$$
\begin{aligned}
& \text { rep } \\
& \text { len_vol } \\
& \text { cyc_vol } \\
& \text { spl_turn } \\
& \text { vol_turn } \\
& \text { off_len } \\
& \text { off_cyc } \\
& \text { off_spl } \\
& \text { off_vol } \\
& \text { off_turn } \\
& \text { det_len } \\
& \text { det_vol } \\
& \text { det_turn }
\end{aligned}
$$

Saturday, March 17, 200126

Dependent Variable: fvalue $\begin{array}{llllll}5 & 1 & 2 & 3 & 4 & 5\end{array}$

6100011000210003300013000230003

$999019029031201120212031501 \quad 15021503$

$\begin{array}{llllllllll}9 & 11 & 12 & 13 & 21 & 22 & 23 & 31 & 32 & 33\end{array}$

$9 \begin{array}{llllllllll}11 & 12 & 13 & 21 & 22 & 23 & 31 & 32 & 33\end{array}$

$8 \quad 1010 \quad 1020 \quad 1030 \quad 1040 \quad 3010 \quad 3020 \quad 3030 \quad 3040$

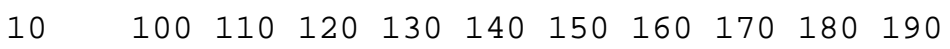

$\begin{array}{lllllllllllll}12 & 11 & 12 & 13 & 21 & 22 & 23 & 31 & 32 & 33 & 41 & 42 & 43\end{array}$

$\begin{array}{lllllllllllll}12 & 11 & 12 & 13 & 21 & 22 & 23 & 31 & 32 & 33 & 41 & 42 & 43\end{array}$

$\begin{array}{lllllllllllll}12 & 11 & 12 & 13 & 21 & 22 & 23 & 31 & 32 & 33 & 41 & 42 & 43\end{array}$

$10 \quad 1010 \quad 1020 \quad 1030 \quad 1040 \quad 1050 \quad 3010 \quad 3020 \quad 3030 \quad 3040 \quad 3050$

$\begin{array}{llllllllllllllll}15 & 11 & 12 & 13 & 21 & 22 & 23 & 31 & 32 & 33 & 41 & 42 & 43 & 51 & 52 & 53\end{array}$

$\begin{array}{llllllllllllllll}15 & 11 & 12 & 13 & 21 & 22 & 23 & 31 & 32 & 33 & 41 & 42 & 43 & 51 & 52 & 53\end{array}$

Number of observations 24300

The SAS system

$23: 44$

The GLM Procedure

$>$ F Source

Sum of

Squares

DF

Mean Square

F Value

$\operatorname{Pr}$ 


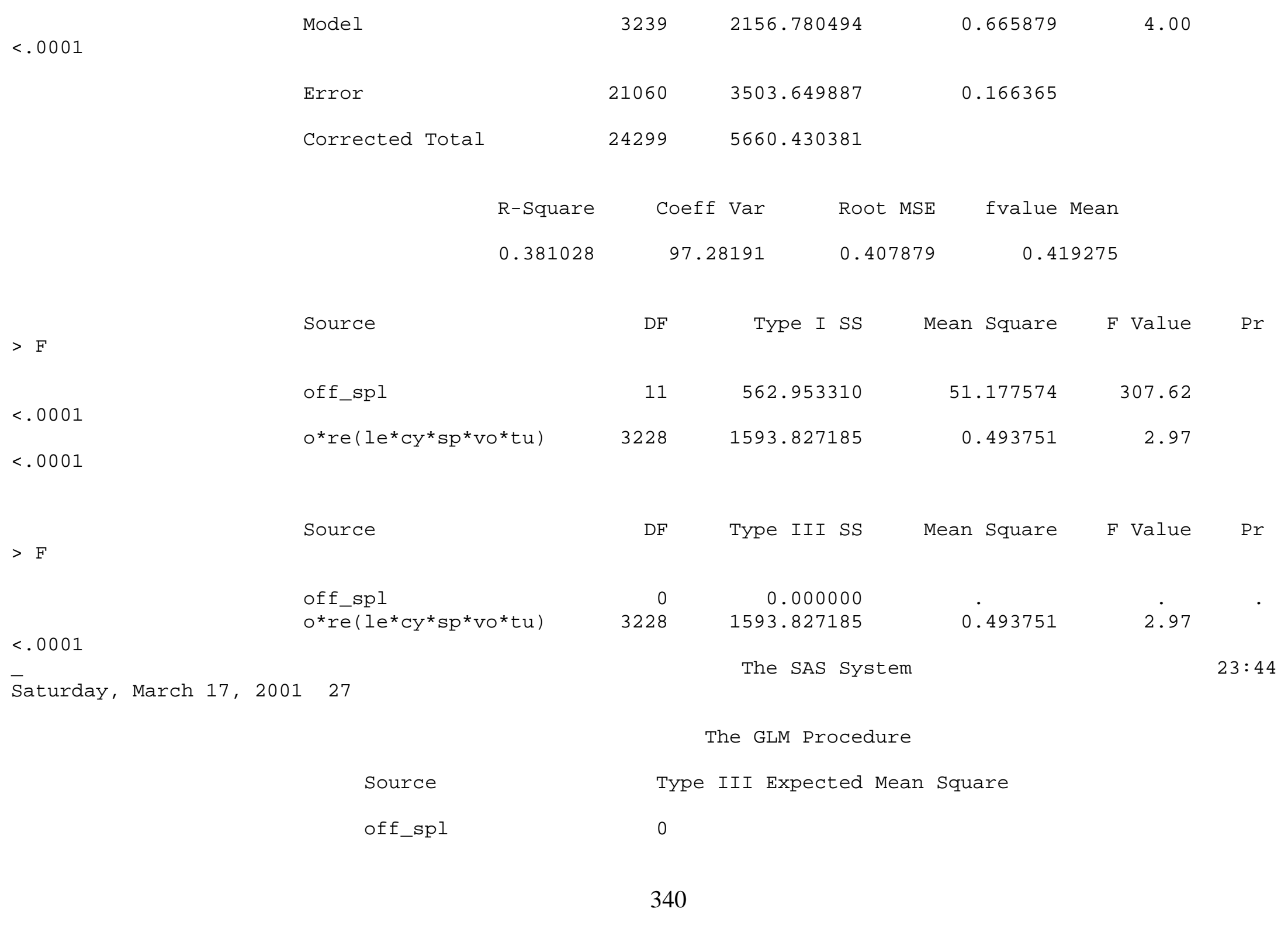




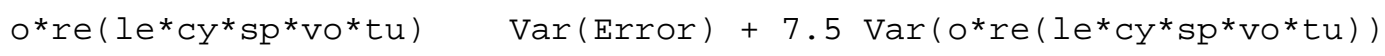

The SAS System

$23: 44$

The GLM Procedure

Student-Newman-Keuls Test for fvalue

NOTE: This test controls the Type I experimentwise error rate under the complete null hypothesis but not under partial null

hypotheses.

$\begin{array}{lr}\text { Alpha } & 0.05 \\ \text { Error Degrees of Freedom } & 3228 \\ \text { Error Mean Square } & 0.493751 \\ \text { Harmonic Mean of Cell Sizes } & 1800\end{array}$

NOTE: Cell sizes are not equal.

$\begin{array}{ccccccccc}\text { Number of Means } & 2 & 3 & 4 & 5 & 6 & 9 & 9 \\ 11 & 12 & & & & & & \end{array}$

$0.07415360 .0754434 \quad 0.0766015$

Means with the same letter are not significantly different.

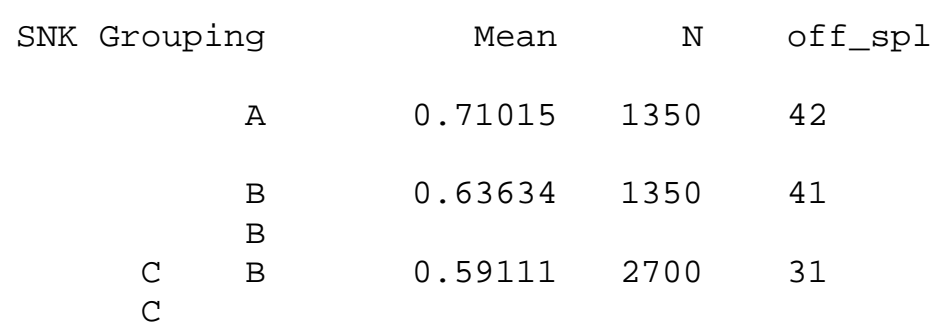




$\begin{array}{llll} & 0.57182 & 2700 & 32 \\ \text { D } & 0.47988 & 1350 & 43 \\ \text { E } & 0.39795 & 2700 & 33 \\ \text { E } & & & \\ \text { E } & 0.35926 & 2700 & 21 \\ \text { F } & 0.30160 & 2700 & 22 \\ \text { F } & & & \\ \text { F } & 0.29604 & 2700 & 23 \\ \text { G } & 0.24240 & 1350 & 13 \\ \text { G } & & & \\ \text { G } & 0.23067 & 1350 & 11 \\ \text { G } & & & \\ \text { G } & 0.21197 & 1350 & 12 \\ & \text { The SAS } & \text { System } & \end{array}$

The GLM Procedure

Class Level Information

\begin{tabular}{|c|c|c|}
\hline Class & Levels & Values \\
\hline length & 2 & 10003000 \\
\hline cycle & 3 & $90 \quad 120 \quad 150$ \\
\hline split & 3 & 123 \\
\hline vol & 3 & 123 \\
\hline turn & 3 & 123 \\
\hline offset 2 & 4 & 1234 \\
\hline
\end{tabular}




$$
\begin{aligned}
& \text { rep } \\
& \text { len_vol } \\
& \text { cyc_vol } \\
& \text { spl_turn } \\
& \text { vol_turn } \\
& \text { off_len } \\
& \text { off_cyc } \\
& \text { off_spl } \\
& \text { off_vol } \\
& \text { off_turn } \\
& \text { det_len } \\
& \text { det_vol } \\
& \text { det_turn }
\end{aligned}
$$

Saturday, March 17, 200130

Dependent Variable: fvalue $\begin{array}{llllll}5 & 1 & 2 & 3 & 4 & 5\end{array}$

6100011000210003300013000230003

$999019029031201 \quad 1202 \quad 120315011502 \quad 1503$

$\begin{array}{llllllllll}9 & 11 & 12 & 13 & 21 & 22 & 23 & 31 & 32 & 33\end{array}$

$\begin{array}{llllllllll}9 & 11 & 12 & 13 & 21 & 22 & 23 & 31 & 32 & 33\end{array}$

$8 \quad 1010 \quad 10201030 \quad 1040 \quad 3010 \quad 3020 \quad 3030 \quad 3040$

$10100110 \quad 120130 \quad 140 \quad 150 \quad 160 \quad 170 \quad 180 \quad 190$

$\begin{array}{lllllllllllll}12 & 11 & 12 & 13 & 21 & 22 & 23 & 31 & 32 & 33 & 41 & 42 & 43\end{array}$

$\begin{array}{lllllllllllll}12 & 11 & 12 & 13 & 21 & 22 & 23 & 31 & 32 & 33 & 41 & 42 & 43\end{array}$

$\begin{array}{lllllllllllll}12 & 11 & 12 & 13 & 21 & 22 & 23 & 31 & 32 & 33 & 41 & 42 & 43\end{array}$

$101010 \quad 1020 \quad 1030 \quad 1040 \quad 1050 \quad 3010 \quad 3020 \quad 3030 \quad 3040 \quad 3050$

$\begin{array}{llllllllllllllll}15 & 11 & 12 & 13 & 21 & 22 & 23 & 31 & 32 & 33 & 41 & 42 & 43 & 51 & 52 & 53\end{array}$

$\begin{array}{llllllllllllllll}15 & 11 & 12 & 13 & 21 & 22 & 23 & 31 & 32 & 33 & 41 & 42 & 43 & 51 & 52 & 53\end{array}$

Number of observations 24300

The SAS system

$23: 44$

The GLM Procedure

$>$ F Source

Sum of

Squares

DF

Mean Square

F Value

$\operatorname{Pr}$ 


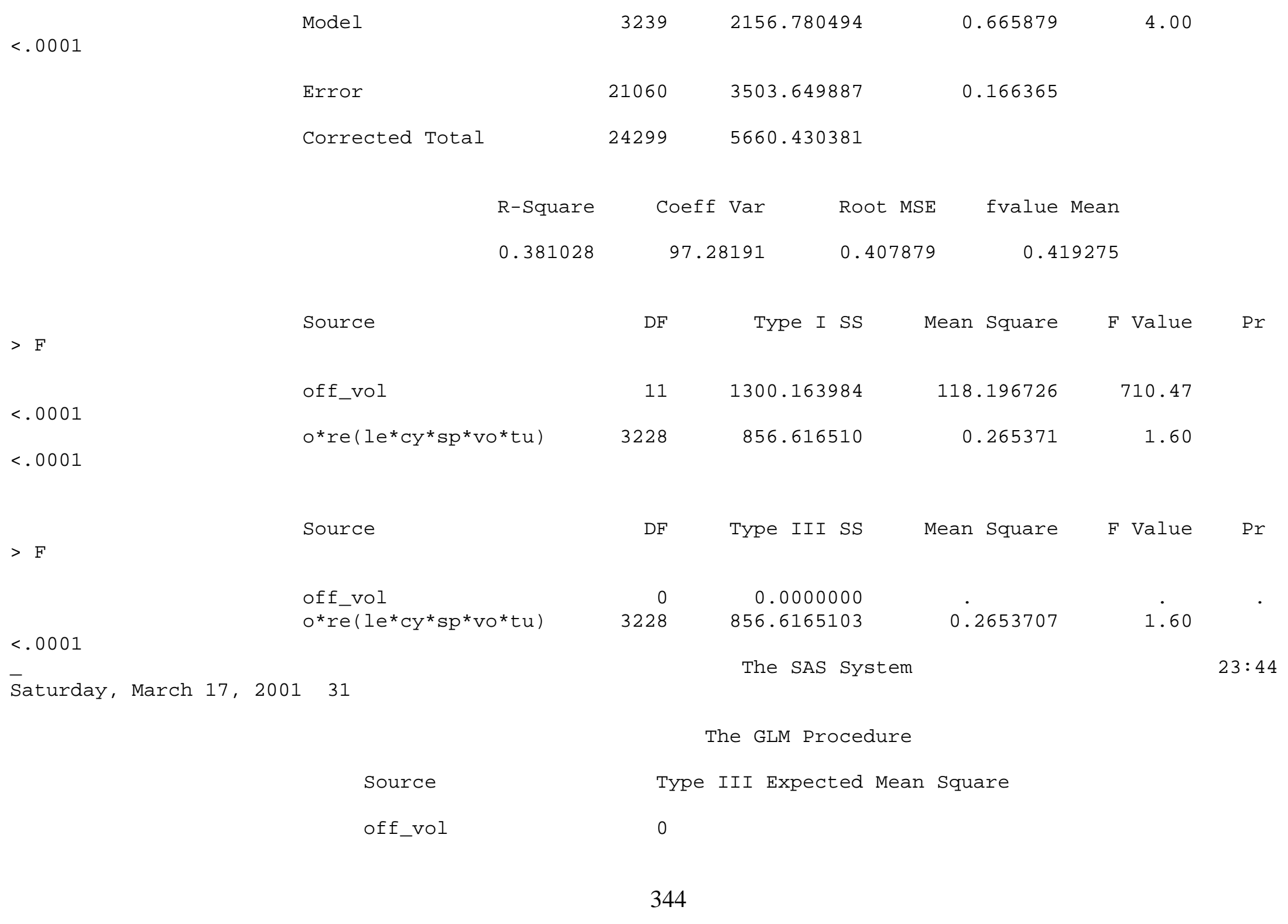




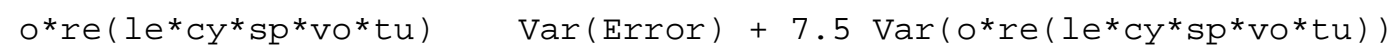

The SAS system

$23: 44$

The GLM Procedure

Student-Newman-Keuls Test for fvalue

NOTE: This test controls the Type I experimentwise error rate under the complete null hypothesis but not under partial null

hypotheses.

$\begin{array}{lr}\text { Alpha } & 0.05 \\ \text { Error Degrees of Freedom } & 3228 \\ \text { Error Mean Square } & 0.265371 \\ \text { Harmonic Mean of Cell Sizes } & 1800\end{array}$

NOTE: Cell sizes are not equal.

Number of Means

11

10

Critical Range $0.03366790 .0402632 \quad 0.0441368 \quad 0.04686710 .04896340 .05065910 .05207890 .0532975$

Means with the same letter are not significantly different.

SNK Grouping

$\begin{array}{rrr}\text { Mean } & \mathrm{N} & \text { off_vol } \\ 0.83899 & 1350 & 41 \\ 0.74394 & 1350 & 42 \\ 0.73900 & 2700 & 31\end{array}$




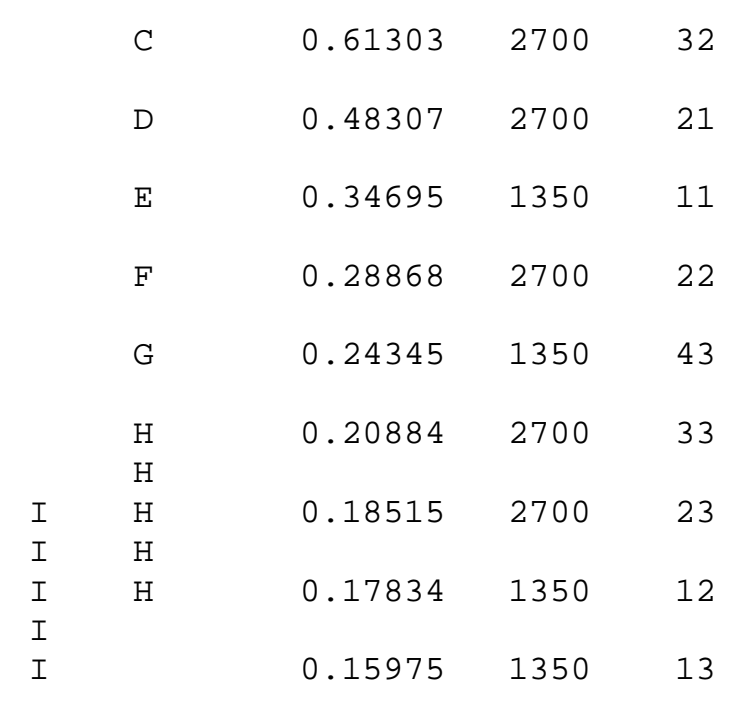




\section{Skewness Model GLM}

16:00 Sunday, March 18, 20011
The SAS system

The GLM Procedure

Class Level Information

\begin{tabular}{|c|c|c|c|}
\hline Class & Levels & Values & \\
\hline length & 2 & 10003000 & \\
\hline cycle & 3 & $90 \quad 120150$ & \\
\hline split & 3 & 123 & \\
\hline vol & 3 & 123 & \\
\hline turn & 3 & 123 & \\
\hline offset 2 & 4 & 1234 & \\
\hline det_loc & 5 & $\begin{array}{lllll}1 & 2 & 3 & 4 & 5\end{array}$ & \\
\hline rep & 5 & 12345 & \\
\hline len_vol & 6 & 1000110002 & 10003300013000230003 \\
\hline Cyc_vol & 9 & 901902903 & $1201 \quad 1202 \quad 1203 \quad 1501 \quad 1502 \quad 1503$ \\
\hline cyc_turn & 9 & $\begin{array}{llll}11 & 12 & 13 & 21\end{array}$ & $\begin{array}{lllll}22 & 23 & 31 & 32 & 33\end{array}$ \\
\hline vol_turn & 9 & $\begin{array}{llll}11 & 12 & 13 & 21\end{array}$ & $\begin{array}{lllll}22 & 23 & 31 & 32 & 33\end{array}$ \\
\hline off_cyc & 10 & $100 \quad 110120$ & $\begin{array}{lllllll}130 & 140 & 150 & 160 & 170 & 180 & 190\end{array}$ \\
\hline off_spl & 12 & $\begin{array}{llll}11 & 12 & 13 & 21\end{array}$ & $\begin{array}{llllllll}22 & 23 & 31 & 32 & 33 & 41 & 42 & 43\end{array}$ \\
\hline
\end{tabular}




$\begin{array}{lcccccccccccccccccc}\text { off_vol } & 12 & 11 & 12 & 13 & 21 & 22 & 23 & 31 & 32 & 33 & 41 & 42 & 43 & & \\ \text { off_turn } & 12 & 11 & 12 & 13 & 21 & 22 & 23 & 31 & 32 & 33 & 41 & 42 & 43 & & \\ \text { det_vol } & 15 & 11 & 12 & 13 & 21 & 22 & 23 & 31 & 32 & 33 & 41 & 42 & 43 & 51 & 52 & 53 \\ \text { det_turn } & 15 & 11 & 12 & 13 & 21 & 22 & 23 & 31 & 32 & 33 & 41 & 42 & 43 & 51 & 52 & 53\end{array}$

16:00 Sunday, March 18, 20012

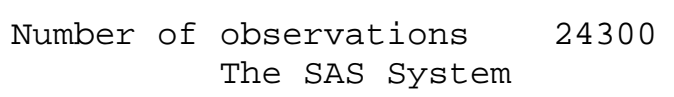

The GLM Procedure

Dependent Variable: diff_skw

$\begin{array}{ll}>\mathrm{F} & \text { Source } \\ <.0001 & \text { Model }\end{array}$

\section{Error}

Corrected Total

R-Square
0.365761

Source

$>\mathrm{F}$

Sum of

Squares

Mean Square

F Value

Pr

4049

12073.61178

2.98187

2.88

20250

20935.91414

1.03387

24299

33009.52592

$\begin{array}{rrr}\text { Coeff Var } & \text { Root MSE } & \text { diff_skw Mean } \\ 548.4090 & 1.016795 & 0.185408\end{array}$

DF

Type I SS

Mean Square

F Value

Pr 


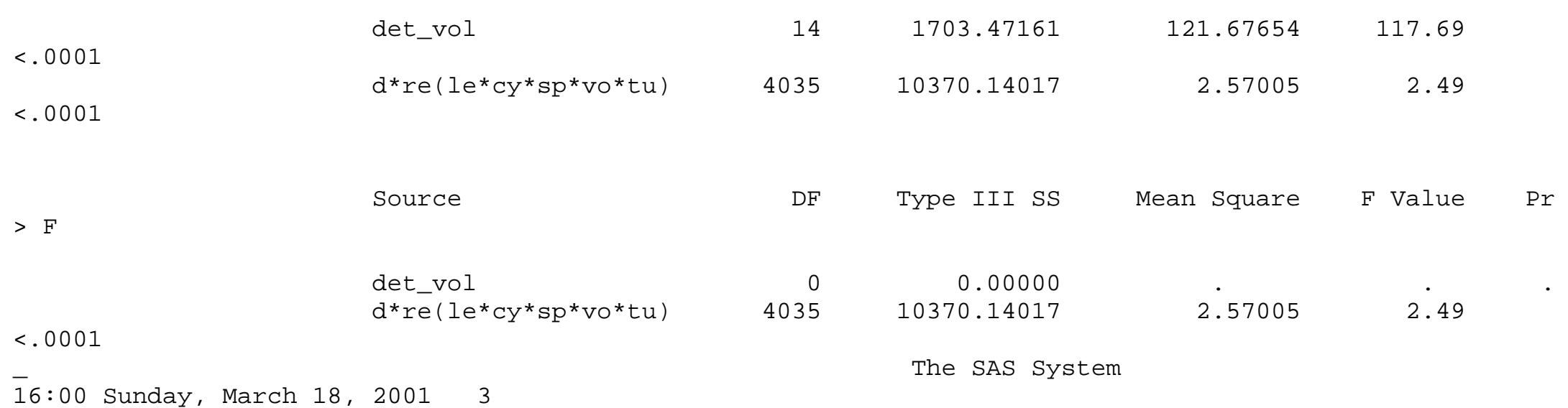

The GLM Procedure

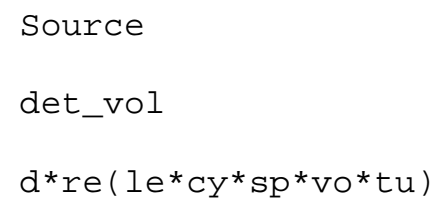

16:00 Sunday, March 18, 2001
Type III Expected Mean Square

0

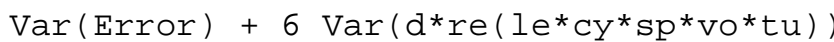

The SAS system

The GLM Procedure

Student-Newman-Keuls Test for diff_skw

NOTE: This test controls the Type I experimentwise error rate under the complete null hypothesis but not under partial null hypotheses.

$\begin{array}{lr}\text { Alpha } & 0.05 \\ \text { Error Degrees of Freedom } & 4035 \\ \text { Error Mean Square } & 2.570047\end{array}$




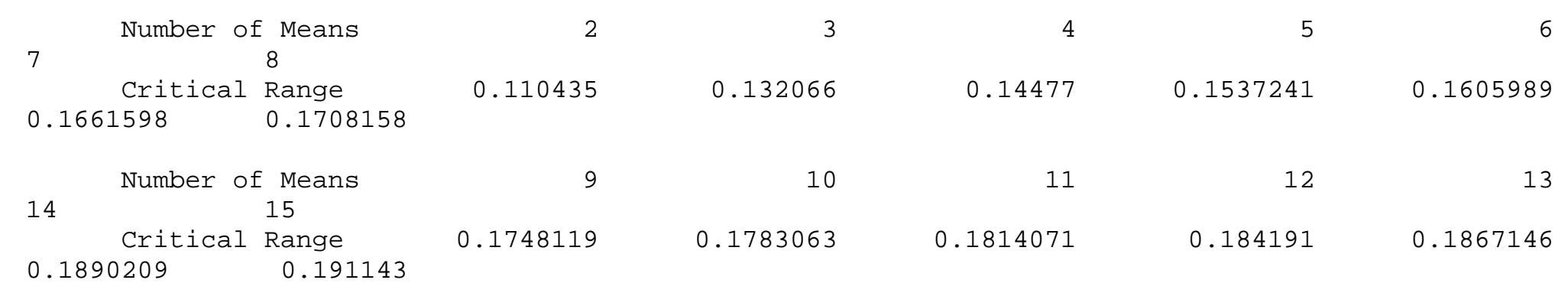

Means with the same letter are not significantly different.

\begin{tabular}{|c|c|c|c|c|}
\hline SNK & Grouping & Mean & $\mathrm{N}$ & det_vol \\
\hline & $\begin{array}{l}\mathrm{A} \\
\mathrm{A}\end{array}$ & 0.61713 & 1620 & 31 \\
\hline & $\begin{array}{l}\text { B } \\
B\end{array}$ & 0.53475 & 1620 & 41 \\
\hline & $\begin{array}{l}\text { B } \\
B\end{array}$ & 0.41301 & 1620 & 21 \\
\hline & $\begin{array}{l}\text { B } \\
\text { B }\end{array}$ & 0.41220 & 1620 & 22 \\
\hline & $\begin{array}{l}\text { B } \\
B\end{array}$ & 0.39686 & 1620 & 51 \\
\hline & B & 0.38865 & 1620 & 12 \\
\hline & $\begin{array}{l}\mathrm{C} \\
\mathrm{C}\end{array}$ & 0.21822 & 1620 & 32 \\
\hline & $\begin{array}{l}\mathrm{D} \\
\mathrm{D}\end{array}$ & 0.11791 & 1620 & 42 \\
\hline & $\begin{array}{l}\mathrm{D} \\
\mathrm{D}\end{array}$ & 0.06014 & 1620 & 13 \\
\hline & $\begin{array}{l}\mathrm{D} \\
\mathrm{D}\end{array}$ & 0.05449 & 1620 & 52 \\
\hline & $\mathrm{D}$ & 0.01189 & 1620 & 53 \\
\hline
\end{tabular}




\begin{tabular}{|c|c|c|c|}
\hline \multicolumn{4}{|c|}{ D } \\
\hline D & 0.01057 & 1620 & 43 \\
\hline$D$ & & & \\
\hline D & -0.00951 & 1620 & 33 \\
\hline D & & & \\
\hline D & -0.01790 & 1620 & 23 \\
\hline & -0.42728 & 1620 & 1 \\
\hline
\end{tabular}


The GLM Procedure

Class Level Information

Class
length
cycle
split
vol
turn
rep
len_vol
cyc_vol
cyc_turn
vol_turn
off_cyc
off_spl
off_vol
off_turn
det_vol


det_turn

$\overline{0} 0: 18$ Sunday, March 18, 2001

2

Dependent Variable: diff_skw

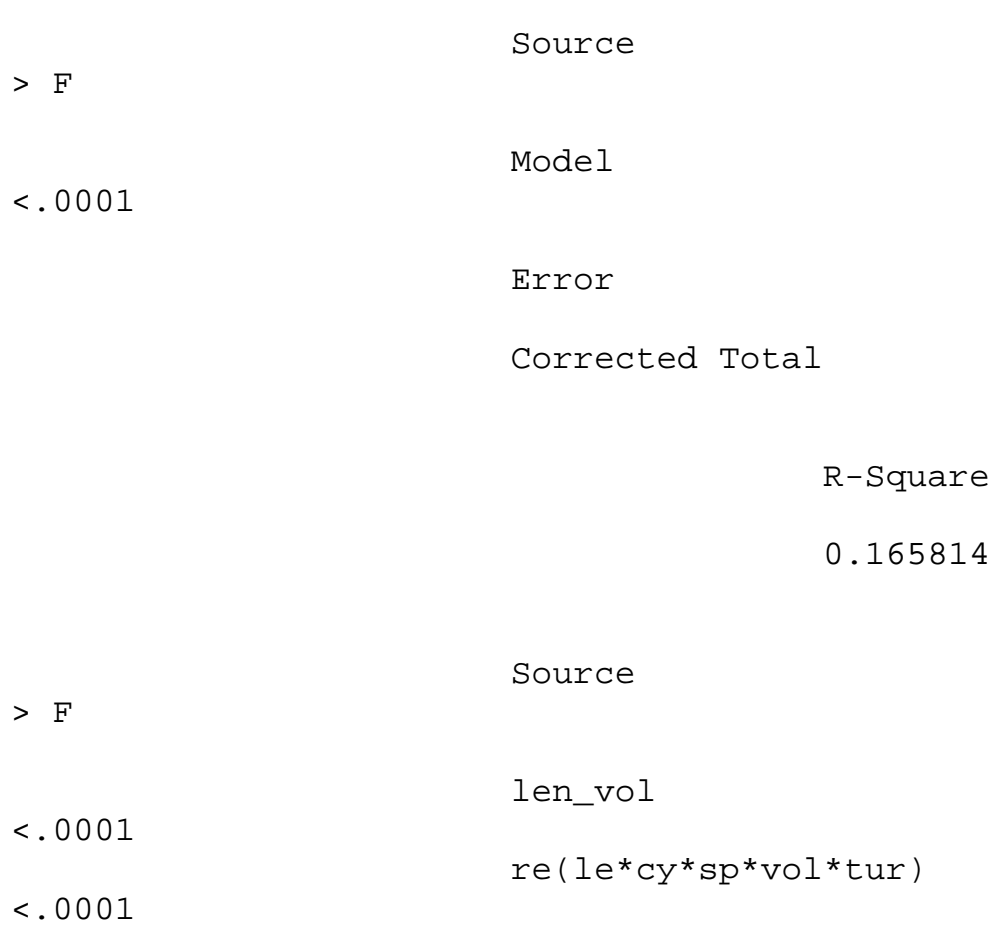

Number of observations The SAS system

24300

The GLM Procedure

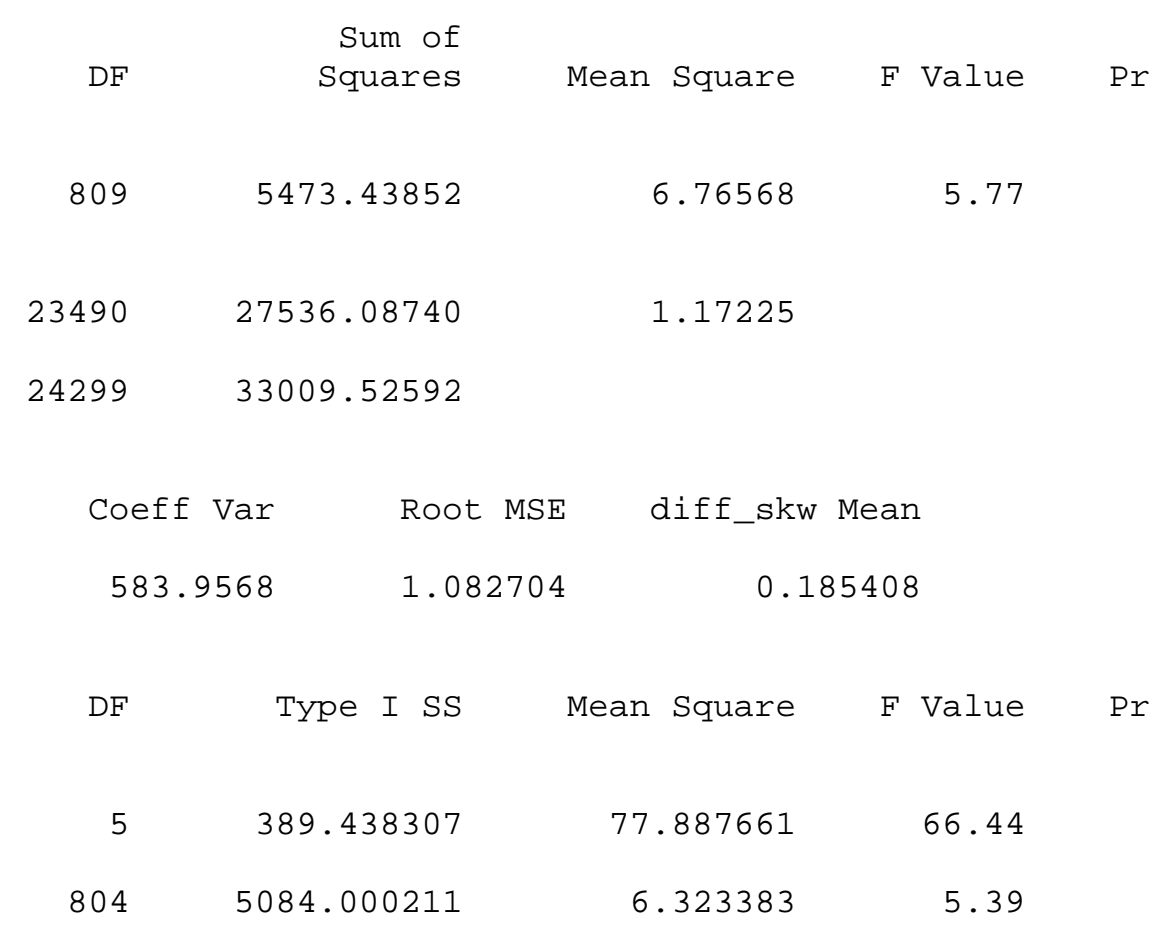




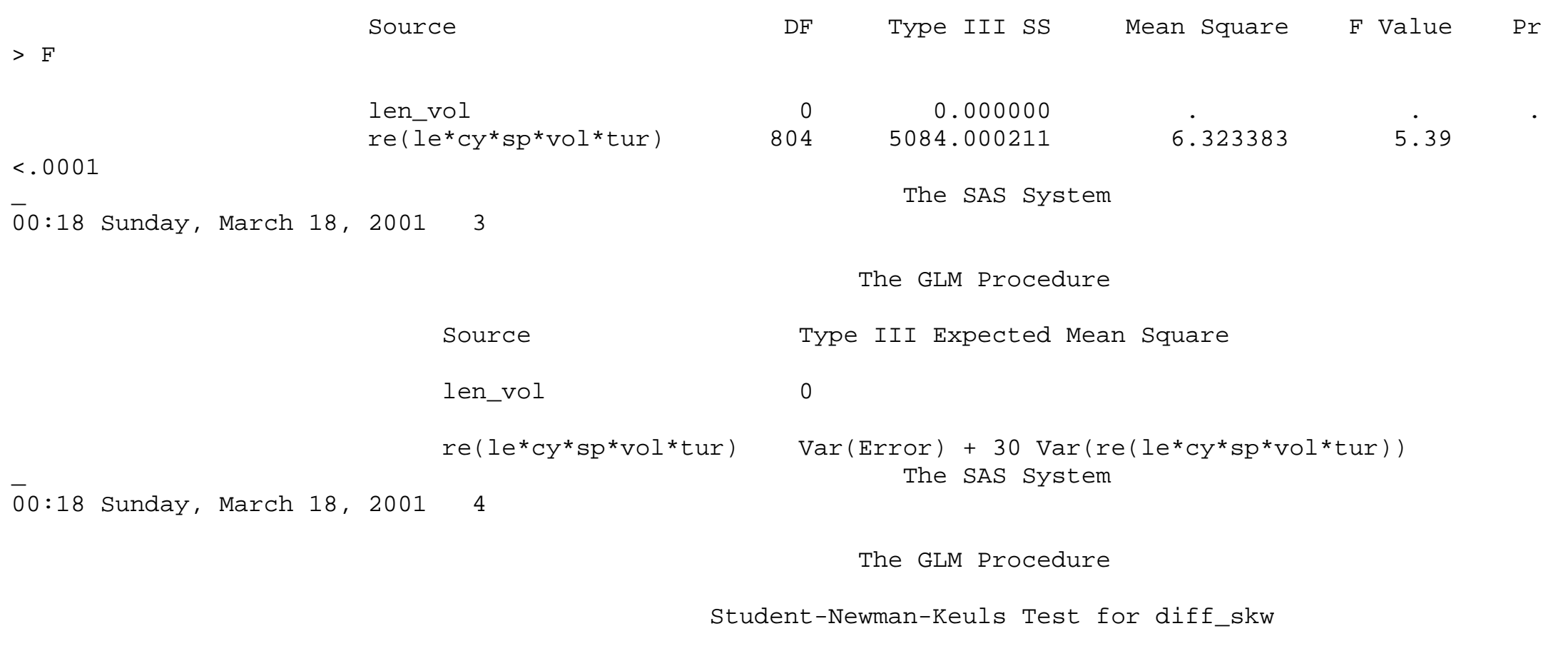

NOTE: This test controls the Type I experimentwise error rate under the complete null hypothesis but not under partial null

hypotheses.

\begin{tabular}{|c|c|c|c|c|c|}
\hline & & $\begin{array}{l}\text { Alpha } \\
\text { Error } \\
\text { Error }\end{array}$ & $\begin{array}{l}\text { Degrees of } \\
\text { Mean Square }\end{array}$ & $\begin{array}{l}0.05 \\
\text { om } 804 \\
6.323383\end{array}$ & \\
\hline & Number of Means & 2 & 3 & 4 & 5 \\
\hline 1596299 & Critical Range & 0.1096895 & 0.1312112 & 0.1438631 & 0.1527783 \\
\hline
\end{tabular}


Means with the same letter are not significantly different.

$\overline{0} 0: 18$ Sunday, March 18, 2001

$\begin{array}{cccc}\text { SNK Grouping } & \text { Mean } & \text { N } & \text { len_vol } \\ \text { A } & 0.31712 & 4050 & 10001 \\ \text { A } & & & \\ \text { A } & 0.29667 & 4050 & 30001 \\ \text { A } & & & \\ \text { A } & 0.24155 & 4050 & 30002 \\ \text { A } & 0.23504 & 4050 & 10002 \\ \text { A } & 0.01288 & 4050 & 10003 \\ \text { B } & 0.00920 & 4050 & 30003 \\ \text { B } & \text { The SAS System } \\ \text { B } & 0.0503 & \end{array}$

The GLM Procedure

Class Level Information

\begin{tabular}{|c|c|c|}
\hline Class & Levels & Values \\
\hline length & 2 & 10003000 \\
\hline cycle & 3 & $90 \quad 120 \quad 150$ \\
\hline split & 3 & 123 \\
\hline vol & 3 & 123 \\
\hline turn & 3 & 123 \\
\hline rep & 5 & 12345 \\
\hline
\end{tabular}




len_vol
cyc_vol
cyc_turn
vol_turn
off_cyc
off_spl
off_vol
off_turn
det_vol
det_turn

$\overline{0} 0: 18$ Sunday, March 18, 2001

6
6100011000210003300013000230003

$999019029031201120212031501 \quad 1502 \quad 1503$

$\begin{array}{lllllllllll}9 & 11 & 12 & 13 & 21 & 22 & 23 & 31 & 32 & 33\end{array}$

$\begin{array}{llllllllll}9 & 11 & 12 & 13 & 21 & 22 & 23 & 31 & 32 & 33\end{array}$

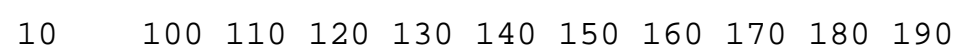

$\begin{array}{lllllllllllll}12 & 11 & 12 & 13 & 21 & 22 & 23 & 31 & 32 & 33 & 41 & 42 & 43\end{array}$

$\begin{array}{lllllllllllll}12 & 11 & 12 & 13 & 21 & 22 & 23 & 31 & 32 & 33 & 41 & 42 & 43\end{array}$

$\begin{array}{llllllllllllll}12 & 11 & 12 & 13 & 21 & 22 & 23 & 31 & 32 & 33 & 41 & 42 & 43\end{array}$

$\begin{array}{llllllllllllllll}15 & 11 & 12 & 13 & 21 & 22 & 23 & 31 & 32 & 33 & 41 & 42 & 43 & 51 & 52 & 53\end{array}$

$\begin{array}{llllllllllllllll}15 & 11 & 12 & 13 & 21 & 22 & 23 & 31 & 32 & 33 & 41 & 42 & 43 & 51 & 52 & 53\end{array}$

Number of observations 24300 The SAS System

The GLM Procedure

Dependent Variable: diff_skw

$\begin{array}{ll}>\mathrm{F} & \text { Source } \\ <.0001 & \text { Model } \\ & \text { Error }\end{array}$

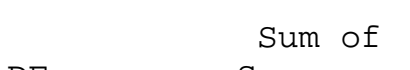

809

Mean Square

$\operatorname{Pr}$

23490
DF Squares

F Value
5473.43852

27536.08740

6.76568

5.77

1.17225 


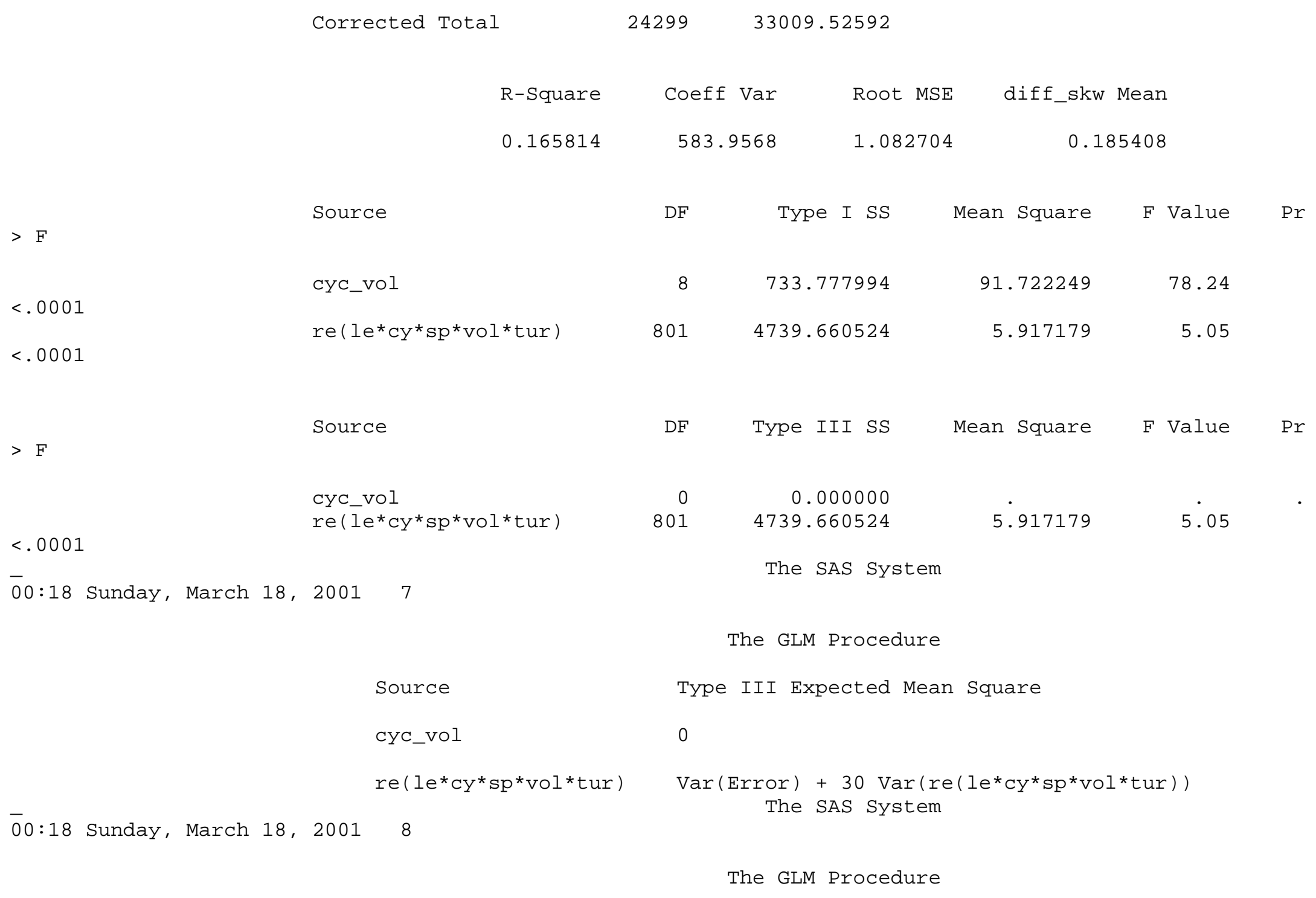


NOTE: This test controls the Type I experimentwise error rate under the complete null hypothesis but not under partial null

\section{hypotheses.}

$\begin{array}{lr}\text { Alpha } & 0.05 \\ \text { Error Degrees of Freedom } & 801 \\ \text { Error Mean Square } & 5.917179\end{array}$

Number of Means

Critical Range 0.1299558

0.2011933
2 0.2059159
3

0.1554541
4

0.1704438
5

0.1810063
0.1891239

Means with the same letter are not significantly different.

\begin{tabular}{|c|c|c|c|c|}
\hline \multicolumn{2}{|c|}{ Grouping } & Mean & $\mathrm{N}$ & Cyc_vol \\
\hline & A & 0.54656 & 2700 & 901 \\
\hline & B & 0.31890 & 2700 & 1502 \\
\hline & B & & & \\
\hline & B & 0.29752 & 2700 & 1201 \\
\hline & B & & & \\
\hline & B & 0.24643 & 2700 & 1202 \\
\hline & B & & & \\
\hline $\mathrm{C}$ & B & 0.14955 & 2700 & 902 \\
\hline $\mathrm{C}$ & & & & \\
\hline $\mathrm{C}$ & & 0.07660 & 2700 & 1501 \\
\hline $\mathrm{C}$ & & & & \\
\hline $\mathrm{C}$ & & 0.05429 & 2700 & 1503 \\
\hline $\mathrm{C}$ & & & & \\
\hline $\mathrm{C}$ & & -0.00428 & 2700 & 1203 \\
\hline
\end{tabular}


$\overline{0} 0: 18$ Sunday, March 18, 2001

9

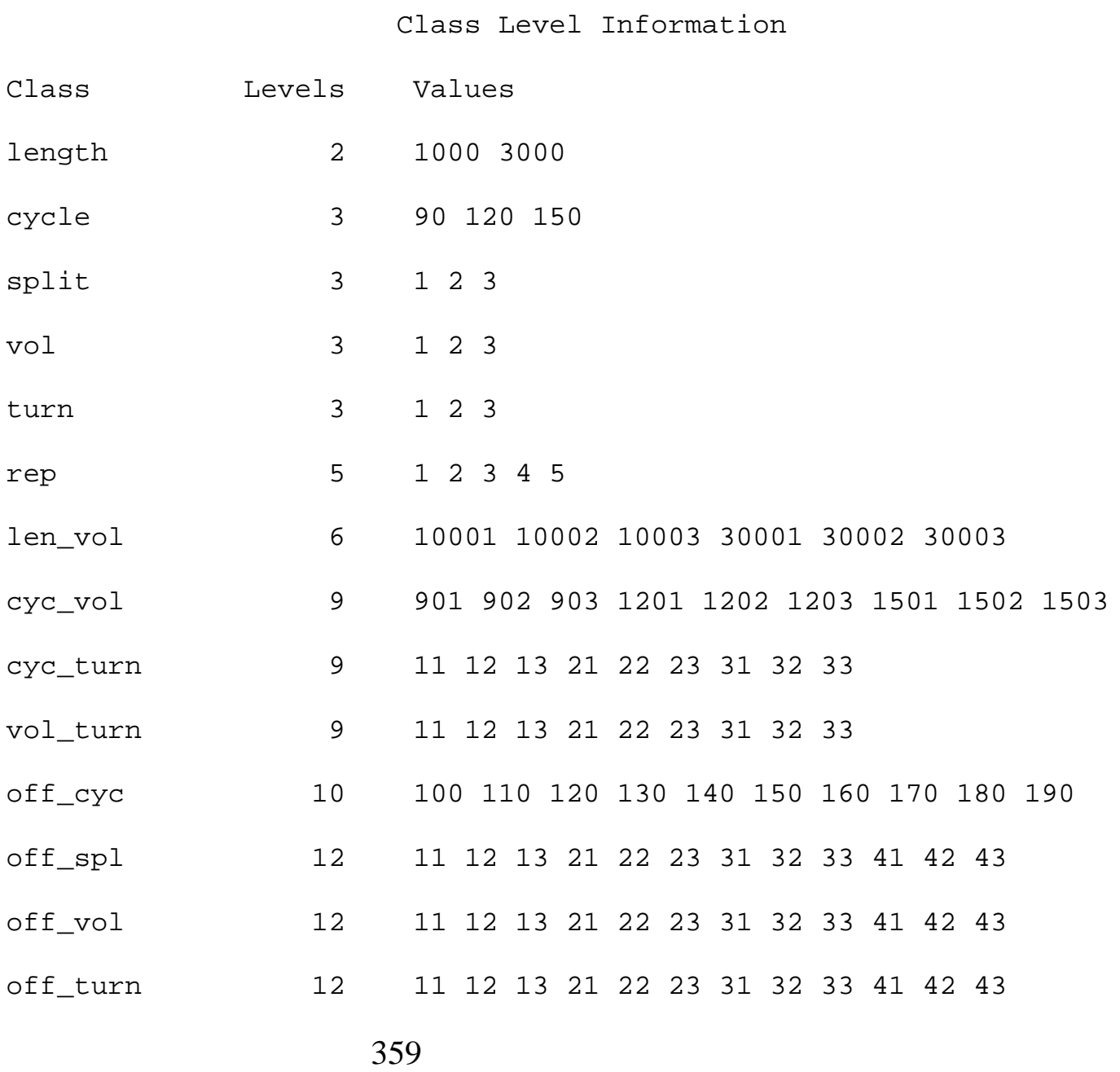




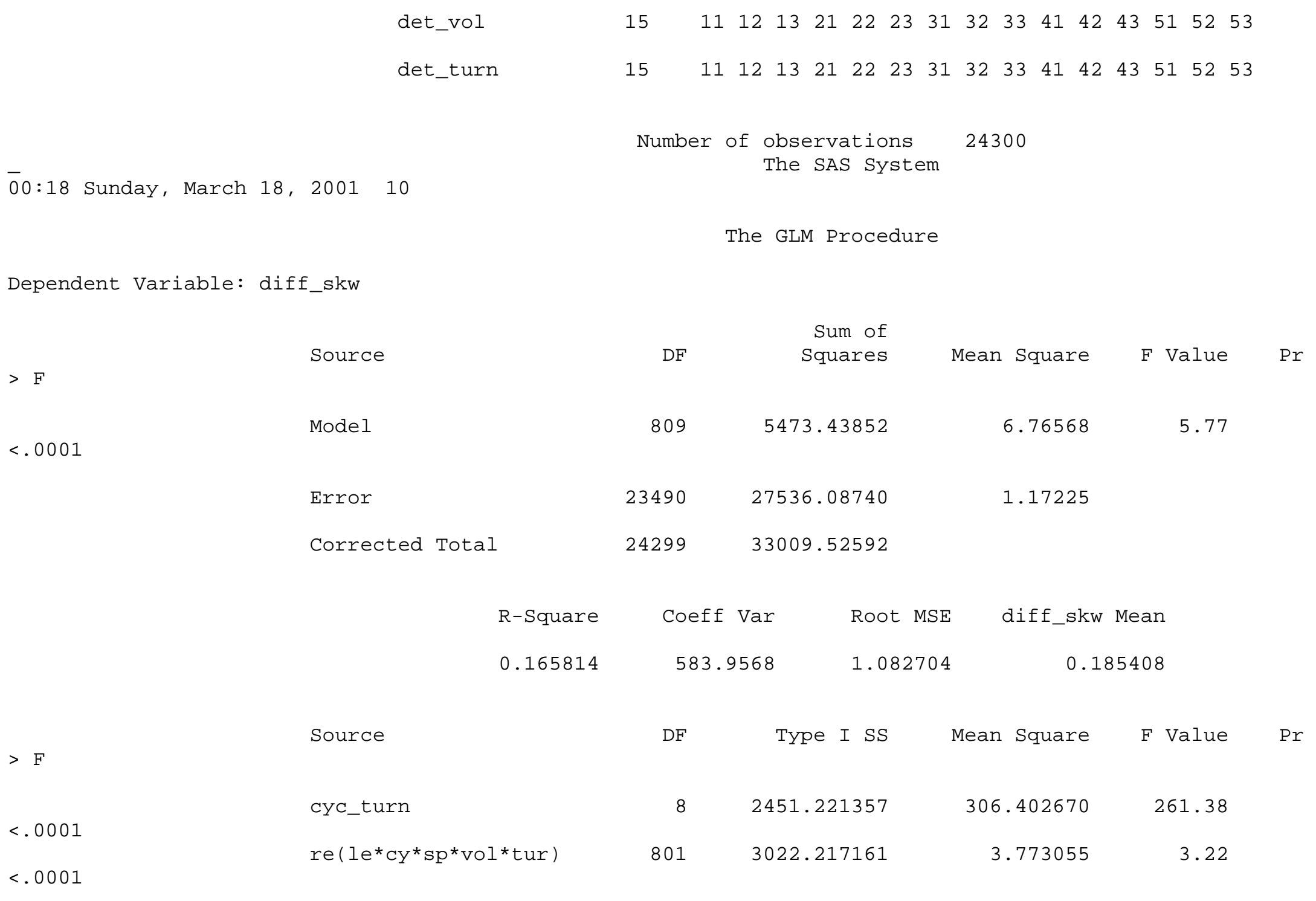




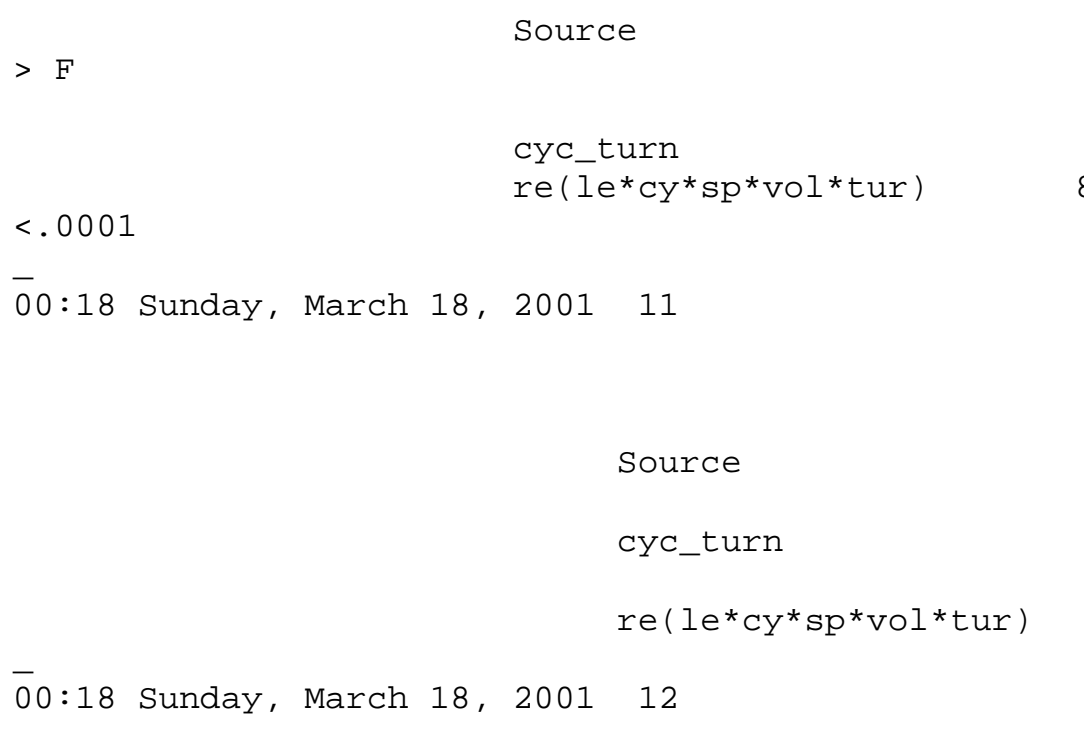

$\mathrm{DF}$

$\begin{array}{rr}0 & 0.000000 \\ 801 & 3022.017161\end{array}$

3022.217161

The SAS System

The GLM Procedure

Type III Expected Mean Square

0

$\operatorname{Var}($ Error $)+30 \operatorname{Var}\left(r e\left(1 e^{*} \mathrm{Cy}{ }^{*} \mathrm{sp}{ }^{*} \operatorname{Vol}{ }^{*} \operatorname{tur}\right)\right)$

The SAS system

The GLM Procedure

Student-Newman-Keuls Test for diff_skw

NOTE: This test controls the Type I experimentwise error rate under the complete null hypothesis but not under partial null

hypotheses.

$\begin{array}{lr}\text { Alpha } & 0.05 \\ \text { Error Degrees of Freedom } & 801 \\ \text { Error Mean Square } & 3.773055\end{array}$

Number of Means

2

3

4

5

6 
Critical Range 0.1037731

0.1241342

0.1361038

0.1445383

0.1510204

0.1562654

$0.1606582 \quad 0.1644293$

Means with the same letter are not significantly different.

SNK Groupin



$F$
$F$
$F$
$F$

00:18 Sunday, March 18, 200113

$\begin{array}{ccc}\text { Mean } & \mathrm{N} & \text { cyc_turn } \\ 0.77516 & 2700 & 11 \\ 0.52115 & 2700 & 12 \\ 0.37260 & 2700 & 13 \\ 0.34088 & 2700 & 21 \\ 0.13693 & 2700 & 22 \\ -0.02952 & 2700 & 23 \\ -0.10452 & 2700 & 31 \\ -0.16739 & 2700 & 32 \\ -0.17660 & 2700 & 33 \\ \text { The SAS } & \text { System } & \end{array}$

The GLM Procedure

Class Level Information

$\begin{array}{lrl}\text { Class } & \text { Levels } & \text { Values } \\ \text { length } & 2 & 1000 \quad 3000 \\ \text { cycle } & 3 & 90120 \quad 150\end{array}$




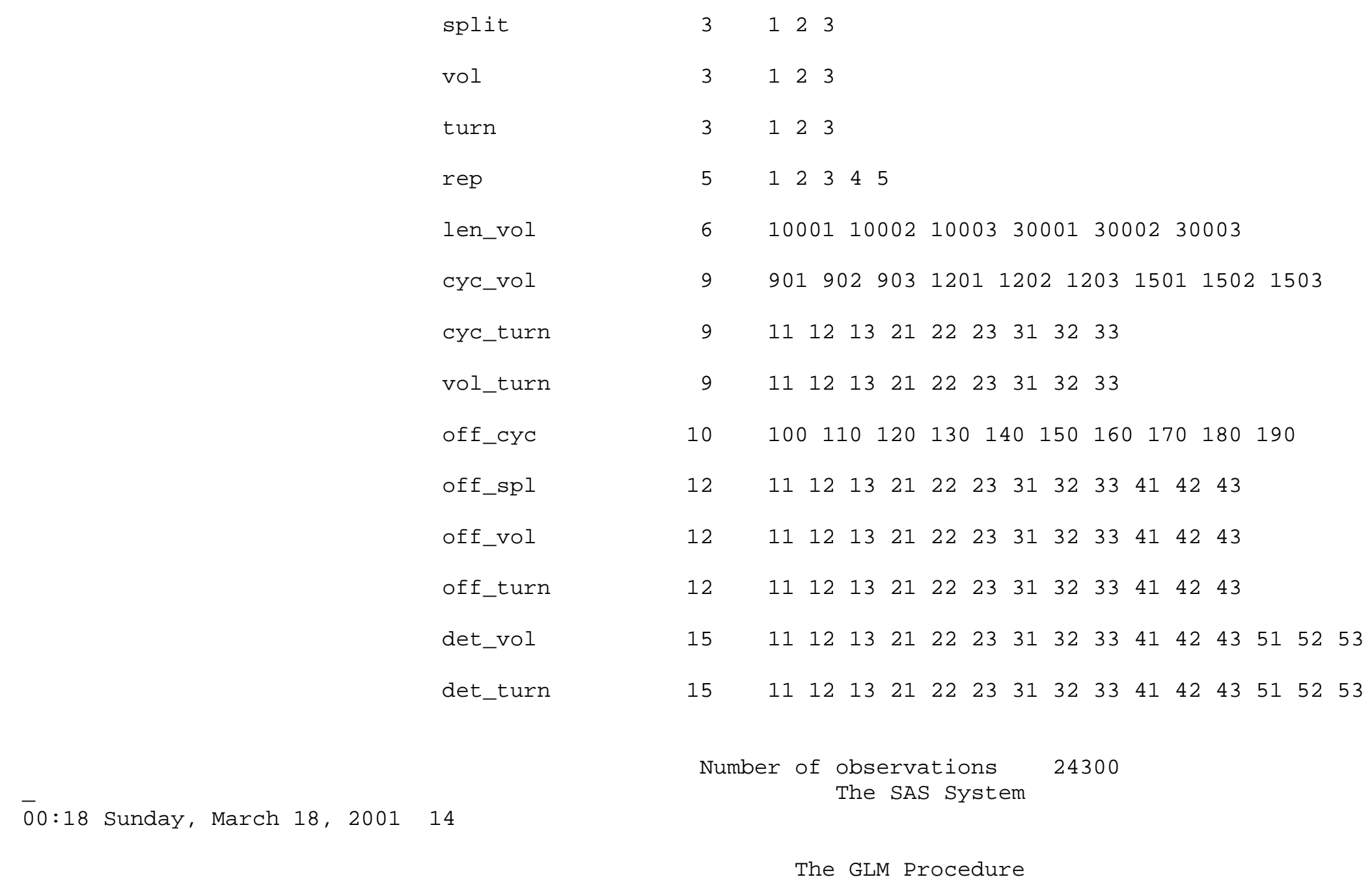




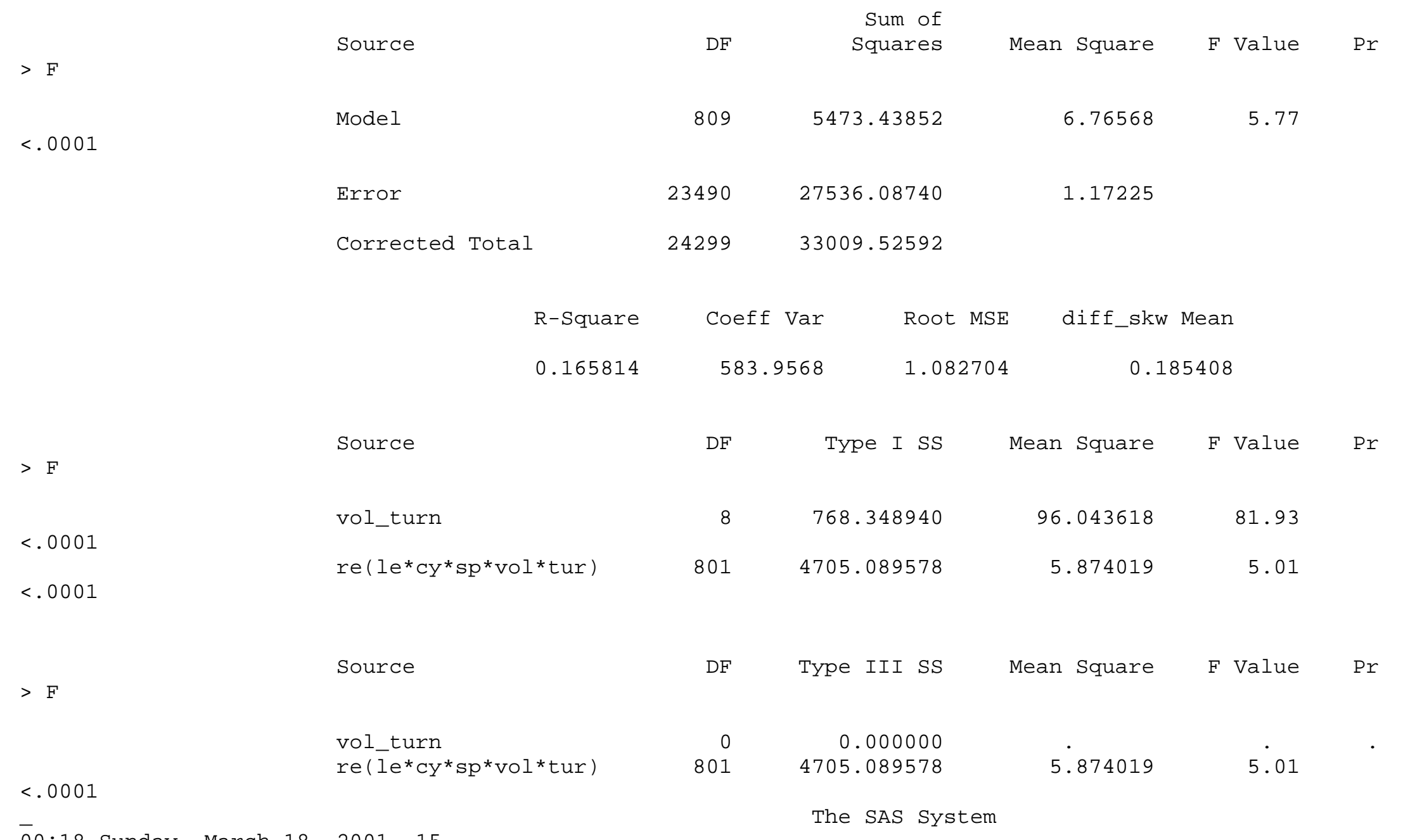

The GLM Procedure 
vol_turn

$r e\left(l e^{*} \mathrm{cy}^{\star} \mathrm{sp}^{*} \operatorname{vol}{ }^{*} \operatorname{tur}\right)$

00:18 Sunday, March 18, 2001
0

$\operatorname{Var}($ Error $)+30 \operatorname{Var}\left(r e\left(1 e^{*} \mathrm{cy}{ }^{\star} \mathrm{sp}{ }^{\star} \operatorname{Vol}{ }^{*} \operatorname{tur}\right)\right)$

The SAS system

The GLM Procedure

Student-Newman-Keuls Test for diff_skw

NOTE: This test controls the Type I experimentwise error rate under the complete null hypothesis but not under partial null

hypotheses.

$\begin{array}{lr}\text { Alpha } & 0.05 \\ \text { Error Degrees of Freedom } & 801 \\ \text { Error Mean Square } & 5.874019\end{array}$

Number of Means

8

0.2004582
Critical Range

0.2051635
0.129481

0.1548861

3

0.169821

0.1803449
Means with the same letter are not significantly different.

SNK Grouping

$\begin{array}{rrr}\text { Mean } & \text { N } & \text { vol_turn } \\ 0.47176 & 2700 & 11 \\ 0.44895 & 2700 & 21 \\ 0.31687 & 2700 & 12\end{array}$

B $\quad$ A 
$\overline{0} 0: 18$ Sunday, March 18, 200117

Class
length
cycle
split
vol
turn
offset2
rep
len_vol
Cyc_vol

$\begin{array}{llccc}\text { B } & \text { C } & 0.18949 & 2700 & 22 \\ & \text { C } & & & \\ \text { D } & \text { C } & 0.13205 & 2700 & 13 \\ \text { D } & \text { C } & & & \\ \text { D } & \text { C } & 0.09081 & 2700 & 31 \\ \text { D } & \text { C } & & & \\ \text { D } & \text { C } & 0.07645 & 2700 & 23 \\ \text { D } & & -0.01567 & 2700 & 32 \\ \text { D } & & & \\ \text { D } & & -0.04202 & 2700 & 33 \\ \text { D } & & \text { The SAS System } & \end{array}$

The GLM Procedure

Class Level Information

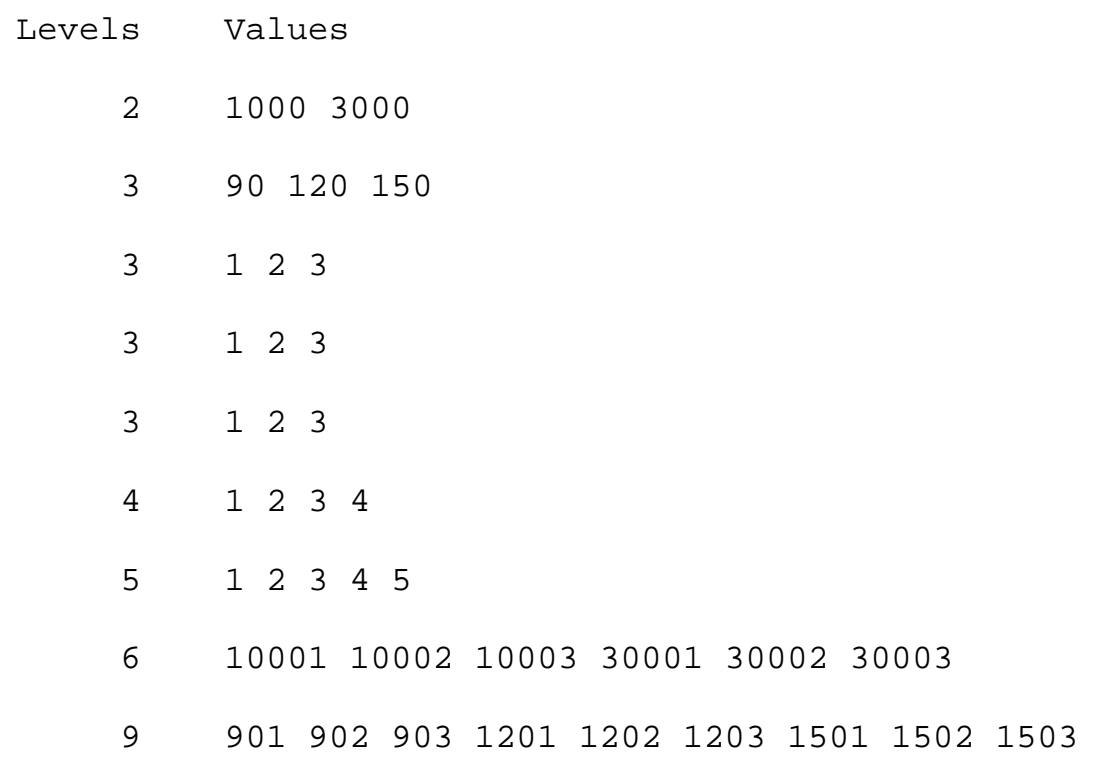




$\begin{array}{lccccccccccccccccccc}\text { cyc_turn } & 9 & 11 & 12 & 13 & 21 & 22 & 23 & 31 & 32 & 33 & & & \\ \text { vol_turn } & 9 & 11 & 12 & 13 & 21 & 22 & 23 & 31 & 32 & 33 & & & \\ \text { off_cyc } & 10 & 10 & 110 & 120 & 130 & 140 & 150 & 160 & 170 & 180 & 190 & \\ \text { off_spl } & 12 & 11 & 12 & 13 & 21 & 22 & 23 & 31 & 32 & 33 & 41 & 42 & 43 & & \\ \text { off_vol } & 12 & 11 & 12 & 13 & 21 & 22 & 23 & 31 & 32 & 33 & 41 & 42 & 43 & & \\ \text { off_turn } & 12 & 11 & 12 & 13 & 21 & 22 & 23 & 31 & 32 & 33 & 41 & 42 & 43 & & \\ \text { det_vol } & 15 & 11 & 12 & 13 & 21 & 22 & 23 & 31 & 32 & 33 & 41 & 42 & 43 & 51 & 52 & 53 \\ \text { det_turn } & 15 & 11 & 12 & 13 & 21 & 22 & 23 & 31 & 32 & 33 & 41 & 42 & 43 & 51 & 52 & 53\end{array}$

$\overline{0} 0: 18$ Sunday, March 18, 200118

Number of observations 24300
The SAS System

The GLM Procedure

Dependent Variable: diff_skw

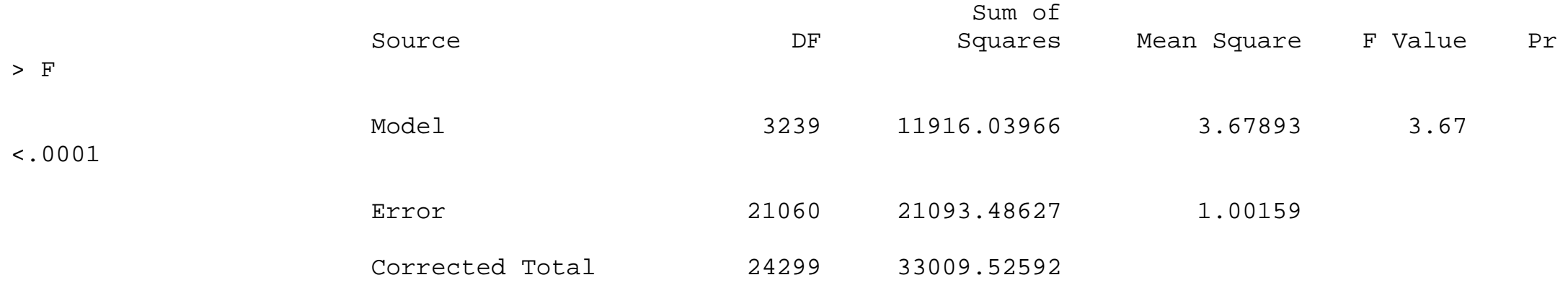

Coeff Var Root MSE diff_skw Mean

367 


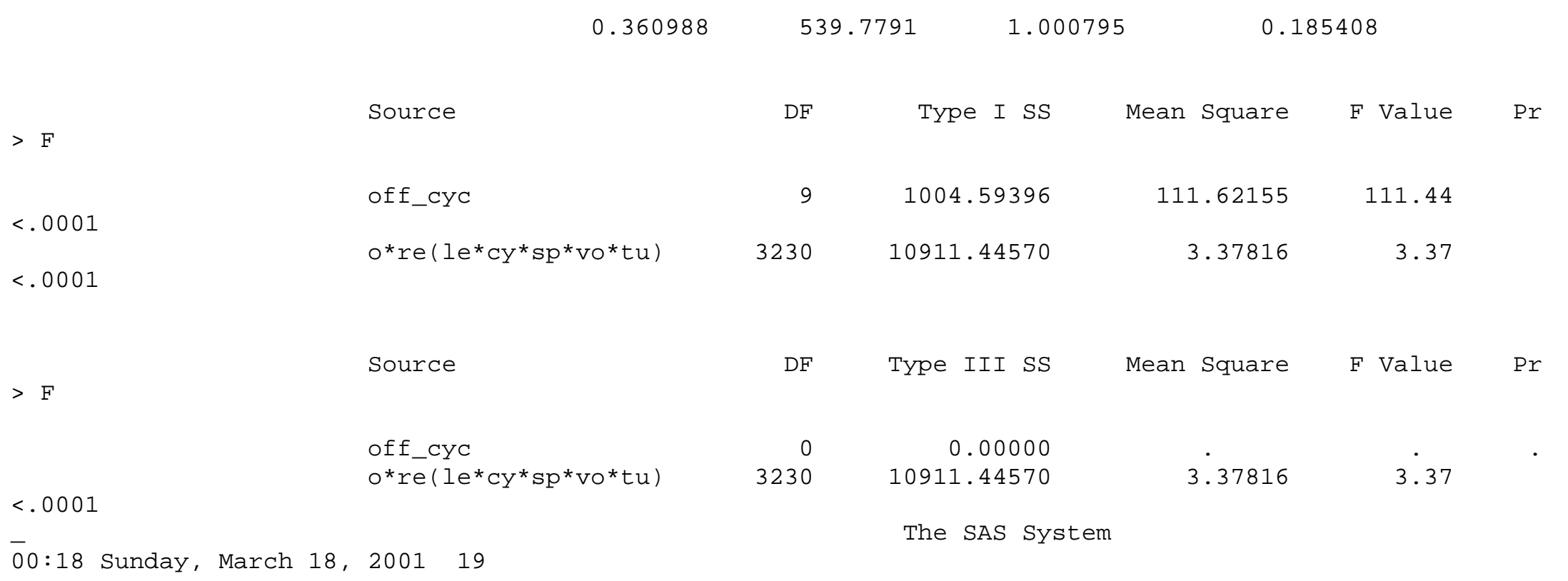

The GLM Procedure

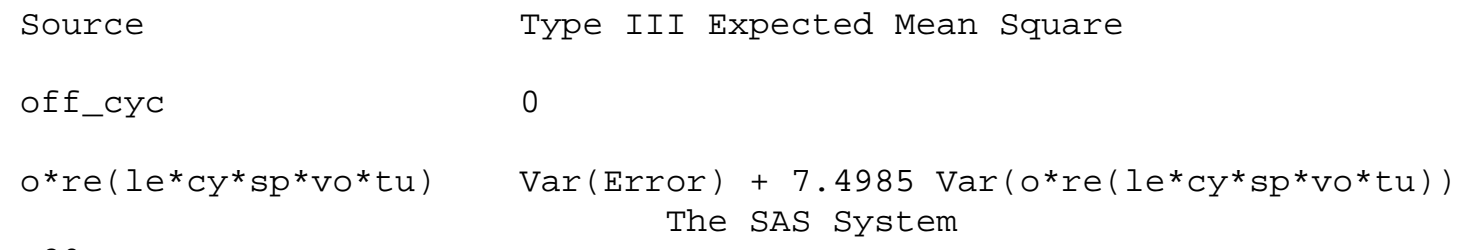

The GLM Procedure

Student-Newman-Keuls Test for diff_skw

NOTE: This test controls the Type I experimentwise error rate under the complete null hypothesis but not under partial null 
hypotheses.

$\begin{array}{lr}\text { Alpha } & 0.05 \\ \text { Error Degrees of Freedom } & 3230 \\ \text { Error Mean Square } & 3.378157 \\ \text { Harmonic Mean of Cell Sizes } & 2250\end{array}$

NOTE: Cell sizes are not equal.

$\begin{array}{lccccc}\text { Number of } \begin{array}{l}\text { Means } \\ 8\end{array} & 2 & 3 & 4 & 5 & 7 \\ \text { Critical Range } & 0.1074422 & 0.1284892 & 0.1408508 & 0.1495636 & 0.1562536 \\ 0.1661959 & 0.1700848 & 0.1734855 & & \end{array}$

Means with the same letter are not significantly different.

\begin{tabular}{|c|c|c|c|c|c|}
\hline \multirow[t]{15}{*}{ SNK } & \multicolumn{2}{|c|}{ Grouping } & Mean & $\mathrm{N}$ & off_cyc \\
\hline & & A & 0.66530 & 1350 & 190 \\
\hline & & B & 0.36566 & 2700 & 150 \\
\hline & & B & & & \\
\hline & & B & 0.34408 & 2700 & 180 \\
\hline & & B & & & \\
\hline & $\mathrm{C}$ & B & 0.30229 & 2700 & 120 \\
\hline & \multicolumn{5}{|c|}{ (6) } \\
\hline & \multirow[t]{7}{*}{$\mathrm{C}$} & $\mathrm{D}$ & 0.20905 & 2700 & 160 \\
\hline & & D & & & \\
\hline & & D & 0.15675 & 2700 & 130 \\
\hline & & D & & & \\
\hline & & $\mathrm{D}$ & 0.11910 & 1350 & 100 \\
\hline & & D & & & \\
\hline & & $\mathrm{D}$ & 0.11717 & 2700 & 110 \\
\hline
\end{tabular}


$\overline{0} 0: 18$ Sunday, March 18, 2001 21

Class
length
cycle
split
vol
turn
offset2
rep
len_vol
cyc_vol
cyc_turn
vol_turn
off_cyc
off_spl

$\begin{array}{rrrr}E & -0.06633 & 2700 & 140 \\ E & & & \\ E & -0.15222 & 2700 & 170 \\ & \text { The SAS } & \text { System } & \end{array}$

The GLM Procedure

Class Level Information

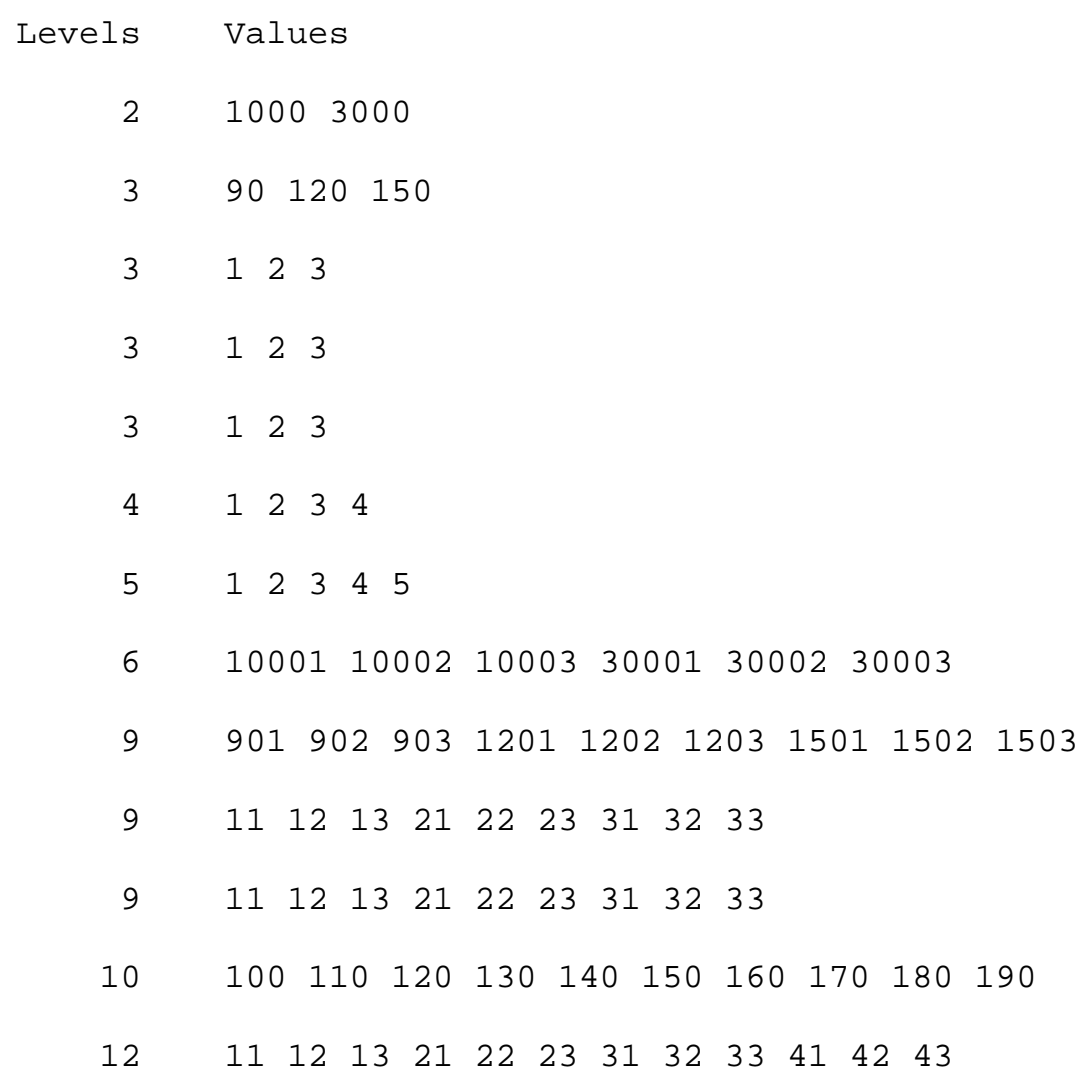




$$
\begin{aligned}
& \text { off_vol } \\
& \text { off_turn } \\
& \text { det_vol } \\
& \text { det_turn }
\end{aligned}
$$

$\overline{0} 0: 18$ Sunday, March 18, 2001

22

Dependent Variable: diff_skw

$\begin{array}{ll}>F & \text { Source } \\ & \\ <.0001 & \text { Model } \\ & \text { Error } \\ & \text { Corrected Total }\end{array}$

Corrected Total

Source $\begin{array}{llllllllllll}11 & 12 & 13 & 21 & 22 & 23 & 31 & 32 & 33 & 41 & 42 & 43\end{array}$

$\begin{array}{llllllllllll}11 & 12 & 13 & 21 & 22 & 23 & 31 & 32 & 33 & 41 & 42 & 43\end{array}$

$\begin{array}{lllllllllllllll}11 & 12 & 13 & 21 & 22 & 23 & 31 & 32 & 33 & 41 & 42 & 43 & 51 & 52 & 53\end{array}$

$\begin{array}{lllllllllllllll}11 & 12 & 13 & 21 & 22 & 23 & 31 & 32 & 33 & 41 & 42 & 43 & 51 & 52 & 53\end{array}$

Number of observations

24300

The GLM Procedure

Sum of

DF Squares

Mean Square F Value

Pr

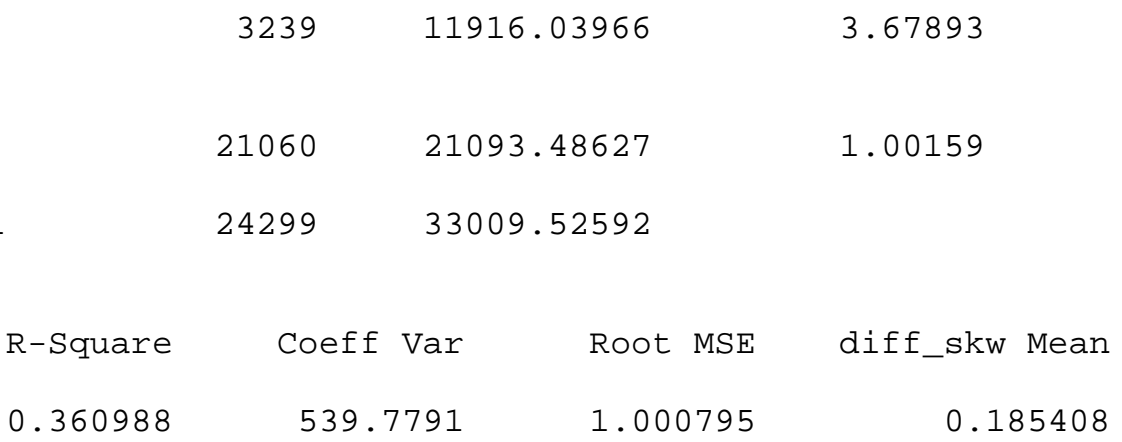

$\mathrm{DF}$

Type I SS

Mean Square

F Value

$\operatorname{Pr}$ 


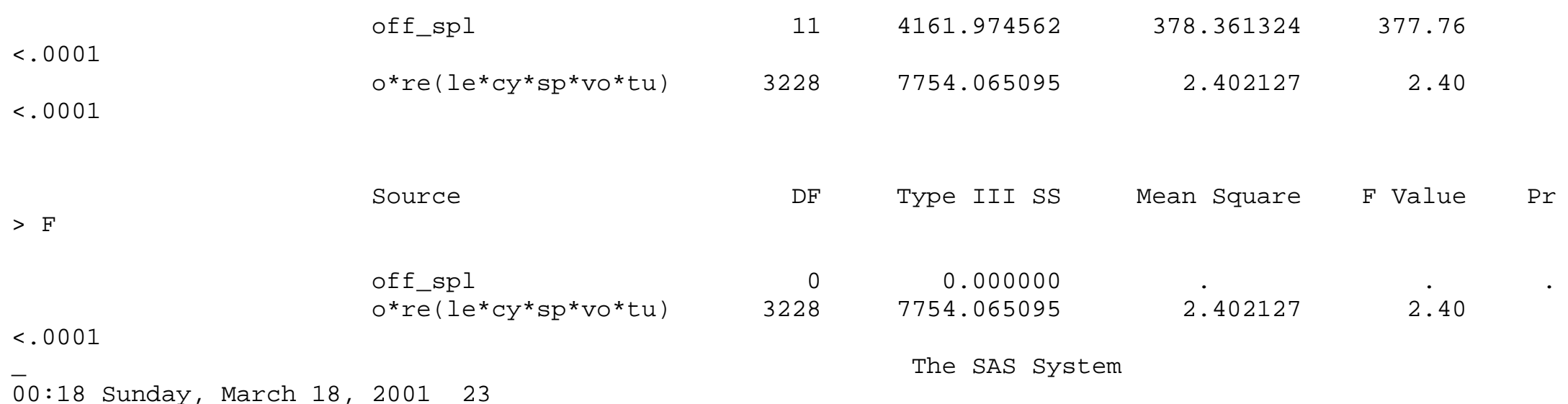

The GLM Procedure

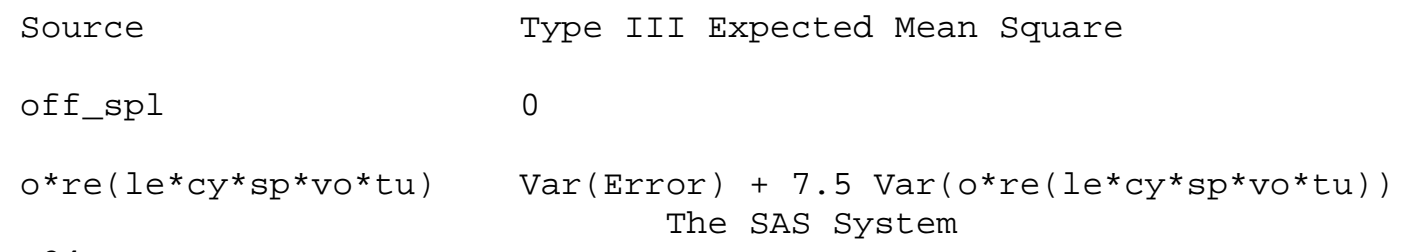

$\overline{0} 0: 18$ Sunday, March 18, 200124

The GLM Procedure

Student-Newman-Keuls Test for diff_skw

NOTE: This test controls the Type I experimentwise error rate under the complete null hypothesis but not under partial null

hypotheses.

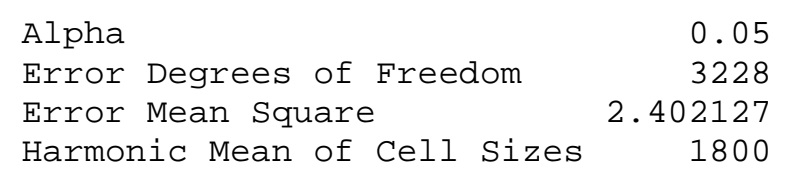


NOTE: Cell sizes are not equal.

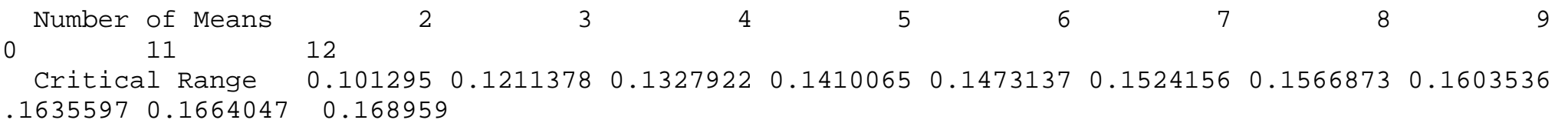

Means with the same letter are not significantly different.

\begin{tabular}{|c|c|c|c|c|}
\hline SNK & Grouping & Mean & $\mathrm{N}$ & off_spl \\
\hline & A & 1.05657 & 1350 & 41 \\
\hline & B & 0.90610 & 2700 & 31 \\
\hline & $\mathrm{C}$ & 0.75365 & 1350 & 42 \\
\hline & $\mathrm{D}$ & 0.24939 & 2700 & 32 \\
\hline & $\mathrm{D}$ & & & \\
\hline & $\mathrm{D}$ & 0.15692 & 1350 & 11 \\
\hline & $\mathrm{D}$ & & & \\
\hline & $\mathrm{D}$ & 0.15607 & 2700 & 21 \\
\hline & $\mathrm{E}$ & -0.11384 & 1350 & 12 \\
\hline & $\mathrm{E}$ & & & \\
\hline & $\mathrm{E}$ & -0.12101 & 2700 & 22 \\
\hline & $\mathrm{E}$ & & & \\
\hline & $\mathrm{E}$ & -0.13642 & 2700 & 23 \\
\hline & $\mathrm{E}$ & & & \\
\hline & $\mathrm{E}$ & -0.14345 & 2700 & 33 \\
\hline & $\mathrm{E}$ & & & \\
\hline & $\mathrm{E}$ & -0.16031 & 1350 & 13 \\
\hline & $\mathrm{E}$ & & & \\
\hline & $\mathrm{E}$ & -0.17698 & 1350 & 43 \\
\hline
\end{tabular}


$\overline{0} 0: 18$ Sunday, March 18, 2001

25

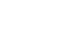

\section{Class}

length

cycle

split

vol

turn

offset 2

rep

len_vol

cyc_vol

cyc_turn

vol_turn

off_cyc

off_spl

off_vol

off_turn
The SAS system

The GLM Procedure

Class Level Information

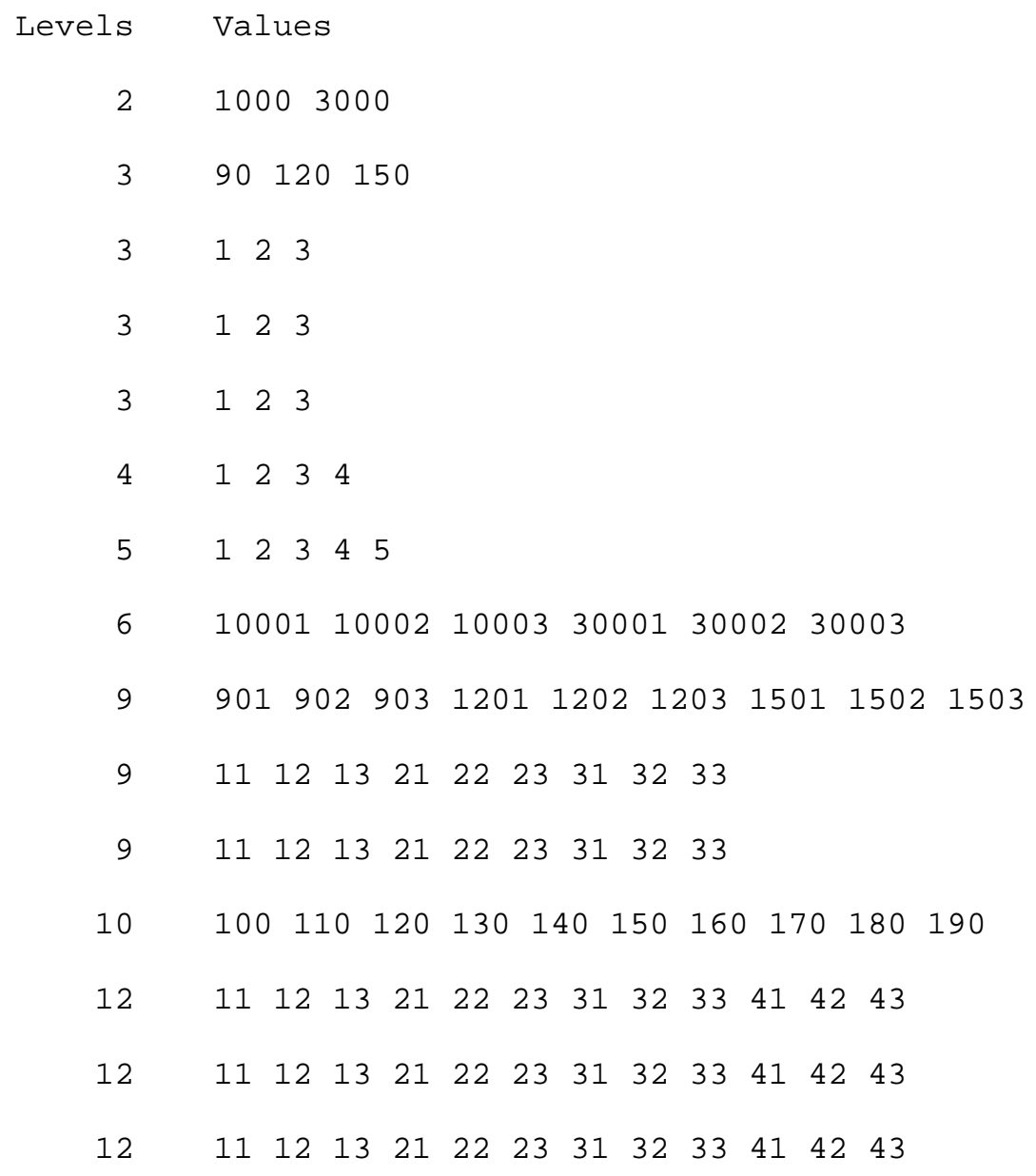




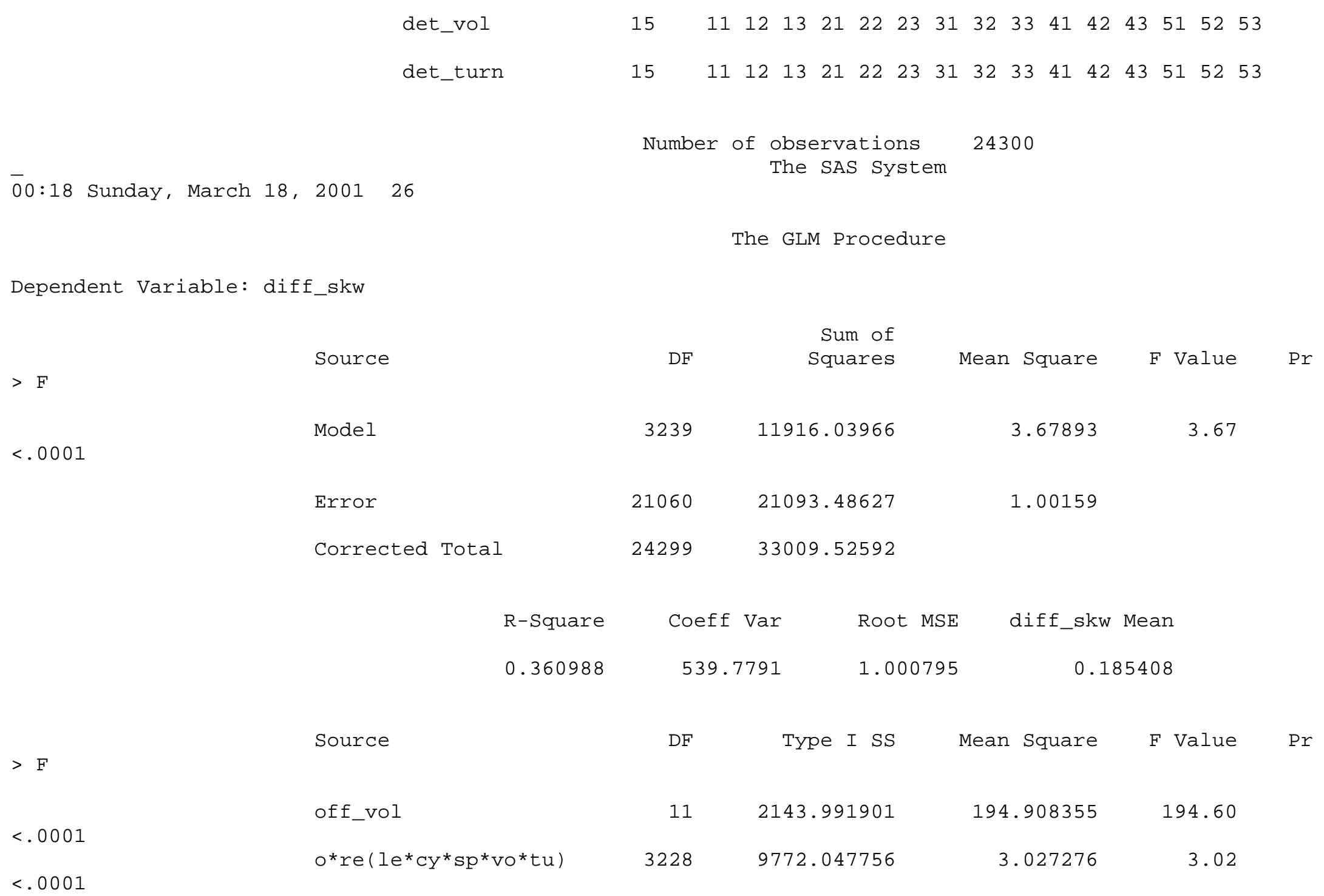




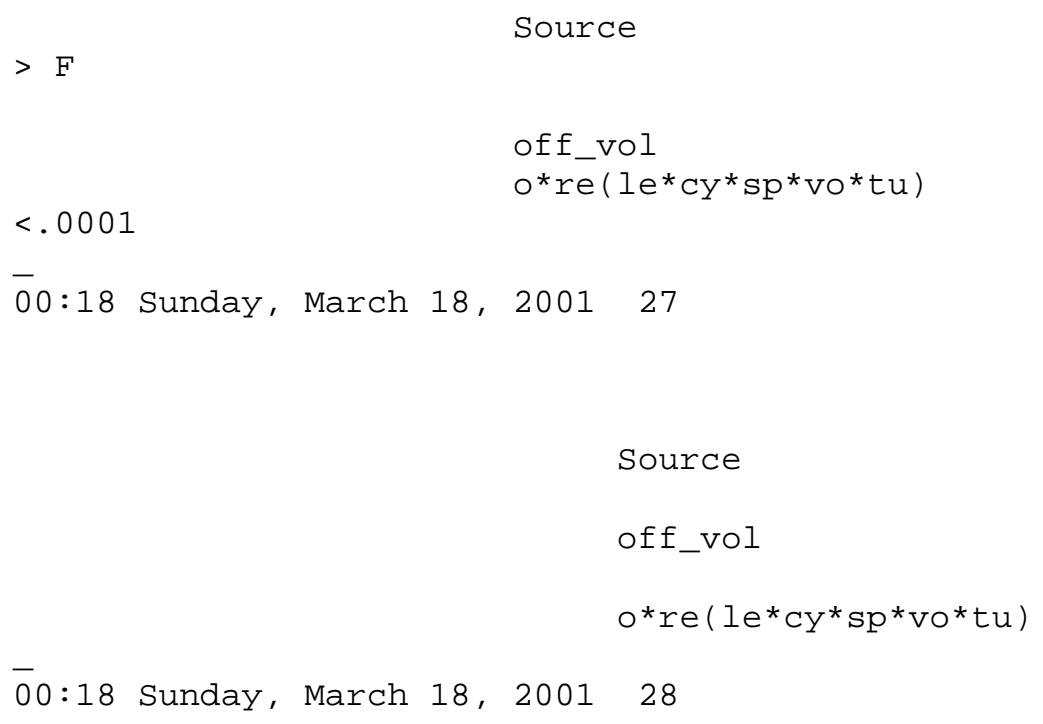




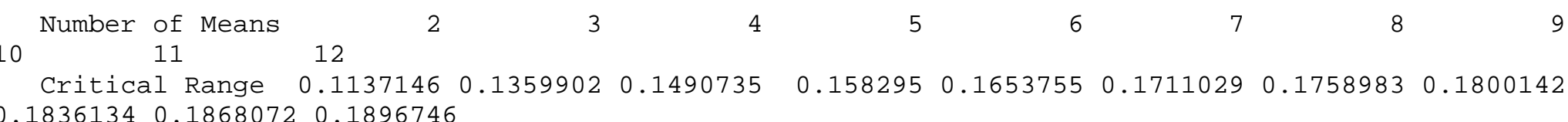
0.18361340 .18680720 .1896746

Means with the same letter are not significantly different.

\begin{tabular}{|c|c|c|c|c|}
\hline \multirow{21}{*}{ SNK } & \multirow{2}{*}{$\begin{array}{r}\text { Grouping } \\
\text { A }\end{array}$} & Mean & $\mathrm{N}$ & off_vol \\
\hline & & 0.83695 & 1350 & 41 \\
\hline & B & 0.68626 & 1350 & 42 \\
\hline & B & & & \\
\hline & B & 0.58699 & 2700 & 31 \\
\hline & $\mathrm{C}$ & 0.39955 & 2700 & 32 \\
\hline & $\mathrm{D}$ & 0.11003 & 1350 & 43 \\
\hline & $\mathrm{D}$ & & & \\
\hline & $\mathrm{D}$ & 0.02549 & 2700 & 33 \\
\hline & $\mathrm{D}$ & & & \\
\hline & $\mathrm{D}$ & -0.01599 & 2700 & 22 \\
\hline & $\mathrm{D}$ & & & \\
\hline & $\mathrm{D}$ & -0.02362 & 1350 & 12 \\
\hline & $\mathrm{D}$ & & & \\
\hline & $\mathrm{D}$ & -0.03007 & 2700 & 23 \\
\hline & $\mathrm{D}$ & & & \\
\hline & $\mathrm{D}$ & -0.03465 & 1350 & 13 \\
\hline & $\mathrm{D}$ & & & \\
\hline & $\mathrm{D}$ & -0.05531 & 2700 & 21 \\
\hline & $\mathrm{D}$ & & & \\
\hline & $\mathrm{D}$ & -0.05896 & 1350 & 11 \\
\hline
\end{tabular}

$\overline{0} 0: 18$ Sunday, March 18, 200129

The GLM Procedure 
Class Level Information

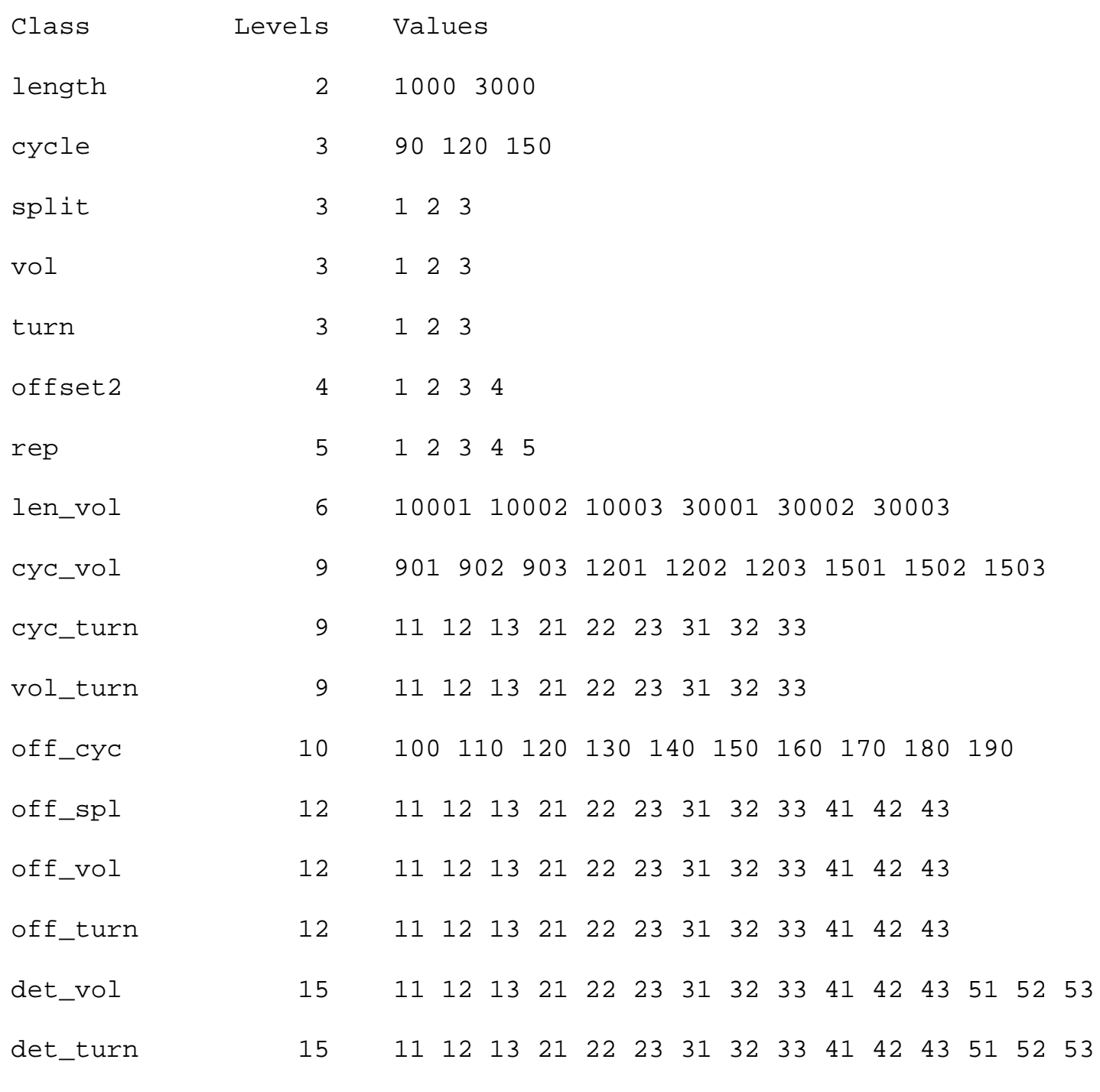


$\overline{0} 0: 18$ Sunday, March 18, 200130

Dependent Variable: diff_skw

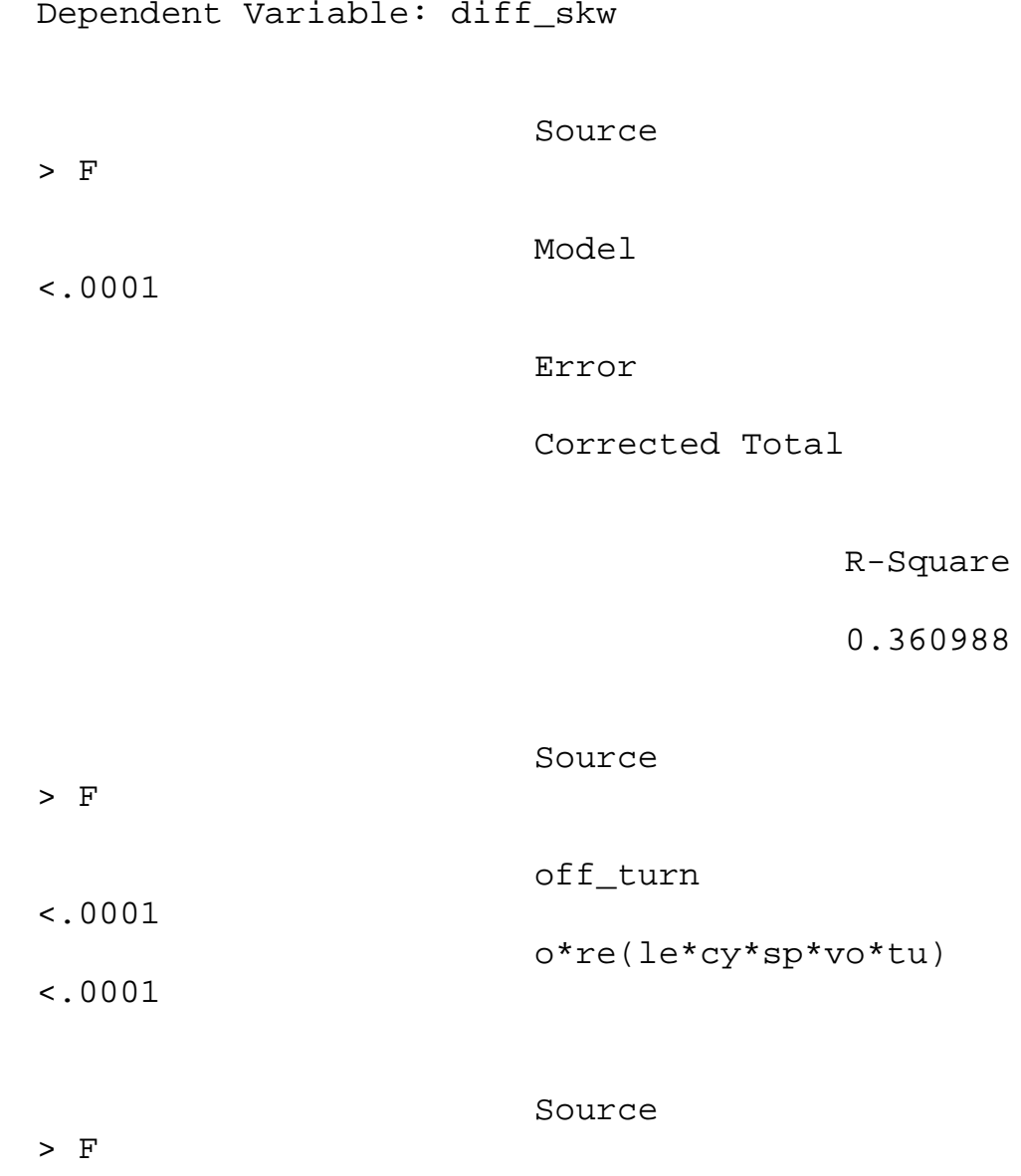

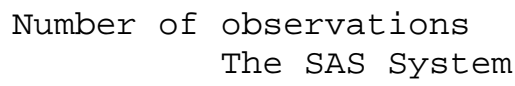

The GLM Procedure

$\mathrm{DF}$

Type III SS
24300

$\begin{array}{rrrrr}\text { DF } & \begin{array}{r}\text { Sum of } \\ \text { Squares }\end{array} & \text { Mean Square } & \text { F Value } & \text { Pr } \\ 3239 & 11916.03966 & 3.67893 & 3.67 \\ 21060 & 21093.48627 & 1.00159 & \\ 24299 & 33009.52592 & & \end{array}$

$\begin{array}{rrr}\text { Coeff Var } & \text { Root MSE } & \text { diff_skw Mean } \\ 539.7791 & 1.000795 & 0.185408\end{array}$

$\begin{array}{rrrrr}\text { DF } & \text { Type I SS } & \text { Mean Square } & \text { F Value } & \text { Pr } \\ 11 & 1781.25914 & 161.93265 & 161.68 \\ 3228 & 10134.78052 & 3.13965 & 3.13\end{array}$

Mean Square

F Value

Pr 
The GLM Procedure

\section{Source \\ off_turn \\ $o * r e\left(l e{ }^{*} \mathrm{Cy}^{\star} \mathrm{sp} \mathrm{*}^{*} \mathrm{vo} t u\right)$}

$\overline{0} 0: 18$ Sunday, March 18, 2001
Type III Expected Mean Square

0

$\operatorname{Var}($ Error $)+7.5 \operatorname{Var}\left(0^{\star} r e\left(1 \mathrm{e}^{\star} \mathrm{Cy}^{\star} \mathrm{Sp}^{\star} \mathrm{Vo}^{\star} \mathrm{tu}\right)\right)$

The SAS System

The GLM Procedure

Student-Newman-Keuls Test for diff_skw

NOTE: This test controls the Type I experimentwise error rate under the complete null hypothesis but not under partial null

hypotheses .

$\begin{array}{lr}\text { Alpha } & 0.05 \\ \text { Error Degrees of Freedom } & 3228 \\ \text { Error Mean Square } & 3.139647 \\ \text { Harmonic Mean of Cell Sizes } & 1800 \\ & \\ \text { NOTE: Cell sizes are not equal. }\end{array}$

Number of Means $11 \quad 12$

2 3

4

5

6

7

8

Critical Range $0.1158058 \quad 0.13849120 .15181510 .16120620 .16841690 .17424960 .17913320 .1833248$ $0.1869902 \quad 0.1902427 \quad 0.1931629$ 
Means with the same letter are not significantly different.

00:18 Sunday, March 18, 2001

33

SNK






E
E
E

A

$B$

B

C

C

D

D

D

D

A
B
B
B
C
C
C
D
D
D
D
D
G
G
G
G
G
G
G

G

G

G

G

\begin{tabular}{|c|c|}
\hline Mean & $\mathrm{N}$ \\
\hline 0.83415 & 1350 \\
\hline 0.53374 & 1350 \\
\hline 0.51314 & 2700 \\
\hline 0.36356 & 2700 \\
\hline 0.26536 & 1350 \\
\hline 0.13533 & 2700 \\
\hline 0.09503 & 1350 \\
\hline 0.03378 & 2700 \\
\hline-0.03167 & 2700 \\
\hline-0.07253 & 1350 \\
\hline-0.10348 & 2700 \\
\hline-0.13973 & 1350 \\
\hline
\end{tabular}

The GLM Procedure

Class Level Information

Class Levels Values 


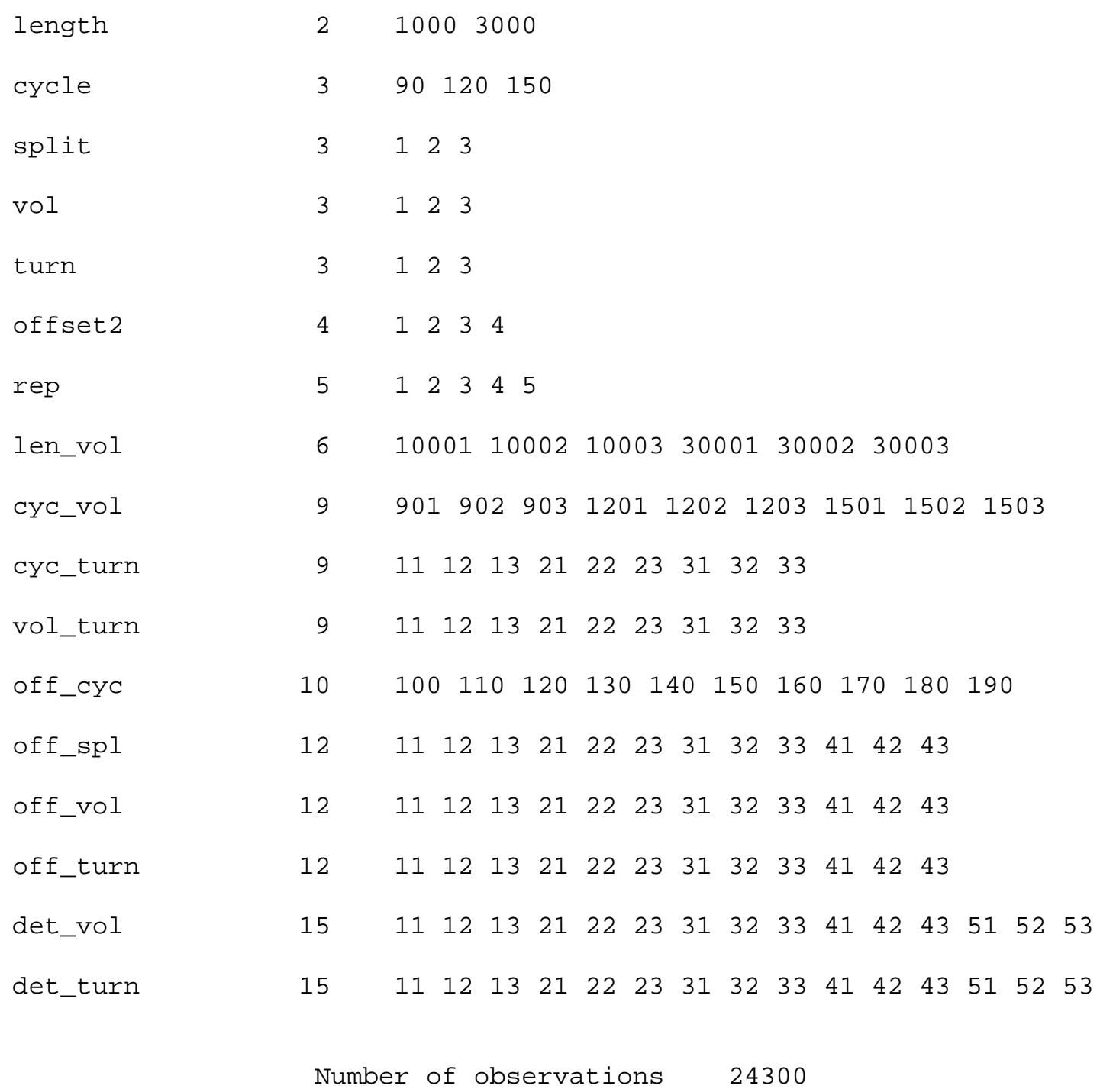


The GLM Procedure

Dependent Variable: diff_skw

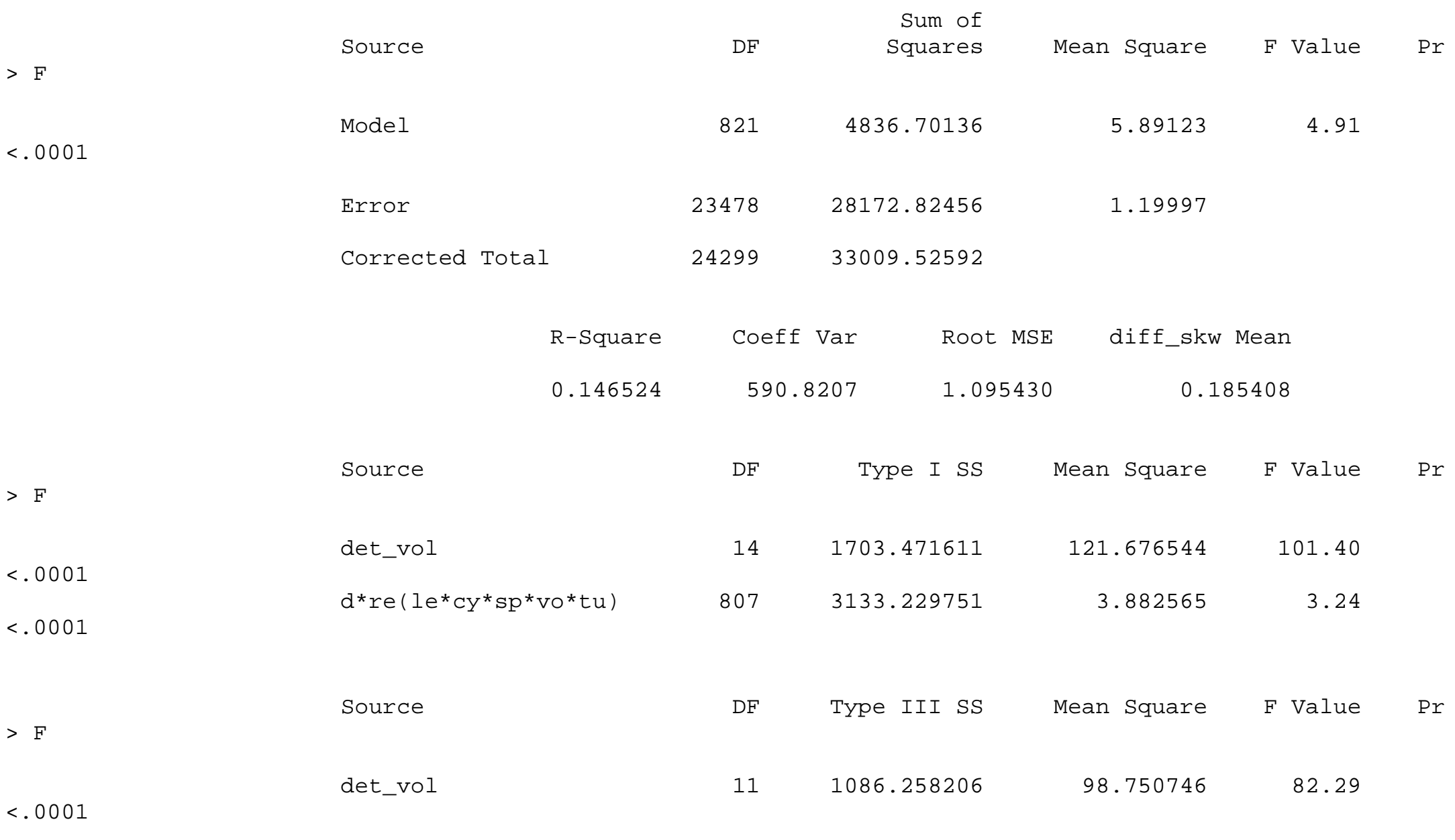


$<.0001$

$d{ }^{\star} r e\left(l e e^{*} c{ }^{*} s p{ }^{*}\right.$ vo*tu $)$

00:18 Sunday, March 18, 2001

35
807

3133.229751

3.882565

3.24

The SAS system

The GLM Procedure

Student-Newman-Keuls Test for diff_skw

NOTE: This test controls the Type I experimentwise error rate under the complete null hypothesis but not under partial null

hypotheses.

\begin{tabular}{|c|c|c|c|c|c|}
\hline & & $\begin{array}{l}\text { Alpha } \\
\text { Error } \\
\text { Error }\end{array}$ & $\begin{array}{l}\text { Degrees of } \\
\text { Mean Square }\end{array}$ & 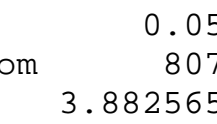 & \\
\hline Number of Means & 2 & 3 & 4 & 5 & 6 \\
\hline $\begin{array}{cc}\text { Critical } & \text { Range } \\
0.2046406 & 0.210393\end{array}$ & 0.1358992 & 0.1625633 & 0.1782382 & 0.1892834 & 0.1977721 \\
\hline $\begin{array}{c}\text { Number of Means } \\
15\end{array}$ & 9 & 10 & 11 & 12 & 13 \\
\hline $\begin{array}{cl}\text { Critical } & \text { Range } \\
0.2329004 & 0.2355256\end{array}$ & 0.2153314 & 0.2196508 & 0.2234866 & 0.2269267 & 0.2300478 \\
\hline
\end{tabular}

Means with the same letter are not significantly different.

\begin{tabular}{|c|c|c|c|c|}
\hline SNK & Grouping & Mean & $\mathrm{N}$ & det_vol \\
\hline & A & 0.61713 & 1620 & 31 \\
\hline & A & & & \\
\hline & B & 0.53475 & 1620 & 41 \\
\hline
\end{tabular}




\begin{tabular}{|c|c|c|c|c|}
\hline B & & & & \\
\hline B & & 0.41301 & 1620 & 21 \\
\hline B & & & & \\
\hline B & & 0.41220 & 1620 & 22 \\
\hline B & & & & \\
\hline B & & 0.39686 & 1620 & 51 \\
\hline B & & & & \\
\hline B & & 0.38865 & 1620 & 12 \\
\hline & C & 0.21822 & 1620 & 32 \\
\hline & C & & & \\
\hline D & C & 0.11791 & 1620 & 42 \\
\hline D & C & & & \\
\hline D & C & 0.06014 & 1620 & 13 \\
\hline D & C & & & \\
\hline D & C & 0.05449 & 1620 & 52 \\
\hline D & & & & \\
\hline D & & 0.01189 & 1620 & 53 \\
\hline D & & & & \\
\hline D & & 0.01057 & 1620 & 43 \\
\hline D & & & & \\
\hline D & & -0.00951 & 1620 & 33 \\
\hline D & & & & \\
\hline D & & -0.01790 & 1620 & 23 \\
\hline & $E$ & -0.42728 & 1620 & 11 \\
\hline & & The SAS & ystem & \\
\hline
\end{tabular}

The GLM Procedure

Class Level Information

$\begin{array}{lrl}\text { Class } & \text { Levels } & \text { Values } \\ \text { length } & 2 & 10003000 \\ \text { cycle } & 3 & 90120150\end{array}$




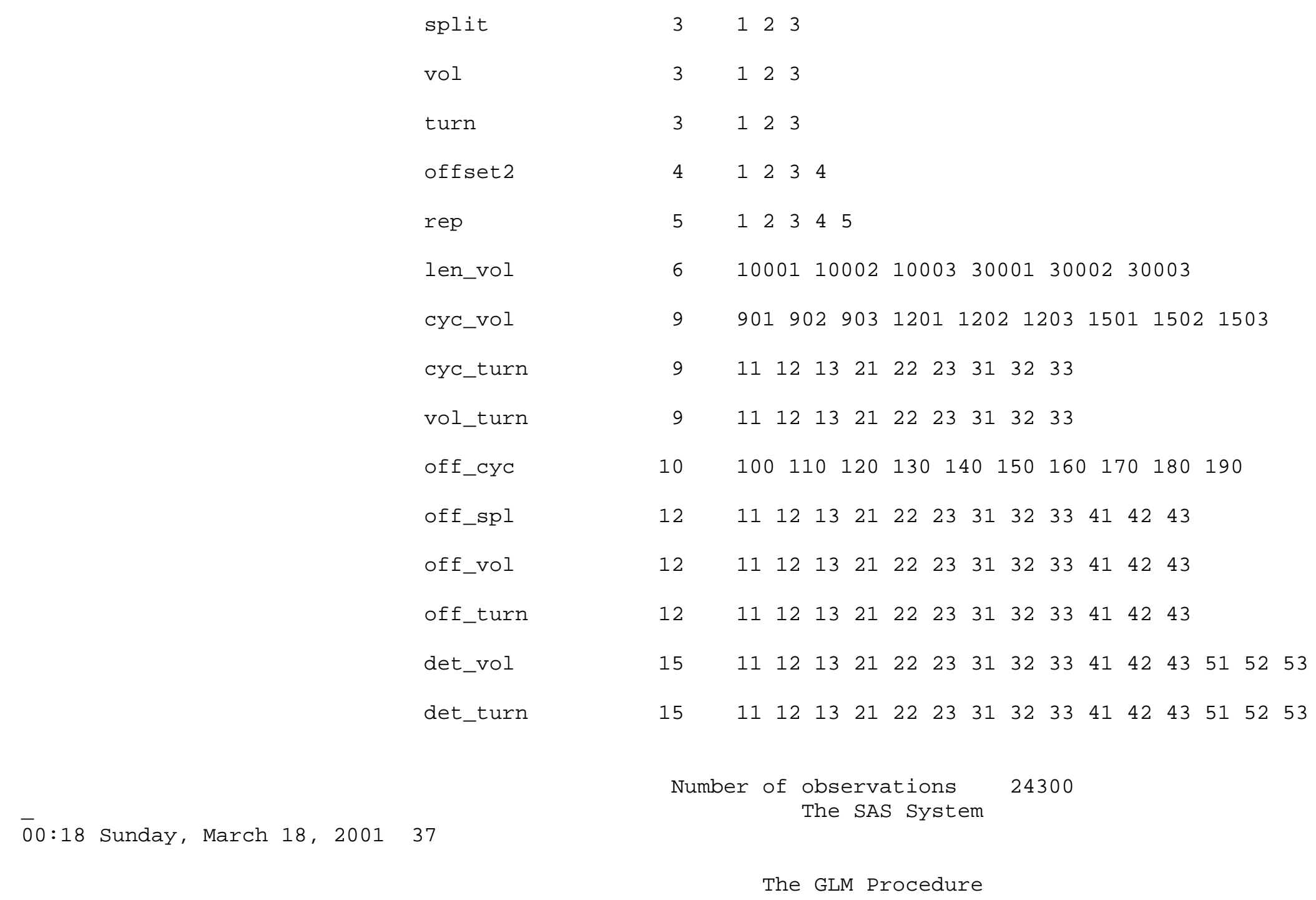


Dependent Variable: diff_skw

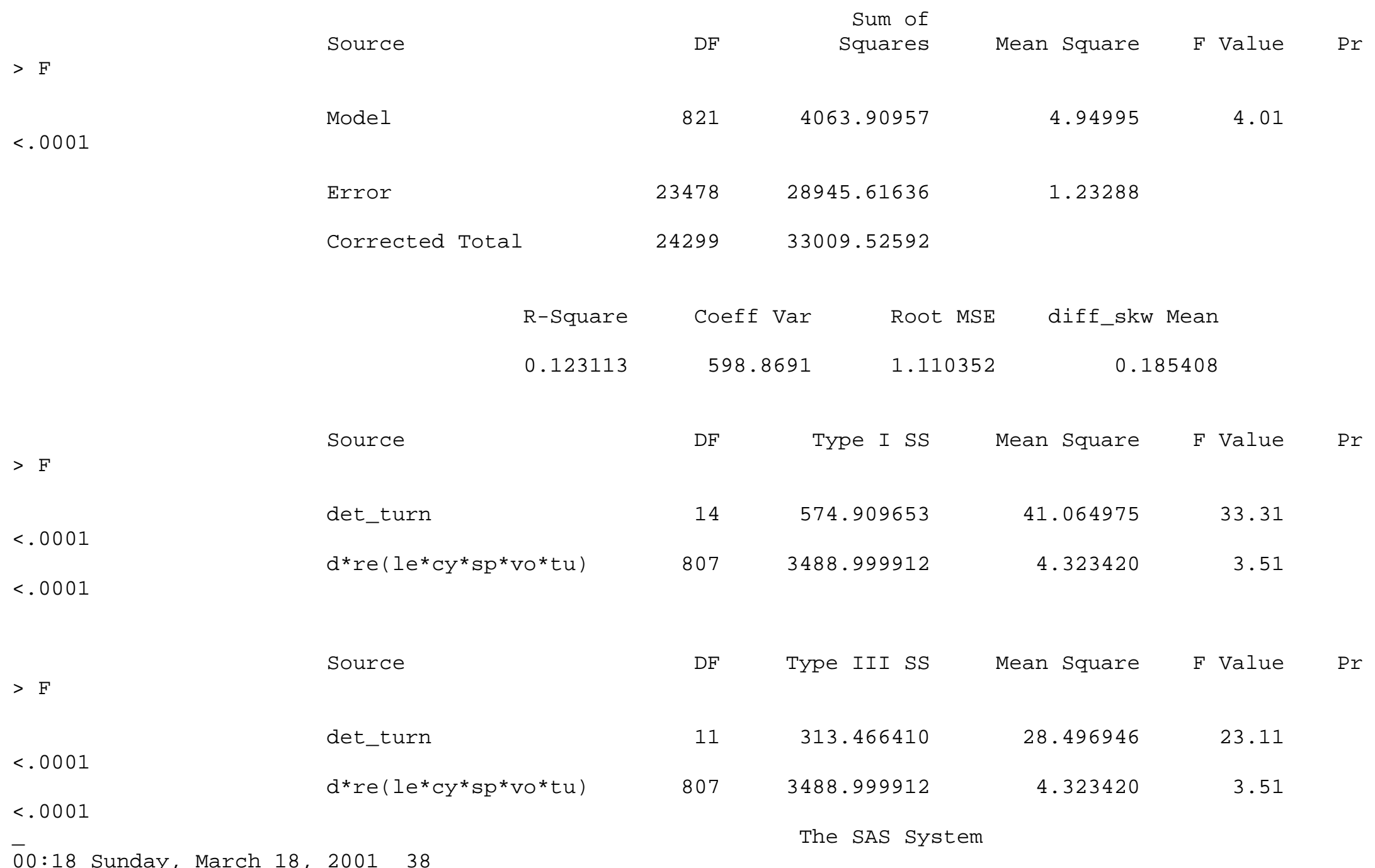


The GLM Procedure

Student-Newman-Keuls Test for diff_skw

NOTE: This test controls the Type I experimentwise error rate under the complete null hypothesis but not under partial null

hypotheses.

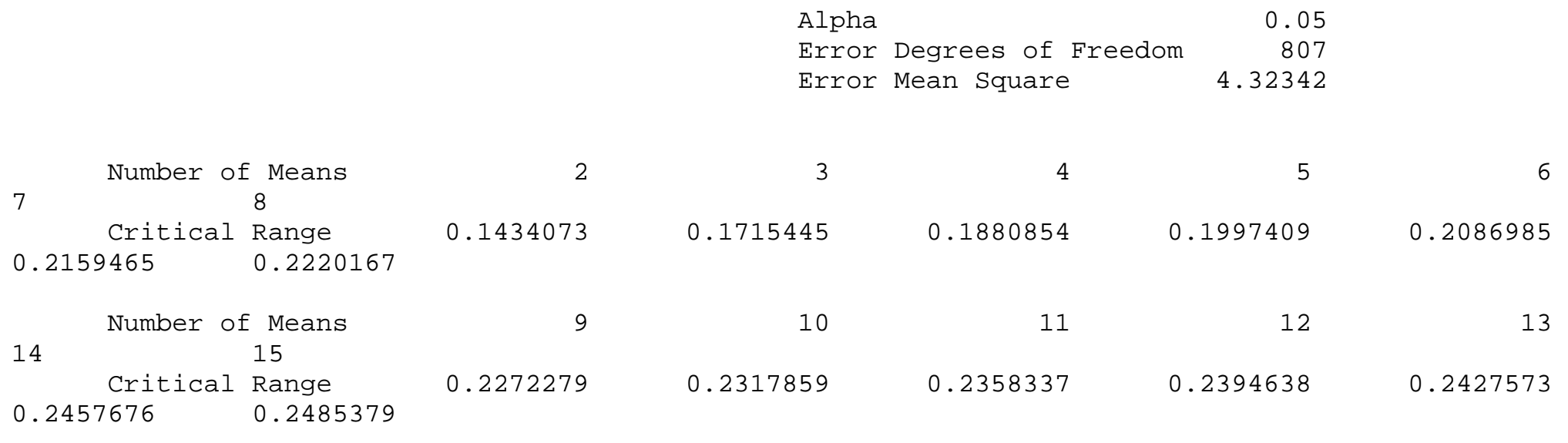

Means with the same letter are not significantly different.

\begin{tabular}{|c|c|c|c|c|c|}
\hline \multirow[t]{5}{*}{ SNK } & \multicolumn{2}{|c|}{ Grouping } & Mean & $\mathrm{N}$ & det_turn \\
\hline & $\mathrm{A}$ & & 0.43035 & 1620 & 21 \\
\hline & A & & & & \\
\hline & A & & 0.42777 & 1620 & 31 \\
\hline & $\mathrm{A}$ & & & & \\
\hline B & A & & 0.36214 & 1620 & 41 \\
\hline B & A & & & & \\
\hline B & $\mathrm{A}$ & $\mathrm{C}$ & 0.27866 & 1620 & 51 \\
\hline B & $\mathrm{A}$ & $\mathrm{C}$ & & & \\
\hline
\end{tabular}




$\begin{array}{llllll}\text { B } & \text { A } & \text { C } & 0.26282 & 1620 & 22 \\ \text { B } & \text { A } & \text { C } & & & \\ \text { B } & \text { A } & \text { C } & 0.25398 & 1620 & 32 \\ \text { B } & & \text { C } & & & \\ \text { B } & \text { D } & \text { C } & 0.19922 & 1620 & 42 \\ \text { B } & \text { D } & \text { C } & & & \\ \text { B } & \text { D } & \text { C } & 0.18693 & 1620 & 11 \\ & \text { D } & \text { C } & & & \\ & \text { D } & \text { C } & 0.14408 & 1620 & 33 \\ & \text { D } & \text { C } & & & \\ & \text { D } & \text { C } & 0.11638 & 1620 & 52 \\ & \text { D } & \text { C } & & & \\ & \text { D } & \text { C } & 0.11415 & 1620 & 23 \\ & \text { D } & \text { C } & & & \\ & \text { D } & \text { C } & 0.10188 & 1620 & 43 \\ & \text { D } & \text { C } & & & \\ & \text { D } & \text { C } & 0.06820 & 1620 & 53 \\ & \text { D } & & -0.01458 & 1620 & 12 \\ \text { E } & \text { D } & & & & \\ \text { E } & & & & & \\ \text { E } & & & & & \end{array}$

\section{Discriminant Analysis Code}

data offsets;

INFILE 'n: \offsets.inp';

input length 1-6 offset 7-12 cycle 13-18 split 19-24 turn 25-30 vol 31-36 det_loc 37-42 cnt_skw 43-50 occ_skw 51-57;

IF offset=5 THEN offset $2=3$;

ELSE IF offset $=6$ THEN offset $2=2$;

ELSE offset $2=$ offset;

IF split=1 THEN split2=0.33;

ELSE IF split=2 THEN split $2=0.5$;

ELSE split2=0.67;

run;

data ftrn 


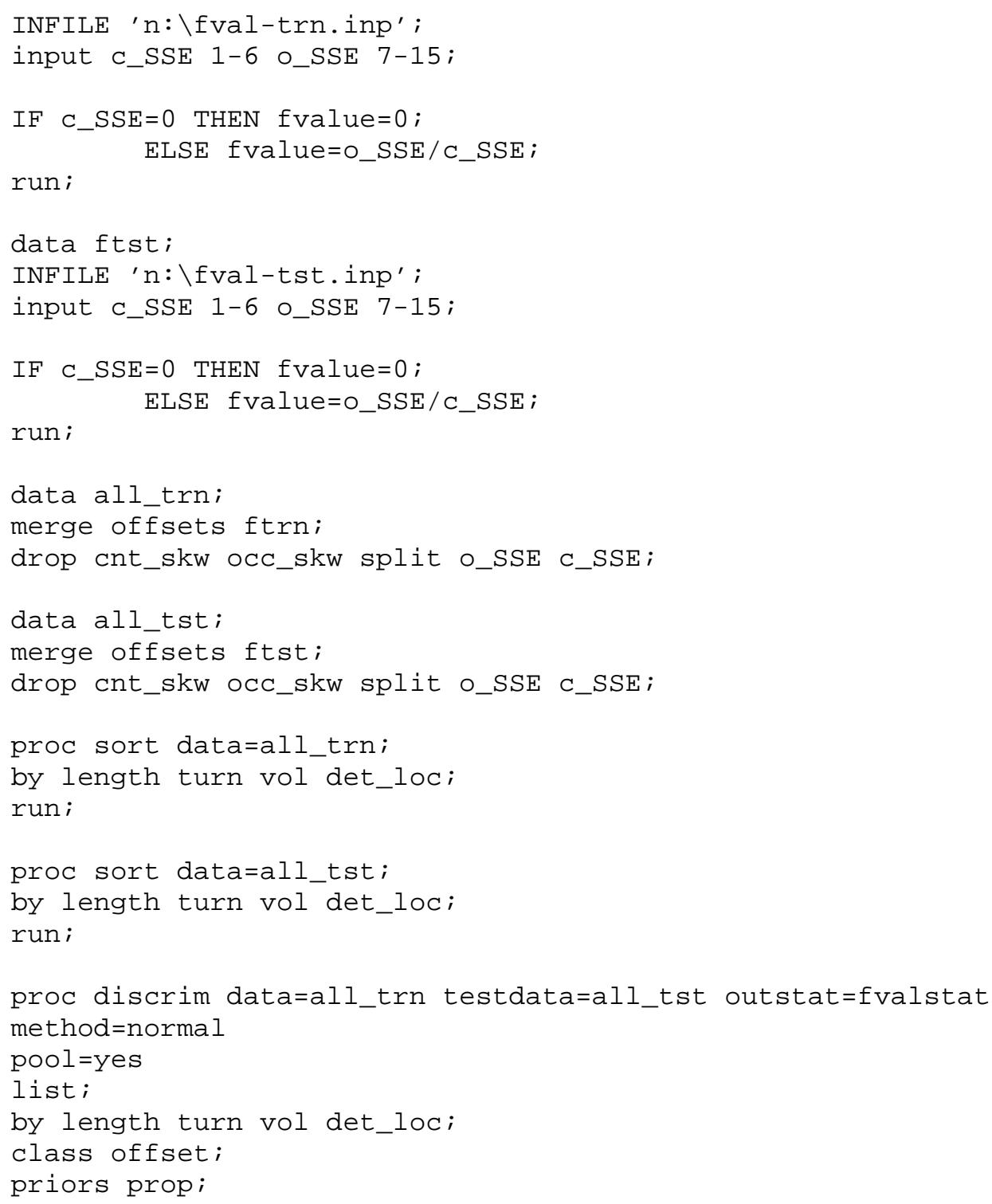


var fvalue

run;

\section{Sample Discriminant Output}

LENGTH $=1000$ TURN $=1$ VOL=1 DET LOC $=1$

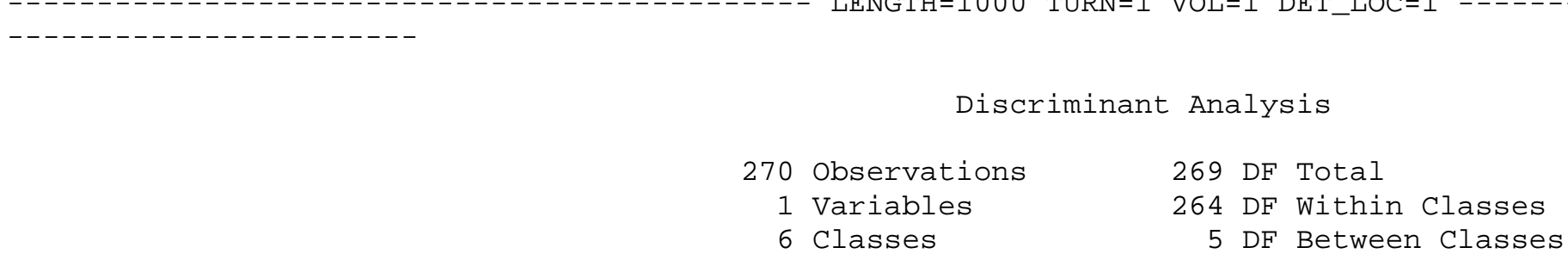

Class Level Information

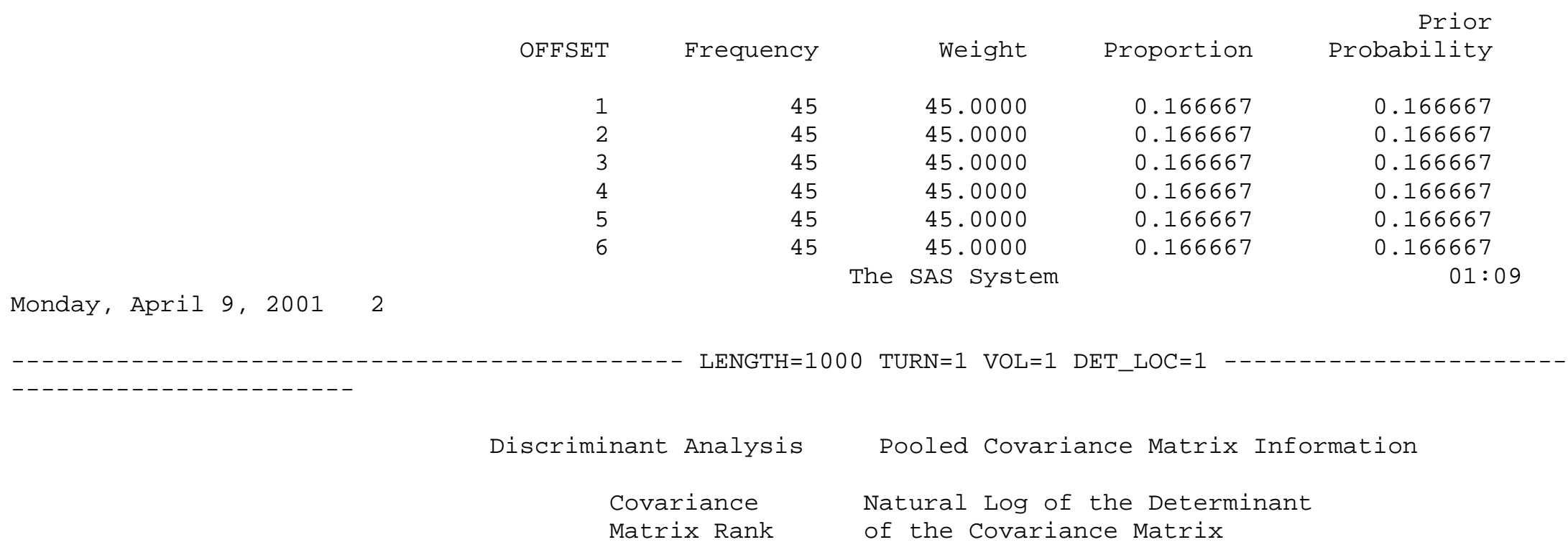




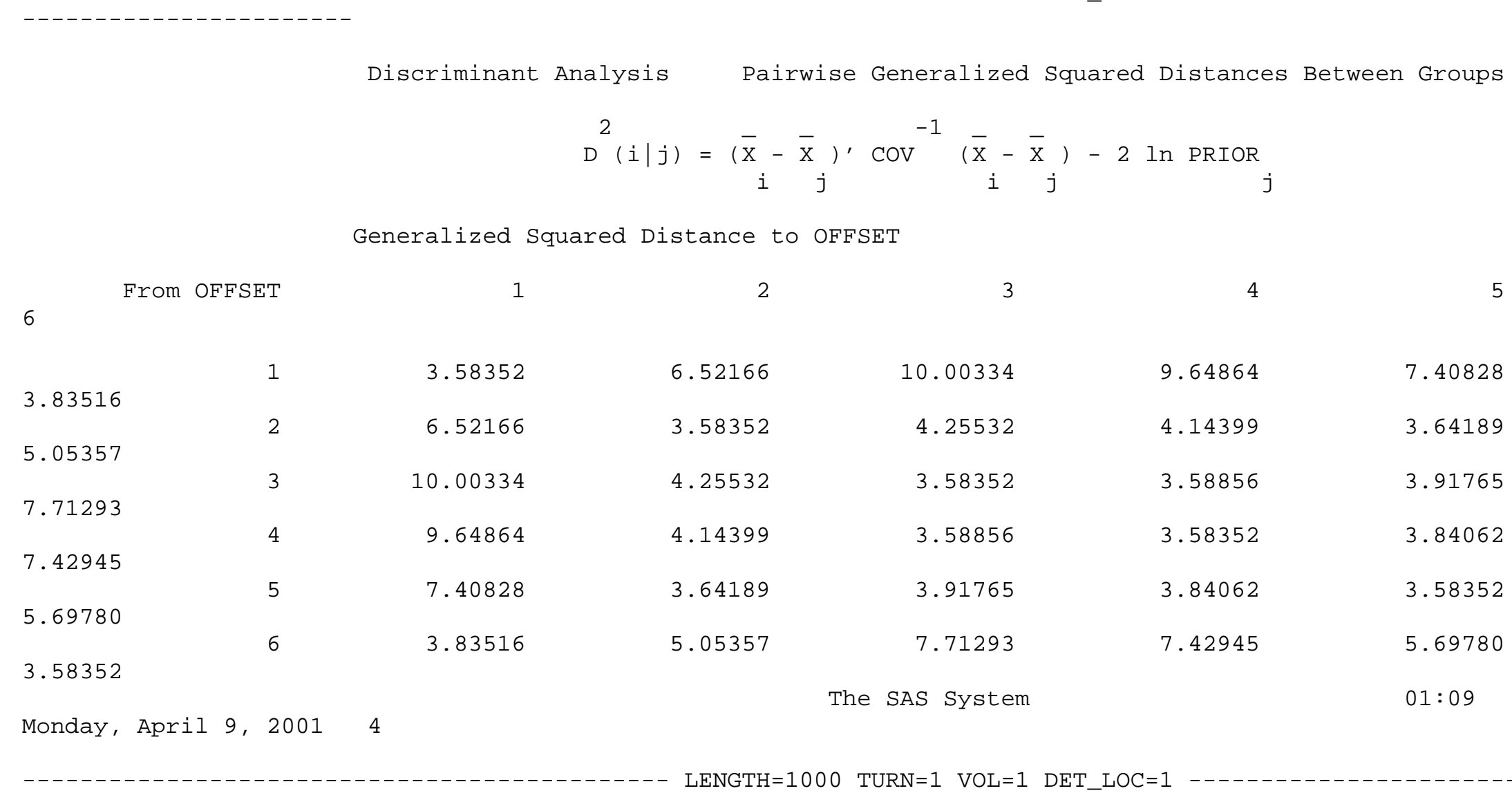

\section{Discriminant Analysis Linear Discriminant Function}

$$
\text { Constant }=-.5 \overline{\mathrm{X}}^{\prime} \operatorname{COV}^{-1} \overline{\mathrm{X}}+\ln \text { PRIOR } \quad \text { Coefficient Vector }=\operatorname{COV}^{-1} \overline{\mathrm{X}}
$$


OFFSET

$\begin{array}{cccccc}{ }^{\text {CONSTANT }} & -1.95801 & -4.41547 & -6.62893 & -6.41065 & -4.99809 \\ 3.37309 & 1.79409 & 7.12729 & 9.67748 & 9.45661 & 7.87900\end{array}$

3.35488 each OFFSET:

Generalized Squared Distance Function:

$$
\mathrm{D}^{2}(\mathrm{X})=(\mathrm{X}-\overline{\mathrm{X}})^{\prime} \mathrm{COV}^{-1}(\mathrm{X}-\overline{\mathrm{X}})-2 \ln \text { PRIOR }
$$

Posterior Probability of Membership in

(X) )

$$
\text { j j j j j }
$$$$
\operatorname{Pr}(j \mid X)=\exp \left(-.5 \mathrm{D}^{2}(\mathrm{X})\right) / \mathrm{SUM} \exp \left(-.5 \mathrm{D}^{2}\right.
$$

Number of Observations and Percent Classified into OFFSET:

From OFFSET
Total

45
1

44
2

0
3

0 


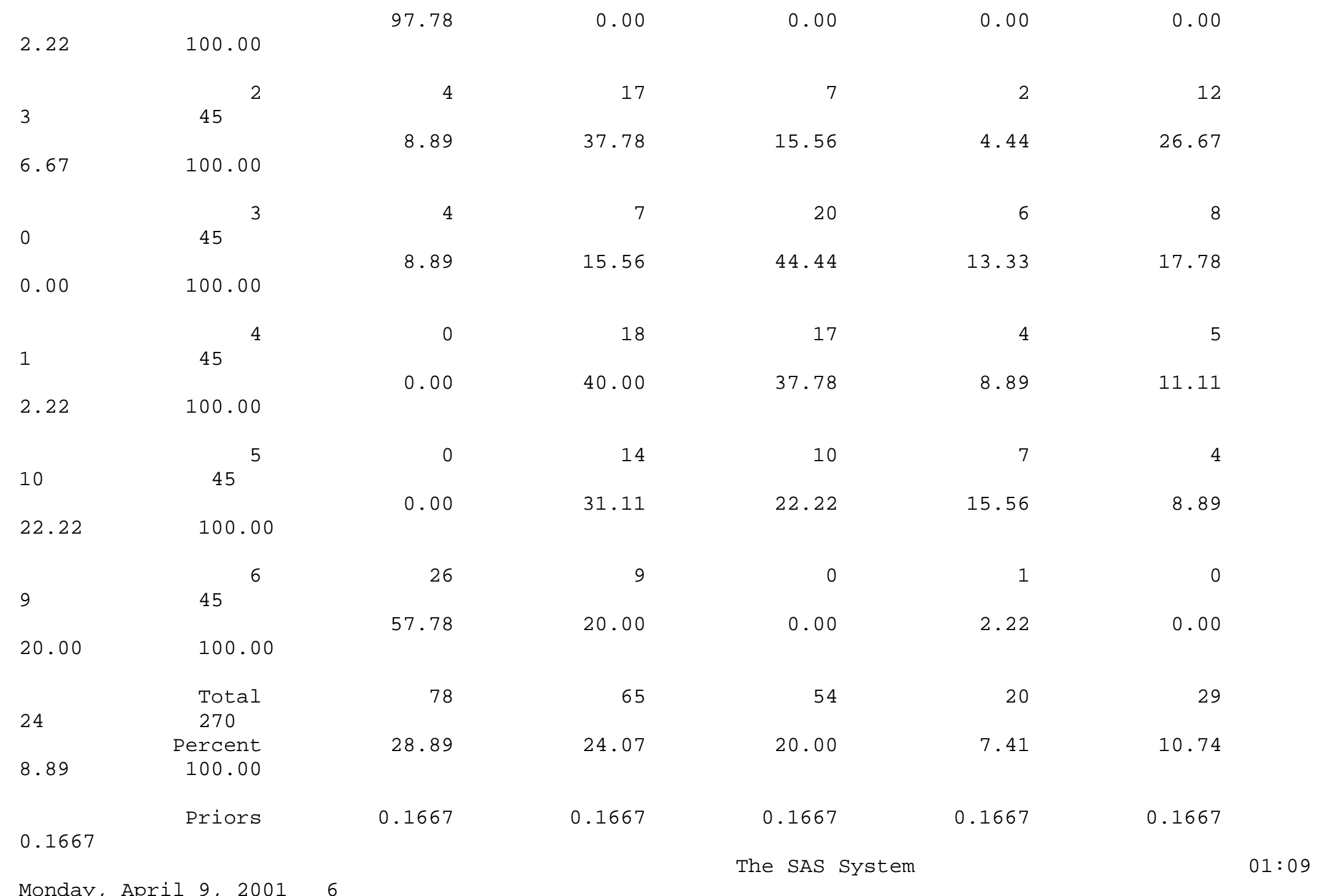




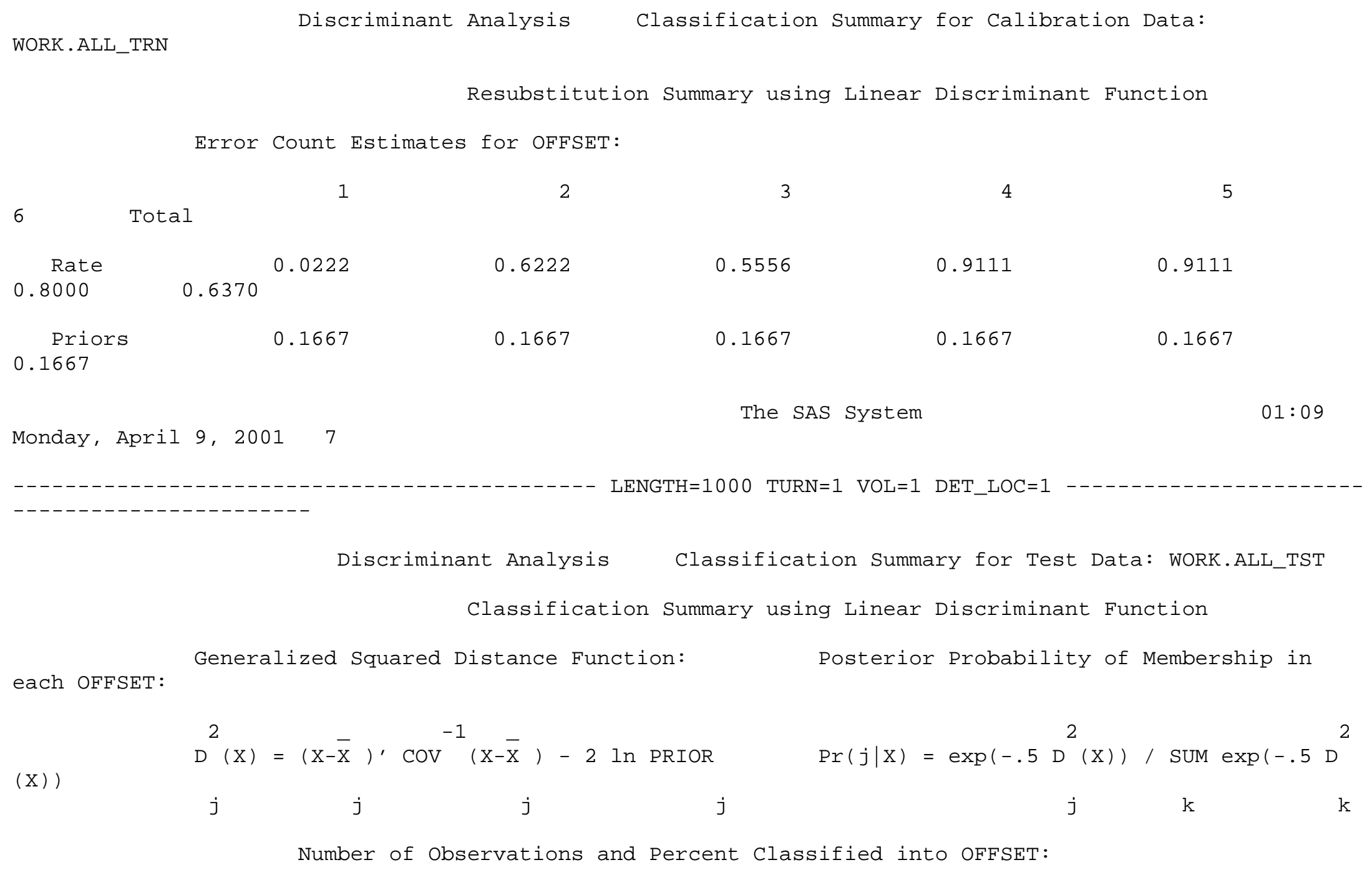




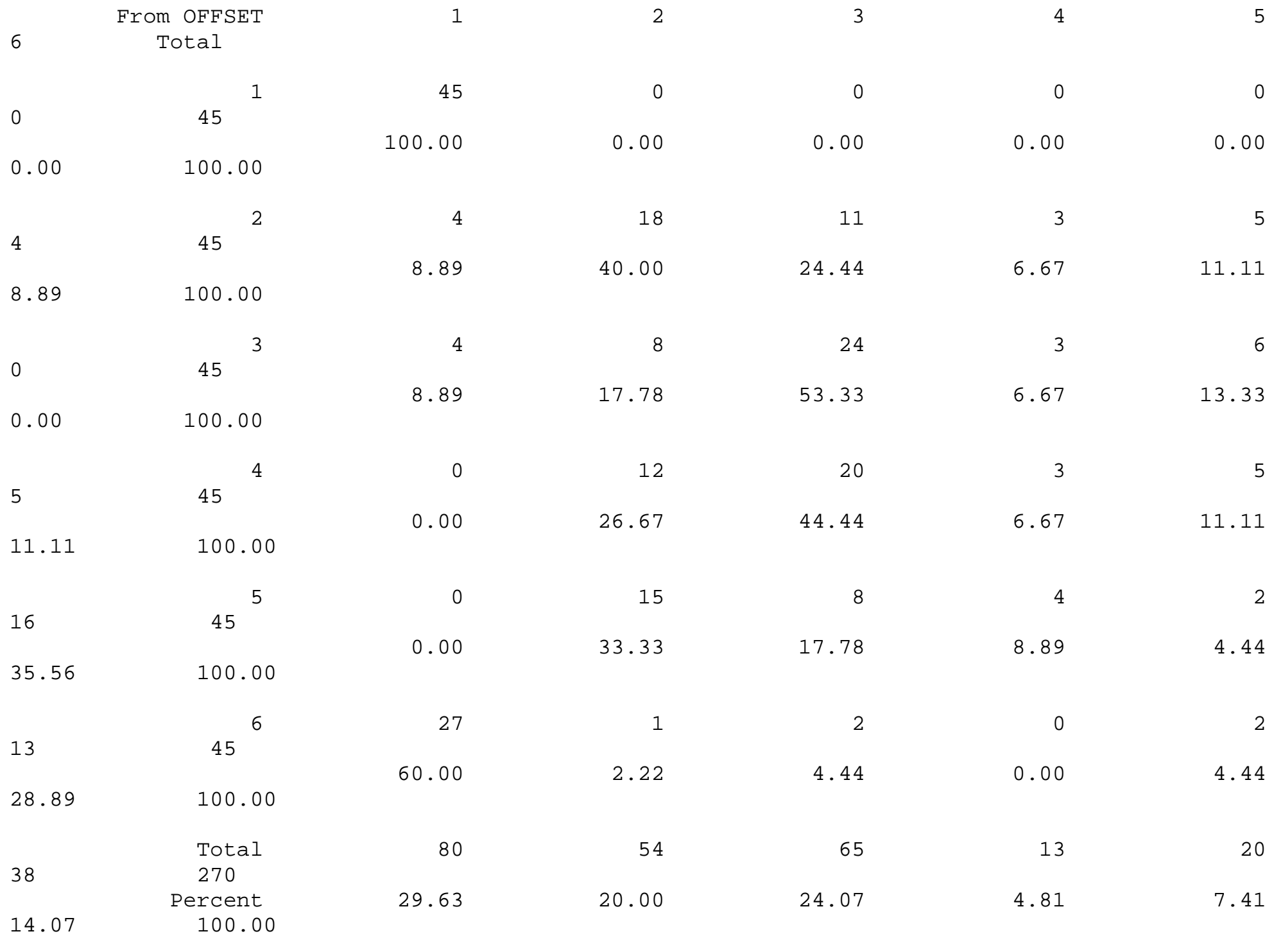




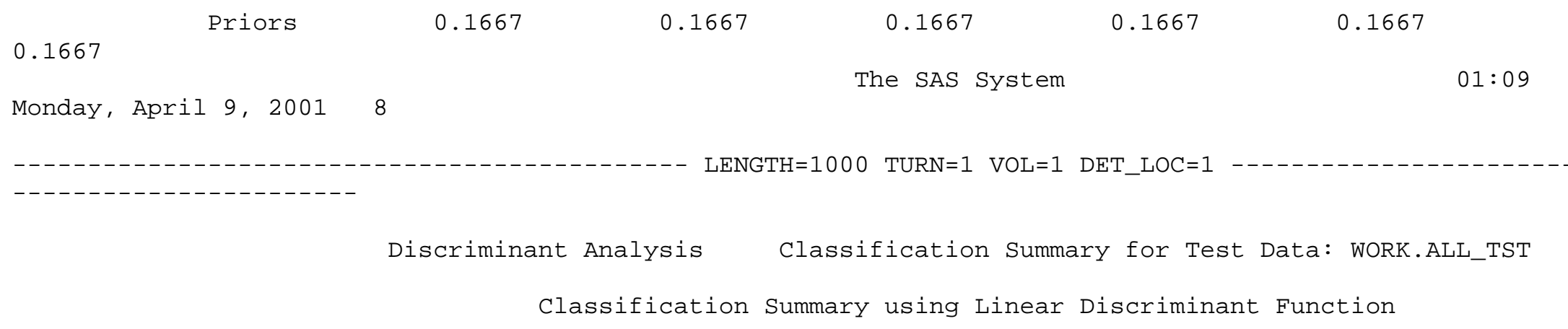

Error Count Estimates for OFFSET:

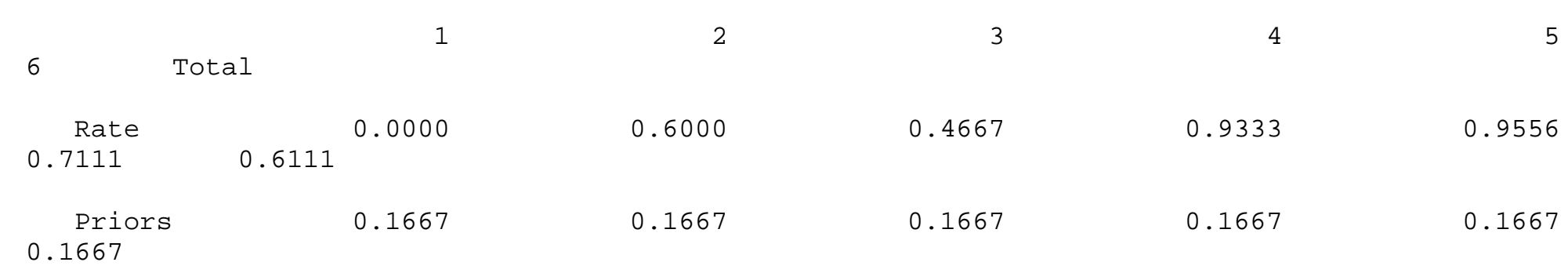


Appendix D-Glossary 
ATC: Advanced Transportation Controller

ASN: Abstract Syntax Notation

CORSIM: Corridor Simulation Program

DCE: Data Communications Equipment

DOT: Department of Transportation

DTE: Data Terminal Equipment

EMS: Expected Mean Squares

MSE: Mean Square Error

G/C ratio: Green per Cycle Ratio

HDLC: High-level Data Link Control

ICONS: Software Package for Communicating With and Managing Traffic Signal Controller in Real-Time

MOE: Measures of Effectiveness

NEMA: The National Electrical Manufacturers Association

NTCIP: National Transportation Communication for ITS Protocol

OPAC: Optimized Policies for Adaptive Control

PDU: protocol data unit

PRO-TRACTS: Purdue Real-time Offset Transitioning Algorithm for Coordinating Traffic Signals 
RHODES: Real-Time, Hierarchical, Optimized, Distributed and Effective System

SCATS: Sydney Coordinated Adaptive Traffic System

SCOOT: Split, Cycle, Offset Optimization Technique

SNMP: Simple Network Management Protocol

STMP: Simple Transportation Management Protocol

SYNCHRO: Software Package for Optimizing Cycle, Splits, and Offsets

TEA-21: Transportation Equity Act for the $21^{\text {st }}$ century

TOD: Time of Day

TRANSYT: Traffic Network Study Tool

TRP: Traffic Responsive Program

TS1: Traffic Control Systems (a NEMA standard)

TS2: Traffic Controller Assemblies with NTCIP Requirements (a NEMA standard)

UTCS: Urban Traffic Control Software 